Portland State University

PDXScholar

Fall 1-14-2020

\title{
Modeling Spatiotemporal Patterns of PM2.5 at the Sub-Neighborhood Scale Using Low-Cost Sensor
} Networks

Philip Jeffrey Orlando

Portland State University

Follow this and additional works at: https://pdxscholar.library.pdx.edu/open_access_etds

Part of the Environmental Sciences Commons

Let us know how access to this document benefits you.

\section{Recommended Citation}

Orlando, Philip Jeffrey, "Modeling Spatiotemporal Patterns of PM2.5 at the Sub-Neighborhood Scale Using Low-Cost Sensor Networks" (2020). Dissertations and Theses. Paper 5366.

https://doi.org/10.15760/etd.7239

This Thesis is brought to you for free and open access. It has been accepted for inclusion in Dissertations and Theses by an authorized administrator of PDXScholar. Please contact us if we can make this document more accessible: pdxscholar@pdx.edu. 
Modeling Spatiotemporal Patterns of $\mathrm{PM}_{2.5}$ at the Sub-Neighborhood Scale Using Low-Cost Sensor Networks

\author{
by \\ Philip Jeffrey Orlando
}

A thesis submitted in partial fulfillment of the requirements for the degree of

\author{
Master of Science \\ in \\ Environmental Science and Management
}

Thesis Committee:

Linda George, Chair

Meenakshi Rao

Kelly Gleason

Lynne Messer

Portland State University

2019 


\begin{abstract}
Epidemiological research has demonstrated an adverse relationship between fine particulate matter $\left(\mathrm{PM}_{2.5}\right)$ and human health. While $\mathrm{PM}_{2.5}$ continues to pose a signficant global health risk, there is still the need to further characterize exposures at the intra-urban scale. Land use regression is a statistical modeling technique which is used to predict air pollution concentrations at high resolution from a limited number of monitoring sites. However, the existing regulatory monitoring networks are typically not dense enough to apply these techniques. We explored the potential of using low-cost $\mathrm{PM}_{2.5}$ sensor networks to overcome the limitations of the existing regulatory monitoring infrastructure, and identified the need to determine sensor-specific correction factors based on the local $\mathrm{PM}_{2.5}$ source profile. Once calibrated, a land use regression model $\left(\mathrm{R}^{2}=0.89\right)$ was developed using the low-cost sensor network $(n \approx 20)$, alongside several land use and meteorological variables, to predict daily particulate matter concentrations at a $50 \mathrm{~m}$ spatial resolution during a two year period within Portland, Oregon. From this model, we assessed the relative strengths of expected sources and sinks of fine particulate matter, focusing specifically on the role that the urban canopy may play in mitigating $\mathrm{PM}_{2.5}$ exposure. This model showed a modest but observable spatial pattern in $\mathrm{PM}_{2.5}$, but attributed the majority of $\mathrm{PM}_{2.5}$ variation to temporal predictors (e.g. ambient background $\mathrm{PM}_{2.5}$, wind speed, temperature). Neither proxies for traffic-related sources, or vegetationrelated sinks were identified as significant predictors of $\mathrm{PM}_{2.5}$. Our research also demonstrated the importance of sensor placement, as a considerably different set of predictors was selected after the inclusion of four additional monitoring
\end{abstract}


sites. Future work will apply this method to four cities with a varying degree of canopy cover to assess differences in intra-urban gradients of $\mathrm{PM}_{2.5}$ and to further characterize the influence of vegetation. 


\section{Acknowledgements}

First of all, I would like to express my sincere gratitude to my advisor Dr. Linda George for her continuous patience, guidance, and expertise throughout my graduate study and research. I never would have imagined pursuing a graduate degree without her inspiring wisdom and dedication to science.

Besides my advisor, I would like to thank the rest of my thesis committee: Dr. Meenakshi Rao, Dr. Kelly Gleason, and Dr. Lynne Messer for their time, encouragement, and insightful feedback.

My sincere thanks also goes to the remaining members of the Canopy Continuum project: Dr. Vivek Shandas and Dr. Todd Rosenstiel for their helpful comments and support.

Another special thanks goes to Meenakshi Rao, as well as, Dr. Yangdong Pan and Jackson Voelkel for cultivating my appreciation of computer science and for teaching me how to harness it to tackle environmental problems.

I thank my fellow labmates in the Sustainable Atmospheres Research lab: Devin Wilde, Dat Vo, Kirsten Sarle, Modi Raduma, Patrick Léon Gross, and Kellen McInerney for their field and laboratory assistance.

I would like to thank my partner, Judit Takács, for her continual moral support and patience while I was often absorbed by my graduate work. 
Last but not least, I would like to thank my parents: Shannon and Jeff Orlando for their endless encouragement and support throughout my entire life. 


\section{Contents}

$\begin{array}{ll}\text { Abstract } & \text { i }\end{array}$

Acknowledgements $\quad$ iii

List of Tables viii

List of Figures $\quad$ ix

1 Introduction $\quad 1$

1.1 Background ............................ 1

1.1.1 Particulate Matter and Human Health . . . . . . . . . . . 1

1.1.2 Characterizing Particulate Matter Pollution . . . . . . . . . 2

1.1.3 Urban Air Quality and Traffic . . . . . . . . . . . . . . . . 4

1.1.4 Influence of Vegetation on Air Quality . . . . . . . . . 5

1.2 Modeling Intra-Urban $\mathrm{PM}_{2.5} \ldots \ldots \ldots$. . . . . . . . . 7

1.2.1 The Lack of Spatially Resolved $\mathrm{PM}_{2.5} \ldots \ldots$. . . . . . . . 7

1.2.2 Land Use Regression Modeling of $\mathrm{PM}_{2.5} \ldots \ldots$. . . . . . 9

1.2.3 Opportunities and Challenges of Low-Cost Sensors . . . . 12

1.3 Research Objectives . . . . . . . . . . . . . 14

2 Methodology 15

2.1 Low-Cost Sensor Selection . . . . . . . . . . . . . . . . . . 15

2.1.1 Sensor Performance . . . . . . . . . . . . . . 15

2.1.2 PurpleAir Device Ownership and Open Data Philosophy . 17

2.2 Laboratory Evaluations . . . . . . . . . . . . . . . . 17

2.3 PurpleAir Data Acquisition and Storage . . . . . . . . . . . . . . . 19

2.4 Opportunistic Ambient Calibration of PurpleAir Networks . . . . 21

2.5 Data Recovery and Sensor Lifetime . . . . . . . . . . . . . . . 23

2.6 Sensor Allocation . . . . . . . . . . . . . . . 23

2.7 Data Compilation . . . . . . . . . . . . . . . 31

2.7.1 Spatial and Temporal Predictors of $\mathrm{PM}_{2.5} \ldots \ldots 33$ 
Road and Rail Density . . . . . . . . . . . . . . . . . . 34

NDVI and EVI . . . . . . . . . . . . . . . . . 34

Population Density . . . . . . . . . . . . . . . . 36

GridMET Meteorological Variables . . . . . . . . . . . . . . 36

Land Use and Land Cover . . . . . . . . . . . . . . . 37

Planetary Boundary Layer Height . . . . . . . . . . . . . 38

Nephelometer $\mathrm{PM}_{2.5} \ldots \ldots \ldots \ldots \ldots$

Latitude and Longitude . . . . . . . . . . . . . . . . . . 39

2.7 .2 Focal Statistics . . . . . . . . . . . . . . . . . . . . . . . 39

2.7 .3 Data Extraction . . . . . . . . . . . . . . . . . . . . 41

2.8 Predictor Selection . . . . . . . . . . . . . . . . . . . . . 42

2.9 Modeling and Validation . . . . . . . . . . . . . . . 44

3 Results and Discussion $\quad 47$

3.1 Laboratory Evaluations . . . . . . . . . . . . . . . . . 47

3.2 Opportunistic Ambient Calibration . . . . . . . . . . . . . . 53

3.3 Predictor Selection . . . . . . . . . . . . . . . . . . . 59

3.4 Leave-One-Out-Cross-Validation . . . . . . . . . . . . . . 60

3.5 Model Validation . . . . . . . . . . . . . . . . . . . . 62

3.6 Standardized Regression Coefficients . . . . . . . . . . . . . . . . 69

3.6.1 Temporal Predictors . . . . . . . . . . . . . . . . 69

Nephelometer $\mathrm{PM}_{2.5} \ldots \ldots \ldots \ldots$

Planetary Boundary Layer Height . . . . . . . . . . . . 70

Season . . . . . . . . . . . . . . . 70

3.6.2 Spatiotemporal Predictors . . . . . . . . . . . . . 71

Temperature . . . . . . . . . . . . . . . . . 71

Wind Speed . . . . . . . . . . . . . . . . . . . . . . . . . 72

Relative Humidity . . . . . . . . . . . . . . . . . . . . 72

3.6.3 Spatial Predictors . . . . . . . . . . . . . . . 73

Elevation . . . . . . . . . . . . . . . . . . 73

Developed Medium Intensity LULC . . . . . . . . . . . . . 73

Longitude . . . . . . . . . . . . . . . . . . . 74

3.7 Spatial Patterns of Model Residuals . . . . . . . . . . . . . . 76

3.8 The Importance of Sensor Placement $\ldots \ldots \ldots \ldots$

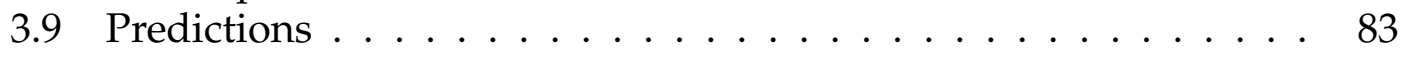

3.9.1 Land Use Regression . . . . . . . . . . . . . . . . . . . 83

3.9.2 Random Forest . . . . . . . . . . . . . . . . . . 85

3.9.3 Final Model Predictions Over Time . . . . . . . . . . . 89 
4 Summary and Future Work 92

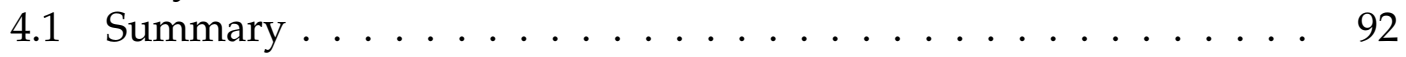

4.2 Future Work . . . . . . . . . . . . . . . . . . . . 94

$\begin{array}{ll}\text { Bibliography } & 97\end{array}$

$\begin{array}{ll}\text { Appendix A Source Code } & 117\end{array}$

Appendix B ThingSpeak PurpleAir Field Descriptions 346

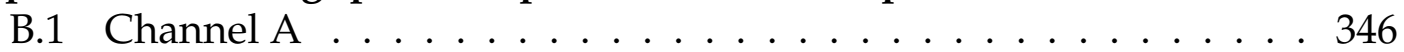

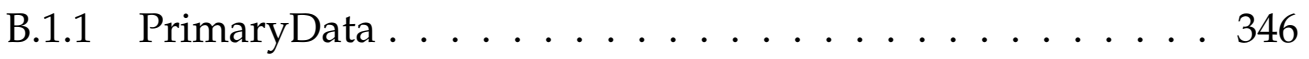

B.1.2 SecondaryData . . . . . . . . . . . . . 347

B.2 Channel B . . . . . . . . . . . . . . . 347

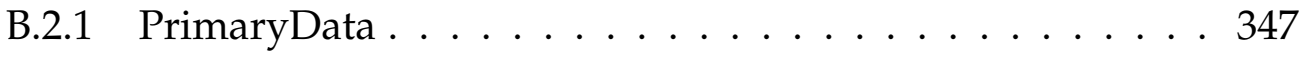

B.2.2 SecondaryData . . . . . . . . . . . . . . 348 


\section{List of Tables}

2.1 Table of Predictors . . . . . . . . . . . . . . . 46

3.1 Lab Evaluations Summary Statistics . . . . . . . . . . . . . . . . . 49

3.2 VIF Scores . . . . . . . . . . . . . . . . . . 60

3.3 LOOCV Summary Table ............................ 63

3.4 Beta Coefficients of Final Model . . . . . . . . . . . . . . . . . 75

3.5 Beta Coefficients of Additional Model . . . . . . . . . . . . . . 82 


\section{List of Figures}

2.1 PurpleAir $\mathrm{R}^{2}$ as a Function of Distance . . . . . . . . . . . . 25

2.2 Albuquerque Site Map . . . . . . . . . . . . . . . . . 27

2.3 Boise Site Map . . . . . . . . . . . . . . . . . . . . . . 28

2.4 Sacramento Site Map . . . . . . . . . . . . . . . . . . . . . . . . . . 29

2.5 Tacoma Site Map . . . . . . . . . . . . . . . . . . . . . . 30

2.6 Workflow Diagram . . . . . . . . . . . . . . . . . 33

2.7 Focal Buffers of RLIS Streets . . . . . . . . . . . . . . . . . . . 41

$3.1 \mathrm{R}^{2}$ Distributions from Lab Evaluations . . . . . . . . . . . . . . 49

3.2 Slope Distributions from Lab Evaluations . . . . . . . . . . . 50

3.3 Scatter Plot of PurpleAir Primary Sensor and DustTrak . . . . . . 51

3.4 Scatter Plot of PurpleAir Secondary Sensor and DustTrak . . . . . 52

$3.5 \mathrm{R}^{2}$ Distributions from Field Evaluations . . . . . . . . . . . . 55

3.6 Slope Distributions from Field Evaluations . . . . . . . . . . 56

3.7 Scatter Plot of PurpleAir Primary Sensor and Nephelometer . . . 57

3.8 Scatter Plot of PurpleAir Secondary Sensor and Nephelometer . . 58

3.9 LOOCV Observation Bar Plot . . . . . . . . . . . . . . . . 62

3.10 Scatter Plot Comparing Test Data and LUR Predictions . . . . . 66

3.11 LUR Test Assumptions . . . . . . . . . . . . . . . . . . . . . 67

3.12 Scatter Plot Comparing Test Data and RF Predictions . . . . . . 68

3.13 Map of LUR Standard Error . . . . . . . . . . . . . . . . 77

$3.14 \mathrm{PM}_{2.5}$ from both LUR Model Predictions . . . . . . . . . . . . 87

3.15 Scatter Plot Comparing LUR Model Predictions . . . . . . . . . 88

$3.16 \mathrm{PM}_{2.5}$ from both Random Forest Model Predictions . . . . . . . . 89

3.17 Predictions During the Wildfire Season . . . . . . . . . . . . 90

3.18 Predictions During the Winter Season . . . . . . . . . . . . 91 


\section{Chapter 1}

\section{Introduction}

\subsection{Background}

\subsubsection{Particulate Matter and Human Health}

Epidemiological studies have demonstrated an adverse relationship between ambient particulate matter (PM) exposure and human health (Kloog et al., 2013; Brunekreef and Holgate, 2002; Dockery et al., 1993). Adverse health effects from PM exposure include cardiovascular and respiratory morbidity and mortality (Le Tertre et al., 2002; Schwartz, 1994; Pope and Dockery, 2006). The Global Burden of Disease (GBD) comparative risk assessment conducted by the World Health Organization (WHO) attributed approximately 3.2 million year2010 deaths to ambient $\mathrm{PM}_{2.5}$ (PM with a diameter of $2.5 \mu m$ or smaller) exposure, and ranked this pollutant as the sixth largest overall risk factor for shortened life expectancy globally (Lim et al., 2012; Apte et al., 2015; Fann et al., 2012; Cohen et al., 2005). To provide further context, the global health burden from ambient $\mathrm{PM}_{2.5}$ is greater than the cumulative health risks of other wellestablished global health threats including HIV-AIDS and malaria which contributed 1.5 million and 1.2 million year-2010 deaths respectively (Lim et al., 
2012; Apte et al., 2015). While ambient PM continues to pose a significant global health burden, there is the need to further characterize higher granularity PM exposure gradients, specifically at the intra-urban scale. Health studies typically rely on inter-city estimates from a limited number of monitoring sites to develop a single exposure estimate for a given city. These single estimates are then assigned to an entire metropolitan area, despite the potential for intra-city heterogeneity in PM sinks and sources. This likely contributes to the underestimation of PM-related health effects in the vicinity of sources (Ross et al., 2007; Jerrett et al., 2005; Hoek et al., 2002).

\subsubsection{Characterizing Particulate Matter Pollution}

PM is a mixture of solid and liquid particles suspended in the air that vary in shape, number, chemical composition and size. PM is often described by three major size categories, the largest being coarse respirable $\mathrm{PM}$, or $\mathrm{PM}_{10}$. These are particles with a diameter less than $10 \mu \mathrm{m}$ but greater than $2.5 \mu \mathrm{m}$, and are mostly derived from biogenic or geogenic sources including the suspension or resuspension of dust, soil, windstorms, volcanoes, pollen, mold, spores, and sea salt. $\mathrm{PM}_{10}$ is also attributed to anthropogenic sources including agricultural activities and mining (Pope and Dockery, 2006). Smaller than coarse PM are fine PM, or $\mathrm{PM}_{2.5}$. These are particles that have a diameter less than or equal to $2.5 \mu \mathrm{m}$. Fine PM are derived primarily from anthropogenic sources, usually the direct result from some form of combustion. This includes vehicle emissions, wood burning, coal burning, and industrial processes (steel mills, cement plants, paper mills). In addition to primary sources of fine PM, there are also 
secondary sources, where particles form as the result of some chemical transformation in the atmosphere. Secondary fine PM is often the result of nitrogen and sulfur oxides transforming into nitrates and sulfates, or through the formation of secondary organic aerosols (SOAs) from volatile organic compound emissions (VOCs) (Pope and Dockery, 2006). Even smaller than fine PM are the ultrafine PM. While their definition appears to range slightly throughout the literature, these are typically particles which have an aerodynamic diameter less than $0.1 \mu m$ (Pope and Dockery, 2006; Oberdorster, Oberdorster, and Oberdorster, 2005; EPA, 2004). Similar to fine PM, ultrafines are primarily derived from some source of combustion. However, ultrafines have an extremely short lifetime (minutes to hours), and will rapidly coagulate or condense to form larger $\mathrm{PM}_{2.5}$ particles, which can remain suspended in the atmosphere and have much longer residence time (days to weeks). Due to these properties, ultrafine PM are often indicative of freshly-emitted PM from local sources.

The human health impacts from PM exposure have been shown to vary depending on the size of the particle. Generally, the smaller the particles, the deeper into the lungs they can infiltrate, leading to more severe health effects. While ultrafine PM may be more capable than fine PM of transferring from the lung to the blood and other parts of the body, there has been a focus on monitoring only fine and coarse PM throughout regulatory agencies due to the cost and reproducibility of ultrafine measurements. Fine PM, in contrast to larger particles, can remain suspended in the atmosphere for longer periods of time, can be breathed more deeply into the lungs, and are more capable of infiltrating indoor environments. In addition, $\mathrm{PM}_{10}$ levels throughout the US are generally 
within compliance, whereas $\mathrm{PM}_{2.5}$ remains a regulatory challenge.

\subsubsection{Urban Air Quality and Traffic}

Air pollution can vary across spatiotemporal scales (Gilliland Frank et al., 2005; Beckerman et al., 2013). Spatially, local-scale variations are the result of primary sources, whereas regional-scale variations occur from secondary reactions and transport mechanisms. The majority of temporal variation is the result of diurnal traffic patterns or meteorology (Beckerman et al., 2013). Generally, pollutants directly emitted from mobile sources (traffic-related emissions), or indirectly via photochemical reactions, still dominate the urban environment despite significant improvements in fuel, engine, and emission control technology over the last few decades (Fenger, 1999; Hoek et al., 2000; Nyberg et al., 2000). It is imperative that we characterize air pollution gradients within cities as population density and traffic emissions are high within these environments and exposure is not expected to be experienced evenly (Vardoulakis et al., 2003; Rao et al., 2014). The spatial variability of primary air pollution is higher near major intersections and busy streets within the built environment, where traffic emissions are higher and ventilation is reduced due to the local topography (Harrison, Jones, and Barrowcliffe, 2004; Vardoulakis et al., 2003). In contrast, the spatial variability of secondary pollutants is driven by meteorology and generally regional in scale. Many studies have identified an increase in respiratory and cardiovascular problems from living near major roadways (Jerrett et al., 2008; Brugge, Durant, and Rioux, 2007; Zhou and Levy, 2007). Identifying intra-urban pollution gradients will become increasingly relevant as rising rates 
of urbanization and high density development result in greater human exposure to near-road environments. Currently, roughly $80 \%$ of the US population currently live within metropolitan areas (and approximately half of the global population), and the global urban population is expected to increase to approximately 68\% by the year 2050 (Bureau, 2012; United Nations, 2018).

A meta-analysis conducted by Karner, Eisinger, and Niemeier, 2010 found that many air pollutants within cities have strong pollution gradients, decaying within $150 \mathrm{~m}$ - $200 \mathrm{~m}$ from the source, and reaching background levels between 200 m - 1000 m (Karner, Eisinger, and Niemeier, 2010). These fine-scale gradients from road sources are well documented for $\mathrm{NO}, \mathrm{NO}_{2}, \mathrm{PNC}, \mathrm{CO}$, and $\mathrm{PM}_{10}$ (Karner, Eisinger, and Niemeier, 2010; Rao et al., 2014; Zhou and Levy, 2007). However, patterns in $\mathrm{PM}_{2.5}$ mass concentration as distance from road sources increases are less pronounced. While $\mathrm{PM}_{2.5}$ is a component of traffic-related emissions, mass-based measurements do not always capture this influence. The spatial patterns of $\mathrm{PM}_{2.5}$ are mixed, either decreasing very gradually from traffic sources, or showing no clear trend at all (Karner, Eisinger, and Niemeier, 2010; Kendrick, Koonce, and George, 2015).

\subsubsection{Influence of Vegetation on Air Quality}

There is an increasing body of evidence that suggests that access to greenery can improve human health. A pioneering study conducted by Ulrich showed that hospital patients recovering from surgery were discharged sooner and required less pain medication if they had a view of greenspace from their hospital bed. This was in comparison to patients whose hospital room window 
only provided a view of a brick wall (Ulrich, 1984). Subsequent studies have shown that access to greenspace is associated with reduced morbidity (Maas et al., 2009), mortality (Mitchell and Popham, 2007), and lower obesity rates (Bell, Wilson, and Liu, 2008). In addition, recent studies have shown an association between urban greenery (specifically trees) and air quality, suggesting that the urban canopy has a small yet significant effect on reducing pollutants including $\mathrm{NO}_{2}, \mathrm{PM}_{10}, \mathrm{O}_{3}$, and $\mathrm{SO}_{2}$ (Donovan et al., 2011; Rao et al., 2014; Nowak, Crane, and Stevens, 2006). While some studies found that vegetation may only improve the average air quality by $<1-2 \%$, this statistic accounts for multiple pollutants, and the actual magnitude of pollution mitigation can be substantial, typically on the order of $10^{2}-10^{3}$ metric tons per year in a given city (Nowak, Crane, and Stevens, 2006). Even when focusing on just a single pollutant, for example $\mathrm{NO}_{2}$, a recent study in Portland, Oregon attributed pollution removal by trees to providing a $\$ 7$ million USD annual benefit in reduced occurrences of respiratory complications (Rao et al., 2014). Given that trees are a fundamental component of the urban environment, with a $35 \%$ average tree cover in US cities (Nowak and Greenfield, 2012), and that rates of urbanization are expected to rapidly increase over the next several decades, it is crucial that we continue to assess the potential role the urban canopy may play in reducing even more harmful air pollutants such as $\mathrm{PM}_{2.5}$.

Trees can affect atmospheric PM concentrations by removal (Beckett, FreerSmith, and Taylor, 2000) and emission (e.g. pollen). Particles that physically deposit on the leaf surface can also be resuspended, often by precipitation or when leaves, twigs, and branches fall to the ground. While some of the particles 
can be absorbed into the tree, most are confined to the plant surface and are ultimately resuspended. As a result, trees are considered only temporary sinks for many atmospheric particles (Nowak et al., 2013). Most of the studies related to PM and urban trees have focused on $\mathrm{PM}_{10}$ (Nowak et al., 2013), and while some studies have evaluated the removal rate and suspension of $\mathrm{PM}_{2.5}$ by specific tree species (Beckett, Freer-Smith, and Taylor, 2000; Freer-Smith, El-Khatib, and Taylor, 2004; Freer-Smith, Beckett, and Taylor, 2005; Pullman, 2008), few have estimated the effect of trees on $\mathrm{PM}_{2.5}$ concentrations at the city-scale (Nowak et al., 2013). There is some evidence suggesting $\mathrm{PM}_{2.5}$ mitigation by urban trees is considerably lower relative to $\mathrm{PM}_{10}$ in terms of mass, but the health benefits are significantly higher (Nowak, Crane, and Stevens, 2006; Nowak et al., 2013). The benefits the urban canopy provides with $\mathrm{PM}_{2.5}$ removal is still not well understood, and only a limited number of studies have attempted to evaluate this effect at the intra-urban scale (Nowak et al., 2013; Jeanjean, Monks, and Leigh, 2016; Tallis et al., 2011). This is due, in part, to the challenges inherent to creating spatially resolved $\mathrm{PM}_{2.5}$ exposures surfaces at this resolution.

\subsection{Modeling Intra-Urban $\mathbf{P M}_{2.5}$}

\subsubsection{The Lack of Spatially Resolved $\mathbf{P M}_{2.5}$}

The lack of spatially resolved $\mathrm{PM}_{2.5}$ at the city-scale is mostly due to the practical constraints of air quality monitoring networks. Due to the high cost, cumbersome design, and the skilled labor required to establish and maintain researchgrade air quality instrumentation, the current distribution of monitoring stations is confined to a limited number of near-road and urban background sites 
within a given city (Vardoulakis, Solazzo, and Lumbreras, 2011). For example, within our Portland, Oregon study area, approximately 4-6 $\mathrm{PM}_{2.5}$ monitoring stations were operational during the two year study period. This network, maintained by Oregon Department of Environmental Quality (ODEQ) consisted of a couple rural ambient background sites, urban ambient background sites, and a single near-road site. Reference monitoring stations, typically maintained by local or regional air quality management agencies, are primarily established to assess regulatory compliance and develop a regional air quality index. However, the use of these monitors in epidemiological research, specifically their use in modeling spatially resolved PM surfaces at sub-neighborhood scales (0.01 $1.0 \mathrm{~km}$ ), is largely an afterthought. This focus on compliance ultimately results in the potential lack of representativeness of fixed air quality monitoring sites in epidemiological studies. In order to account for the spatial heterogeneity of air pollution sources in urban areas, several modeling techniques have been developed to spatially interpolate $\mathrm{PM}_{2.5}$ in order to better estimate the human health effects from $\mathrm{PM}_{2.5}$ exposure (Vardoulakis, Solazzo, and Lumbreras, 2011). These include land use regression (LUR) and dispersion modeling techniques (Briggs et al., 2000; Vardoulakis, Solazzo, and Lumbreras, 2011; Hoek et al., 2008; Zhou and Levy, 2008). For the purpose of this study, we applied LUR techniques to develop spatially resolved $\mathrm{PM}_{2.5}$ exposures necessary for assessing the relative power of land use sources and sinks, with a focus on the influence of vegetation. 


\subsubsection{Land Use Regression Modeling of $\mathbf{P M}_{2.5}$}

Land use regression (LUR) is a statistical modeling approach which is used to spatially predict continuous air pollution concentrations at high resolution from a limited number of monitoring sites (Briggs et al., 2000; Rao et al., 2014). Landuse and land-cover (LULC) variables are extracted within an appropriate buffer distance from each monitoring location using spatial analysis software. A multiple linear regression model is then developed using the monitored air pollution data as a response variable. The model is then assessed to see how well it meets its test assumptions. These assumptions include (1) a linear relationship exists between the response variable and predictors, (2) model residuals are normally distributed, (3) variation of observations around the line of best fit is constant (homoscedasicity), (4) and strong multicollinearity among predictors is not observed. This method is rooted in the principles that the environmental conditions for the air pollutant response variable of interest can be determined from a limited number of readily available predictors; and that the relationship between the response variable and predictors can be evaluated using a small sample of 'training' data (Briggs et al., 2000). After validation, the LUR model can be used to make spatial predictions of air pollution in between and beyond the original monitoring network used during the training phase. Briefly, LUR models of air pollutants typically rely on predictors including population density, land use, and traffic-related variables to characterize mean pollutant concentrations within several buffer distances at specific monitoring sites. Typical buffer sizes range between 50 m - 3000 m (Eeftens et al., 2012; Jerrett et al., 2007; Rao et al., 2014; Liu et al., 2016). Once the model performance has been evaluated, this 
technique can be used to make air quality predictions without the presence of a monitoring station, essentially extrapolating pollutant exposure throughout the remaining study area by relying on the patterns observed within the predictor variables.

While land use regression models are capable of providing spatially resolved air pollutant surfaces at fine scales, they still rely on observational data from a limited number of monitoring sites for model development, calibration, and validation (Jerrett et al., 2005; Vardoulakis, Solazzo, and Lumbreras, 2011). As a result, the performance of LUR models depends on monitoring locations which provide a representative sample for the remaining study area that is to be predicted. In addition, regression mapping techniques are based on assumptions regarding their predictors, or independent variables.

Numerous LUR studies have been conducted to assess fine-scale spatially resolved $\mathrm{NO}_{2}$ exposures because low-cost passive sampler technology has enabled the deployment of high-density monitoring networks (Rao et al., 2014; Briggs et al., 2000; Eeftens et al., 2012; Hoek et al., 2008). In contrast, LUR models for particulate matter are less numerous because they necessitate a more intensive monitoring campaign, despite evidence suggesting that adverse health effects are tied more closely to PM exposure than nitrogen oxides (Eeftens et al., 2012; Hoek et al., 2008; Pope et al., 2002; Sarnat et al., 2001). The ESCAPE Project (European Study of Cohorts for Air Pollution Effects), a pioneering LUR study focusing on $\mathrm{PM}_{2.5}$, produced one of the largest spatially resolved PM databases in Europe, and relied on a total of 20 - 40 monitoring sites within 
each of the 20 European cities examined (Eeftens et al., 2012). However, existing regulatory monitoring networks throughout our five US cities of interest within this study are not dense enough to develop LUR models predicting intraurban $\mathrm{PM}_{2.5}$ (e.g. Portland $\mathrm{n}=4$, Boise $\mathrm{n}=2$, Albuquerque $\mathrm{n}=5$, Tacoma $\mathrm{n}=2$, and Sacramento $\mathrm{n}=7$ ) (according to the EPA's AirData Active $\mathrm{PM}_{2.5}$ Map) (US EPA, 2016). Due to the aforementioned limitations of deploying high-density networks with research-grade monitoring equipment, we opted to explore the potential of commercially available low-cost $\mathrm{PM}_{2.5}$ sensors to achieve the recommended network density of $\geq 20$ per city.

Previously developed LUR models of $\mathrm{PM}_{2.5}$ within the ESCAPE project have shown moderate to good explained variance, ranging from $R^{2}=35 \%$, to $R^{2}=$ $94 \%$, with a median of $R^{2}=71 \%$ (Eeftens et al., 2012). This range in variance explained is partially due to limited availability of relevant GIS predictor variables across cities. As a result, no single model could be applied to each city. Instead, area-specific predictors were selected for each city's model due to these differences in available predictors. Better performing models were observed to have local traffic data available, specifically traffic intensity, and models without local or limited traffic data performed below the median variance explained (71\%). Traffic variables were selected in 18 of the 20 models developed. Less important predictors included residential land use, population density, industrial land use and ports, and natural land use (Eeftens et al., 2012). The differences between final model $\mathrm{R}^{2}$ and Leave-One-Out Cross-Validation (LOOCV) $\mathrm{R}^{2}$ was less than $15 \%$ for most models, indicating overall model stability (Eeftens et al., 2012). 


\subsubsection{Opportunities and Challenges of Low-Cost Sensors}

Optical Particle Counters (OPCs), also referred to as photometers, are commonly used for particle monitoring because of their ability to provide continuous data, ease of operation, small size, and relatively low cost. This is in contrast to gravimetric techniques which, despite being the most accurate form of measurement, do not provide continuous data and are costly to routinely operate and maintain. Briefly, gravimetric PM samplers actively pull an air sample at a constant flow rate through a series of impactors to collect size-selected PM (e.g. $\mathrm{PM}_{2.5}$ or $\mathrm{PM}_{10}$ ) on a filter paper over a sample period of $\geq 24$ hours. Once a measurable amount of $\mathrm{PM}_{2.5}$ has been collected after this sample period, the filters are processed and weighed in a lab. The total $\mathrm{PM}_{2.5}$ mass is divided by the total volume of air that was sampled throughout the entire sample interval to determine a mean mass density (e.g. $10 \mathrm{\mu gm}^{-3}$ ). This method requires a technician to routinely install and collect filters at the beginning and end of each sample period. In contrast to collecting and weighing PM samples, OPCs rely on a laser beam and light receptor (usually a photodiode) to measure the light scattered from an individual illuminated aerosol particle due to Mie scattering (Chun et al., 2001; Kim, 2004; Kohli and Mittal, 2015). The particle number concentration (PNC) is then determined by counting the number of scattered light pulses within a specific time interval. The particle diameter is also determined by measuring the height of these light pulses. The PNC is typically discretized into regulatory or epidemiologically relevant size bins (e.g. $\mathrm{PM}_{2.5}$, $\mathrm{PM}_{10}$ ). Once discretized, PNC can be used to approximate PM mass density, but these standalone values should be interpreted carefully. OPCs cannot be used to 
determine aerosol type and some concessions must be made before PNC can be converted into a mass density measurement. For example, OPCs are not able to account for several aerosol properties, including particle density, shape, refractive index, and absorption. As a result, assumptions are made regarding each of these properties, as OPCs report light-scattering equivalent diameters instead of the true physical diameter and mass density (Kobayashi et al., 2014; Kohli and Mittal, 2015). Examples of some of these assumptions include aerosol particles having an aerodynamic diameter (particles are assumed to be spherical), with a constant density of $1 \mathrm{~g} \mathrm{~cm}^{-3}$ (density of water), and a constant refractive index or absorption. As a result, OPCs may be more adept at approximating PM mass of certain sources than others, which often depends on source of PM used during the factory calibration. This information is often not disclosed by OPC manufacturers, as the exact algorithms used to convert PNC to particle mass are proprietary.

Despite the challenges of using OPCs to derive $\mathrm{PM}_{2.5}$ mass density, the potential of these low-cost sensor networks in producing spatially resolved exposure maps at finer scales relative to the existing monitoring infrastructure is promising. If individual correction factors can be established which correspond to the ambient $\mathrm{PM}_{2.5}$ source profile of a specific urban area, then low-cost sensor networks may provide an advantage over traditional monitoring techniques for LUR modeling. 


\subsection{Research Objectives}

This thesis focuses on the development of (a) an opportunistic ambient calibration method for correcting low-cost $\mathrm{PM}_{2.5}$ sensor networks in order to use them for intra-urban modeling and (b) a LUR modeling technique which relies on this corrected sensor network data to predict spatially resolved $\mathrm{PM}_{2.5}$ throughout Portland, Oregon in order to assess the drivers of $\mathrm{PM}_{2.5}$ intra-urban variability, with focus on vegetation. This thesis is part of a larger epidemiological study entitled the Canopy Continuum Project, funded by the USFS. The method developed within this thesis will be subsequently applied to four additional cities within the USFS Canopy Continuum study (Albuquerque, New Mexico, Boise, Idaho, Sacramento, California, and Tacoma, Washington).

The analytical goal of this study is to develop a predictive model to produce spatially resolved $\mathrm{PM}_{2.5}$ surfaces at a spatial resolution of $50 \mathrm{~m}$ and a daily tem-

poral resolution throughout a 2 year period. These fine-scale exposures will be developed for later use in an epidemiological study which characterizes the effects of several environmental stressors, including urban heat, $\mathrm{NO}_{2}$, and $\mathrm{PM}_{2.5}$ on prenatal health, and assesses the extent the urban canopy may play in helping mitigate prenatal exposure to these environmental stressors. 


\section{Chapter 2}

\section{Methodology}

\subsection{Low-Cost Sensor Selection}

\subsubsection{Sensor Performance}

We selected the PurpleAir-II SD (PurpleAir) for this study due to their previously demonstrated performance and relatively low-cost (\$250). The South Coast Air Quality Management District's (SCAQMD) Air Quality Sensor Performance Evaluation Center (AQ-SPEC) has created both lab and field evaluation reports comparing several PurpleAir monitors against Federal Equivalent Methods (FEM). They observed good correlations with FEMs during lab ( $\mathrm{R}^{2}$ $\approx 0.99)$ and field $\left(\mathrm{R}^{2} \approx 0.95\right)$ evaluations of $\mathrm{PM}_{1}$ and $\mathrm{PM}_{2.5}$ (AQ-SPEC South Coast AQMD, 2017b; AQ-SPEC South Coast AQMD, 2017a). AQ-SPEC also observed moderate to good accuracy within the $0-250 \mathrm{\mu gm}^{-3}$ range from the PurpleAir monitors across multiple temperature and relative humidity profiles. The PurpleAir monitors outperformed competing sensors that cost 2-4 times more (MetOne E-Sampler, Alphasense OPC-N2, Dylos). The low-cost and good accuracy of the PurpleAir monitors allowed us to installed a higher density network in each of the five cities. The PurpleAir monitors are also WiFi capable, 
and transmit data to the ThingSpeak IoT cloud platform every minute. This enabled the real-time monitoring of the network via a Python script which notified us when a specific sensor lost WiFi connection or power, and data collection was interrupted (Appendix A: purpleair_watchdog.py). We could then notify the appropriate volunteer monitor hosts to troubleshoot the device and establish a connection, improving long-term data recovery. The PurpleAir-II SD model also includes local storage in the form of a micro-SD card, providing a backup if the device loses connection to its WiFi network, or was not available at a specific location altogether. While this appeared to be a promising feature, the current firmware version (2.50i) of the PurpleAir-II SD had issues with data logging, and the microcontroller would intermittently lose connection with the SD card module. This produced data gaps and error messages were logged within the main data file, creating further data processing issues during analysis. Upon this discovery, we modified our sensor hosting requirements to require a WiFi connection to avoid local storage issues.

Each PurpleAir monitor contains two Plantower PMS5003 sensors. These are low-cost sensors (\$20-\$40) that approximate PM mass using an optical scattering technique. The sensors record PM mass density concentrations for 1.0 $\mu \mathrm{m}, 2.5 \mu \mathrm{m}, 5.0 \mu \mathrm{m}$, and $10 \mu \mathrm{m}$ size categories using a proprietary algorithm and come pre-calibrated from factory. The PMS5003 has additional fields that report number density concentrations of particles ranging in size from 0.3 to 10.0 $\mu m$. In addition to the two Plantower PMS5003 sensors, the PurpleAir monitors contain a Arduino ESP8266 microcontroller for wireless data communication and logging, a BME280 pressure, temperature, and humidity sensor, an SD card 
reader, and a real time clock to keep time while disconnected from the main power supply. The devices also have an IP68 waterproof rating, and are designed for long-term outdoor use.

\subsubsection{PurpleAir Device Ownership and Open Data Philosophy}

PurpleAir upholds an open data philosophy that is not matched by competing low-cost air quality sensor manufacturers. Many sensor companies consider data as a service, renting monitors and providing data access via monthly subscription (AirAdvice, Apis Inc, MetOne, Alphasense OPC-N2, etc.). While this may benefit the use-cases of some consumers (device troubleshooting/maintenance is often included within this service), this was disadvantageous for our research purposes. In contrast, any data collected from PurpleAir monitors is freely available to all users, independent of device ownership. Data from third party monitors are accessible via their application program interface (API). After field calibration, this allowed us to incorporate data from additional third party PurpleAir monitors into our existing network.

\subsection{Laboratory Evaluations}

We conducted laboratory evaluations of 65 PurpleAir monitors within a $25{ }^{\circ} \mathrm{C}$ temperature controlled chamber at ambient humidity, and measured their response to multiple PM sources. The low-cost PurpleAir monitors were compared against a TSI DustTrak DRX 8433 Aerosol Monitor (also an optical scattering instrument). PurpleAir monitors were exposed to PM generated from smoke generated from lit matches, and smoke created by snuffing a candle 
within the test chamber. Only one specific PM source was evaluated during a given test. Approximately 8-10 PurpleAir monitors were suspended from the lid of the $160 \mathrm{~L}$ stainless steel test chamber using baling wire. The DustTrak reference monitor was placed on the floor of the chamber to ensure adequate ventilation between itself and the sensors hanging above. During the candle and match smoke tests, a $100 \mathrm{~mL}$ beaker was placed on the floor of the chamber, opposite to the sampling inlet of the DustTrak monitor. A match or candle was lit and snuffed inside of the beaker and the chamber lid was immediately closed. The chamber would rapidly saturate with PM well above the maximum effective range reported by the low-cost sensor $\left(500 \mu \mathrm{gm}^{-3}\right)$ manufacturer. The PM would then slowly decay to zero as it was ventilated with particle-free air (zero-air pushed through a $0.3 \mu \mathrm{m}$ particle filter) at a constant flow-rate (inlet pressure of $30 \mathrm{psi})$. During this time, the PM levels within the chamber were monitored using the analog port on the DustTrak, an analog to digital convert (A/D converter), and a Python script which provided a real-time 1-second estimate of the $\mathrm{PM}_{2.5}$ mass density. The entire process, from $\mathrm{PM}$ saturation to below-ambient concentration, required approximately 1-2 hours per test. The slow and steady decay of the PM within the chamber allowed us to compare each PurpleAir monitor to the DustTrak reference across a wide range of PM concentrations, throughout the entirety of the low-cost sensors' effective range $\left(0-500 \mathrm{\mu gm}^{-3}\right)$. However, since we were primarily interested in sensor performance across a environmentally relevant range, we developed individual linear models for each sensor by comparing 1-minute averages against the reference 
instrument between 0 - $150 \mu \mathrm{gm}^{-3}$. This method allowed us to capture a nearcontinuous concentration range instead of just a few discrete points that a typical span calibration would provide. Comparing the low-cost sensors across a continuous PM range from multiple PM sources helped characterize the linearity of the low-cost sensors against our reference monitor.

\subsection{PurpleAir Data Acquisition and Storage}

PurpleAir provides a lightweight JSON API (http ://www . purpleair . com/j son) for requesting the most recent observation from each monitor, but accessing historical data is not as straightforward. While historical data is stored by PurpleAir and made available via a wagtail which requires a manual download (http: //www .purpleair.com/sensorlist), they do not provide a programmatic interface for downloading historical data from their platform. Instead, historical data is accessible through the ThingSpeak cloud platform. Programmatically downloading historical data is still free and open, but it is significantly more challenging than accessing the real-time data from the PurpleAir JSON API.

This added difficulty can be explained by two main factors. The primary factor is the API rate limit set by ThingSpeak. The size of each request is limited to a maximum of 8000 observations. This equates to about one week's worth of data from a single node per request. Therefore, accessing historical data from multiple devices over a long period of time (especially in the case of initial database population) required a dynamic method of scheduling a sequence of API requests based on a given start and end date. To overcome this obstacle, we developed a suite of R/Python/Bash/SQL software which dynamically identified 
new sensors as they are installed within our study areas, and included them into our data acquisition queue. Our system then iteratively made requests no larger than 8000 observations across the entire lifetime of each monitor within the network. The results from each request were then processed and stored in our PostgreSQL database with the PostGIS extension. This preserved the spatial coordinates associated with each PurpleAir monitor, and allowed us to perform spatial SQL queries throughout analysis. Once our initial database was populated, a similar script was executed from a Linux server (Xubuntu 18.04) each day at 1:00 AM using the 'cron' task-scheduling software. This script would upload the previous day's data into the database, including any new sensors that were installed throughout the network. It is worth noting that there are no API rate limits in terms of request frequency (requests per unit time), and we found success through multi-threading our pull requests. So long as each request was no larger than 8000 observations, we could pull ThingSpeak data from every available processor core simultaneously. This reduced our initial database population time by $1 / 8^{\text {th }}-1 / 12^{\text {th }}$ depending on the available hardware ( 8 cores versus 12 cores).

In addition to the API request size limit, another challenge we encountered was the field encoding and total field limit of the PurpleAir data when stored on the ThingSpeak platform. Relations within the ThingSpeak API are limited to a total of eight fields, and their names are encoded (e.g. 'field1' instead of 'PM1.0 (CF=ATM) ug/m3'). However, the PurpleAir monitors record 32 fields. Therefore, requesting all of the available data from a given PurpleAir monitor required four separate API requests, achieved through a combination of 
ThingSpeak primary and secondary API keys included within the PurpleAir JSON API. These four subsets were combined through SQL-like table joins using an R script. A key provided by PurpleAir's technical support team was required before these data could be fully processed (Appendix B). Once these fields were decoded, our suite of R/Python/Bash/SQL software automated the acquisition, processing, and storage of historical PurpleAir data (Appendix A: purpleair_id_key.py; create_observation.sql; thingspeakCollect.R; uploadDailyThingspeak. R; uploadHistoricalThingspeak.R).

\subsection{Opportunistic Ambient Calibration of PurpleAir Networks}

Since the response of each PMS5003 sensor is dependent on the PM source (in addition to the intra-model variation observed), an opportunistic ambient calibration method was developed with the goal of applying regionally-specific correction factors to each individual PMS5003 sensor within the PurpleAir network (Portland, OR). Given that $\mathrm{PM}_{2.5}$ is not just a single pollutant, but a complex mixture of particles from multiple primary and secondary sources, an ambient field calibration method was required to best reflect the local PM source profile throughout each city. To determine locally relevant correction factors for each PMS5003 sensor in the Portland network, we compiled 5-minute nephelometerderived $\mathrm{PM}_{2.5}$ data provided by the Oregon Department of Environmental Quality (ODEQ) throughout the duration of our study period (September 2017 - March 2019). Nephelometers, being an optical scattering instrument themselves, initially provide only a Bscat value (the extinction of light due to back 
scattering), and are subject to the same response issues as any other opticalbased PM measurement (e.g. PurpleAir). However, a linear model was developed by ODEQ to correct these Bscat values. Nephelometers and 24 hour $\mathrm{PM}_{2.5}$ gravimetric samplers (FRM) were co-located to develop these correction factors. We used the 5-minute $\mathrm{PM}_{2.5}$ data provided by the ODEQ's FRM-corrected nephelometers to compare with our low-cost sensor network as a means of quality scaffolding. Depending on the day, there were 4-6 active nephelometers throughout the entire study period. We used these nephelometers to identify periods of time where $\mathrm{PM}_{2.5}$ levels were regionally homogeneous. This consisted of 15-minute periods (a total of three 5-minute observations per nephelometer) where the relative standard deviation (RSD) of the ODEQ reference monitors was less than or equal to $10 \%$. Once windows of regionally homogeneous PM were identified, we compared the atmospheric $\mathrm{PM}_{2.5}$ channels ('pm2_5_atm' as opposed to 'pm2_5_cf_1' which is recommended for indoor use) from both PMS5003 sensors within a given PurpleAir monitor against the ambient background concentration (the mean of the active nephelometers) using an Ordinary Least Squares regression (OLS) to determine sensor-specific correction factors. These correction factors were stored as a new relation in our Postgres database to be queried during the modeling phase. Sensors with an $R^{2}$ value below 0.90 were deemed faulty or unreliable, and were excluded from the modeling phase. This method incorporated periods of time throughout the entire study duration, allowing us to capture PM across a range of seasonal sources (wildfires, wood heating, traffic, temperature inversions, etc.) and develop comprehensive correction factors that account for all regionally relevant sources. 


\subsection{Data Recovery and Sensor Lifetime}

Between July 2017 and December 2018 data quality issues from 7\% of the PurpleAir network were observed. While this was an improvement over the Shinyei sensors (AirAdvice monitors) we have used during previous studies, the PMS5003 within the PurpleAir are not immune to optical contamination and laser diode degradation. PurpleAir's technical support team recommended cleaning the optics with compressed air and/or applying a vacuum to the sample inlets. Compressed air revived one out of three faulty sensors. Data recovery over this period was greater than $90 \%$ and the remaining network has shown no signs of further degradation. Plantower now provides a dual-laser model (PMS6003) which has effectively doubled the lifetime of the sensor by alternating diodes between measurements. Modifications to the sensor chassis and sampling path design have also demonstrated improvements to mitigating dust accumulation in the optics in the previous models (PMS1003, PMS3003).

\subsection{Sensor Allocation}

The Portland, Oregon sensor network was the first in the Canopy Continuum study, and PurpleAir monitors were placed opportunistically. A sensor allocation and volunteer recruitment method was developed for the remaining four cities (Albuquerque, New Mexico, Boise, Idaho, Tacoma, Washington, and Sacramento, California). These cities were previously selected within the Canopy Continuum project because they represent a varying degree of canopy 
cover necessary to characterize the potential role of vegetation in mitigating environmental stressors. Our sensor allocation approach first characterized the spatial autocorrelation of the existing Portland PurpleAir network to identify the minimum distance necessary to capture spatially heterogeneous $\mathrm{PM}_{2.5}$. In other words, we plotted the $\mathrm{R}^{2}$ between every sensor pair as a function of distance and observed when the $\mathrm{R}^{2}$ began to drop below 0.75 . We identified a minimum placement distance of $2 \mathrm{~km}$ from these results (Figure 2.1). Sensor allocation in the remaining cities relied on a random sampling approach, stratifying by population density. For each city, a population density surface with a spatial resolution of $2 \mathrm{~km}^{2}$ was derived via areal interpolation of American Community Survey (ACS) data at the census block group level. Census data was retrieved using the tidycensus $\mathrm{R}$ package (Walker, 2019). In short, a $2 \mathrm{~km}^{2}$ grid was created using the spatial extent defined by the bounding box of a given city's Metropolitan/Micropolitan Statistical Area shapefile (US Census Bureau, 2015). A spatial intersection was applied to each grid cell and its corresponding census block group(s) to determine area-based weights. For example, if a grid cell encompassed 50\% of a given block group, the weight for that block group would be 0.5 . If a grid cell was completely within a single block group, the weight would be 1.0. These weights were then applied to each block group's population count, assuming that the population density within a given block group was evenly distributed. The sum of these weighted population counts, determined by the relative area of each block group within that cell, was divided by the total area of the cell $\left(2 \mathrm{~km}^{2}\right)$ to calculate its population density. We then iterated through each grid cell/block group pair to create population density 
surfaces throughout each city.

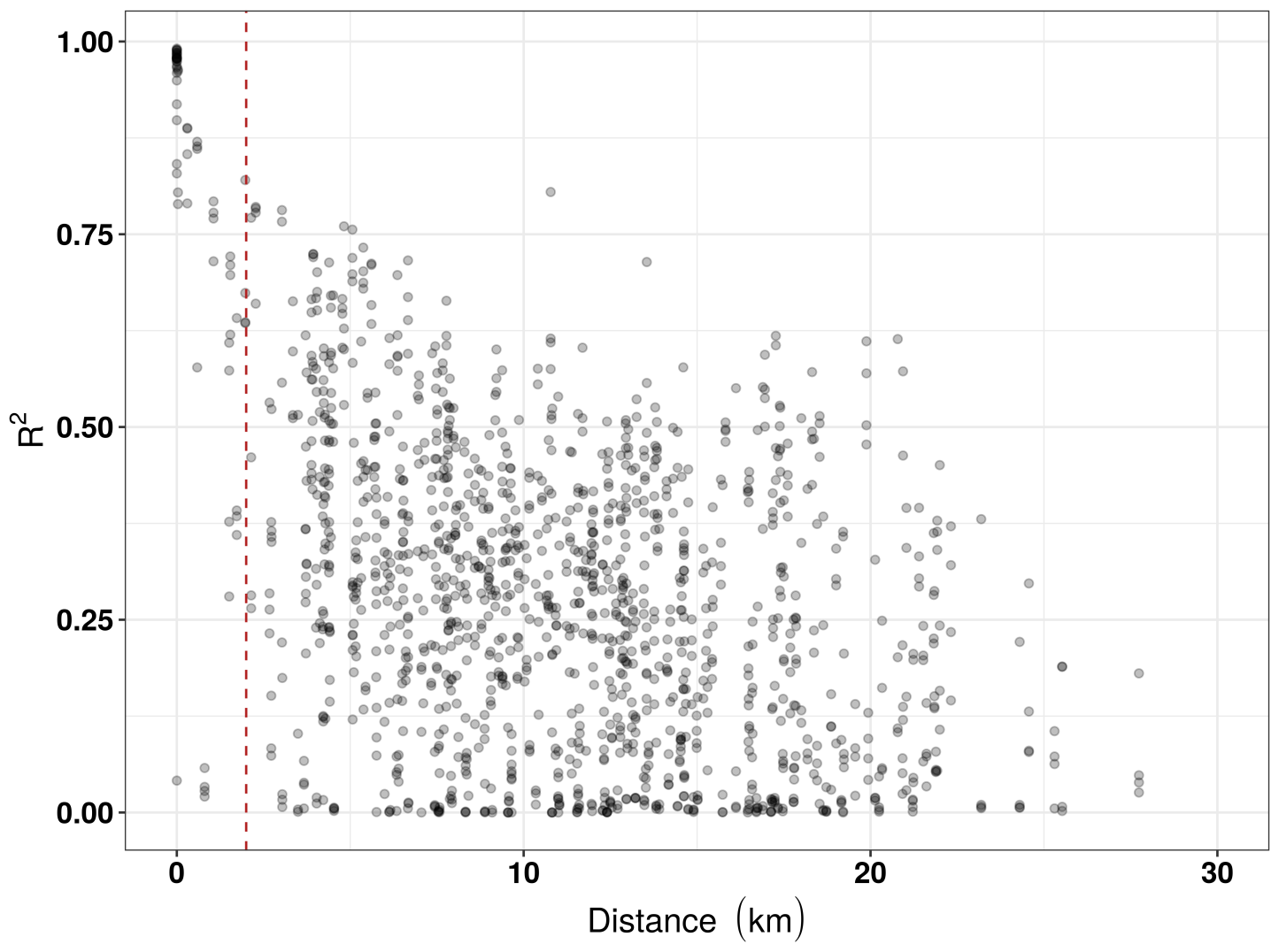

FIGURE 2.1: Scatter plot of the Portland PurpleAir network spatial autocorrelation as a function of distance.

These gridded population density layers consisted of approximately 200-500 individual $2 \mathrm{~km}^{2}$ grid cells depending on the study area. With only a limited number of PurpleAir sensors available to deploy in each city (Albuquerque $n=$ 30; Tacoma $n=25$; Boise and Sacramento $n=20$ ), a random sampling approach was employed to prioritize areas of interest during network installation. These randomly assigned target areas were stratified by population density deciles. 
Given that the primary goal of this study was to generate PM exposure maps for future analysis in an epidemiological study, the majority of the target areas were randomly assigned to the most densely populated deciles. In other words, we attempted to recruit more volunteers to host PurpleAir monitors who lived in more densely populated neighborhoods, and fewer volunteers from suburban and rural neighborhoods. The total number of monitors deployed in a given city was determined by the size of its Metropolitan Statistical Area (MSA), as well as, the number of pre-existing $3^{\text {rd }}$ party PurpleAir monitors. After the random stratified selection process, target areas were manually adjusted for adjacency to other target areas, as well as, any existing $3^{\text {rd }}$ party PurpleAir monitors.

While this method provided a quantifiable approach to sensor allocation, the structure of each city's network was ultimately limited by volunteer availability. We adopted the 'some data is better than no data' philosophy, and placed the majority of our sensors wherever there were willing and able participants. Sensors were typically installed 6-8 ft above ground in the front or backyard of a volunteer residence. Occasionally, sensors were sited outside commercial or government offices. This was especially the case in target areas with relatively low population densities. Sensors were placed at least $30 \mathrm{ft}$ away from hyperlocal sources including barbeques, laundry vents, and fireplaces.

Web maps with the gridded population density and sensor target areas (outlined in bold) were developed using leaflet $\mathrm{R}$ package (Cheng, Karambelkar, and Xie, 2018). Static maps are included below and hyperlinks to the original web maps are included within the figure captions (Figure 2.2; Figure 2.3; Figure 2.4; Figure 2.5). 


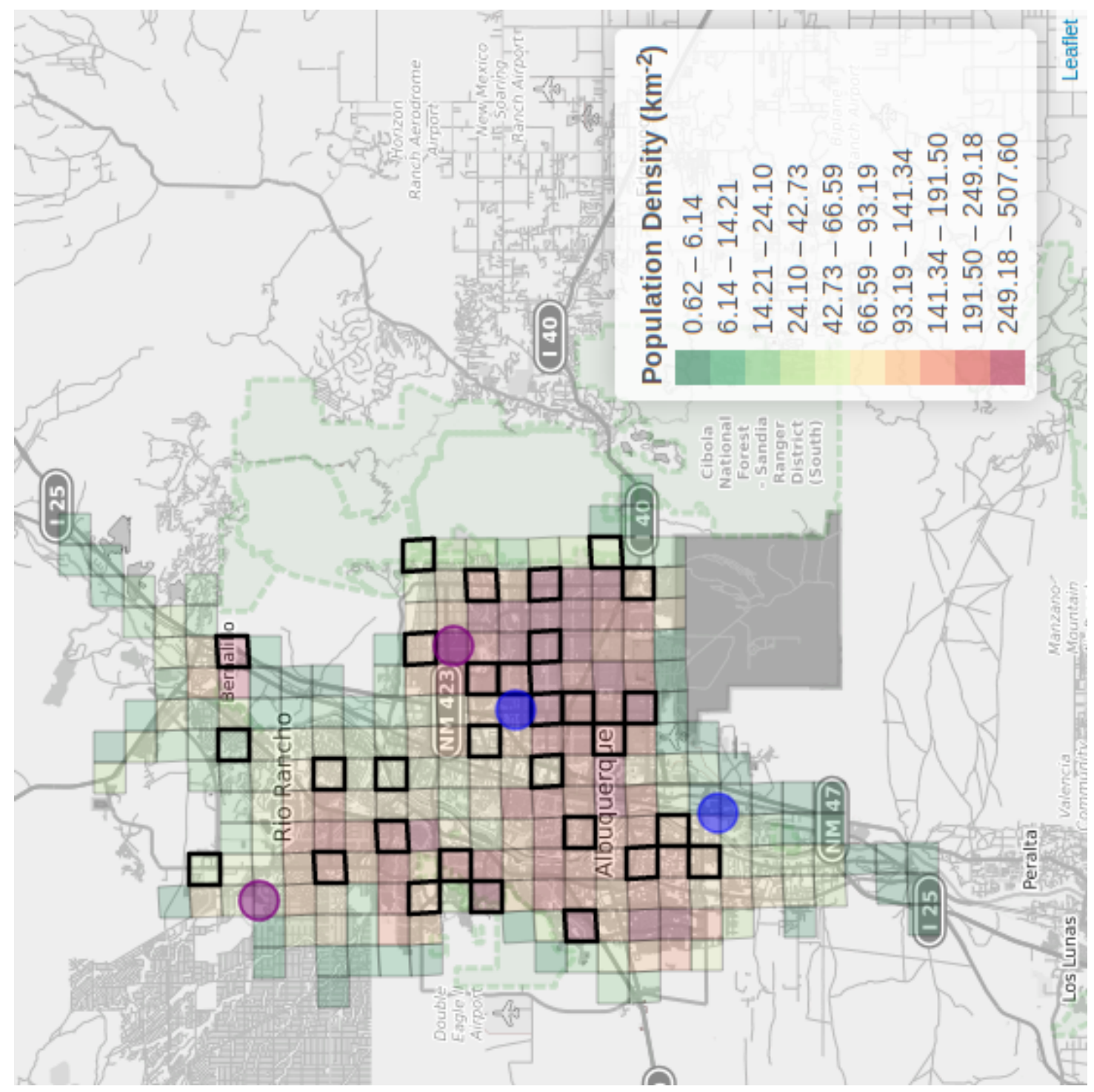

FIGURE 2.2: Sensor allocation map developed for Albuquerque, New Mexico. Areas prioritized for sensor allocation are highlighted in bold. The web map can be viewed here: http://web. $\mathrm{pdx} . \mathrm{edu} /{ }^{\sim}$ porlando/albuquerque.html 


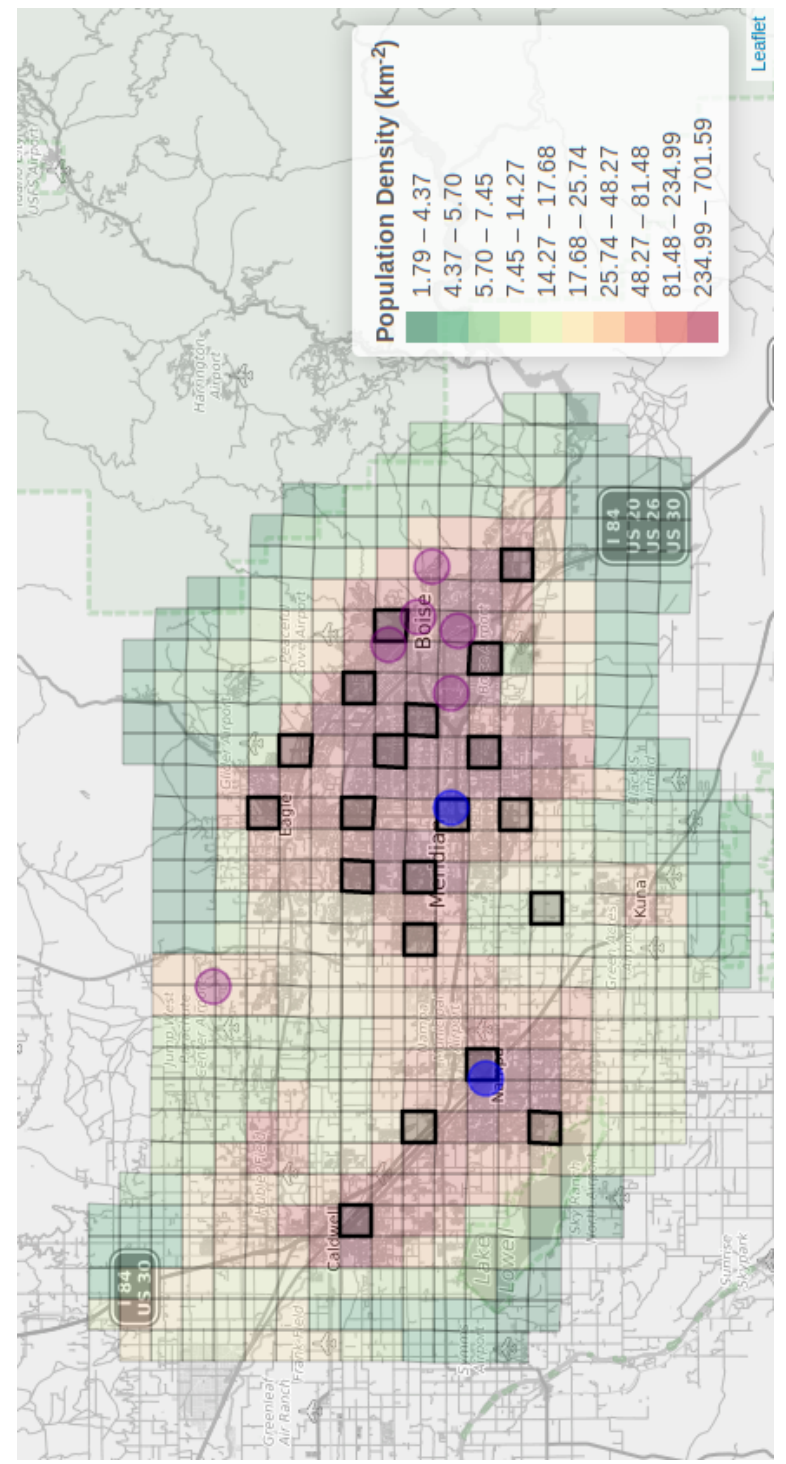

FIGURE 2.3: Sensor allocation map developed for Boise, Idaho. Areas prioritized for sensor allocation are highlighted in bold. The web map can be viewed here: http://web.pdx.edu/ porlando/ boise.html 


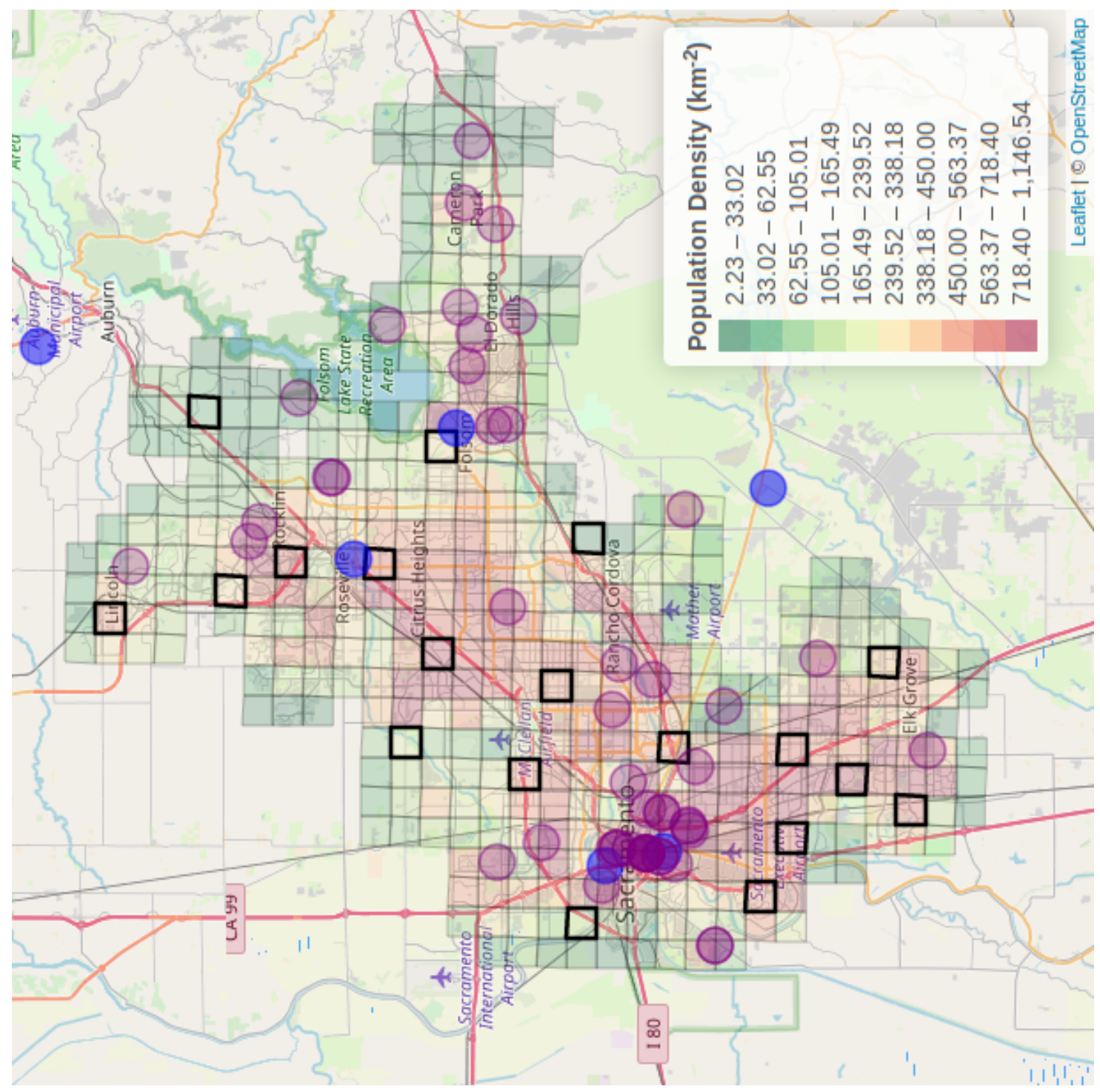

FIGURE 2.4: Sensor allocation map developed for Sacramento, California. Areas prioritized for sensor allocation are highlighted in bold. The web map can be viewed here: http://web.pdx.edu/ porlando/sacramento.html 


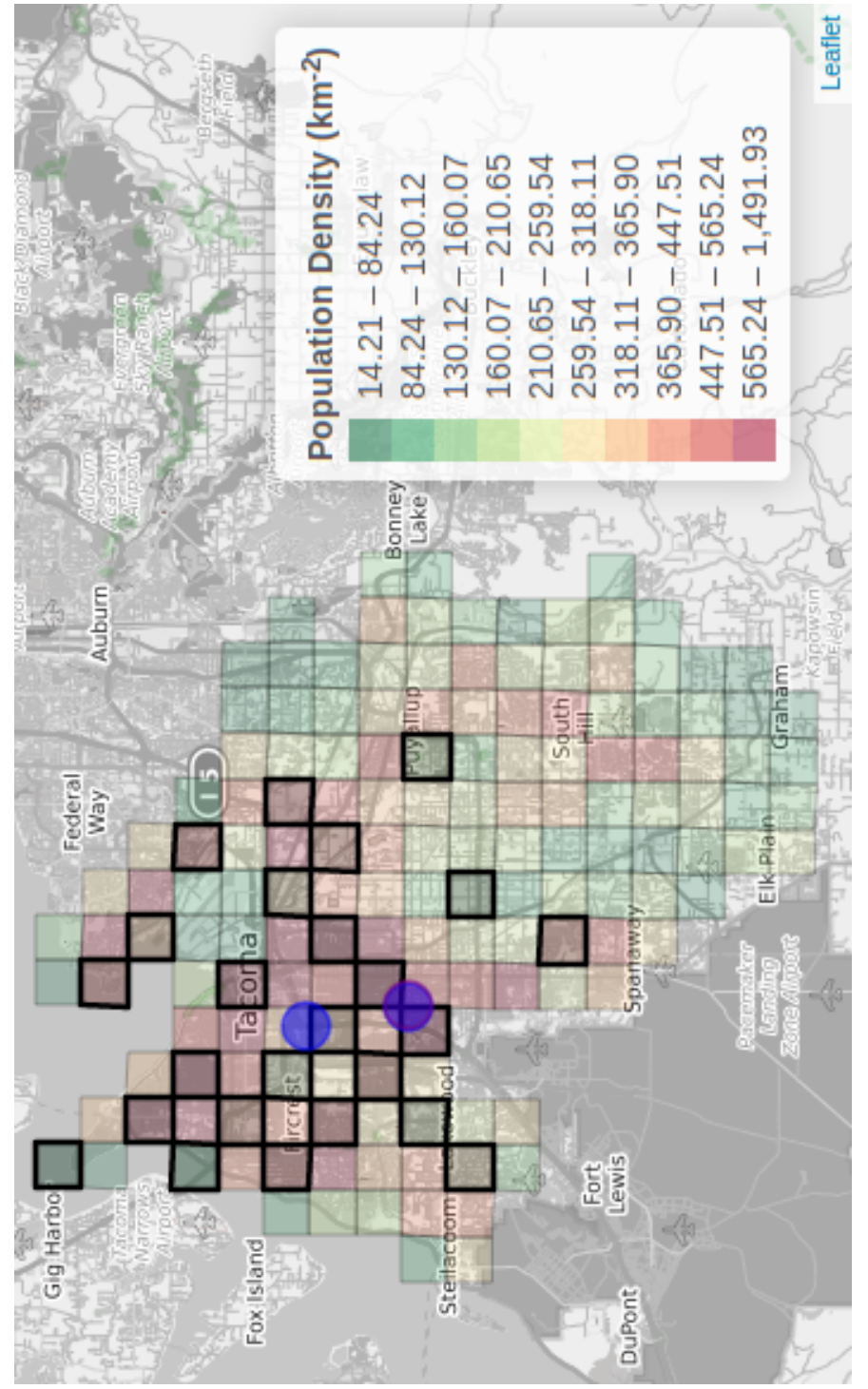

FIGURE 2.5: Sensor allocation map developed for Tacoma, Washington. Areas prioritized for sensor allocation are highlighted in bold. The web map can be viewed here: http://web.pdx.edu/ porlando/tacoma.html 


\subsection{Data Compilation}

All data compilation and spatial analysis were performed using the R Statistical Programming Language (R Core Team, 2019), and its corresponding spatial libraries. These include 'sp' (Pebesma and Bivand, 2005), 'sf' (Pebesma, 2018), 'raster' (Hijmans, 2019), 'rgdal' (Bivand, Keitt, and Rowlingson, 2019), and 'rgeos' (Bivand and Rundel, 2019). The following workflow diagram highlights the various data acquisition and processing scripts necessary to develop LUR models with corrected PurpleAir network data (Figure 2.6). 


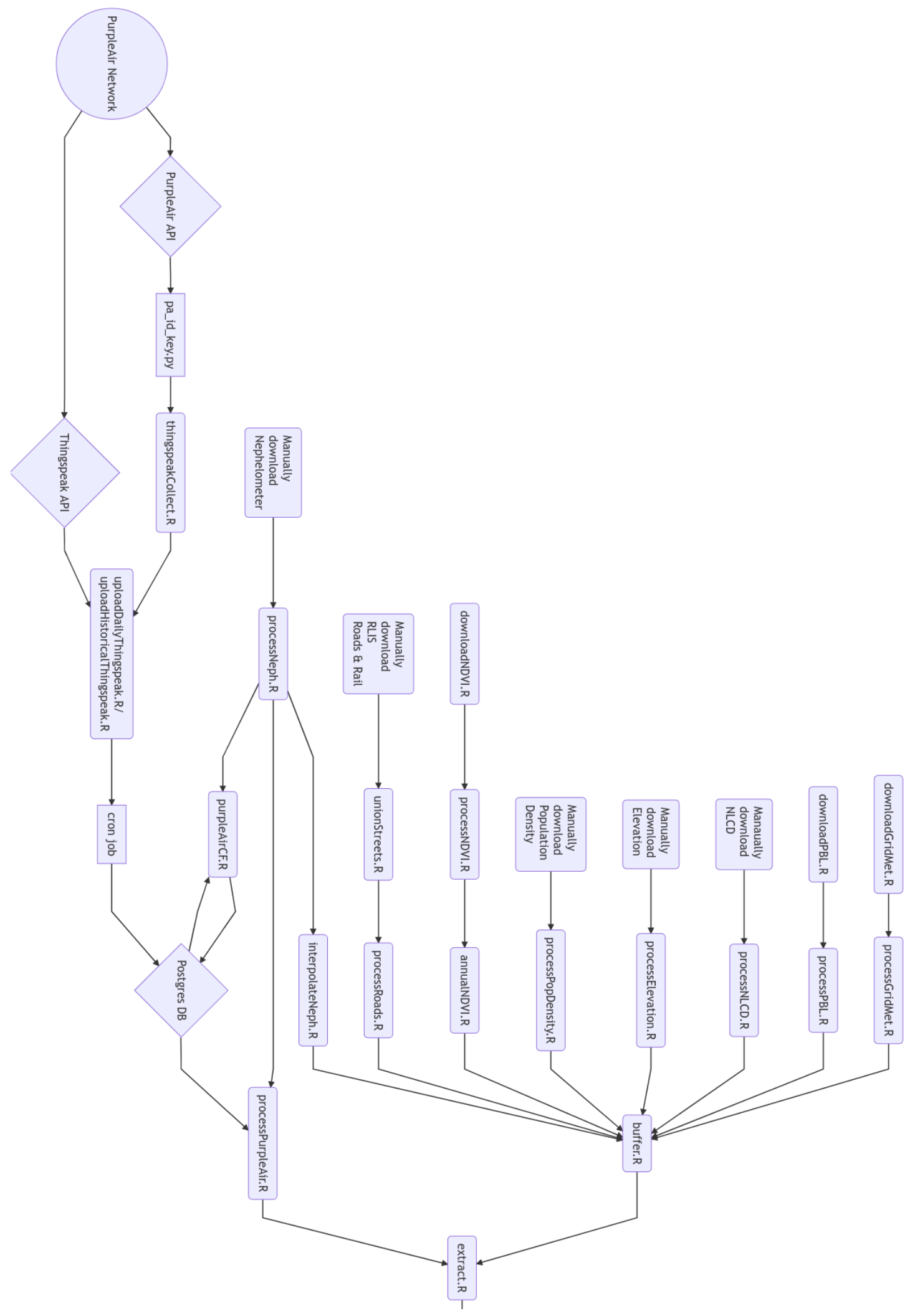


FIGURE 2.6: A workflow diagram highlighting the various data compilation scripts necessary to develop a LUR model with corrected PurpleAir data. All relevant source code is included within Appendix A.

\subsubsection{Spatial and Temporal Predictors of $\mathbf{P M}_{2.5}$}

Several land-use (LU) and meteorological variables were evaluated to predict daily $\mathrm{PM}_{2.5}$ surfaces at a spatial resolution of $50 \mathrm{~m}$. Land-use variables were included as surrogates for potential PM sources in attempt to characterize the spatial heterogeneity of PM within the study area. This included proxies of traffic-related PM such as road (freeways, major arterials, streets, etc.) and rail density. Other spatial predictors were included to account for potential area sources (population density, LULC), or sinks (NDVI, EVI). Meteorological variables were included to characterize the temporal variation of PM. For example, PM concentrations typically increase as the planetary boundary layer (PBL) decreases as the effective mixing volume for PM dilution decreases during low boundary height scenarios. In addition, wind speed has been shown to affect PM concentrations. As wind speed increases, PM generated from urban sources 
is readily removed, diluting overall PM levels throughout the urban core (Chudnovsky et al., 2014). The network of ODEQ PM monitors (nephelometers) were also included as a temporal scaling factor of PM. This allowed us to capture temporal patterns in PM that would otherwise be unaccounted for by meteorological variables alone (e.g. wildfires).

\section{Road and Rail Density}

Road and rail data were obtained from Metro's Regional Land Information System (RLIS) data resource center (Oregon Metro, 2018). This included GIS shapefiles of the 'Streets', 'Arterials' 'Major Arterials', 'Freeways', 'Railroads', and 'Railyards' feature classes. Originally vector data, each feature class was discretized into a new raster layer with a spatial resolution of $50 \mathrm{~m}$. These data were also reprojected to match the Coordinate Reference System (CRS) of the study area, and clipped by its extent (Appendix A: processRoads. R). For Portland, the bounding box was defined by Metro's urban growth boundary extent (UGB). RLIS data is not available for the other study areas, and a national-scale data sources should be used instead during future modeling endeavors.

\section{NDVI and EVI}

Satellite-derived Normalized Difference Vegetation Index (NDVI) and Enhanced Vegetation Index (EVI) data were obtained from the $250 \mathrm{~m}$ MODIS 16-day product (MOD13Q1) (Didan, 2015b).These data were programmatically downloaded using the MODIS R package (Mattiuzzi and Detsch, 2019) (Appendix A: downloadNDVI.R). The NDVI was determined using red and near 
infrared surface reflectances, which has been shown to provide a temporal indication of vegetation cover and its phenological state (Tucker, 1979; Tucker and Sellers, 1986). The EVI layer is more sensitive over densely vegetated areas, explaining further variation in vegetation density beyond the range of the NDVI. Both of these layers are derived from bi-directional surface reflectances, with masking algorithms for water, clouds (and their shadows), and aerosols (Didan, 2015b). Each MODIS layer was reprojected to match the CRS of the study area, clipped by the study extent, and resampled via bilinear interpolation to match the $50 \mathrm{~m}$ spatial resolution of the model. Originally, we explored a temporal cubic spline interpolation method to derive daily NDVI/EVI observations from each 16-day MODIS overpass (Fritsch and Carlson, 1980). While this technique produced promising results with the $1 \mathrm{~km}$ resolution MODIS product (MOD13A2), (Didan, 2015a), it did not scale to the $250 \mathrm{~m}$ resolution product, and our processing script unexpectedly terminated without explanation (Appendix A: interpolateNDVI.R). This was likely due to issues with cloud masking and excessive missing values at this resolution. Instead of interpolating daily NDVI/EVI from the $250 \mathrm{~m}$ product, we opted to develop annual aggregates (Appendix A: annualNDVI.R).

Elevation data were retrieved from NASA's Advanced Spaceborne Thermal Emission and Reflection Radiometer (ASTER) digital elevation map (DEM) product. This is a global DEM with a spatial resolution of $30 \mathrm{~m}$ provided in GeoTiff format (NASA, 2009). This layer was reprojected to match the CRS of our study area, clipped by our study extent, and resampled from $30 \mathrm{~m}$ to $50 \mathrm{~m}$ (Appendix A: processElevation.R). 


\section{Population Density}

Population density data was obtained from NASA's Gridded Population of the World (GPW) collection (version 4) (Doxsey-Whitfield et al., 2015). This product models the distribution of the global human population on a continuous raster layer for use in social, economic, and Earth science disciplines. This is a 30-arc second resolution product (approximately $1 \mathrm{~km}$ ) provided in GeoTIFF format. This global raster layer was reprojected to match the CRS of our study area, and then clipped by its extent. It was also resampled from $1 \mathrm{~km}$ to $50 \mathrm{~m}$ via bilinear interpolation (Appendix A: processPopDensity.R).

\section{GridMET Meteorological Variables}

Meteorological data was obtained from the University of Idaho's Gridded Surface Meteorological Data (GridMET). The GridMET data is a combination of high-resolution spatial data $(4 \mathrm{~km})$ from the Parameter-elevation Regressions on Independent Slopes Model (PRISM) and the high-temporal resolution data from the National Land Data Assimilation System (NLDAS) which generates spatially and temporally continuous fields of several meteorological variables (Abatzoglou, 2013; Daly, 2006; Mesinger et al., 2006). The GridMET product provides estimates of daily precipitation totals, minimum and maximum air temperature and relative humidity, specific humidity, mean wind direction and mean wind speed. However, the GridMET product was not designed to capture microclimates that occur at more granular resolutions than its native resolution of the two parent data sources (Abatzoglou, 2013). The wind variables are limited to a $32 \mathrm{~km}$ spatial resolution and are not capable of capturing any influences 
of terrain on wind fields (Abatzoglou, 2013). An R script was developed to programmatically download daily GridMET data via wget calls to a HTTP server (Appendix A: downloadGridMET.R). As with the previous predictors, the GridMET data was reprojected to match the CRS of our study area, clipped by our study extent, and resampled to $50 \mathrm{~m}$ to match the resolution of our modeled surfaces. A bilinear interpolation was applied to all scalar variables, however, wind direction was resampled using the nearest neighbor method because the bilinear method is incapable of vector averaging (Appendix A: processGridMET.R).

\section{Land Use and Land Cover}

Land Use and Land Cover (LULC) data was obtained from the USGS's National Land Cover Database (NLCD) 2011 product. The NLCD provides a $30 \mathrm{~m}$ spatial resolution categorical raster layer with fifteen different LULC attributes. This includes open water, low, medium, and high intensity developed land, deciduous, evergreen, and mixed forest lands (Homer et al., 2015). This raster was reprojected to match the CRS of our study area, clipped by its extent, and resampled from $30 \mathrm{~m}$ to $50 \mathrm{~m}$ using the nearest neighbor method. This single categorical layer was decomposed into individual presence/absence rasters for each LULC attribute via one-hot encoding. For example, if the 'open water' LULC was observed within a raster cell, a value of 1 would be assigned to this cell. If a LULC was not observed within a cell, then a value of 0 would be assigned. One-hot encoding the NLCD raster into individual layers was necessary for creating focal statistics (Appendix A: processNLCD.R). 


\section{Planetary Boundary Layer Height}

Planetary boundary layer height (PBL) data was obtained from NOAA's NARR product (Mesinger et al., 2006). An R script was used to programmatically download 3-hourly PBL netCDF data throughout the study duration (September 2017 - March 2019) via wget calls to NOAA's FTP server (Appendix A: downloadPBL.R). The NARR PBL grid resolution is approximately 0.3 degrees, which corresponds to $32 \mathrm{~km}$ at the lowest latitudes. Each 3-hourly grid was aggregated into a single mean value throughout the study area. These 3-hour means were then used to determine the daily maximum and minimum PBL height values. The minimum and maximum PBL values were used as temporal scaling factors, and were extrapolated into continuous $50 \mathrm{~m}$ resolution spatial grids corresponding to the extent and CRS of our study area (Appendix A: processPBL.R).

\section{Nephelometer $\mathbf{P M}_{2.5}$}

Nephelometer-derived $\mathrm{PM}_{2.5}$ data with a 5-minute temporal resolution were provided by ODEQ. In addition to the use of these data for determining correction factors for our PurpleAir sensor network, these data were also included as a temporal scaling factor to account for changes in the ambient background concentration due to regional sources (e.g. wildfires). The 5-minute $\mathrm{PM}_{2.5}$ values from 4-6 active nephelometers were aggregated into daily mean and median values (Appendix A: processNeph.R). Similar to the PBL compilation, these values were extrapolated into a continuous $50 \mathrm{~m}$ resolution surface corresponding to the extent and CRS of our study area (Appendix A: interpolateNeph.R). 


\section{Latitude and Longitude}

Continuous fields for both latitude and longitude were generated using $R$ and its spatial libraries (raster, rgdal, rgeos, sp, sf, etc.). These variables were included to account for the potential effects of spatial autocorrelation described by Tobler's $1^{\text {st }}$ law of geography (Tobler, 1970).

\subsubsection{Focal Statistics}

Focal statistics were determined for each spatial (and temporal-spatial) predictor at $50 \mathrm{~m}, 100 \mathrm{~m}, 300 \mathrm{~m}, 500 \mathrm{~m}$, and $1000 \mathrm{~m}$ buffer sizes (Appendix A: buffer.R). These buffer distances were adopted from the LUR methods developed by the ESCAPE Project (Eeftens et al., 2012). The population density variable also included 1250 m, 1500 m, 2000 m, and $2500 \mathrm{~m}$ focal buffer distances. Briefly, this method iterates through each cell within a given $50 \mathrm{~m}$ resolution spatial grid and determines the mean value of all adjacent cells within a prescribed radius (neighborhood). The target cell is then assigned the mean value of this neighborhood. This process is then repeated across the sequence of desired buffer distances. For a graphical representation of the new layers created from applying focal statistics to the 'Streets' layer, see Figure 2.7. For the predictors which are purely spatial (NLCD, Roads and Rail, Elevation) focal statistics at each neighborhood size were performed only once. However, for the temporal spatial-predictors (NDVI, EVI, GridMET), this operation was repeated for each daily observation. This resulted in a total of 33 thousand individual raster layers, stored as GeoTIFFs, which consumed approximately 100 GB of local disk 
storage. Despite being rasterized, this method was also computationally expensive. Determining thousands of focal buffers required the use of a high-memory compute server provided by PSU Research Computing, and consumed geq500 GB of memory while running on all 24 available processor cores. Processing time was on the order of 4-6 hours depending on the total number of predictors selected for modeling.

The temporal predictors lacking any spatial component (e.g. scaling factors including PBL and Nephelometer PM) were not included in the focal statistics pipeline, as these layers only consisted of a single mean value for each day.

Focal statistics for the GridMET wind direction data were handled differently than the other variables. This layer, with the inclusion of wind speed, was converted from degrees to radians, and then transposed into its respective $u$ (zonal velocity) and $v$ (meridional velocity) components. Focal statistics were then performed on the $u$ and $v$ components (unit-vector averaging), and converting back into degrees using the arc-tangent (Appendix A: buffer.R). 


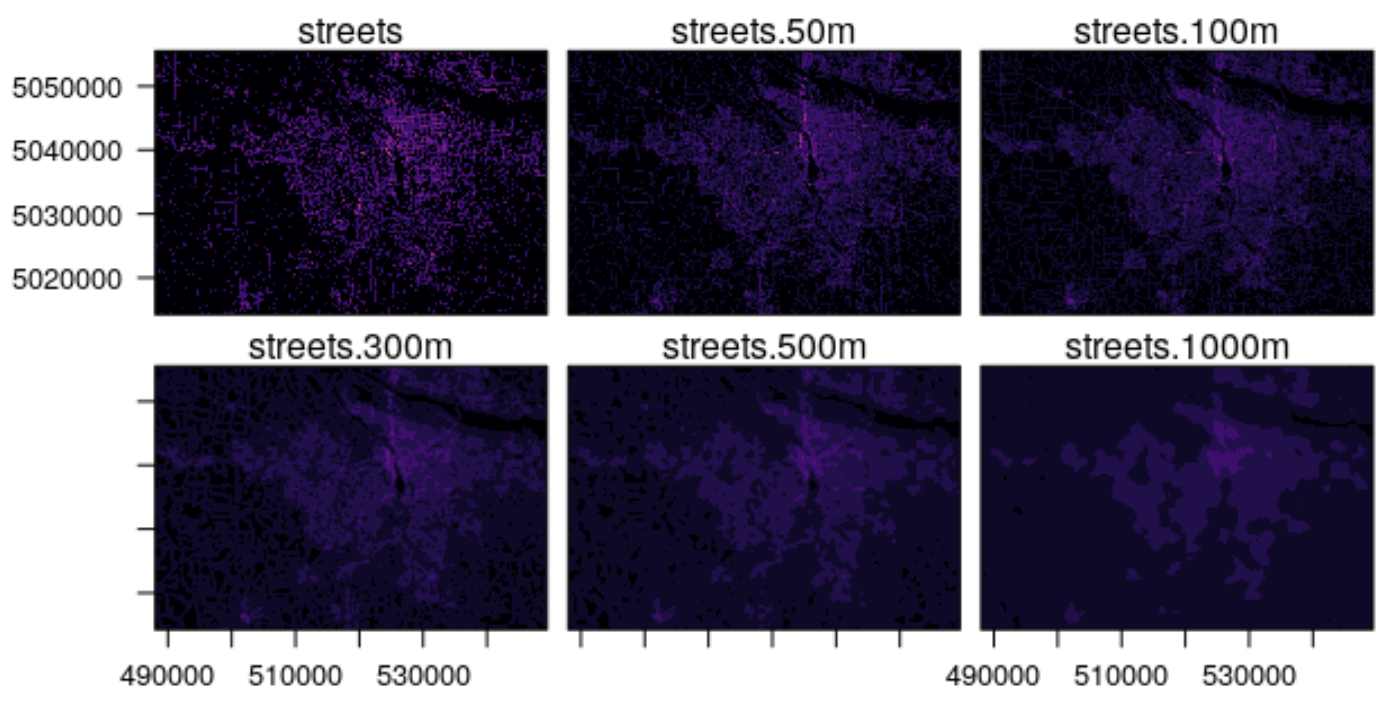

FIGURE 2.7: A graphical representation of applying focal buffers to the RLIS 'streets' layer. The buffer distances are appended to the layer name in each panel, e.g. the $1 \mathrm{~km}$ buffer is named 'streets.1000m'. The original rasterized RLIS 'streets' layer does not have a buffer size appended to its layer name.

\subsubsection{Data Extraction}

After generating focal statistics across our sequence of buffer distances, variables, and days, we extracted the grid cells values that spatially intersected with the corrected PurpleAir response variable (Appendix A: extract.R). Iterating the raster extraction process across each day within our study duration, the end result was a single large matrix (data frame) with the corrected PurpleAir $\mathrm{PM}_{2.5}$ response variable and all relevant predictors at each buffer size. This matrix 
consisted of 211 variables and 11,546 observations. Additional categorical variables were added to this matrix including 'Weekday/Weekend', 'Day of Week', 'Season', and 'Month'. This matrix was used during the modeling phase. The study duration consisted of 575 consecutive days and, given the 11,546 unique observations, the average number of active PurpleAir monitors on a given day was approximately 20. This is comparable to the network sizes within the ESCAPE Project (Eeftens et al., 2012). The Portland network did grow overtime as PurpleAir monitors were incrementally added by our research group as well as other $3^{\text {rd }}$ parties. The network size was closer to 10 active nodes during the first few months of the study, and grew to more than 35 nodes by its end. Some of these were active for a short period of time, but most remained active once initially established.

\subsection{Predictor Selection}

To avoid issues of multicollinearity, only a single focal buffer distance was selected for each predictor variable during the modeling phase. A correlation matrix was produced for each variable against the response data and the focal buffer with the strongest correlation with the response variable was then selected for modeling. If multiple focal buffers had the same correlation coefficient, then the finest spatial resolution buffer was chosen to capture as much spatial heterogeneity as possible. Variables with nonintuitive correlations, e.g. expected sources yielding a negative correlation, or expected sinks yielding a positive correlation, were excluded from the model. 
An additional correlation matrix which compared all of the previously selected predictors was generated to explore groups which covaried. A complete linkage hierarchical clustering algorithm, a built-in parameter within the R 'corrplot' library (Wei and Simko, 2017), was used to identify clustered predictors (Defays, 1977). Once these groups were identified, only a single predictor within each group was selected for the modeling phase. For example, if the NLCDderived 'Deciduous Forest', 'Mixed Forest', 'Evergreen Forest' were clustered with 'NDVI', then the most generalizeable predictor was selected for the final model (e.g. the NDVI layer can be used to explain all of the other vegetation layers).

These predictor selection criteria were established to meet the multicollinearity assumption of a traditional multiple linear regression. We relied on a Variance Inflation Factor (VIF) below 5 to meet this assumption. VIF scores were determined using the 'car' R library to assess multicollinearity (Fox et al., 2007). Although the multicollinearity assumption only applies to multiple linear regression model, the same variable selection procedure was applied to the Random Forest model for direct comparison. Model variables were also tested for significance, and only predictor coefficient's p-value less than 0.1 were selected for the final model (Appendix A: modeling.R).

The initial predictors selected for the modeling phase are included within Table 2.1. This table details the variable description, abbreviation, and respective data sources. The expected source and sink proxies for each predictor are also included. 


\subsection{Modeling and Validation}

For this study, we compared the performance of a traditional LUR model (multiple linear regression) against a decision-tree-based machine learning model (Random Forest) (Liaw and Wiener, 2002; Breiman, 2001). Of the 11,546 total observations, we randomly subset $70 \%$ during the training phase for each model. The remaining 30\%, or 'test' data, was reserved for model validation. In addition to this 70/30 holdout approach, we also employed a leave-oneout cross-validation (LOOCV) method which has been widely used in previous LUR studies (Yang et al., 2017; Eeftens et al., 2012; Saucy et al., 2018). With this approach, a single PurpleAir node was excluded from the training data and a model was developed using the $n-1$ remaining sites. The subsequent model was then used to predict all of the observations for this single node, and the model performance was evaluated by observing the cross validation $(\mathrm{CV}) \mathrm{R}^{2}$ and CV RMSE. This process was then repeated for each individual node, excluding nodes with fewer than 100 observations. The LOOCV method allowed us to evaluate how well a given model predicted for a specific sensor over a consecutive period of time, instead of predicting a random subset of days and sensors simultaneously. In addition, this method allowed us to compare an entire ensemble of predictions (a new model was developed for each node that was left out), and identify any locations that a model was consistently under/over predicting.

An ensemble method was used during the LUR model prediction phase. Specifically, each of the $n-1$ models developed during the LOOCV phase were 
used to predict an individual $\mathrm{PM}_{2.5}$ surface. This ensemble of predictions was then aggregated to determine a single mean $\mathrm{PM}_{2.5}$ surface each day. The Random Forest method was too computationally expensive to rely on the LOOCV technique when making predictions. Instead, the results from the single model which included all available monitoring sites generated from the 70/30 holdout process was used during the prediction phase. 


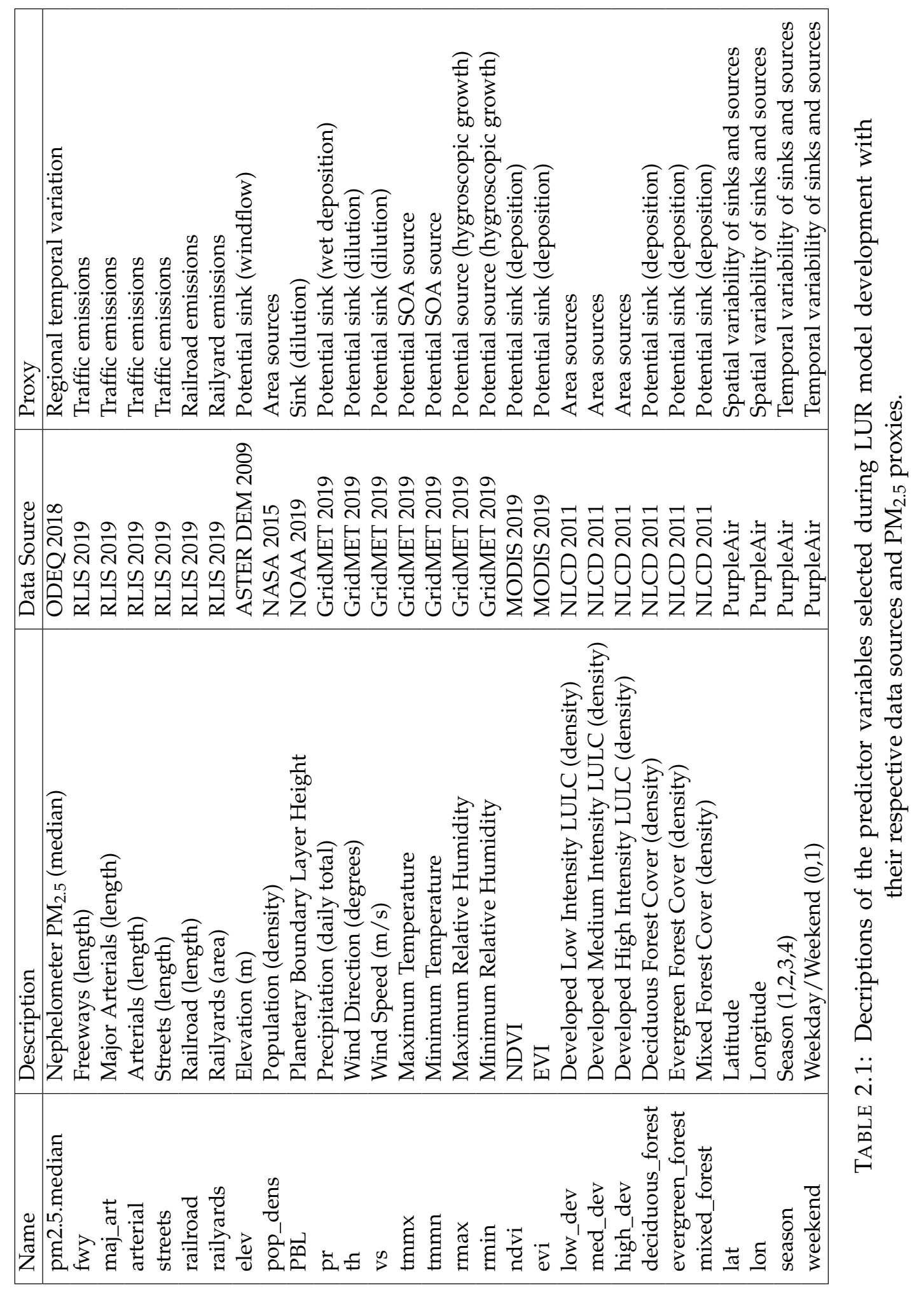




\section{Chapter 3}

\section{Results and Discussion}

\subsection{Laboratory Evaluations}

Strong linear relationships between the low-cost PurpleAir monitors and the reference DustTrak DRX Aerosol Monitor Model 8533 were observed for both the match and candle smoke tests. The mean $\mathrm{R}^{2}$ of the candle smoke evaluations was $0.958 \pm 0.14$, whereas the mean $\mathrm{R}^{2}$ of the match smoke evaluations was $0.942 \pm 0.17$ Table 3.1. Despite following the same protocol between tests, some sensors did not perform linearly with the reference instrument (Figure 3.1). However, these cases occurred infrequently and meaningful calibrations with $\mathrm{R}^{2}$ values greater than 0.90 occurred for more than $80 \%$ of all test periods. While the strength of DustTrak-PurpleAir relationship was independent of the PM source, the slopes differed depending on the PM source. The mean slope of the models generated from the match smoke tests was $1.35 \pm 0.64$. This was approximately half the value observed from the candle smoke tests which yielded a mean slope of $2.66 \pm 0.75$ (Figure 3.2). These results indicate two challenges with low-cost PM sensors for LUR. The first was that intra-model 
variation does occur with the Plantower sensors, despite the manufacturer's effort to pre-calibrate each sensor. This means that two sensors, when exposed to the same PM sample, will produce a slightly different response. The slopes from the match smoke tests are more narrowly distributed, while the candle smoke tests have a wider distribution ranging from 2-3 and appear bimodal. This was somewhat unexpected because multiple different types of matches were used throughout these laboratory evaluations, whereas only a single candle was used. This could potentially be explained by match smoke PM having similar physical properties as Plantower's PM source during factory calibration (e.g. absorption, profile, shape, etc.). The second challenge presented by these results is that the Plantower/PurpleAir response is dependent on the PM source. This suggests that laboratory calibrations alone, while a reliable screening tool for faulty sensors, are not enough to determine meaningful correction factors for field-deployed sensors necessary to produce epidemiologically relevant $\mathrm{PM}_{2.5}$ concentrations. These results support the development of an ambient calibration method of PurpleAir networks for determining field-specific correction factors. Two scatter plots were included to highlight the PurpleAir's response across the entire PM range of a typical test for both the primary and secondary sensors (A and B sensors) (Figure 3.3; Figure 3.4). 


\begin{tabular}{|l|c|c|c|c|c|c|}
\hline PM Source & $\mathrm{R}^{2}$ & $\mathrm{R}^{2}$ SD & Slope & Slope SD & Intercept & Intercept SD \\
\hline Candle & 0.958 & \pm 0.14 & 2.7 & \pm 0.78 & 0.34 & \pm 2.83 \\
Match & 0.942 & \pm 0.17 & 1.35 & \pm 0.64 & 3.98 & \pm 5.24 \\
Field & 0.958 & \pm 0.02 & 0.46 & \pm 0.06 & 1.63 & \pm 0.31 \\
\hline
\end{tabular}

TABLE 3.1: Linear model summary statistics of both laboratory and field evaluations. Lab evaluations are comparing the DustTrak against PurpleAir monitors grouping by the PM source (candle and match smoke). The field evaluations are comparing the mean nephelometer $\mathrm{PM}_{2.5}$ against PurpleAir monitors. $\mathrm{SD}=$ standard deviation.

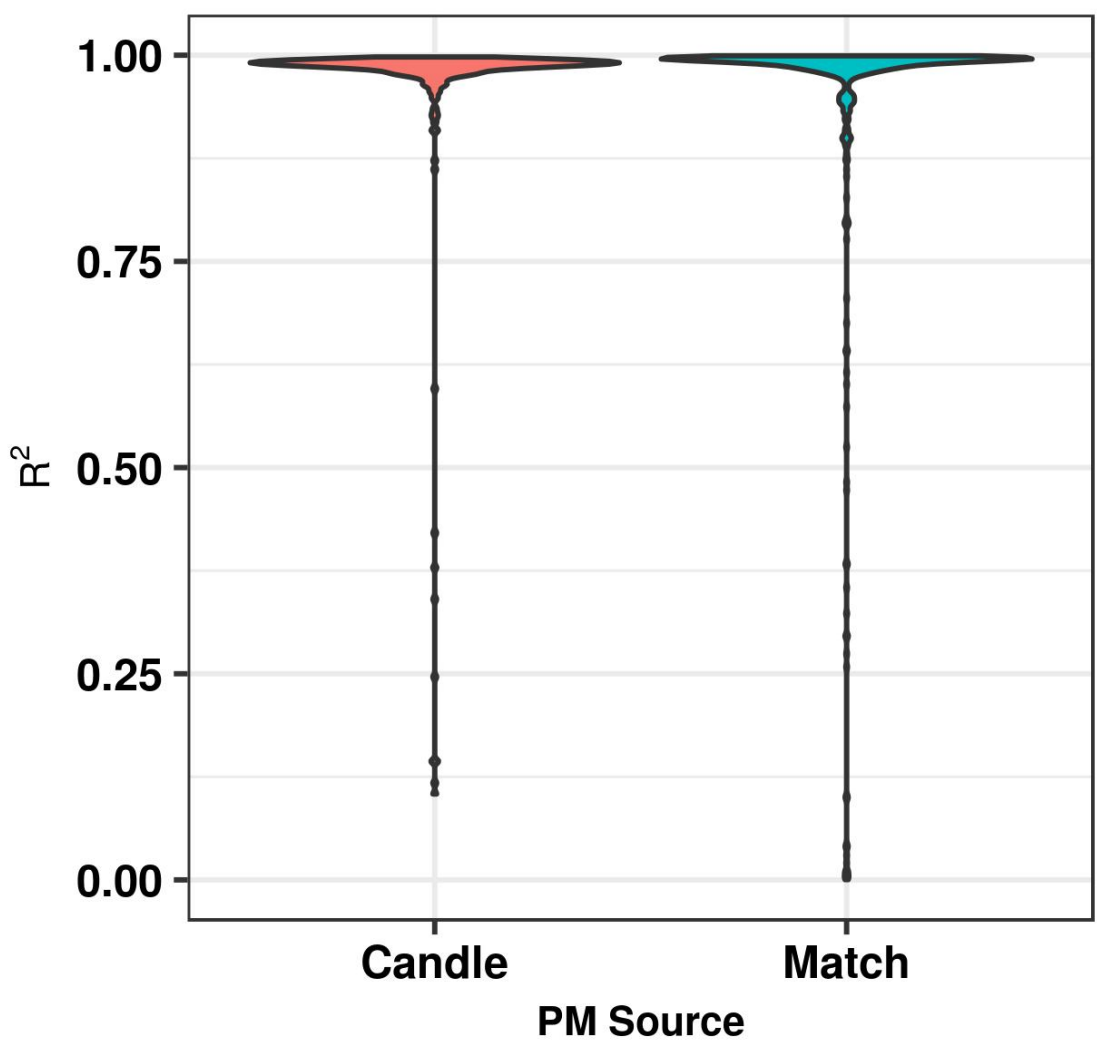

FIGURE 3.1: Distribution of $\mathrm{R}^{2}$ values from linear models comparing DustTrak reference instrument against PurpleAir monitors during laboratory evaluations. 


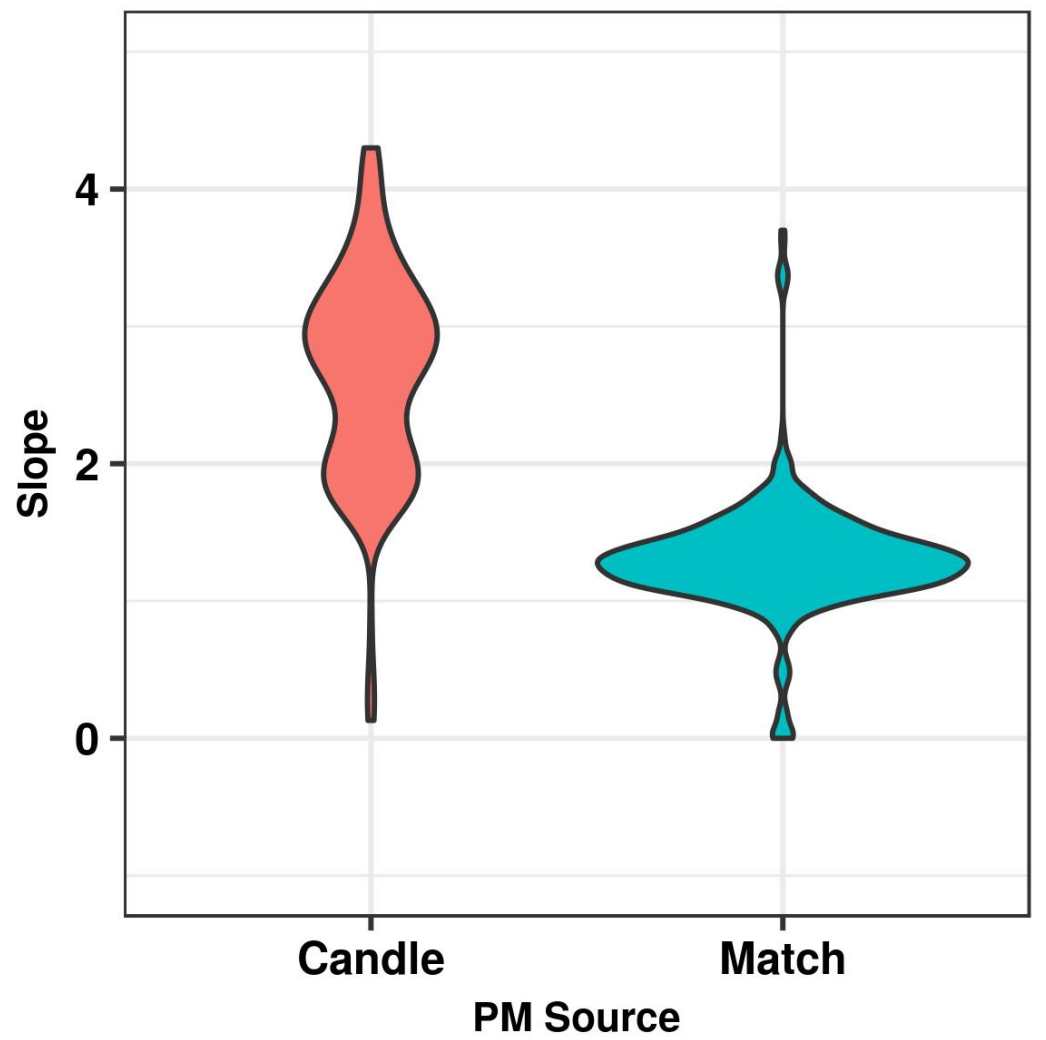

FIGURE 3.2: Distribution of Slope values from linear models comparing DustTrak reference instrument against PurpleAir monitors during laboratory evaluations. 


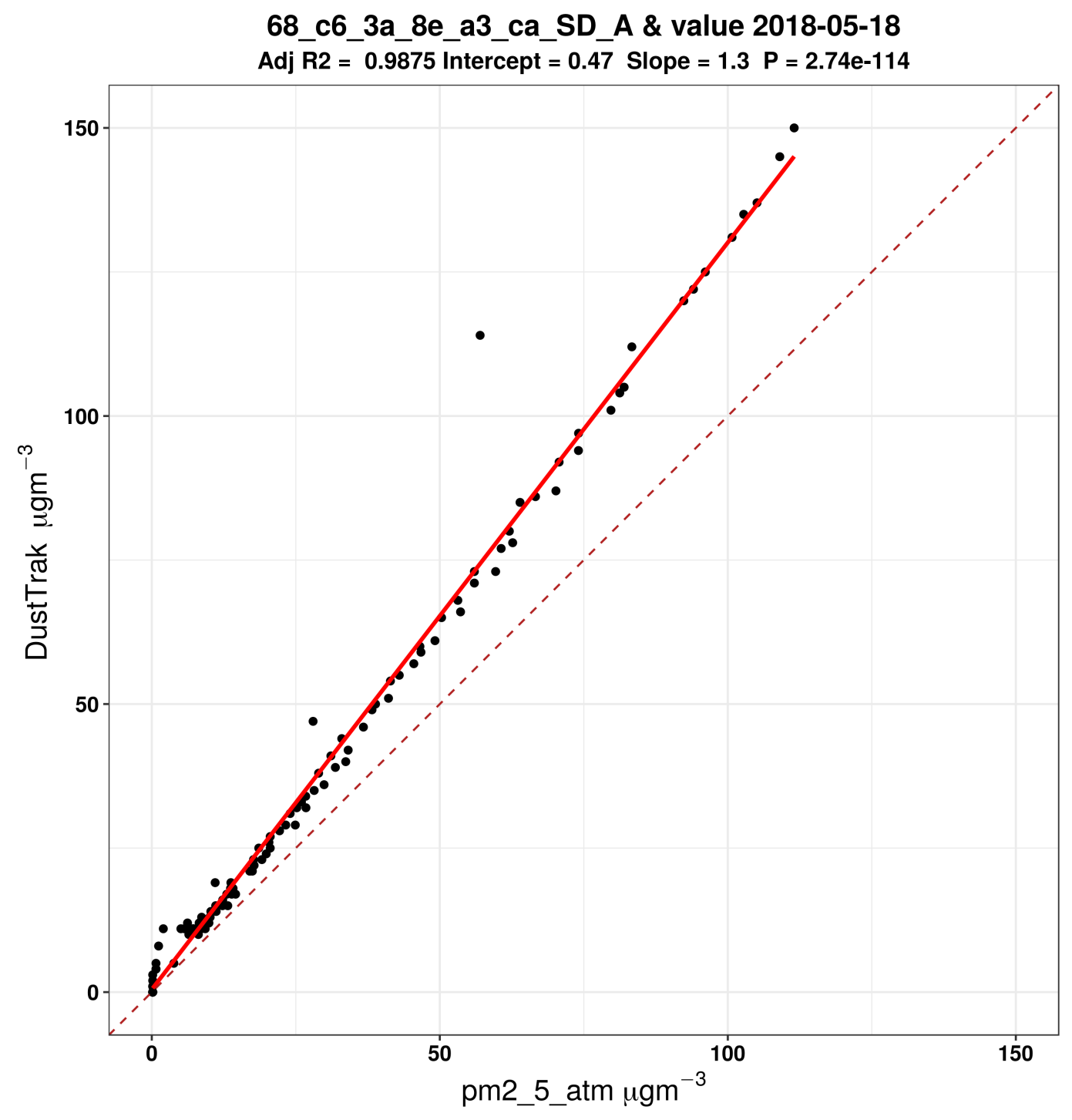

FIGURE 3.3: Scatter plot of laboratory evaluation comparing DustTrak reference instrument against PurpleAir primary sensor (Device ID: 68:C6:8E:A3:CA). The "pm2_5_atm" label refers to the $\mathrm{PM}_{2.5}$ mass-density channel of the PurpleAir. 


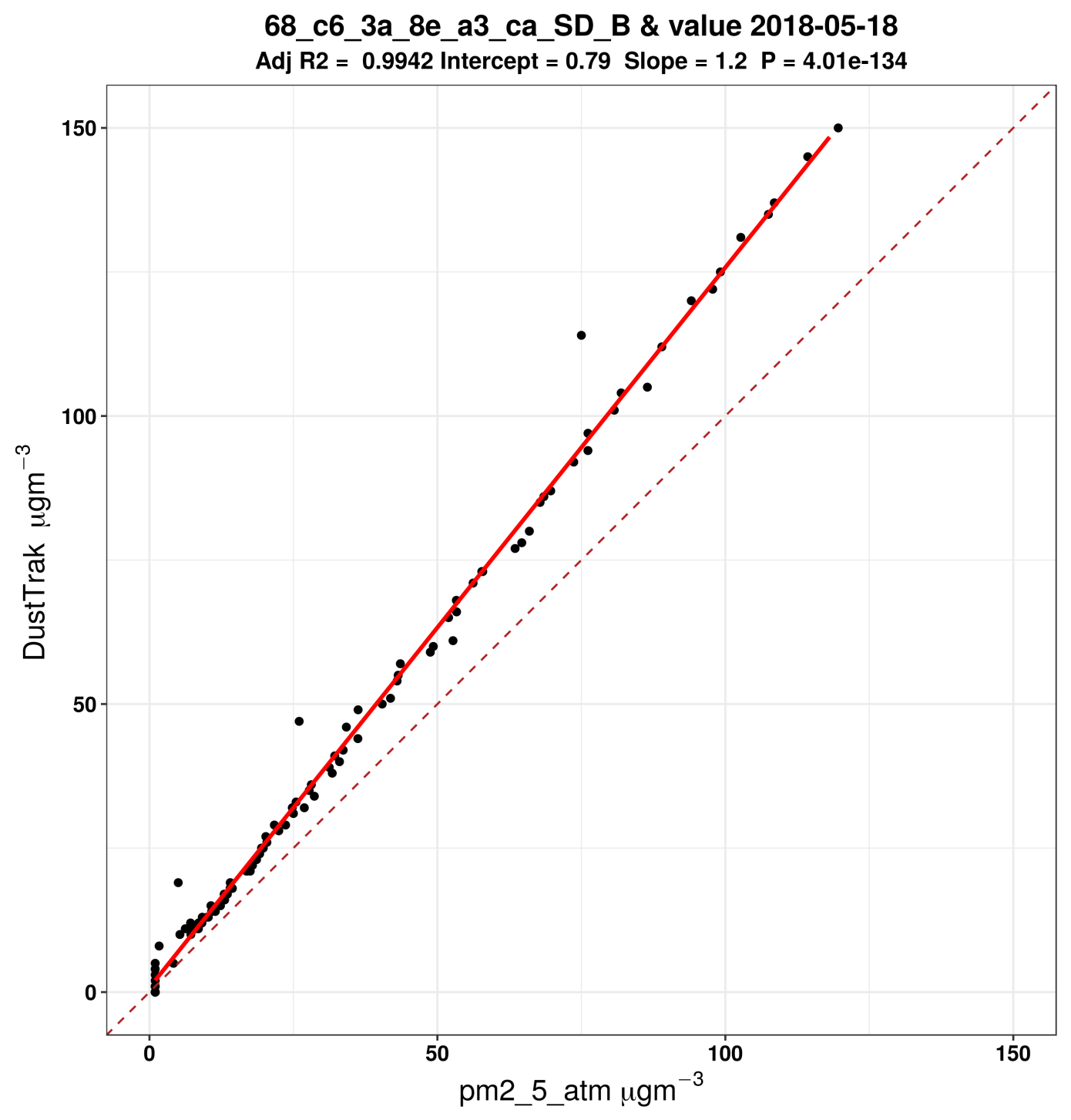

FIGURE 3.4: Scatter plot of laboratory evaluation comparing DustTrak reference instrument against PurpleAir secondary sensor (Device ID: 68:C6:8E:A3:CA). The "pm2_5_atm" label refers to the $\mathrm{PM}_{2.5}$ mass-density channel of the PurpleAir. 


\subsection{Opportunistic Ambient Calibration}

Based on the laboratory calibration findings, we sought a way to calibrate sensors in-situ with ambient particulate matter. Based on other studies (Eeftens et al., 2012; Zhou and Levy, 2007), $\mathrm{PM}_{2.5}$ levels can be mainly driven by regional sources thereby limiting small-scale variability. We tested the idea of calibrating the sensors during regional homogeneity events. Homogeneity was assessed using the 4-6 ODEQ nephelometers in the Portland metro area. These nephelometers have been calibrated against the federal reference method. Originally 5-minute resolution data, the nephelometers were aggregated to 15-minute means to identify periods when $\mathrm{PM}_{2.5}$ was determined to be regionally homogeneous (the relative standard deviation of all active nephelometers within the Portland metropolitan area was $\leq 10 \%)$. Once these periods were identified, linear models were generated for each sensor, and those with an $\mathrm{R}^{2}<0.90$, or those with $<100$ observations were excluded from the modeling phase (Appendix A: homogeneousPM.R). Violin plots were created to graphically assess the distribution of $\mathrm{R}^{2}$ and slope values during the field calibrations. The $\mathrm{R}^{2}$ values from the field evaluations were not as narrowly distributed below 1.0 relative to the laboratory evaluations, and considerable left-skew of the $\mathrm{R}^{2}$ distribution was observed. This suggests that the some sensors were affected by local PM sources that were otherwise not captured by the network of nephelometers. Figure 3.5 shows the distribution of $R^{2}$ for the 138 sensors with the nephelometers during homogeneity events. We chose an $\mathrm{R}^{2}$ of 0.90 as our quality filter. After applying the quality filter to the 138 unique sensors observed throughout 
the Portland network, a total of $61(48 \%)$ sensors were considered viable for the modeling phase. These 61 sensors corresponded to 35 individual locations. There were 26 nodes which both the primary and secondary sensors passed our quality standards, and there were 9 remaining nodes which only had a single passing sensor. The 35 site total was accumulated throughout the entire 2-year study period, but, on average, there were 20 active sites on any given day. The slopes of the 61 sensors were then graphically assessed via violin plot. The slopes from the field evaluations ranged between 0.31 and 0.80 , with a mean of 0.46 (Figure 3.6; Table 3.1). These coefficients are comparable to those determined by other field evaluations, where PurpleAir monitors were co-located with FRM/FEM instruments (AQ-SPEC South Coast AQMD, 2017a).

Scatter plots illustrating the relationship between primary and secondary sensors from a select PurpleAir node against the homogeneous mean $\mathrm{PM}_{2.5}$ ambient background concentration were included to highlight the observed intramodel variation in slopes (Figure 3.6). The 'STAR Lab Creston-Kenilworth' monitor was an extreme example, and exhibited a 30\% difference in slopes between the primary and secondary sensors, despite both sensors producing relatively high $R^{2}$ values. The primary sensor produced an $R^{2}$ of 0.970 , a slope of 0.45 , and a RMSE of 1.19. A mean $\mathrm{PM}_{2.5}$ range of 0-50 $\mu \mathrm{gm}^{-3}$ was observed from the nephelometer network during the evaluation period, with the majority of observations limited to $0-25 \mu g m^{-3}$. The secondary sensor yielded an $\mathrm{R}^{2}$ of 0.967 , a slope of 0.31 , and a RMSE of 1.25 , and encompassed a similar range in nephelometer PM as the primary sensor. Model intercepts were 1.3 and 1.2 $\mu \mathrm{gm}^{-3}$ for the primary and secondary sensor respectively (Figure 3.7; Figure 
$3.8)$.

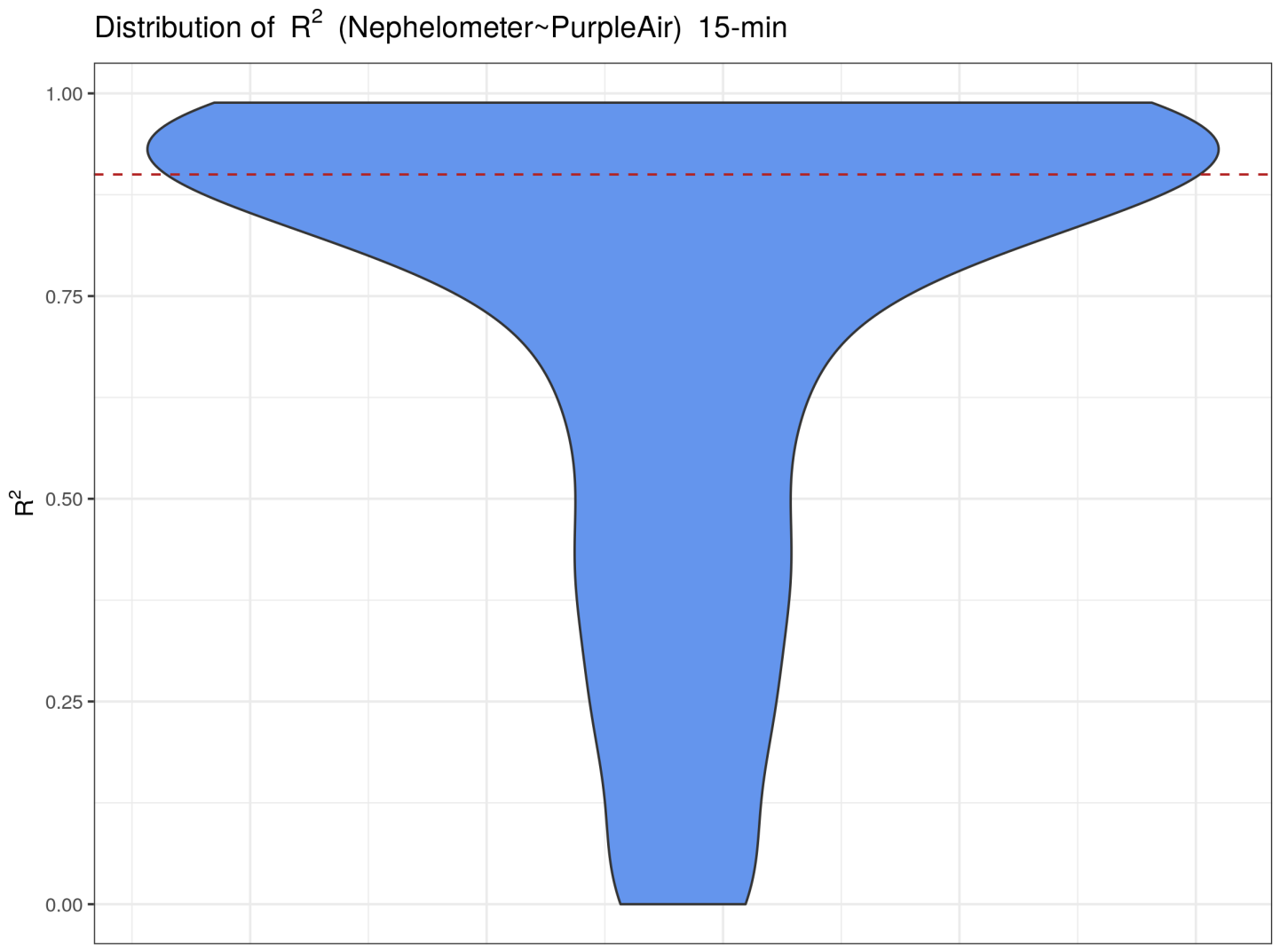

FIGURE 3.5: Distribution of $\mathrm{R}^{2}$ values from linear models comparing PurpleAir network to ODEQ nephelometer-PM during 15minute periods when PM was regionally homogeneous (Relative Standard Deviation $\leq 10 \%$ 


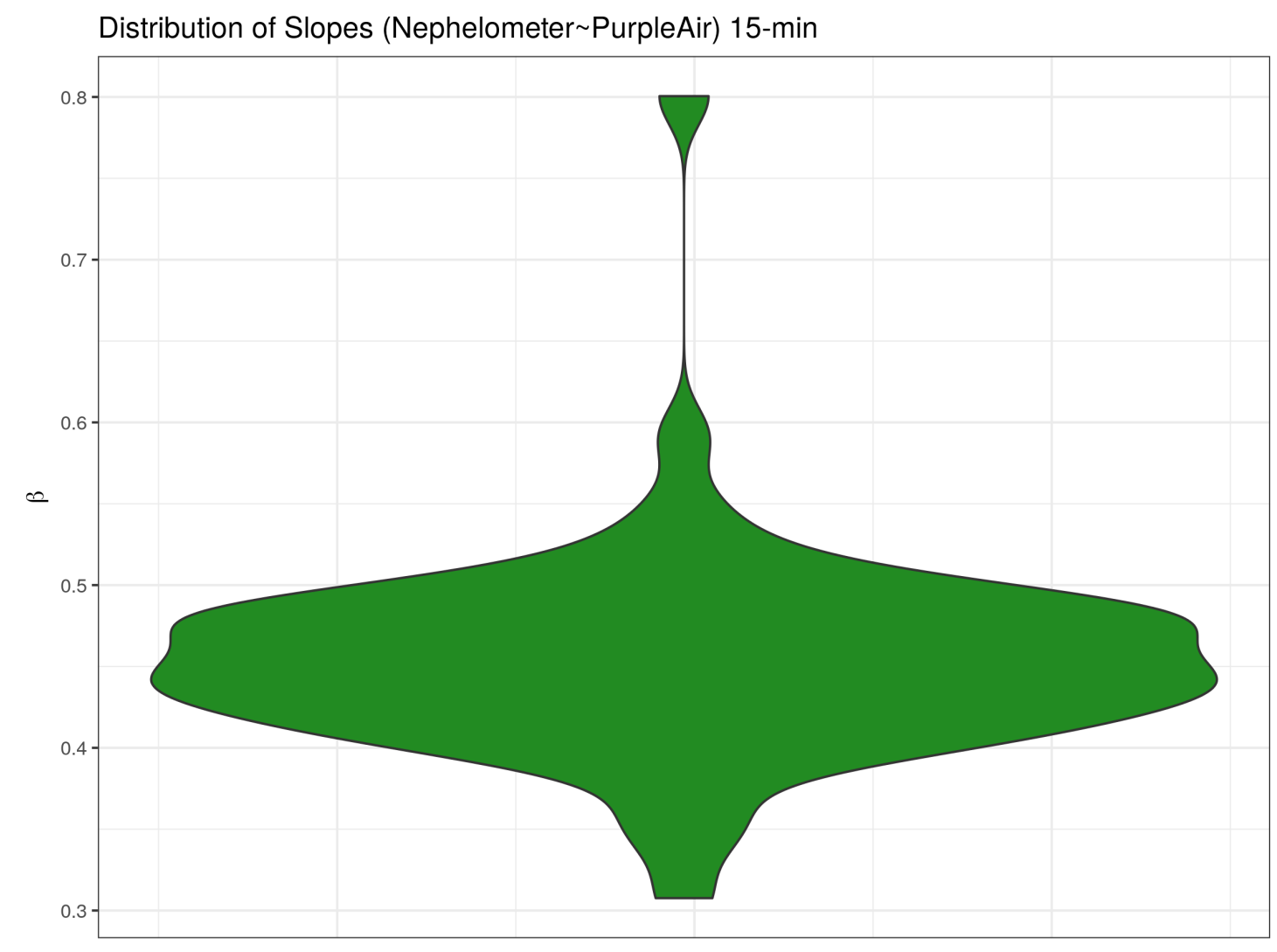

FIGURE 3.6: Distribution of slope values from linear models comparing PurpleAir network to ODEQ nephelometer-PM during 15minute periods when PM was regionally homogeneous (Relative Standard Deviation $\leq 10 \%$. Only the PurpleAir monitors with an $R^{2} \geq 0.90$ were included within this plot. 


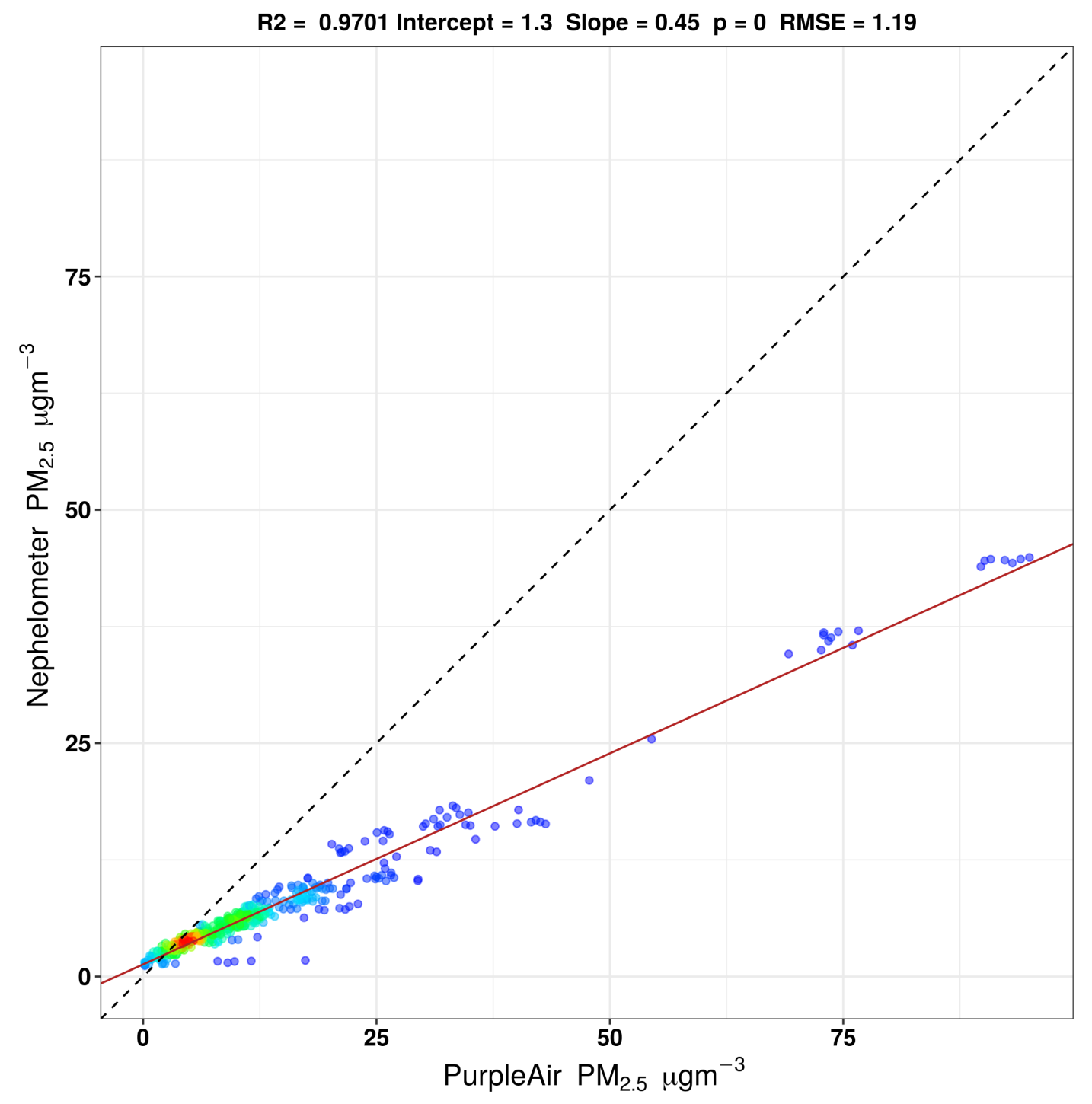

FIGURE 3.7: Scatter plot of a select field evaluation comparing mean regional Nephelometer $\mathrm{PM}_{2.5}$ against PurpleAir primary sensor (STAR Lab Creston-Kenilworth). The relative point density is captured by the colorscale. 


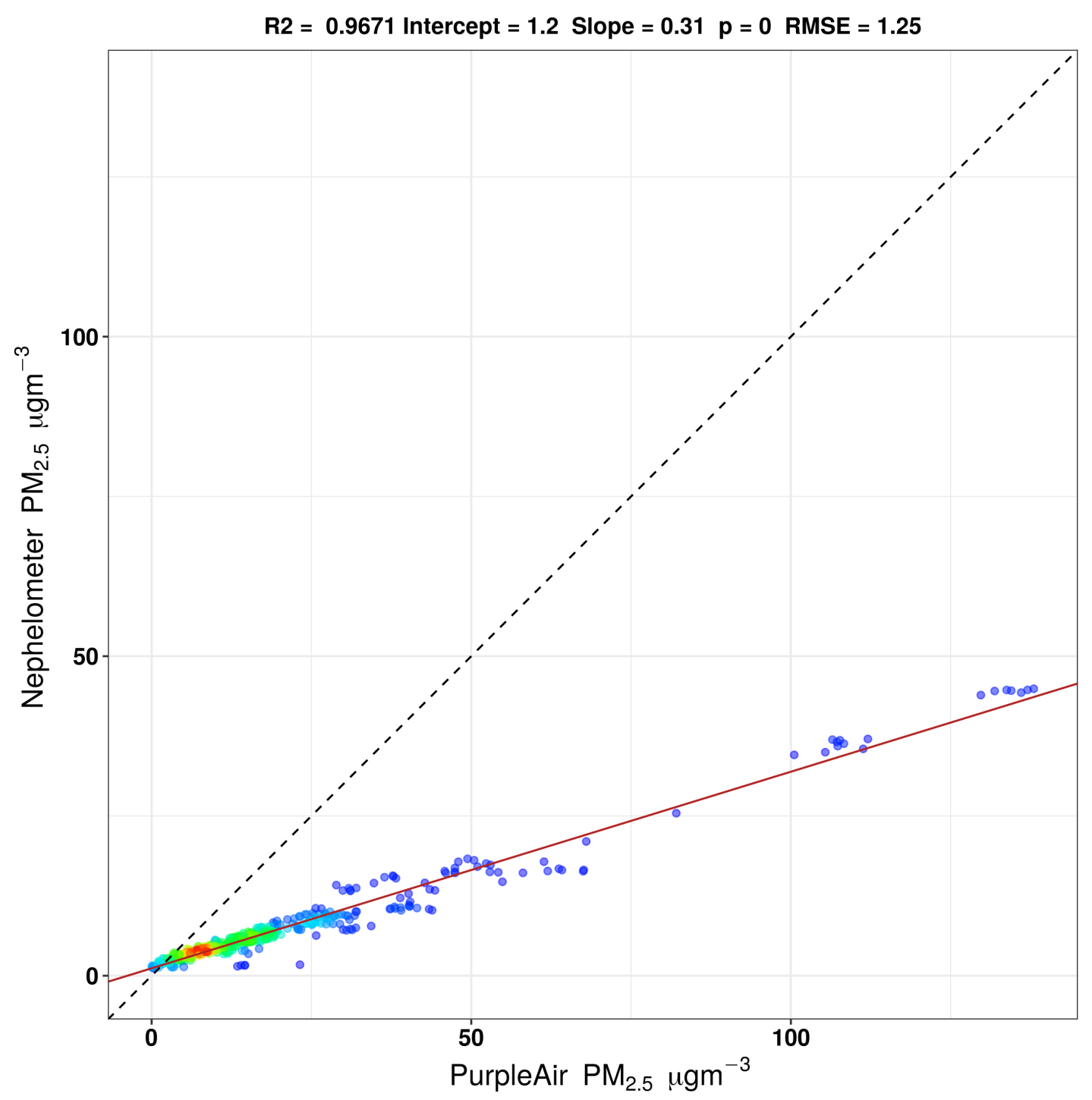

FIGURE 3.8: Scatter plot of a select field evaluation comparing mean regional Nephelometer $\mathrm{PM}_{2.5}$ against PurpleAir secondary sensor (STAR Lab Creston-Kenilworth). The relative point density is captured by the colorscale. 


\subsection{Predictor Selection}

The goal of the modeling effort is to predict $\mathrm{PM}_{2.5}$ at high spatial resolution and identify the land-use or physical drivers of variability. The next step for model development was to identify variables for the inclusion in the model. Purposeful variable selection was performed partly by software and partly by hand. We relied on previous literature and our understanding of the physical properties of $\mathrm{PM}_{2.5}$ to derive our set of proxies for expected sources and sinks (Eeftens et al., 2012; Rao et al., 2014; Brokamp et al., 2017; Saucy et al., 2018; Wang and Ogawa, 2015; Chudnovsky et al., 2014). A list of all initial predictors that were considered are outlined in Table 2.1. Only a single buffer for each spatial predictor was selected for the subsequent modeling phases to avoid issues regarding multicollinearity. We then narrowed down our selected predictors by exploring clusters which covaried. Finally, a stepwise Akaike Information Criterion (AIC) reduction method was employed to further narrow the set of predictors into a final parsimonious model, referred to as the 'final model'. We relied on the hybrid selection method, which is a combination of both forward and backward selection techniques (Venables and Ripley, 2002). Of the 28 predictors initially considered, only 9 were included within our final explanatory model. It is worth noting that the spatial and spatiotemporal variables each had multiple buffer distances, and the total number of initial predictors was considerably higher than 28 prior to the selection of a single buffer per variable. The 9 final predictors were selected based on correlation strength with the response variable, VIF scores below 5, intuitive signs of model coefficients (i.e. potential sinks 
have negative coefficients, and potential sources have positive coefficients), and coefficient p-values below 0.10 . The majority of the VIF scores of the predictors within the final model were below 2 . The 'Season' variable exhibited the highest VIF score of 3.50. This was followed by a score of 2.93 for the maximum daily temperature ('tmmx.50m') and 2.16 for daily mean wind speed ('vs.50m').

TABLE 3.2: Summary table of the VIF scores from the final model predictors.

\begin{tabular}{cccc}
\hline \hline & GVIF & Df & GVIF $^{\wedge}(1 /(2 *$ Df $))$ \\
\hline Median Nephelometer $\mathrm{PM}_{2.5}$ & 1.163 & 1 & 1.078 \\
Elevation $(50 \mathrm{~m})$ & 1.824 & 1 & 1.351 \\
Minimum PBL Height & 1.923 & 1 & 1.387 \\
Maximum Relative Humidity (50 m) & 1.466 & 1 & 1.211 \\
Maximum Temperature (50 m) & 2.934 & 1 & 1.713 \\
Mean Wind Speed (50 m) & 2.160 & 1 & 1.470 \\
Developed Medium Intensity (100 m) & 1.900 & 1 & 1.378 \\
Season & 3.497 & 3 & 1.232 \\
Longitude & 1.167 & 1 & 1.081 \\
\hline
\end{tabular}

\subsection{Leave-One-Out-Cross-Validation}

Before conducting the LOOCV with the LUR model, the total number of observations for each PurpleAir node (both primary and secondary sensors) was determined. Nodes with fewer than 100 observations were excluded from the LOOCV modeling phase to prevent individual model performance from being unduly influenced by outliers. Overall, 32 of the 34 PurpleAir nodes had more than 100 observations and were used for LOOCV (the primary and secondary sensor data from each node were aggregated). There were 20 nodes which were 
established earlier on in the study and captured more than 200 daily observations (Figure 3.9).

The mean $\mathrm{R}^{2}$ from these $32 \mathrm{LOOCV}$ models was 0.87 , and ranged between 0.73 and 0.97. The interquartile range in $\mathrm{R}^{2}$ was between 0.84 and 0.90 . Model RMSE ranged between 1.31 and $3.71 \mu \mathrm{gm}^{-3}$. Model slopes ranged between 0.67 - 1.06 with a mean of 0.87 , and the mean intercept was approximately 0 for most models (Table 3.3). The two nodes which produced LOOCV $\mathrm{R}^{2}$ below 0.80 ('Haig St', 'Upper Multnomah Village') each had fewer than 200 observations. Differences between LOOCV $R^{2}$ and final model $R^{2}$ were typically below $10 \%$, indicating overall model stability. 


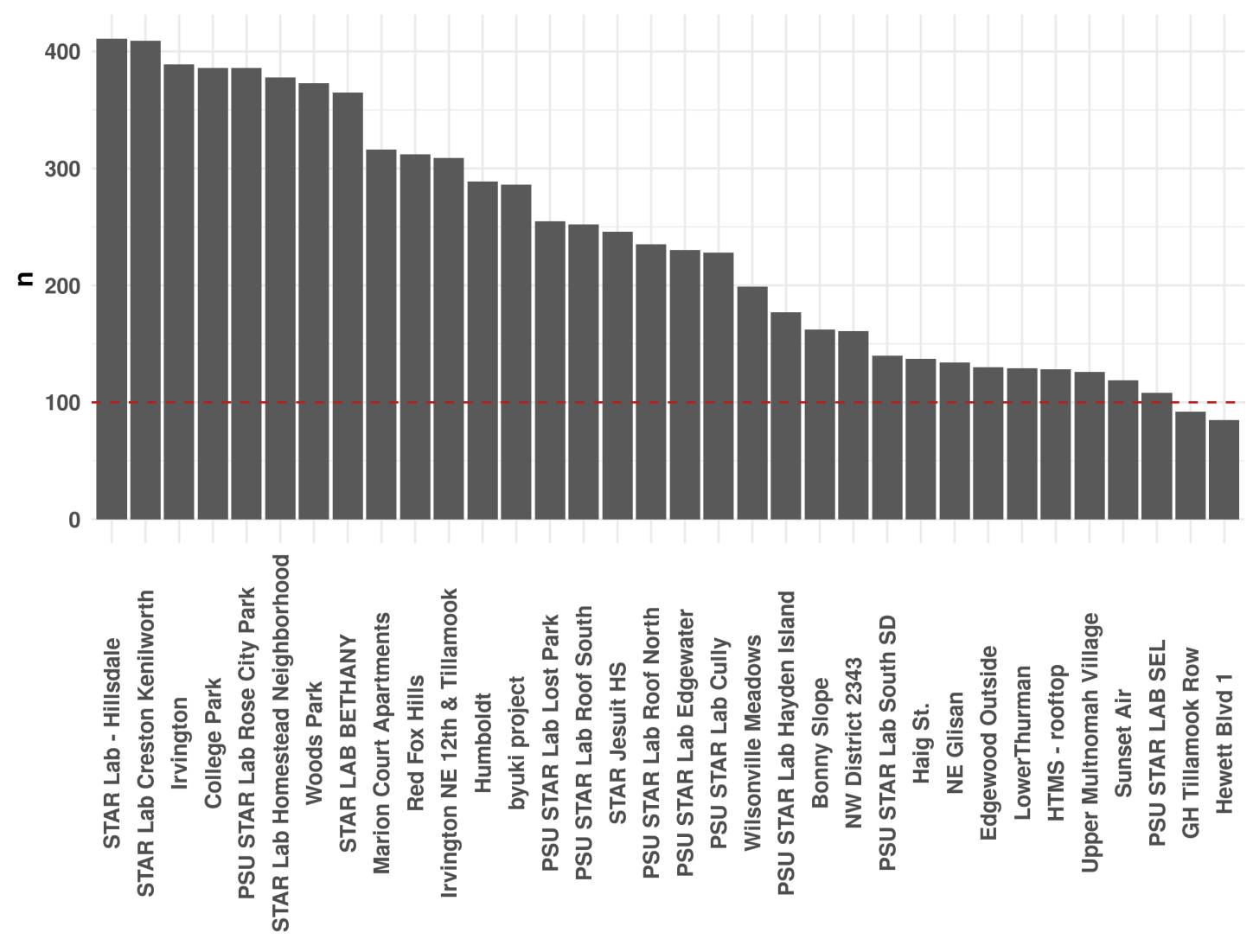

FIGURE 3.9: A bar plot assessing the number of observations for each PurpleAir monitor used during LOOCV. Nodes with fewer than 100 observations (bars below the dotted red line) were not included during the LOOCV.

\subsection{Model Validation}

Individual predictions from each of the $n-1$ LUR models developed during the LOOCV phase were aggregated to produce a mean ensemble prediction. This ensemble prediction was then compared against the 'test' data (the 30\% random subset of the corrected PurpleAir data held out during the model training 


\begin{tabular}{|l|c|c|c|c|}
\hline Stat & $\mathrm{R}^{2}$ & RMSE & Slope & Intercept \\
\hline Min & 0.73 & 1.31 & 0.67 & -1.23 \\
$1^{\text {st }}$ Quartile & 0.84 & 1.64 & 0.82 & 0.73 \\
Median & 0.88 & 2.00 & 0.88 & 0.91 \\
Mean & 0.87 & 2.04 & 0.87 & 0.95 \\
$3^{\text {rd }}$ Quartile & 0.90 & 2.29 & 0.94 & 1.30 \\
Max & 0.97 & 3.71 & 1.06 & 2.64 \\
\hline
\end{tabular}

TABLE 3.3: Summary statistics from the LOOCV phase. A validation regression was generated with each LOOCV model and the 'test' data to evaluate model stability.

phase) to assess overall model performance. Specifically, a validation regression was generated, modeling the observed $\mathrm{PM}_{2.5}$ from the 'test' data as a function of the LUR predictions. The LUR model explained $0.89 \%$ of the variance in the observed data and had a root mean squared error (RMSE) of $\pm 2.39 \mathrm{\mu gm}^{-3}$. The slope of this model was 0.98 , which suggests that the model slightly underpredicts the corrected PurpleAir 'test' data. Despite the good model fit, a graphical assessment of the residuals shows that the model appears to perform better at lower PM concentrations, where more training data was available. While a disproportionate number of days with low pollution should generally be considered a benefit, for modeling purposes this left room for improvement when predicting $\mathrm{PM}_{2.5}$ above the $20 \mu \mathrm{gm}^{-3}$ threshold. At lower concentrations, between 0-20 $\mathrm{ggm}^{03}$, the residuals were within the $\pm 5 \mu \mathrm{gm}^{-3}$ range. However, LUR model performance appeared to decline as the predicted range exceeded this threshold. There are instances where the model both overpredicts and underpredicts $\mathrm{PM}_{2.5}$, and this was only exacerbated when concentrations are above $20 \mathrm{\mu gm}^{-3}$ (Figure 3.10). Instances when the observed $\mathrm{PM}_{2.5}$ levels were much 
higher than the predicted levels suggest days when the regional monitoring network was unable to capture some local PM source at a given PurpleAir node.

The test assumptions of the LUR model were graphically assessed and can be viewed in Figure 3.11. The 'Residuals vs Fitted' panel shows the red trendline appears to approximate the zero line, which indicates that the linearity assumption is being met. The 'Normal Q-Q' panel shows that the residuals are normally distributed between the -2 and 2 theoretical quantiles, but the normality assumption is not met across the entire range of model residuals. The 'ScaleLocation' panel shows the red trendline increasing as the fitted values increase. This means that the LUR model does not meet the homoscedascity assumption. However, since we were mostly interested in our ability to predict $\mathrm{PM}_{2.5}$ concentrations that typically occur within the urban environment $\left(0-20 \mu g m^{-3}\right)$, we were willing to move forward with the model prediction phase, acknowledging that the spatial variability of exceptionally high PM events (e.g. forest fires) must be interpreted with caution. Finally, the 'Residuals vs Leverage' panel is used to evaluate potenitally influential outliers that located beyond Cook's distance. Despite our lack of normally distributed residuals, no data points were observed to exceed this distance.

In addition to the LUR model, we explored using a random forest (RF) model for its expectedly higher predictive power and its ability to account for some of the limitations inherent to LUR. Land use regression models have difficulty capturing non-linear relationships and complex interactions, but random forests (RF) are resistant to these problems. Random forests are often preferred 
in prediction analyses due to their increased accuracy and resistance to multicollinearity and complex interactions relative to linear regression (Brokamp et al., 2017; Hastie et al., 2005). Our RF model performed slightly better than the LUR model, despite the computational limitations which prohibited an ensemble approach. This single model predicted $0.93 \%$ of the variance in the observed data and had a RMSE of $\pm 1.84 \mu \mathrm{gm}^{-3}$, or $23 \%$ lower than the LUR model (Figure 3.12). The slope of this CV model was 1.0, and the intercept was near zero (-0.07). The residuals from the RF model also appear to be more tightly grouped along the 1:1 line relative to the LUR model. There were still some persistent outliers observed in the residuals of this model. Despite the promising summary statistics generated during the random forest model validation phase, we encountered some limitations while developing spatial predictions and with overall model interpretability that prevented us from relying on this technique instead of LUR. The limitations that we experienced with RF will be discussed in Section 3.9. 


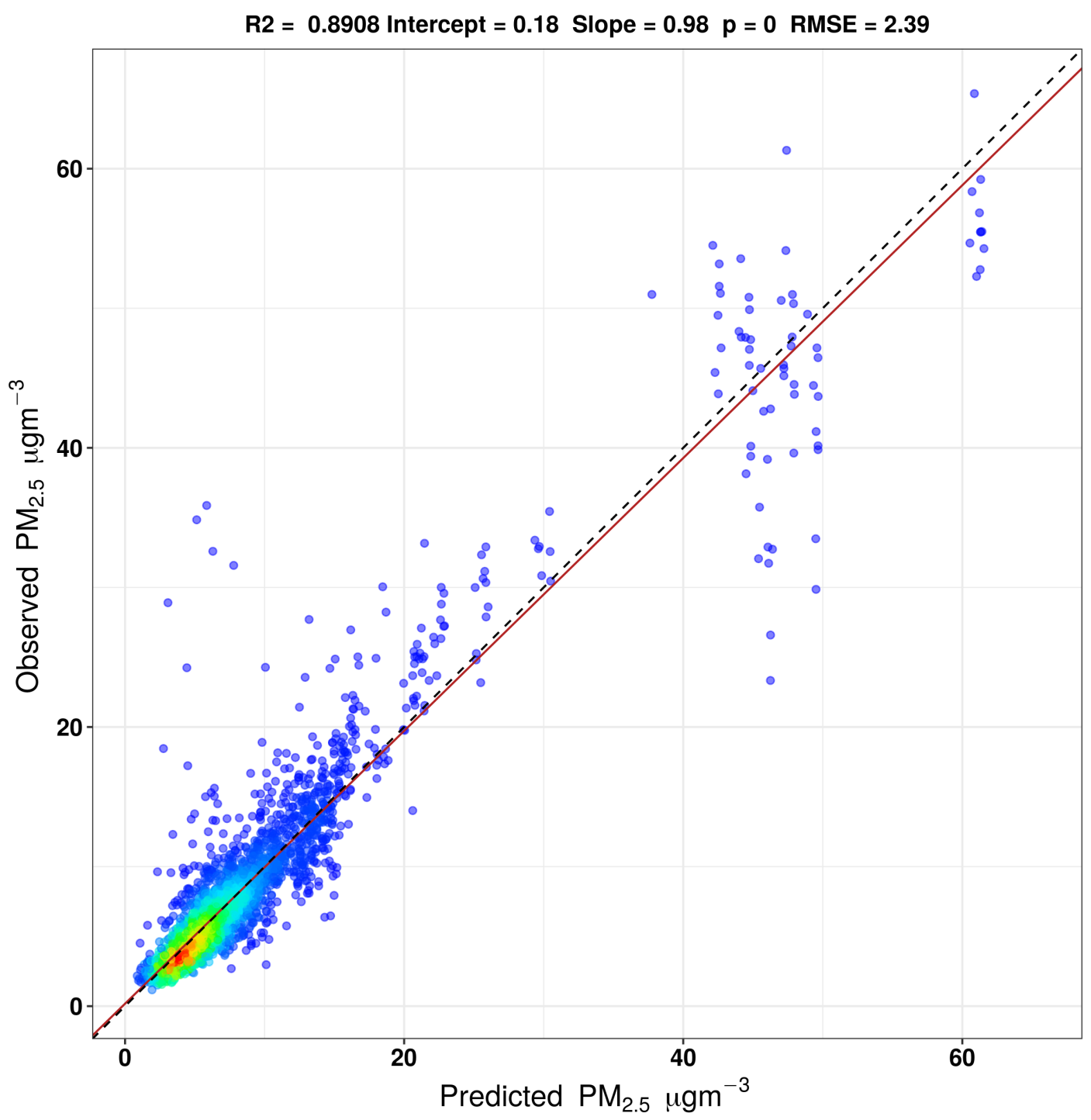

FIGURE 3.10: Scatter plot comparing the observed 30\% holdout data as a function of the LUR mean ensemble predicted $\mathrm{PM}_{2.5}$. 

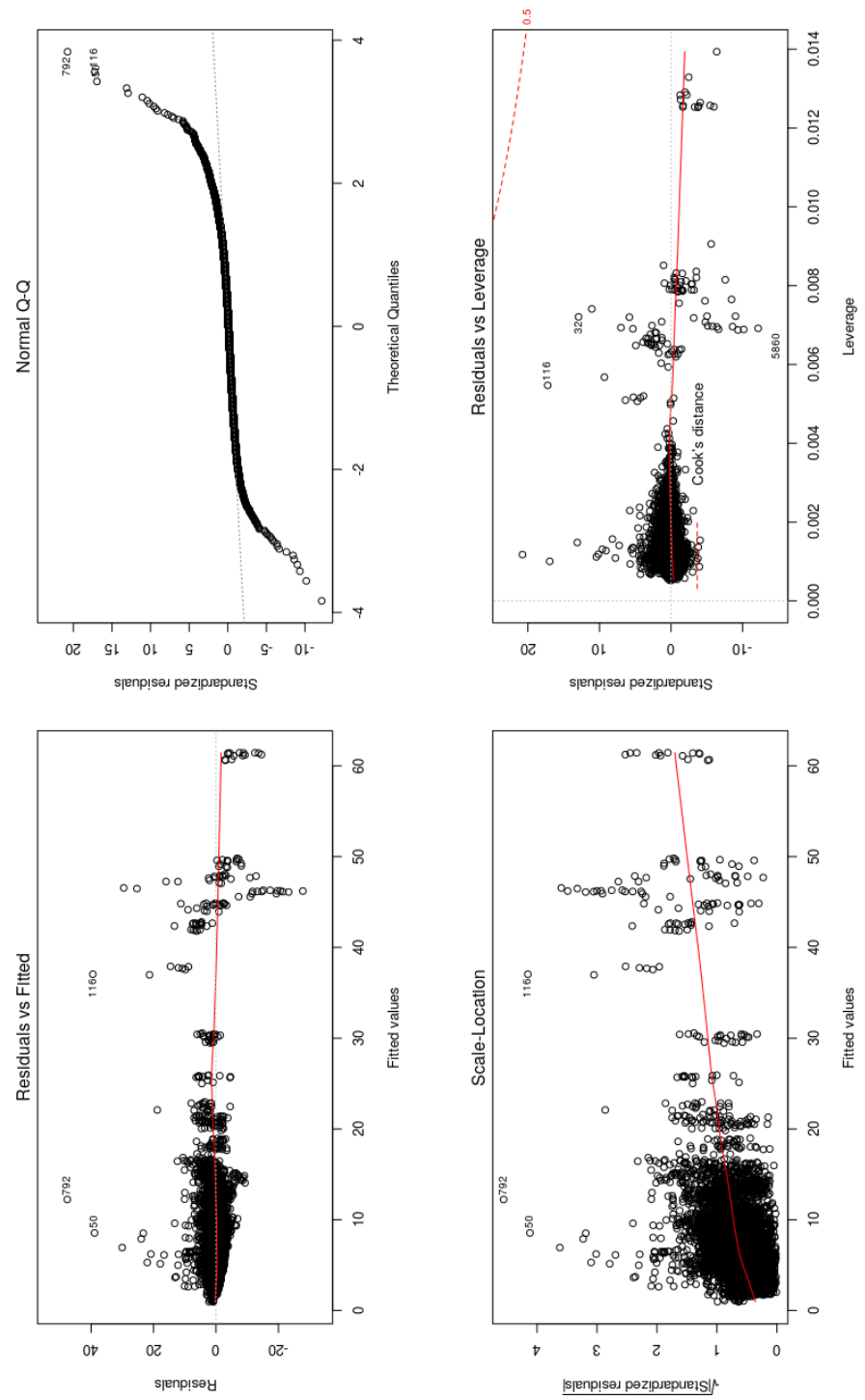

FIGURE 3.11: A graphical assessement of the LUR model's ability to meet multiple linear regression test assumptions. 


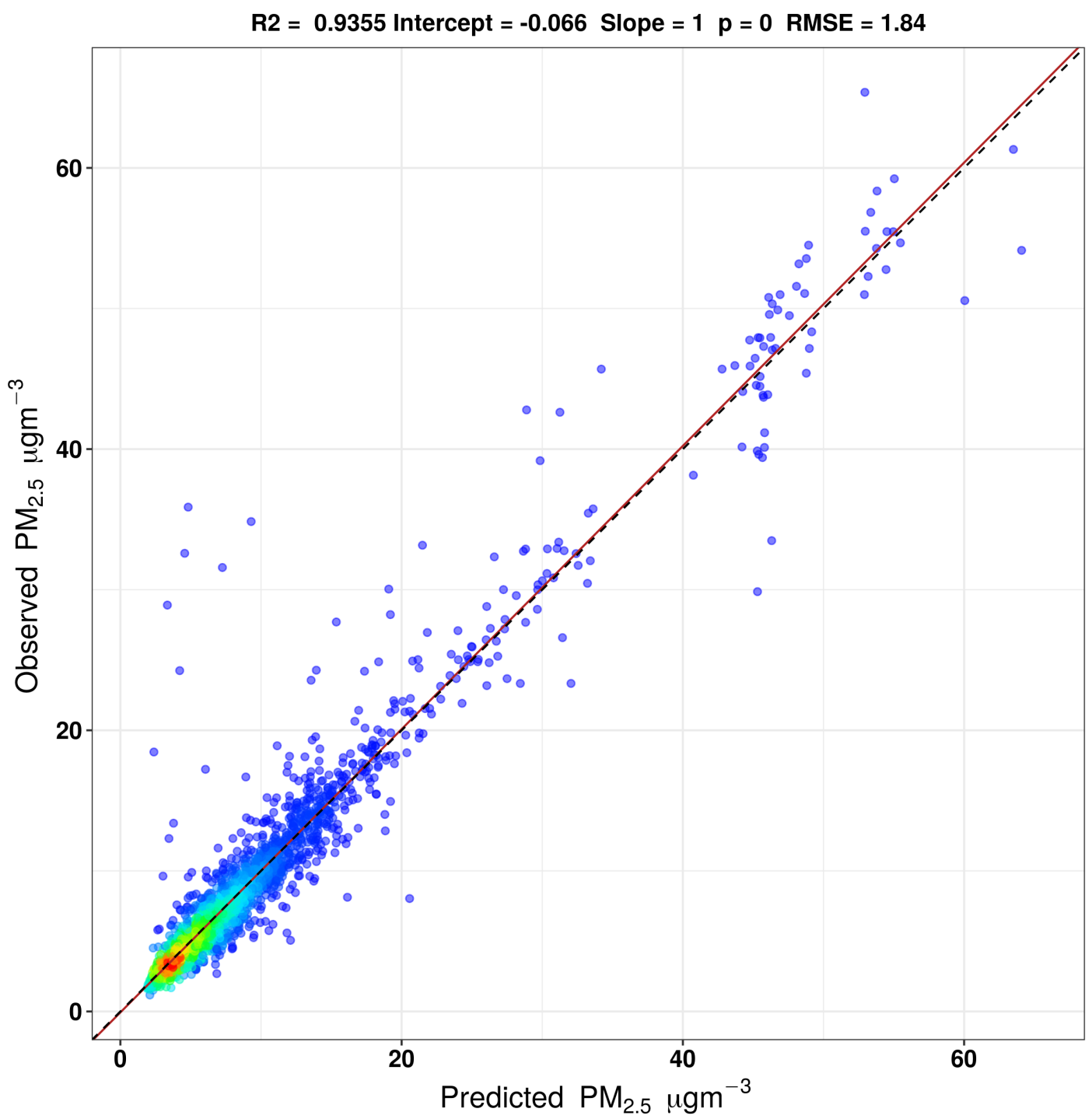

FIGURE 3.12: Scatter plot comparing the observed 30\% holdout data as a function of the Random Forest predicted $\mathrm{PM}_{2.5}$ 


\subsection{Standardized Regression Coefficients}

\subsubsection{Temporal Predictors}

\section{Nephelometer $\mathbf{P M}_{2.5}$}

The final model included the median $\mathrm{PM}_{2.5}$ of the nephelometer network (the ODEQ regional monitoring network), elevation, the minimum planetary boundary layer height, the maximum relative humidity and temperature, mean wind speed, developed medium intensity LULC, season, and longitude. The standardized regression coefficients (betas) of the final LUR model show that the median nephelometer $\mathrm{PM}_{2.5}$ value ('pm2.5.median') had the strongest association with $\mathrm{PM}_{2.5}$ concentrations observed from the PurpleAir network. A one standard deviation increase in median nephelometer $\mathrm{PM}_{2.5}$ corresponded to a 0.908 increase in standard deviation of the PurpleAir network. The unstandardized coefficient for the median nephelometer $\mathrm{PM}_{2.5}$ indicates a 0.834 increase in PurpleAir $\mathrm{PM}_{2.5}$ for every $1.0 \mu \mathrm{gm}^{-3}$ increase in the ambient background concentration. The daily median nephelometer $\mathrm{PM}_{2.5}$ concentration can be interpreted as a temporal scaling factor, and the variation in the PurpleAir network can largely be explained simply by the variation in the ambient background concentration. This was not surprising, as Karner et al. previously identified little to no spatial variation with intra-urban $\mathrm{PM}_{2.5}$ (Karner, Eisinger, and Niemeier, 2010). Instead, meteorology mostly drives the temporal variation, while the spatial variability of this pollutant typically occurs at regional scales (Table 3.4). The association of the remaining 8 predictors and $\mathrm{PM}_{2.5}$ was much weaker, each with beta values below 0.10 . 


\section{Planetary Boundary Layer Height}

The minimum planetary boundary layer height ('pbl.min') was shown to have the $7^{\text {th }}$ highest beta value. An increase in one standard deviation in minimum PBL height resulted in a 0.015 decrease in the standard deviation of our response network. This variable corresponds to the minimum atmospheric volume available for dilution of urban sources. PBL was primarily included to capture days when meteorological conditions were stable, and temperature inversions were expected to trap surface-emitted $\mathrm{PM}_{2.5}$, resulting in elevated concentrations during the cold season (Table 3.4). It was surprising that this variable did not have as strong an association with $\mathrm{PM}_{2.5}$ as some of the other temporal predictors (e.g. wind speed and maximum daily temperature).

\section{Season}

A categorical 'Season' variable was included to account for recurring seasonal patterns in $\mathrm{PM}_{2.5}$ variation over the 2 year study period. While the the fall season had the fourth highest beta coefficient in the final model, the other two seasons (spring and summer) had some of the lowest associations with the PurpleAir response network (Table 3.4).

Another categorical variable was considered during the initial predictor selection phase to account for patterns in human activity (traffic, area sources) on weekends versus weekdays. This variable was also included to account for the lack of traffic intensity data that has been widely used throughout previous LUR studies. Weekdays were expected to have higher $\mathrm{PM}_{2.5}$ levels due to increase traffic intensity during the work week commute relative to weekends. 
However, this variable was not determined to be significant during the LUR model development.

\subsubsection{Spatiotemporal Predictors}

\section{Temperature}

The daily maximum temperature at a $50 \mathrm{~m}$ resolution had the second highest beta value. A one standard deviation increase in maximum daily temperature at $50 \mathrm{~m}$ ('tmmx.50m') resulted in a 0.073 decrease in standard deviation of the $\mathrm{PM}_{2.5}$ levels from our response network. Several potential proxies were considered with the inclusion of temperature data in the final LUR model. On the one hand, higher temperatures correspond with a larger mixing volume, providing a surrogate for increased dilution. However, on the other hand, higher temperatures also enable higher rates of secondary organic aerosol formation, potentially leading to higher $\mathrm{PM}_{2.5}$ levels in our response network (Wang and Ogawa, 2015). Additionally, higher temperatures are also associated with the wild fire season within the Portland, Oregon, and some of the most polluted days observed within the training data occurred during the warm season. Despite the uncertainties regarding which proxy would potentially drive the association between temperature and $\mathrm{PM}_{2.5}$, we chose to include 'tmmx.50m' in the final LUR model due to its coefficient's significance. Here, we can interpret this negative beta coefficient to be due to the influence from wildfires, and perhaps slightly by SOA formation. It is worth noting that although temperature was the second most important predictor its beta was only $8 \%$ of the nephelometerderived $\mathrm{PM}_{2.5}$ beta (Table 3.4). In other words, the majority of the variation in 
our PurpleAir data was explained by the ambient background concentration of the nephelometers.

\section{Wind Speed}

Wind speed was identified to be somewhat associated with $\mathrm{PM}_{2.5}$, and had the $3^{\text {rd }}$ highest beta coefficient. This predictor was included as a surrogate for dilution, as $\mathrm{PM}_{2.5}$ from local sources are readily removed from the urban environment was wind speeds increase. As this predictor increased by one standard deviation, the response variable was observed to decrease by 0.063 standard deviations (Table 3.4). During data compilation, wind direction was determined to be too coarse of a spatial resolution and was excluded from the modeling phase.

\section{Relative Humidity}

The maximum relative humidity at $50 \mathrm{~m}$ ('rmax') was shown to be one of the least associated predictors with the PurpleAir $\mathrm{PM}_{2.5}$. An increase of one standard deviation of this variable was associated with only a 0.011 decrease in the response network. Similar to the maximum temperature layer, relative humidity was included to account for multiple potential affects on $\mathrm{PM}_{2.5}$ concentrations. Generally, when relative humidity increases, $\mathrm{PM}_{2.5}$ mass density is observed to also increase due to hygroscopic growth. However, after a certain relative humidity threshold, $\mathrm{PM}_{2.5}$ levels have been observed to decline as hygroscopic growth produces particles which are too large to remain suspended in 
the air, ultimately depositing (Wang and Ogawa, 2015). Despite these uncertainties, this variable was included within the final LUR model due to its significant p-value (Table 3.4).

\subsubsection{Spatial Predictors}

\section{Elevation}

Elevation at a $50 \mathrm{~m}$ resolution ('elev.50m') was shown to be the most influential spatial predictor within the final LUR model. Most of the spatial variation observed in the model predictions approximated this elevation layer. This predictor had the $5^{\text {th }}$ highest beta value, and an increase by one standard deviation in elevation resulted in a 0.03 decrease in standard deviation of our response network. This variable was a proxy for dilution, as wind speeds are typically higher at higher elevations and less shielded by surface roughness. The inclusion of this variable was an effort to capture some of the finer-scale topographical effects on $\mathrm{PM}_{2.5}$ that were unaccounted for by the GridMET wind speed product. The majority of primary urban $\mathrm{PM}_{2.5}$ is emitted near the surface, and can become trapped at lower elevations during stable meteorological conditions.

\section{Developed Medium Intensity LULC}

The developed medium intensity LULC variable at the $100 \mathrm{~m}$ buffer size ('med_dev' or 'developed_medium_intensity.100m') was shown to have the $6^{\text {th }}$ highest beta. A one standard deviation increase in medium developed LULC corresponded to a 0.023 increase in standard deviation of the response network. In other words, the more medium developed LULC observed within $100 \mathrm{~m}$ of 
a given PurpleAir site, the more likely $\mathrm{PM}_{2.5}$ levels were to be slightly higher. This variable was included as a proxy for area sources in effort to characterize the magnitude, however limited, of fine-scale $\mathrm{PM}_{2.5}$ gradients. While the ASTER population density layer was originally prioritized over the NLCD predictors as general proxy for all area sources, its coefficient was determined to be insignificant. The inclusion of medium density development LULC was considered only after the population density layer was determined to no longer be a significant predictor. Surprisingly, during the beginning of the predictor selection phase, high density developed land use was not positively associated with $\mathrm{PM}_{2.5}$ across the range of buffer sizes, despite the expectation that more area sources would occur within this LULC class. Also, low density developed land use did initially have a positive correlation with our response network, but this variable did not yield a significant coefficient $p$-value during the first round of modeling. (Table 3.4). While no traffic-related predictors were included within the final model, graphically speaking, the medium density LULC layer appears to approximate the density of major roads throughout the region.

\section{Longitude}

Longitude ('lon') was the second least associated with the PurpleAir response network. A one standard deviation increase in longitude resulted in a 0.013 increase in the standard deviation of $\mathrm{PM}_{2.5}$ (Table 3.4). This suggests that the variability of various $\mathrm{PM}_{2.5}$ sources and sinks have only a weak association with longitude throughout the Portland, Oregon study area. 
TABLE 3.4: Coefficients (1) and standardized (beta) coefficients (2) of the final model with standard error in parentheses

\begin{tabular}{|c|c|c|}
\hline & \multicolumn{2}{|c|}{ Dependent variable: } \\
\hline & \multicolumn{2}{|c|}{$\mathrm{Y}$} \\
\hline & (1) & (2) \\
\hline Median Nephelometer $\mathrm{PM}_{2.5}$ & $\begin{array}{c}0.834^{* * *} \\
(0.004)\end{array}$ & $\begin{array}{c}0.908^{* * *} \\
(0.004)\end{array}$ \\
\hline Elevation (50 m) & $\begin{array}{c}-0.003^{* * *} \\
(0.001)\end{array}$ & $\begin{array}{c}-0.030^{* * *} \\
(0.001)\end{array}$ \\
\hline Minimum PBL Height & $\begin{array}{c}-0.0003^{* * *} \\
(0.0001)\end{array}$ & $\begin{array}{c}-0.015^{* * *} \\
(0.0001)\end{array}$ \\
\hline Maximum Relative Humidity (50 m) & $\begin{array}{l}0.007^{* *} \\
(0.003)\end{array}$ & $\begin{array}{c}0.011^{* * *} \\
(0.003)\end{array}$ \\
\hline Maximum Temperature (50 m) & $\begin{array}{c}-0.058^{* * *} \\
(0.005)\end{array}$ & $\begin{array}{c}-0.073^{* * *} \\
(0.005)\end{array}$ \\
\hline Mean Wind Speed (50m) & $\begin{array}{c}-0.242^{* * *} \\
(0.022)\end{array}$ & $\begin{array}{c}-0.063^{* * *} \\
(0.022)\end{array}$ \\
\hline Developed Medium Intensity (100 m) & $\begin{array}{c}0.555^{* * *} \\
(0.132)\end{array}$ & $\begin{array}{c}0.023 \\
(0.132)\end{array}$ \\
\hline Season (Spring) & $\begin{array}{c}-0.186^{* *} \\
(0.090)\end{array}$ & $\begin{array}{l}-0.011 \\
(0.090)\end{array}$ \\
\hline Season (Summer) & $\begin{array}{c}-0.232^{*} \\
(0.131)\end{array}$ & $\begin{array}{l}-0.014 \\
(0.131)\end{array}$ \\
\hline Season (Fall) & $\begin{array}{c}0.718^{* * *} \\
(0.073)\end{array}$ & $\begin{array}{c}0.051 \\
(0.073)\end{array}$ \\
\hline Longitude & $\begin{array}{c}0.00001^{* * *} \\
(0.00000)\end{array}$ & $\begin{array}{c}0.013^{* * *} \\
(0.00000)\end{array}$ \\
\hline
\end{tabular}




\begin{tabular}{lcc} 
Constant & $11.775^{* * *}$ & 0.000 \\
& $(2.705)$ & $(2.705)$ \\
\hline Observations & 8,082 & 8,082 \\
$\mathrm{R}^{2}$ & 0.874 & 0.874 \\
Adjusted $\mathrm{R}^{2}$ & 0.874 & 0.874 \\
Residual Std. Error $(\mathrm{df}=8070)$ & 2.295 & 2.295 \\
F Statistic $(\mathrm{df}=11 ; 8070)$ & $5,091.712^{* * *}$ & $5,091.712^{* * *}$ \\
Note: & ${ }^{*} \mathrm{p}<0.1 ;{ }^{* *} \mathrm{p}<0.05 ; * * * 0.01$
\end{tabular}

\subsection{Spatial Patterns of Model Residuals}

The global Moran's I of the residuals from each predicted raster was evaluated for spatial autocorrelation. On average, this statistic was $0.99 \pm 0.0005$, indicating a lack of complete spatial randomness in model residuals. Instead, the model error was significantly spatially clustered (a Moran's I value of 1.0 indicates perfect clustering of similar values). Therefore, some predictors are contributing to the spatially consistent overprediction/underprediction of $\mathrm{PM}_{2.5}$ throughout the Portland study area. Specifically, high model standard error was observed at both the global maximum and minimum elevations. Model standard error can also be seen increasing towards the Eastern and Western extents of the study area, suggesting potential edge effects, or an undesirable influence from including longitude as a predictor. Areas with consistently low modeled standard error were observed within the developed medium intensity LULC (Figure 3.13). Most of the PurpleAir monitors were located within this LULC which may explain the lower residuals within the urban core. Overall, the LUR model residuals ranged between 0.05 and $0.25 \mu \mathrm{gm}^{-3}$. 
While the Global Moran's I coefficient of 0.99 suggests the omission of specific variables that may contribute to spatially clustered error, or the inclusion of additional variables that may better explain the variation in intra-urban $\mathrm{PM}_{2.5}$, the range in the residuals from the spatially resolved $\mathrm{PM}_{2.5}$ predictions were well below the RMSE of the model (2.39). Therefore, we did not account for spatial autocorrelation of LUR model residuals beyond its initial characterization.

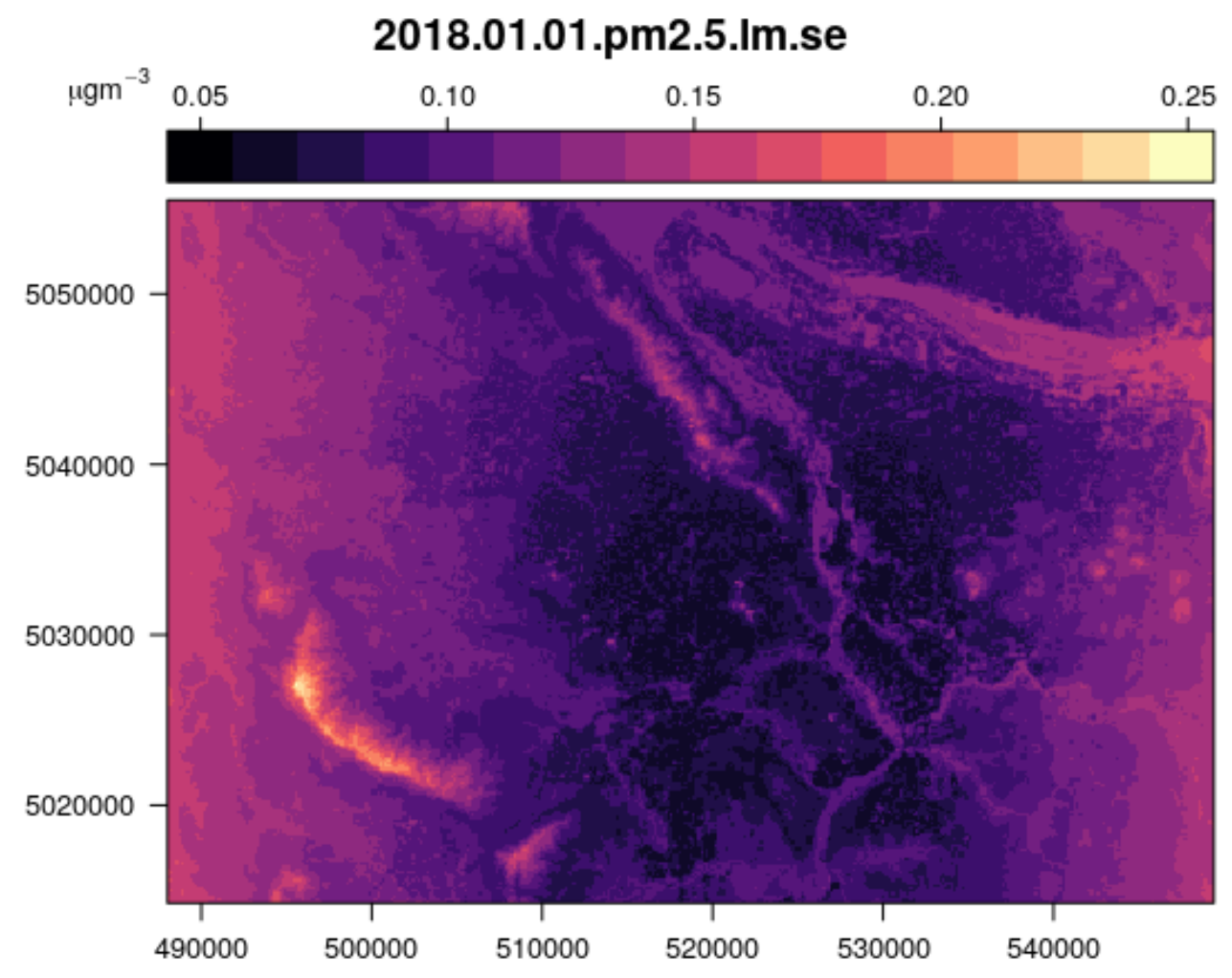

FIGURE 3.13: A map highlighting the spatially clustered standard error from a single LUR model prediction (January $1^{\text {st }}, 2018$ ). All remaining days during the study period exhibited similar patterns in residuals. 


\subsection{The Importance of Sensor Placement}

While we were primarily focused on the anthropogenic drivers of fine-scale $\mathrm{PM}_{2.5}$ spatial gradients, and the influence that vegetation may have on mitigating these exposures, ultimately no clear patterns between traffic or vegetation variables could be established. Our assessment of the relative strengths of the various sources and sinks of $\mathrm{PM}_{2.5}$ has shown that the median ambient background concentration provided by the ODEQ nephelometer network had the strongest association with $\mathrm{PM}_{2.5}$, and the remaining variables were only weakly associated. When comparing the relative strengths of the remaining variables, the effect size of the temporal and spatiotemporal predictors was relatively higher compared to the spatial variables, indicating that the spatial variation of urban $\mathrm{PM}_{2.5}$ is primarily driven by meteorology and regional sources at the $50 \mathrm{~m}$ scale.

In addition to the observed spatial clustering in our model error, we found the LUR model development to be extremely sensitive to the initial placement of the PurpleAir response network. During the opportunistic ambient calibration phase, we explored several different time aggregations of the nephelometer network in order to identify periods when $\mathrm{PM}_{2.5}$ was regionally homogeneous. While we initially selected a 15-minute time resolution to evaluate the RSD of the nephelometer network, we also explored a 1-hour resolution, which produced another set of reasonable correction factors. By implementing a coarser time resolution during the opportunistic ambient calibration, we observed an additional 9 sensors which passed our quality control criterion $\left(R^{2}>0.90\right.$ and 
$n \geq 50$ ). This corresponded to approximately 5 additional locations (each node contains both a primary and secondary sensor) and an increase in the total number of sensors from 61 to 70 . The number of observations used during model development subsequently rose from 11,546 to 12,388 during model development. A new LUR model was developed with these data, and a different set of predictors was selected (despite following the same predictor selection protocol as with the previous model) (Table 3.5). For example, an additional proxy for an anthropogenic source, railroad density, was determined to be a statistically significant predictor with the new data. The log of the railroad density at 500 $\mathrm{m}$ produced a beta of 0.015 . In addition, a vegetation variable, deciduous forest cover, was also identified as a significant predictor. The deciduous forest predictor had a beta of 0.008 , which was the lowest in this model. The relationship between many of the spatial predictors and $\mathrm{PM}_{2.5}$ were weak, often with beta coefficients below 0.05 . Due to the spatial predictors being weakly associated with $\mathrm{PM}_{2.5}$, the addition or removal of a small subset of our response network can lead to a major difference in the predictors selected for the final model. When there are only a few sensors at each LULC to begin with, it becomes difficult to effectively capture the entire variation in each proxy, and model bias increases. The correlations for many of the proxies for anthropogenic sources, e.g. freeways, major arterials, railroads, developed LULC, and $\mathrm{PM}_{2.5}$ were teetering above and below 0 . The sign of the correlation coefficients for some predictors were observed to flip depending on whether the 15-minute or 1-hour RSD nephelometer data were used to correct the response network. For example, freeways at the $50 \mathrm{~m}$ buffer size flipped from a correlation coefficient of 0.01 
for the 15-minute data, to -0.01 for the 1-hour data. In reality, this relationship is effectively null.

Patterns in $\mathrm{PM}_{2.5}$ were predominately explained by temporal factors over spatial ones. Spatially, $\mathrm{PM}_{2.5}$ only varied by $0-5 \mu g m^{-3}$. Temporally, however, $\mathrm{PM}_{2.5}$ levels were observed to increase by tens of micrograms per cubic meter from day to day, especially during wildfire PM events in the summer months, and temperature inversions in the winter months.

If there is a lack of representativeness with the air quality network used to train the model, then model bias for predictions in unrepresented areas will be higher. For example, if only a single sensor is located near railroad that has elevated $\mathrm{PM}_{2.5}$ levels, then the model will predict that locations with a similar railroad density will also be elevated, regardless of the train volume on each particular rail.

In addition to the observed spatial clustering in our model error, we found the LUR model development to be extremely sensitive to the initial placement of the PurpleAir response network. During the opportunistic ambient calibration phase, we explored several different time resolutions of the nephelometer network in order to identify periods when $\mathrm{PM}_{2.5}$ was regionally homogeneous. While we initially selected a 15-minute time resolution to evaluate the RSD of the nephelometer network, we also explored a 1-hour resolution, which produced another reasonable set of correction factors. By implementing this coarser 1-hour resolution during the opportunistic ambient calibration, we actually observed an additional 9 sensors which passed our quality control criterion $\left(R^{2}\right.$ $>0.90$ and $n \geq 50$ ). This corresponded to approximately 5 additional locations 
(each node contains both a primary and secondary sensor) and an increase in the total number of observations from 11,546 to 12,388 during model development. With these data, a new LUR model was developed, and a different set of predictors was selected (following the same predictor selection protocol as with the previous model). For example, an additional proxy for anthropogenic emissions, railroad density, was determined to be a statistically significant predictor with this new data. In addition, a vegetation variable, deciduous forest cover, was also identified as a significant predictor. However, the correlations for many of the spatial predictors with $\mathrm{PM}_{2.5}$ were non-existent, often with correlation coefficients below 0.05 . Therefore, the addition or removal of a small subset of our response network led to a major difference in the predictors selected for the final model. When there are only a few sensors at each LULC to begin with, it becomes difficult to capture the entire extent of the variation with each proxy, and model bias increases. The correlations for many of the proxies for anthropogenic sources, e.g. freeways, major arterials, railroads, developed LULC, and $\mathrm{PM}_{2.5}$ were teetering above and below zero. The sign of the correlation coefficient for some predictors was observed to flip depending on whether the 15 minute or 1 hour RSD nephelometer data were used to correct the response network. For example, freeways at the $50 \mathrm{~m}$ buffer size flipped from a correlation coefficient of 0.01 for the 15-minute data, to -0.01 for the 1 hour data, when in reality, this is effectively a null correlation. 
TABLE 3.5: Coefficients (1) and standardized (beta) coefficients (2) of a second LUR model, with the standard error included within parentheses. This model was developed using the PurpleAir correction factors data developed with hourly nephelometer data (opposed to the 15-minute data).

\begin{tabular}{|c|c|c|}
\hline & \multicolumn{2}{|c|}{ Dependent variable: } \\
\hline & \multicolumn{2}{|c|}{ NA } \\
\hline & (1) & (2) \\
\hline Median Nephelometer $\mathrm{PM}_{2.5}$ & $\begin{array}{c}0.983^{* * *} \\
(0.004)\end{array}$ & $\begin{array}{c}0.920^{* * *} \\
(0.004)\end{array}$ \\
\hline Elevation (50 m) & $\begin{array}{c}-0.003^{* * *} \\
(0.001)\end{array}$ & $\begin{array}{c}-0.020^{* * *} \\
(0.001)\end{array}$ \\
\hline Minimum PBL Height & $\begin{array}{c}-0.0004^{* * *} \\
(0.0001)\end{array}$ & $\begin{array}{c}-0.016^{* * *} \\
(0.0001)\end{array}$ \\
\hline Log Railroad (500 m) & $\begin{array}{c}0.018^{* * *} \\
(0.006)\end{array}$ & $\begin{array}{c}0.015^{* * *} \\
(0.006)\end{array}$ \\
\hline Maximum Relative Humidity (50 m) & $\begin{array}{l}0.009^{* *} \\
(0.004)\end{array}$ & $\begin{array}{c}0.011^{* * *} \\
(0.004)\end{array}$ \\
\hline Maximum Temperature (50 m) & $\begin{array}{c}-0.062^{* * *} \\
(0.006)\end{array}$ & $\begin{array}{c}-0.062^{* * *} \\
(0.006)\end{array}$ \\
\hline Mean Wind Speed (50 m) & $\begin{array}{c}-0.219^{* * *} \\
(0.027)\end{array}$ & $\begin{array}{l}-0.044 \\
(0.027)\end{array}$ \\
\hline Season (Spring) & $\begin{array}{c}-0.306^{* * *} \\
(0.108)\end{array}$ & $\begin{array}{l}-0.015 \\
(0.108)\end{array}$ \\
\hline Season (Summer) & $\begin{array}{c}-0.311^{* *} \\
(0.158)\end{array}$ & $\begin{array}{l}-0.015 \\
(0.158)\end{array}$ \\
\hline Season (Fall) & $\begin{array}{c}0.822^{* * *} \\
(0.089)\end{array}$ & $\begin{array}{c}0.045 \\
(0.089)\end{array}$ \\
\hline
\end{tabular}




\begin{tabular}{lcc} 
Deciduous Forest $(300 \mathrm{~m})$ & $-3.661^{*}$ & -0.008 \\
& $(1.933)$ & $(1.933)$ \\
Developed Medium Intensity $(300 \mathrm{~m})$ & $0.967^{* * *}$ & 0.023 \\
& $(0.255)$ & $(0.255)$ \\
Longitude & $-0.00001^{* *}$ & $-0.009^{* * *}$ \\
& $(0.00001)$ & $(0.00001)$ \\
& & \\
Latitude & $0.00001^{* *}$ & $0.009^{* * *}$ \\
& $(0.00001)$ & $(0.00001)$ \\
Constant & -32.782 & 0.000 \\
& $(26.091)$ & $(26.091)$ \\
\hline Observations & & 8,671 \\
$\mathrm{R}^{2}$ & 8,671 & 0.879 \\
Adjusted $\mathrm{R}^{2}$ & 0.879 & 0.879 \\
Residual Std. Error $(\mathrm{df}=8656)$ & 0.879 & 2.878 \\
F Statistic $(\mathrm{df}=14 ; 8656)$ & 2.878 & $4,500.152^{* * *}$ \\
\hline \hline Note: & $4,500.152^{* * *}$ & \\
& ${ }^{*} \mathrm{p}<0.1 ;{ }^{* *} \mathrm{p}<0.05 ;{ }^{* * *} \mathrm{p}<0.01$ \\
& &
\end{tabular}

\subsection{Predictions}

\subsubsection{Land Use Regression}

Predictions of a select day, October $10^{\text {th }}, 2018$, were mapped in order to characterize the differences between the 1-hour RSD network and 15-minute RSD network for both LUR and RF models. The spatial patterns from the 15-minute RSD LUR model appear to approximate the underlying elevation and developed medium intensity LULC predictors, despite the betas of these variables being ranked only the $5^{\text {th }}$ and $6^{\text {th }}$ most associated with $\mathrm{PM}_{2.5}$. Since the general range 
of $\mathrm{PM}_{2.5}$ levels for any given day is primarily governed by the median nephelometer values, and other temporal scaling factors such as wind speed and temperature, any remaining spatial heterogeneity is typically confined within a $\pm 5 \mathrm{\mu gm}^{-3}$ range from the median. For example, the exact range in predicted $\mathrm{PM}_{2.5}$ on this day was $6.25-8.62 \mu g m^{-3}$, a difference of only $2.37 \mu g m^{-3}$ from the most to least polluted location. The 1-hour RSD LUR model captures similar patterns in elevation as the previous model, but the railroad and deciduous forest variables suggest different hot spots and cold spots. For example, the Western extent of the study area shows elevated $\mathrm{PM}_{2.5}$ levels due to the presence of railroads, whereas the previous model indicated this region should have lower levels. In addition, small pockets of low $\mathrm{PM}_{2.5}$ concentrations scattered throughout the region correspond to the presence of deciduous forest cover. From the addition of these two spatial variables, the predicted range in $\mathrm{PM}_{2.5}$ concentration grew to $5.37-9.62 \mathrm{\mu gm}^{-3}$, a $4.25 \mathrm{\mu gm}^{-3}$ difference between most and least polluted locations (Figure 3.14). The spatial heterogeneity in $\mathrm{PM}_{2.5}$ on a given day is further limited when the model RMSE is taken into consideration. The 15-minute RSD model, previously referred to as the 'final model', had a RMSE of 2.39. When the RMSE is applied to the range in predicted $\mathrm{PM}_{2.5}$, it becomes difficult to interpret most spatial patterns. For example, the cold spots observed on October $10^{\text {th }}, 2018$ could potentially overlap with the hotspots, and vice versa (e.g. $6.25+2.39 \approx 8.62-2.39$ ). In addition to the graphical assessment, we regressed the 1-hour RSD model against the 15-minute RSD model to further assess the relationship between the two models (Figure 3.15). The 15-minute RSD model ('final model') explained less than $1 \%$ of the variation in 
the 1-hour RSD model, indicating that the inclusion of 4 PurpleAir sites has resulted a significantly different set of predictions. Each point within Figure 3.15 represents the two predicted $\mathrm{PM}_{2.5}$ values of a single $50 \mathrm{~m}$ by $50 \mathrm{~m}$ cell within our raster, itself consisting of $1.031 e^{6}$ total cells. The areas where the 1-hour RSD model predicts considerably lower than the 15-minute RSD model are due to the inclusion of the deciduous forest cover LULC. The 1-hour RSD model can also be seen predicting higher than the 15-minute model within the $500 \mathrm{~m}$ railroad buffer.

\subsubsection{Random Forest}

Exposure maps of the random forest $(\mathrm{RF})$ predictions illustrate some of the limitations of using machine learning techniques over traditional LUR models. The range in $\mathrm{PM}_{2.5}$ from the 15-minute and 1-hour RSD RF models was 6.63 - 10.78 $\mu \mathrm{gm}^{-3}$ and $6.74-10.55 \mathrm{\mu gm}^{-3}$ respectively (Figure 3.16). Although the RF model appeared to perform marginally better during the validation phase, with higher validation regression $R^{2}$ and lower RMSE, the RF predictions show unrealistic gradients, and appear to overfit on the latitude and longitude variables. Specifically, explicit vertical thresholds in both the 15-minute, and 1-hour RSD models can be seen due to the inclusion of the longitude predictor. A horizontal threshold in the 1-hour RSD model predictions, while less pronounced, is also evident because this model found latitude to be a significant predictor. These RF results stress the importance of understanding the physical processes that drive $\mathrm{PM}_{2.5}$ variation, as relying solely on the statistical measures of model performance may be misleading. Unlike the traditional LUR approach, RF models are 
essentially a black box and do not provide model coefficients necessary for interpreting the relative strengths of sources and sinks. As with many other machine learning algorithms, random forest is a 'data hungry' technique, and relies on a substantial training set in order to develop a robust stable model. While our training set included more than 10,000 observations, these were generated from a limited number $(n \approx 20)$ of physical locations which repeated over a 2-year period. In comparison, the study area in which we are attempting to predict includes over $1.031 e^{6}$ individual grid cells at the $50 \mathrm{~m}$ resolution. We are likely not capturing enough variation in each of our spatial predictors necessary to develop a meaningful RF model for predicting intra-urban $\mathrm{PM}_{2.5}$. 


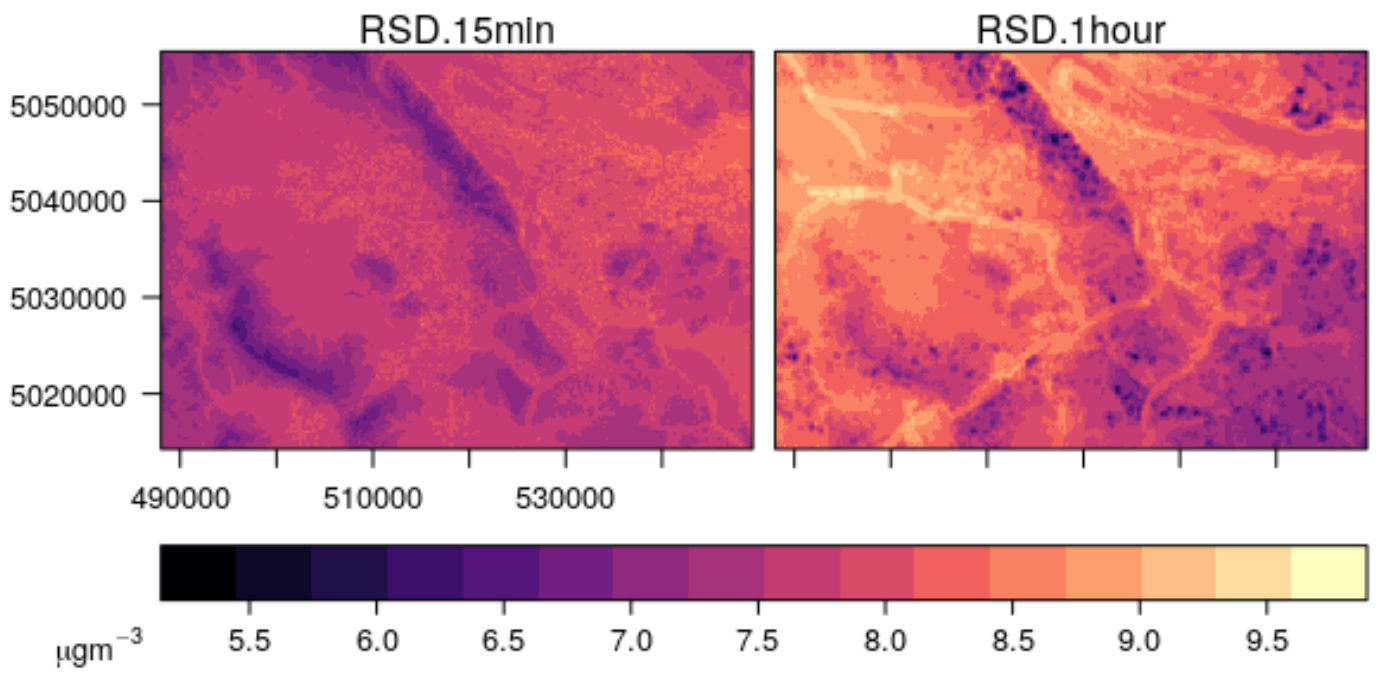

FIGURE 3.14: Spatially resolved $\mathrm{PM}_{2.5}$ predictions of Portland, Oregon on October $10^{\text {th }}, 2018$ from two LUR models that were developed using 15-minute and 1-hour resolution RSD to correct the PurpleAir network. The 1-hour RSD model includes rail density and deciduous forest density predictors that were not selected in the 15-minute model. 


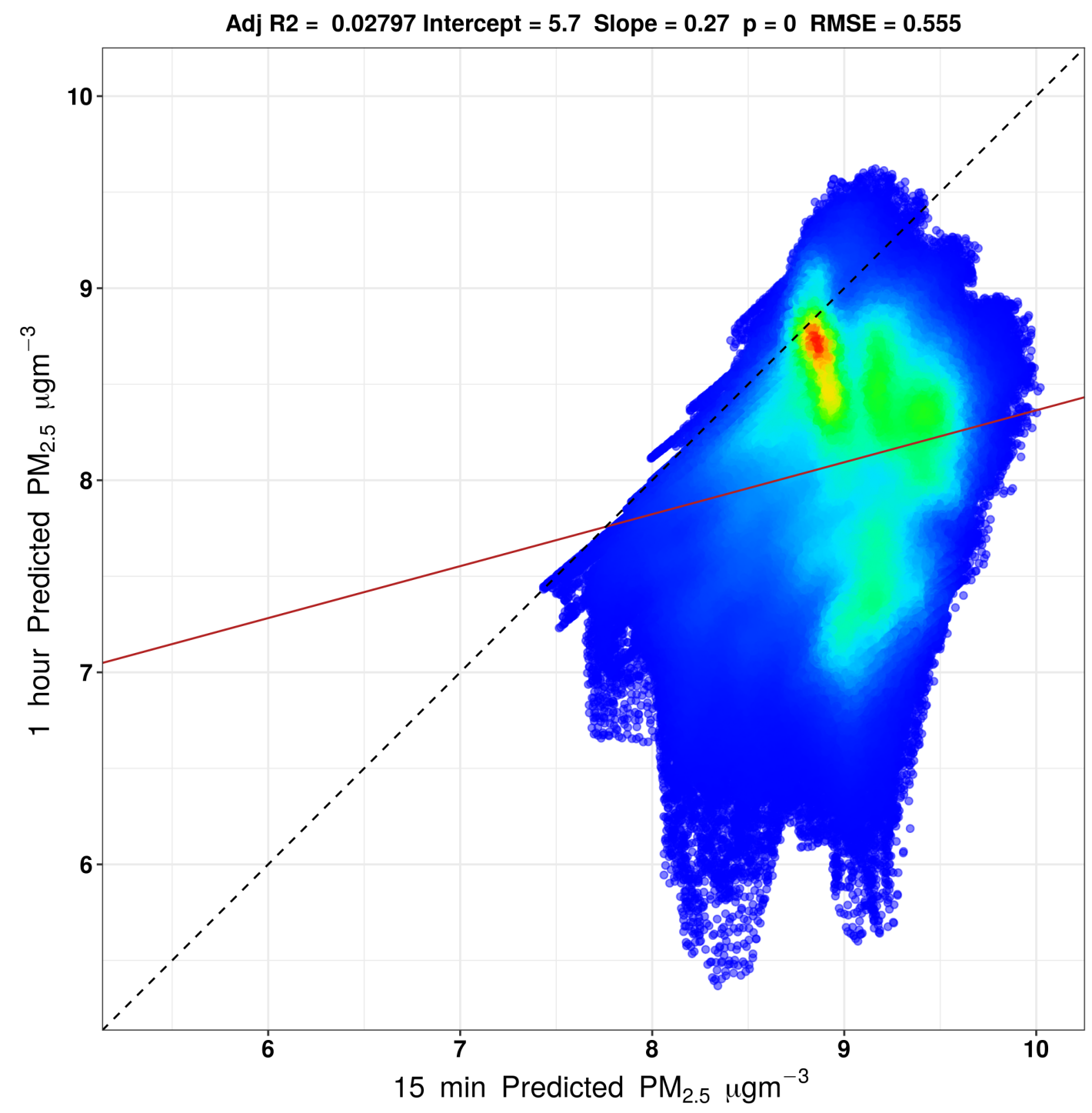

FIGURE 3.15: Scatter plot comparing the predicted $\mathrm{PM}_{2.5}$ on 201810-10 from the 1-hour RSD model as a function of the 15-minute RSD model. 


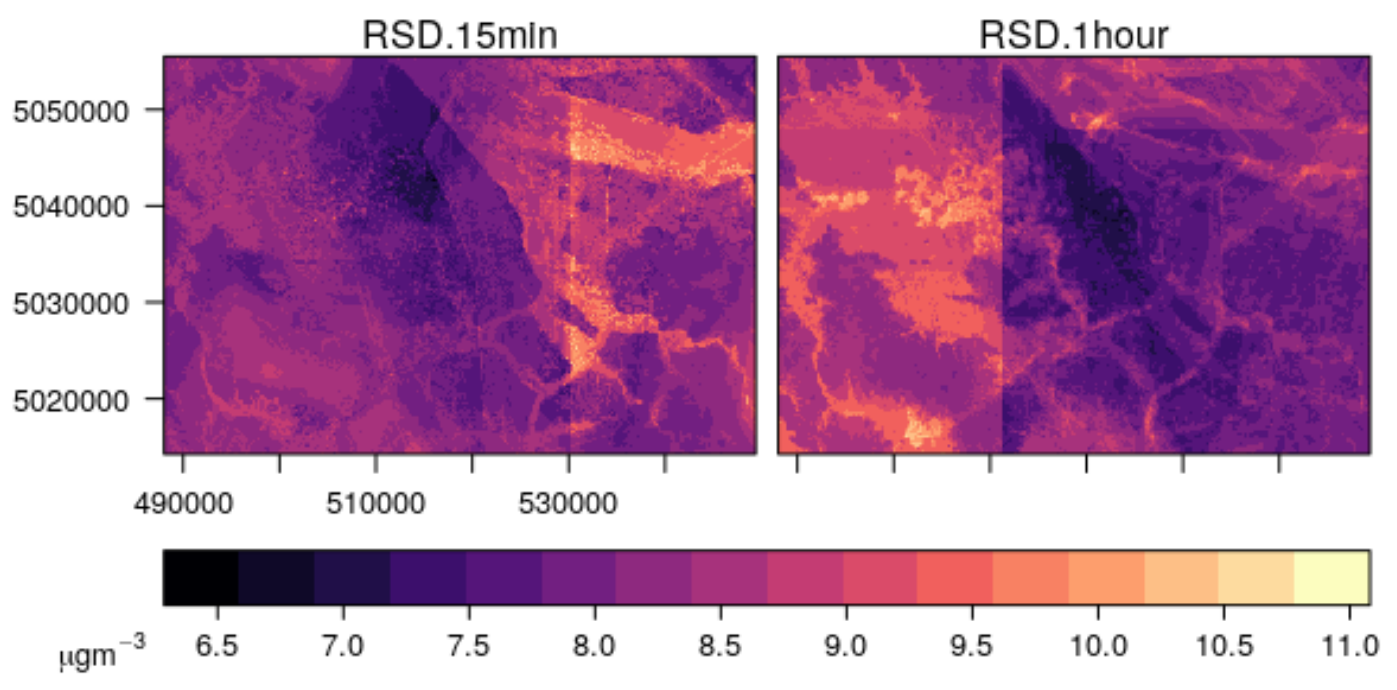

FIGURE 3.16: Spatially resolved $\mathrm{PM}_{2.5}$ predictions of Portland, Oregon on October $10^{\text {th }}, 2018$ from two Random Forest models that were developed using 15-minute and 1-hour resolution RSD to correct the PurpleAir network. The 1-hour RSD model includes rail density and deciduous forest density predictors that were not selected with the 15-minute model.

\subsubsection{Final Model Predictions Over Time}

We used the 'final model' to predict 573 consecutive days (September 2017 March 2019) of $\mathrm{PM}_{2.5}$ throughout the Portland metropolitan area. An animated figure which cycles through each daily prediction can be viewed at the following hyperlink: http://web.pdx.edu/ porlando/animated-pm.gif. This animation allowed us to identify periods of interest, including a forest fire haze event that occurred during August, 2018 and periods with stable meteorology and wood 
burning in December, 2017. The spatial pattern appears to be relatively consistent even on the most polluted days (Figures 3.17, 3.18).

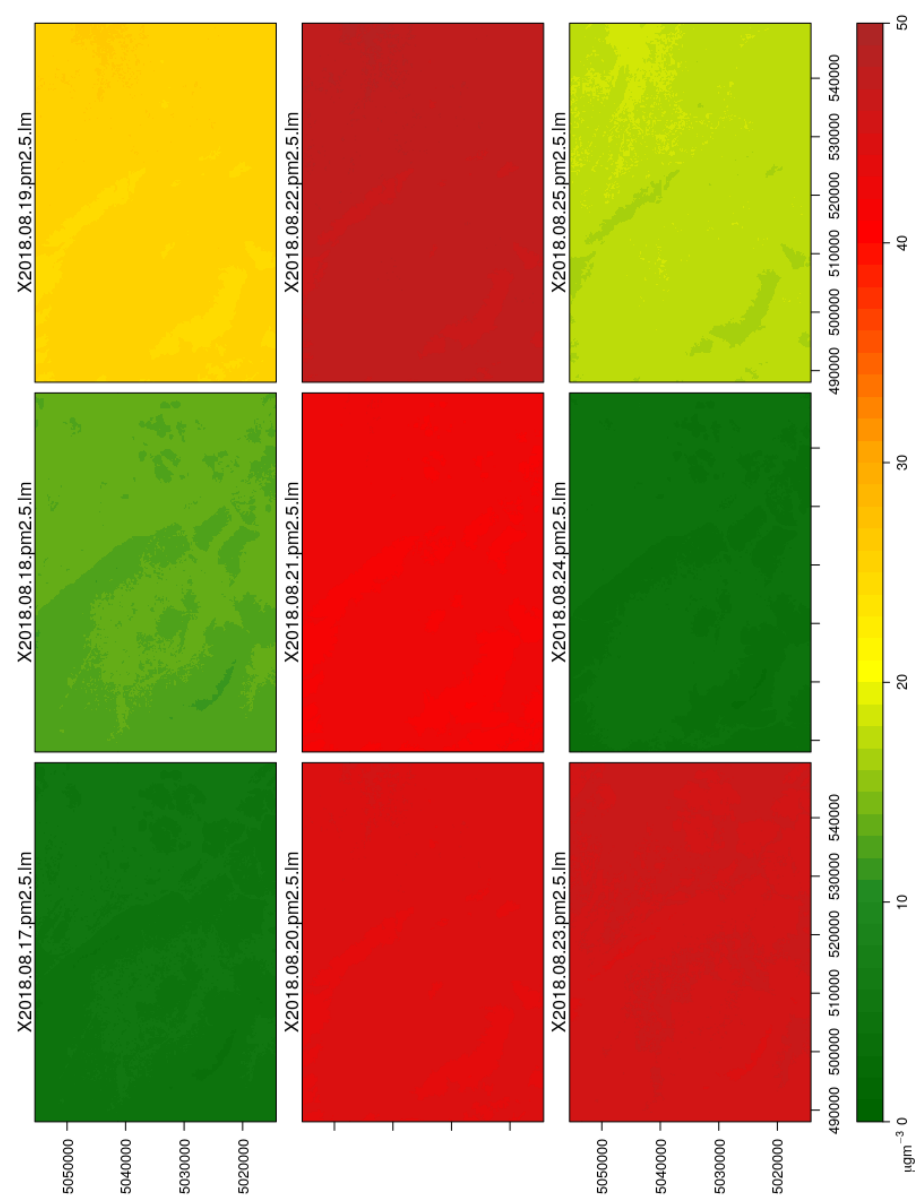

FIGURE 3.17: Spatially resolved $\mathrm{PM}_{2.5}$ predictions of Portland, Oregon capturing a forest fire haze event that occurred between August $17^{\text {th }}-25^{\text {th }}, 2018$ from the 'final model'. 

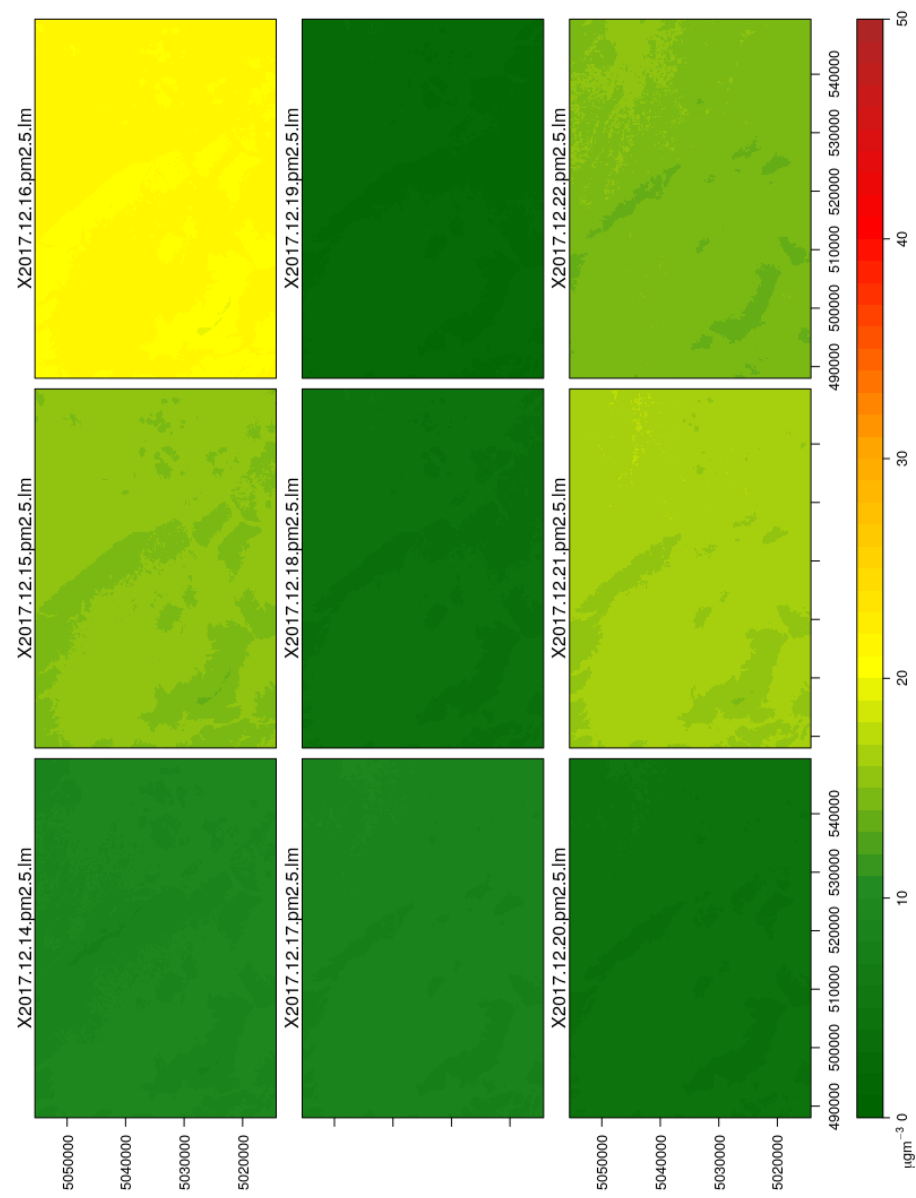

FIGURE 3.18: Spatially resolved $\mathrm{PM}_{2.5}$ predictions of Portland, Oregon during stable meteorological conditions and higher rates of wood-burning between December $14^{\text {th }}-22^{\text {nd }}, 2017$ from the 'final model'. 


\section{Chapter 4}

\section{Summary and Future Work}

\subsection{Summary}

Spatially resolved $\mathrm{PM}_{2.5}$ concentrations at the intra-urban scale are not well defined throughout US cities. This is due to the practical constraints of deploying high-density networks using costly research-grade monitoring equipment, and the focus on compliance at the regional scale from regulatory agencies. Monitoring networks for $\mathrm{PM}_{2.5}$ are scarce, typically between 4-6 monitors per city, and are often deliberately placed away from local sources in order to assess the ambient background concentration. The focus on compliance ultimately results in the potential lack of representativeness of fixed air quality monitoring sites for epidemiological studies, and limits studies which aim to characterize the potential role vegetation may play in mitigating $\mathrm{PM}_{2.5}$ exposure. Land use regression techniques have been developed to account for the spatial heterogeneity of air pollution sources within urban areas, in an effort to provide spatially resolved exposure maps and identify the relative strengths of various sources and sinks. This thesis explored the potential of using low-cost sensors for modeling spatially resolved $\mathrm{PM}_{2.5}$ exposures within Portland, Oregon at a $50 \mathrm{~m}$ resolution 
during a 2 year period. The low-cost sensor network increased the density of the existing monitoring network by a factor of 5 , enabling us to better characterize the potential patterns in sources and sinks throughout the region necessary for producing spatially resolved $\mathrm{PM}_{2.5}$ exposures. However, we observed the spatial predictors of $\mathrm{PM}_{2.5}$ to be only weakly associated with our response network, relative to temporal scaling factors. This was not entirely surprising based on the lack of fine-scale spatial variability in $\mathrm{PM}_{2.5}$ observed by previous studies (Karner, Eisinger, and Niemeier, 2010). In addition, the predicted range of $\mathrm{PM}_{2.5}$ on a given day was typically within the RMSE of the LUR model. While many air pollutants within cities have strong pollution gradients, typically decaying within $150 \mathrm{~m}-200 \mathrm{~m}$ from the source, spatial patterns of $\mathrm{PM}_{2.5}$ are not as distinct. As identified by Karner et al., gradients of $\mathrm{PM}_{2.5}$ with increasing distance from traffic-related sources are mixed, and either decrease very gradually or show no clear trend altogether (Karner, Eisinger, and Niemeier, 2010; Kendrick, Koonce, and George, 2015). We could not identify a significant association between our PurpleAir response network and any of the proxies for traffic sources. The only anthropogenic source associated with $\mathrm{PM}_{2.5}$ was the medium intensity developed LULC. This predictor was observed in both the 1hour and 15-minute RSD models, which may be a sign of developed land use as a proxy for area sources. In contrast, no clear associations between proxies for vegetation and $\mathrm{PM}_{2.5}$ were established during these initial modeling efforts, and only a single vegetation LULC was identified during the additional 1-hour RSD model. We characterized the sensitivity to initial sensor placement when developing LUR models, as an addition of 4 sensors resulted in a considerably 
different set of predictions throughout the study period. Despite the challenges inherent to modeling spatially resolved $\mathrm{PM}_{2.5}$ with low-cost sensor data, this thesis has developed a method for modeling intra-urban $\mathrm{PM}_{2.5}$ that can be generalized to many other cities, and produced a set of exposure maps which are a notable improvement over the alternative exposures generated from spatially interpolating the existing regulatory monitoring sites.

\subsection{Future Work}

We have developed a method for modeling spatially resolved $\mathrm{PM}_{2.5}$ at the subneighborhood scale that can be applied to the remaining four cities within the Canopy Continuum project. Future work will explore the associations between a similar set of land use and meteorological predictors with each of the corresponding low-cost $\mathrm{PM}_{2.5}$ networks deployed throughout Albuquerque, New Mexico, Boise, Idaho, Sacramento, California, and Tacoma, Washington. Since these cities exhibit a varying degree of urban canopy cover, the development of additional LUR models, and the resulting inter-city comparison may provide further insights regarding the potential role of vegetation as a sink for $\mathrm{PM}_{2.5}$. The inclusion of some additional key predictors should also be considered with future models. As outlined by the ESCAPE project, traffic intensity data was shown to be a crucial variable when predicting spatially resolved $\mathrm{PM}_{2.5}$ across many European cities, where PM emissions are higher due to higher levels of diesel engines, and study areas without local traffic intensity resulted in poorer models. While we incorporated road density data of several road classes (streets, arterials, freeways, etc.), the average annual daily traffic volume of 
each road segment was not accounted for. In addition to traffic-intensity data, the performance of ESCAPE models for predicting PM absorbance, a marker of black carbon, were generally higher than that of $\mathrm{PM}_{2.5}$. This suggests that additional monitoring networks may be required in order to characterize anthropogenic sources. While a substantial fraction of European private cars, and nearly all middle and heavy duty vehicles, use diesel instead of gasoline, the inclusion of PM absorbance within our LUR models may better reflect the impact that mobile emissions have on fine-scale PM exposures within the urban environment (Eeftens et al., 2012). The inclusion of particle number concentration, specifically of ultrafine PM, should also be considered in future modeling efforts, as this pollutant is another proxy for emissions from local sources. The two Plantower sensors inside each PurpleAir monitor provide additional mass density measurements for $\mathrm{PM}_{1}$ and $\mathrm{PM}_{10}$. Plantower sensors are also capable of measuring the number density of particles as small as $300 \mathrm{~nm}$ in diameter $\left(\mathrm{PM}_{0.3}\right)$. Future work should consider the inclusion of the various different PM channels recorded by the PurpleAir network in effort to further characterize the land-use and physical drivers of intra-urban PM variability (Appendix B).

Sensor placement, as we previously demonstrated, can have a cascading effect during model development. Two areas of improvement have been identified from these results: (1) a stratified sensor allocation method and (2) an alternative field calibration technique. A stratified sensor allocation method would better approximate the true variation in each of the relevant spatial predictors. The opportunistic placement of the PurpleAir sensors in Portland resulted in a network which overrepresented urban areas near small streets, but lacked data 
for both heavily trafficked and heavily vegetated areas, and did not fully capture the extent of the urban-rural gradient. A previous study which developed fine-scale exposure maps of $\mathrm{NO}_{2}$ throughout Portland, Oregon relied on a stratified random network of 144 passive samplers (Rao et al., 2014). Unfortunately, a $\mathrm{PM}_{2.5}$ monitoring campaign of this caliber still poses many logistical challenges even for low-cost sensors. The PurpleAir monitors are still limited by cost (a PurpleNetwork with 144 sites would cost $\$ 36,000)$, power and telecommunication. Identifying remote locations that still provide access to an $\mathrm{AC}$ power source and a WiFi connection would likely be prohibitive with a stratified random sensor allocation. Our opportunistic ambient calibration method, while a major improvement over using the uncorrected sensor data, recovered only $48 \%$ of the PurpleAir network. An alternative field calibration method, for example a pre- and post-study calibration of each individual sensor against a co-located FRM in the field may return a higher data recovery rate, while enabling the assessment of sensor drift overtime. This is especially important as we demonstrated that the inclusion of a just a few sensors can have a significant impact on the predicted spatial variability. 


\section{Bibliography}

Abatzoglou, John T. (2013). “Development of gridded surface meteorological data for ecological applications and modelling". In: International Journal of Climatology 33.1, pp. 121-131. ISSN: 1097-0088. DOI: 10.1002/ joc .3413. URL: https://rmets . onlinelibrary. wiley.com/doi/abs/10 . 1002 / joc . 3413 (visited on 06/25/2019).

Apte, Joshua S., Julian D. Marshall, Aaron J. Cohen, and Michael Brauer (July 7, 2015). "Addressing Global Mortality from Ambient PM2.5”. In: Environmental Science E Technology 49.13, pp. 8057-8066. ISSN: 0013-936X. DOI: 10.1021/ acs . est . 5b01236. URL: https : / / doi . org/10 . 1021/acs . est . 5b01236 (visited on 06/28/2019).

AQ-SPEC South Coast AQMD, OAR (Aug. 17, 2017a). Field Evaluation Purple Air (PA-II) PM Sensor. AQ-SPEC. URL: http: //www . aqmd .gov/aq-spec (visited on $07 / 09 / 2019)$.

- (Aug. 17, 2017b). Laboratory Evaluation Purple Air (PA-II) PM Sensor. AQSPEC. URL: http: //www . aqmd .gov/aq-spec (visited on 07/09/2019).

Beckerman, Bernardo S., Michael Jerrett, Marc Serre, Randall V. Martin, SeungJae Lee, Aaron van Donkelaar, Zev Ross, Jason Su, and Richard T. Burnett 
(July 2, 2013). “A Hybrid Approach to Estimating National Scale Spatiotemporal Variability of PM2.5 in the Contiguous United States". In: Environmental Science E Technology 47.13, pp. 7233-7241. ISSN: 0013-936X. DOI: 10 . 1021/es400039u. URL: https : / / doi .org/10 . 1021/es400039u (visited on 07/07/2019).

Beckett, K Paul, Peter Freer-Smith, and Gail Taylor (2000). “Effective Tree Species for Local Air Quality Management". In: p. 8.

Beckett, K. Paul, P. H. Freer-Smith, and Gail Taylor (2000). “Particulate pollution capture by urban trees: effect of species and windspeed". In: Global Change Biology 6.8, pp. 995-1003. ISSN: 1365-2486. DOI: 10 .1046/j .1365-2486 . 2000. 00376.x. URL: https: //onlinelibrary.wiley.com/doi/abs/10.1046/j . $1365-2486.2000 .00376 \cdot x$ (visited on 07/04/2019).

Bell, Janice F., Jeffrey S. Wilson, and Gilbert C. Liu (Dec. 1, 2008). “Neighborhood Greenness and 2-Year Changes in Body Mass Index of Children and Youth". In: American Journal of Preventive Medicine 35.6, pp. 547-553. ISSN: 0749-3797. DOI: 10.1016/j . amepre . 2008 .07 .006. URL: http: //www . sciencedirect . com/science/article/pii/S0749379708007344 (visited on 07/04/2019).

Bivand, Roger, Tim Keitt, and Barry Rowlingson (2019). rgdal: Bindings for the 'Geospatial' Data Abstraction Library. URL: https : / CRAN . R-project . org/ package $=$ rgdal.

Bivand, Roger and Colin Rundel (2019). rgeos: Interface to Geometry Engine - Open Source ('GEOS'). URL: https : //CRAN . R-project . org/package=rgeos. 
Breiman, Leo (Oct. 1, 2001). “Random Forests”. In: Machine Learning 45.1, pp. 532. ISSN: 1573-0565. DOI: 10 . 1023/ A : 1010933404324. URL: https : / / doi . org/10 .1023/A : 1010933404324 (visited on 07/10/2019).

Briggs, David J., Cornelis de Hoogh, John Gulliver, John Wills, Paul Elliott, Simon Kingham, and Kirsty Smallbone (May 15, 2000). "A regression-based method for mapping traffic-related air pollution: application and testing in four contrasting urban environments". In: Science of The Total Environment 253.1, pp. 151-167. ISSN: 0048-9697. DOI: 10 . 1016/S0048-9697(00) 00429 - 0. URL: http : / / www . sciencedirect.com/science/article/pii / S0048969700004290 (visited on 06/26/2019).

Brokamp, Cole, Roman Jandarov, M. B. Rao, Grace LeMasters, and Patrick Ryan (Feb. 1, 2017). “Exposure assessment models for elemental components of particulate matter in an urban environment: A comparison of regression and random forest approaches". In: Atmospheric Environment 151, pp. 1-11. ISSN: 1352-2310. DOI: $10.1016 / \mathrm{j}$. atmosenv . 2016 . 11.066. URL: http : / / www . sciencedirect.com/science/article/pii/S1352231016309566 (visited on 07/17/2019).

Brugge, Doug, John L. Durant, and Christine Rioux (Aug. 9, 2007). "Nearhighway pollutants in motor vehicle exhaust: A review of epidemiologic evidence of cardiac and pulmonary health risks". In: Environmental Health 6.1, p. 23. ISSN: 1476-069X. DOI: 10 . $1186 / 1476-069 X-6-23$. URL: https : //doi.org/10.1186/1476-069X-6-23 (visited on 07/03/2019).

Brunekreef, B. and S. T. Holgate (Oct. 19, 2002). “Air pollution and health". In: The Lancet 360.9341, pp. 1233-1242. ISSN: 0140-6736. DOI: 10 . 1016 / S0140- 
6736(02 ) 11274 - 8. URL: http : / / www . sciencedirect . com / science / article/pii/S0140673602112748 (visited on 06/22/2019).

Bureau, US Census (2012). “Patterns of metropolitan and micropolitan population change: 2000 to 2010". In: Census Special Reports.

Cheng, Joe, Bhaskar Karambelkar, and Yihui Xie (2018). leaflet: Create Interactive Web Maps with the JavaScript 'Leaflet' Library. URL: https : //CRAN . R-project. org/package=leaflet.

Chudnovsky, A. A., P. Koutrakis, I. Kloog, S. Melly, F. Nordio, A. Lyapustin, Y. Wang, and J. Schwartz (June 1, 2014). "Fine particulate matter predictions using high resolution Aerosol Optical Depth (AOD) retrievals". In: Atmospheric Environment 89, pp. 189-198. ISSN: 1352-2310. DOI: 10 . 1016 / j . atmosenv . 2014. 02 .019. URL: http: //wwW . sciencedirect . com/science/article/ pii/S1352231014001150 (visited on 03/19/2019).

Chun, Youngsin, Jiyoung Kim, Jae Cheon Choi, Kyung On Boo, Sung Nam Oh, and Meehye Lee (May 1, 2001). "Characteristic number size distribution of aerosol during Asian dust period in Korea". In: Atmospheric Environment 35.15, pp. 2715-2721. ISSN: 1352-2310. DOI: 10 . 1016/S1352-2310 (00) 00404 - 0. URL: http : / / www . sciencedirect.com/science/article/pi / S1352231000004040 (visited on 07/03/2019).

Cohen, A. J., R. H. Anderson, B. Ostro, K. D. Pandey, M. Krzyzanowski, N Künzli, K. Gutschmidt, A. C. Pope III, I Romieu, J.M. Samet, and K. Smith (July 1, 2005). "The Global Burden of Disease Due to Outdoor Air Pollution". In: Journal of Toxicology and Environmental Health, Part A 68.13, pp. 1301-1307. 
ISSN: 1528-7394. DOI: 10.1080/15287390590936166. URL: https ://doi . org/ 10.1080/15287390590936166 (visited on 06/22/2019).

Daly, C. (May 2006). “Guidelines for assessing the suitability of spatial climate data sets". In: International Journal of Climatology 26.6, pp. 707-721. ISSN: 08998418, 1097-0088. DOI: 10 . 1002/ joc .1322. URL: http://doi . wiley .com/10. 1002/joc. 1322 (visited on 06/25/2019).

Defays, D. (Jan. 1, 1977). "An efficient algorithm for a complete link method". In: The Computer Journal 20.4, pp. 364-366. ISSN: 0010-4620. DOI: 10 . 1093/ comjnl/20 . 4 364. URL: https://academic. oup.com/comjnl/article/20/ 4/364/393966 (visited on 07/08/2019).

Didan, K (2015a). MOD13A2 MODIS/Terra Vegetation Indices 16-Day L3 Global $1 \mathrm{~km}$ SIN Grid V006, NASA EOSDIS LP DAAC.

Didan, K. (2015b). “MOD13Q1 MODIS/Terra vegetation indices 16-day L3 global 250m SIN grid V006". In: NASA EOSDIS Land Processes DAAC.

Dockery, D.W., A.C. Pope III, X. Xu, J. D. Spengler, J. H. Ware, Martha E. Fay, Benjamin G. Ferris, and Frank E. Speizer (Dec. 9, 1993). “An Association between Air Pollution and Mortality in Six U.S. Cities". In: New England Journal of Medicine 329.24, pp. 1753-1759. ISSN: 0028-4793. DOI: 10 . 1056 / NEJM199312093292401. URL: https : / / doi . org / 10 . 1056 / NEJM199312093292401 (visited on 06/22/2019).

Donovan, Geoffrey H., Yvonne L. Michael, David T. Butry, Amy D. Sullivan, and John M. Chase (Jan. 1, 2011). “Urban trees and the risk of poor birth outcomes". In: Health \& Place. Health Geographies of Voluntarism 17.1, pp. 390393. ISSN: 1353-8292. DOI: 10.1016/j . healthplace. 2010.11 .004. URL: http: 
// www . sciencedirect . com/science/article / pii / S1353829210001656 (visited on 07/04/2019).

Doxsey-Whitfield, Erin, Kytt MacManus, Susana B. Adamo, Linda Pistolesi, John Squires, Olena Borkovska, and Sandra R. Baptista (July 3, 2015). “Taking Advantage of the Improved Availability of Census Data: A First Look at the Gridded Population of the World, Version 4". In: Papers in Applied Geography 1.3, pp. 226-234. ISSN: 2375-4931. DOI: 10 .1080/23754931 .2015.1014272. URL: https : / / doi .org/ 10 . 1080/23754931 . 2015 . 1014272 (visited on 07/08/2019).

Eeftens, Marloes, Rob Beelen, Kees de Hoogh, Tom Bellander, Giulia Cesaroni, Marta Cirach, Christophe Declercq, Audrius Dedelè, Evi Dons, Audrey de Nazelle, Konstantina Dimakopoulou, Kirsten Eriksen, Grégoire Falq, Paul Fischer, Claudia Galassi, Regina Gražulevičienė, Joachim Heinrich, Barbara Hoffmann, Michael Jerrett, Dirk Keidel, Michal Korek, Timo Lanki, Sarah Lindley, Christian Madsen, Anna Mölter, Gizella Nádor, Mark Nieuwenhuijsen, Michael Nonnemacher, Xanthi Pedeli, Ole Raaschou-Nielsen, Evridiki Patelarou, Ulrich Quass, Andrea Ranzi, Christian Schindler, Morgane Stempfelet, Euripides Stephanou, Dorothea Sugiri, Ming-Yi Tsai, Tarja YliTuomi, Mihály J Varró, Danielle Vienneau, Stephanie von Klot, Kathrin Wolf, Bert Brunekreef, and Gerard Hoek (Oct. 16, 2012). “Development of Land Use Regression Models for PM2.5, PM2.5 Absorbance, PM10 and PMcoarse in 20 European Study Areas; Results of the ESCAPE Project". In: Environmental Science E Technology 46.20, pp. 11195-11205. ISSN: 0013-936X. DOI: 
10.1021/es301948k. URL: https : //doi.org/10.1021/es301948k (visited on 11/16/2018).

EPA, US (2004). “Air quality criteria for particulate matter". In: US Environmental Protection Agency, Research Triangle Park.

Fann, N., A. D. Lamson, S. C. Anenberg, K. Wesson, D. Risley, and B. J. Hubbell (Jan. 1, 2012). "Estimating the National Public Health Burden Associated with Exposure to Ambient PM2.5 and Ozone". In: Risk Analysis 32.1, pp. 8195. ISSN: 0272-4332. DOI: $10.1111 / \mathrm{j} .1539-6924.2011$.01630 .x. URL: https: // onlinelibrary . wiley . com/doi /full/10 . 1111/j . 1539-6924 . 2011. 01630.x (visited on 06/22/2019).

Fenger, Jes (Dec. 1, 1999). "Urban air quality". In: Atmospheric Environment 33.29, pp. 4877-4900. ISSN: 1352-2310. DOI: 10 . 1016 / S1352 - 2310(99) 00290 - 3. URL: http : / / www . sciencedirect.com/science/article/pii / S1352231099002903 (visited on 07/03/2019).

Fox, John, Gregor Gorjanc Friendly, Spencer Graves, Richard Heiberger, Georges Monette, Henric Nilsson, Brian Ripley, Sanford Weisberg, Maintainer John Fox, and MASS Suggests (2007). “The car package". In: R Foundation for Statistical Computing.

Freer-Smith, P. H., K. P. Beckett, and Gail Taylor (Jan. 1, 2005). “Deposition velocities to Sorbus aria, Acer campestre, Populus deltoides $\times$ trichocarpa 'Beaupré', Pinus nigra and $\times$ Cupressocyparis leylandii for coarse, fine and ultra-fine particles in the urban environment". In: Environmental Pollution 133.1, pp. 157-167. ISSN: 0269-7491. DOI: 10 . 1016 / j . envpol . 2004.03 . 
031. URL: http : / / wwW . sciencedirect . com / science / article / pii / S0269749104001228 (visited on 07/04/2019).

Freer-Smith, P. H., A. A. El-Khatib, and Gail Taylor (June 1, 2004). “Capture of Particulate Pollution by Trees: A Comparison of Species Typical of Semi-Arid Areas (Ficus Nitida and Eucalyptus Globulus) with European and North American Species". In: Water, Air, and Soil Pollution 155.1, pp. 173-187. ISSN: 1573-2932. DOI: 10.1023/B:WATE.0000026521.99552.fd. URL: https ://doi . org/10.1023/B:WATE.0000026521.99552.fd (visited on 07/04/2019).

Fritsch, F. and R. Carlson (Apr. 1, 1980). "Monotone Piecewise Cubic Interpolation". In: SIAM Journal on Numerical Analysis 17.2, pp. 238-246. ISSN: 00361429. DOI: 10 . 1137/0717021. URL: https : / / epubs . siam.org/doi/abs/10 . 1137/0717021 (visited on 06/25/2019).

Gilliland Frank, Avol Ed, Kinney Patrick, Jerrett Michael, Dvonch Timothy, Lurmann Frederick, Buckley Timothy, Breysse Patrick, Keeler Gerald, de Villiers Tracy, and McConnell Rob (Oct. 1, 2005). "Air Pollution Exposure Assessment for Epidemiologic Studies of Pregnant Women and Children: Lessons Learned from the Centers for Children's Environmental Health and Disease Prevention Research". In: Environmental Health Perspectives 113.10, pp. 14471454. DOI: 10 . 1289 /ehp . 7673. URL: https : / / ehp . niehs . nih . gov / doi / full/10.1289/ehp. 7673 (visited on 07/07/2019).

Harrison, Roy M., Alan M. Jones, and Roger Barrowcliffe (Dec. 1, 2004). "Field study of the influence of meteorological factors and traffic volumes upon suspended particle mass at urban roadside sites of differing geometries". In: Atmospheric Environment 38.37, pp. 6361-6369. ISSN: 1352-2310. DOI: 10 . 
1016/j . atmosenv . 2004.07 .030. URL: http: / / www . sciencedirect . com/ science/article/pii/S1352231004007058 (visited on 06/28/2019).

Hastie, Trevor, Robert Tibshirani, Jerome Friedman, and James Franklin (2005). “The elements of statistical learning: data mining, inference and prediction". In: The Mathematical Intelligencer 27.2, pp. 83-85.

Hijmans, Robert J. (2019). raster: Geographic Data Analysis and Modeling. URL: https://CRAN.R-project.org/package=raster.

Hoek, Gerard, Bert Brunekreef, Arnoud Verhoeff, Joop van Wijnen, and Paul Fischer (Aug. 1, 2000). "Daily Mortality and Air Pollution in the Netherlands". In: Journal of the Air \& Waste Management Association 50.8, pp. 13801389. ISSN: 1096-2247. DOI: 10 . 1080/10473289 . 2000 . 10464182. URL: https : //doi .org/10.1080/10473289.2000.10464182 (visited on 07/03/2019).

Hoek, Gerard, Bert Brunekreef, Sandra Goldbohm, Paul Fischer, and Piet A van den Brandt (Oct. 19, 2002). "Association between mortality and indicators of traffic-related air pollution in the Netherlands: a cohort study". In: The Lancet 360.9341, pp. 1203-1209. ISSN: 0140-6736. DOI: 10 . 1016 / S0140-6736 (02 ) 11280 - 3. URL: http : / / WwW . sciencedirect. com/science/article/pii / S0140673602112803 (visited on 07/03/2019).

Hoek, Gerard, Rob Beelen, Kees de Hoogh, Danielle Vienneau, John Gulliver, Paul Fischer, and David Briggs (Oct. 1, 2008). "A review of land-use regression models to assess spatial variation of outdoor air pollution". In: Atmospheric Environment 42.33, pp. 7561-7578. ISSN: 1352-2310. DOI: $10.1016 / \mathrm{j}$. atmosenv . 2008 . 05 . 057. URL: http : / / www . sciencedirect . com/science / article/pii/S1352231008005748 (visited on 07/05/2019). 
Homer, C., J. Dewitz, L. Yang, S. Jin, P. Danielson, G. Xian, J. Coulston, N. Herold, J. Wickham, and K. Megown (2015). "Completion of the 2011 National Land Cover Database for the conterminous United States-representing a decade of land cover change information". In: Photogrammetric Engineering \& Remote Sensing 81.5, pp. 345-354.

Jeanjean, A. P. R., P. S. Monks, and R. J. Leigh (Dec. 1, 2016). “Modelling the effectiveness of urban trees and grass on PM2.5 reduction via dispersion and deposition at a city scale". In: Atmospheric Environment 147, pp. 1-10. ISSN: 1352-2310. DOI: 10 . 1016 / j . atmosenv . 2016 . 09 . 033. URL: http : / / www . sciencedirect.com/science/article/pii/S1352231016307336 (visited on 07/05/2019).

Jerrett, M., M. A. Arain, P. Kanaroglou, B. Beckerman, D. Crouse, N. L. Gilbert, J. R. Brook, N. Finkelstein, and M. M. Finkelstein (Feb. 1, 2007). “Modeling the Intraurban Variability of Ambient Traffic Pollution in Toronto, Canada". In: Journal of Toxicology and Environmental Health, Part A 70.3, pp. 200-212. ISSN: 1528-7394. DOI: 10 .1080/15287390600883018. URL: https ://doi . org/ $10.1080 / 15287390600883018$ (visited on $07 / 07 / 2019$ ).

Jerrett, Michael, Richard T Burnett, Renjun Ma, C Arden Pope III, Daniel Krewski, K Bruce Newbold, George Thurston, Yuanli Shi, Norm Finkelstein, Eugenia E Calle, and others (2005). "Spatial analysis of air pollution and mortality in Los Angeles". In: Epidemiology, pp. 727-736.

Jerrett, Michael, Shankardass Ketan, Berhane Kiros, Gauderman W. James, Künzli Nino, Avol Edward, Gilliland Frank, Lurmann Fred, Molitor Jassy N., Molitor John T., Thomas Duncan C., Peters John, and McConnell Rob 
(Oct. 1, 2008). "Traffic-Related Air Pollution and Asthma Onset in Children: A Prospective Cohort Study with Individual Exposure Measurement". In: Environmental Health Perspectives 116.10, pp. 1433-1438. DOI: 10.1289 / ehp . 10968. URL: https://ehp.niehs.nih.gov/doi/10.1289/ehp. 10968 (visited on $07 / 04 / 2019)$.

Karner, Alex A., Douglas S. Eisinger, and Deb A. Niemeier (July 15, 2010). “Near-Roadway Air Quality: Synthesizing the Findings from Real-World Data". In: Environmental Science \& Technology 44.14, pp. 5334-5344. ISSN: 0013-936X. DOI: 10 . 1021/es100008x. URL: https : / / doi .org/10 . 1021 / es100008x (visited on 06/28/2019).

Kendrick, Christine M., Peter Koonce, and Linda A. George (Dec. 1, 2015). “Diurnal and seasonal variations of $\mathrm{NO}, \mathrm{NO} 2$ and PM2.5 mass as a function of traffic volumes alongside an urban arterial". In: Atmospheric Environment 122, pp. 133-141. ISSN: 1352-2310. DOI: 10 . 1016 / j . atmosenv . 2015 . 09 . 019. URL: http : / / wwW . sciencedirect . com / science / article / pii / S1352231015303605 (visited on 07/03/2019).

Kim, Y. S. (2004). "Dust particles in the free atmosphere over desert areas on the Asian continent: Measurements from summer 2001 to summer 2002 with balloon-borne optical particle counter and lidar, Dunhuang, China". In: Journal of Geophysical Research 109 (D19). ISSN: 0148-0227. DOI: 10 . 1029/ 2002JD003269. URL: http://doi.wiley. com/10.1029/2002JD003269 (visited on $07 / 03 / 2019)$.

Kloog, I., B. Ridgway, P. Koutrakis, B. A. Coull, and J. D. Schwartz (July 2013). "Long- and Short-Term Exposure to PM2.5 and Mortality". In: Epidemiology 
(Cambridge, Mass.) 24.4, pp. 555-561. ISSN: 1044-3983. DOI: 10 . 1097 / EDE . Ob013e318294beaa. URL: https : //www .ncbi .nlm.nih.gov/pmc/articles/ PMC4372644/ (visited on 06/22/2019).

Kobayashi, Hiroshi, Masahiko Hayashi, Koichi Shiraishi, Yoshinobu Nakura, Takayuki Enomoto, Kazuhiko Miura, Hiroshi Takahashi, Yasuhito Igarashi, Hiroaki Naoe, Naoki Kaneyasu, Tomoaki Nishizawa, and Nobuo Sugimoto (Nov. 1, 2014). “Development of a polarization optical particle counter capable of aerosol type classification". In: Atmospheric Environment 97, pp. 486492. ISSN: 1352-2310. DOI: $10.1016 / \mathrm{j}$. atmosenv . 2014 . 05.006 . URL: http: // wWw . sciencedirect . com / science / article / pii / S1352231014003537 (visited on $07 / 03 / 2019)$.

Kohli, Rajiv and Kashmiri L. Mittal (Nov. 12, 2015). Developments in Surface Contamination and Cleaning, Vol. 1: Fundamentals and Applied Aspects. GoogleBooks-ID: WZDmBQAAQBAJ. William Andrew. 898 pp. ISBN: 978-0-32331270-7.

Le Tertre, A., S. Medina, E. Samoli, B. Forsberg, P. Michelozzi, A. Boumghar, J. M. Vonk, A. Bellini, R. Atkinson, J. G. Ayres, J. Sunyer, J. Schwartz, and K. Katsouyanni (Oct. 1, 2002). "Short-term effects of particulate air pollution on cardiovascular diseases in eight European cities". In: Journal of Epidemiology $\mathcal{E}$ Community Health 56.10, pp. 773-779. ISSN: 0143-005X, 1470-2738. DOI: 10. 1136/jech. 56 . 10.773. URL: https : / / jech. bmj . com/content/56/10/773 (visited on 06/22/2019). 
Liaw, Andy and Matthew Wiener (2002). "Classification and Regression by randomForest". In: R News 2.3, pp. 18-22. URL: https : //CRAN.R-project. org/ doc/Rnews/.

Lim, Stephen S, Theo Vos, Abraham D Flaxman, Goodarz Danaei, Kenji Shibuya, Heather Adair-Rohani, Mohammad A AlMazroa, Markus Amann, H Ross Anderson, Kathryn G Andrews, and others (2012). “A comparative risk assessment of burden of disease and injury attributable to 67 risk factors and risk factor clusters in 21 regions, 1990-2010: a systematic analysis for the Global Burden of Disease Study 2010". In: The lancet 380.9859, pp. 2224-2260.

Liu, Chao, Barron H. Henderson, Dongfang Wang, Xinyuan Yang, and Zhongren Peng (Sept. 15, 2016). “A land use regression application into assessing spatial variation of intra-urban fine particulate matter (PM2.5) and nitrogen dioxide (NO2) concentrations in City of Shanghai, China". In: Science of The Total Environment 565, pp. 607-615. ISSN: 0048-9697. DOI: 10 .1016/j . scitotenv . 2016.03.189. URL: http://www . sciencedirect.com/science/ article/pii/S004896971630609X (visited on 07/07/2019).

Maas, J., R. A. Verheij, S. de Vries, P. Spreeuwenberg, F. G. Schellevis, and P. P. Groenewegen (Dec. 1, 2009). “Morbidity is related to a green living environment". In: Journal of Epidemiology \& Community Health 63.12, pp. 967-973. ISSN: 0143-005X, 1470-2738. DOI: 10 . 1136/ jech . 2008 . 079038. URL: https : //jech.bmj.com/content/63/12/967 (visited on 07/04/2019).

Mattiuzzi, Matteo and Florian Detsch (2019). MODIS: Acquisition and Processing of MODIS Products. URL: https ://CRAN . R-project . org/package=MODIS. 
Mesinger, F., G. DiMego, E. Kalnay, K. Mitchell, P. C. Shafran, W. Ebisuzaki, D. Jović, J. Woollen, E. Rogers, E.H. Berbery, M. B. Ek, Y. Fan, R. Grumbine, W. Higgins, H. Li, Y. Lin, G. Manikin, D. Parrish, and W. Shi (Mar. 1, 2006). "North American Regional Reanalysis". In: Bulletin of the American Meteorological Society 87.3, pp. 343-360. ISSN: 0003-0007. DOI: 10 . 1175/BAMS-87-3343. URL: https : // journals . ametsoc .org/doi/abs/10 . 1175/BAMS-87-3343 (visited on 06/25/2019).

Mitchell, Richard and Frank Popham (Aug. 1, 2007). “Greenspace, urbanity and health: relationships in England". In: Journal of Epidemiology $\mathcal{E}$ Community Health 61.8, pp. 681-683. ISSN: 0143-005X, 1470-2738. DOI: $10.1136 /$ jech . 2006. 053553. URL: https: / / jech. bmj.com/content/61/8/681 (visited on 07/04/2019).

NASA (2009). ASTER Global Digital Elevation Map. URL: https : //asterweb .jpl . nasa.gov/gdem. asp (visited on 07/10/2019).

Nowak, David J., Daniel E. Crane, and Jack C. Stevens (Apr. 3, 2006). “Air pollution removal by urban trees and shrubs in the United States". In: Urban Forestry E Urban Greening 4.3, pp. 115-123. ISSN: 1618-8667. DOI: 10 . 1016 / j.ufug. 2006 .01.007. URL: http : / / www . sciencedirect . com/science / article/pii/S1618866706000173 (visited on 07/04/2019).

Nowak, David J. and Eric J. Greenfield (July 1, 2012). “Tree and impervious cover in the United States". In: Landscape and Urban Planning 107.1, pp. 2130. ISSN: 0169-2046. DOI: 10.1016/j . landurbplan . 2012.04.005. URL: http: / / wWw . sciencedirect . com / science/article / pii / S0169204612001193 (visited on 07/04/2019). 
Nowak, David J., Satoshi Hirabayashi, Allison Bodine, and Robert Hoehn (July 1, 2013). "Modeled PM2.5 removal by trees in ten U.S. cities and associated health effects". In: Environmental Pollution 178, pp. 395-402. ISSN: 0269-7491. DOI: $10.1016 / \mathrm{j}$. envpol . 2013 .03 . 050. URL: http : / / www . sciencedirect . com / science/article/pii / S0269749113001838 (visited on $07 / 04 / 2019)$.

Nyberg, Fredrik, Per Gustavsson, Lars Järup, Tom Bellander, Niklas Berglind, Robert Jakobsson, and Göran Pershagen (2000). “Urban Air Pollution and Lung Cancer in Stockholm”. In: Epidemiology 11.5, pp. 487-495. ISSN: 10443983. URL: https : / / www . jstor . org / stable / 3703988 (visited on 07/03/2019).

Oberdorster, G., E. Oberdorster, and J. Oberdorster (2005). Nanotoxicology: an emerging discipline evolving from studies of ultrafine particles. Environ Health Perspect 113:823-839.

Oregon Metro (2018). RLIS Discovery: Home. Regional Land Information System. URL: http: //rlisdiscovery . oregonmetro.gov/ (visited on 07/10/2019).

Pebesma, Edzer (2018). “Simple Features for R: Standardized Support for Spatial Vector Data". In: The R Journal 10.1, pp. 439-446. DOI: 10 . 32614/RJ - 2018 009. URL: https://doi .org/10.32614/RJ-2018-009.

Pebesma, Edzer J. and Roger S. Bivand (Nov. 2005). “Classes and methods for spatial data in R". In: $R$ News 5.2, pp. 9-13. URL: https : //CRAN . R-project. org/doc/Rnews/. 
Pope, A. C. III and D. W. Dockery (June 1, 2006). “Health Effects of Fine Particulate Air Pollution: Lines that Connect". In: Journal of the Air \& Waste Management Association 56.6, pp. 709-742. ISSN: 1096-2247. DOI: 10.1080/10473289. 2006.10464485. URL: https://doi.org/10.1080/10473289.2006.10464485 (visited on 06/22/2019).

Pope, C. Arden III, Richard T. Burnett, Michael J. Thun, Eugenia E. Calle, Daniel Krewski, Kazuhiko Ito, and George D. Thurston (Mar. 6, 2002). “Lung Cancer, Cardiopulmonary Mortality, and Long-term Exposure to Fine Particulate Air Pollution". In: JAMA 287.9, pp. 1132-1141. ISSN: 0098-7484. DOI: 10 . 1001 / jama . 287 . 9.1132. URL: https: / / jamanetwork . com/ journals / jama/fullarticle/194704 (visited on 07/06/2019).

Pullman, Marcie (Aug. 21, 2008). “Conifer PM2.5 Deposition and Resuspension in Wind and Rain Events". In: URL: https : / / ecommons . cornell . edu / handle/1813/11239 (visited on 07/04/2019).

R Core Team (2019). R: A Language and Environment for Statistical Computing. Vienna, Austria: R Foundation for Statistical Computing. URL: https ://www . R-project.org/.

Rao, Meenakshi, Linda A. George, Todd N. Rosenstiel, Vivek Shandas, and Alexis Dinno (Nov. 1, 2014). "Assessing the relationship among urban trees, nitrogen dioxide, and respiratory health". In: Environmental Pollution 194, pp. 96-104. ISSN: 0269-7491. DOI: 10 . 1016 / j . envpol . 2014.07 . 011. URL: http : / / ww . sciencedirect . com/science/article/pii / S0269749114003030 (visited on 03/26/2019). 
Ross, Zev, Michael Jerrett, Kazuhiko Ito, Barbara Tempalski, and George D. Thurston (Apr. 1, 2007). “A land use regression for predicting fine particulate matter concentrations in the New York City region". In: Atmospheric Environment 41.11, pp. 2255-2269. ISSN: 1352-2310. DOI: 10 .1016/ j . atmosenv . 2006. 11.012. URL: http : / / wWw . sciencedirect. com/science/article/pii/ S1352231006011150 (visited on 06/28/2019).

Sarnat, J. A., Schwartz J, Catalano P J, and Suh H H (Oct. 1, 2001). “Gaseous pollutants in particulate matter epidemiology: confounders or surrogates?" In: Environmental Health Perspectives 109.10, pp. 1053-1061. DOI: 10 . 1289 / ehp.011091053. URL: https://ehp.niehs.nih.gov/doi/abs/10.1289/ehp. 011091053 (visited on 07/06/2019).

Saucy, Apolline, Martin Röösli, Nino Künzli, Ming-Yi Tsai, Chloé Sieber, Toyib Olaniyan, Roslynn Baatjies, Mohamed Jeebhay, Mark Davey, Benjamin Flückiger, Rajen Naidoo, Mohammed Dalvie, Mahnaz Badpa, and Kees de Hoogh (July 10, 2018). “Land Use Regression Modelling of Outdoor NO2 and PM2.5 Concentrations in Three Low Income Areas in the Western Cape Province, South Africa". In: International Journal of Environmental Research and Public Health 15.7, p. 1452. ISSN: 1660-4601. DOI: 10 . 3390 / i jerph15071452. URL: http: //www . mdpi . com/1660-4601/15/7/1452 (visited on 03/19/2019). Schwartz, J. (Sept. 1, 1994). "Air pollution and hospital admissions for the elderly in Detroit, Michigan." In: American Journal of Respiratory and Critical Care Medicine 150.3, pp. 648-655. ISSN: 1073-449X. DOI: 10 . 1164 / ajrccm . 150 . 3.8087333. URL: https : //www . atsjournals . org/doi/abs/10 . 1164/ ajrccm.150.3.8087333 (visited on 06/22/2019). 
Tallis, Matthew, Gail Taylor, Danielle Sinnett, and Peter Freer-Smith (Nov. 30, 2011). "Estimating the removal of atmospheric particulate pollution by the urban tree canopy of London, under current and future environments". In: Landscape and Urban Planning 103.2, pp. 129-138. ISSN: 0169-2046. DOI: 10 . 1016/j . landurbplan. 2011 .07 .003. URL: http: //www. sciencedirect . com/ science/article/pii/S0169204611002349 (visited on 07/05/2019).

Tobler, W. R. (June 1, 1970). “A Computer Movie Simulating Urban Growth in the Detroit Region". In: Economic Geography 46 (sup1), pp. 234-240. ISSN: 0013-0095. DOI: 10 . 2307 / 143141. URL: https : / / www . tandfonline . com / doi/abs/10.2307/143141 (visited on 06/25/2019).

Tucker, C. J. and P. J. Sellers (Nov. 1, 1986). “Satellite remote sensing of primary production". In: International Journal of Remote Sensing 7.11, pp. 1395-1416. ISSN: 0143-1161. DOI: 10 .1080/01431168608948944. URL: https ://doi .org/ 10. 1080/01431168608948944 (visited on 06/25/2019).

Tucker, Compton J. (May 1, 1979). “Red and photographic infrared linear combinations for monitoring vegetation". In: Remote Sensing of Environment 8.2, pp. 127-150. ISSN: 0034-4257. DOI: 10 . 1016/0034-4257 (79) 90013 - 0. URL: http://www. sciencedirect.com/science/article/pii/0034425779900130 (visited on 06/25/2019).

Ulrich, R. S. (Apr. 27, 1984). "View through a window may influence recovery from surgery". In: Science 224.4647, pp. 420-421. ISSN: 0036-8075, 1095-9203. DOI: 10.1126/science.6143402. URL: https://science. sciencemag . org/ content/224/4647/420 (visited on 07/04/2019). 
United Nations, Population Devision of the Department of Economic \{and\} Social Affairs (2018). World population prospects: the 2019 revision and world urbanization prospects: Press Release. UN.

US Census Bureau (2015). TIGER/Line Shapefiles(machine-readable data files)/preparedby the U.S. Census Bureau, 2015.

US EPA (Aug. 17, 2016). Interactive Map of Air Quality Monitors. US EPA. URL: https : / /ww . epa.gov/ outdoor-air-quality-data/interactive-mapair-quality-monitors (visited on 07/09/2019).

Vardoulakis, Sotiris, Efisio Solazzo, and Julio Lumbreras (Sept. 1, 2011). “Intraurban and street scale variability of BTEX, NO2 and O3 in Birmingham, UK: Implications for exposure assessment". In: Atmospheric Environment 45.29, pp. 5069-5078. ISSN: 1352-2310. DOI: 10 . $1016 / \mathrm{j}$. atmosenv . 2011.06 . 038. URL: http : / / www . sciencedirect . com/science/article/pii / S1352231011006315 (visited on 06/28/2019).

Vardoulakis, Sotiris, Bernard E. A Fisher, Koulis Pericleous, and Norbert Gonzalez-Flesca (Jan. 1, 2003). "Modelling air quality in street canyons: a review". In: Atmospheric Environment 37.2, pp. 155-182. ISSN: 1352-2310. DOI: 10.1016/S1352-2310(02)00857-9. URL: http: //www . sciencedirect. com/ science/article/pii/S1352231002008579 (visited on 06/28/2019).

Venables, W. N. and B. D. Ripley (2002). Modern Applied Statistics with S. Fourth. New York: Springer. URL: http://www.stats . ox. ac . uk/pub/MASS4.

Walker, Kyle (2019). tidycensus: Load US Census Boundary and Attribute Data as 'tidyverse' and 'sf'-Ready Data Frames. URL: https : / CRAN . R-project .org/ package=tidycensus. 
Wang, Jianhua and Susumu Ogawa (Aug. 2015). “Effects of Meteorological Conditions on PM2.5 Concentrations in Nagasaki, Japan". In: International Journal of Environmental Research and Public Health 12.8, pp. 9089-9101. ISSN: 16617827. DOI: 10 . 3390/ijerph120809089. URL: https : //www . ncbi . nlm . nih . gov/pmc/articles/PMC4555266/ (visited on 07/08/2019).

Wei, Taiyun and Viliam Simko (2017). R package "corrplot": Visualization of a Correlation Matrix. URL: https://github.com/taiyun/corrplot.

Yang, Xiaofan, Yixuan Zheng, Guannan Geng, Huan Liu, Hanyang Man, Zhaofeng Lv, Kebin He, and Kees de Hoogh (July 1, 2017). "Development of PM2.5 and NO2 models in a LUR framework incorporating satellite remote sensing and air quality model data in Pearl River Delta region, China". In: Environmental Pollution 226, pp. 143-153. ISSN: 0269-7491. DOI: 10.1016/ j . envpol . 2017 .03 . 079. URL: http: //www . sciencedirect .com/science/ article/pii/S0269749116312131 (visited on 11/16/2018).

Zhou, Ying and Jonathan I. Levy (May 22, 2007). "Factors influencing the spatial extent of mobile source air pollution impacts: a meta-analysis". In: BMC Public Health 7.1, p. 89. ISSN: 1471-2458. DOI: 10.1186/1471-2458-7-89. URL: https://doi .org/10.1186/1471-2458-7-89 (visited on 07/03/2019).

- (Apr. 1, 2008). "The impact of urban street canyons on population exposure to traffic-related primary pollutants". In: Atmospheric Environment 42.13, pp. 3087-3098. ISSN: 1352-2310. DOI: 10 . $1016 / \mathrm{j}$. atmosenv . 2007 . 12 . 037. URL: http : / / www . sciencedirect . com/science/article/pii / S1352231007011636 (visited on 07/05/2019). 


\section{Appendix A}

\section{Source Code}

\section{Source/animateStack.R}

\# load the necessary packages

if ( ! require (pacman)) \{

install . packages ("pacman")

library (pacman)

\}

p_load(raster, rasterVis, viridis, animation, classint, pbapply, parallel)

mods $<-c(" 1 \mathrm{~m} "$

, $\mathrm{rf} "$

, "xgb"

)

pblapply (mods, function (mod) \{

s $<-$ stack(list.files("./data/predicted_raster", paste0 (mod, " $\backslash \backslash$. tif \$"), full.names $=\mathrm{T})$ )

$\mathrm{r}<-\mathrm{s}[[1]]$

\# breaks <- seq $(0,100,5)$

breaks <- c( $\operatorname{seq}(0,50,1)$, Inf $)$ \# focus on lower level variation

\# cols <- colorRampPalette (c("darkgreen", "lightgreen", "yellow", "orange", "red", "maroon"))(length(breaks)-1)

\# cols <- magma (length (breaks) -1 )

cols <- colorRampPalette (c("darkgreen", "forestgreen", "yellow", " orange", "red", "\#A62A2A")) (length (breaks)-1) 


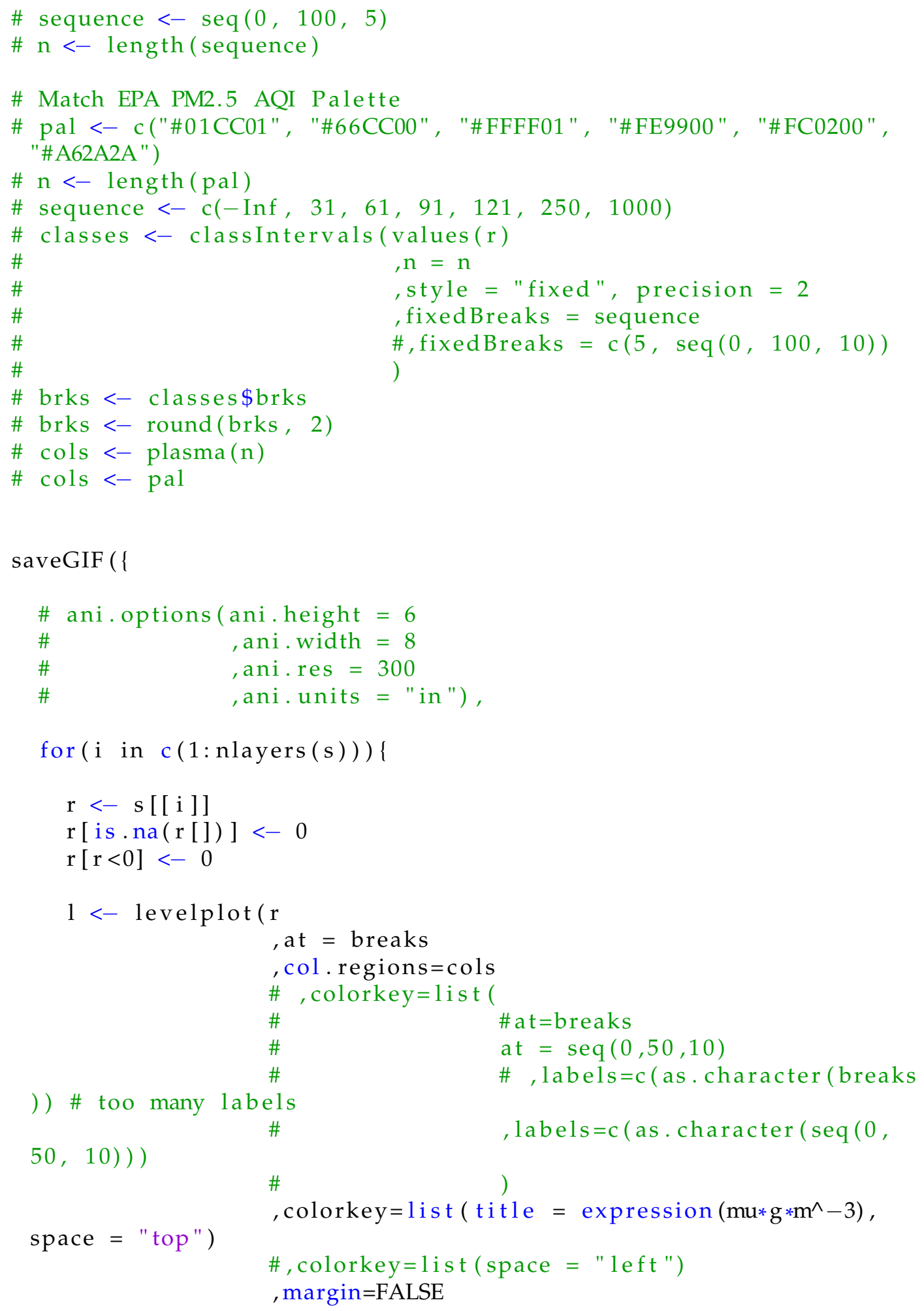




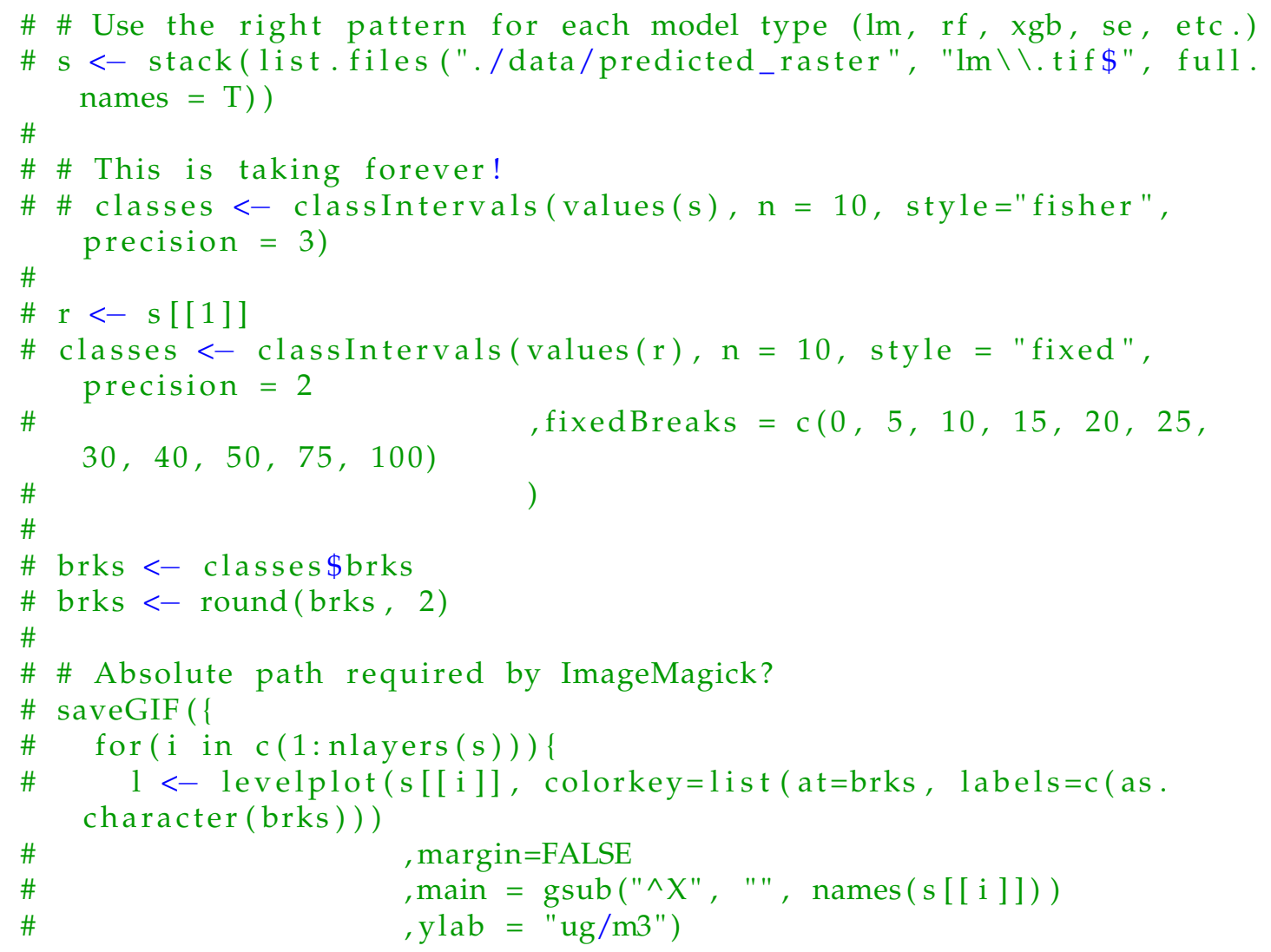




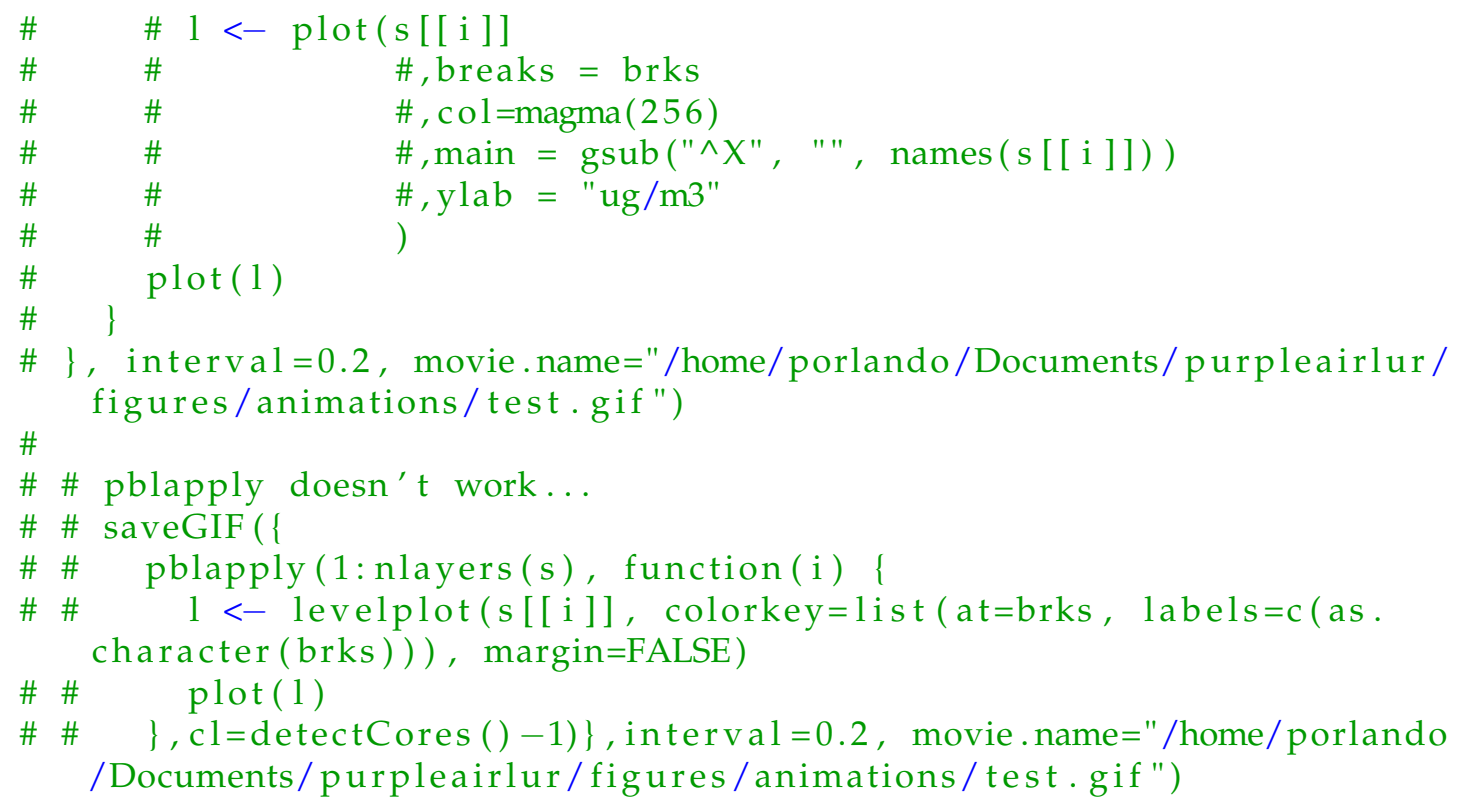

\section{Source/annualNDVI.R}

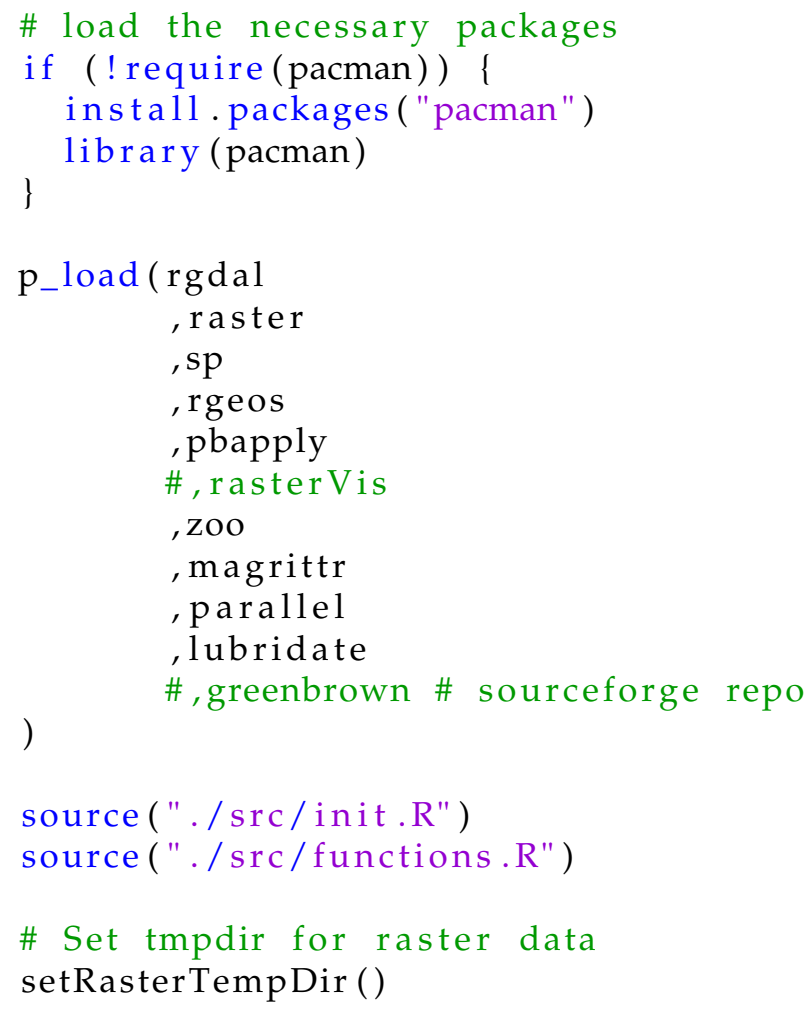




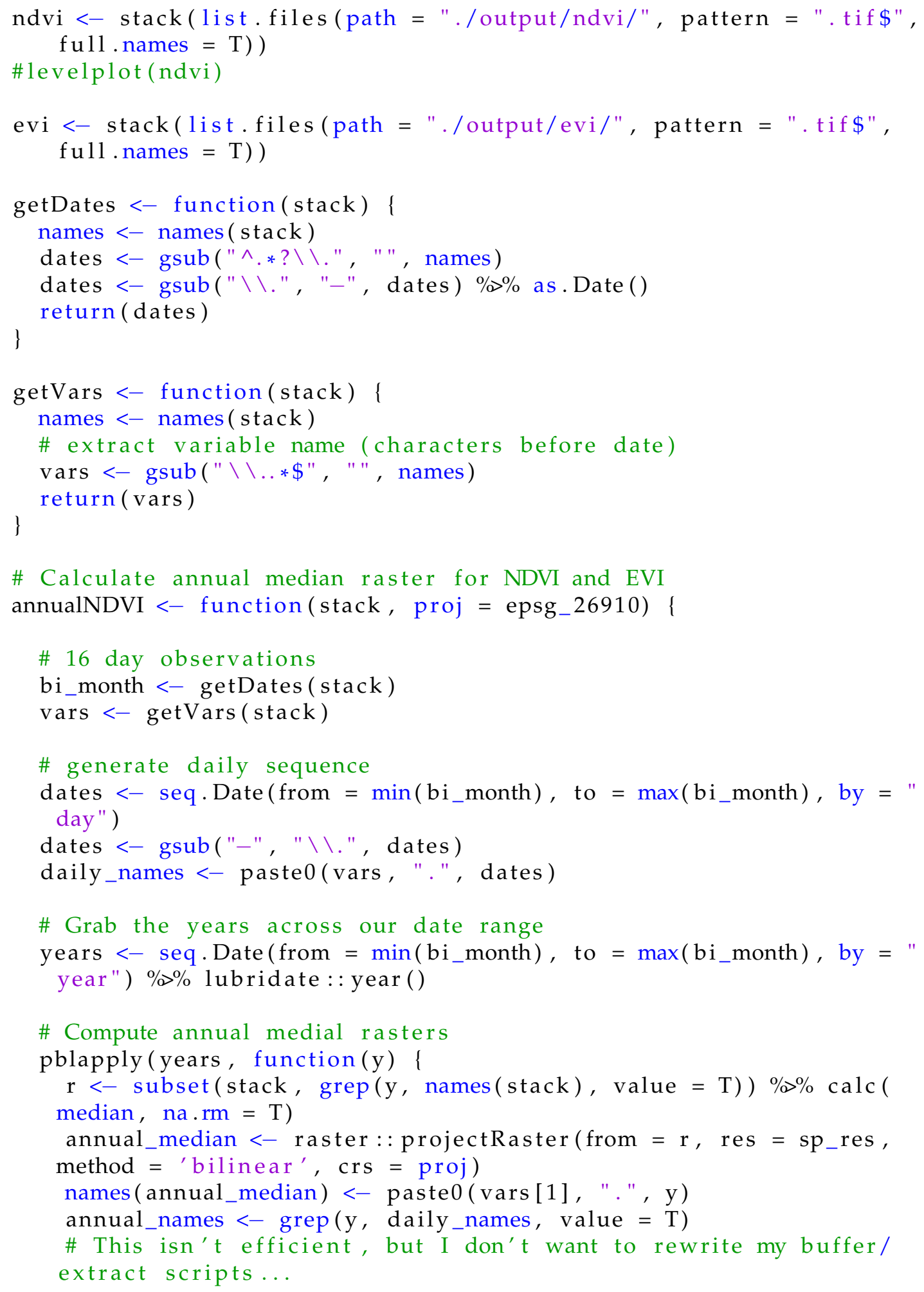




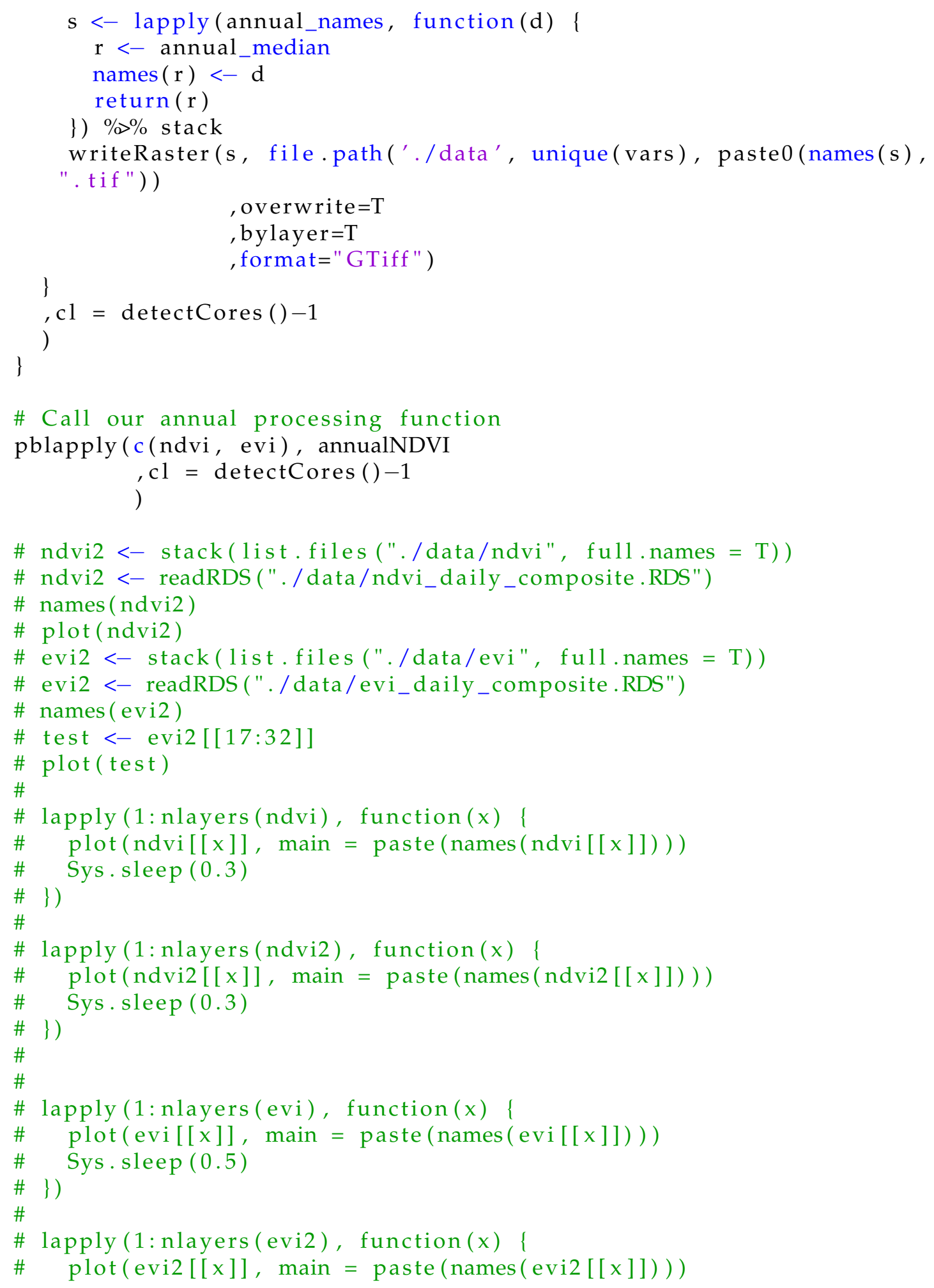


\# $\quad$ Sys.sleep (0.5)

\# \})

\section{Source/compareCF.R}

\# Compare CF from 1 hour and 15 minute data

\# load the necessary packages

if (!require (pacman)) \{

install . packages("pacman")

library (pacman)

\}

p_load (dplyr, ggplot2)

pm_1h $<-$ readRDS("./data/homogenousPM/lm_results_1-hour.RDS") \%\% dplyr:: select (r.squared, slope, id, n_obs, sigma)

pm_15m <- readRDS( './data/homogenousPM/lm_results_15-min.RDS') \% \% dplyr:: select (r.squared, slope, id, n_obs, sigma)

pm_wide $<-$ full_join $($ pm_1h, pm_15m, by $=$ "id", suffix $=c(" .1 \mathrm{~h} ", " .15$ $\left.\mathrm{m}^{\prime \prime}\right)$ )

pm_wide\$slope_diff $<-$ abs (pm_wide\$slope.1h - pm_wide\$slope.15m)

ggplot(filter (pm_wide, r.squared $.1 \mathrm{~h}>=0.9 \& \mathrm{r}$. squared $.15 \mathrm{~m}>=0.90)$, aes $(x=$ as.factor $($ id $), y=$ slope_diff $))+$ geom_col ()

pm_wide $\% \%$ filter $(\mathrm{r}$.squared.1h $>=0.9 \&$ r.squared $.15 \mathrm{~m}>=0.90) \% \%$ dplyr:: select (slope_diff) $\% \%$ boxplot () dplyr:: select (slope_diff) $\% \%$ summary () 


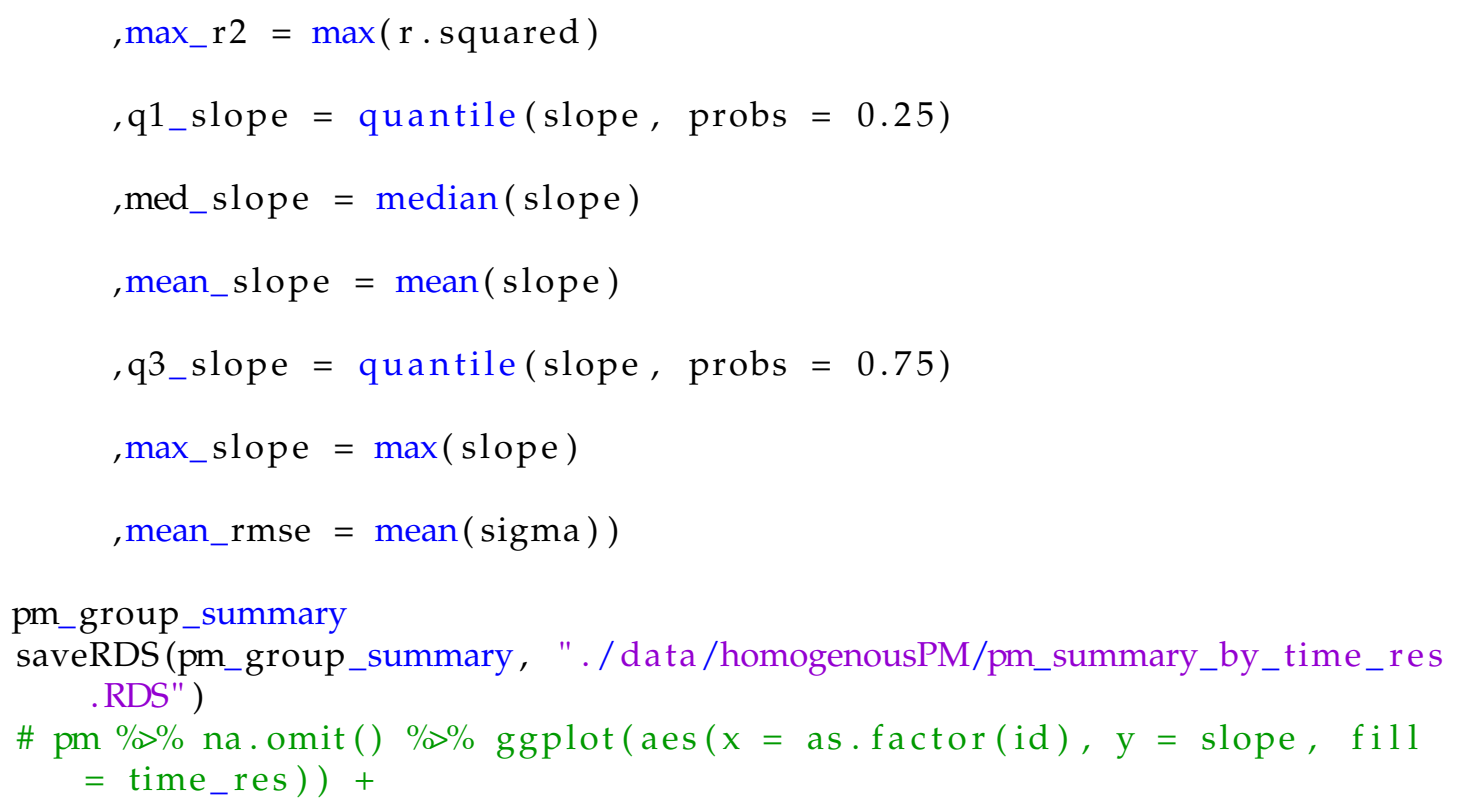

\section{Source/downloadGridMET.R}

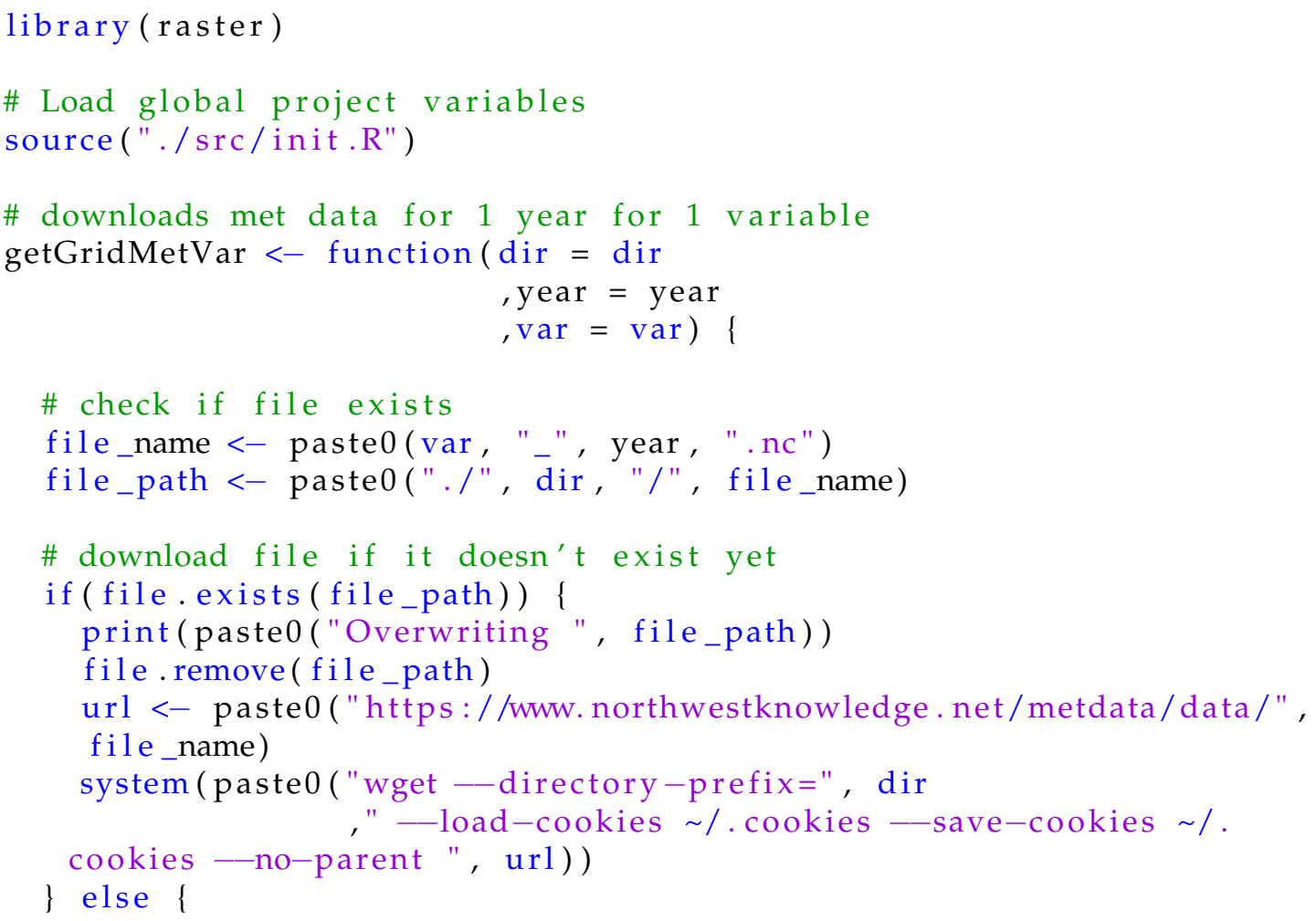




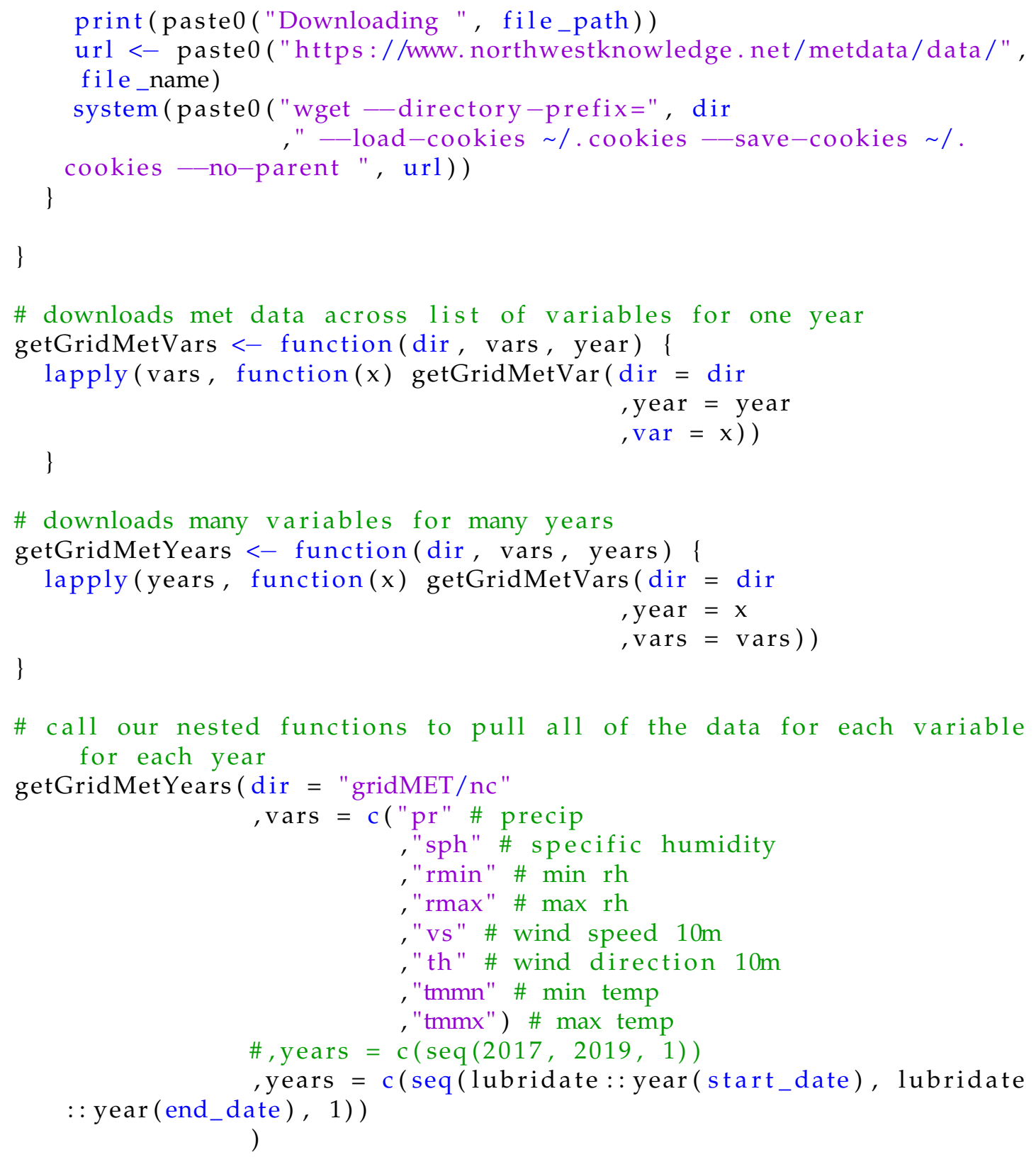

\section{Source/downloadNDVI.R}

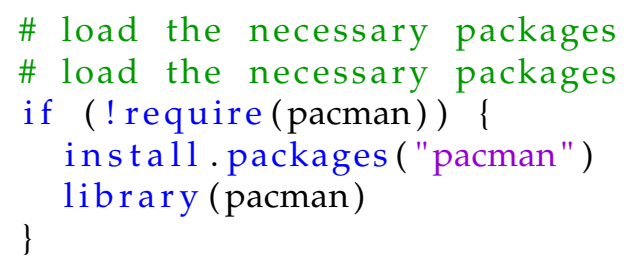




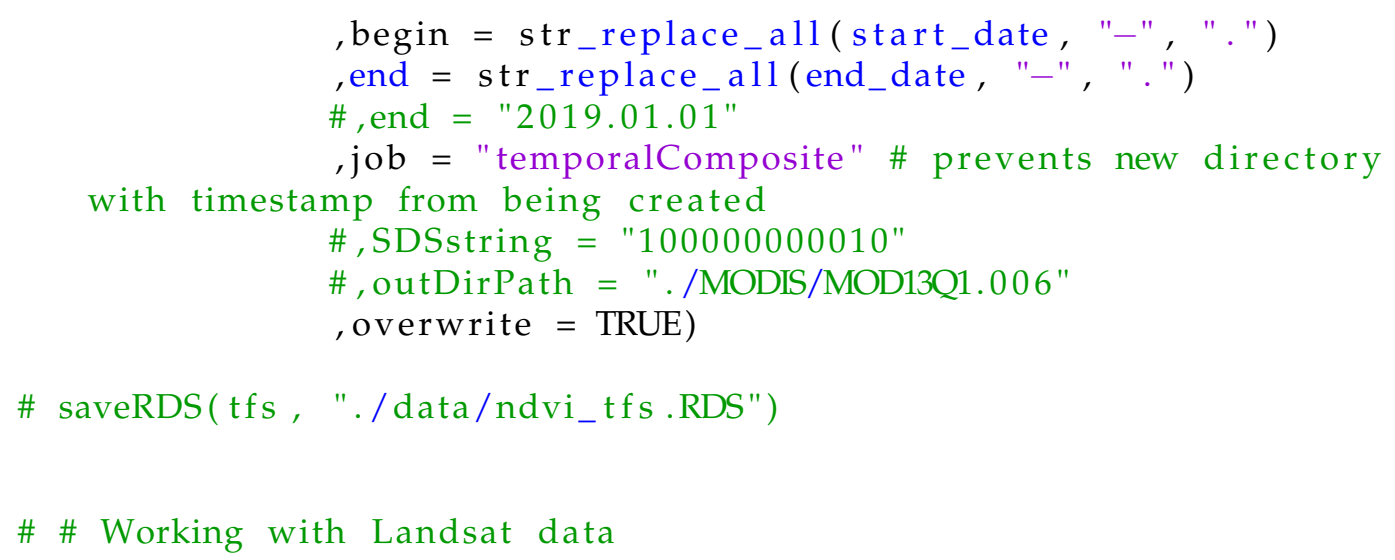




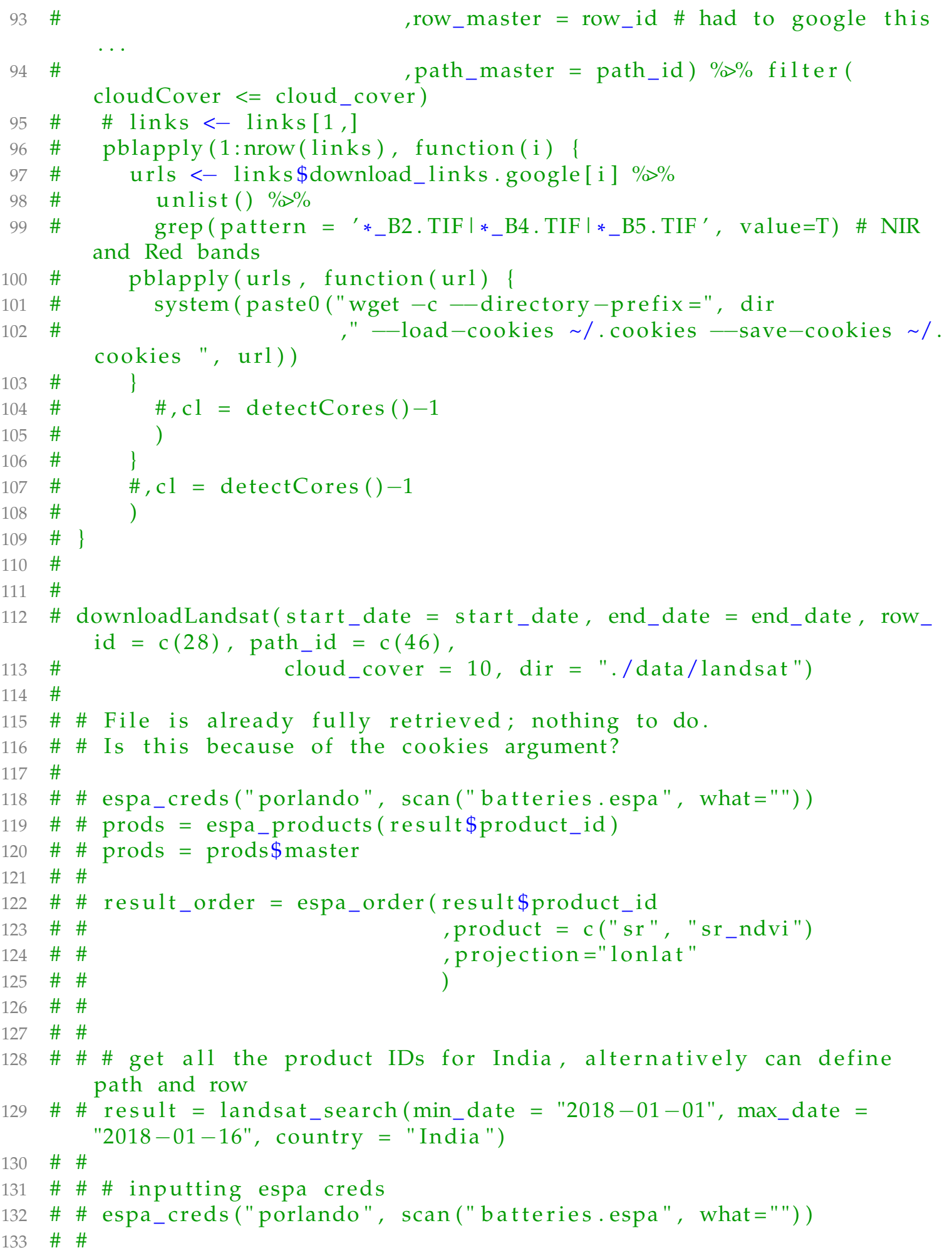


\# \# \# getting available products

\# \# prods = espa_products (result\$product_id)

\# \# prods $=$ prods $\$$ master

\# \#

\# \# \# placing an espa order

\# \# result_order = espa_order $($ result\$product_id, product $=\mathrm{c}(" \mathrm{sr} ", " \mathrm{sr}$ _ndvi"),

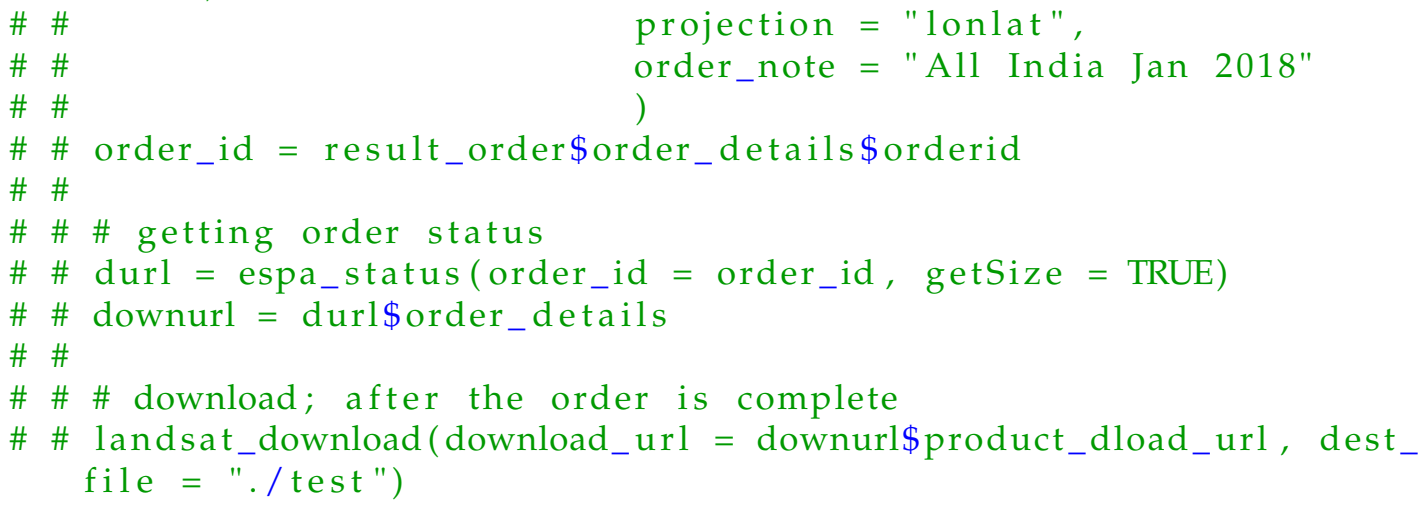




\section{Source/downloadPBL.R}

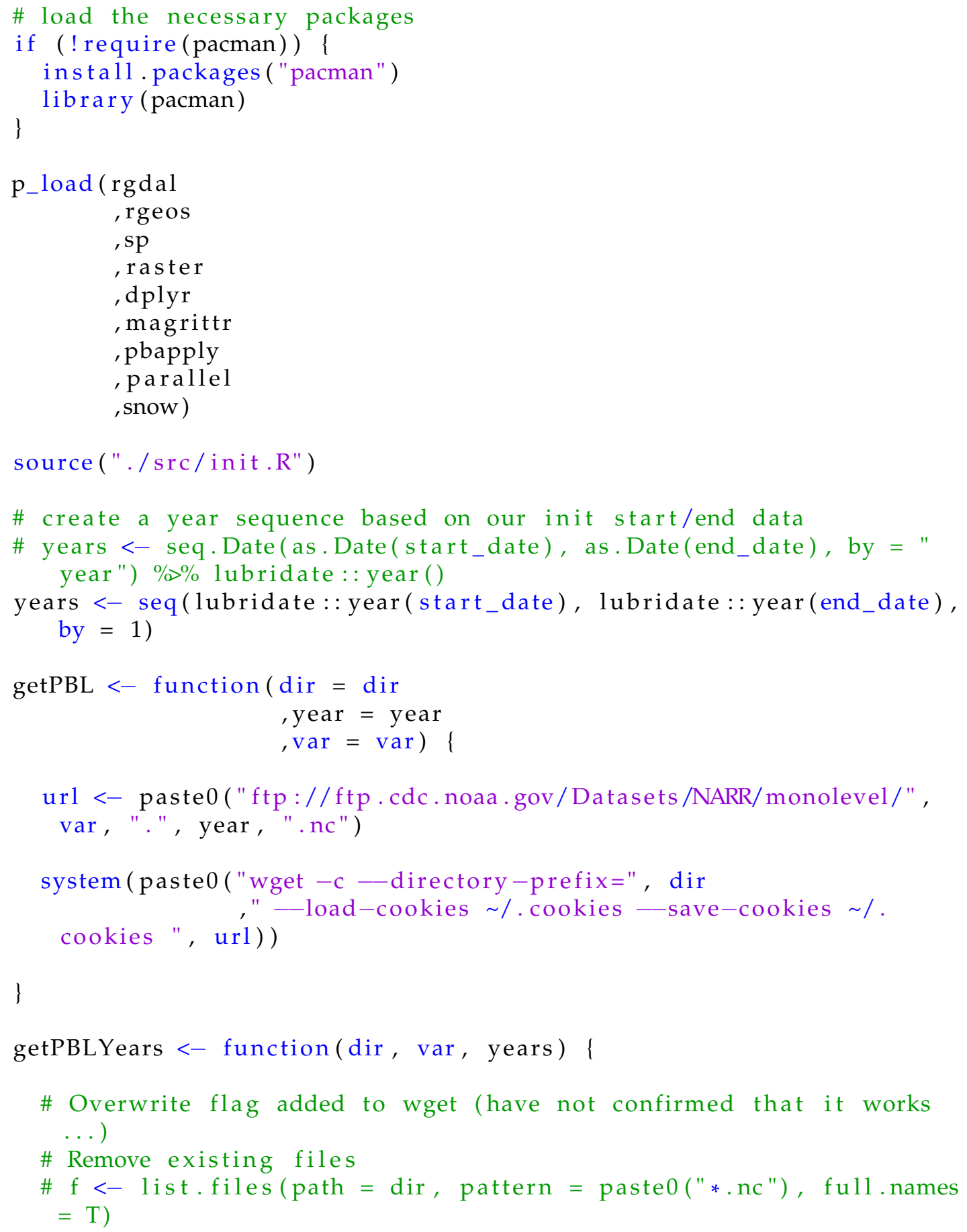




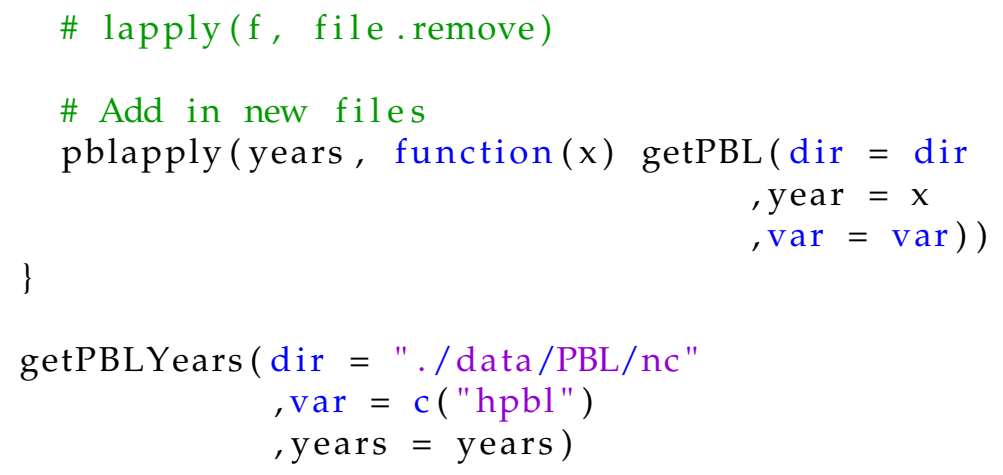

\section{Source/edaPredictors.R}

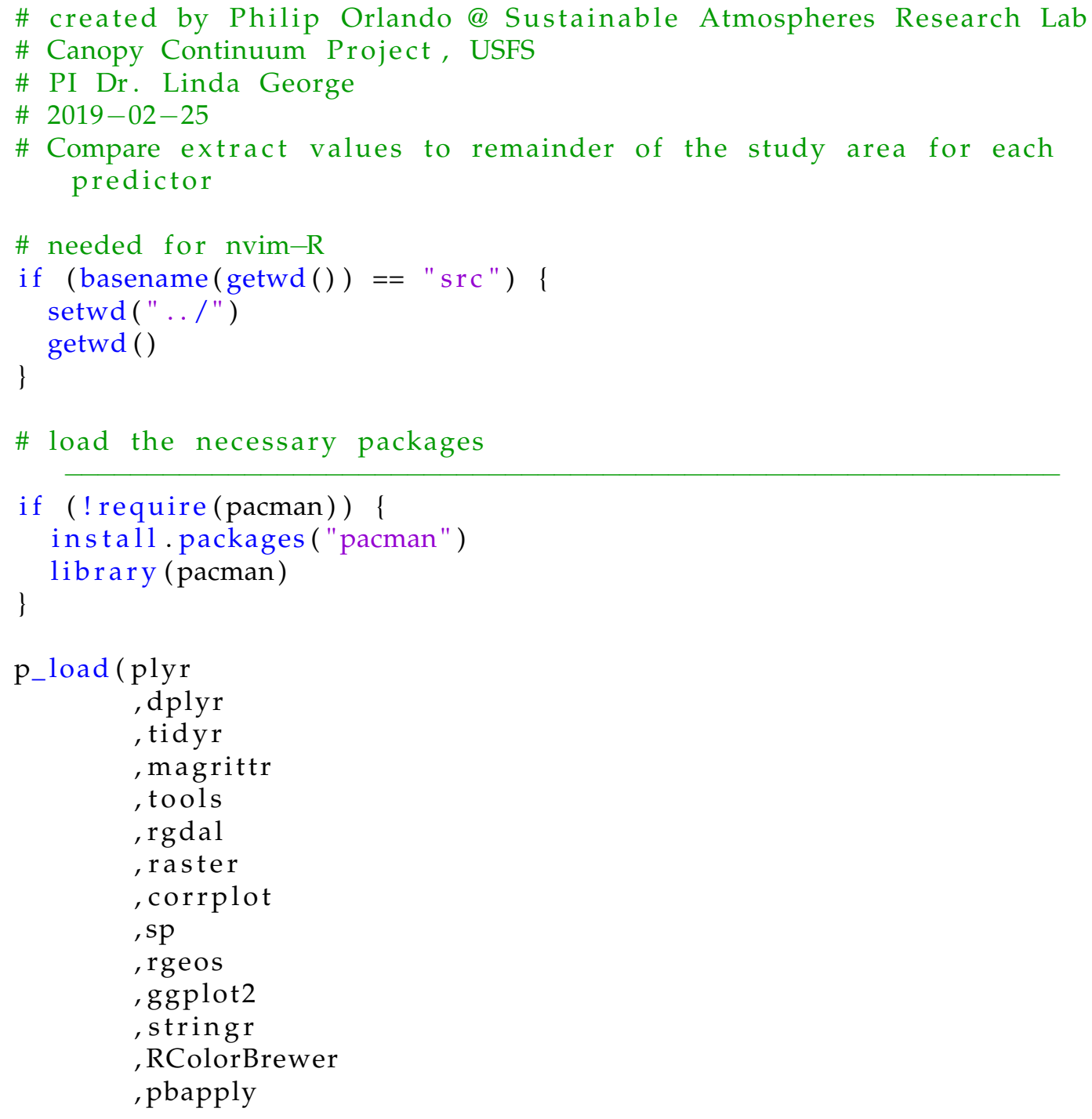




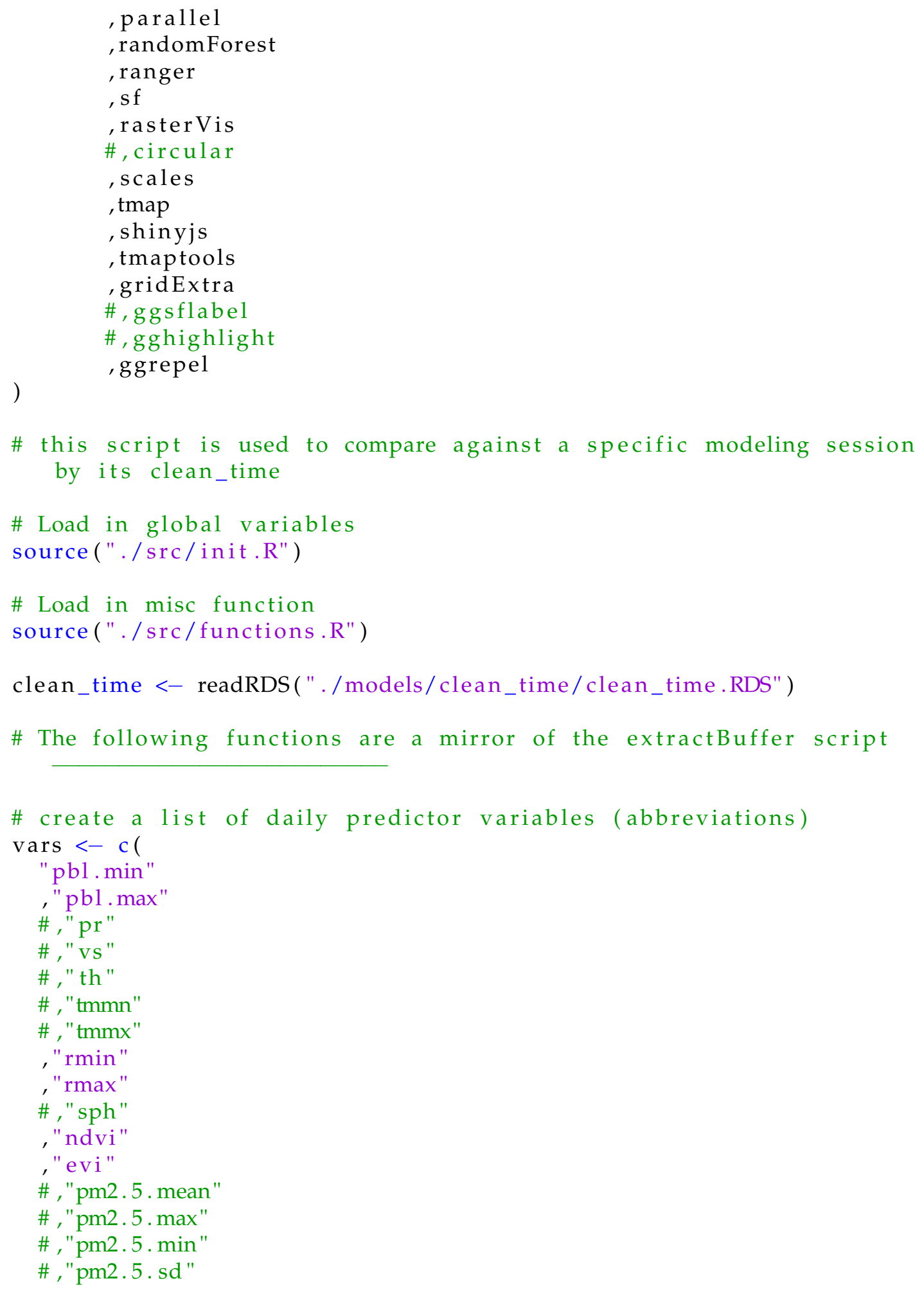




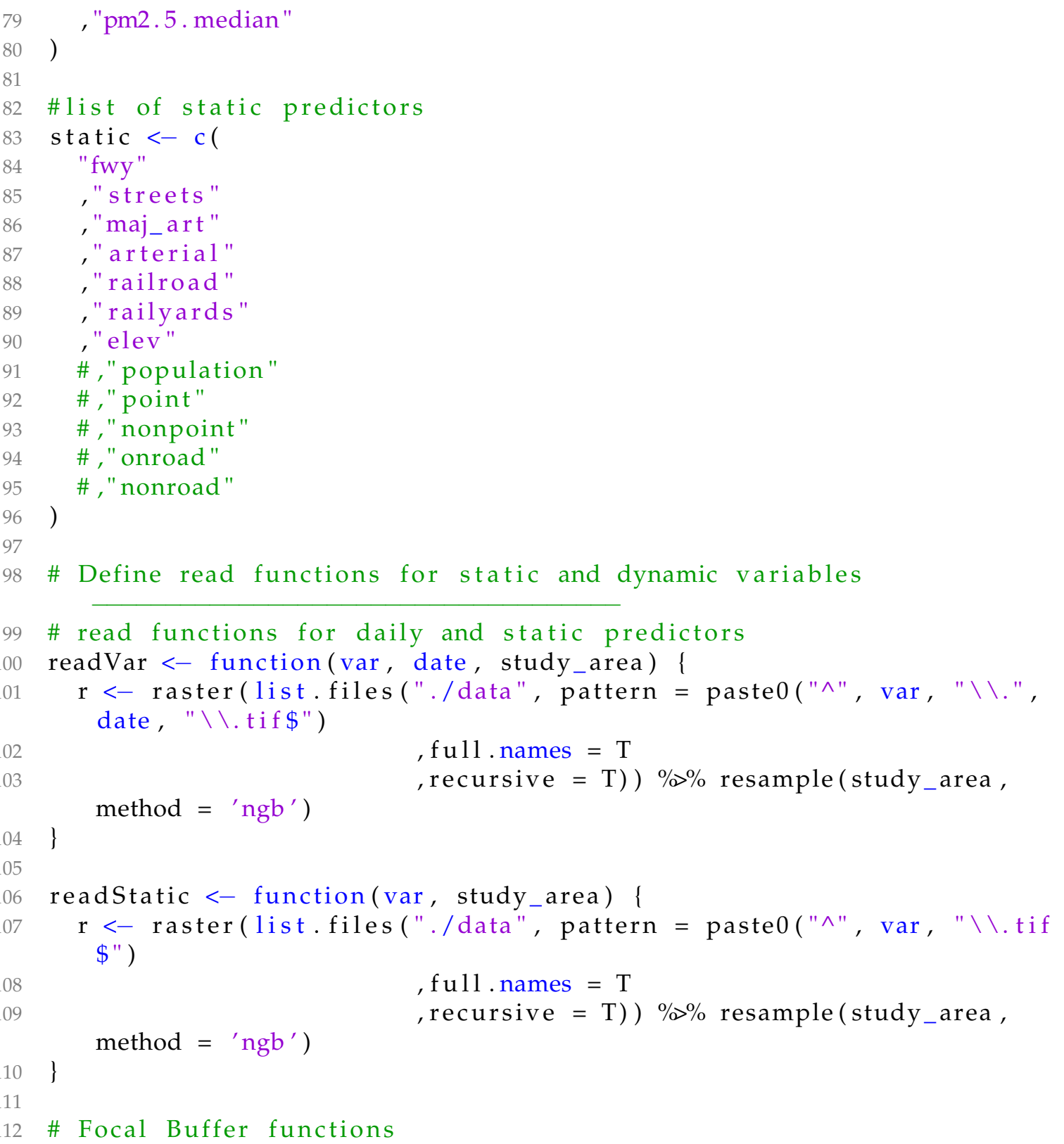

\# focal buffers credited to Jackson Voelkel @ SUPR

\# Buffer sequence

\# buffers <- c ( $\operatorname{seq}(500,5000,500), \operatorname{seq}(6000,10000,1000))$

\# buffers <- c(1000, seq $(0,10000,2500))$ \# $1 \mathrm{~km}$ buffer isn't the same as $0 \mathrm{~km}$ !

\# Read in buffers that matched their creation in extractBuffer.R 
buffers <- readRDS(paste0("./data/buffers/", clean_time, "_buffers. RDS") )

\# Run a single moving window focalBufferRaster <- function (rast, bufferDist) \{ \# skip wind direction and PBL during buffering if (!grepl("^th|pbl.*|pm $2 \backslash \backslash .5 \backslash \backslash . * "$, names( rast $)))$ \{ buf $<-$ focal (rast ,focalWeight (rast, bufferDist, type=" circle") \# assigns NA instead of 0 to edge effects , na.rm $=\mathrm{T}$

) names(buf) <- paste0 (names(rast), ".", bufferDist, "m") return (buf) 


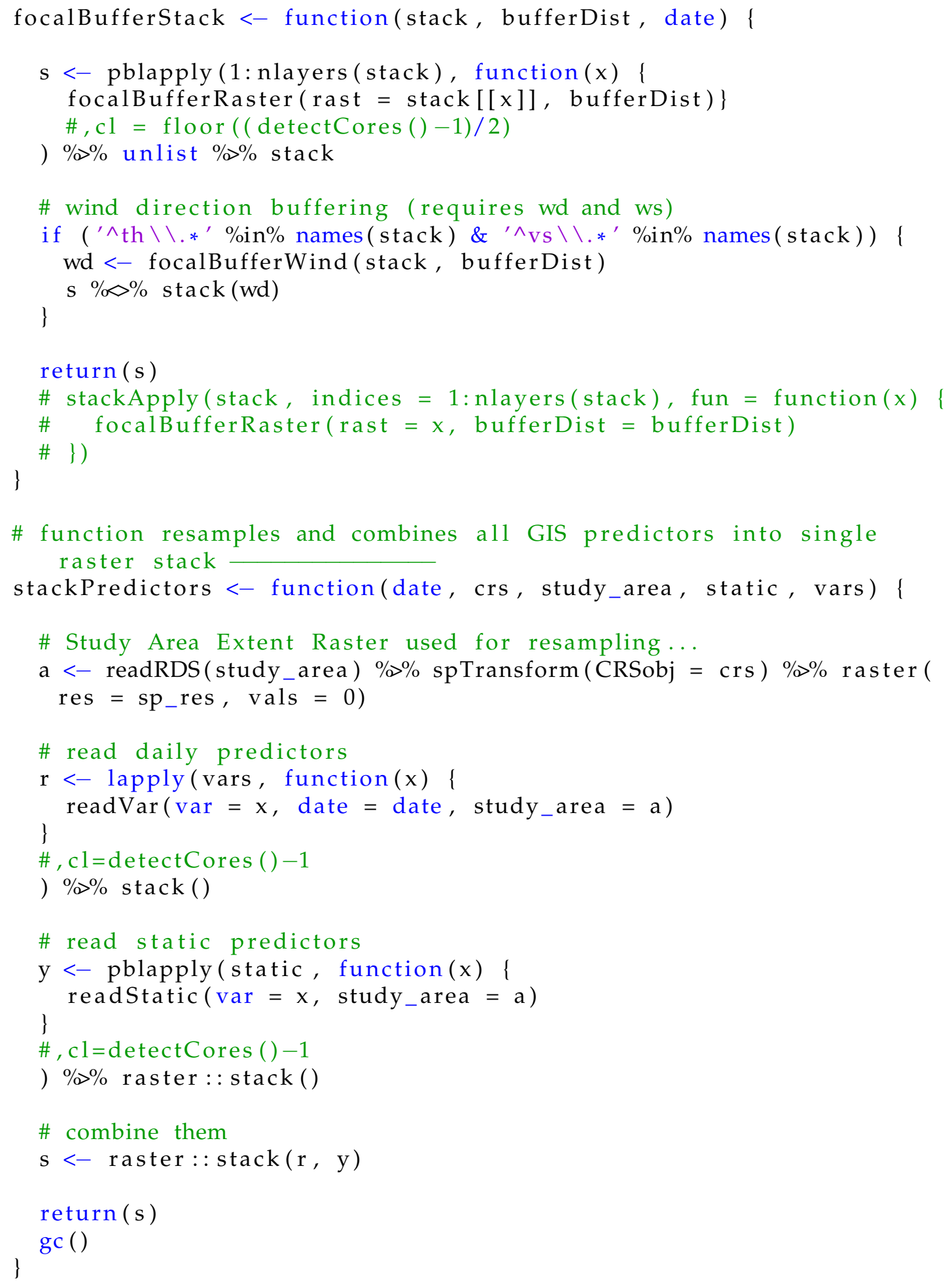


\# Run all of the focal buffers at once!

createBuffers <- function(stack, buffers, date) \{

\# clear buf dir before writing new files?

\# try (system (paste0("rm ./data/buf/", date, "*", bufferDist, "m. tif ")), silent=T)

\# file.remove(paste0 ("./data/buf/", date, "*", bufferDist, "m.tif") , showWarnings $=\mathrm{F}$ )

\# file.remove(paste0 ("./data/buf/", date, ".*", bufferDist, "m\\. tif "), showWarnings $=F$ )

$\# \mathrm{f}<-$ list.files $($ path $=" . /$ data $/$ buf", pattern $=$ paste0 $($ date, $" . *$. tif"), full.names $=\mathrm{T}$ )

\# lapply(f, file.remove)

pblapply (buffers , function $(x)$ i focalBufferStack (stack, bufferDist $=x$, date $=$ date $)$ \}

$\#, \mathrm{cl}=\operatorname{ceiling}((\operatorname{detectCores}()-1) / 2)$

)

\}

\# Raster processing functions

\# Combine all predictors for a given day

extractPredictors <- function(date, crs, study_area, bb, vars, static , pm_df, scale_neph = scale_neph $)\{$

\# Study Area Extent Raster used for resampling...

a $<-$ readRDS(study_area) $\% \%$ spTransform (CRSobj = crs) $\% \%$ raster ( res $=$ sp_res, vals $=0$ )

\# read PurpleAir data

pa $<-$ readRDS $($ pm_df $) \% \%$ spTransform $($ CRSobj $=$ crs $)$

\# Final bounding box

$\mathrm{b}<-\operatorname{readRDS}(\mathrm{bb}) \% \%$ spTransform $($ CRSobj $=\mathrm{crs}) \% \%$ raster $\left(\right.$ res $=\mathrm{sp}_{-}$ res, vals $=0$ )

\# Create Raster Stack

$\mathrm{s}<-$ stackPredictors $($ date $=$ date

, $\mathrm{crs}=\mathrm{crs}$ 


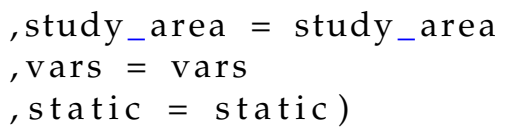




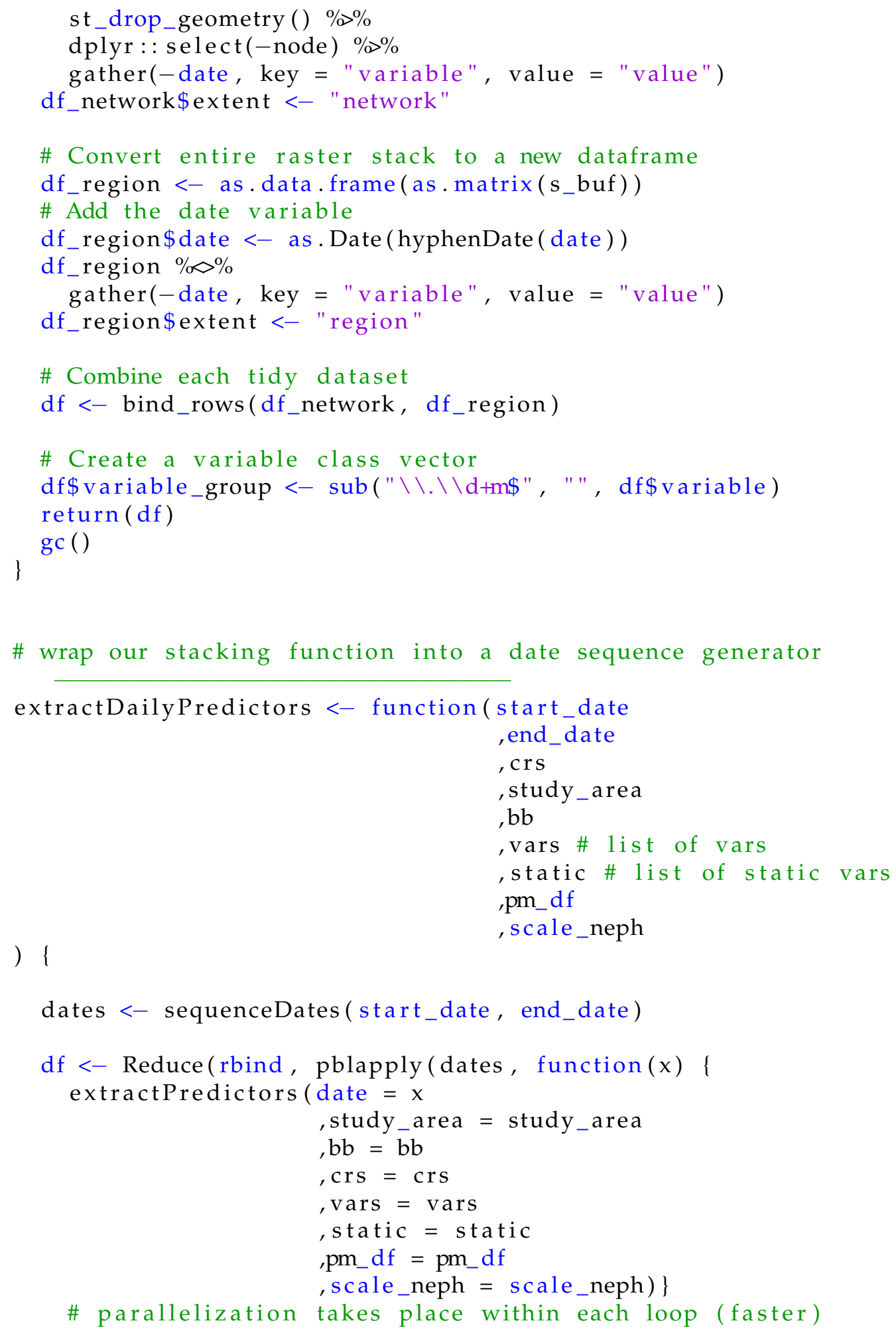




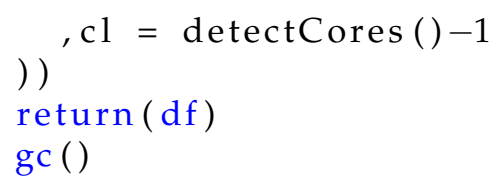




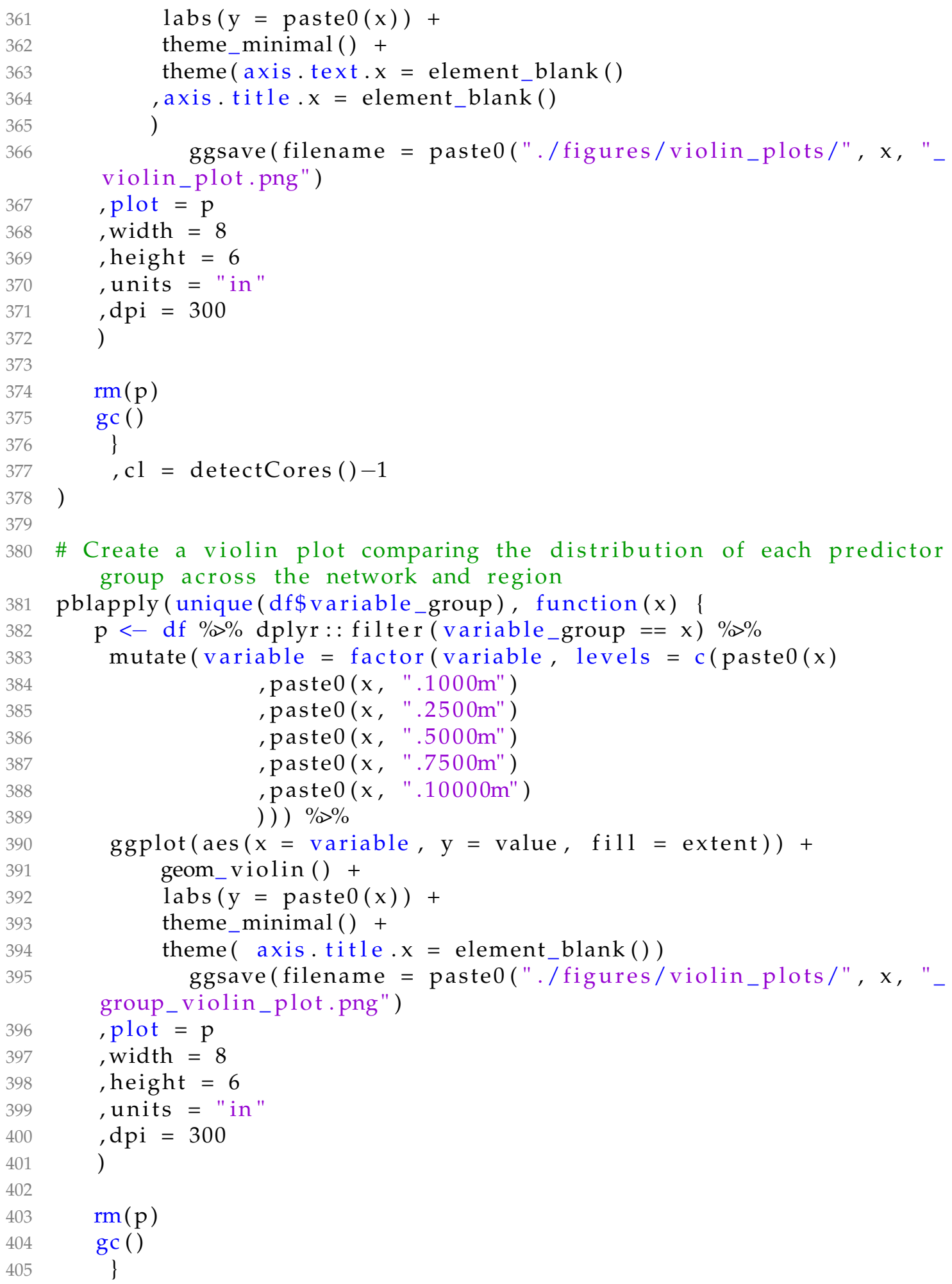




$$
, \mathrm{cl}=\operatorname{detectCores}()-1
$$

)

\section{Source/functions.R}

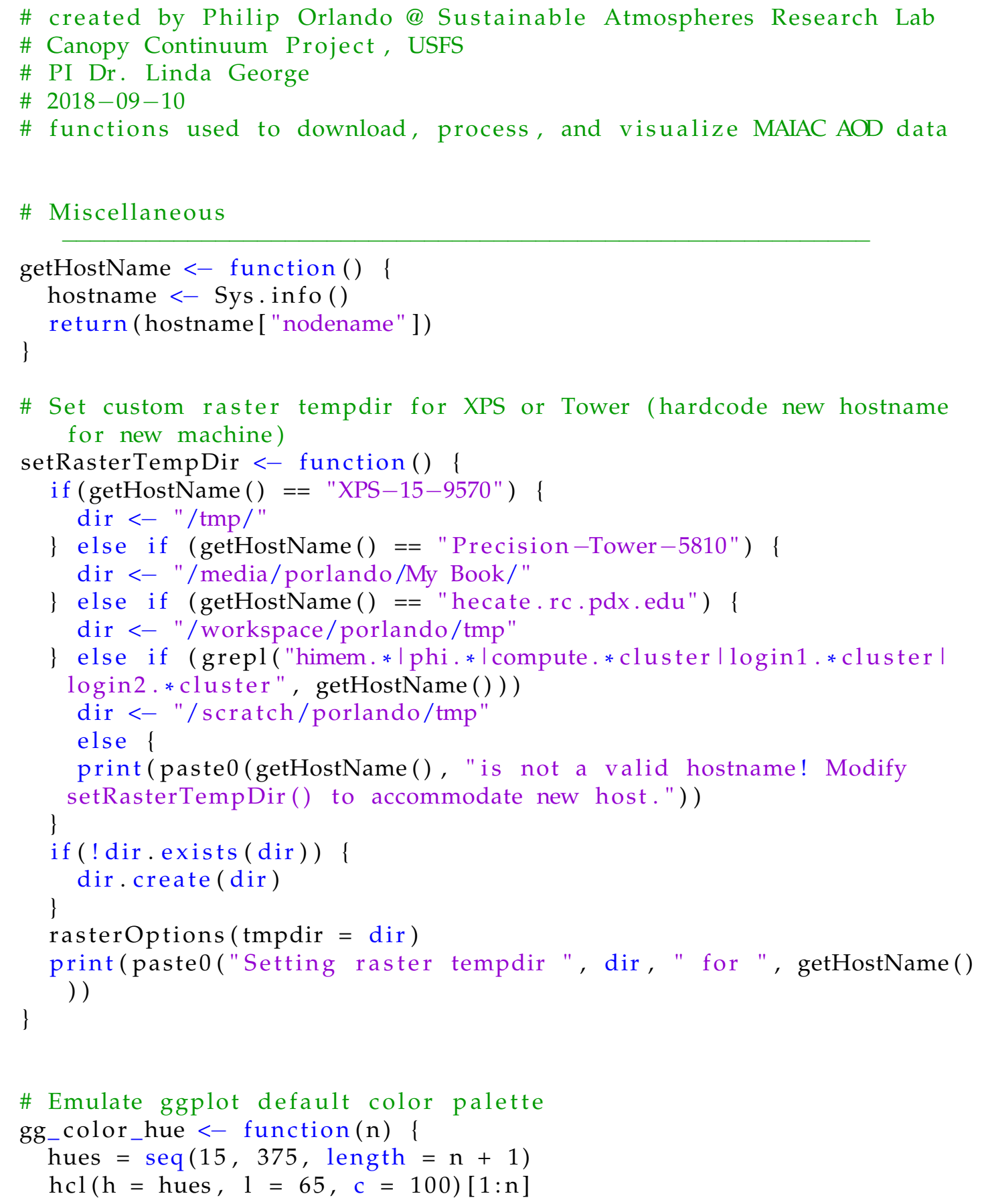




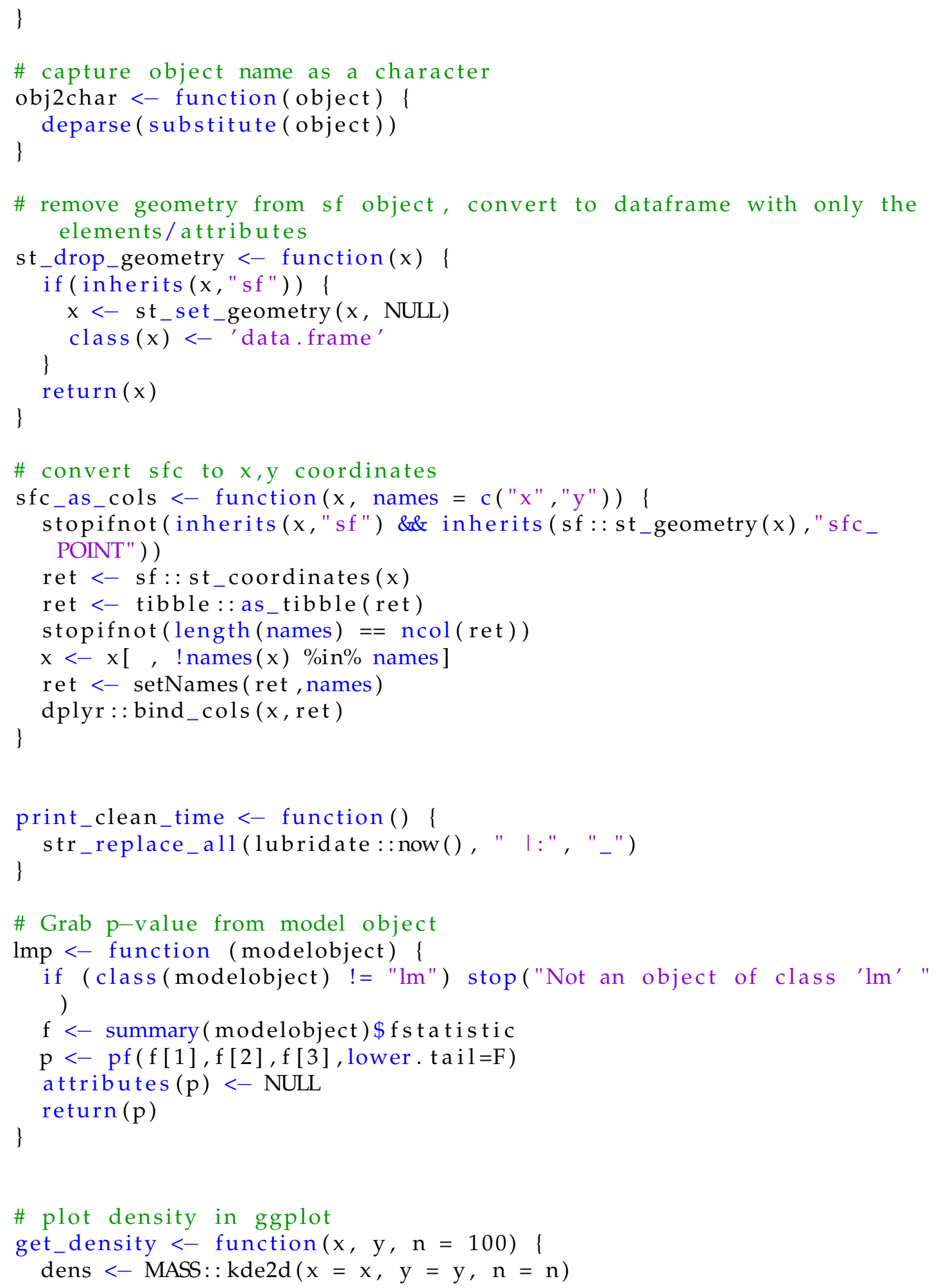




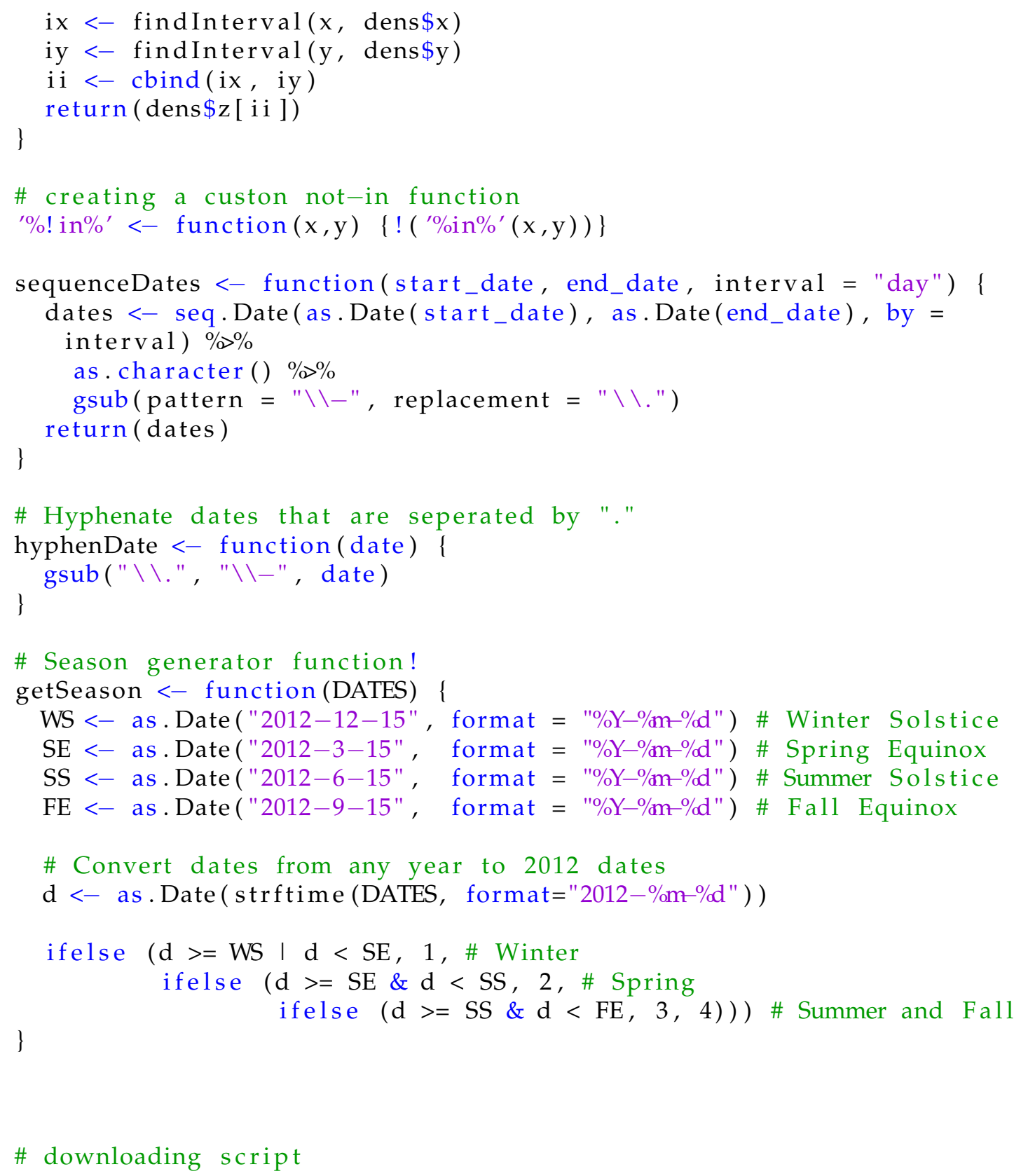




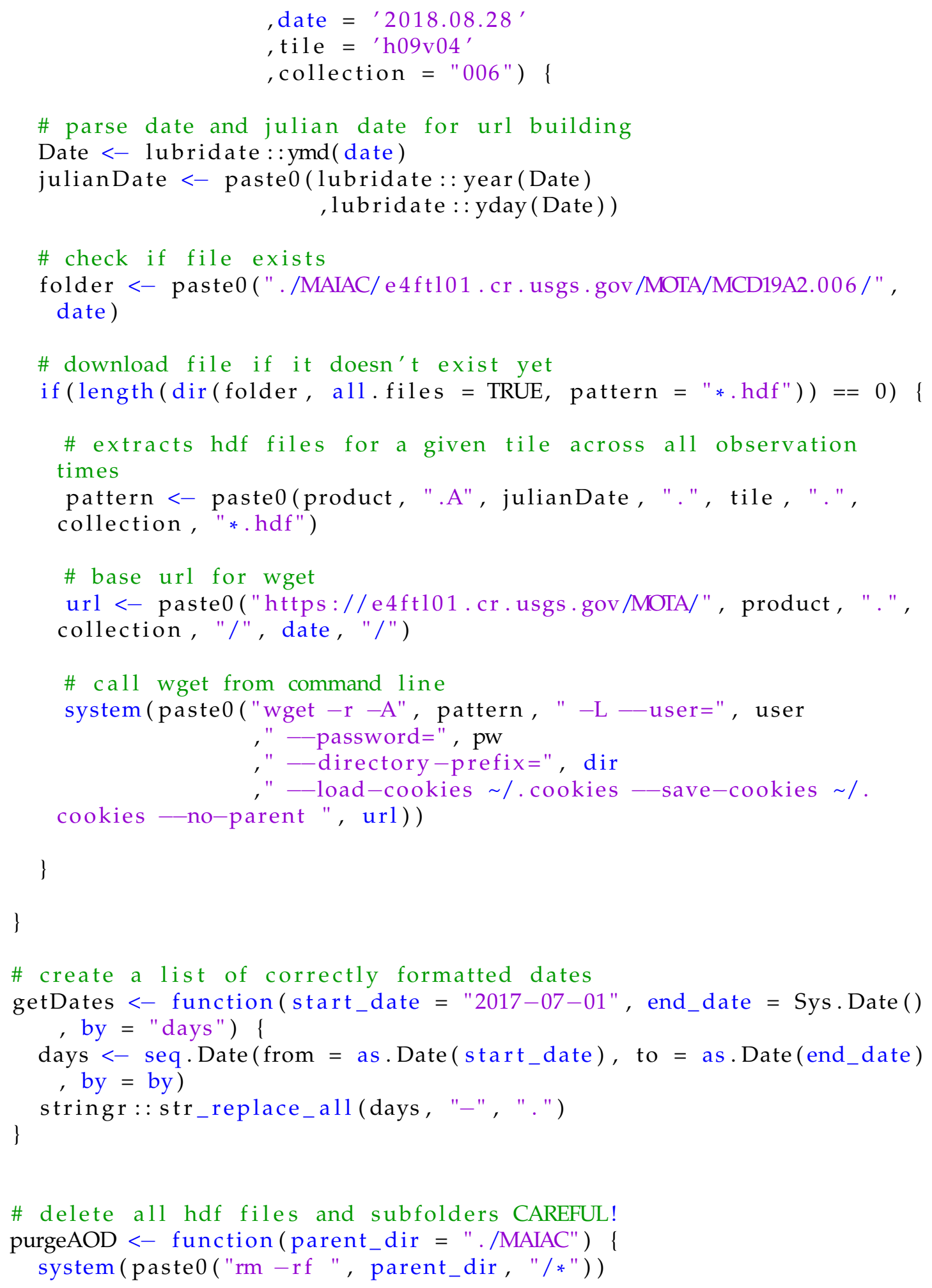


\}

\# processing script

\# process hdf and create raster processAOD <- function(file_path, study_area = portl_buf $)\{$

cat $(" \backslash n ")$

\# extract date from file_path date <- stringr::str_extract (file_path, $\left.[0-9][0-9][0-9][0-9] .[0-9][0-9] .[0-9][0-9]^{\prime}\right)$ date_clean $<-$ stringr::str_replace_all(date, "\\.", "-") \# read in hdf sds $<-$ get_subdatasets(file_path)

\# Grab AOD layers

Optical_Depth_047 = sds[grepl ("grid1km:Optical_Depth_047", sds)] $\% \%$ readGDAL $\% \%$ stack

Optical_Depth_055 = sds[grepl("grid1km:Optical_Depth_055", sds)] $\% \%$ readGDAL $\% \%$ stack

\# More variables...

\# AOD_Uncertainty = sds[grepl("grid1km:AOD_Uncertainty", sds)] \% \% readGDAL $\% \%$ stack

\# FineModeFraction = sds[grepl ("grid $1 \mathrm{~km}:$ FineModeFraction", sds)] $\%$ stack

\# Column_WW $=$ sds $[$ grepl $("$ grid $1 \mathrm{~km}:$ Column_WV", sds $)] \%$ stack

\# AOD_QA $=$ sds [ grepl ("grid $1 \mathrm{~km}:$ AOD_QA", sds)] $\% \% \operatorname{readGDAL~} \% \%$ stack

$\#$ AOD_MODEL $=$ sds $[$ grepl $(" \operatorname{grid} 1 \mathrm{~km}:$ AOD_MODEL", sds $)] \%$ readGDAL $\% \%$ stack

\# Injection_Height $=$ sds[grepl("grid1km:Injection_Height", sds)] $\% \%$ readGDAL $\% \%$ stack

\# Note that some variables such as 'RelAZ' are on a different resolution and must be disaggregated to match -

$\# \cos S Z A=$ sds $[$ grepl $(" \operatorname{grid} 5 \mathrm{~km}: \operatorname{cosSZA} ", \mathrm{sds})] \% \% \operatorname{readGDAL} \% \%$ stack $\% \%$ disaggregate (5)

$\# \cos V Z A=$ sds $[$ grepl $(" \operatorname{grid} 5 \mathrm{~km}: \cos V Z A ", \operatorname{sds})] \% \% \operatorname{readGDAL} \% \%$ stack $\% \%$ disaggregate (5)

\# RelAZ = sds[grepl("grid5km:RelAZ", sds)] \% \% readGDAL \% \% stack $\% \%$ disaggregate (5)

\# Scattering_Angle = sds[grepl("grid5km:Scattering_Angle", sds)] $\% \%$ readGDAL $\% \%$ stack $\% \%$ disaggregate $(5)$

\# Glint_Angle $=$ sds [grepl("grid5km:Glint_Angle", sds)] \% \% readGDAL $\% \%$ stack $\% \%$ disaggregate (5) 


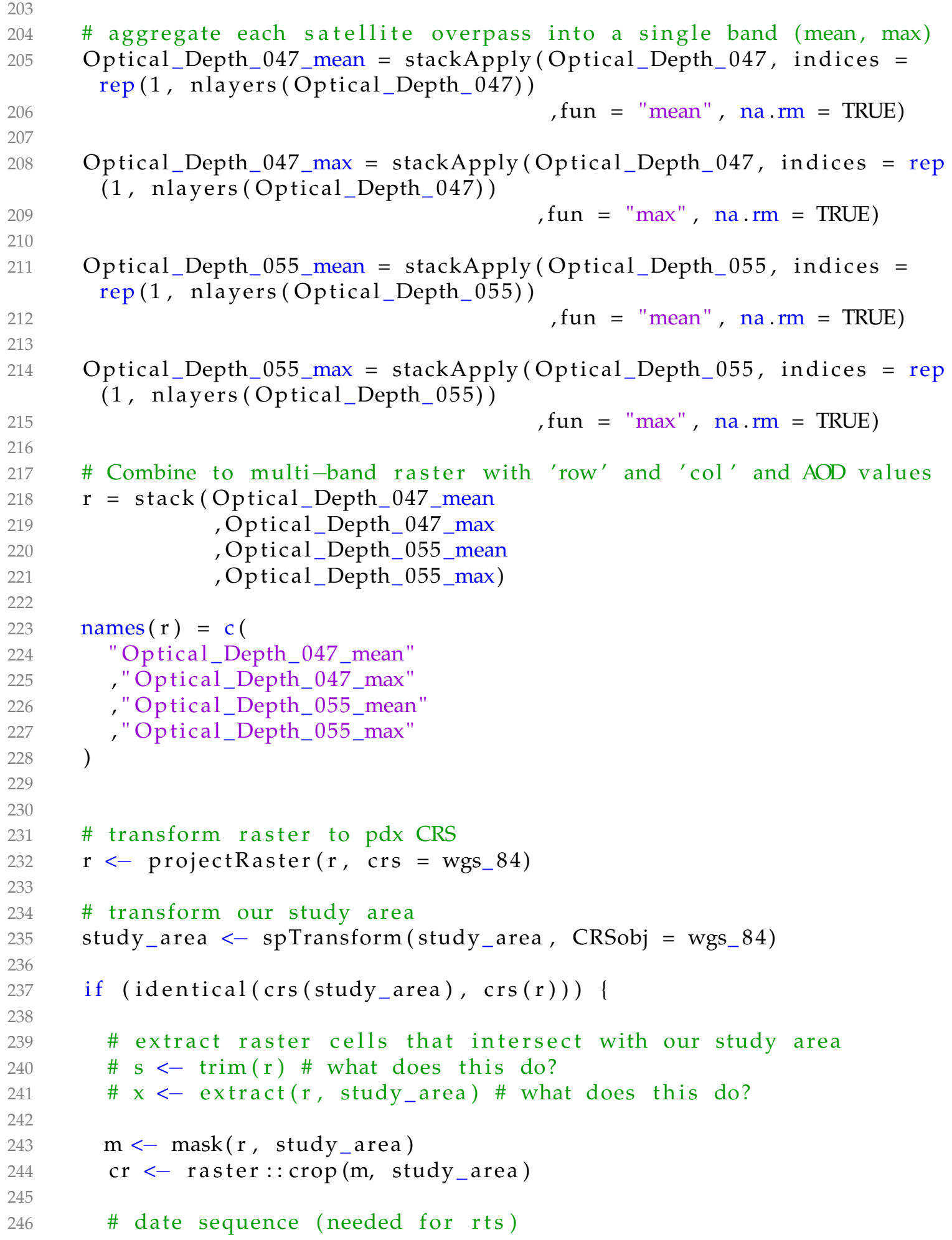




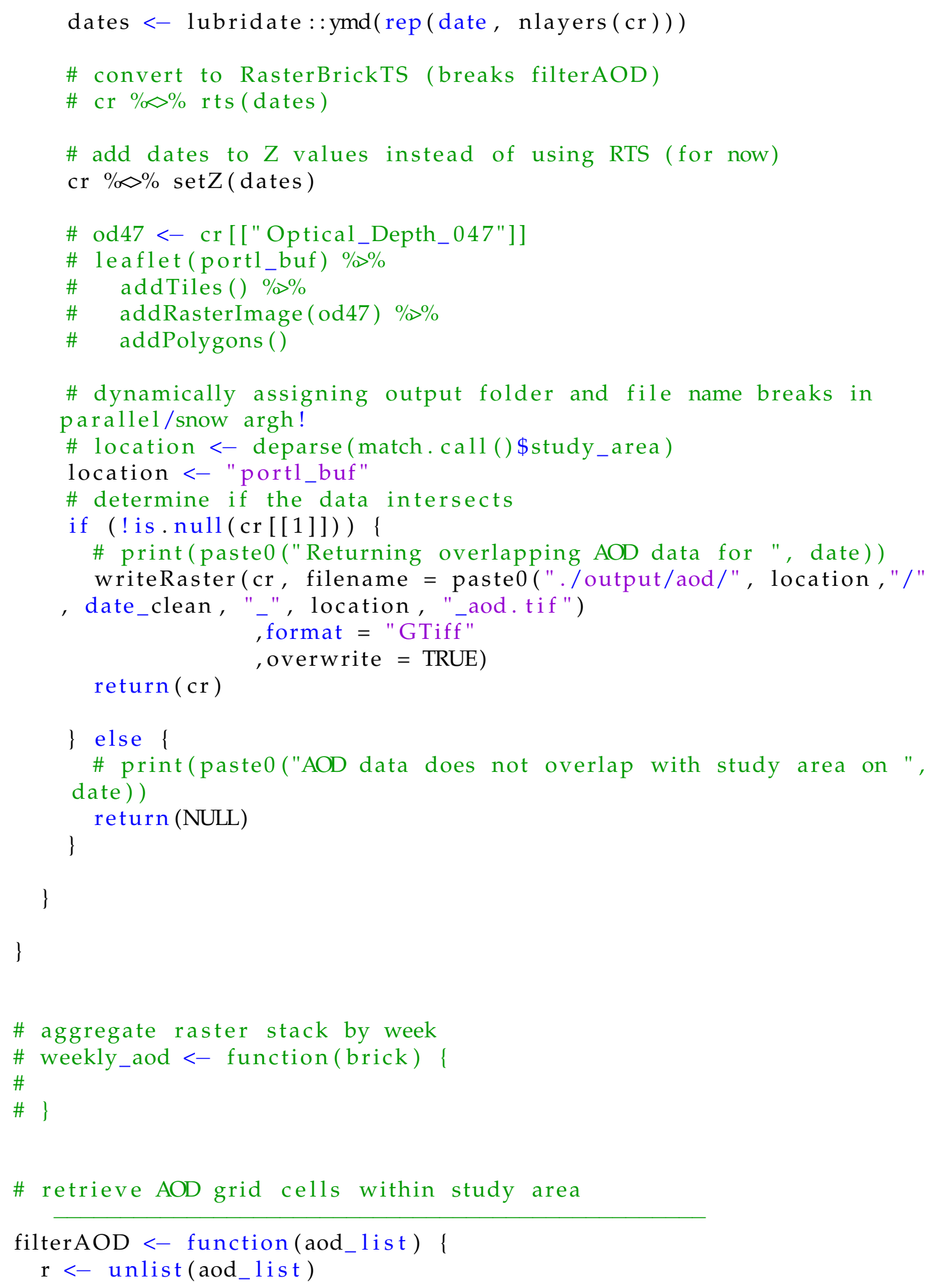




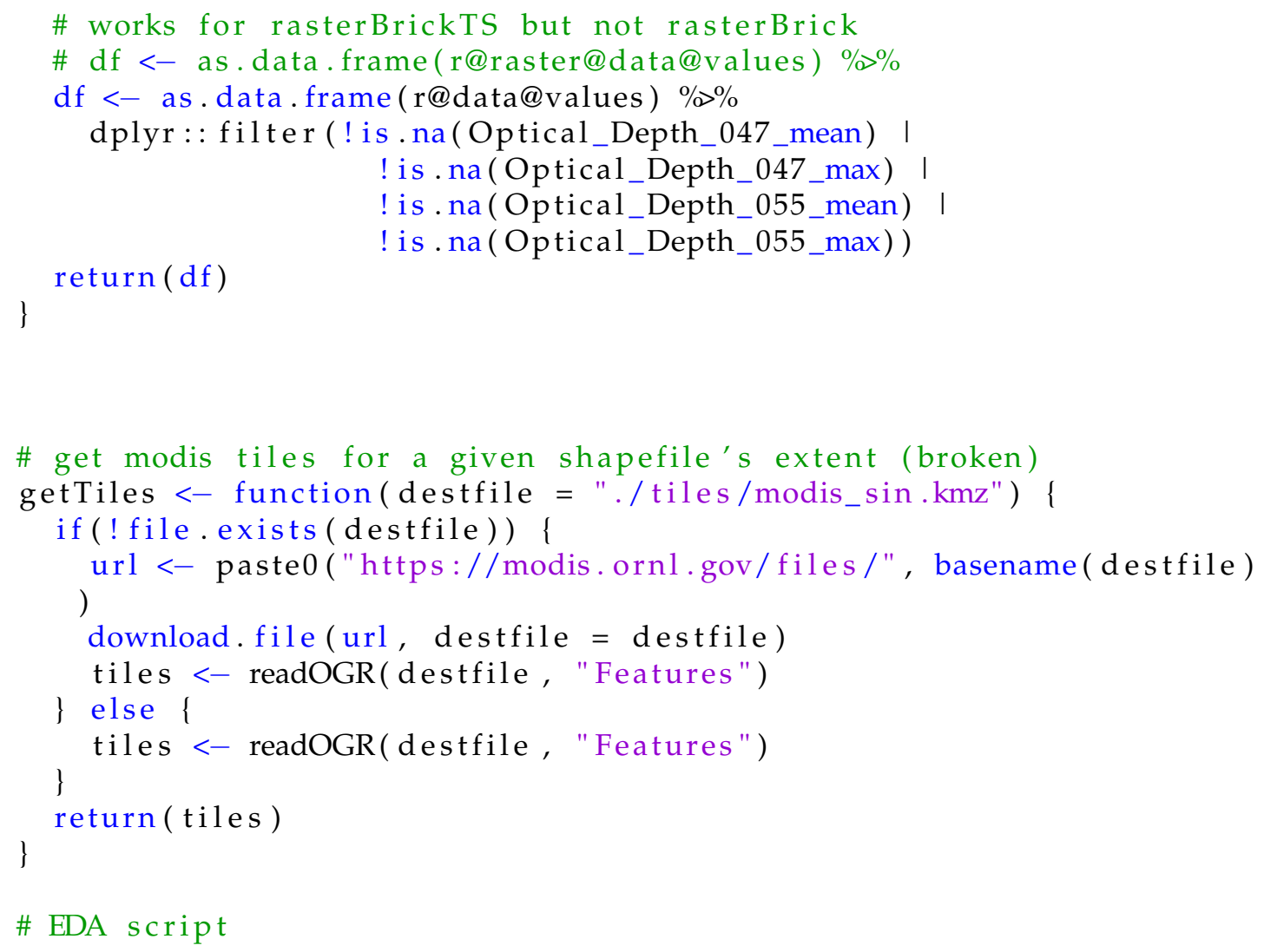




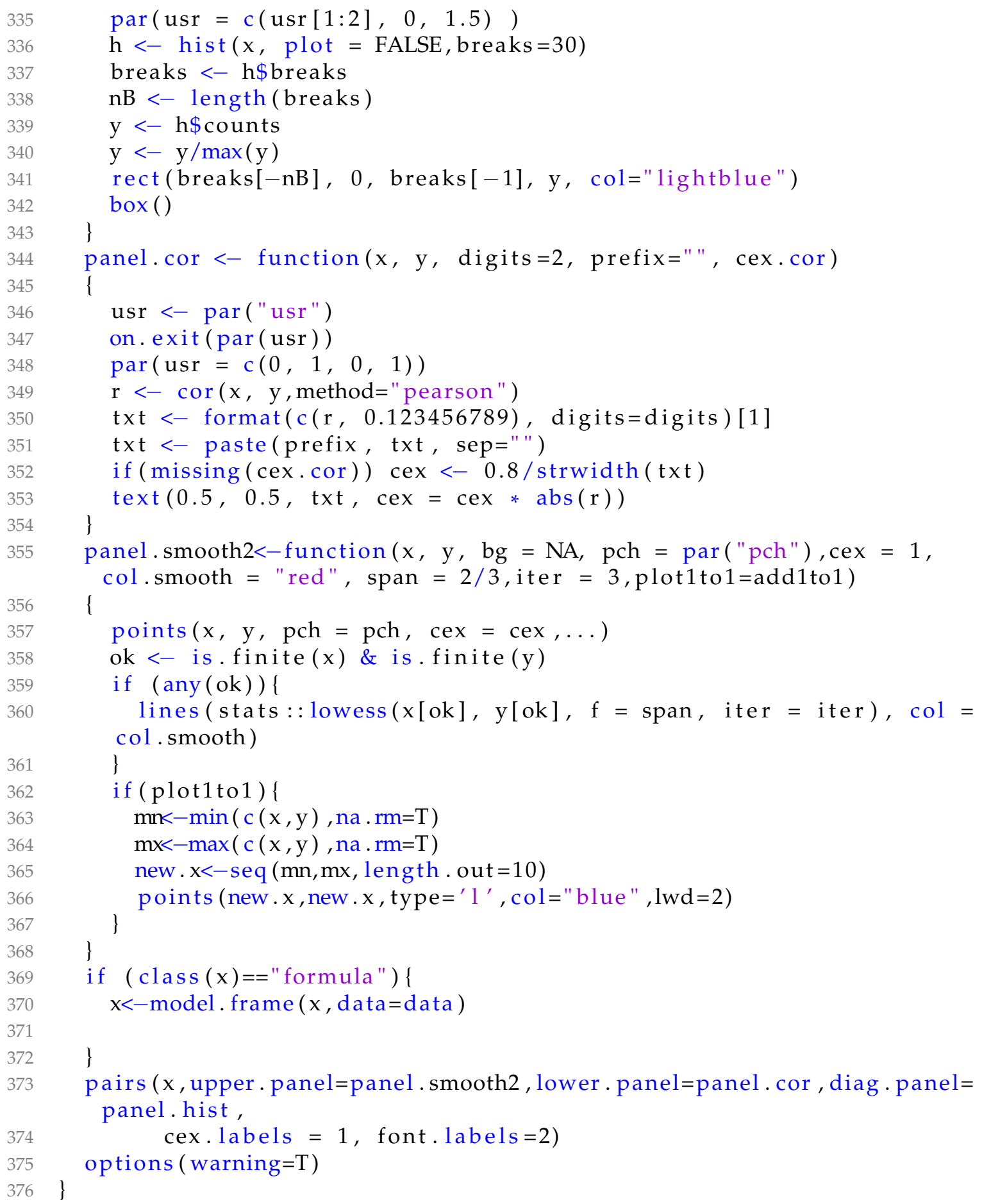

\section{Source/ggRegression.R}


\# Define Test Regression Plot

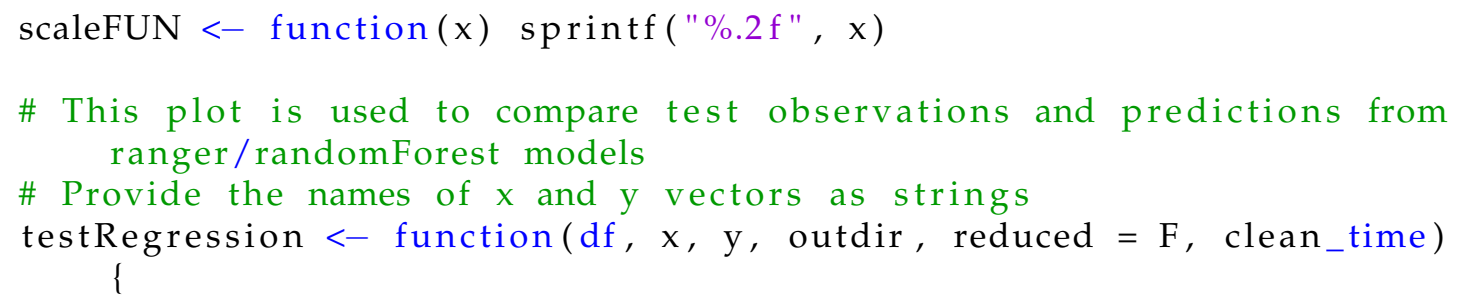


" Slope =", signif (mod_sum\$coef [[2]], 2),

$" \mathrm{p}="$, signif(as.numeric (broom: : glance (
" RMSE =", signif (rmse, 3))) +

\# geom_smooth $(\mathrm{se}=\mathrm{F}$, method $=$ "lm", col = "firebrick") + geom_abline $($ intercept $=\bmod \$$ coef [[1]]

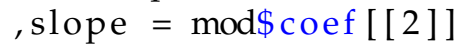




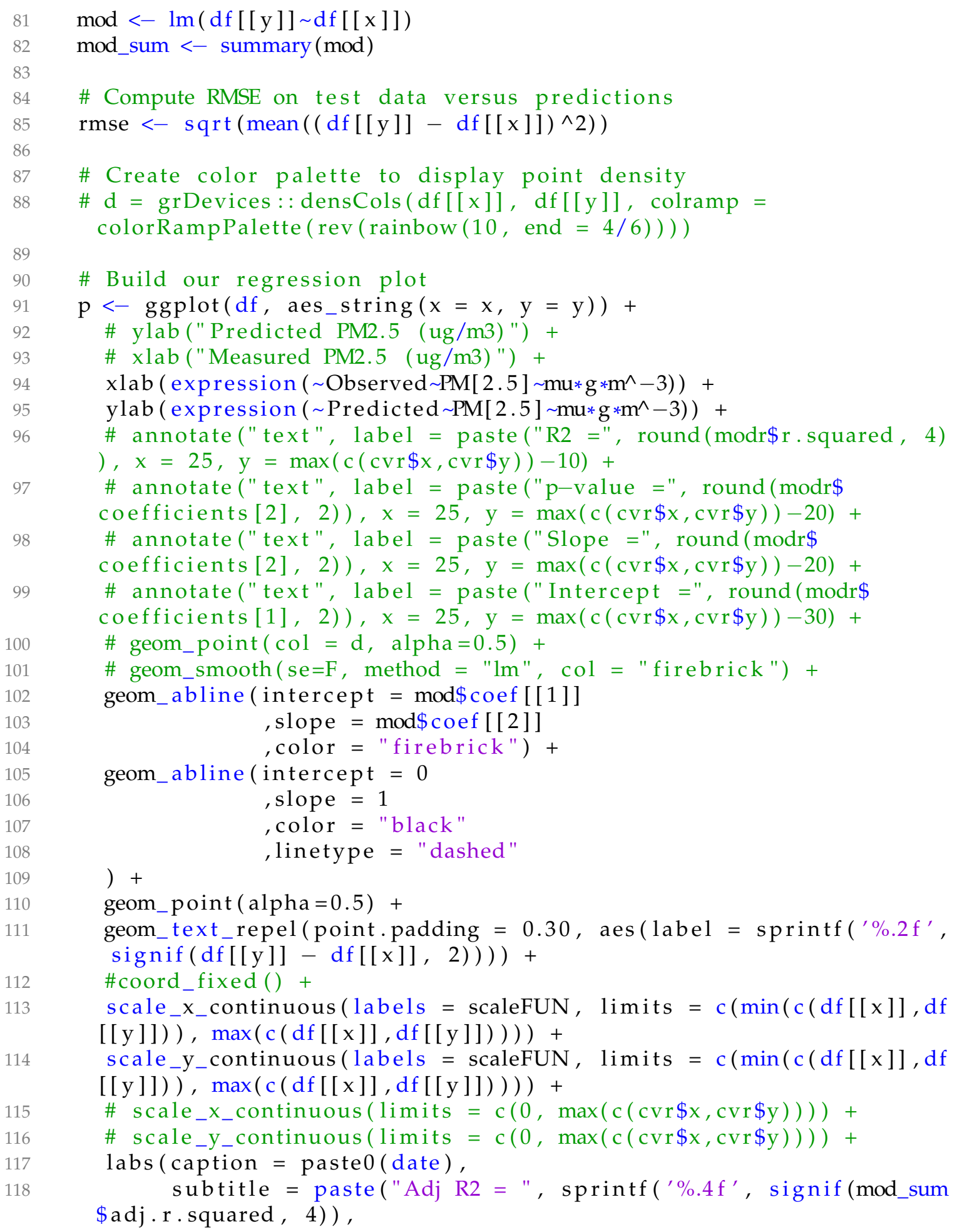


$\operatorname{sum} \$ \operatorname{coef}[[1]], 2))$, coef $[[2]], 2))$, broom : : glance (mod) \$p. value ), 3)),

"Intercept $=", \operatorname{sprintf}\left({ }^{\prime} \% .2 \mathrm{f}^{\prime}\right.$, signif $\left(\bmod _{-}\right.$ "Slope $="$, sprintf $\left({ }^{\prime} \% .2 f f^{\prime}\right.$, signif(mod_sum\$ $" \mathrm{p}="$, sprintf( $\left(\% .3 \mathrm{f}^{\prime}\right.$, signif(as.numeric ( )) + " RMSE $="$, sprintf('\%.3f', signif (rmse, 3))

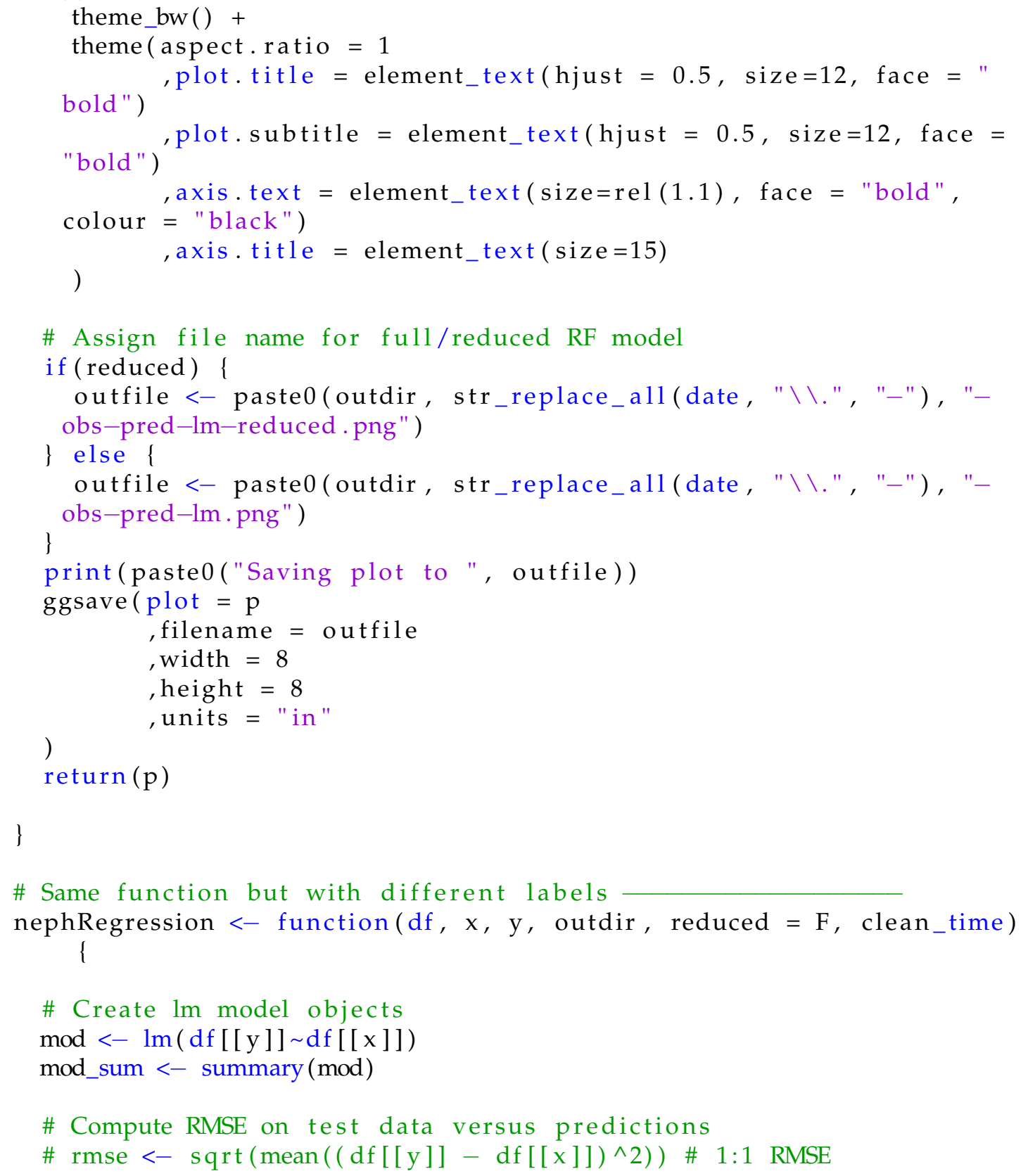


rmse $<-$ glance (mod) \$sigma \# Model RMSE

\# Create color palette to display point density

$\mathrm{d}=\operatorname{grDevices}:$ : densCols $(\mathrm{df}[[\mathrm{x}]], \mathrm{df}[[\mathrm{y}]]$, colramp $=$ colorRampPalette $(\operatorname{rev}(\operatorname{rainbow}(10$, end $=4 / 6))))$

\# Build our regression plot

$\mathrm{p}<-\operatorname{ggplot}(\mathrm{df}$, aes_string $(\mathrm{x}=\mathrm{x}, \mathrm{y}=\mathrm{y}))+$

\# ylab("Predicted PM2.5 (ug/m3)") +

\# xlab ("Measured PM2.5 (ug/m3)") +

xlab (expression ( PurpleAir $\sim$ PM[2.5] $\left.\left.\sim \mathrm{mu} * \mathrm{~g} * \mathrm{~m}^{\wedge}-3\right)\right)+$

ylab (expression $(\sim$ Nephelometer PM[ 2.5] mu*g*m^-3)) +

\# annotate ("text", label = paste $(" \mathrm{R} 2=$ ", round $(\operatorname{modr} \$ \mathrm{r}$. squared, 4)

)$, x=25, y=\max (c(\operatorname{cvr} \$ x, \operatorname{cvr} \$ y))-10)+$

\# annotate ("text", label = paste $(" \mathrm{p}-$ value $="$, round $(\operatorname{modr} \$$ coefficients $[2], 2)), x=25, y=\max (c(\operatorname{cvr} \$ x, c v r \$ y))-20)+$

\# annotate ("text", label = paste ("Slope $=$ ", round $($ modr $\$$ coefficients $[2], 2)), x=25, y=\max (c(\operatorname{cvr} \$ x, c v r \$ y))-20)+$ \# annotate ("text", label $=$ paste $(" I n t e r c e p t="$, round $(\operatorname{modr} \$$ coefficients $[1], 2)), x=25, y=\max (c(\operatorname{cvr} \$ x, c v r \$ y))-30)+$

geom_point $(\operatorname{col}=\mathrm{d}$, alpha $=0.5)+$

\#coord_fixed () +

scale_x_continuous $(\operatorname{limits}=c(\min (\mathrm{c}(\mathrm{df}[[\mathrm{x}]], \mathrm{df}[[\mathrm{y}]])), \max (\mathrm{c}(\mathrm{df}[[$ $\mathrm{x}]], \mathrm{df}[[\mathrm{y}]]))))+$

scale_y_continuous $(\operatorname{limits}=c(\min (\mathrm{c}(\mathrm{df}[[\mathrm{x}]], \mathrm{df}[[\mathrm{y}]])), \max (\mathrm{c}(\mathrm{df}[[$ $\mathrm{x}]], \operatorname{df}[[\mathrm{y}]]))))+$

\# scale_x_continuous $(1$ imits $=c(0, \max (\mathrm{c}(\mathrm{cvr} \$ \mathrm{x}, \mathrm{cvr} \$ \mathrm{y}))))+$

\# scale_y_continuous $($ limits $=c(0, \max (c(\operatorname{cvr} \$ x, c v r \$ y))))+$

labs (subtitle = paste $(" R 2="$, signif (mod_sum\$adj.r.squared, 4),

"Intercept =", signif (mod_sum\$coef [[1]], 2),

" Slope =", signif (mod_sum\$coef [[2]], 2),

) \$p. value ), 3) ,

" $\mathrm{p}="$, signif (as . numeric (broom:: glance (mod 


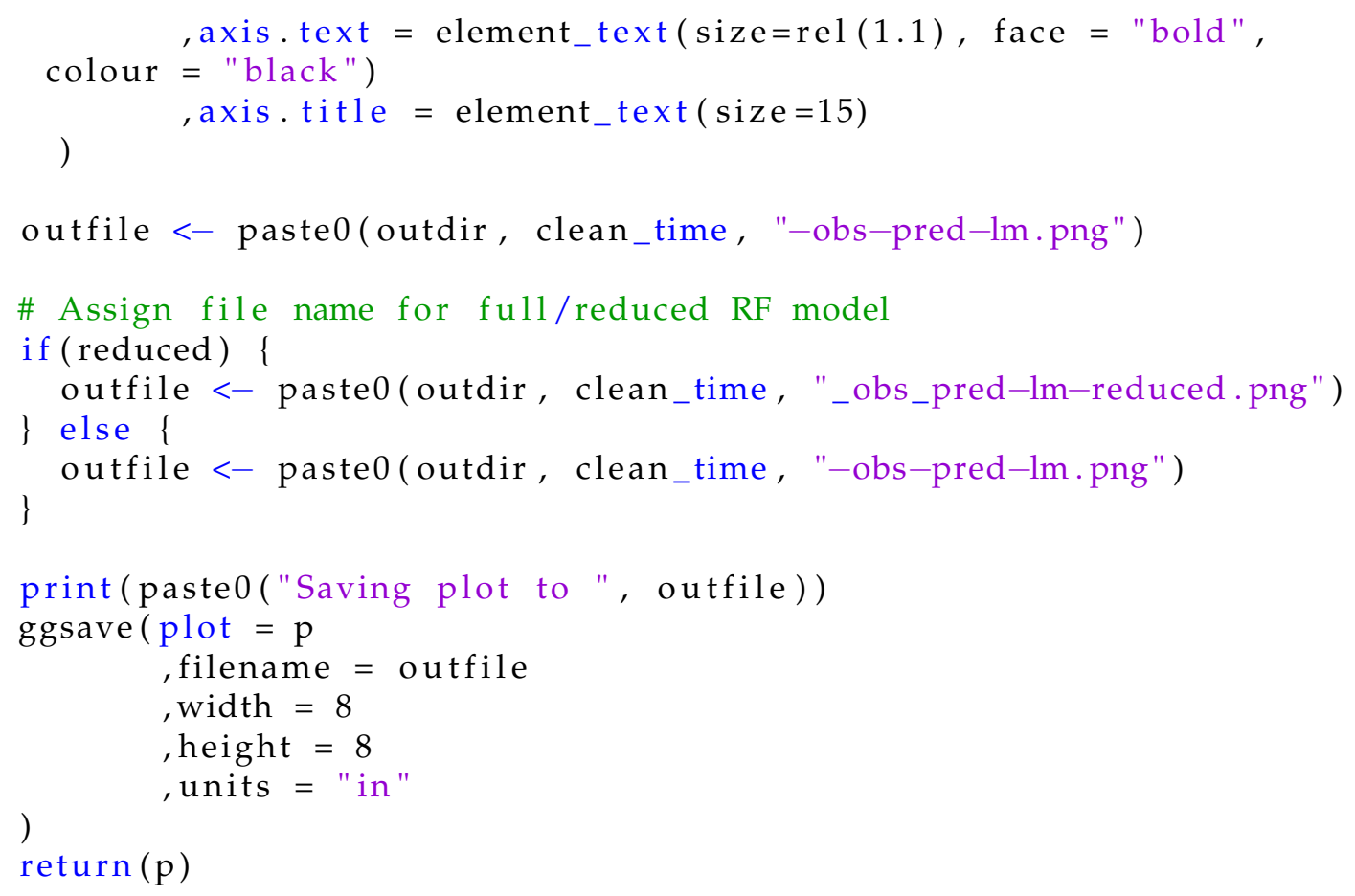

\section{Source/homogeneousPM.R}

\# created by Philip Orlando @ Sustainable Atmopsheres Research Lab 


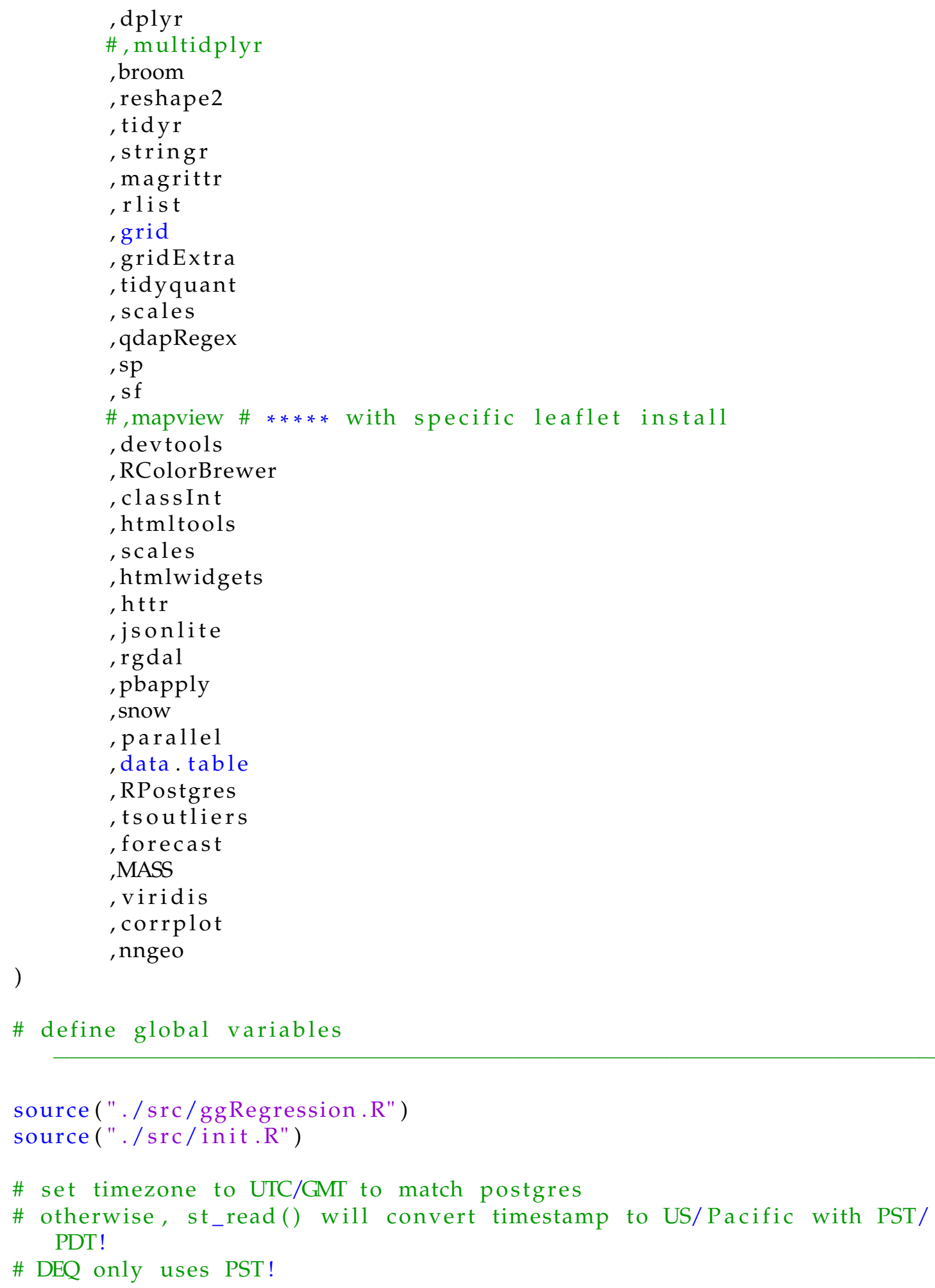




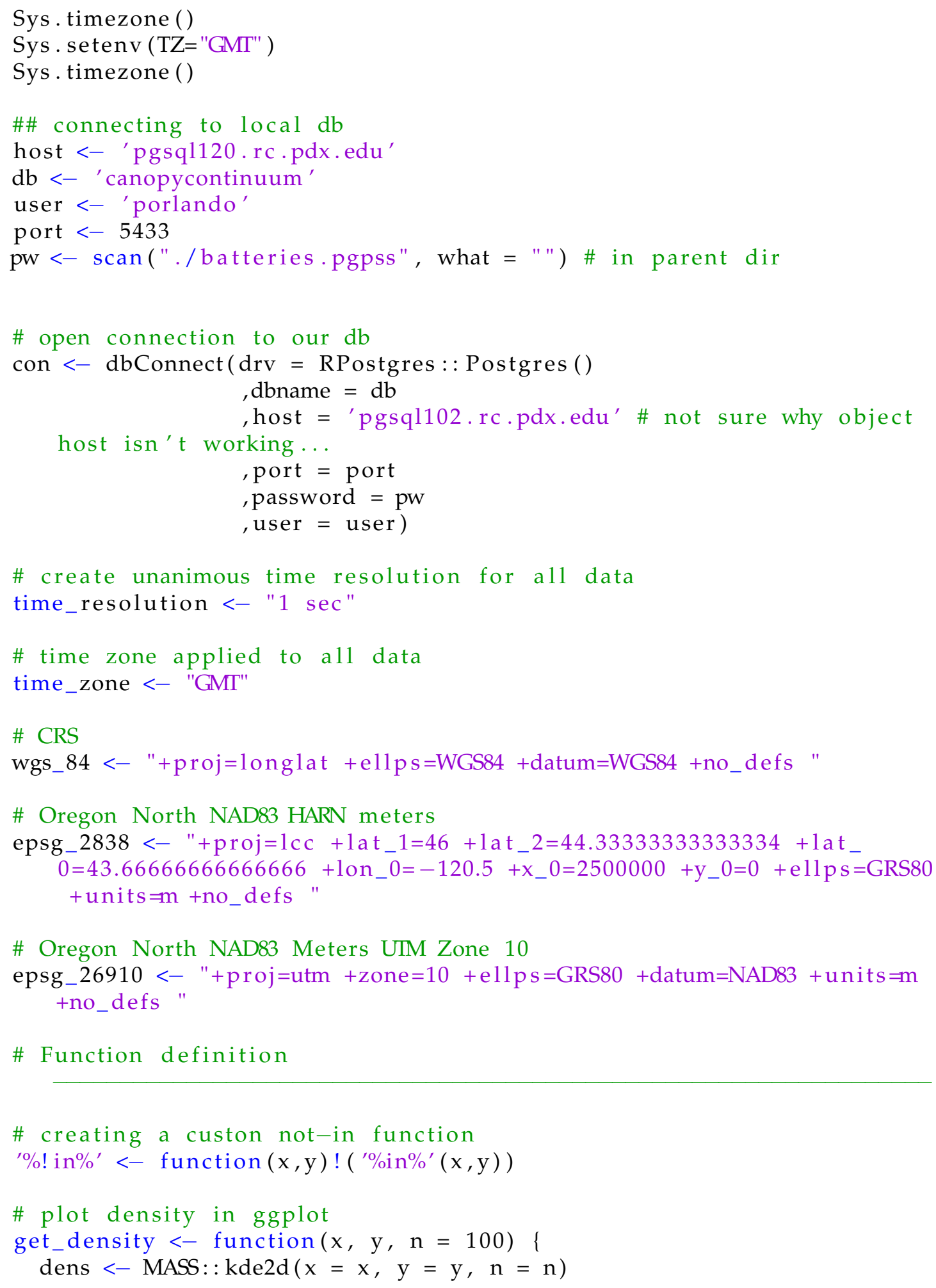




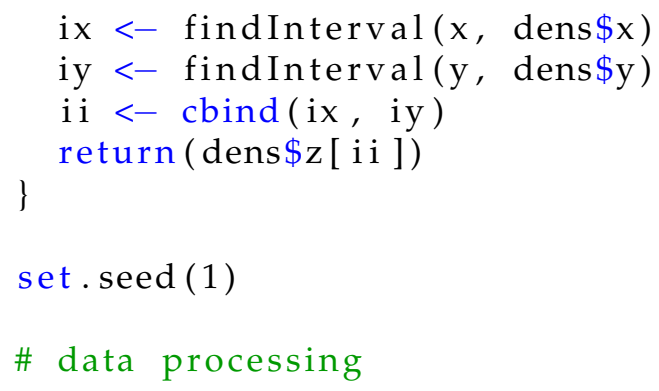




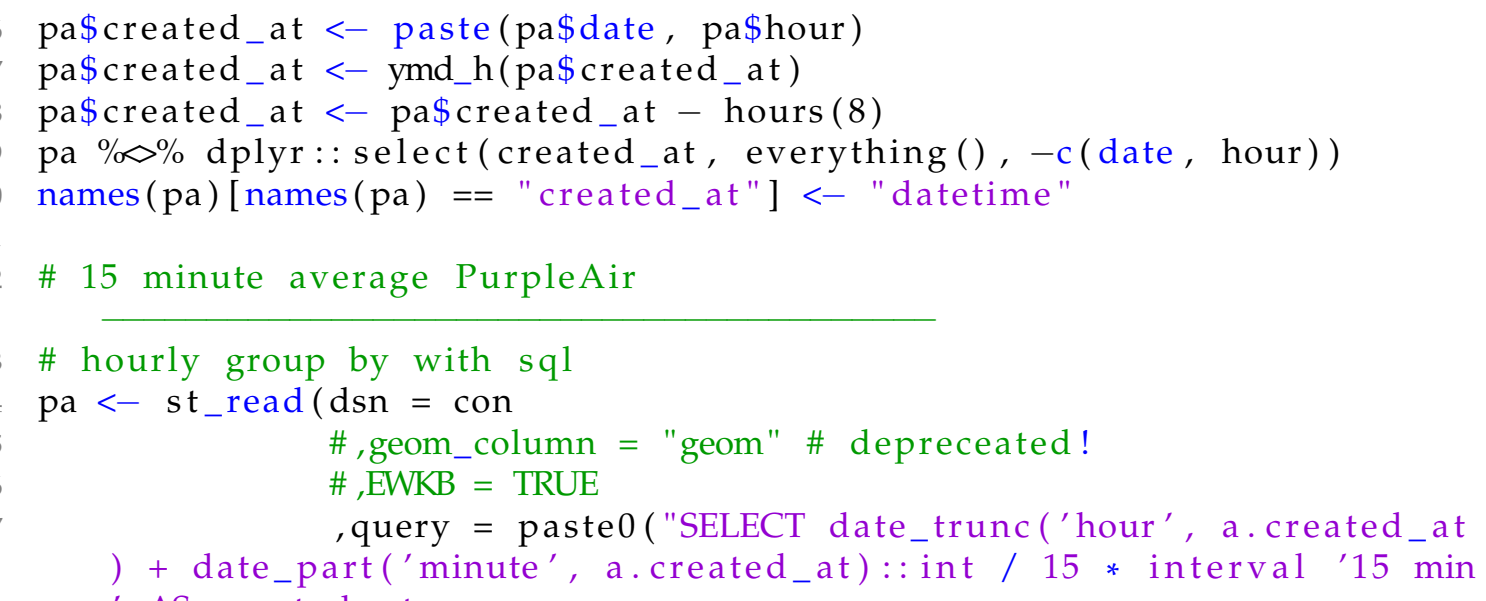

atm) pm2_5_atm, a.geom

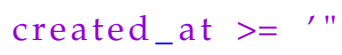




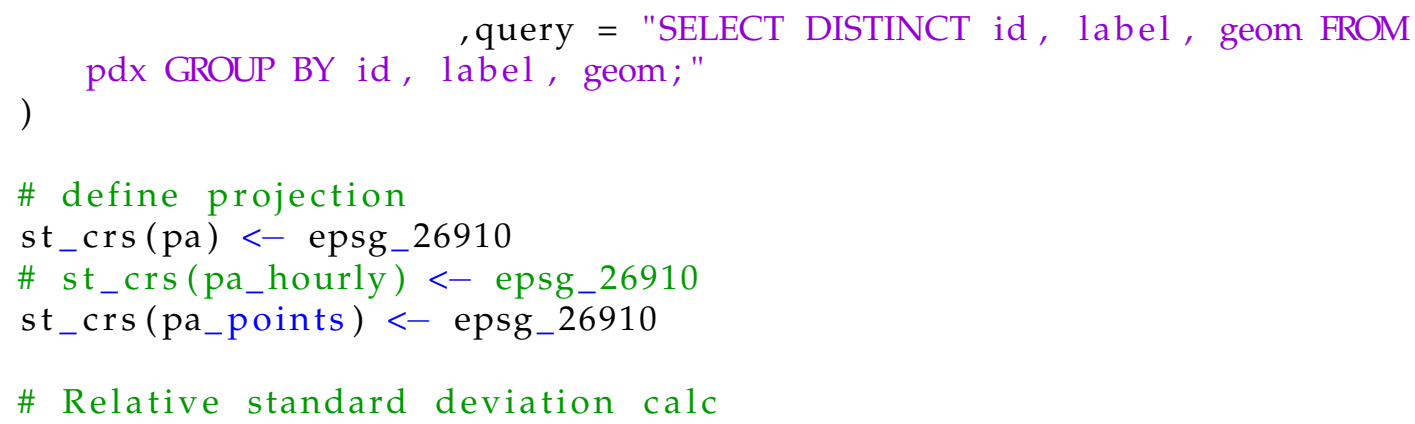


\# For 15-minute data frm_mean $\% \%$ filter $($ pm2_5_rsd $<=$ rsd_threshold $) \% \%$ filter $($ n_mean $>=$ n_min \& n_mean $<=6) \% \%$ st_drop_geometry () $\% \%$ nrow () frm_mean $\% \diamond \%$ filter $\left(\right.$ pm2_5_rsd $<=$ rsd_threshold) $\% \%$ filter $\left(n \_\right.$mean $>=$ n_min \& n_mean $<=6$ ) $\% \%$ st_drop_geometry ()

\# Calc percent difference

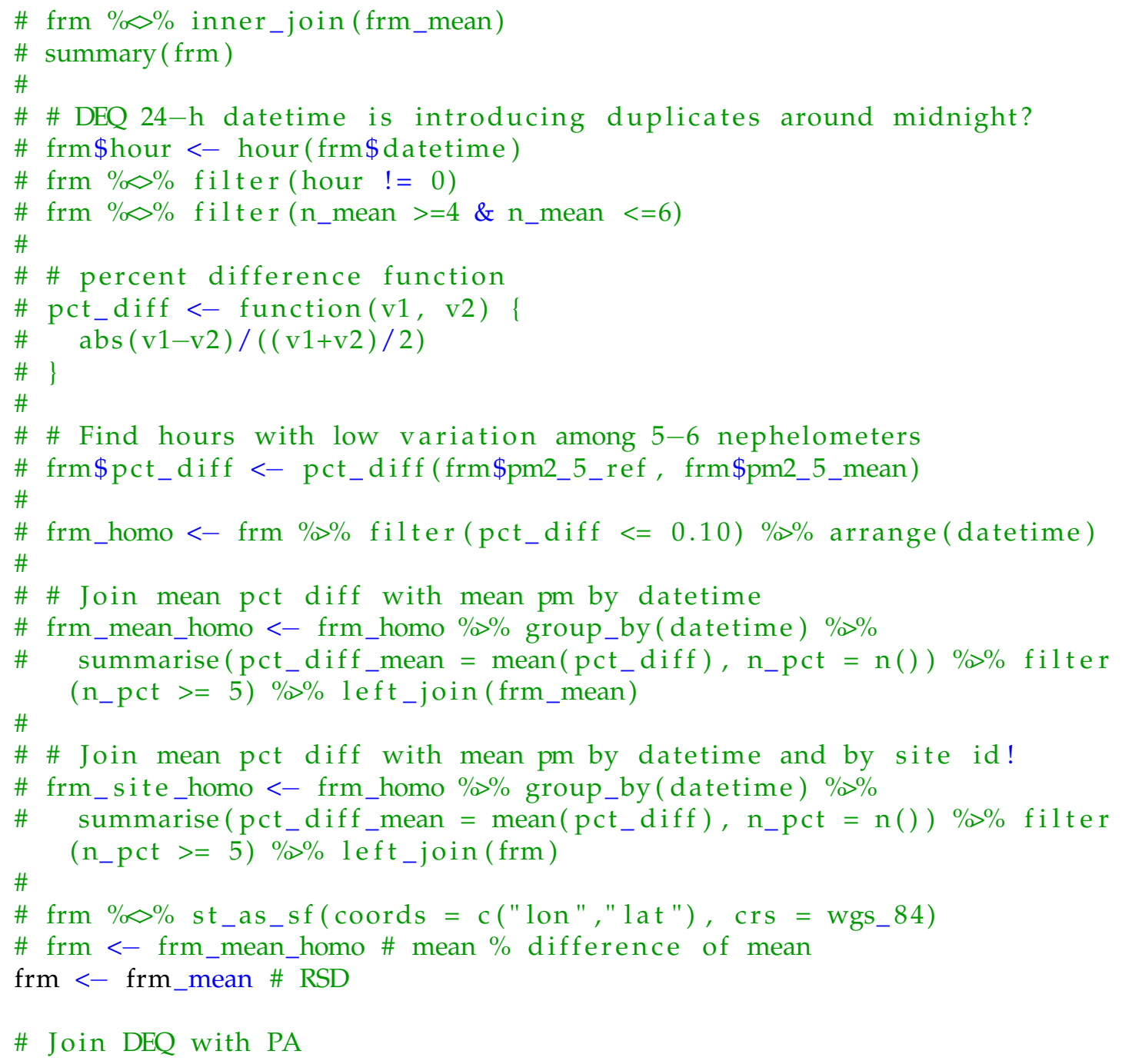




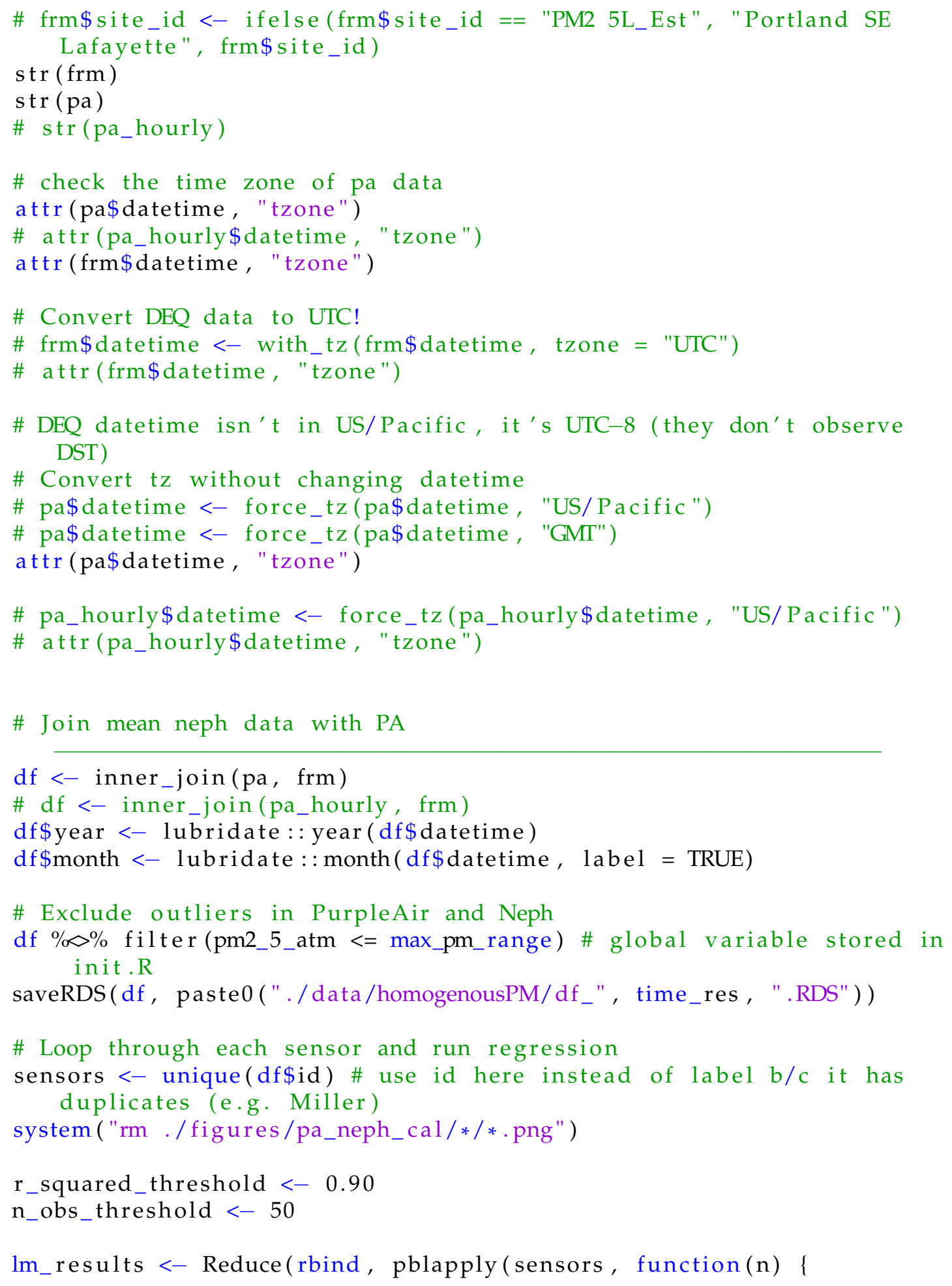




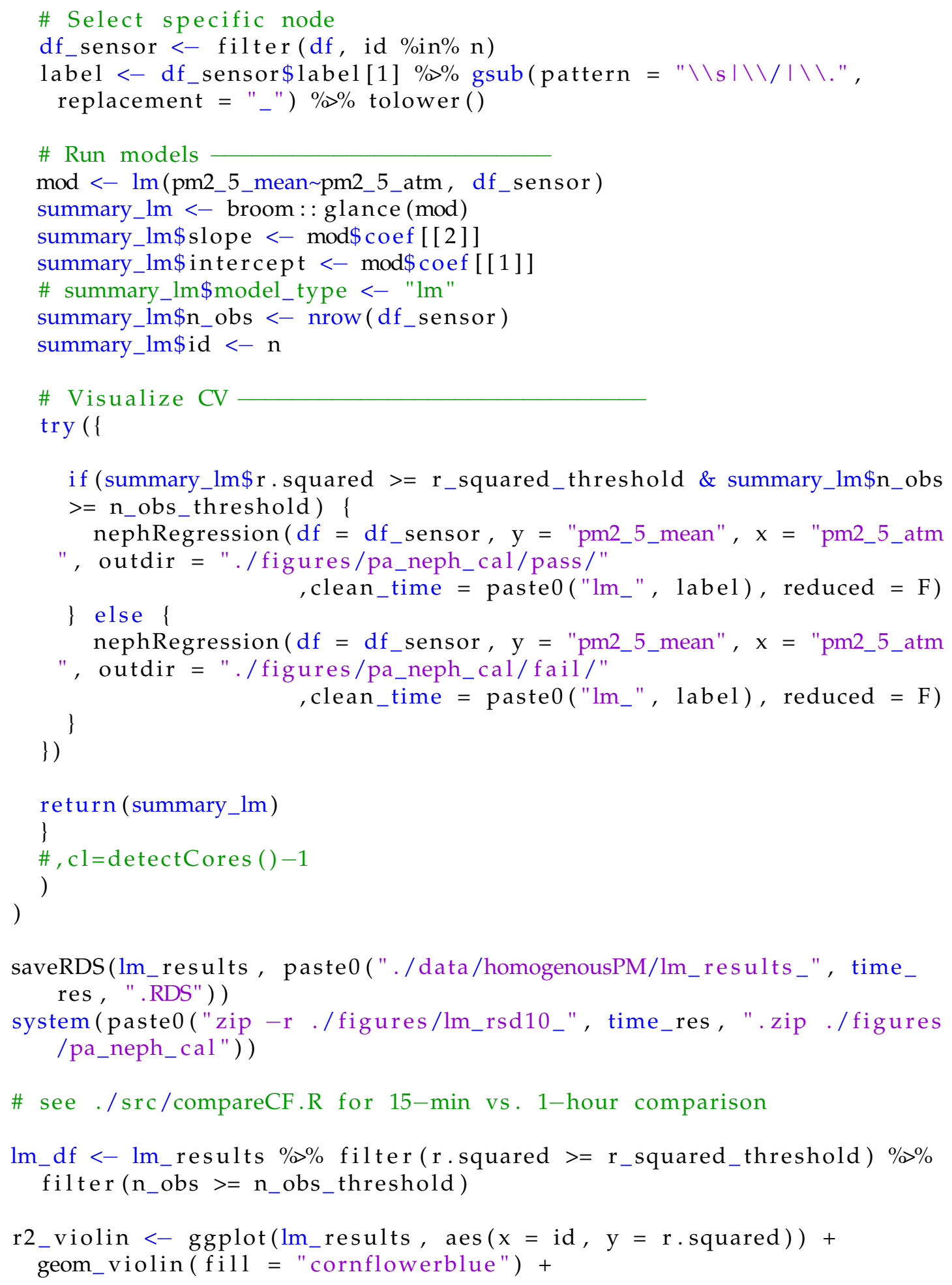


geom_hline (yintercept $=r_{-}$squared_threshold, color $=$"firebrick", linetype $=2)+$ $y l a b\left(\right.$ expression $\left.\left(R^{\wedge} 2\right)\right)+$

\# ggtitle (paste0 (expression (Distribution of $\mathrm{R}^{\wedge} 2$ ), " (Nephelometer PurpleAir)")) + ggtitle (bquote ('Distribution of ' $\mathrm{R}^{\wedge} 2 \sim^{\prime}$ (Nephelometer PurpleAir) ' . ( time_res)) ) +

theme_bw () +

theme $($ axis.title.x = element_blank () , axis.text. $x=$ element_blank () , axis.ticks.x = element_blank())

r2_violin

png (filename $=$ paste0 $(" . /$ figures/pa_neph_violins/r2_", time_res, ". png") 
\# Connect CF to each PurpleAir and upload to DB

\# Checkpoint our homogenous PM results for our CF script saveRDS (lm_df, "./data/cf /homogenous_pm_cf .RDS")

\# Apply best method from EDA to pdx_cf

\# Do this after storing data in DB (store even bad sensors CF for now ...)

\# pdx_cf $\% \diamond \%$

\# group_by(id, label) $\% \%$

\# $\quad$ slice (which.max $(r$.squared $)) \% \%$

\# filter $(\mathrm{r}$. squared $>=0.80) \% \%$ arrange $($ label $)$

\# Append geometry and convert to simplefeatures

pdx_cf $<-$ inner_join $\left(l_{-}\right.$df $_{\text {, pa_points })} \% \% \mathrm{st}_{-}$as_sf ()

\# Prepare colnames for postgres (remove periods)

names $\left(p d x_{-} c f\right)<-$ gsub $\left(x=\right.$ names $\left(p d x_{-} c f\right)$, pattern $\left.=" \backslash \backslash . ", "{ }_{-}\right) \%$ tolower ()

\# names(pdx_cf) [names $\left(p d x \_c f\right)==$ "id"] <- "label"

\# HTMS Roof was returning Inf and NaN

pdx_cf $\% \diamond \%$ na.omit ()

saveRDS (pdx_cf, "./data/cf/pdx_cf .RDS")

\# open connection to our db

con $<-$ dbConnect $(\mathrm{drv}=$ RPostgres : : Postgres ()

, dbname $=\mathrm{db}$ , host $=$ 'pgsq1102.rc.pdx.edu' \# not sure why object

host isn't working...

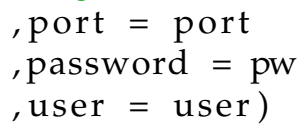

\# Find a better way to do this... append = TRUE is not ideal, but append $=$ FALSE and overwrite $=$ TRUE converts numeric to real datatype!?

\# Write to db as lookup table

\# clear table before writing new data

res $<-$ dbSendQuery (con, statement $=$ "delete from pdx_cf;")

dbClearResult ( res)

res <- dbSendQuery(con, statement = "vacuum full pdx_cf;")

dbClearResult ( res) 


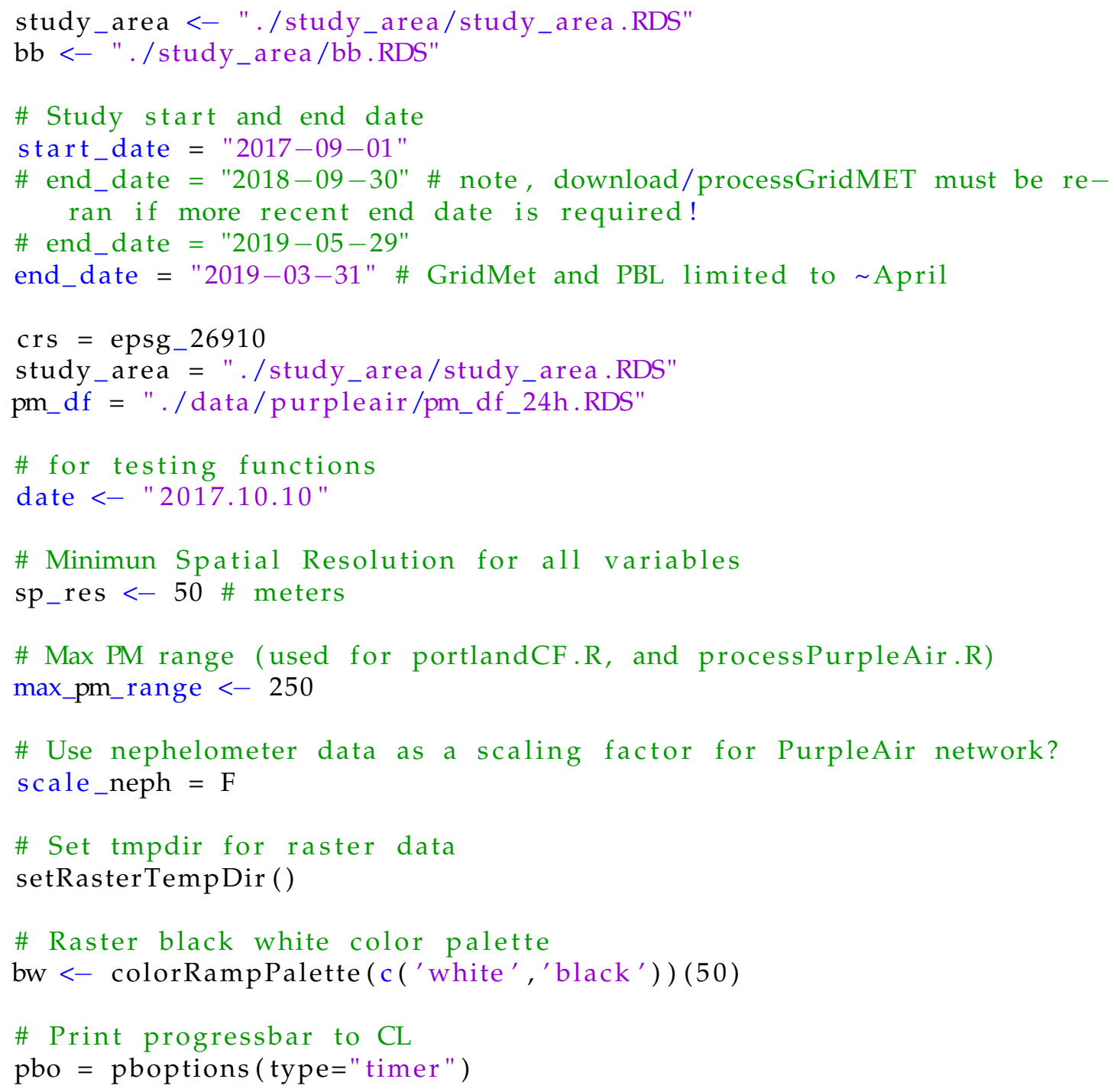

\section{Source/interpolateNDVI.R}

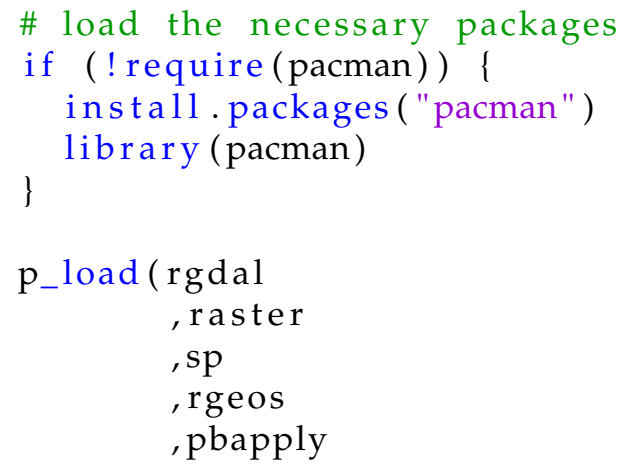




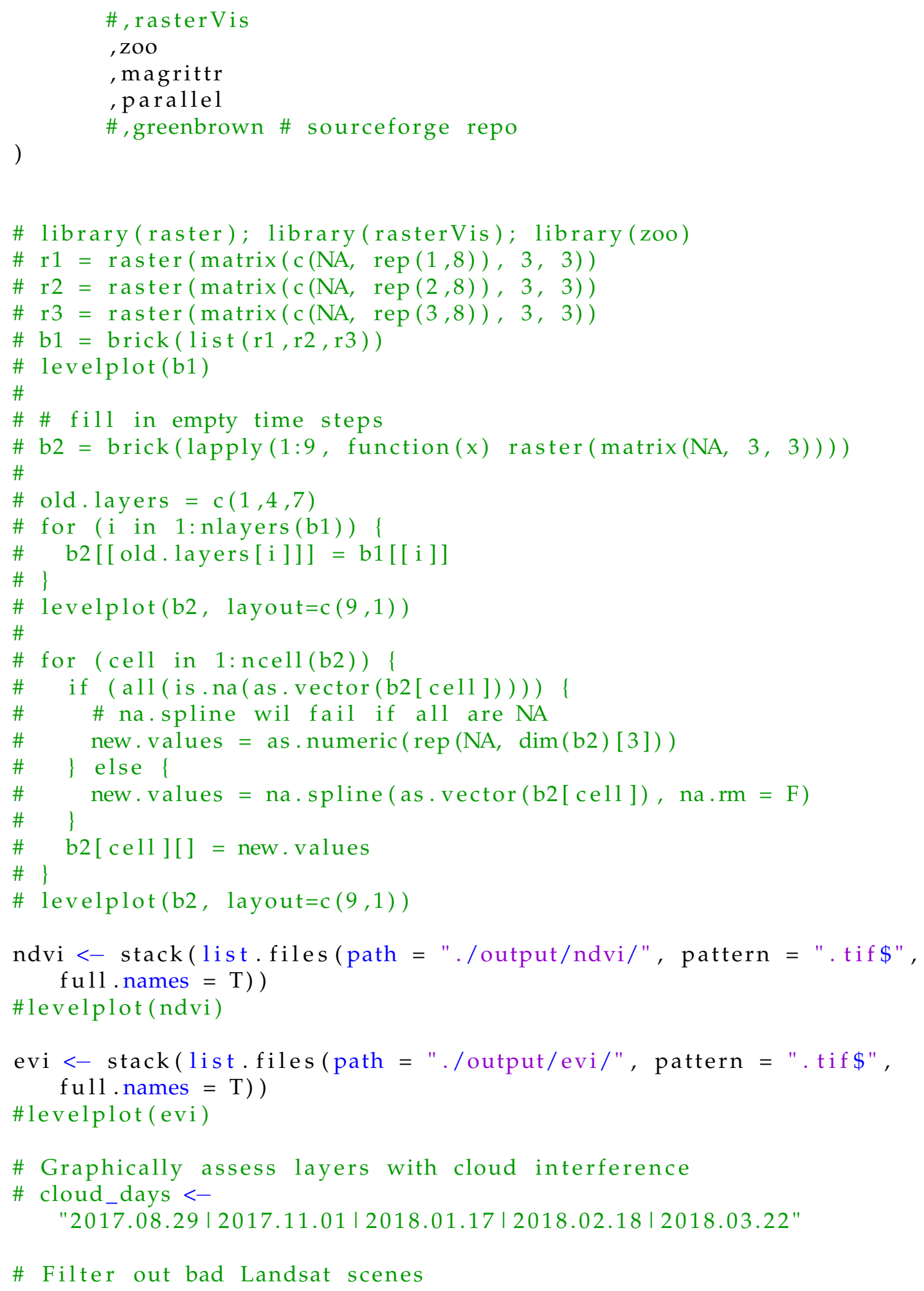




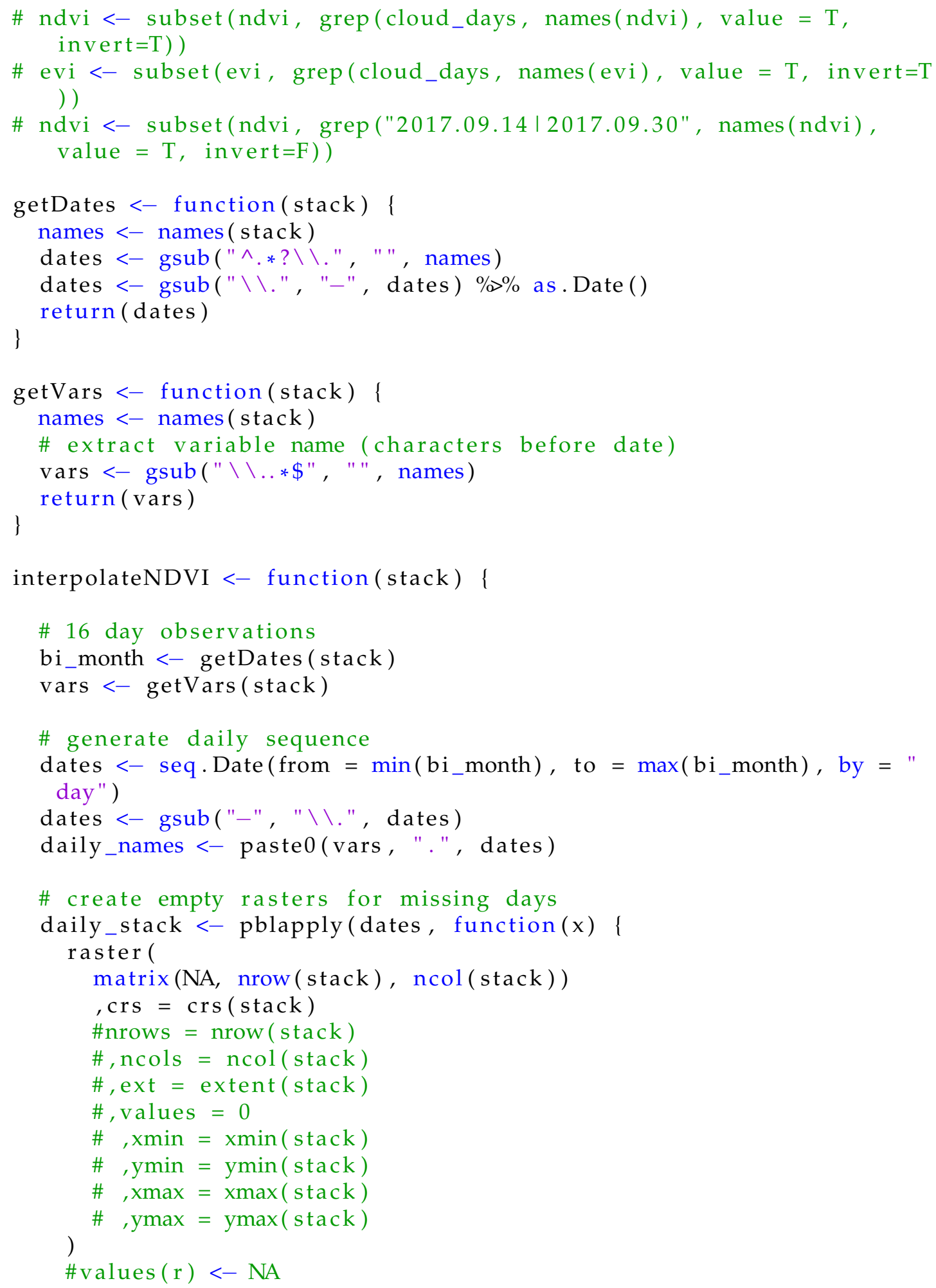




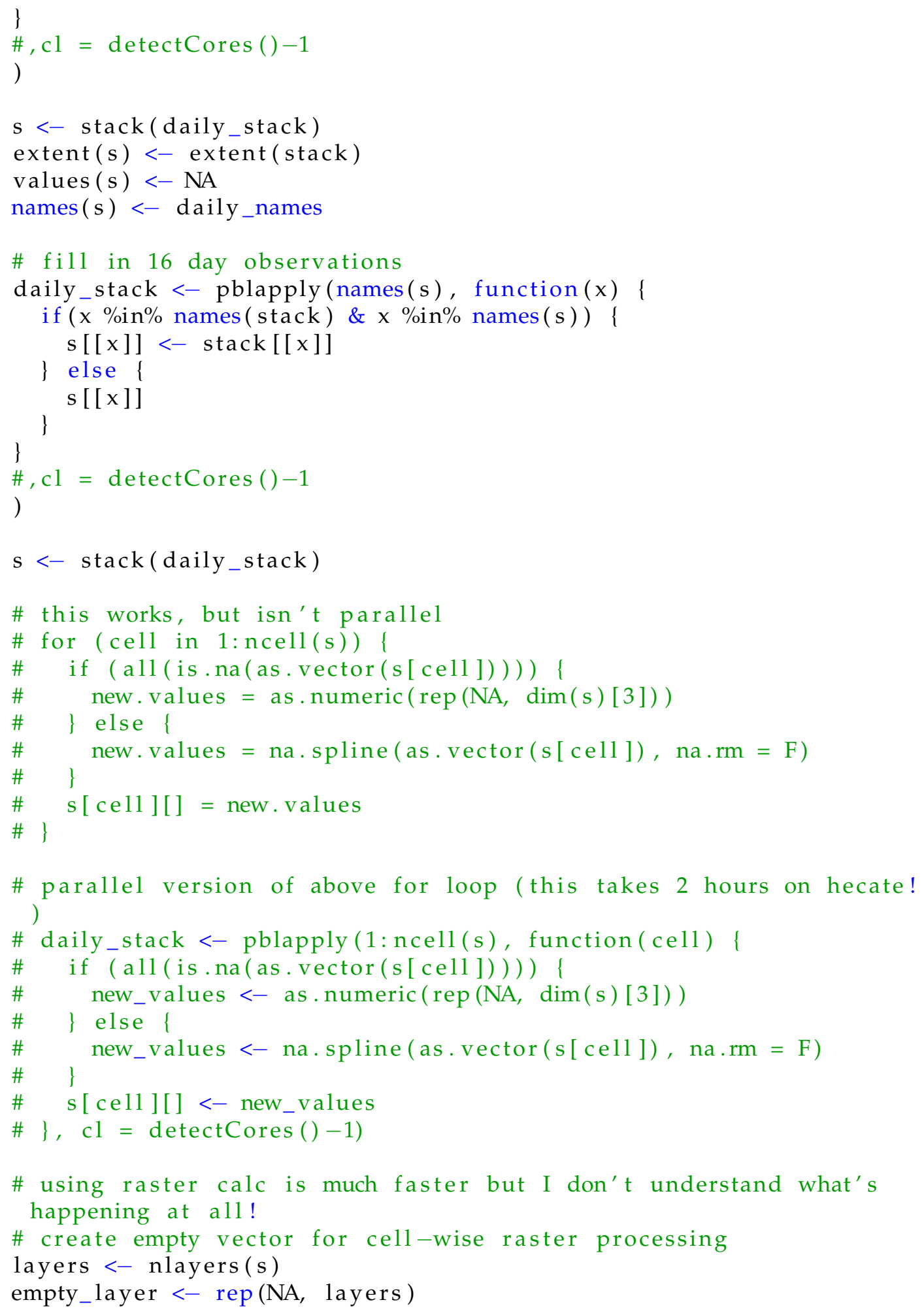




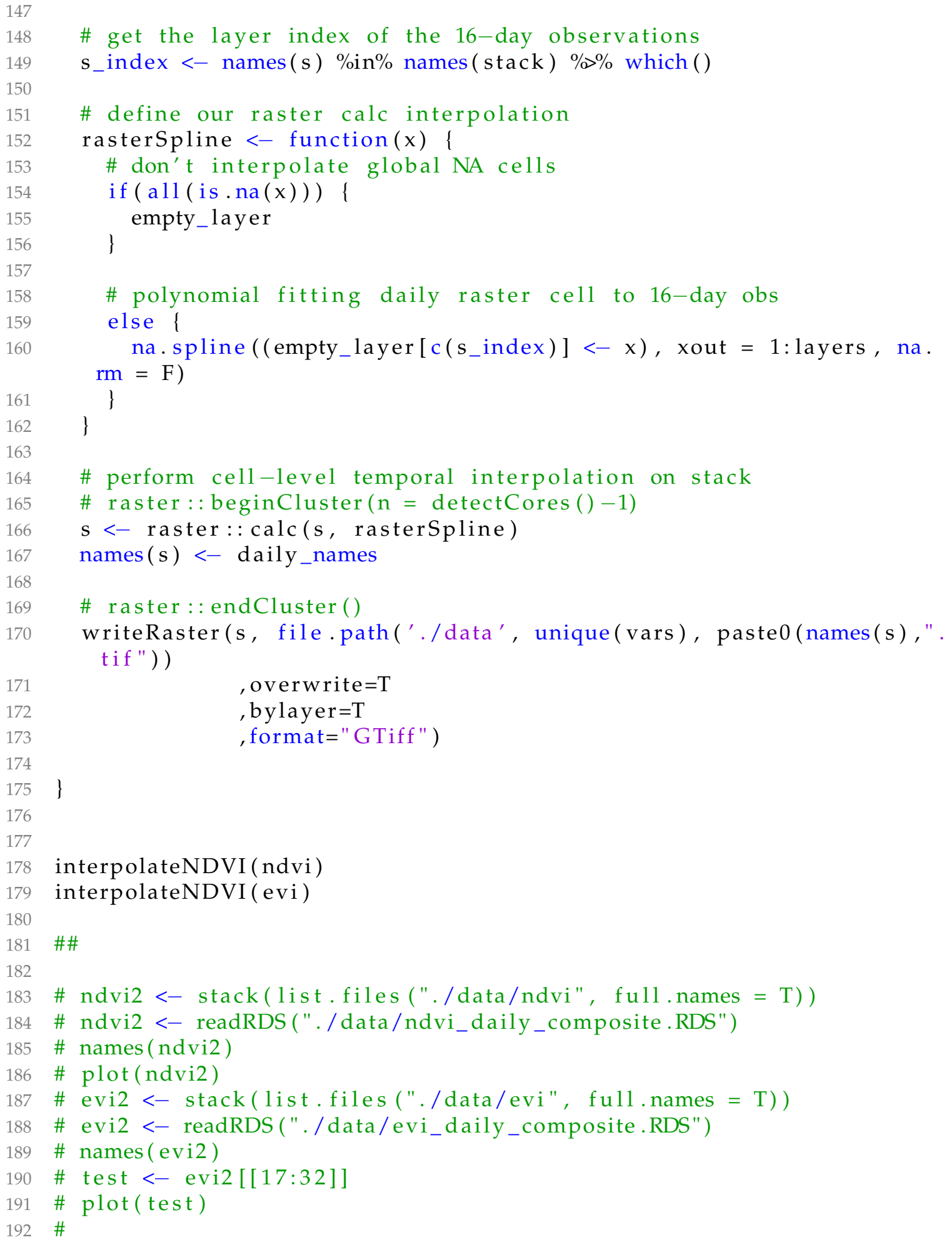




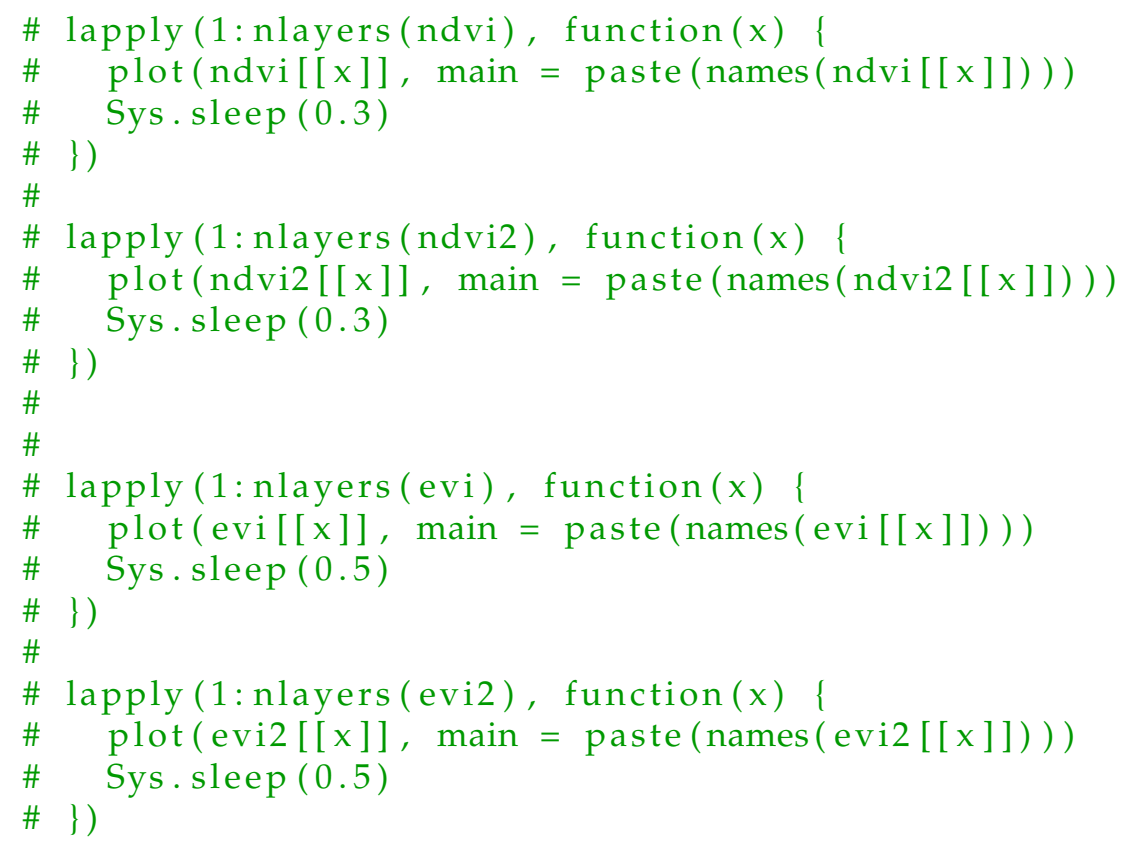

\section{Source/interpolateNeph.R}

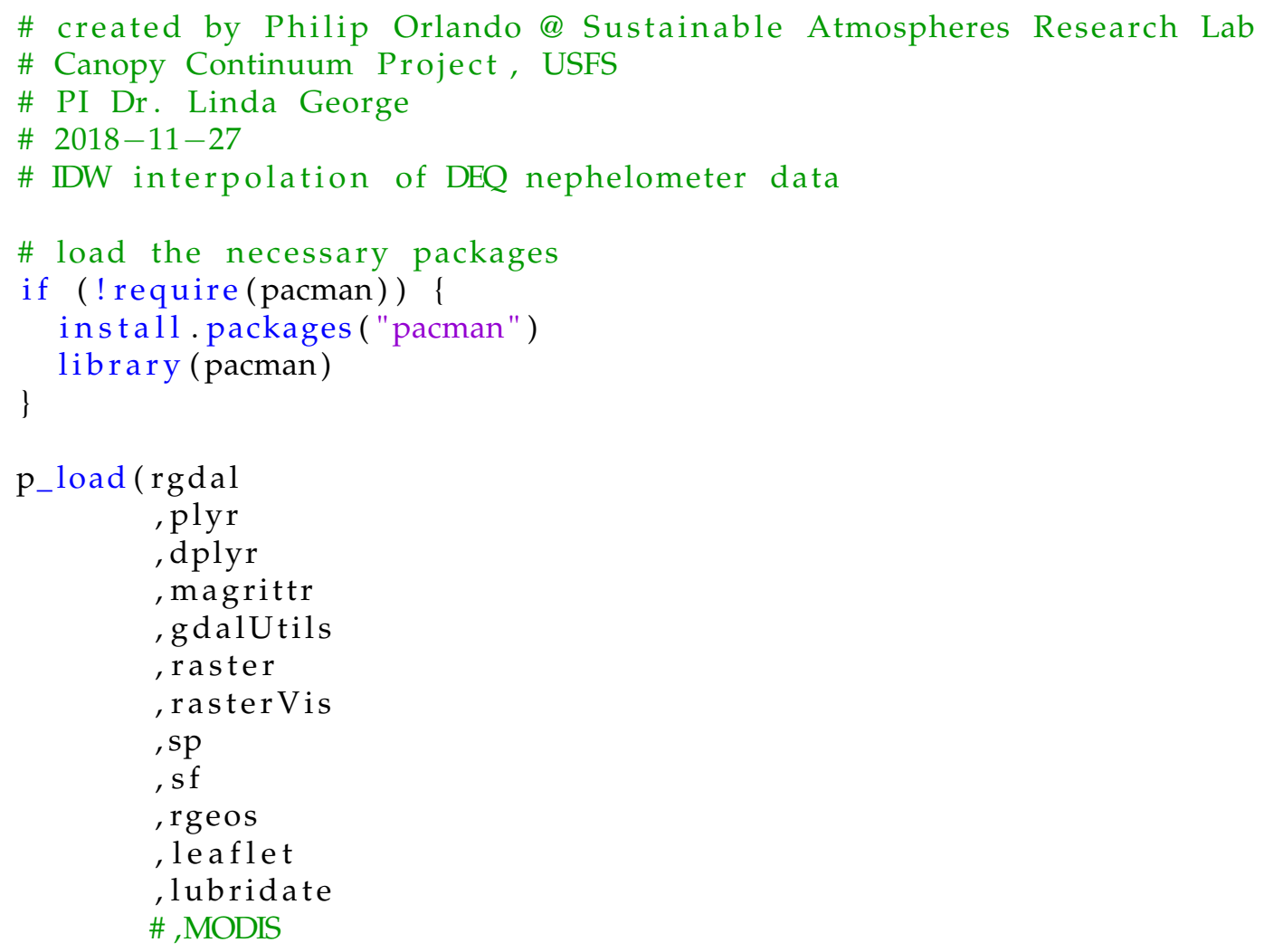




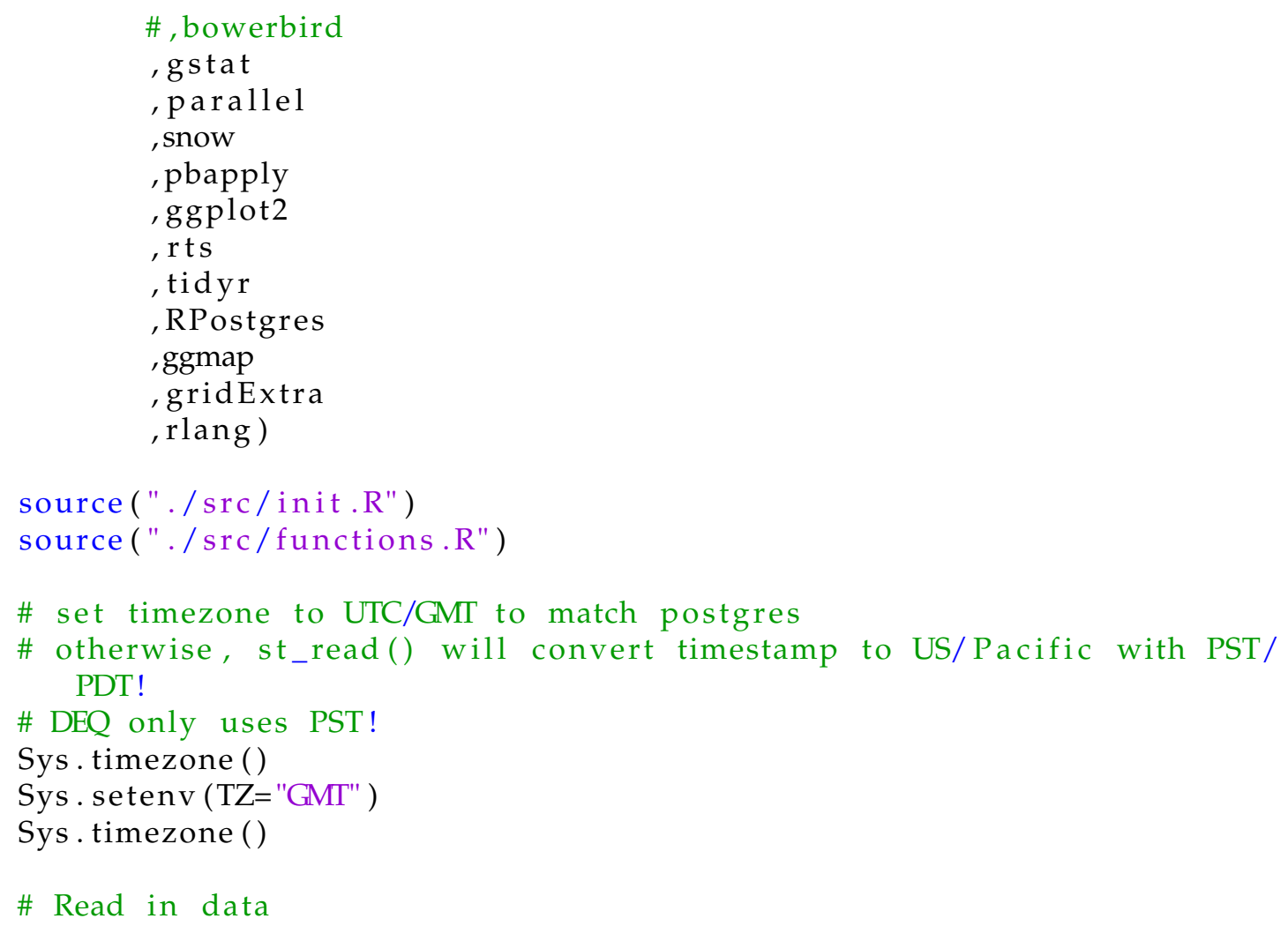


writeRaster(idwr, paste0 (outdir, "/", layer_name, ".tif"), format = "GTiff", overwrite $=\mathrm{T}$ )

\}

\# Determine mean daily PM from the entire network (one value for all cells each day like PBL)

neph_mean $<-$ function (col_name, pm_data, study_area, day, outdir) \{ pm_df $<-$ dplyr::filter $($ pm_data, date $>=$ day \& date $<=$ day) $\% \%$ dplyr:: select (date, col_name, geometry) $\% \%$ na.omit () $\% \%$ st_drop_geometry () \#pm_df $\% \diamond \%$ as ("Spatial") \#pm_df \% $\%$ spTransform $($ CRSobj = crs (study_area $))$ daily_mean <- dplyr: : summarise $\left(\mathrm{pm} \_d f\right.$, mean $=$ mean $($ eval $($ parse $($ text $=$ $($ col_name $)))$, na.rm $=\mathrm{T})$ )

$\mathrm{r}<-$ raster (study_area, $\mathrm{crs}=\mathrm{crs}$, res $=$ sp_res, vals $=$ daily_mean [[1]])

layer_name $<-$ paste0 (col_name, ".", gsub("-", "\\.", day)) names $(\mathrm{r})<-$ layer_name \#plot $(\mathrm{r})$ writeRaster (r, paste0(outdir, "/", layer_name, ".tif"), format = " GTiff", overwrite $=\mathrm{T}$ )

\}

\# Call our ground-station nephelometer interpolation across each day (function wrapper)

interpolate_neph <- function (pm_data, day, vars, study_area, outdir) \{

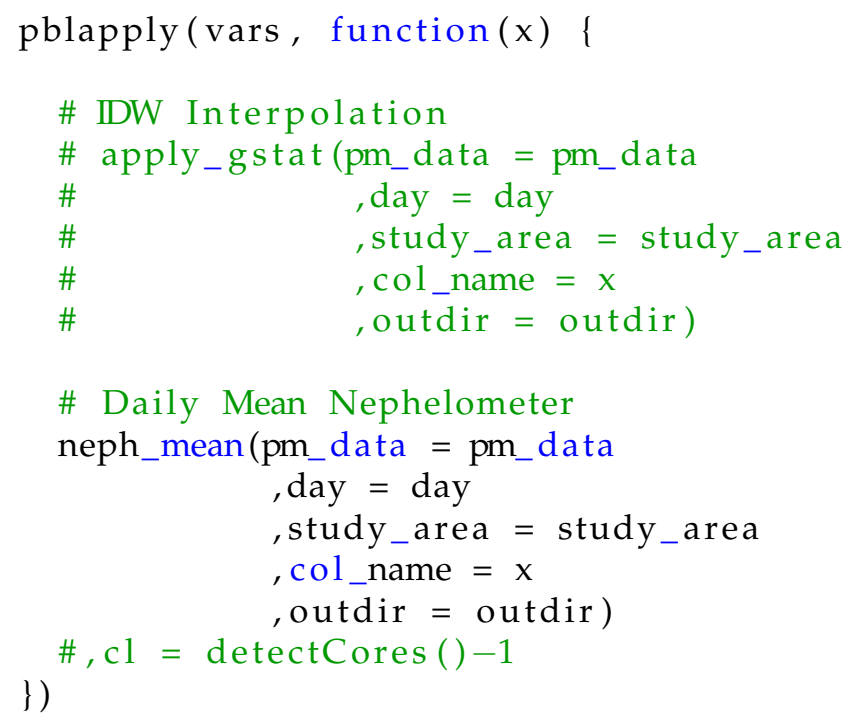




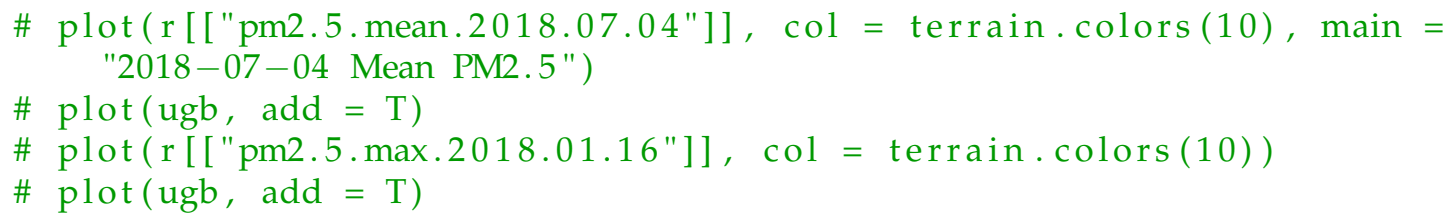

\section{Source/purpleairQAQC.R}

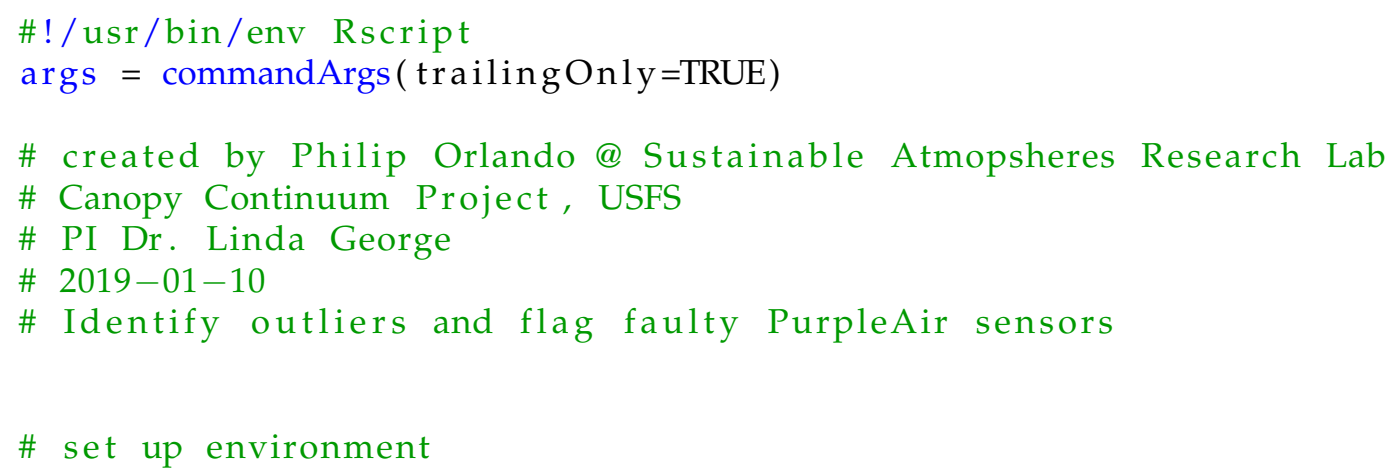




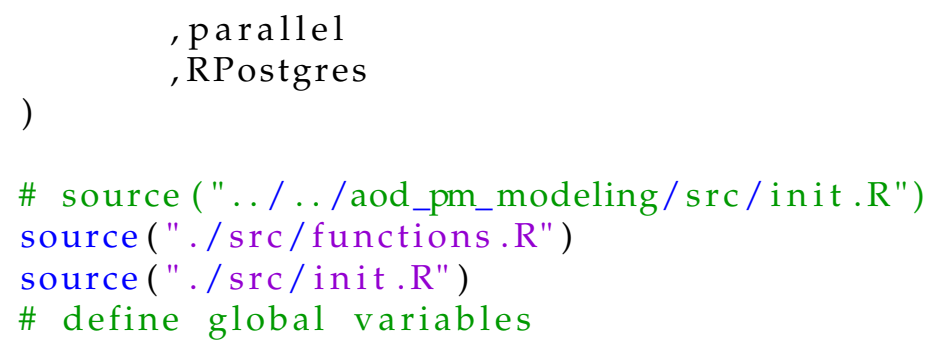




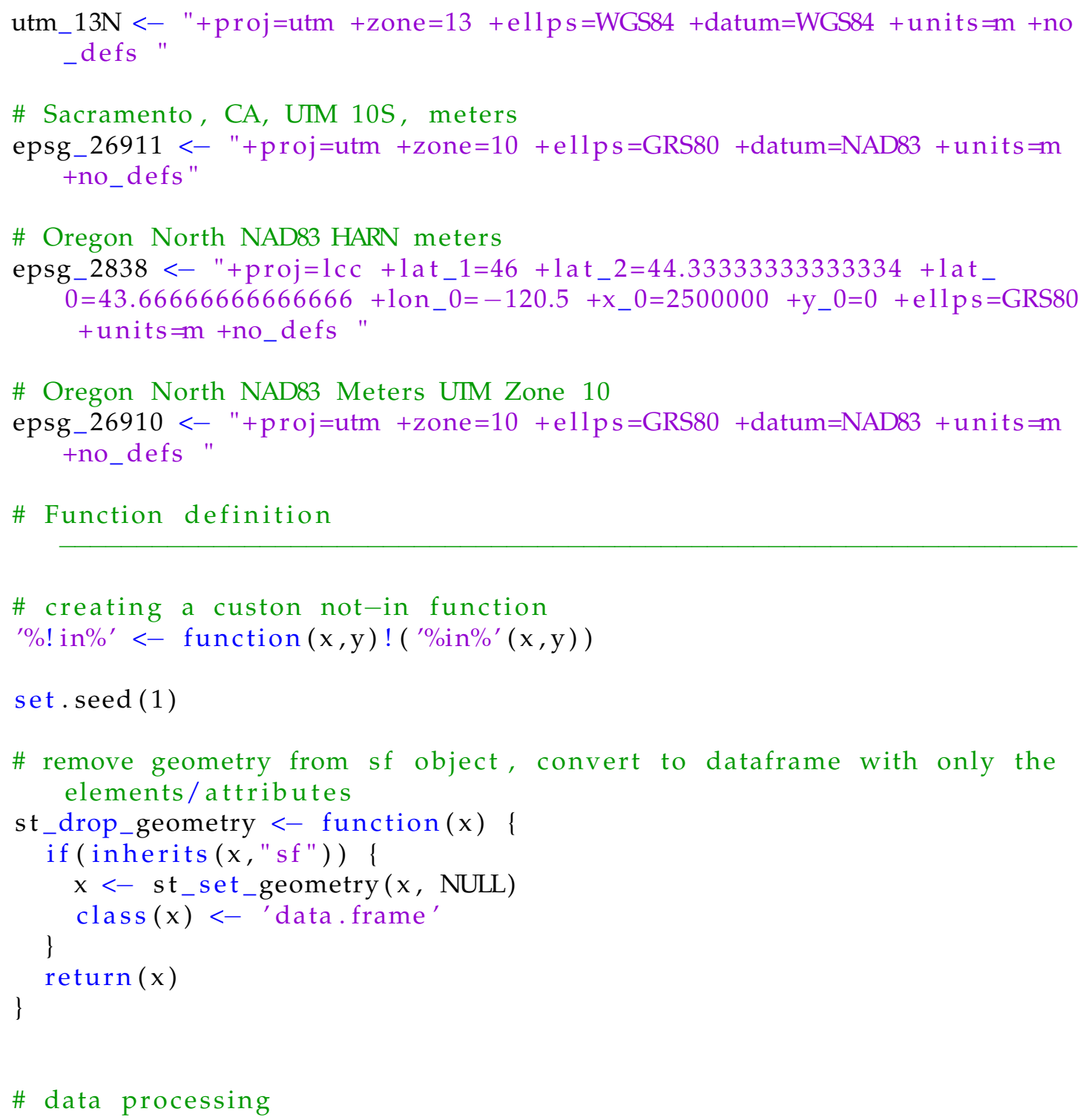


\# $\quad$, query $=$ paste0 $($ "SELECT DISTINCT * FROM pdx WHERE created_at $>=$ '", start_date, "' AND created_at $<=$ '", end_date, "';") 


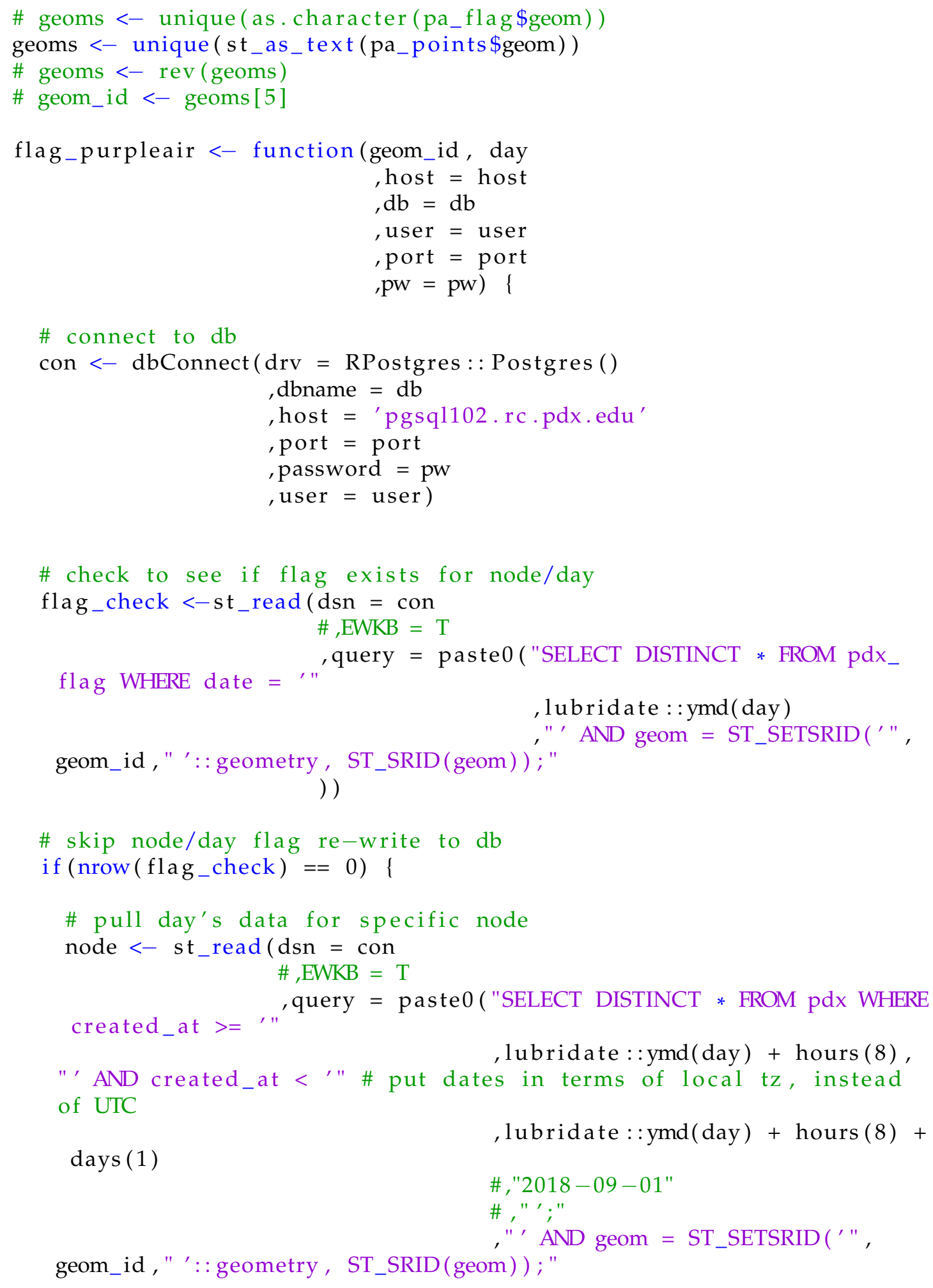




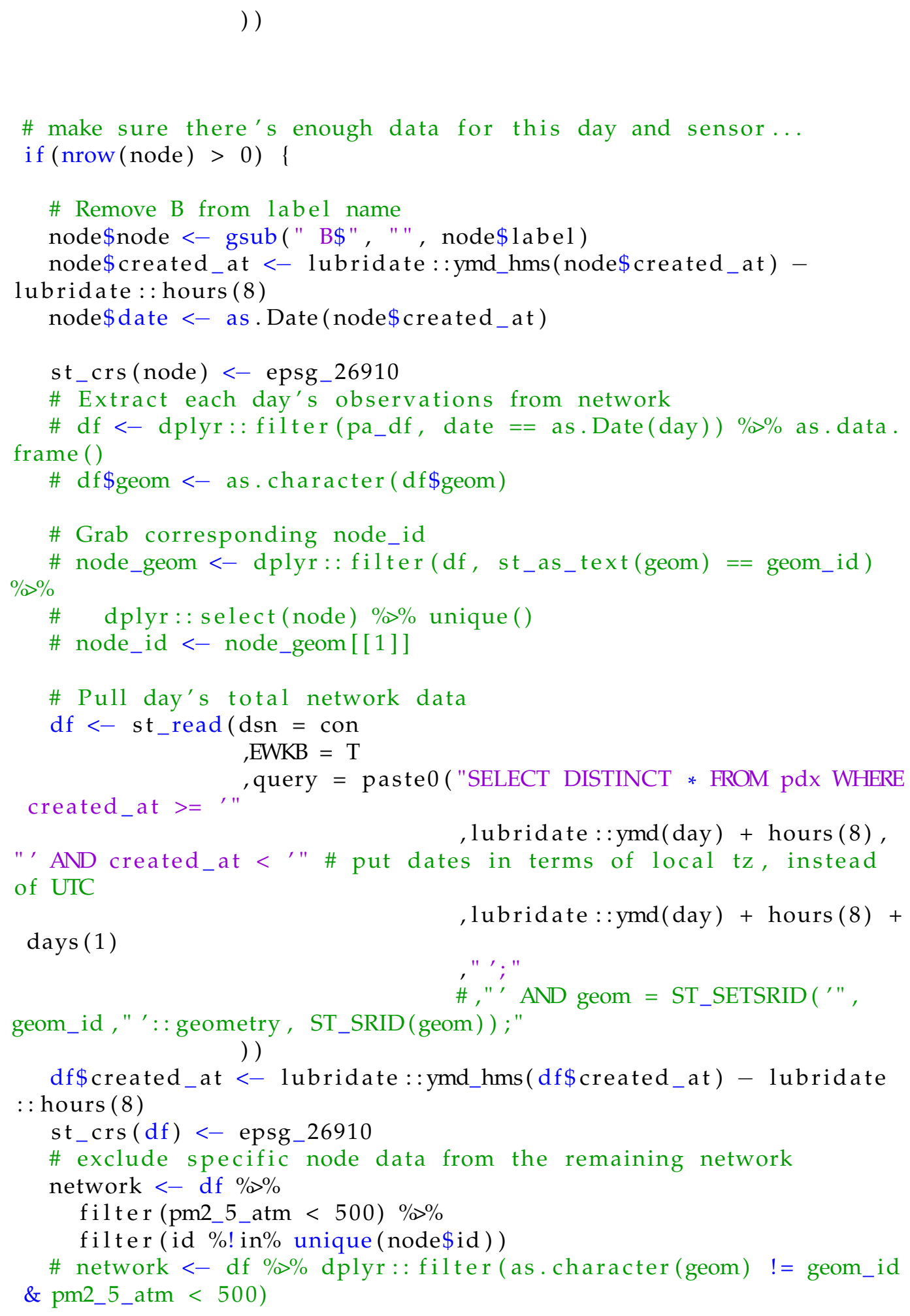




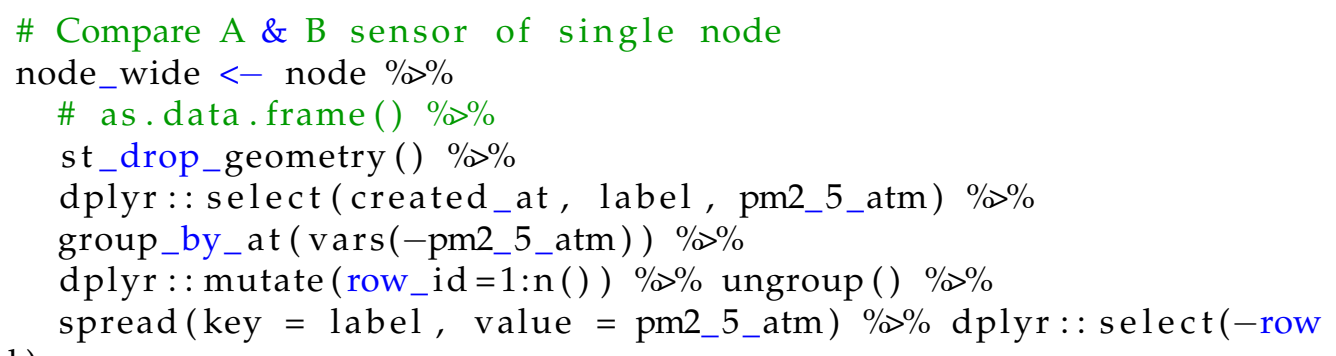




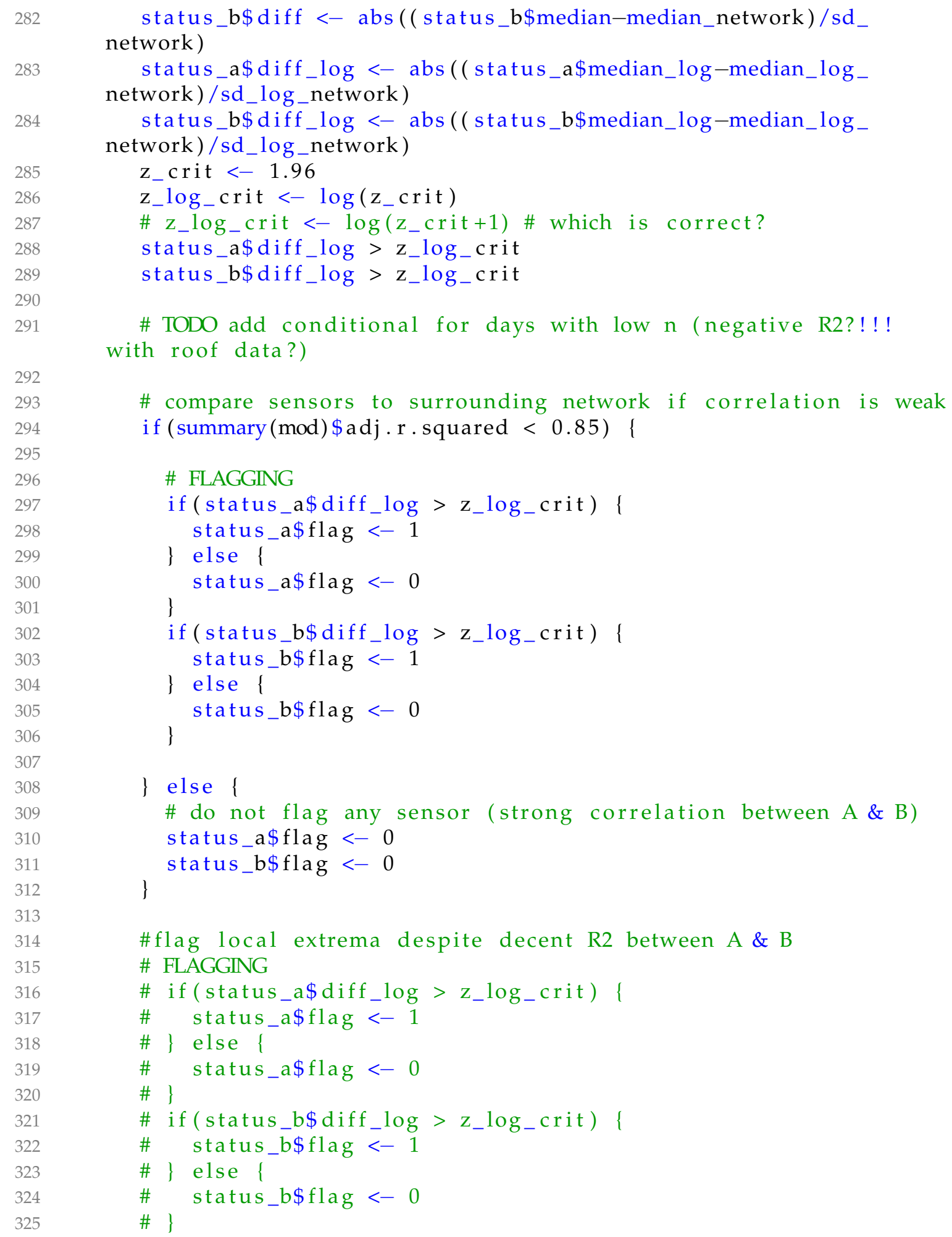




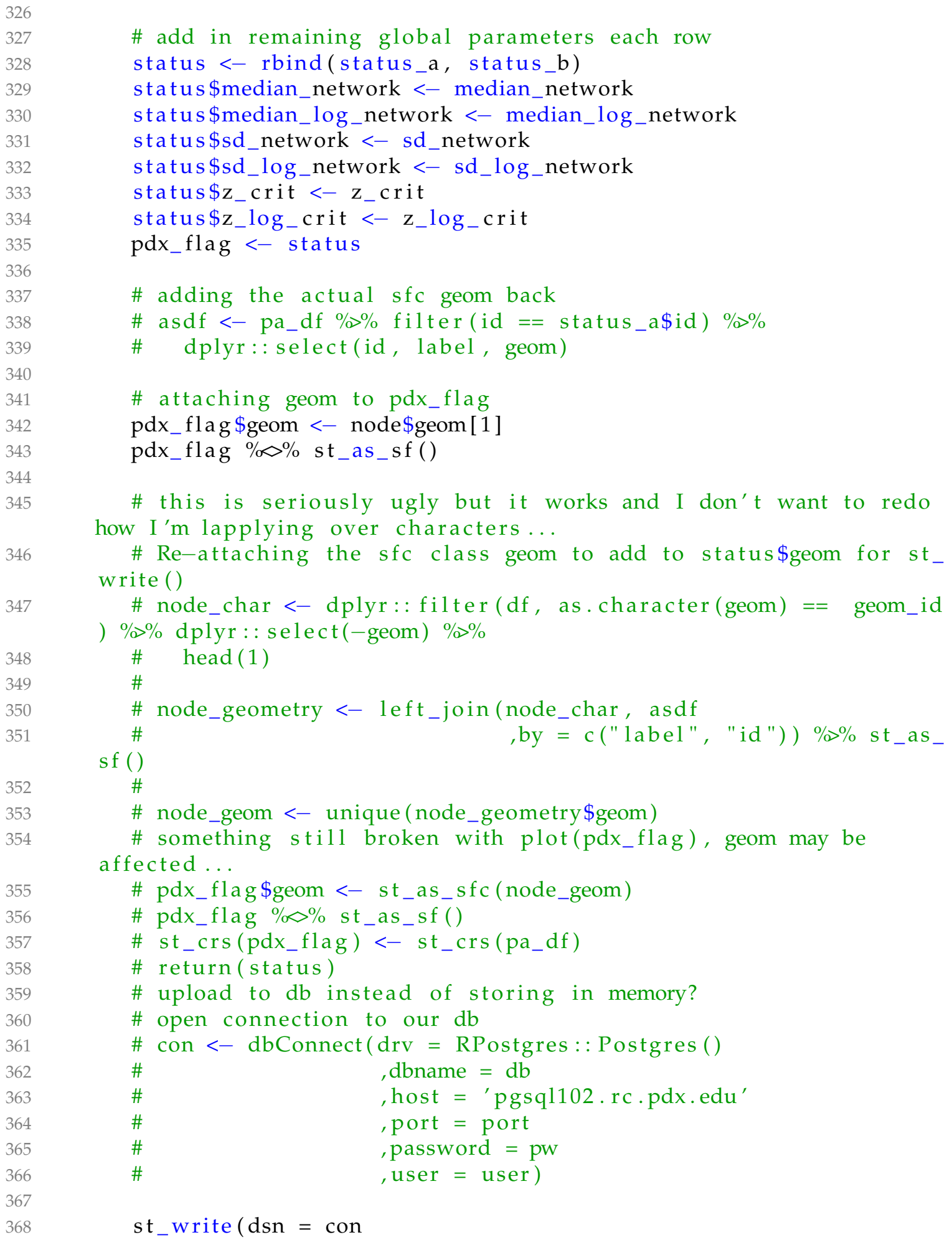




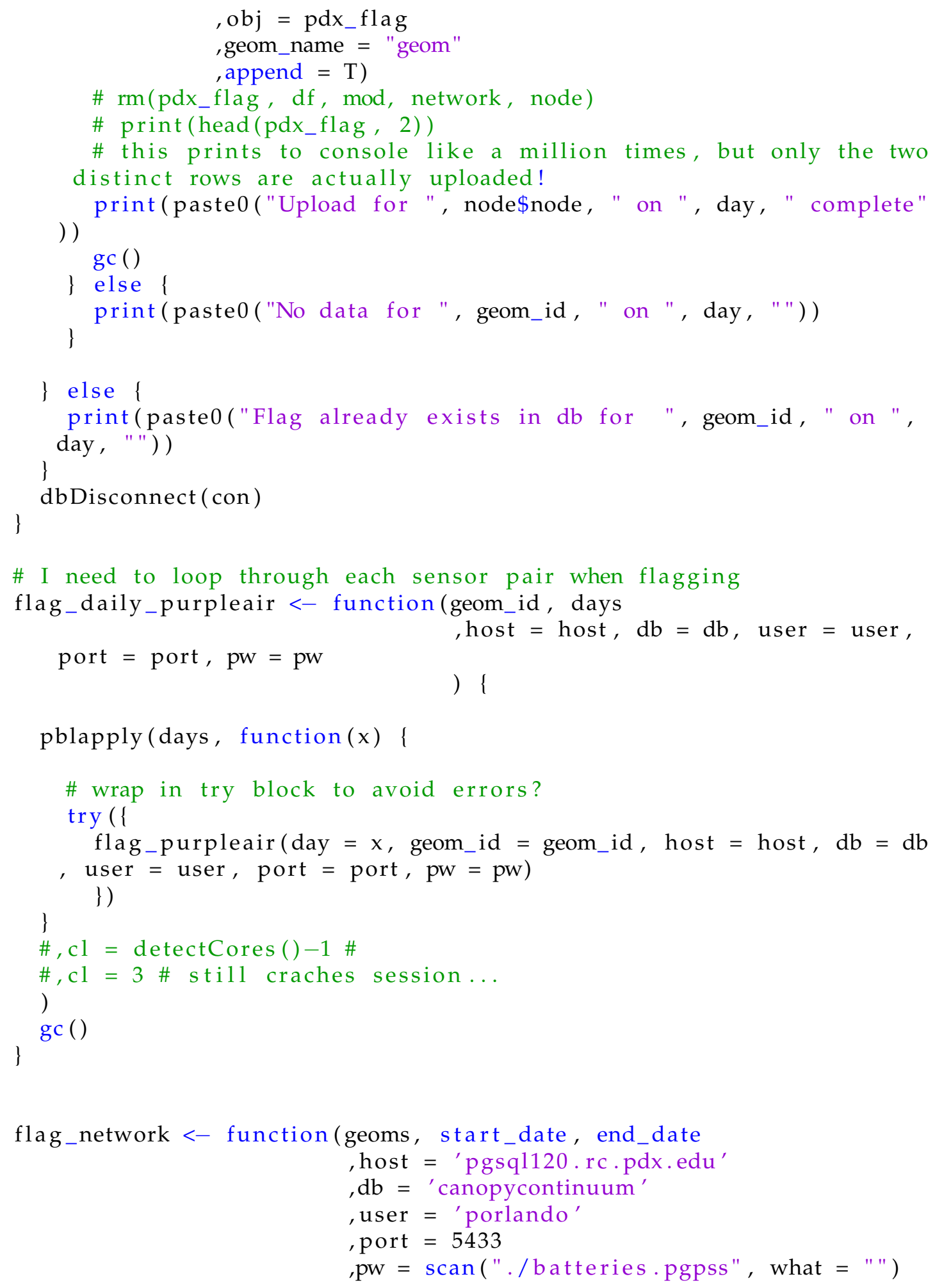




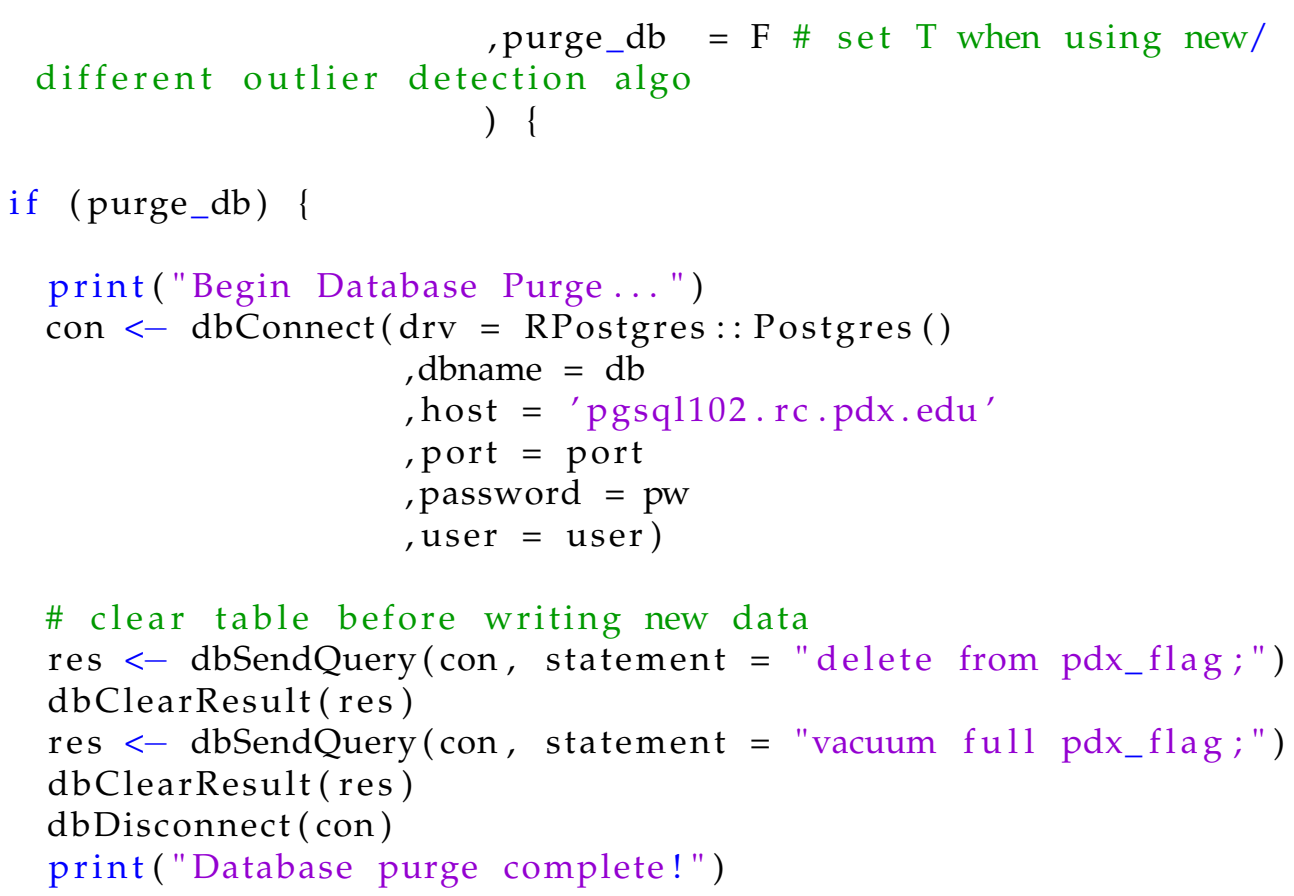




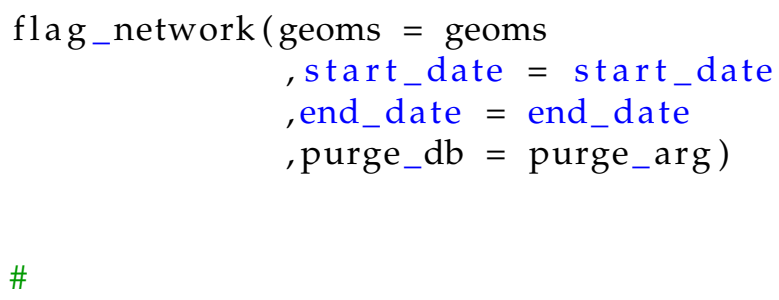

\section{Source/purpleAirCF.R}

\# created by Philip Orlando @ Sustainable Atmopsheres Research Lab \# Canopy Continuum Project, USFS

\# PI Dr. Linda George

\# 2018-09-17

\# determine correction factors for Portland PurpleAir network

\# set up environment

\# load the necessary packages

if (! require (pacman) ) \{ 


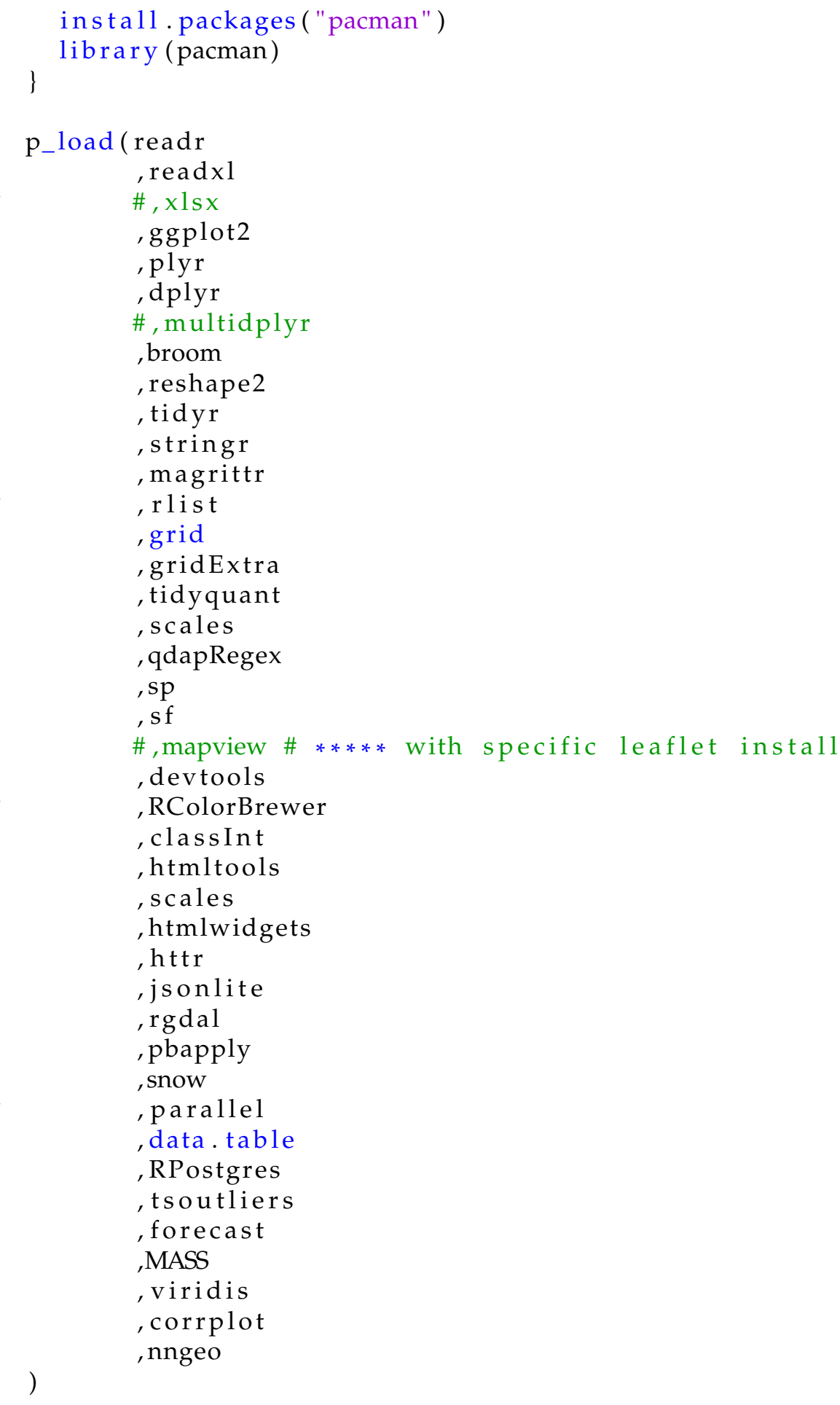


\# define global variables

\# set timezone to UTC/GMT to match postgres

\# otherwise, st_read() will convert timestamp to US/Pacific with PST/ PDT!

\# DEQ only uses PST!

Sys . timezone ()

Sys . setenv $(\mathrm{TZ}=$ "GMT" )

Sys . timezone ()

\#\# connecting to local db

host <- 'pgsq1120.rc.pdx.edu'

$\mathrm{db}<-$ 'canopycontinuum'

user <- 'porlando'

port $<-5433$

pw $<-\operatorname{scan}("$. / batteries.pgpss", what $="$ ") \# in parent dir

\# open connection to our db

con $<-$ dbConnect $(\mathrm{drv}=$ RPostgres : : Postgres ()

, dbname $=\mathrm{db}$

, host $=$ 'pgsq1102.rc.pdx.edu' \# not sure why object

host isn't working...

, port $=$ port

, password = pw

, user = user)

\# create unanimous time resolution for all data

time_resolution $<-" 1$ sec"

\# time zone applied to all data

time_zone <- "GMT"

\# CRS

wgs_84 <- "+proj=longlat +ellps=WGS84 +datum=WGS84 +no_defs "

\# Oregon North NAD83 HARN meters

epsg_2838<- "+proj=1cc + lat_1=46 + lat_2=44.33333333333334+1at_ $0=43.66666666666666+10 n_{-} 0=-120.5+x_{-} 0=2500000+y_{-} 0=0+$ ellps $=$ GRS80

+units=m +no_defs"

\# Oregon North NAD83 Meters UTM Zone 10

epsg_26910<- "+proj=utm +zone=10 +ellps=GRS80 +datum=NAD83 +units=m +no_defs " 
\# Function definition

\# creating a custon not-in function

\% $\%$ ! in $\%{ }^{\prime}<-$ function $(x, y) !\left({ }^{\prime} \%\right.$ in $\%$ ' $\left.(x, y)\right)$

\# plot density in ggplot

get_density $<-$ function $(\mathrm{x}, \mathrm{y}, \mathrm{n}=100)$ \{

dens <- MASS: $\operatorname{kde} 2 d(x=x, y=y, n=n)$

ix $<-$ findinterval $(x$, dens $\$ x)$

iy $<-$ findinterval $(y$, dens $\$ y)$

i i $<-$ cbind (ix, iy)

return (dens $\$ z$ [ i i ] )

\}

set. seed (1)

\# file_path <- "./data/frm/portland_5stn_20180101_to_20180719.csv"

\# read function for the 5-min data that Meena sent over!

read_frm $<-$ function (file_path) \{

\# read in the raw data

$x<-$ read.csv (file_path, header $=$ TRUE, skip $=2$ )

\# delete sub headers

$\# x<-x[-c(1: 2)$,

\# convert date from character to POSIXct

$\mathrm{x} \$$ Date.. Time $<-$ mdy_hm $(\mathbf{x} \$$ Date.. Time, $\mathrm{tz}=$ time_zone $)$

$\mathrm{x}<-\mathrm{x} \% \%$ na.omit ()

\# convert to tidy format, gather on site id

long $<-\mathrm{x} \% \%$

gather (id, pm2_5_atm, $-c($ Date... Time $))$

\# convert characters in pm2_5 data to NA

long \$pm2_5_atm <- as . numeric (long\$pm2_5_atm)

\# exclude NAs (No Data, Zero, DL/Computer, etc. gets converted to NA)

long $<-$ long $\% \%$ na.omit ()

\# alter column names colnames (long) $<-c($ "datetime"

,"site_id"

,"pm2_5_ref") 
GROUP BY id, label, geom;"

)

\# define projection

st_crs (pa) <- epsg_26910

st_crs (pa_points) <- epsg_26910

\# convert from UTC to UTC-8 or PST (same as DEQ)

pa\$created_at $<-1$ lubridate $:: y m d \_h m s($ pa\$created_at $)-$ hours $(8)$

\# read in the frm data (not actually frm, but neph...) provided by Meena

\# frm_files <- c("./data/frm/sel_pm25_5min_20170701_to_20180918.csv")

frm_fíles <- "./data/frm/bhp_hhf_tbc_sel_5min_pm25_20170701_to_ 20180918.csv"

frm $<-$ ldply $($ frm_files, read_frm $)$

frm $\% \diamond \%$ st_as_sf $($ coords $=c(" l o n ", " 1 a t ")$, crs $=$ wgs_84 $)$

\# matching the sel file with the 5 stn id

\# frm\$site_id <- ifelse (frm\$site_id == "PM2 5L_Est", "Portland SE Lafayette", frm\$site_id)

\# make sure the datetime columns match

names $($ pa $)[$ names $($ pa $)==$ "created_at"] $<-$ "datetime"

$\operatorname{str}($ frm $)$

$\operatorname{str}(\mathrm{pa})$

\# check the time zone of pa data

attr (pa\$datetime, "tzone")

attr (frm\$datetime, "tzone")

\# TODO INSERT FLAG DATA SCRIPT HERE

\# added to processPurpleAir in aod_modeling instead...

\# pdx_flag $<-$ st_read $(\mathrm{dsn}=$ con

\# ,geom_column = "geom"

\# $\quad$, query $=$ "SELECT DISTINCT $*$ FROM pdx_flag;")

\#

\# run initial cor plot before creating individual regressions

\# nearby_sensors <- c ("byuki project" 


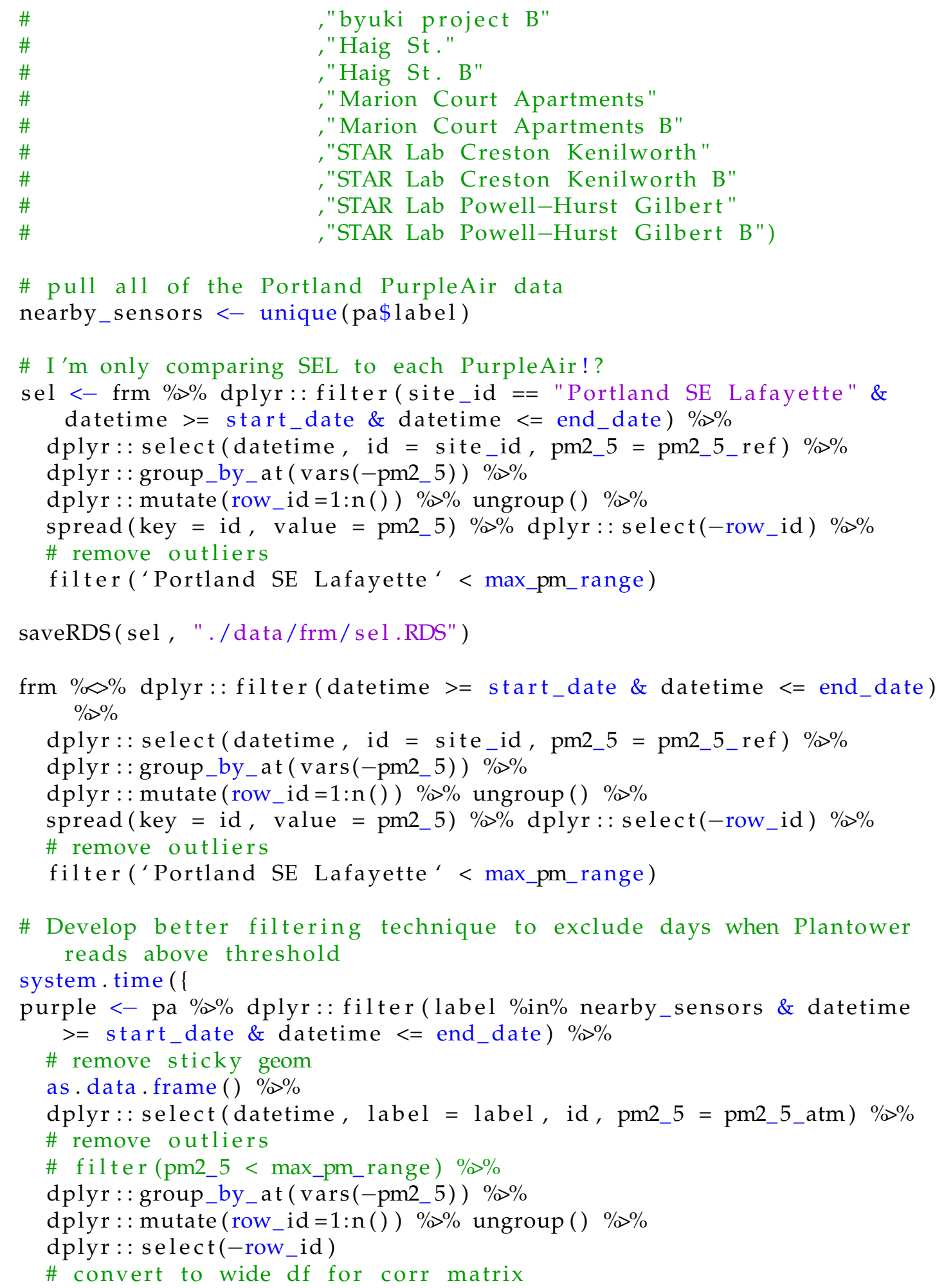




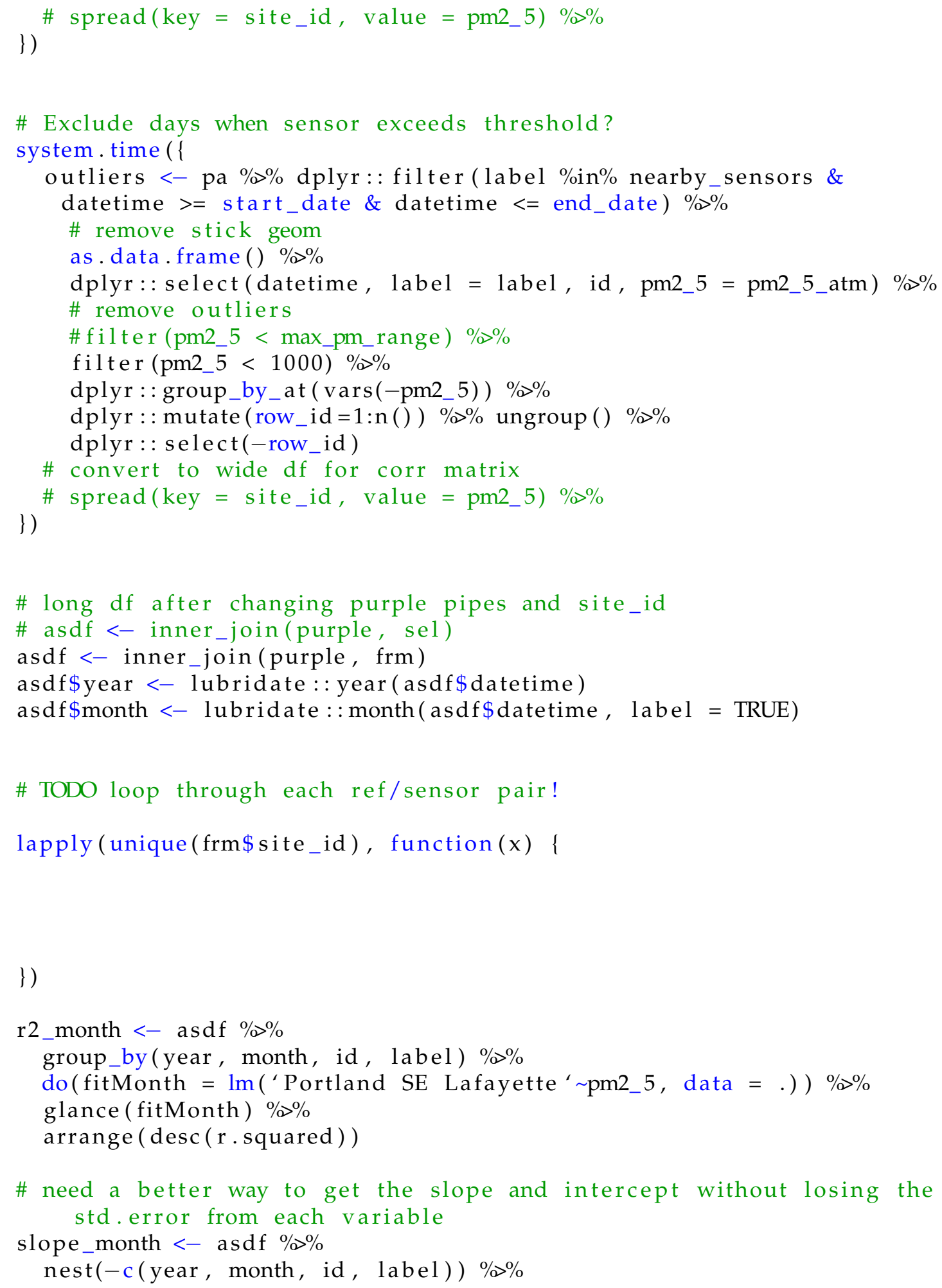




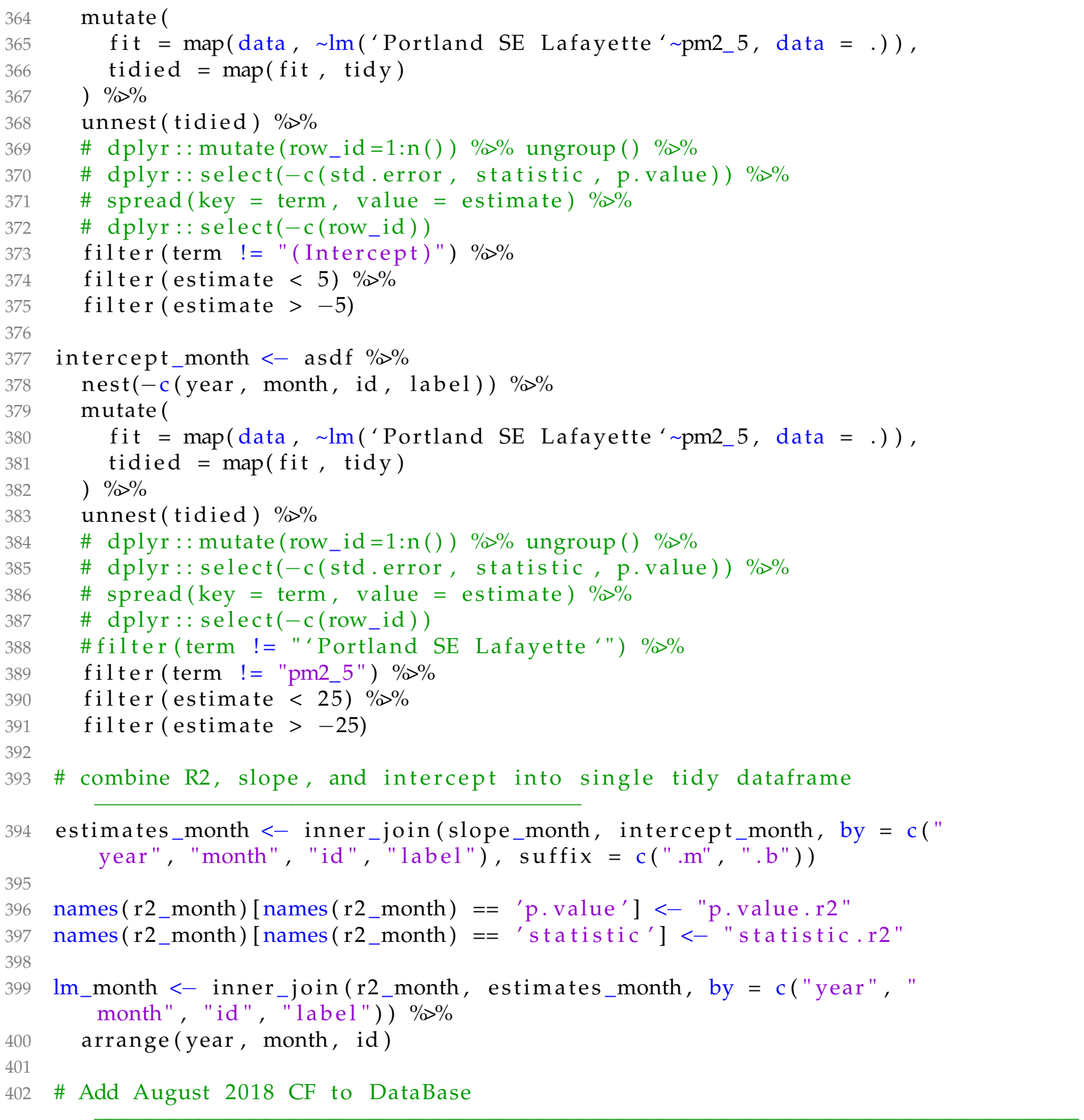




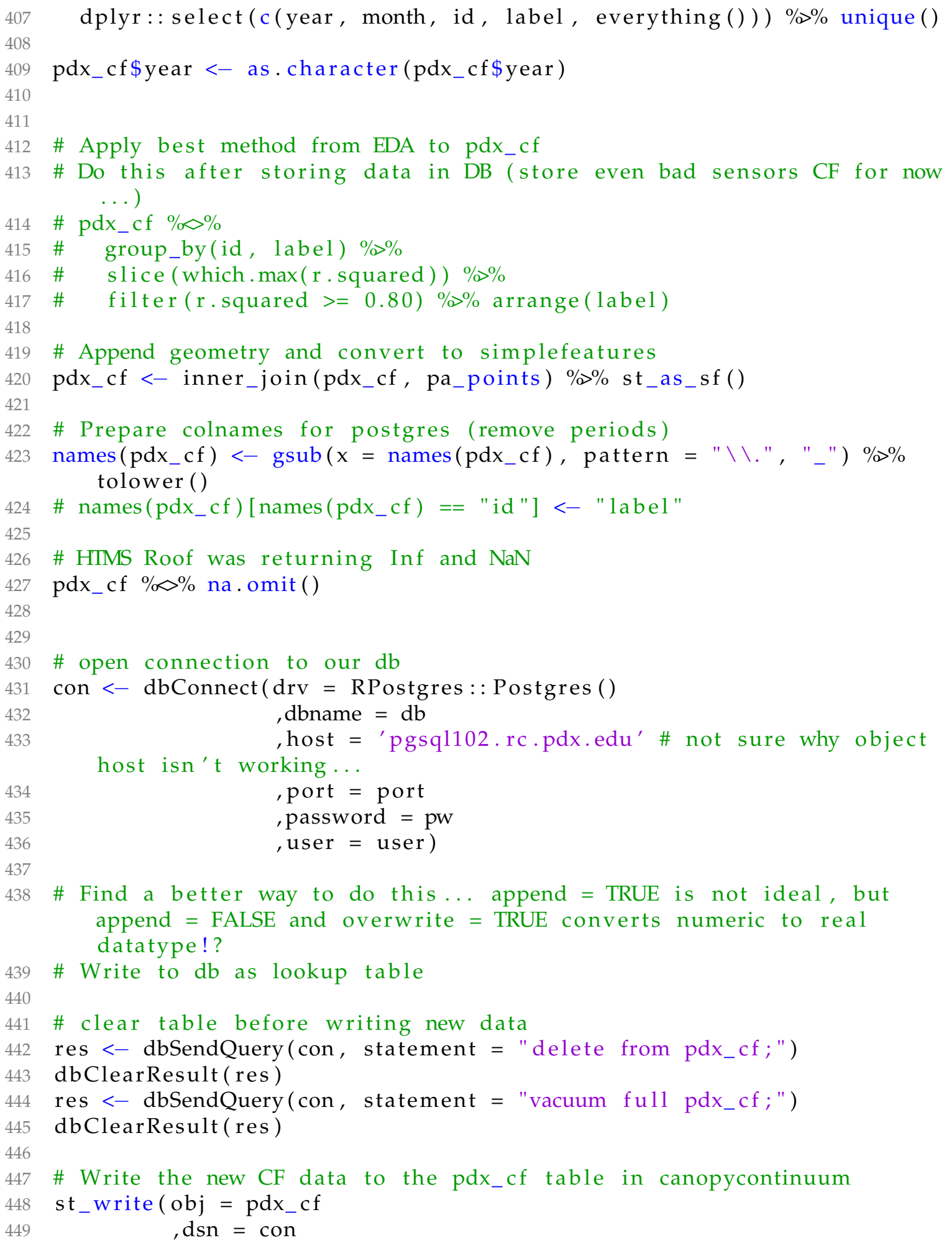


, layer_options = "OVERWRITE=true"

, drop_table = FALSE

, try_drop = FALSE

, debug = TRUE

, append = TRUE

, overwrite = FALSE

, $\mathrm{d}$ ata set_options = "GEOMETRY=AS_WKT"

)

\# \# define global graphics variables

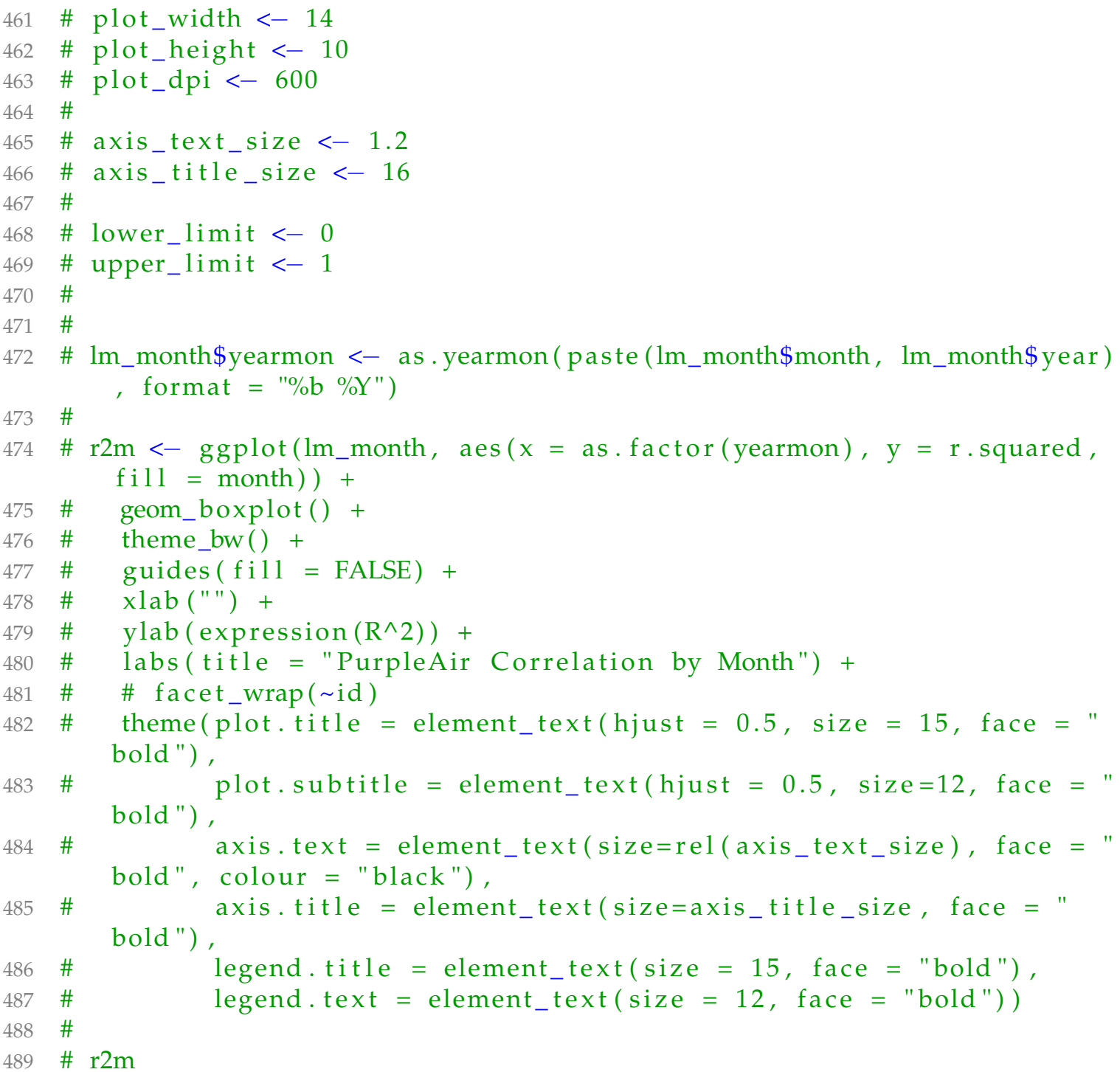




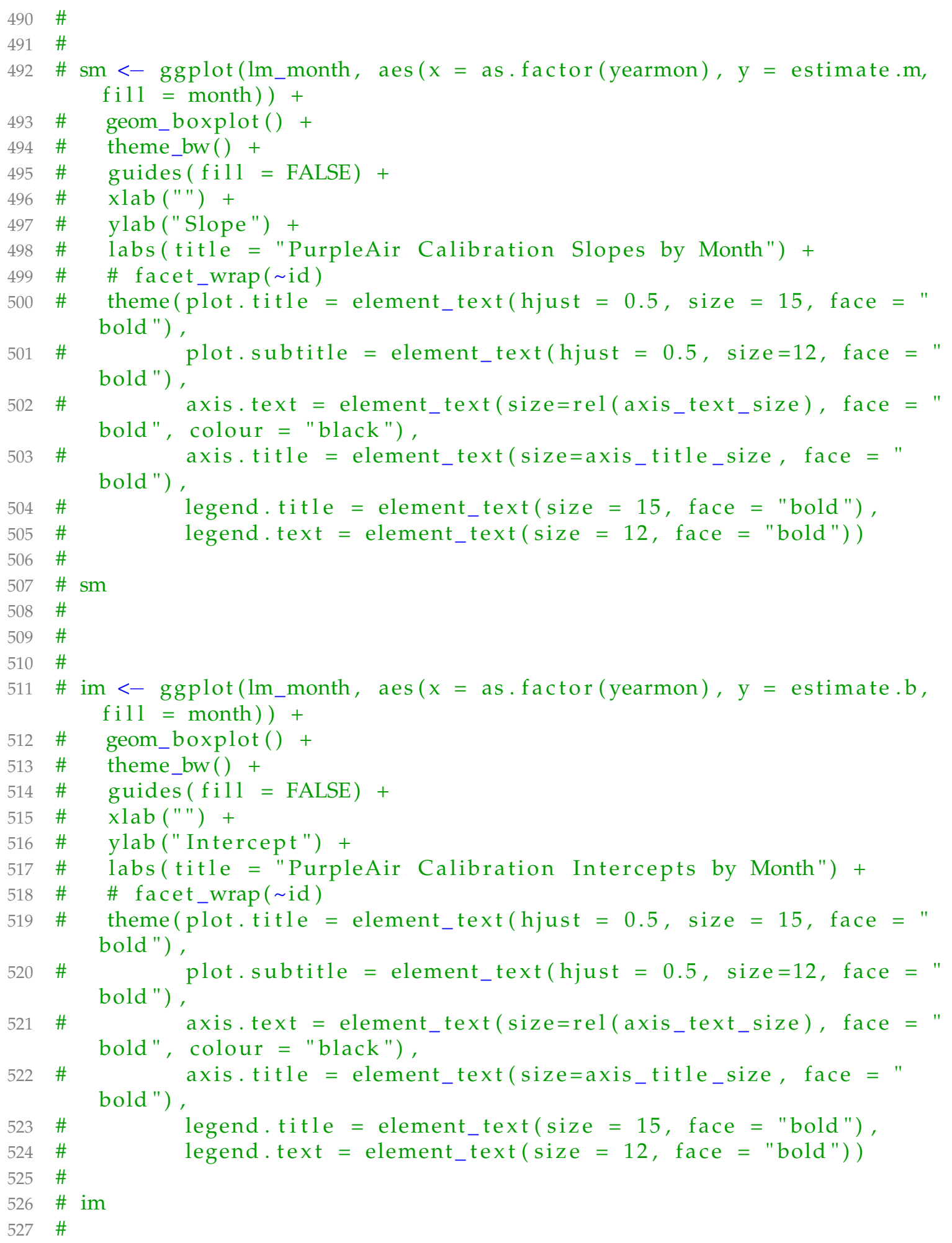




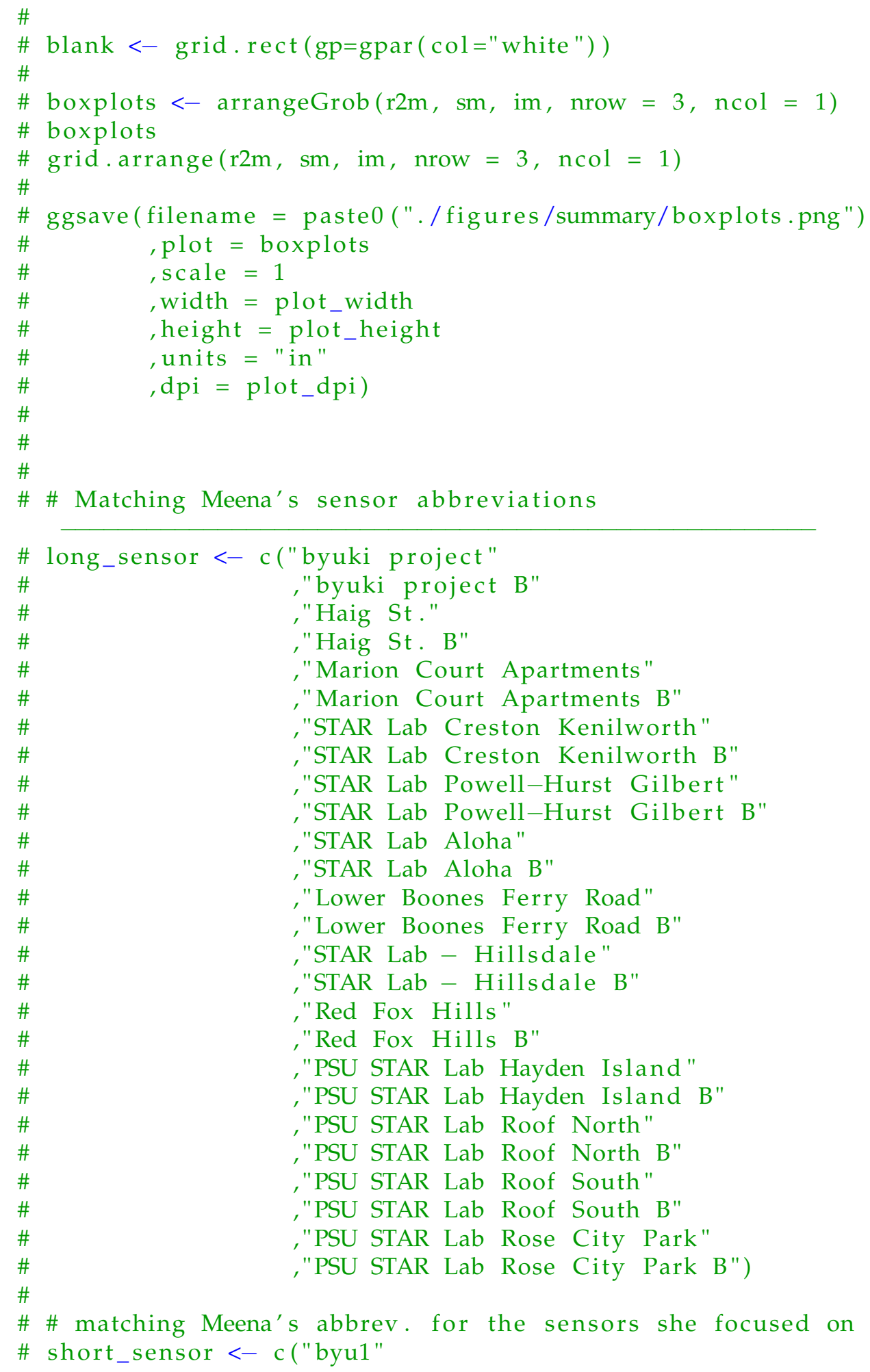




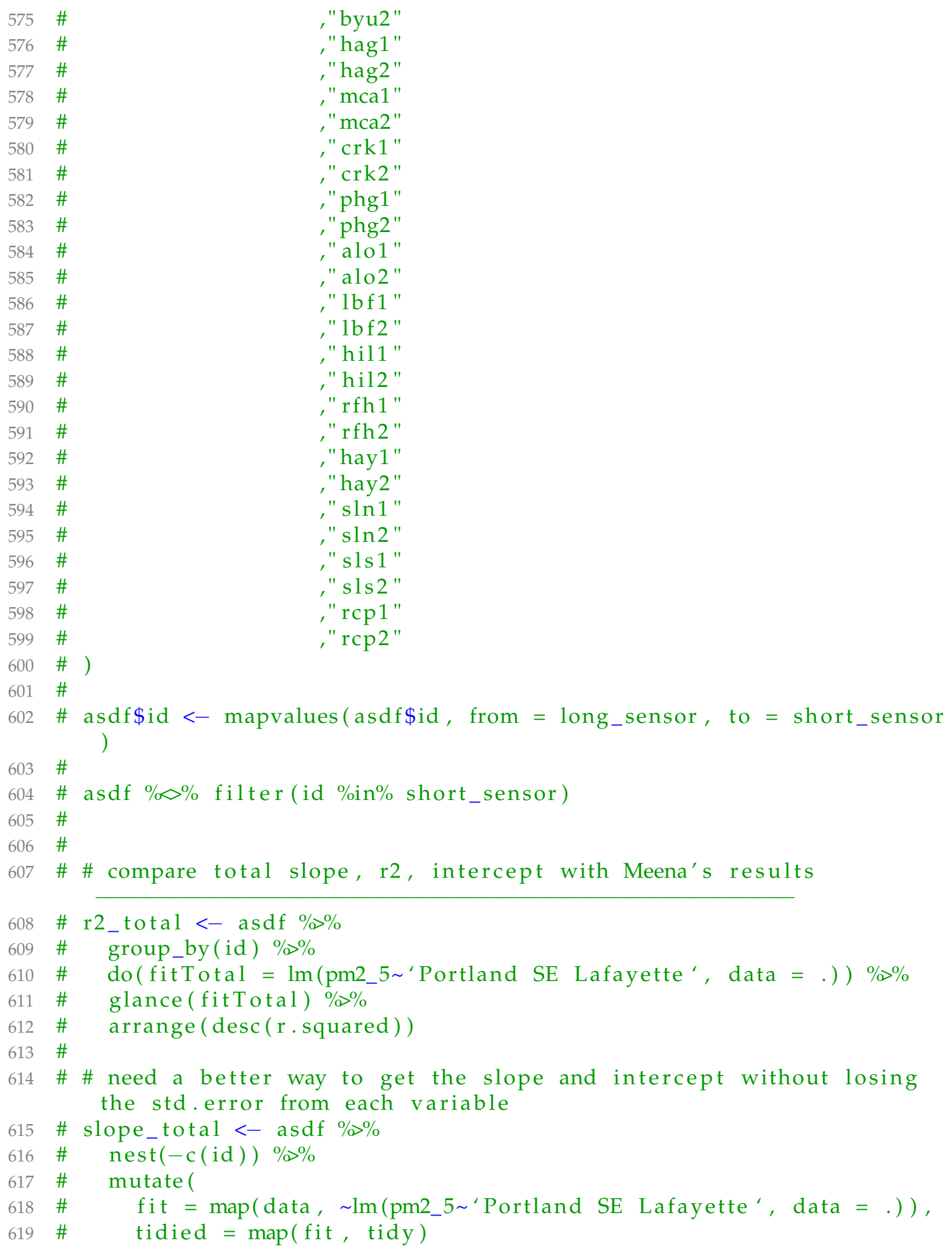




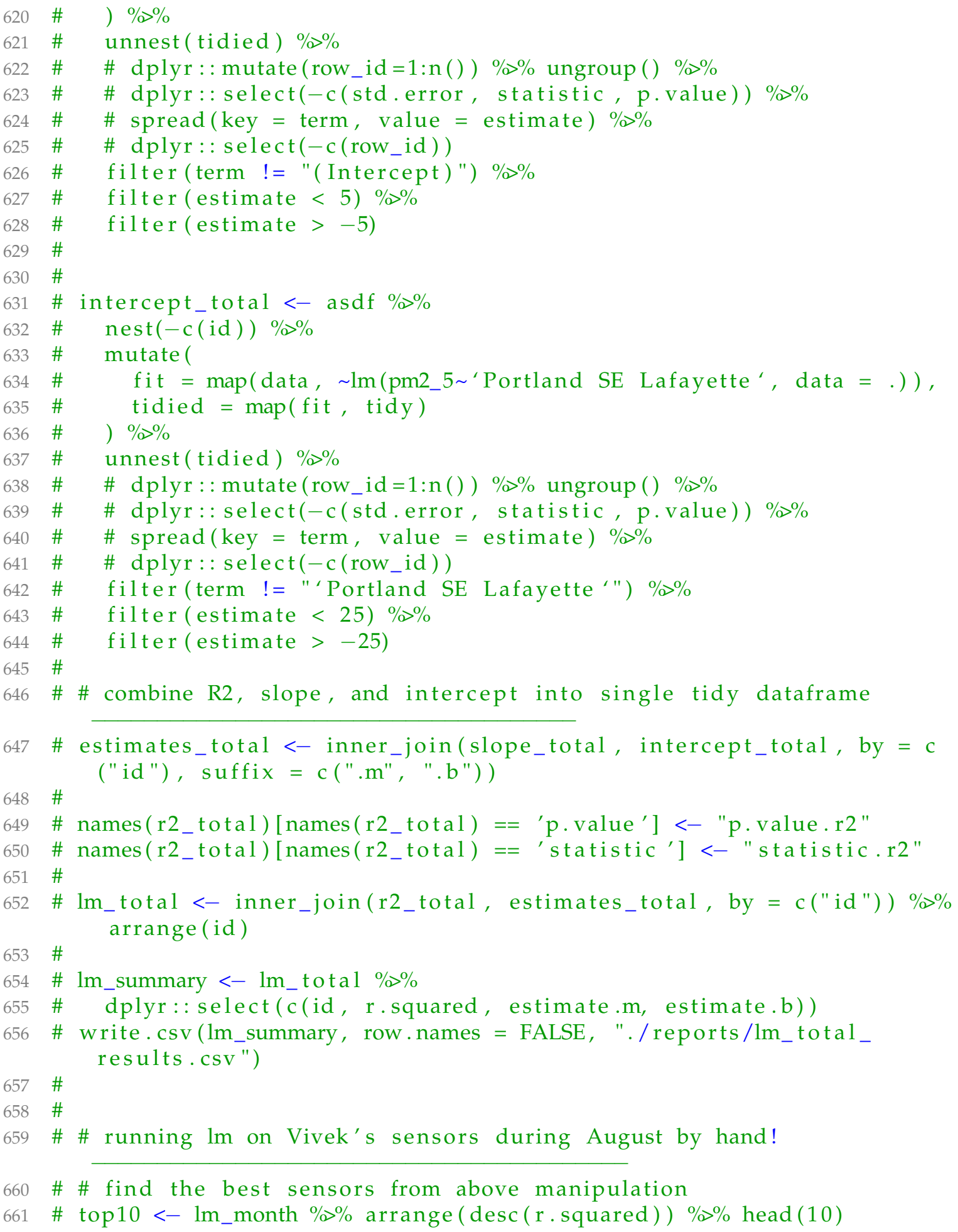


\# a $<-$ asdf $\% \%$ filter (id == "STAR Lab Creston Kenilworth" \& month $==$ "Aug")

\# moda $<-\operatorname{lm}($ a\$pm2_5 a\$'Portland SE Lafayette ')

\# summary (moda)

\#

\# $\mathrm{b}<-$ asdf $\% \%$ filter $(\mathrm{id}==$ "STAR Lab Creston Kenilworth B" \& month $==$ "Aug")

\# modb $<-\operatorname{lm}($ b\$pm2_5 b\$'Portland SE Lafayette ')

\# summary (modb)

\# BEGIN CF EDA

\# \# the slopes vary for a given sensor, despite having strong correlations...

\# gg $<-$ pdx_cf $\% \%$

\# filter(r.squared $>=0.85) \% \%$

\# dplyr:: select(year, month, id, label, r.squared, estimate.m, estimate.b) $\% \%$

\# arrange(label)

\# unique (gg\$label)

\#

\# \# temporary fix is to just use the CF with the strongest correlation, or take the average?

\# max_r2 $<-$ pdx_cf $\% \%$

\# group_by(id, label) $\% \%$

\# $\quad$ slice (which.max (r.squared)) $\% \%$

\# dplyr::select(id, label, r.squared, estimate.m, estimate.b) $\% \%$

\# filter (r.squared $>=0.85$ ) $\% \%$ arrange(label)

\#

\# bad_r2 $<-$ pdx_cf $\% \%$

\# group_by(id, label) $\% \%$

\# $\quad$ slice (which.max(r.squared)) $\% \%$ arrange(r.squared) $\% \%$

\# dplyr::select(id, label, r.squared, estimate.m, estimate.b) $\% \%$

\# filter $(\mathrm{r}$. squared $<0.85) \% \%$ arrange $($ label $)$

\#

\# mean_r2 $<-$ pdx_cf $\% \%$

\# group_by(id, label) $\% \%$

\# filter (r.squared $>=0.85) \% \%$

\# dplyr::select (year = year, month, id, label, r.squared, estimate. m, estimate.b) $\% \%$

\# summarise_if (is . numeric, . funs=mean) $\% \%$

\# arrange (label)

\#

\# diff <- inner_join (mean_r2, max_r2, by = c("id", "label"), suffix = c (". mean", ".max") ) \% \% 


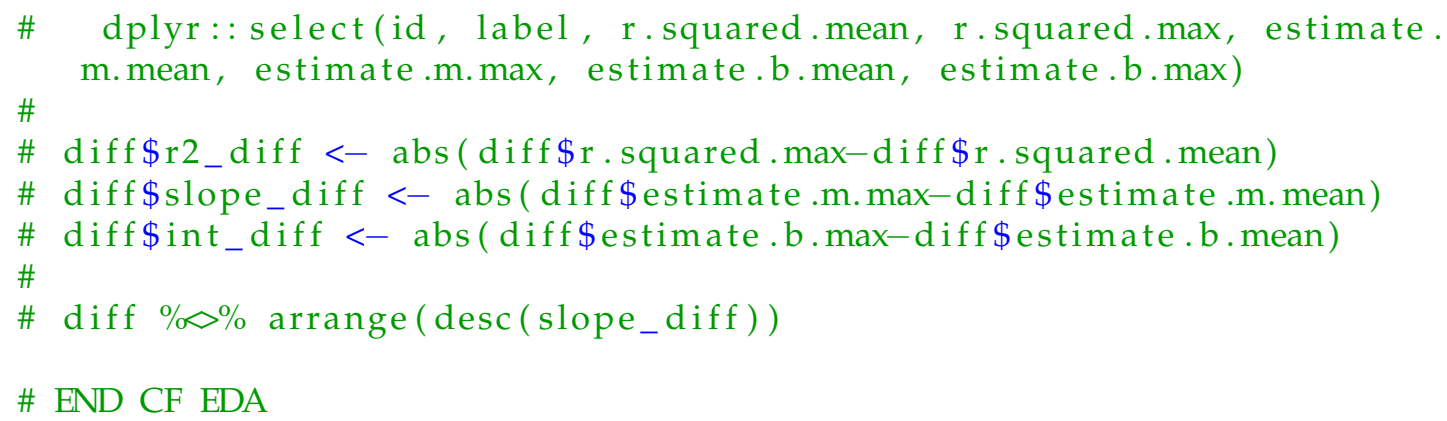

\section{Source/unionStreets.R}

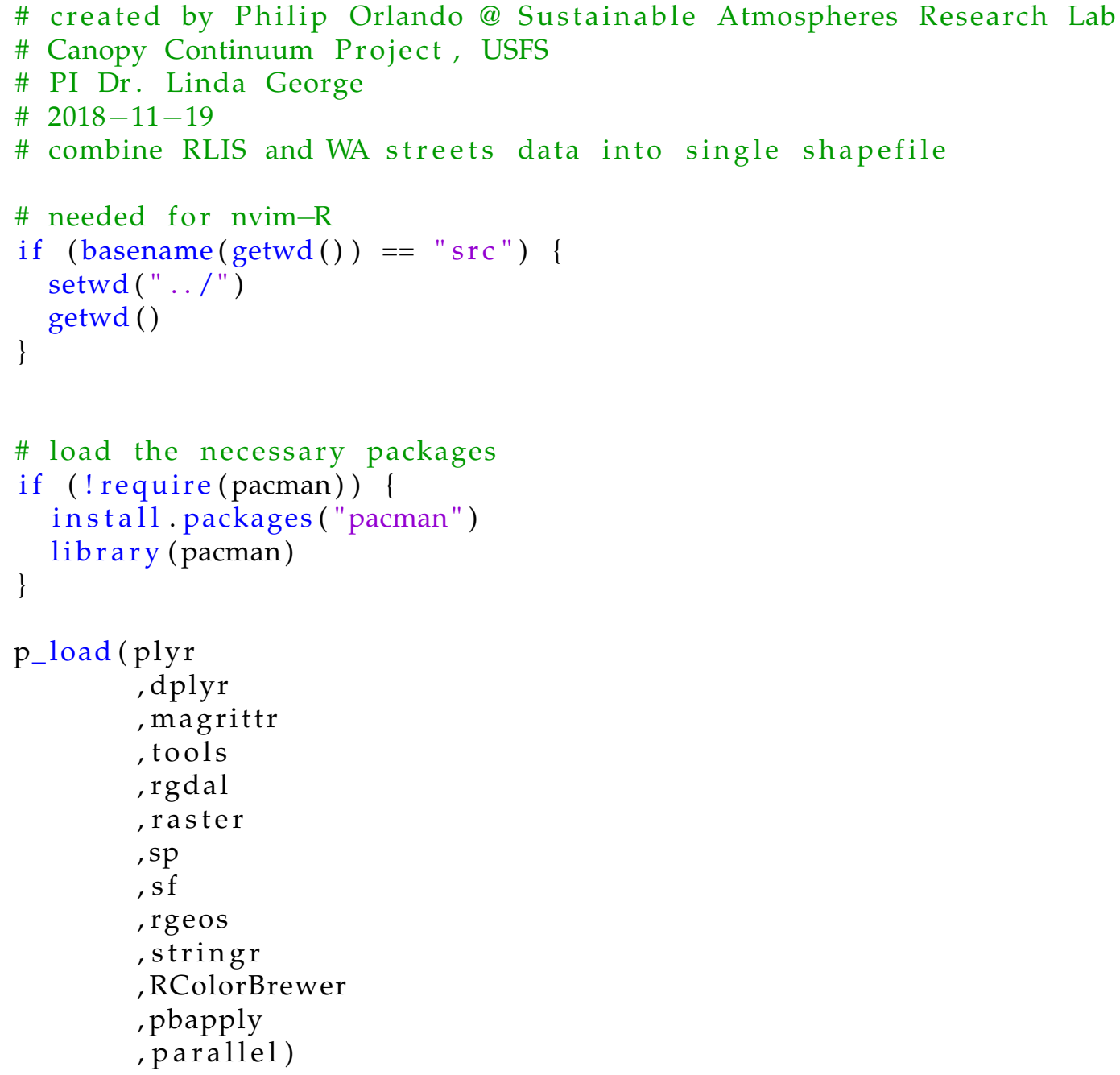


\# Oregon North NAD83 Meters UTM Zone 10

epsg_26910<- "+proj=utm +zone=10 +ellps=GRS80 +datum=NAD83 +units=m +no_defs "

\# Portland Study Area

study_area $<-$ readRDS("./study_area/study_area.RDS")

\# Portland Streets (not from the rlis dir, we write there insteads) pdx <- readOGR("./data/pdx_streets/streets.shp") \% \% spTransform( CRSobj = epsg_26910)

\# Vancouver Streets

vnc $<-$ readOGR("./data/wsdot/WSDOT__Local_Agency_Public_Road_Lines. shp")

vnc $\% \diamond \%$ spTransform (CRSobj = epsg_26910)

vnc $\% \diamond \%$ gIntersection(study_area) \# does this only return lines that intersect the border of our study area, or also lines that are contained by it?

\# Combine them together

$x<-$ raster:: union(pdx, vnc) \# faster but it might be different... writeOGR $(\mathrm{x}, \mathrm{dsn}=" . / \mathrm{data} / \mathrm{rlis} "$, layer $=$ "streets", driver $=$ "ESRI Shapefile", overwrite $=\mathrm{T}$ )

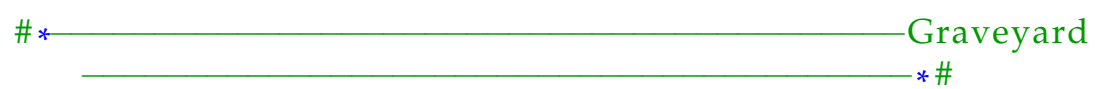




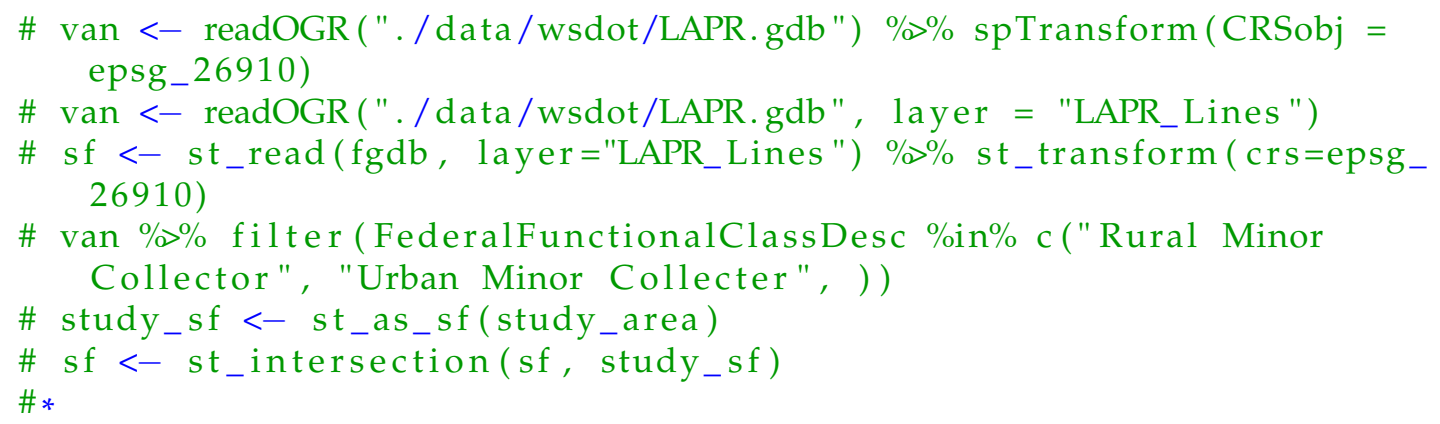

\section{Source/processNDVI.R}

\# created by Philip Orlando @ Sustainable Atmospheres Research Lab

\# Canopy Continuum Project, USFS

\# PI Dr. Linda George

\# 2018-10-18

\# process raw hdf MODIS NDVI data, convert to GeoTiff, perform weekly , monthly averages

\# load the necessary packages

if (! require (pacman) ) \{

install . packages("pacman")

library (pacman)

\}

p_load (rgdal

, dplyr

, magrittr

, gdalUtils

, raster

, sp

, sf

, rgeos

,MODIS

, parallel

, ggplot2

,pbapply

\#,greenbrown \# sourceforge repo

)

source ("./src/init.R")

source ("./src/functions.R")

study_area $<-$ readRDS(study_area) 
\# determine bounding box for portland

\# ndvi_path <- "./MODIS/MOD13A2.006" \# $1 \mathrm{~km}$

ndvi_path <- "./MODIS/MOD13Q1.006" \# $250 \mathrm{~m}$

ndvi_files $<-$ list.files $($ path $=$ ndvi_path, pattern $=" \backslash \backslash$.hdf\$"

, all.files = FALSE, full. names = TRUE

41

subdirectories , recursive $=$ TRUE \# grab files from all

percentNA $<-$ function $(r)\{$ 
\# Not sure how to make use of this layer yet... Only applies to Pixel Reliability $==1$

\# vi_quality <- sds[grepl("16DAY_250m_500m_VI:250m 16 days VI Quality", sds)] $\% \%$ readGDAL $\% \%$ raster

\# 10 other variables (QA, cloud mask, water, climatology, aerosols ?)

\# Exclude Low Quality Pixels from NDVI \& EVI layers pix_rel <- sds[grepl("16DAY_250m_500m_VI:250m 16 days pixel reliability", sds)] $\% \%$ readGDAL $\% \%$ raster values $($ pix_rel $)[$ values $($ pix_rel $)=-1$ ] $<-$ NA \# Fill/No Data: Not Processed

\# values (pix_rel)[values $($ pix_rel) $==1]<-$ NA \# Marginal data: Useful, but look at other QA information values $($ pix_rel $)[$ values $($ pix_rel $)==2$ ] $<-$ NA \# Snow/Ice: Target covered with snow/ice

values $($ pix_rel) [values $($ pix_rel $)==3$ ] <- NA \# Cloudy: Target not visible, covered with cloud

values (pix_rel) [values (pix_rel) == 0] <- 1 \# Good Data: Use with Confidence

ndvi $<-$ ndvi*pix_rel

evi $<-$ evi*pix_rel

print (percentNA (ndvi))

print (percentNA ( evi))

\# Not useful for annual median values, but might be useful for daily interpolation...

na_threshold $<-1.00$ \# decimal percent

if $($ percentNA $($ ndvi $)<$ na_threshold \&\& percentNA $($ evi $)<$ na_threshold ) \{

\# combine NDVI and EVI into a single stack

$\mathrm{r}<-$ stack (ndvi, evi)

names $(r)<-c($ paste0 ("ndvi.", date), paste0("evi.", date))

$\mathrm{r}<-$ raster:: projectRaster $($ from $=r$, method $=$ 'bilinear', $c r s=$ proj)

study_area $<-$ spTransform $($ study_area, CRSobj $=$ proj)

if (identical (crs (study_area), crs (r))) \{

$\mathrm{m}<-\operatorname{mask}(\mathrm{r}$, study_area $)$

cr $<-\operatorname{crop}(\mathrm{m}$, study_area $)$

pblapply(dates, function(x) \{ 


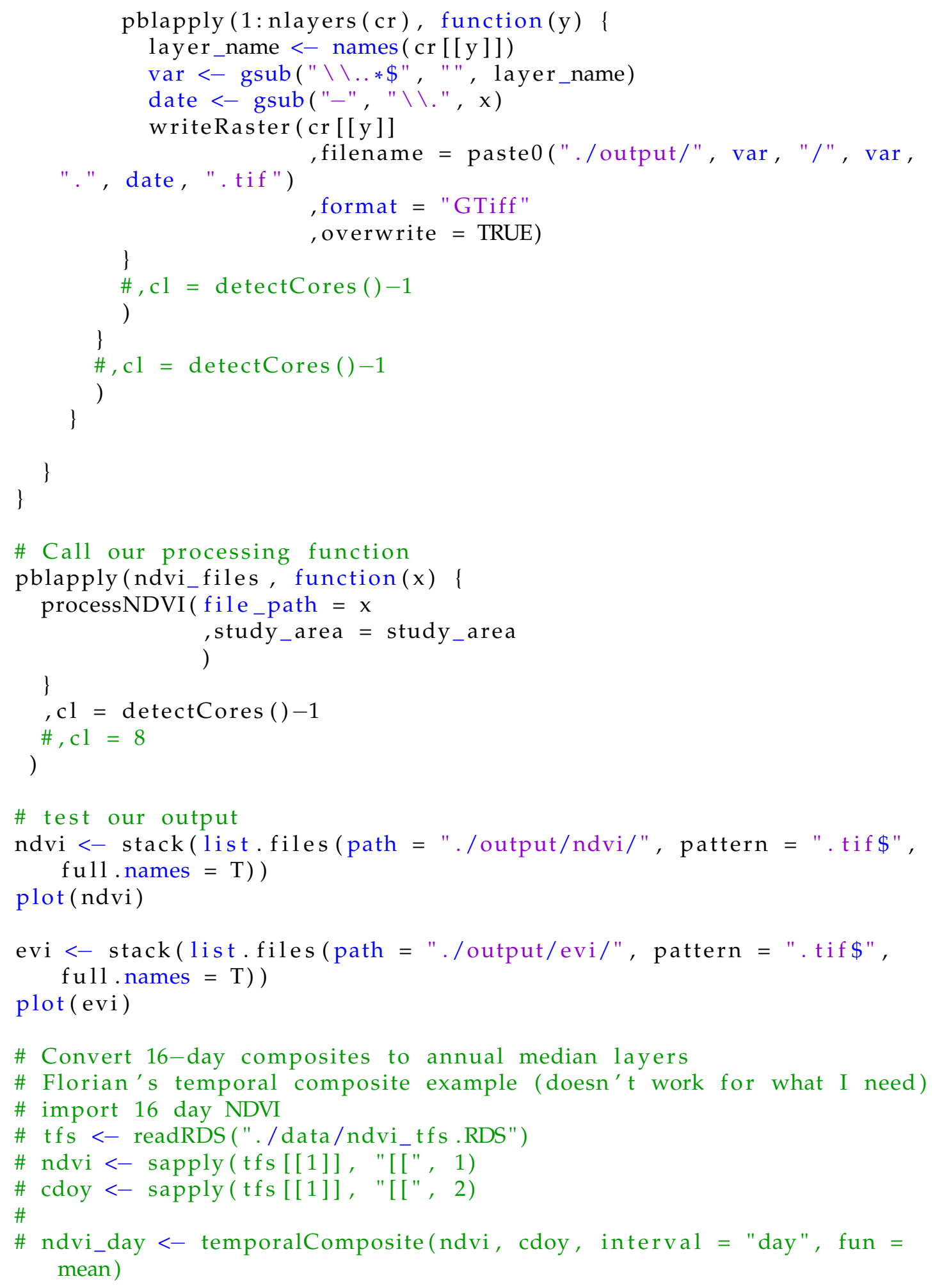


\# saveRDS(ndvi_day, "./data/ndvi_day_composite.RDS")

\# ndvi_day $<-$ readRDS ("./data/ndvi_day_composite.RDS")

\section{Source/processGridMET.R}

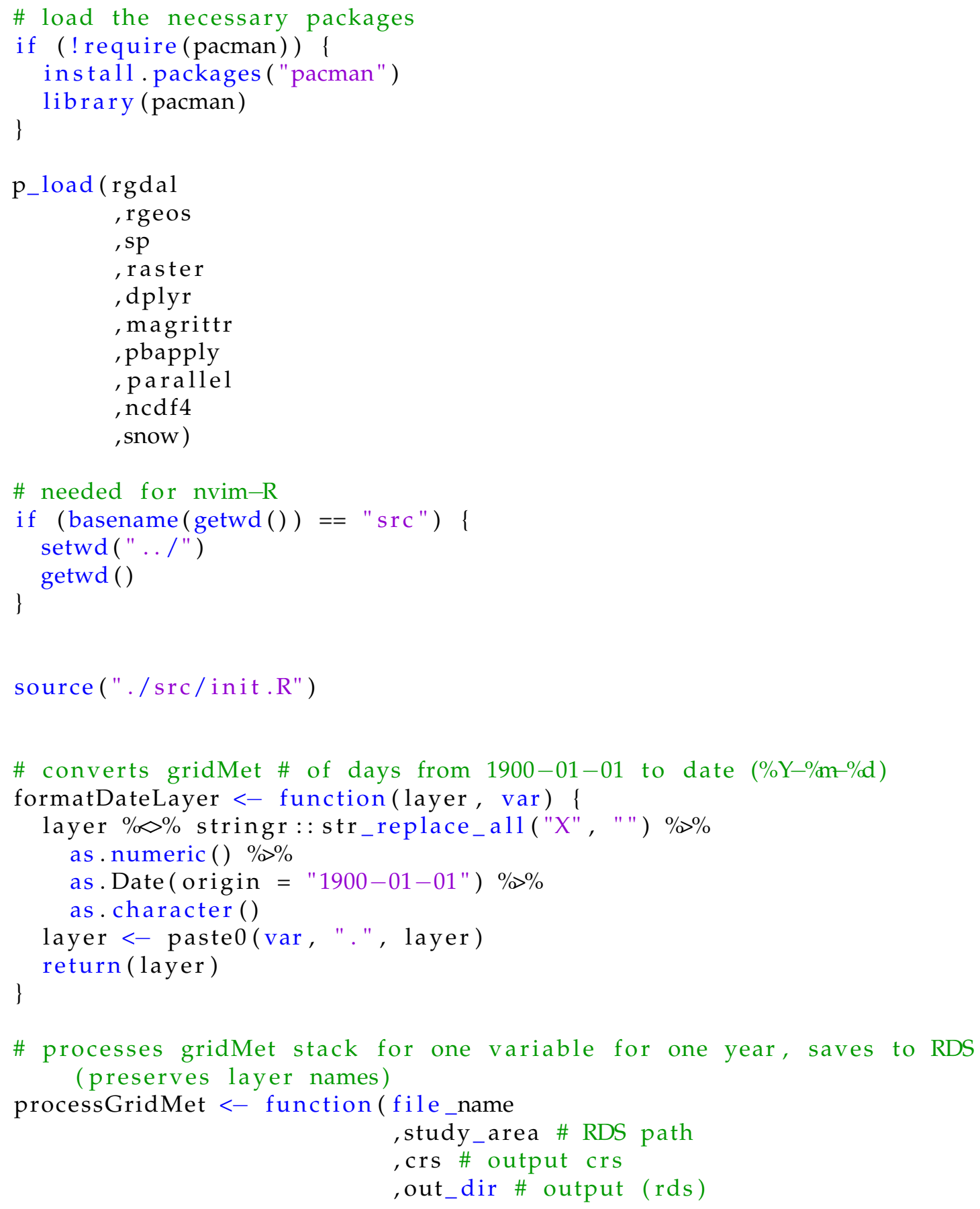




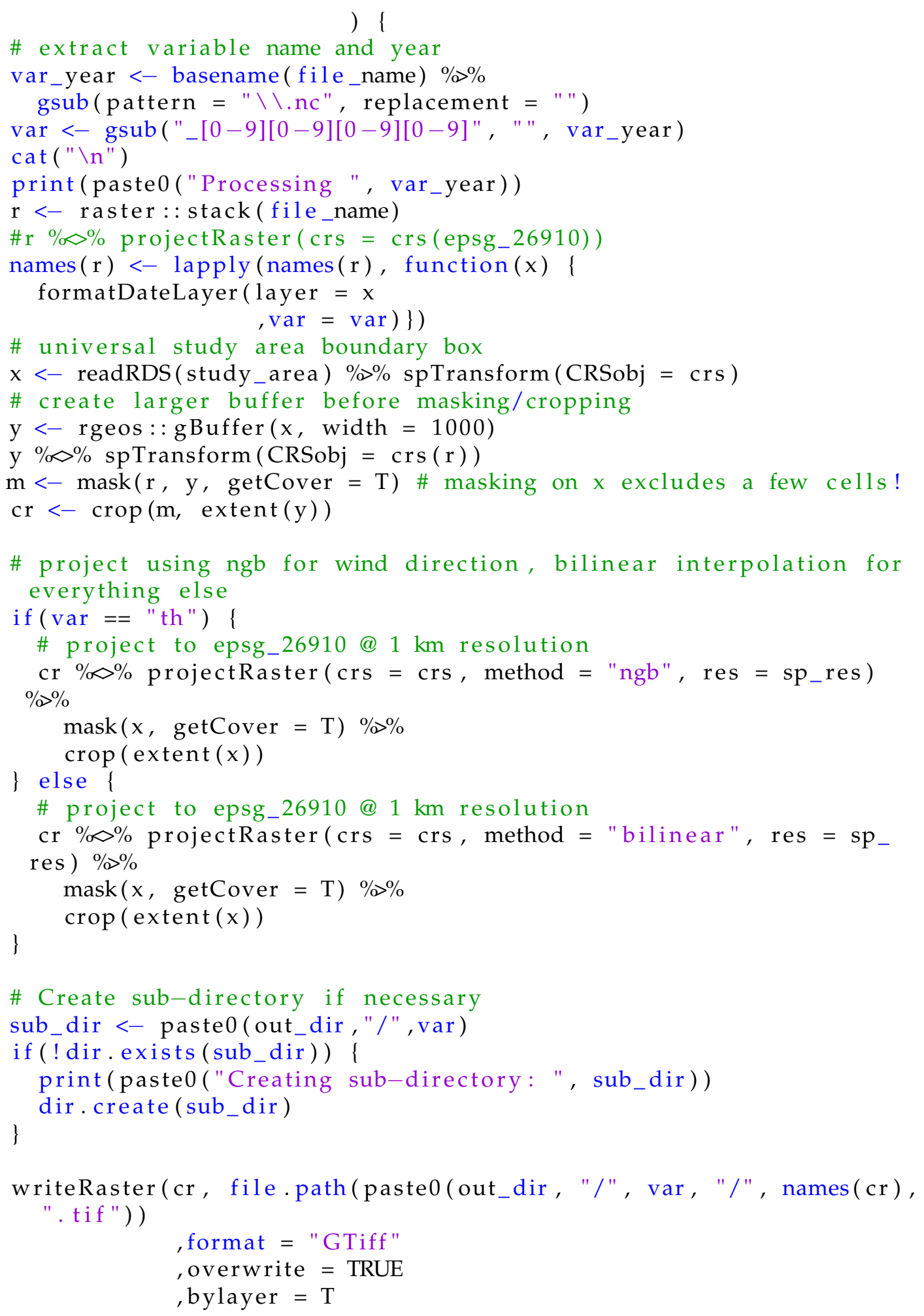




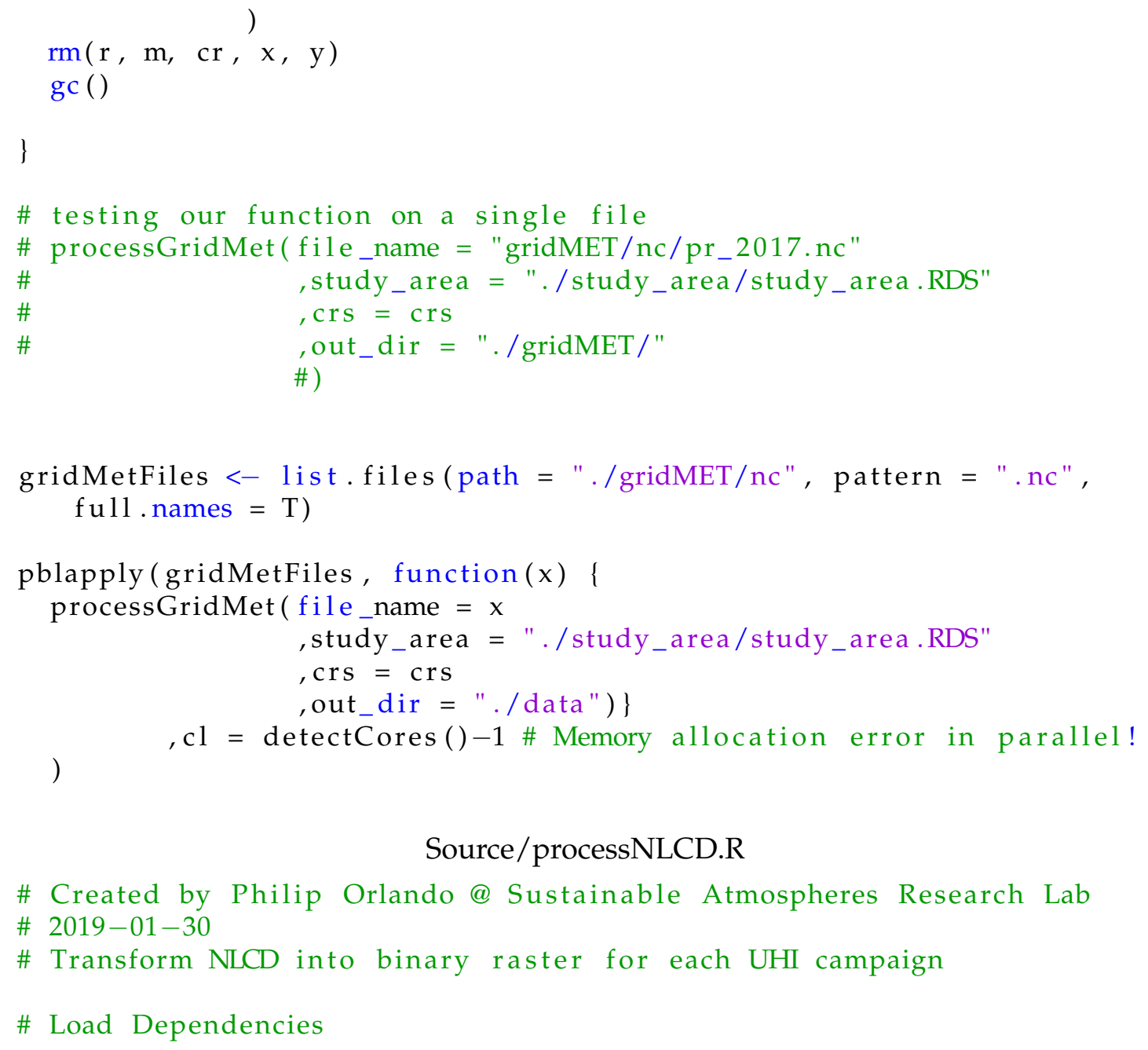

\section{Source/processNLCD.R}

\# Created by Philip Orlando @ Sustainable Atmospheres Research Lab 


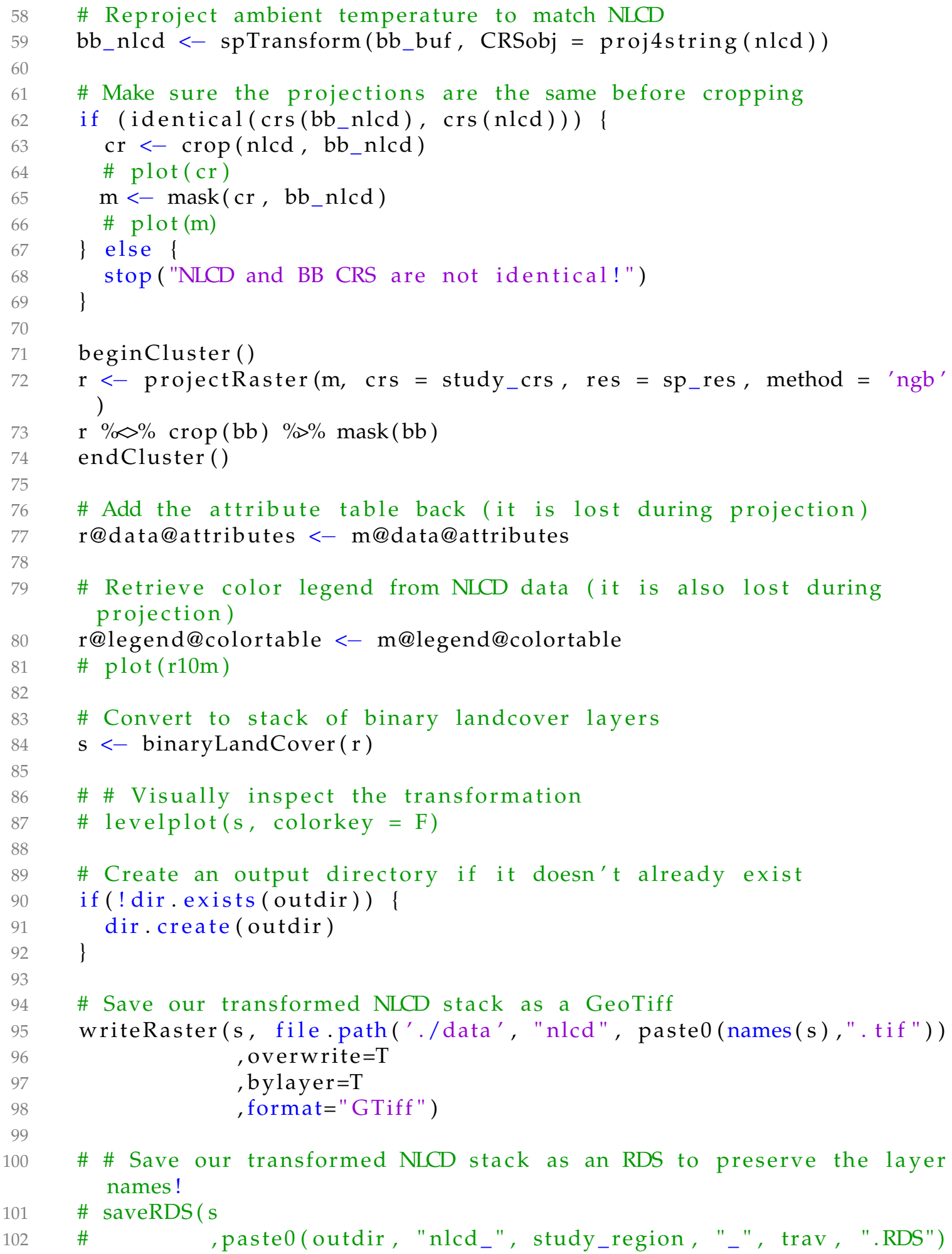




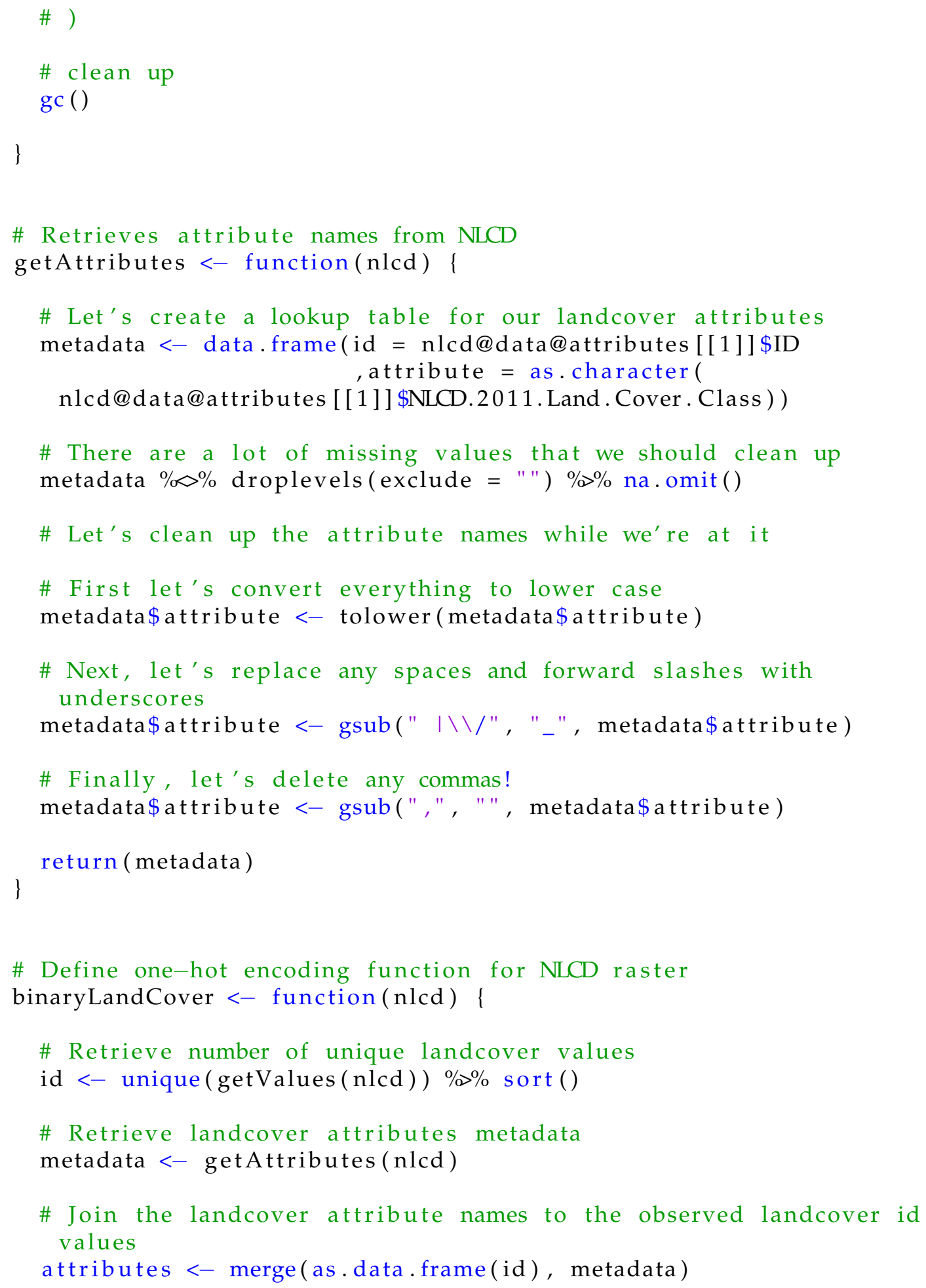


\# Loop through each landcover and create presence/absence layer then name it by LC code

s $<-$ pblapply(id, function $(x)$ \{

\# Create a new raster layer for each landcover class

\# $r<-\operatorname{assign}($ paste0 $(" r ", x), \operatorname{nlcd})$

$\mathrm{r}<-$ nlcd

\# Assign 1 if cell value matches landcover code, 0 otherwise $\mathrm{r}[]<-$ ifelse $(\mathrm{r}[]==\mathrm{x}, 1,0)$

\# attach landcover code to layer name

names $(\mathrm{r})<-\operatorname{paste} 0($ attributes $[$ id $==\mathrm{x}],[2])$

return $(r)$

\}

\# run our loop on multiple cores

, $\mathrm{cl}=\operatorname{detectCores}()-1$

) $\% \%$

\# combine each presence/absence raster into a stack stack ()

\# Create NLCD for Jackson's Lab5

\# processTraverse $($ nlcd $=$ nlcd, study_region $=$ "lab5", trav $=$ "am", outdir = "./data/example/")

\# \# Main processing function

\# processNLCD $<-$ function(nlcd, study_regions, travs, outdir $)\{$

\#

\# \# loop through each study region

\# pblapply(study_regions, function (x) \{

\#

\# \# loop through each traverse for each study region

\# pblapply(travs, function(y) \{

\#

\# \# run our main processing function!

\# $\operatorname{try}($ processTraverse $($ nlcd $=$ nlcd, study_region $=x, \operatorname{trav}=y$, outdir = outdir))

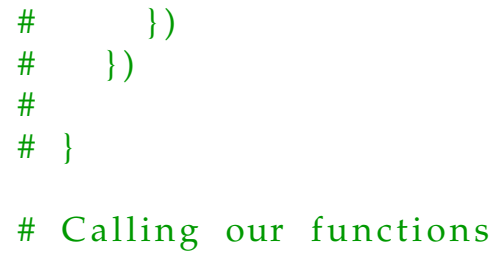




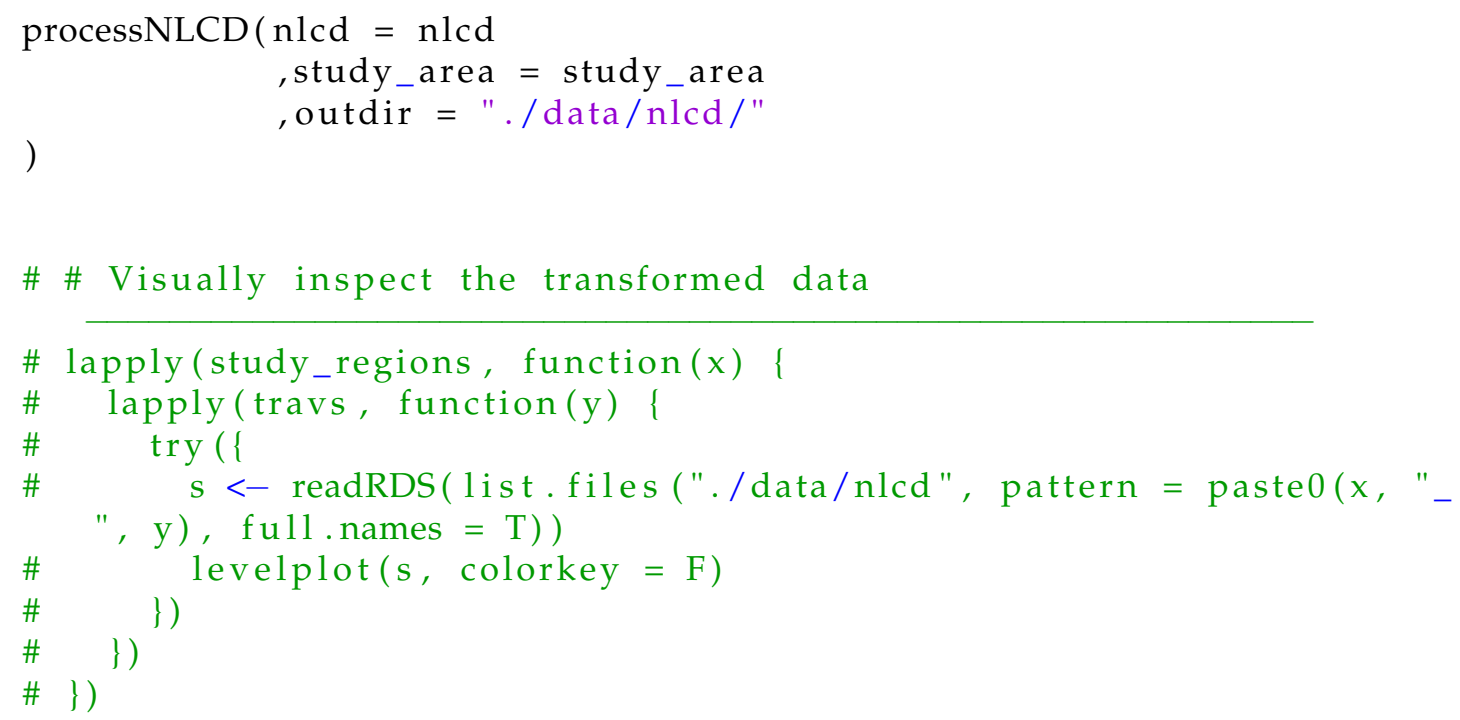

\section{Source/processRoads.R}

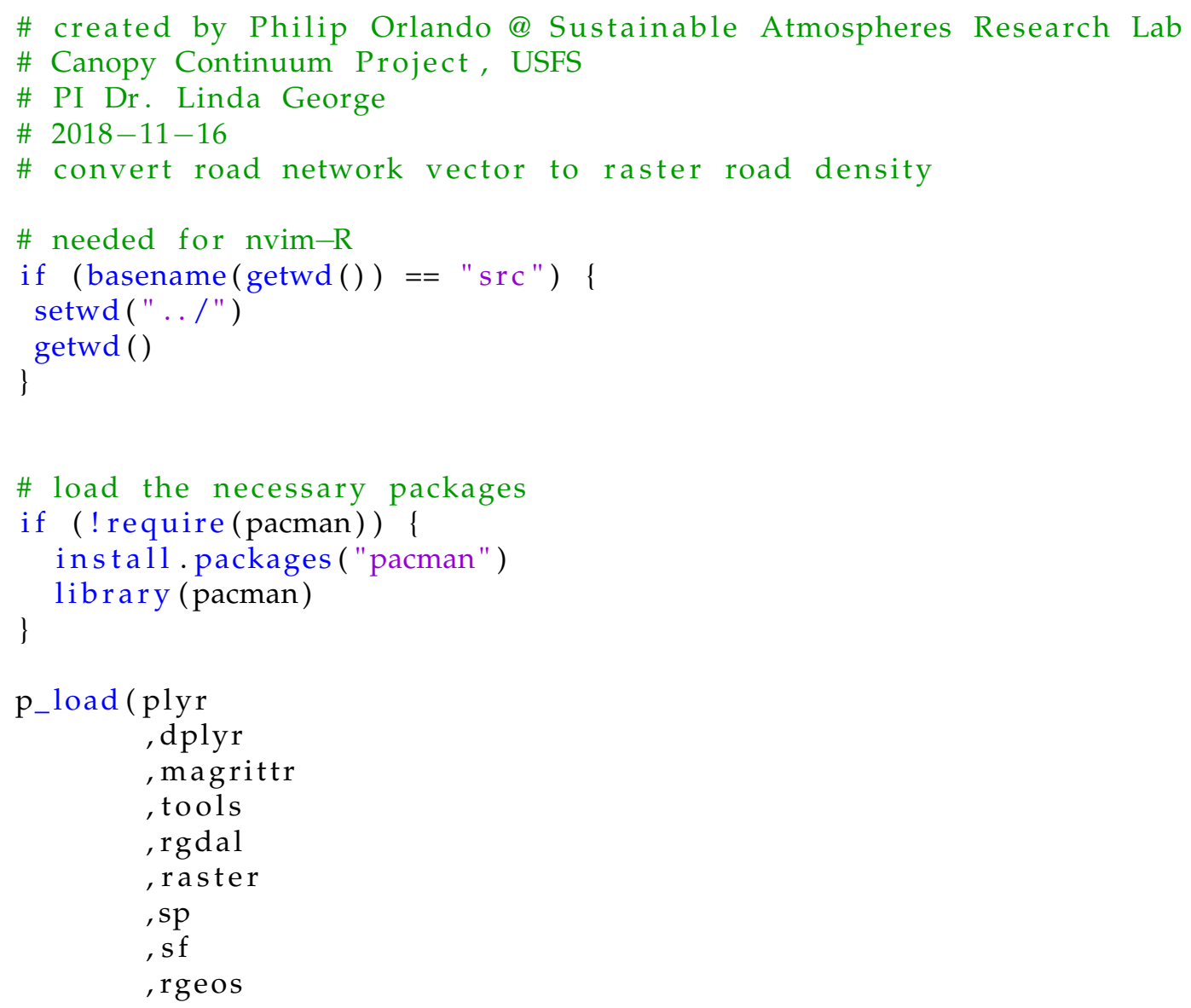




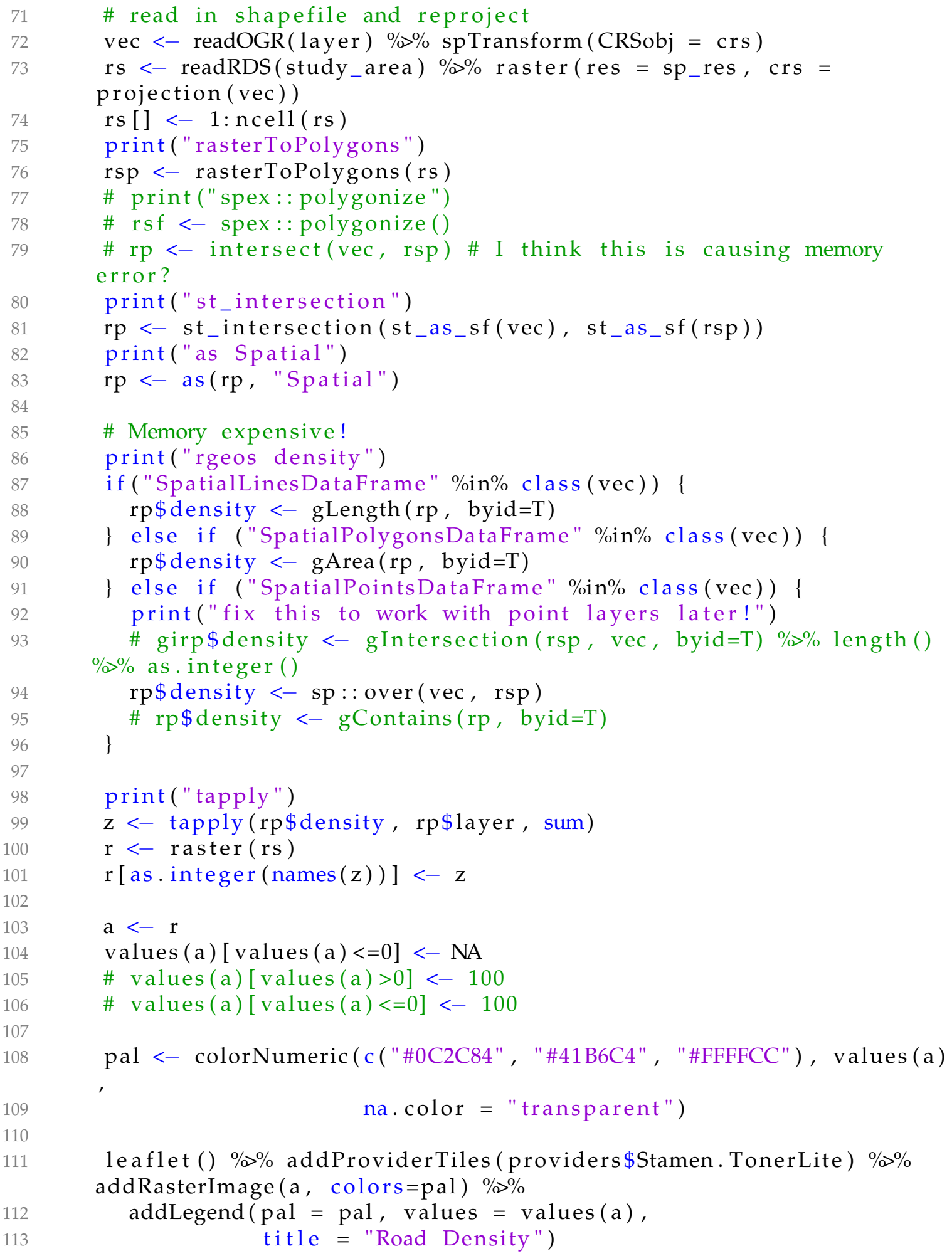




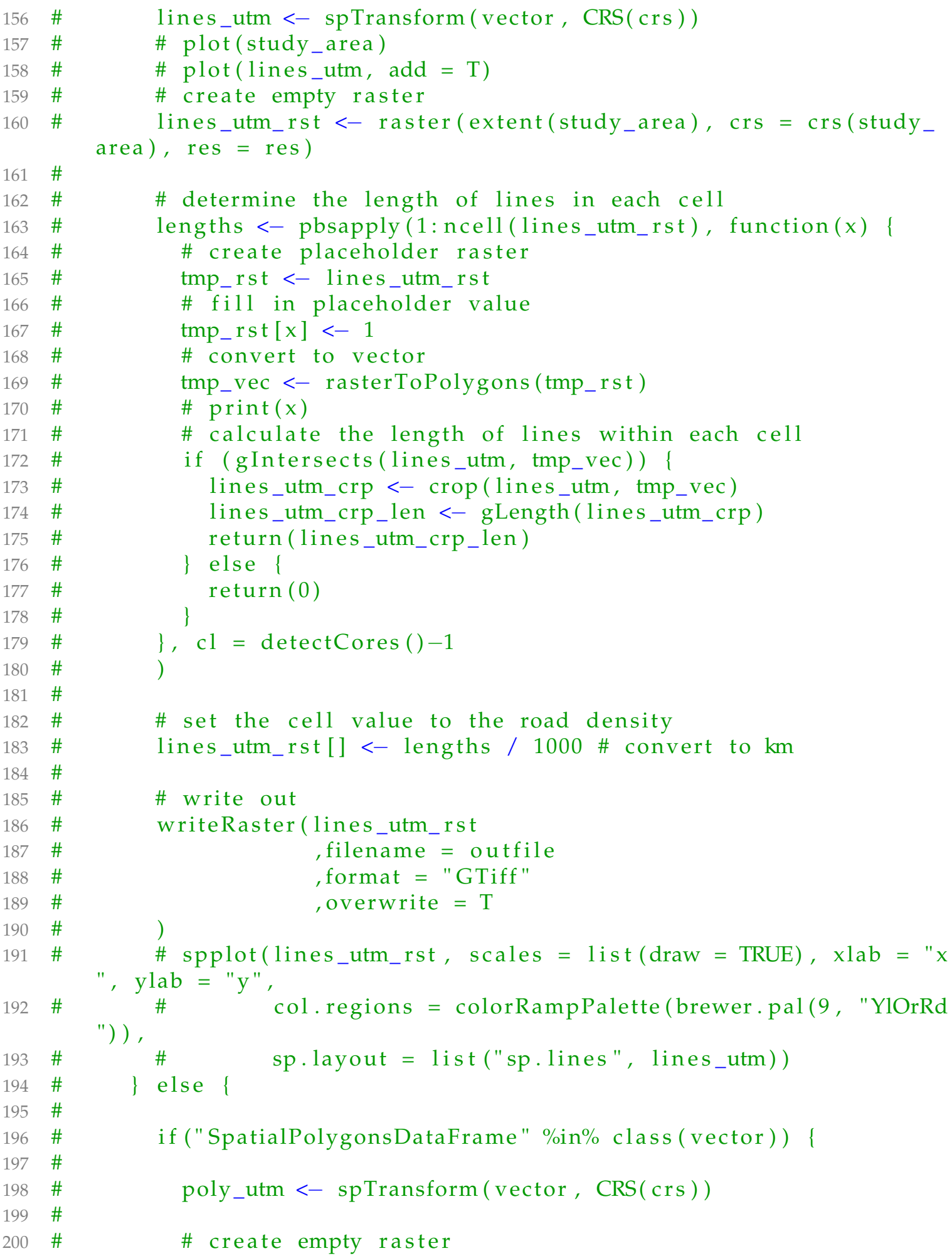




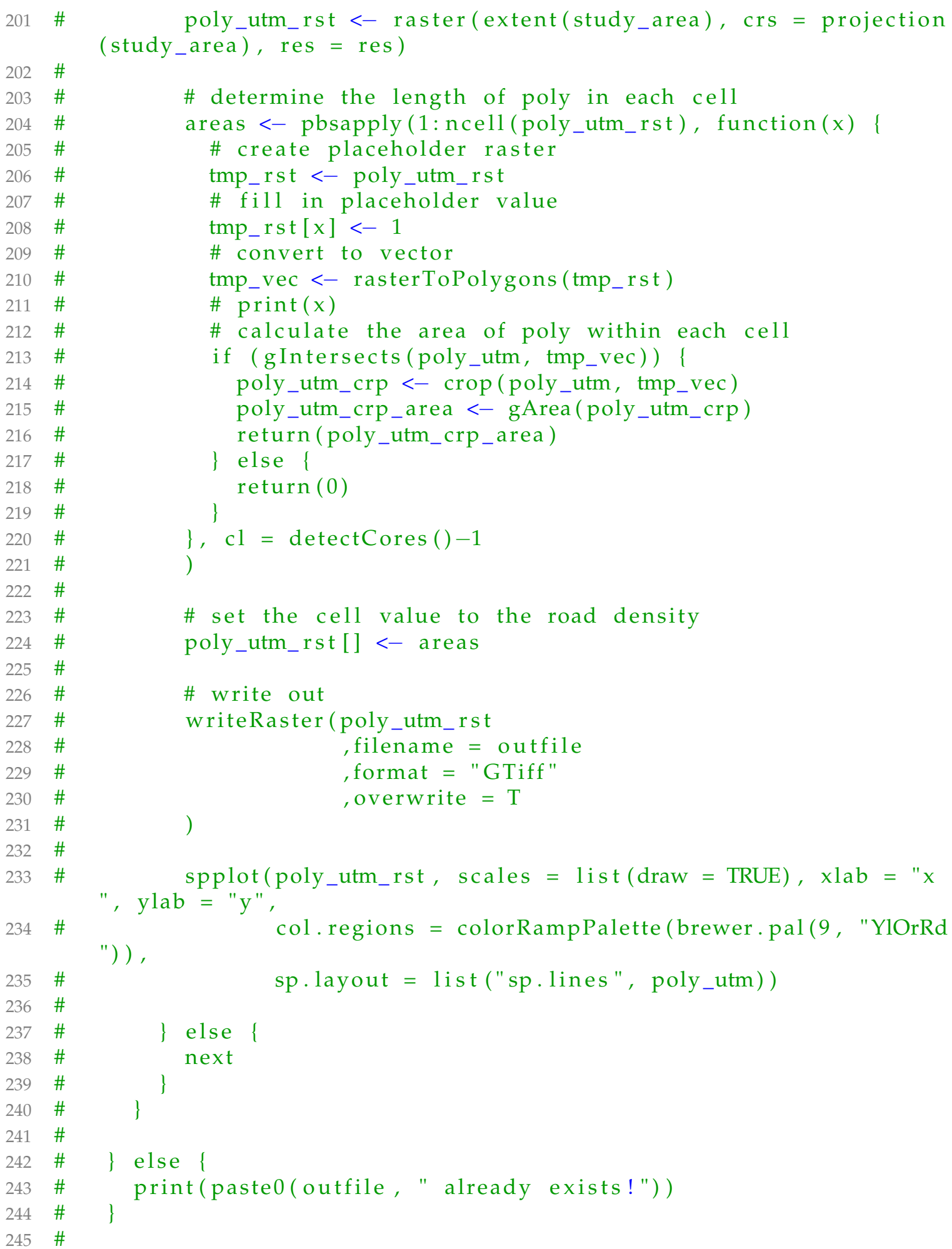




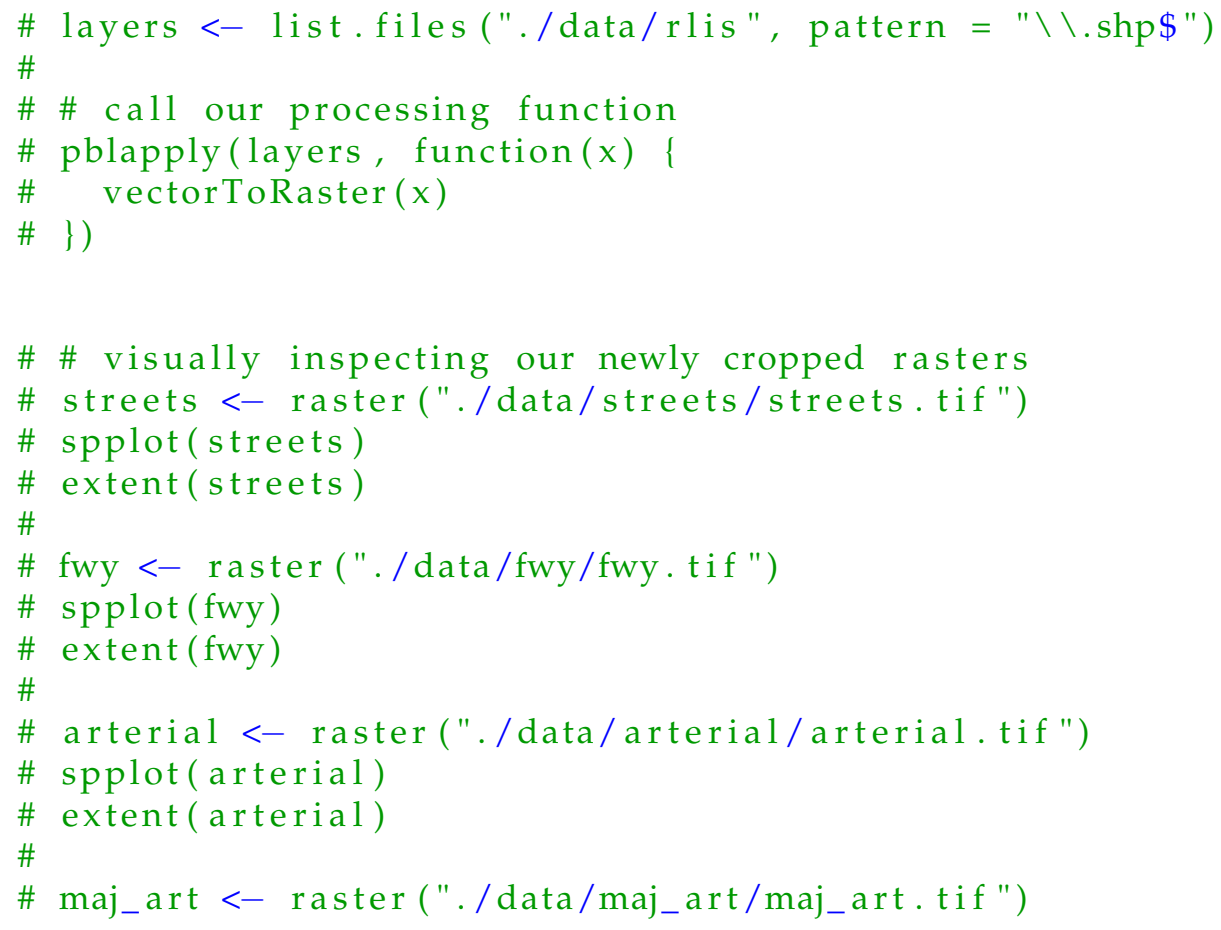




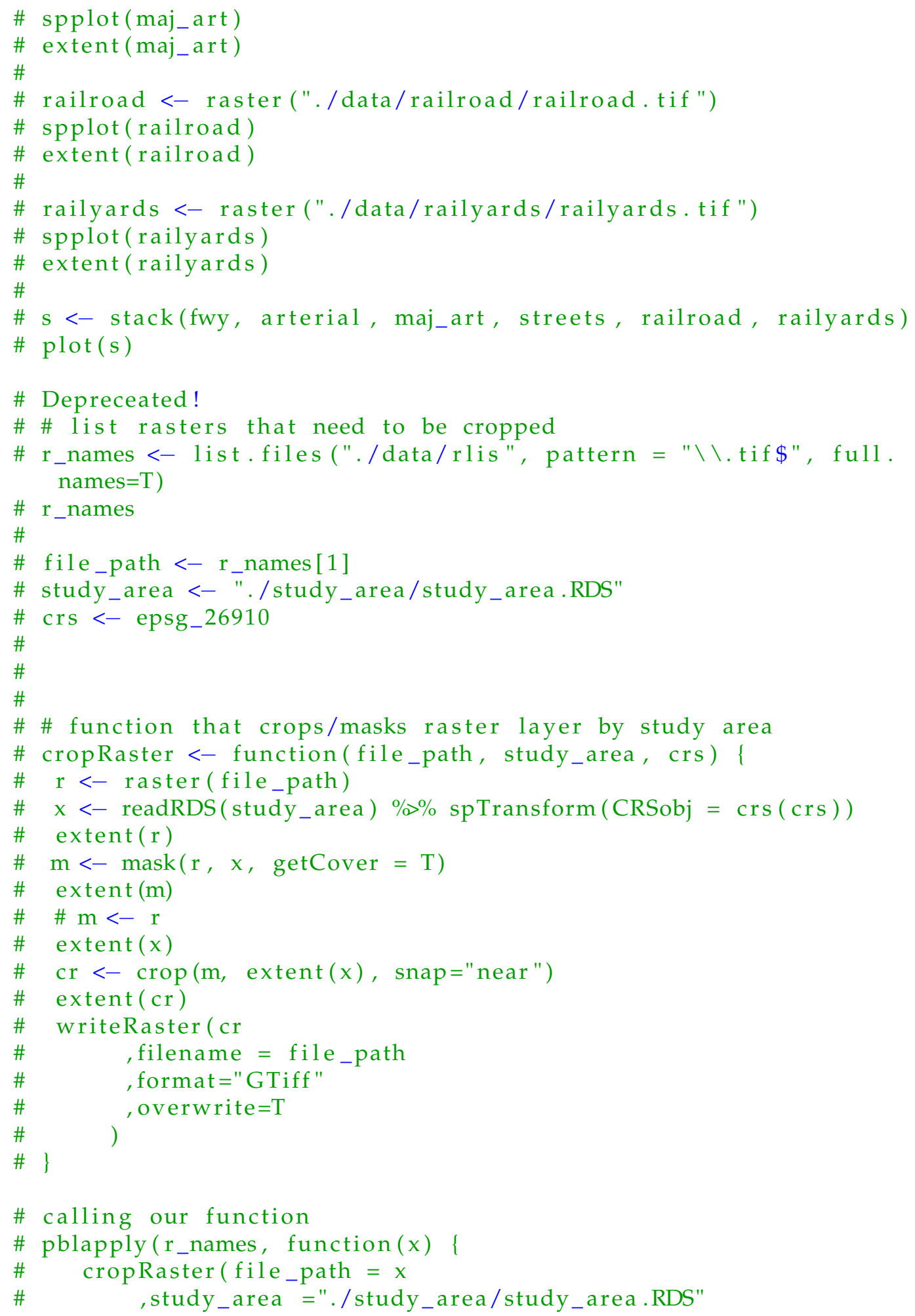


$\begin{array}{ll}334 \# & \text {, crs = epsg_26910 } \\ 335 \# & \text { ) }\})\end{array}$

Source/processPopDensity.R

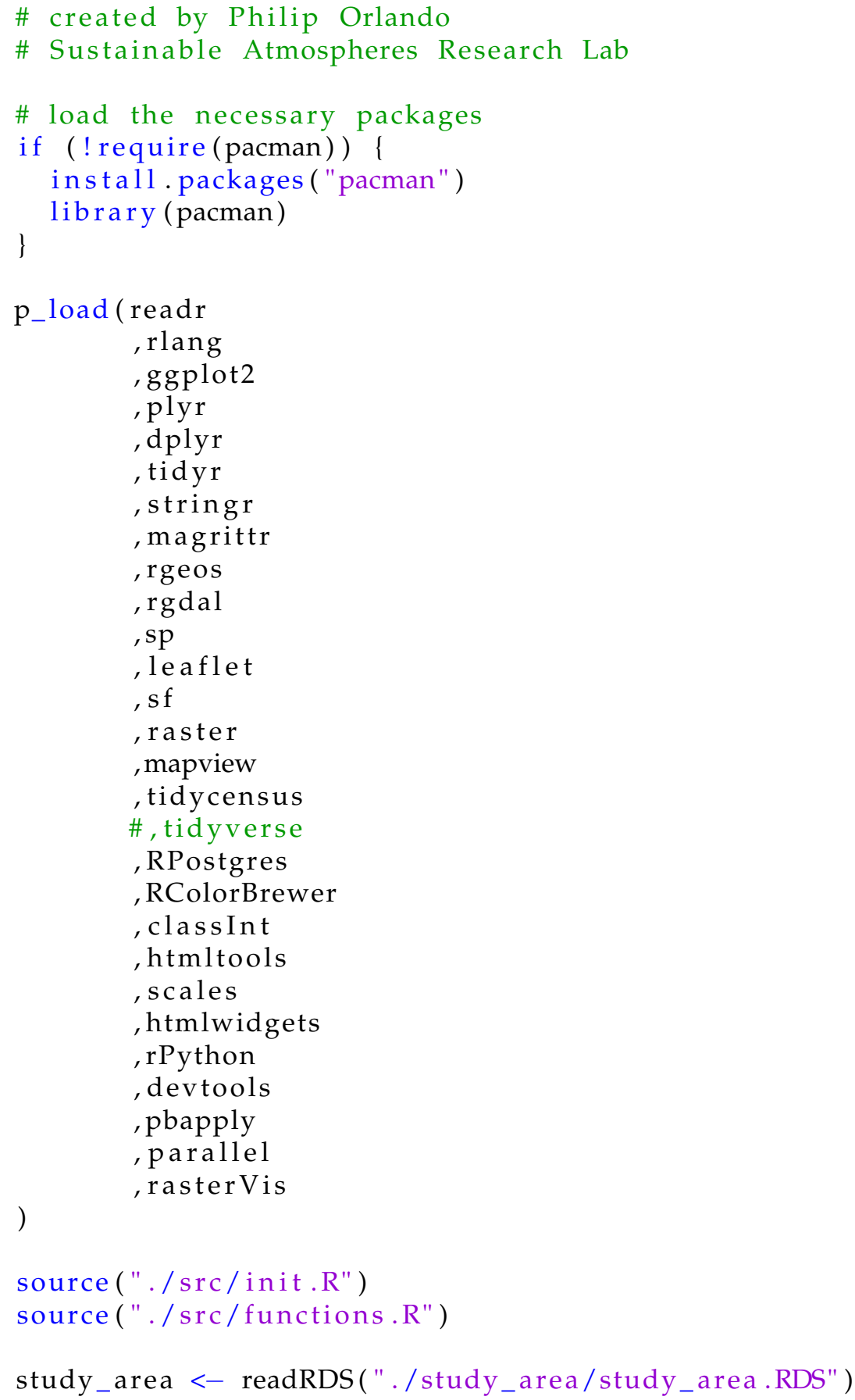


\# GPW v4 data

gpw <- raster ("./data/pop/gpw_v4_population_density_adjusted_to_2015_ unwpp_country_totals_rev11_2015_30_sec.tif")

$\mathrm{f}<-$ paste0 (tempdir (), "/", names(gpw), ".tif")

writeRaster (gpw, f, format $=$ "GTiff", overwrite $=$ T)

e $<-$ bbox (extent (study_area))

tr $<-$ rep (sp_res, 2)

\# $\operatorname{tr}<-\operatorname{rep}(1000,2)$

gdalUtils : : gdalwarp $\left(\mathrm{f}, \mathrm{s}_{-}\right.$srs $=$proj4string (gpw) , $t$ _srs=proj4string ( study_area), sub('\\.tif', ',clipped.tif', f $), \operatorname{tr}=\operatorname{tr}$,

)

cr $<-$ raster (list.files (tempdir (), "_near.* \.tif $\$ "$, full.names=T))

print("Renaming clipped/projected layers")

names $(\mathrm{cr})<-\operatorname{sub}\left({ }^{\prime}\right.$ _clipped $\${ }^{\prime},{ }^{\prime}, '$, names $\left.(\mathrm{cr})\right)$

levelplot (cr, margin $=F)$

lapply(c("near", "cubicspline", "bilinear", "cubic", "lanczos", " average"), function(i) \{

if $(\mathrm{i}==$ "near") \{

$\operatorname{tr}<-\operatorname{rep}(1000,2)$

\} else \{

\}

$\operatorname{tr}<-\operatorname{rep}(50,2)$

cr $<-$ gdalUtils : : gdalwarp $\left(\mathrm{f}, \mathrm{s}_{-}\right.$srs $=$proj4string $(\mathrm{gpw}), \mathrm{t}$-srs=

proj4string(study_area), sub('\\.tif', paste0('_', i, '_clipped. tif '), f), $\operatorname{tr}=\operatorname{tr}$,

TRUE) $\mathrm{r}=\mathrm{i}, \mathrm{te}=\mathrm{c}(\mathrm{e}), \mathrm{multi}=$ TRUE, overwrite $=$

cr $<-$ raster (list.files (tempdir (), paste0 $\left(" \_"\right.$, i, "_clipped $\backslash \backslash . t i f \$ "$

), $\mathrm{full}$. names=T) )

names (cr) <- paste0 ("gpw_", i, "_", unique (tr), "m")

writeRaster (cr, filename $=$ paste0 ("./data/pop/", names (cr), ".tif") , format = "GTiff", overwrite $=\mathrm{T}$ )

if $(\mathrm{i}==$ "bilinear") \{

writeRaster $(\mathrm{cr}$, filename $="$./data/pop/pop_dens.tif", format $="$ GTiff", overwrite $=$ T) \}

rasterVis:: levelplot $(\mathrm{cr}$, main $=\operatorname{names}(\mathrm{cr}), \operatorname{margin}=\mathrm{F})$

\}) print (paste0 ("Processing ", names(cr)))

$\mathrm{r}<-$ raster ("./data/pop/gpw_near_1000m. tif")

s $<-$ stack (list.files ("./data/pop", "50m\\.tif\$", full.names = T) )

plot (gpw, margin $=F$ ) 


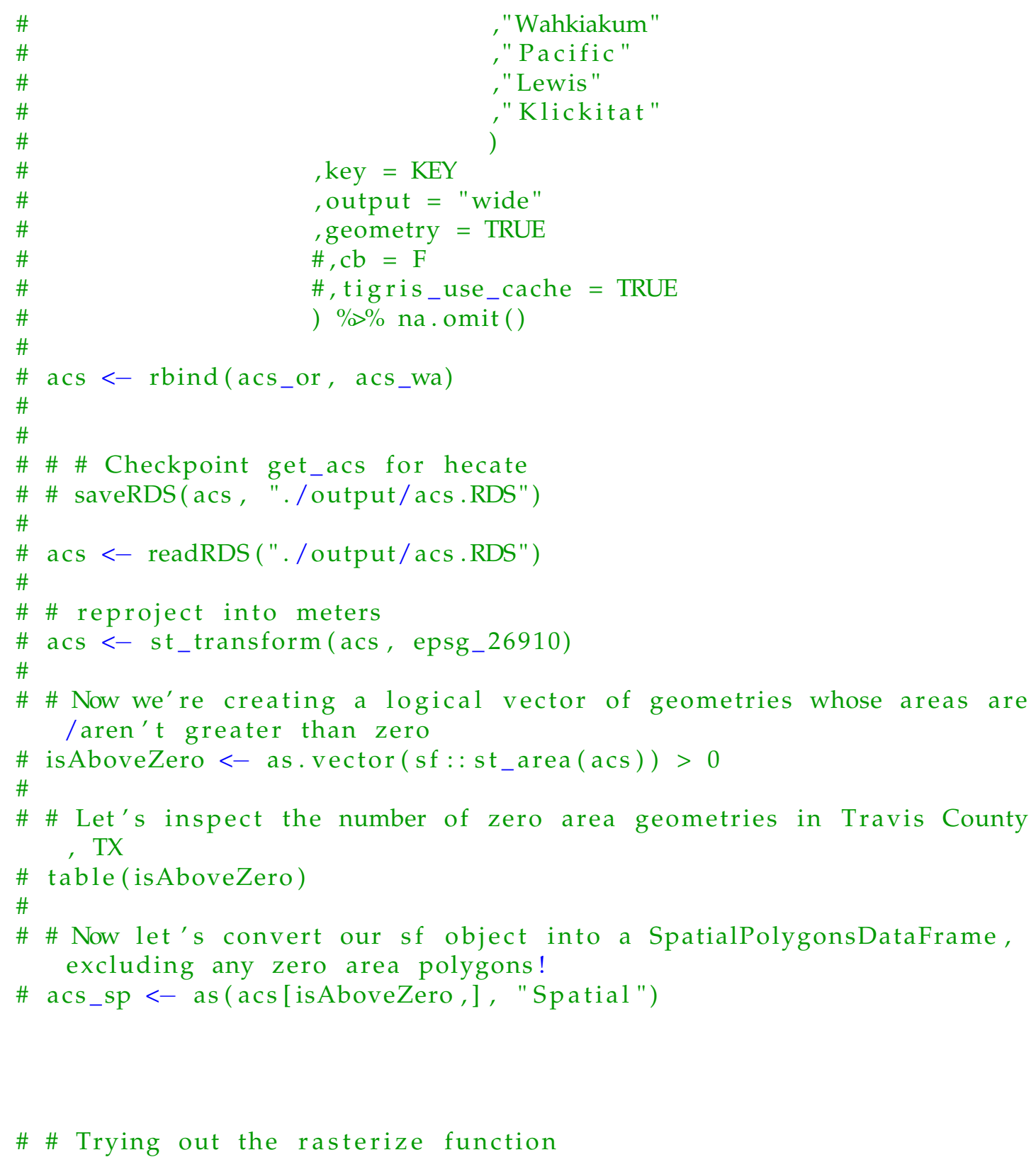

\#

\#

\#

\#

\#

\# , key $=\mathrm{KEY}$

\# ,output = "wide"

\# , geometry = TRUE

\# \#, cb = F

\# \#,tigris_use_cache = TRUE

\# ) $\% \%$ na.omit ()

\# acs $<-$ rbind $($ acs_or, acs_wa)

\#

\#

\# \# \# Checkpoint get_acs for hecate

\# \# saveRDS(acs,"./output/acs.RDS")

\#

\# acs $<-$ readRDS (". / output/acs.RDS")

\#

\# \# reproject into meters

\# acs <- st_transform (acs, epsg_26910)

\#

\# \# Now we're creating a logical vector of geometries whose areas are /aren't greater than zero

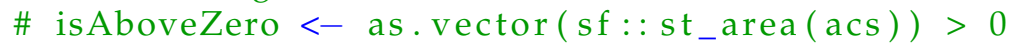

\#

\# \# Let's inspect the number of zero area geometries in Travis County , TX \# table (is AboveZero)

\#

\# \# Now let's convert our sf object into a SpatialPolygonsDataFrame, excluding any zero area polygons!

\# acs_sp <- as (acs [isAboveZero,], "Spatial")

\# \# Trying out the rasterize function

$$
\text { placeholder raster }
$$

\# pop_r <- rasterize $(x=$ acs_sp, y = template_r, field = "B00001_001E

", fun=mean, mask=F, na .rm=T)

\# spplot(acs_sp["B00001_001E"])

\#

\# rasterVis : : levelplot (pop_r, margin=F) 


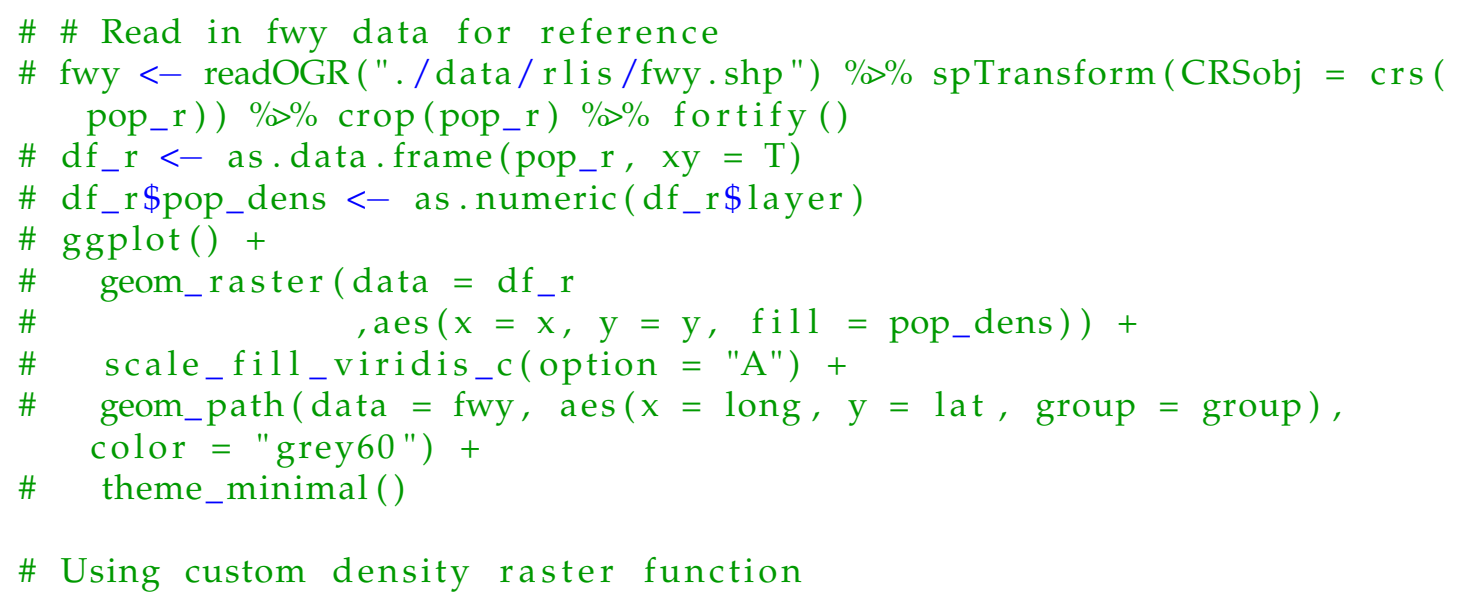




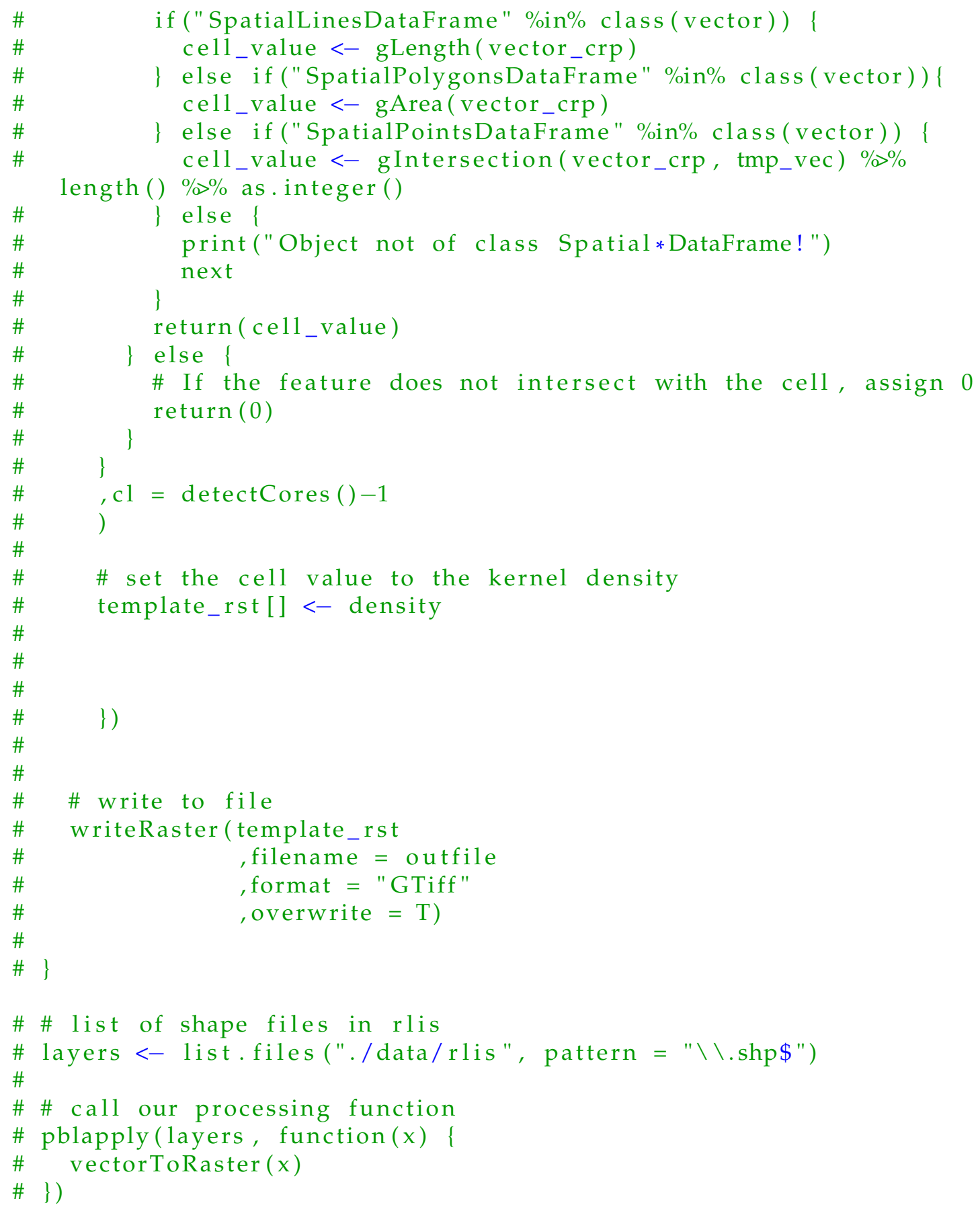




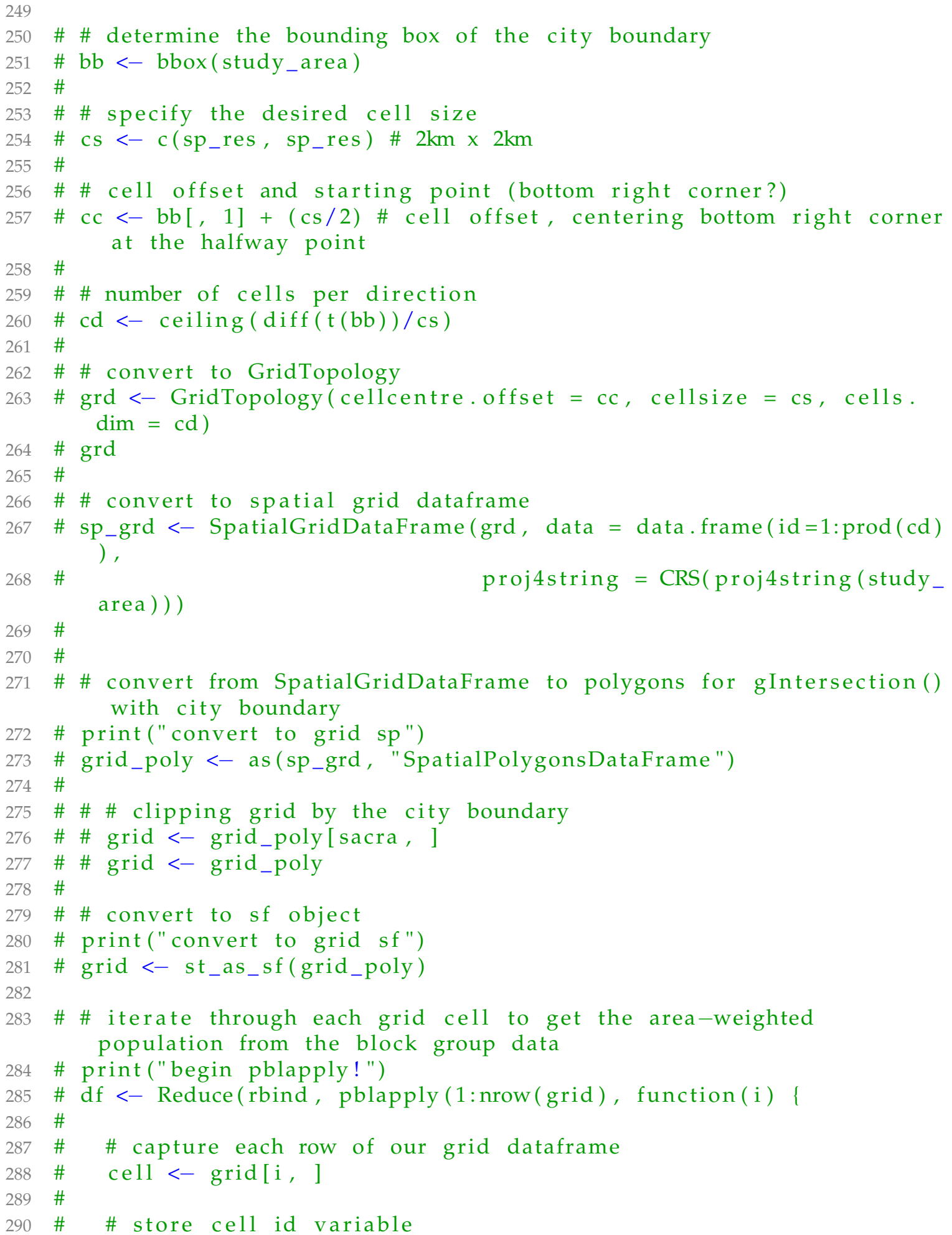




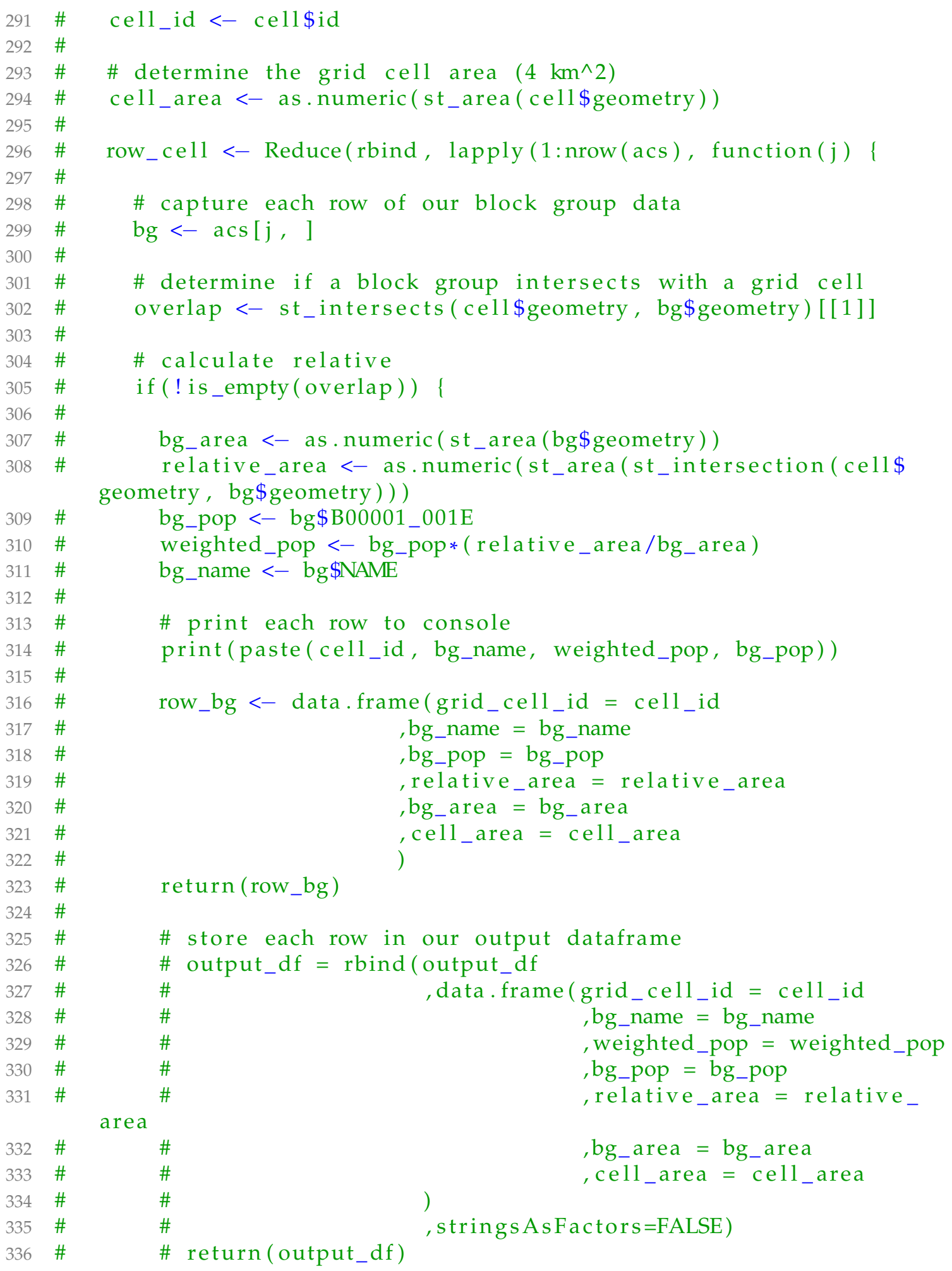




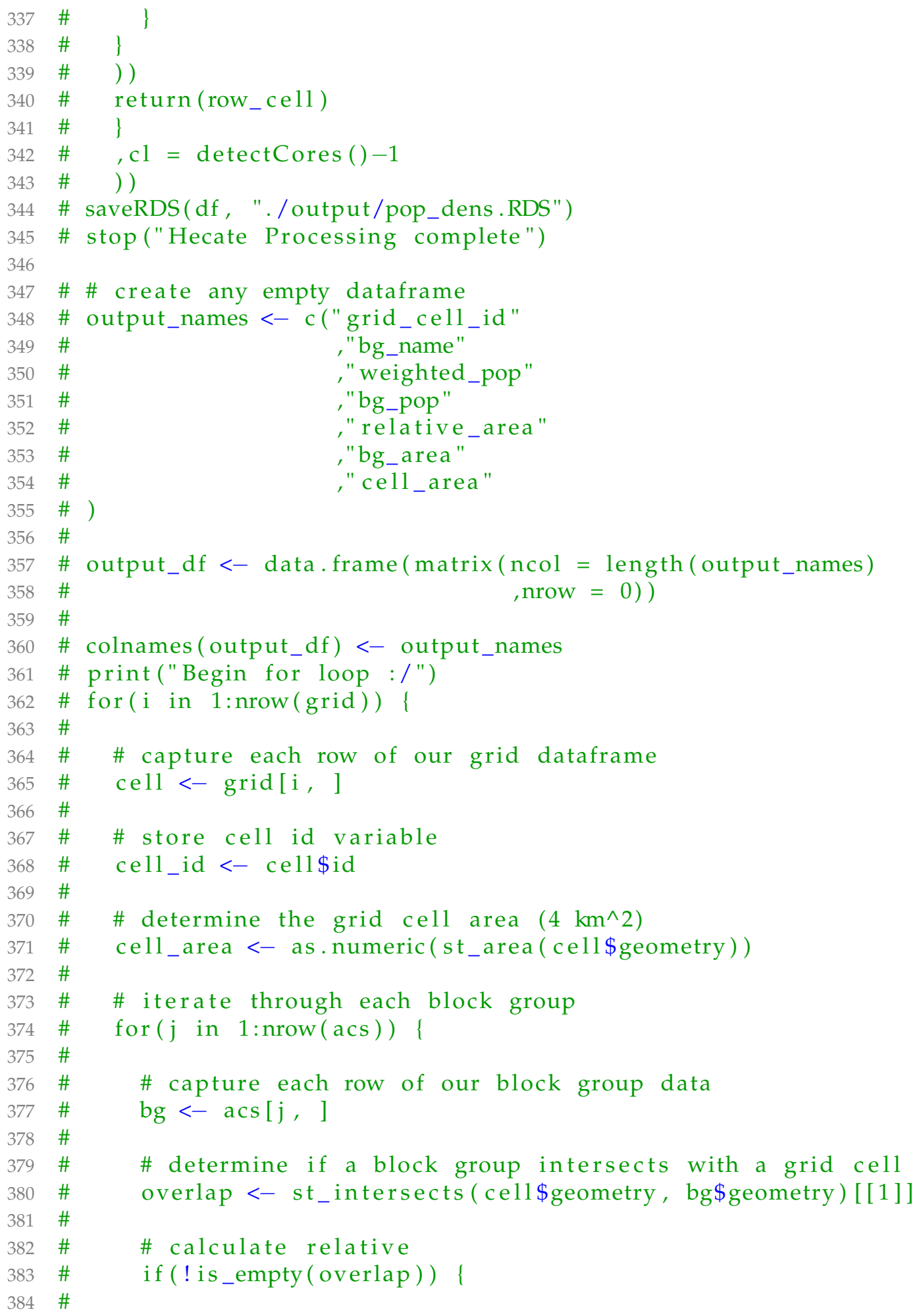




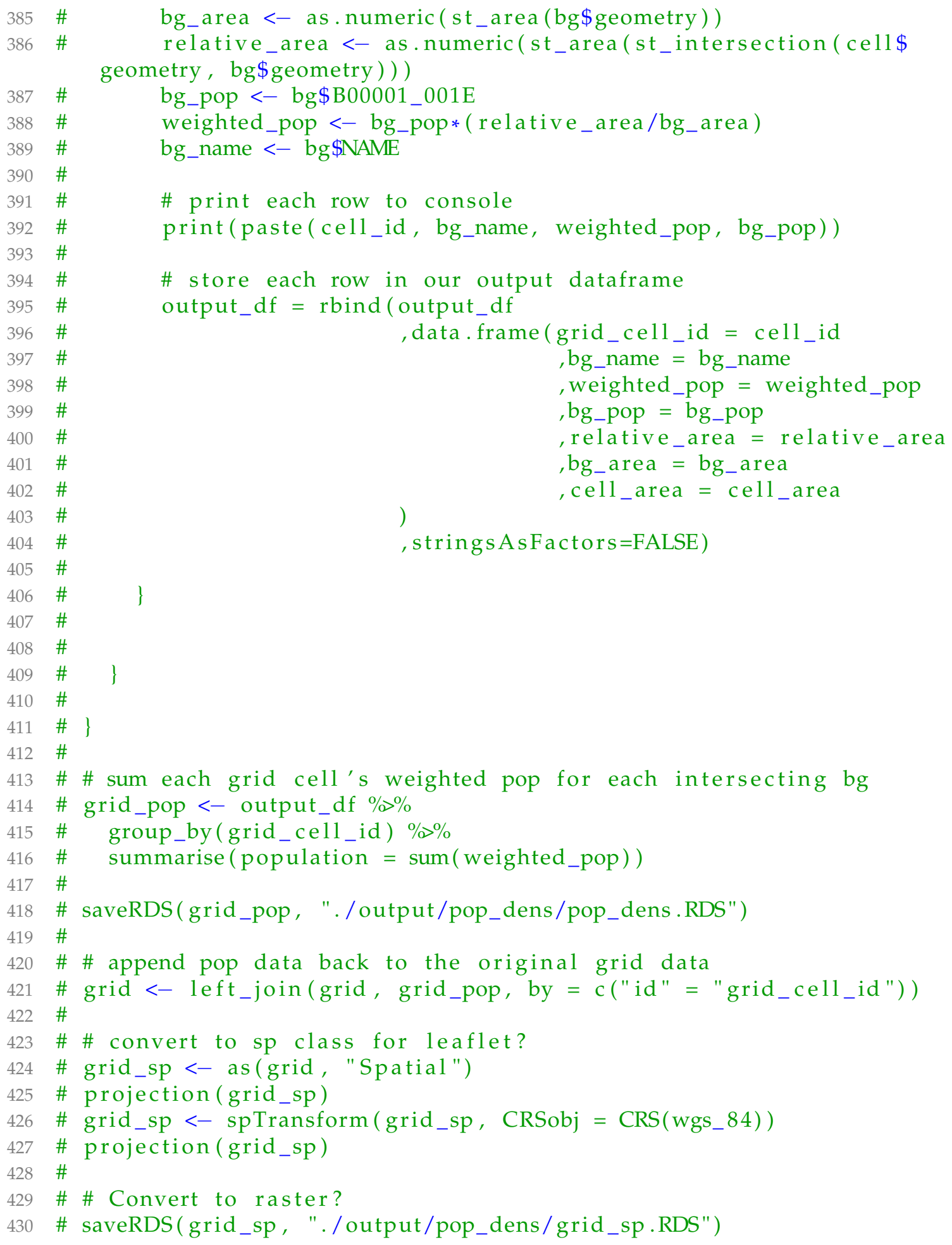




\section{Source/processPBL.R}

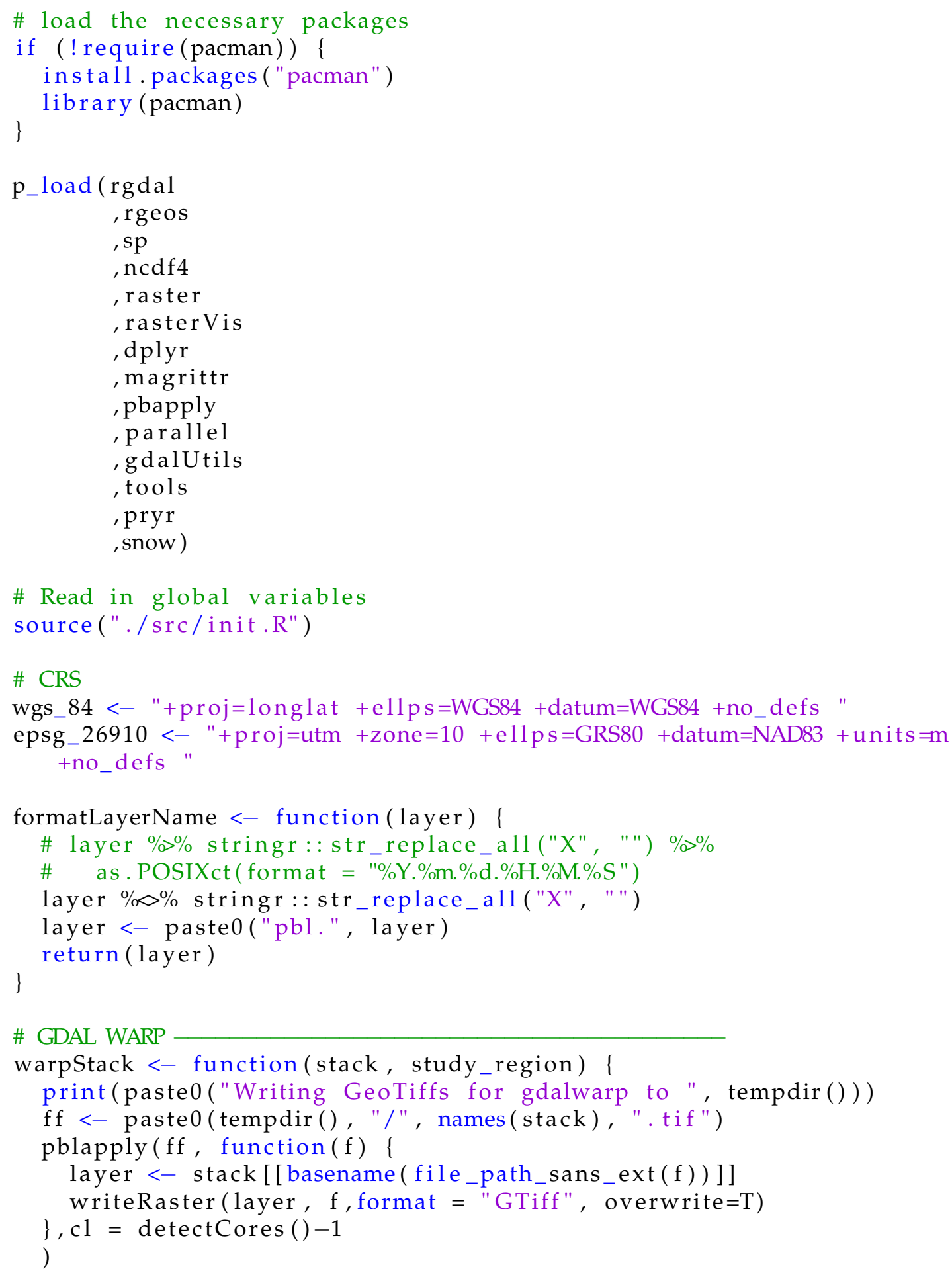




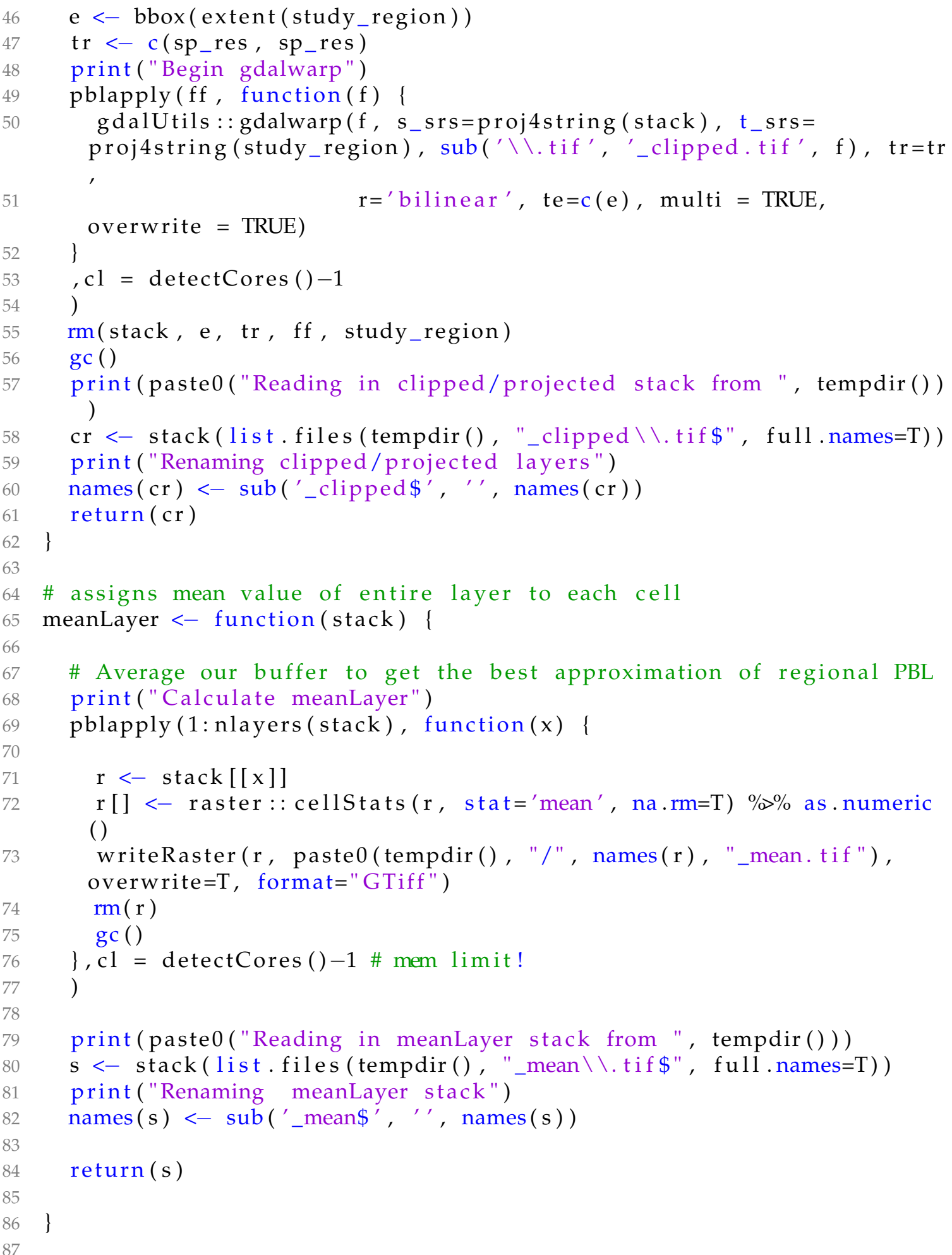




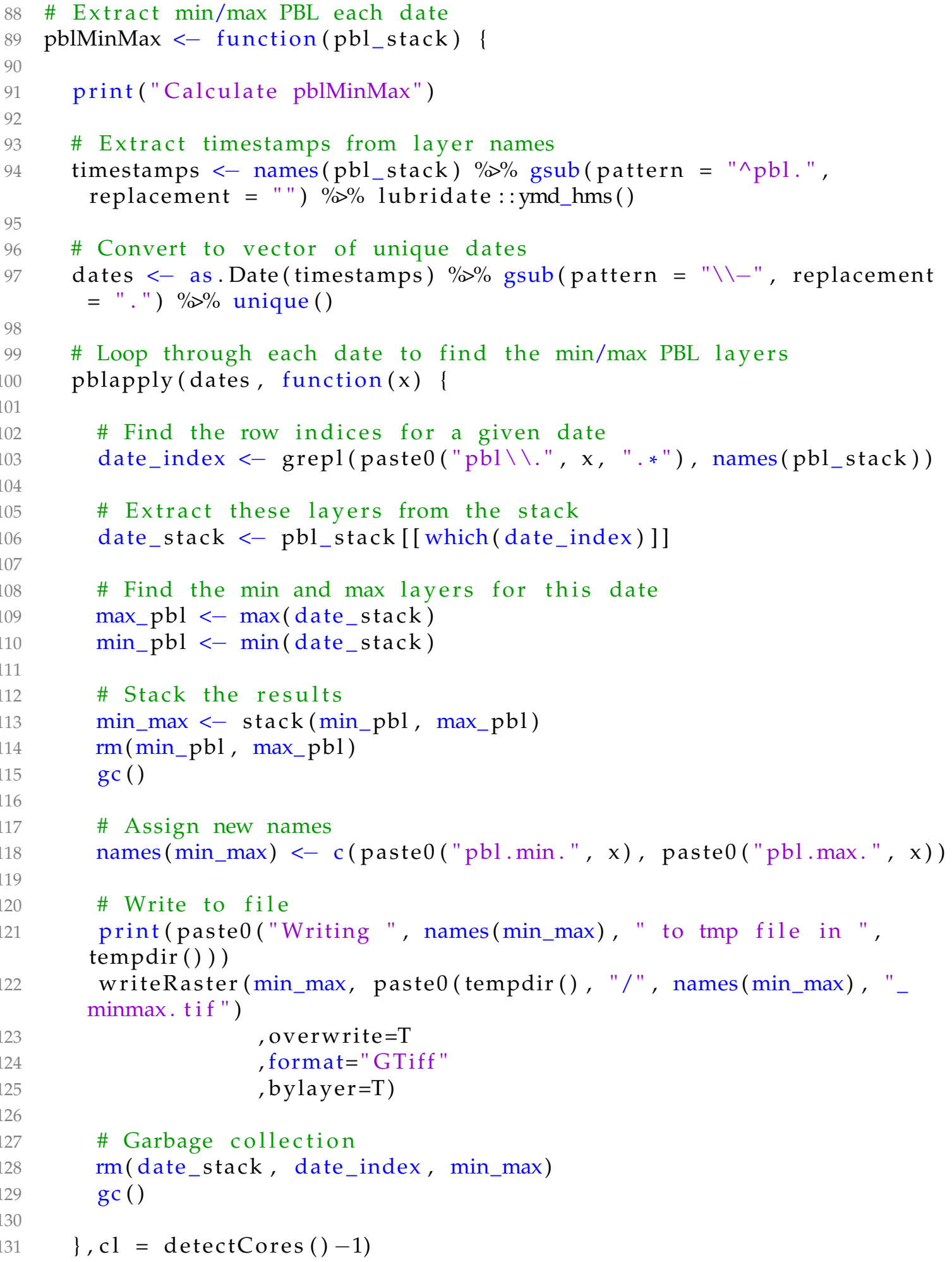




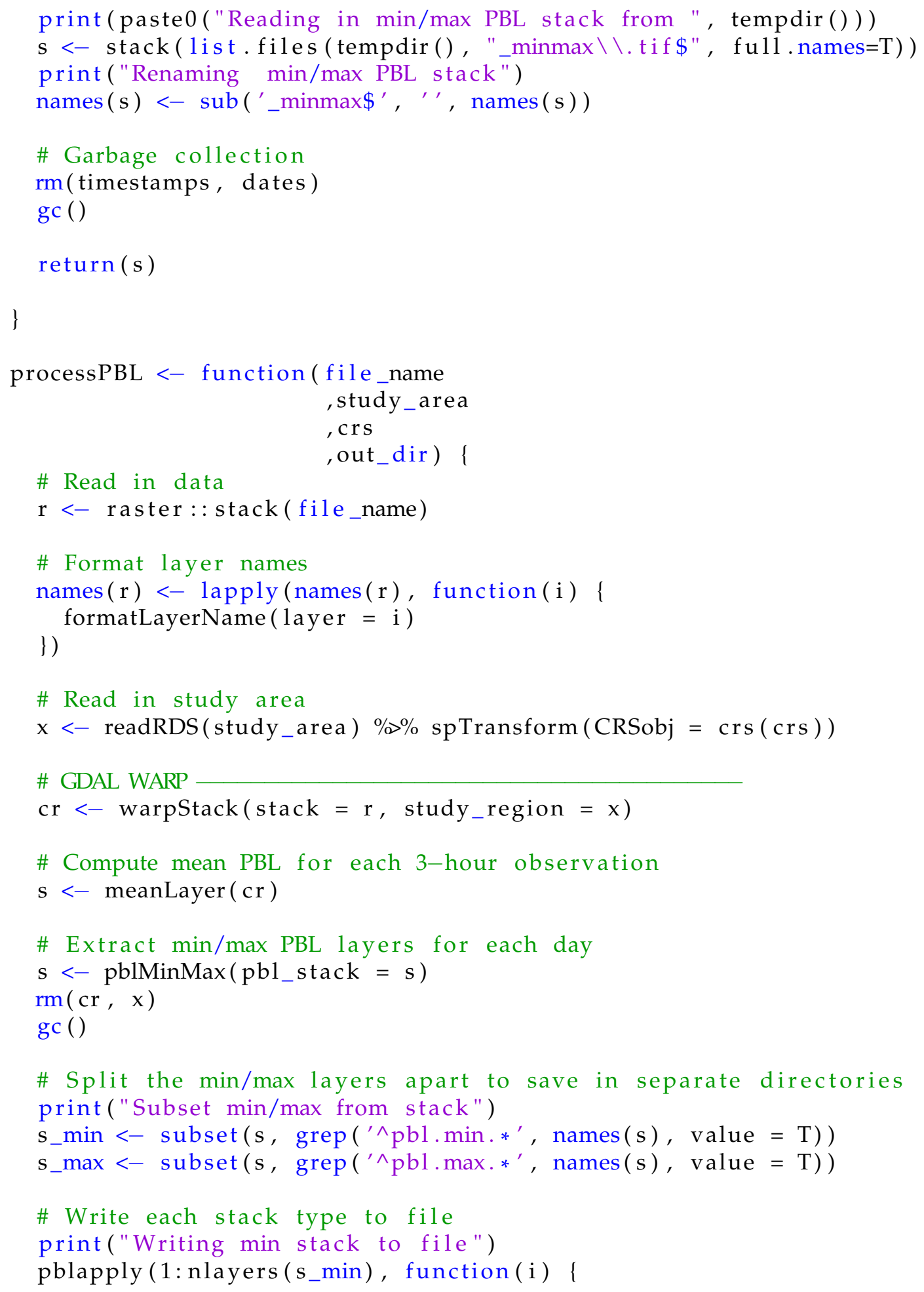


writeRaster(s_min[[i]], file.path(paste0(out_dir, "min/", names(s _min [[i]]),".tif")) ,format = "GTiff" , overwrite $=$ TRUE , bylayer $=\mathrm{T}$

) \}, $\mathrm{cl}=\operatorname{detectCores}()-1$ 
\# $\operatorname{spplot}(\mathrm{a})$

\section{Source/processElevation.R}

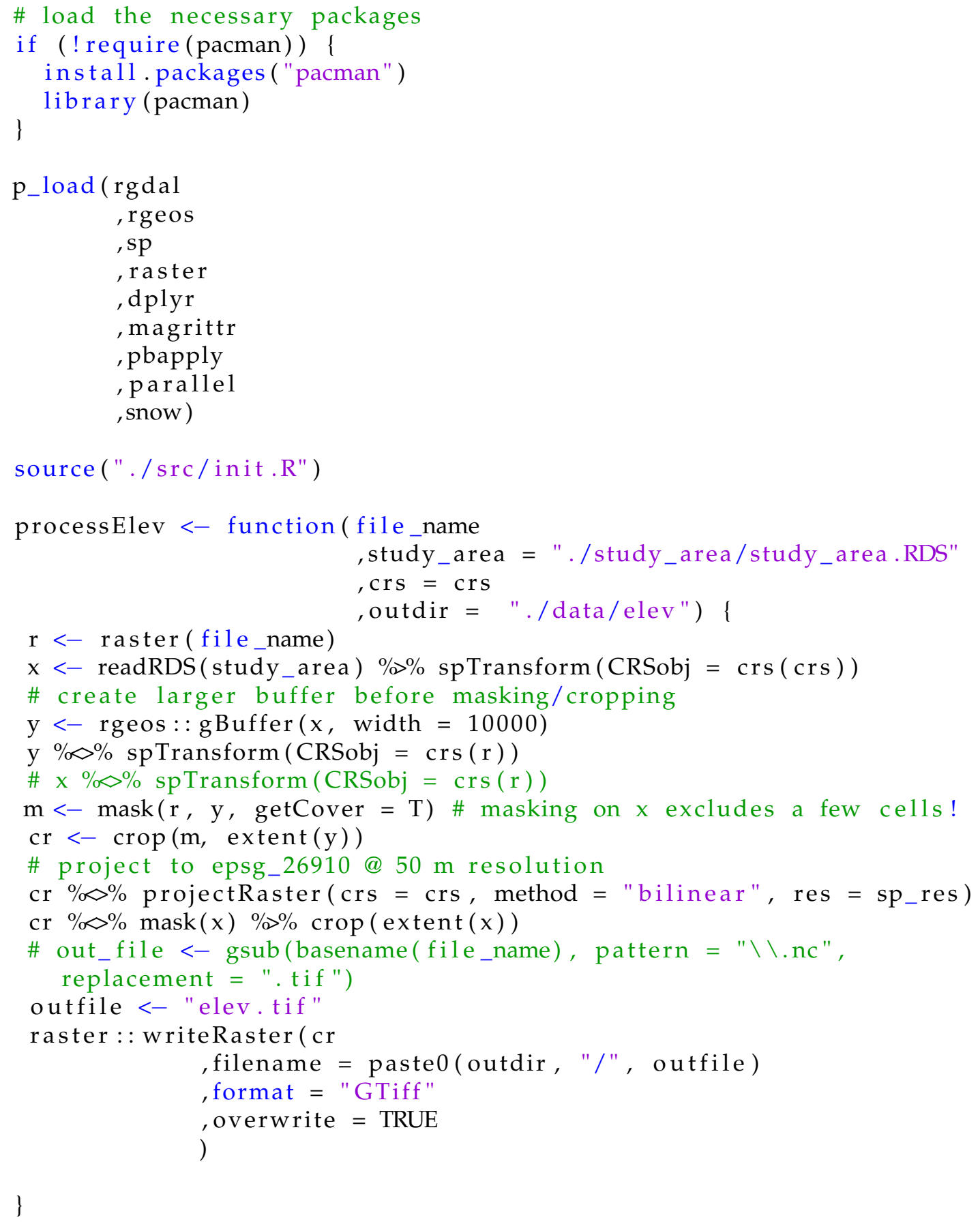


\# I'm using the single elevation layer for each day. It's not going to change that much each

processElev (file_name $=$ "./data/elev/aster_dem_pdx.tif"

, $\mathrm{crs}=\mathrm{crs}$

, outdir $=" . /$ data/elev")

\section{Source/processNeph.R}

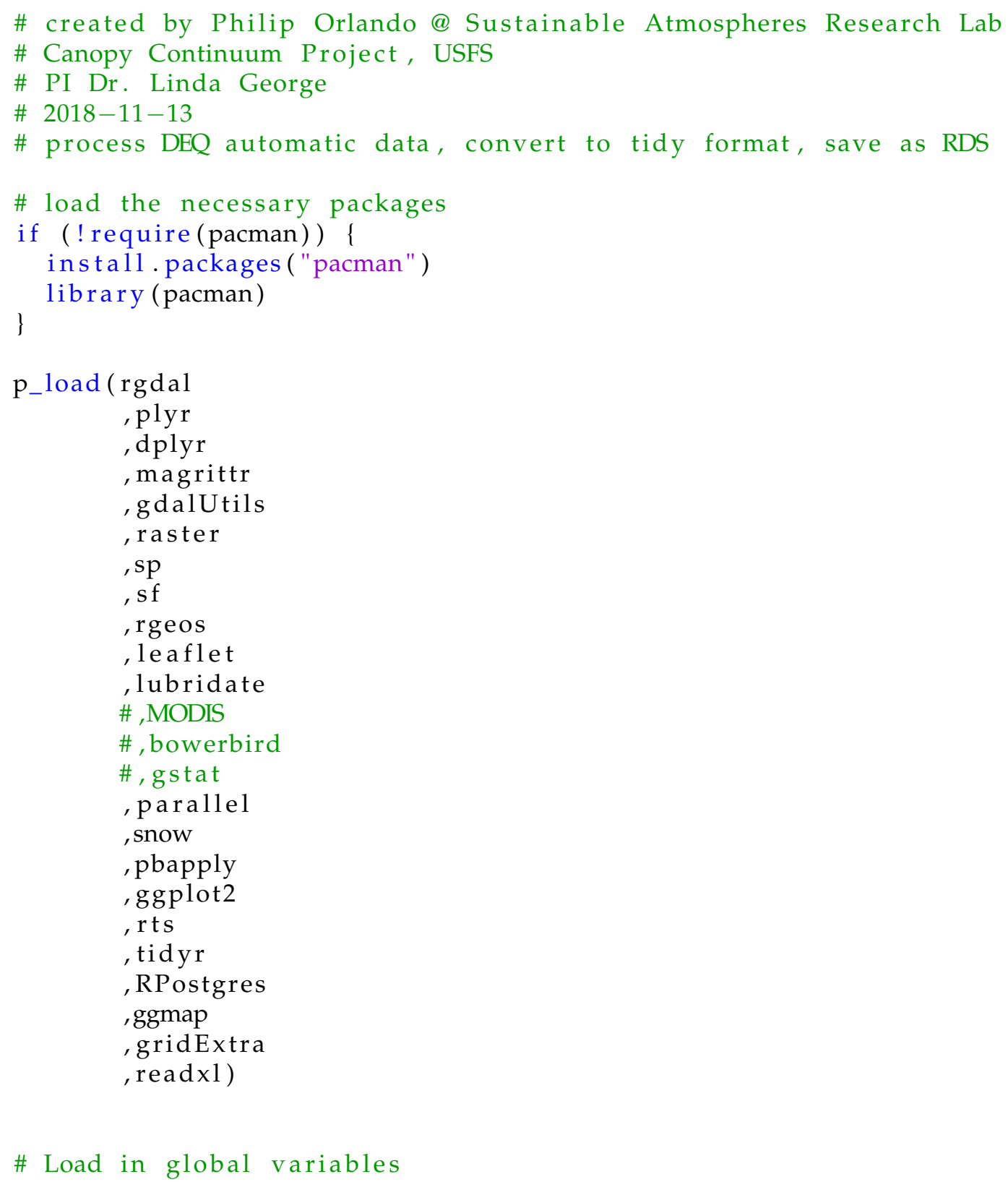




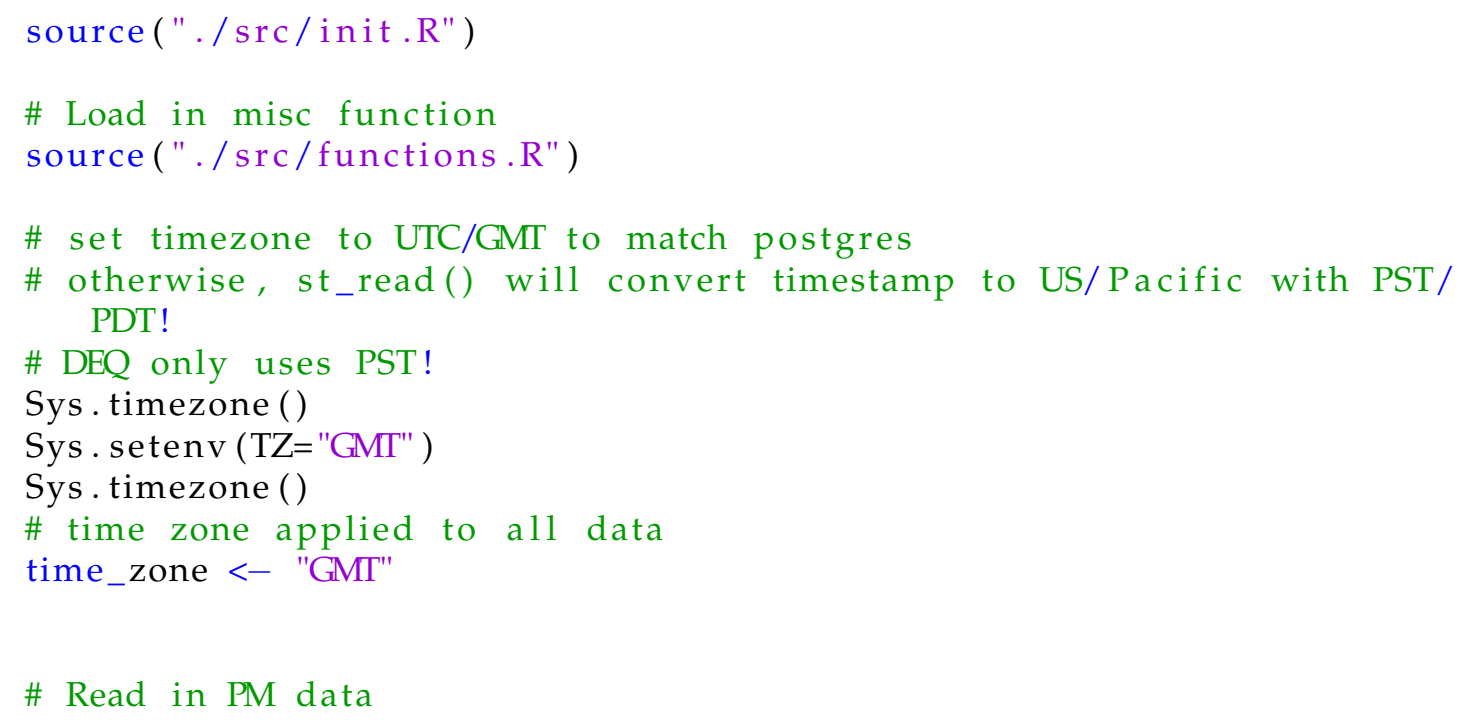




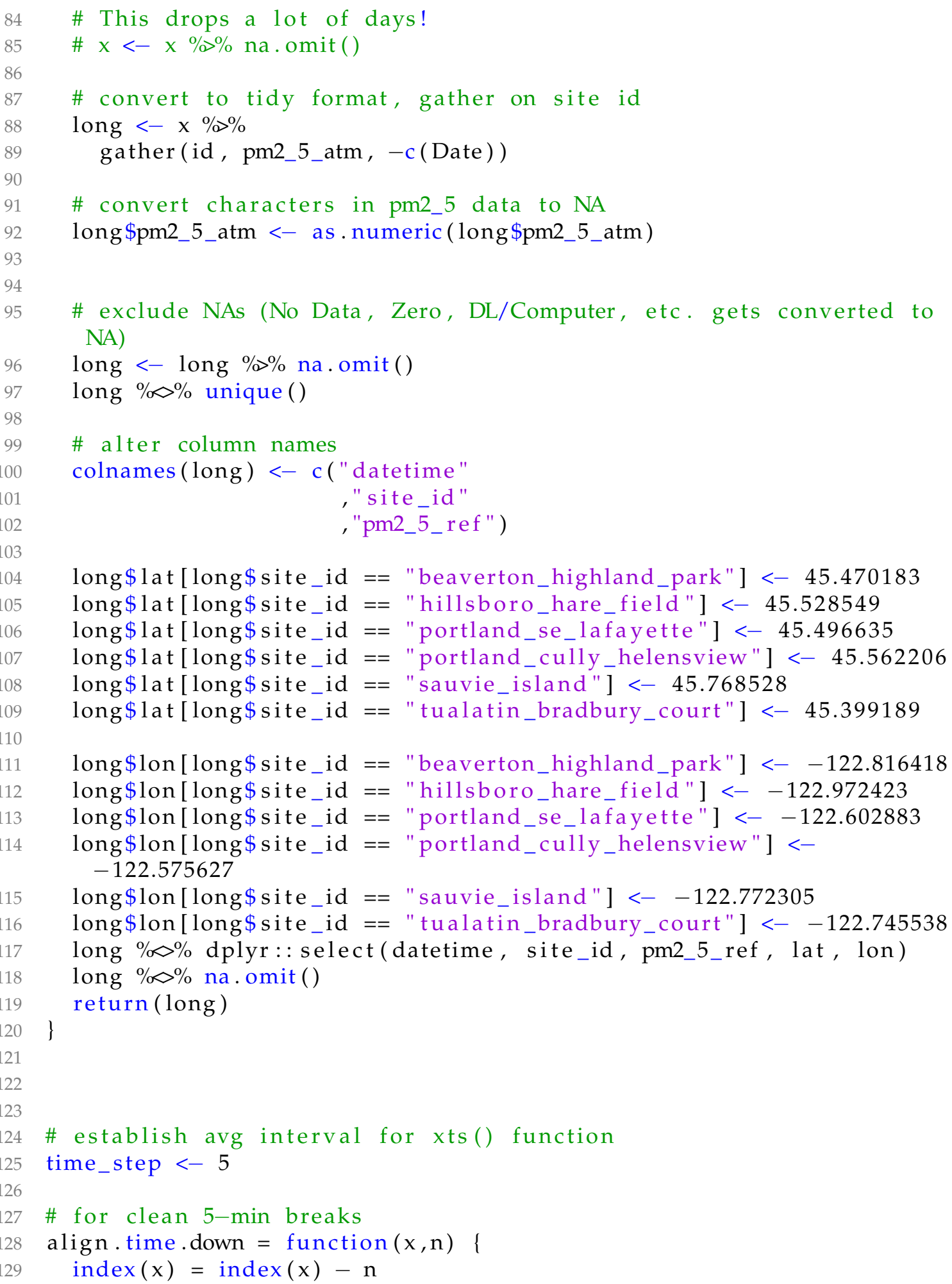




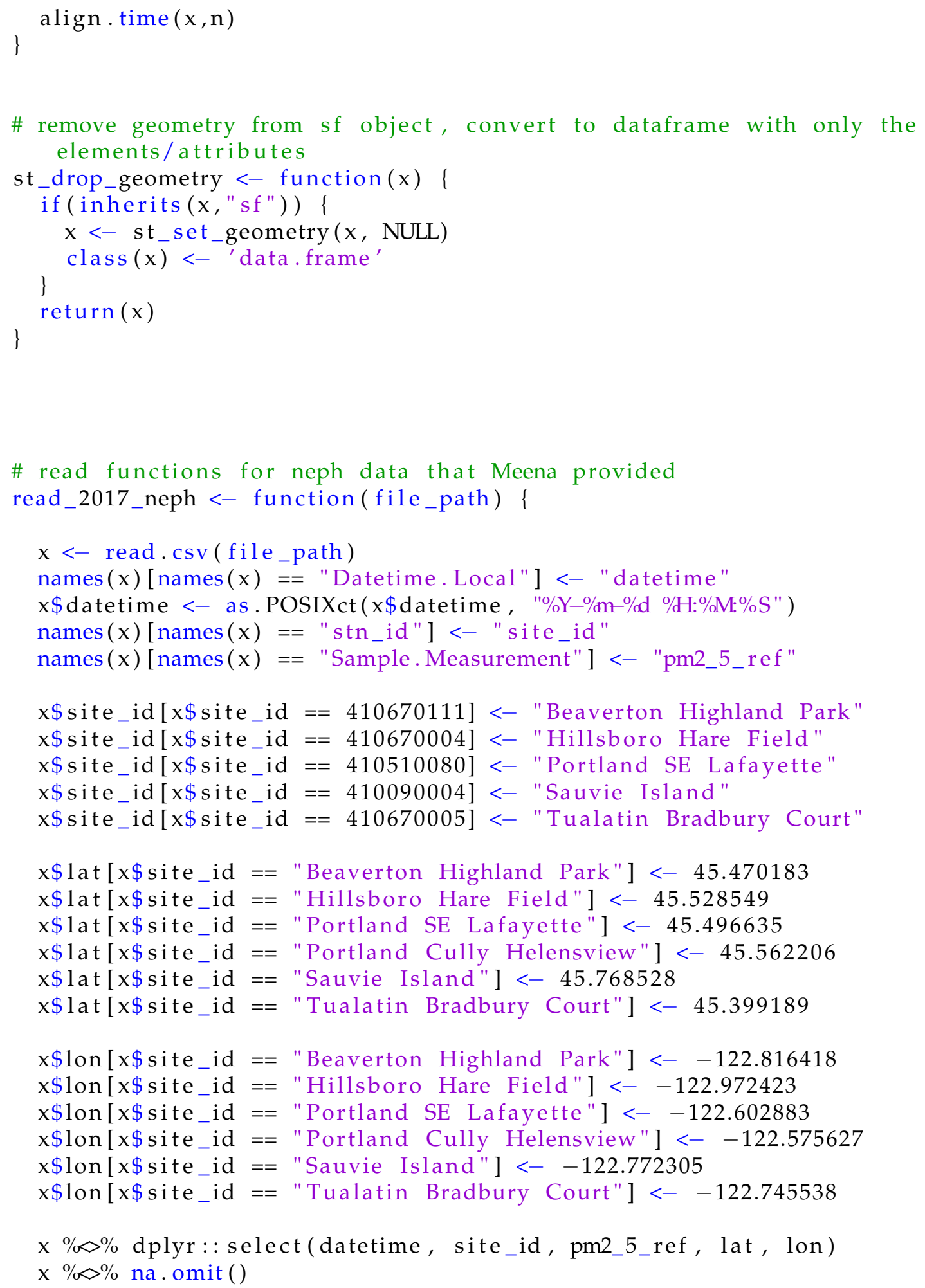




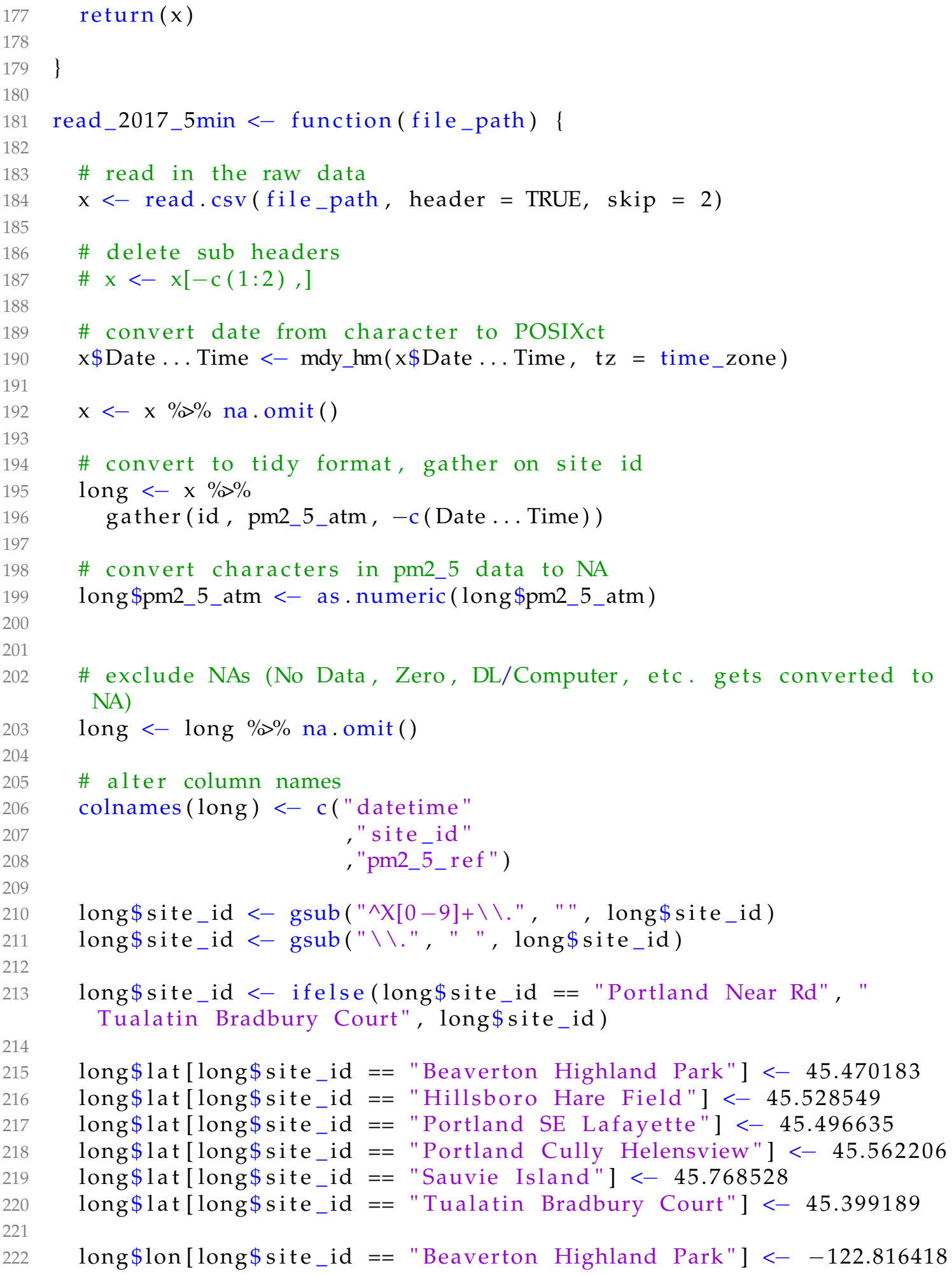




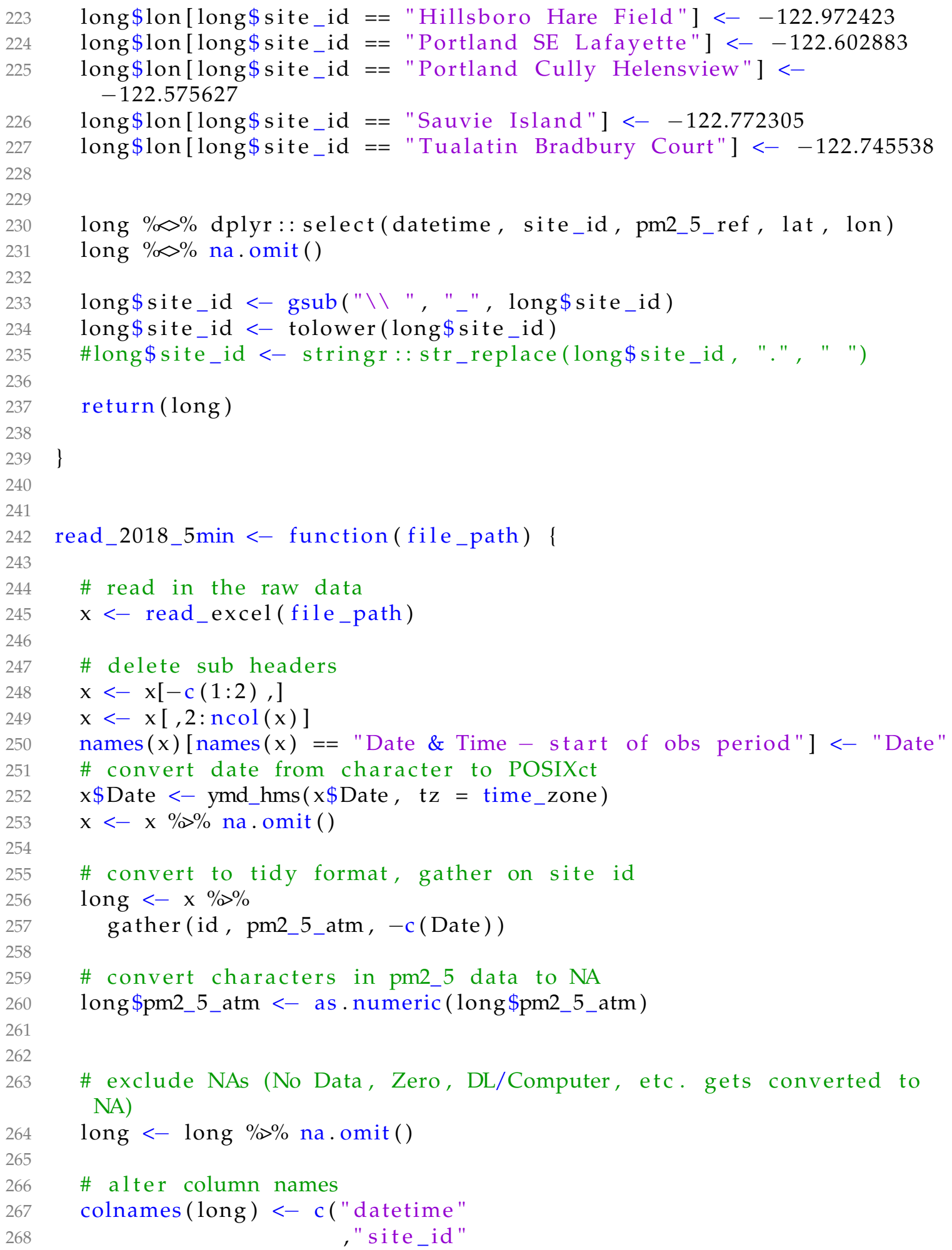




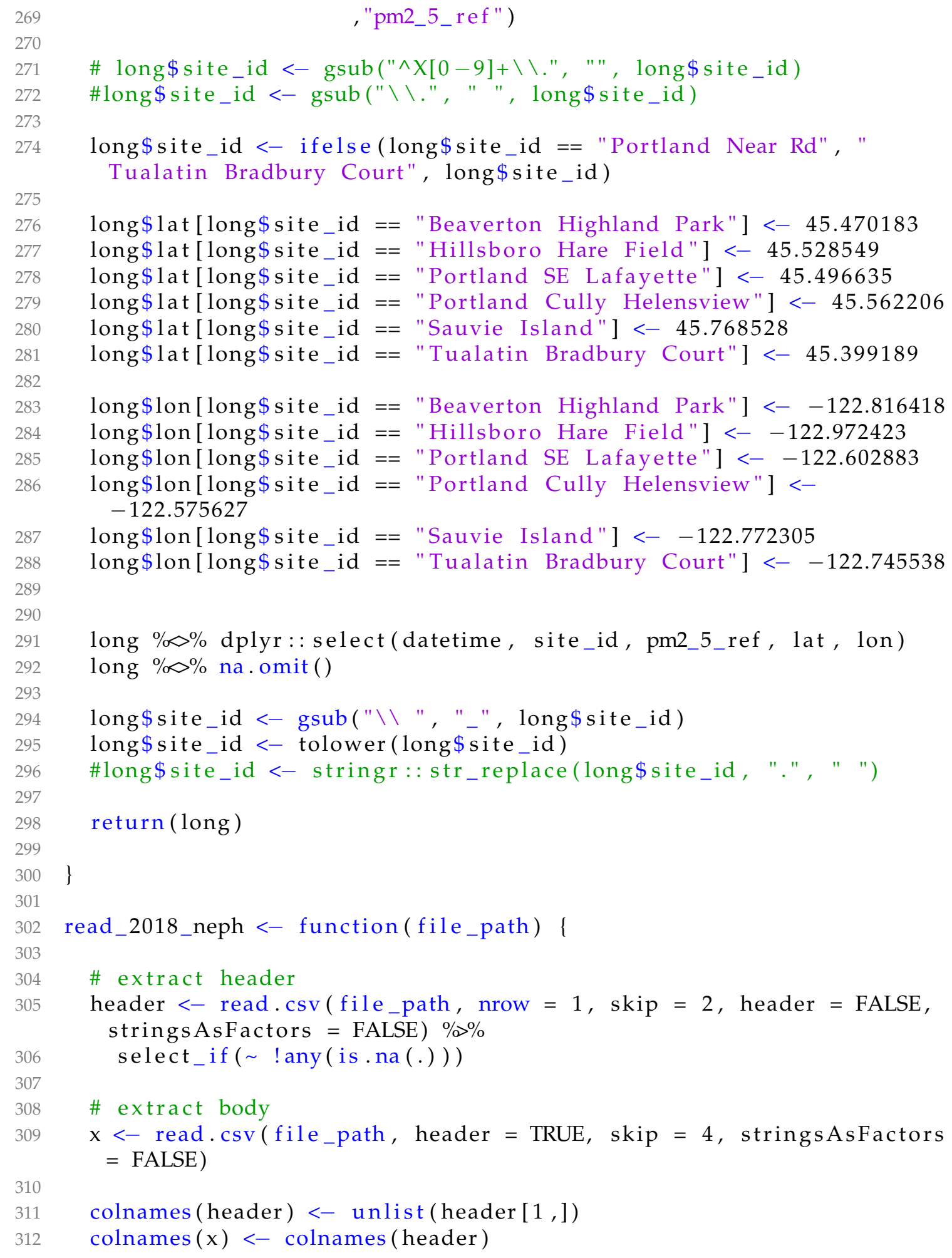




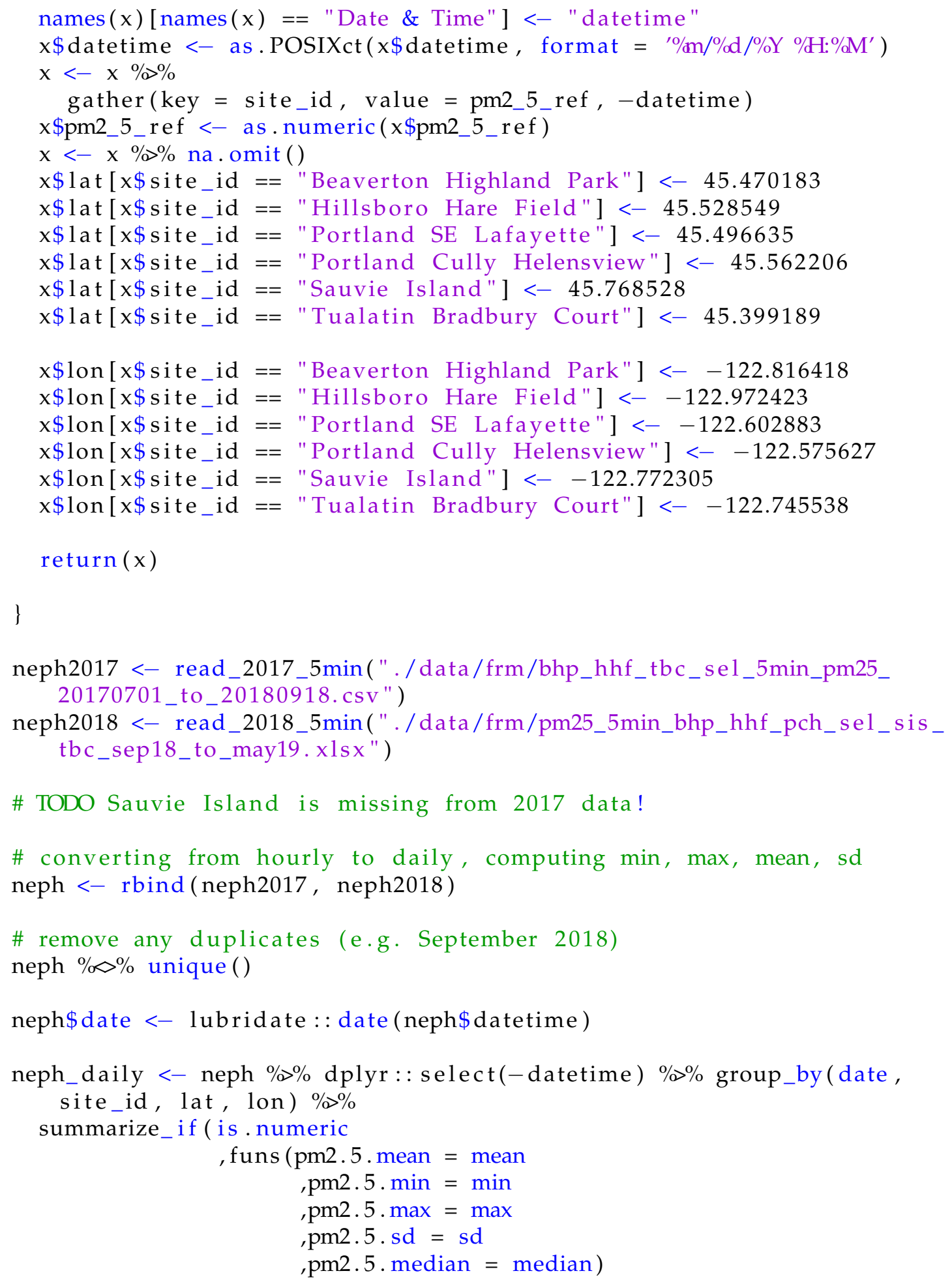




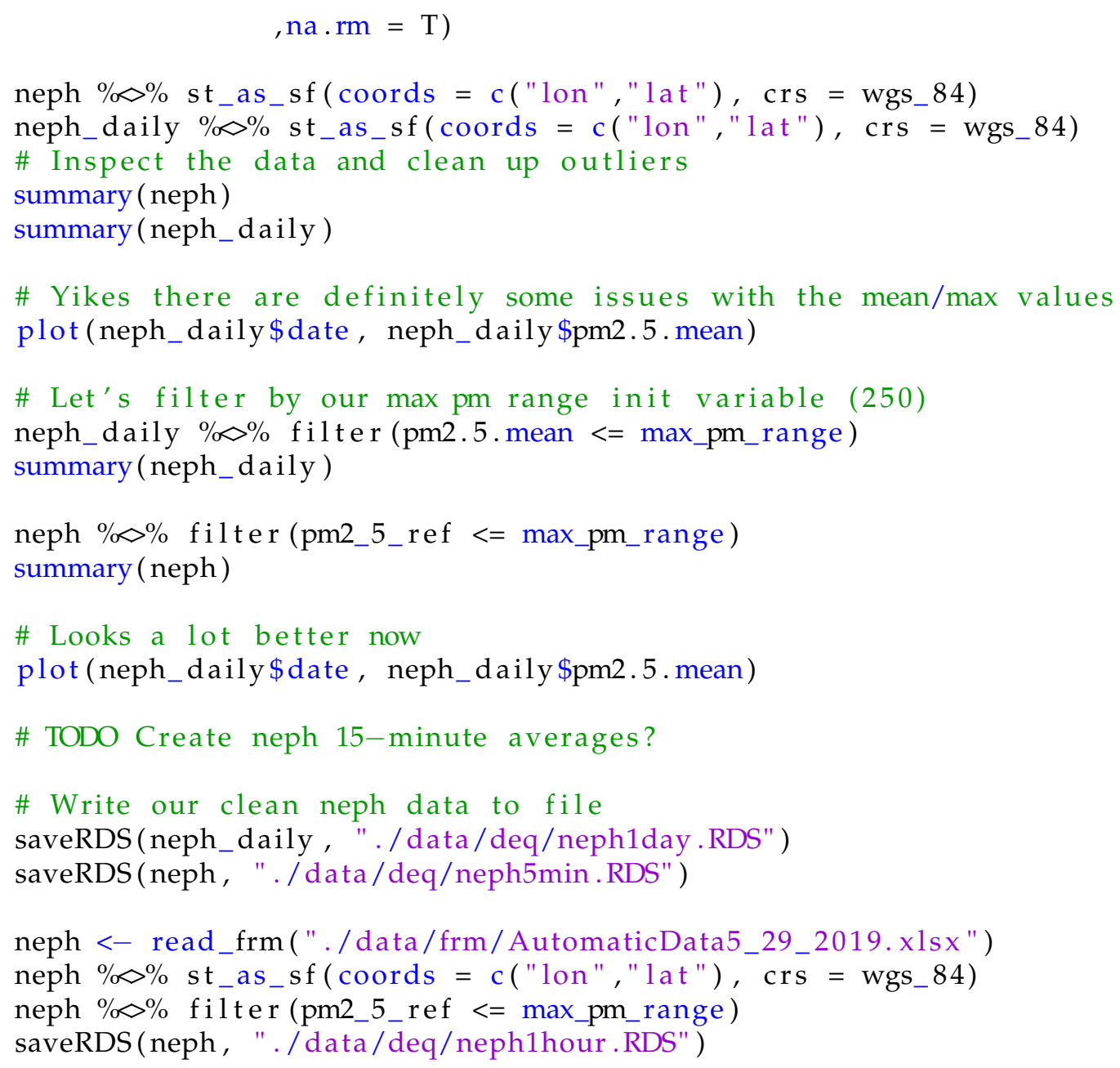

\section{Source/processPurpleAir.R}

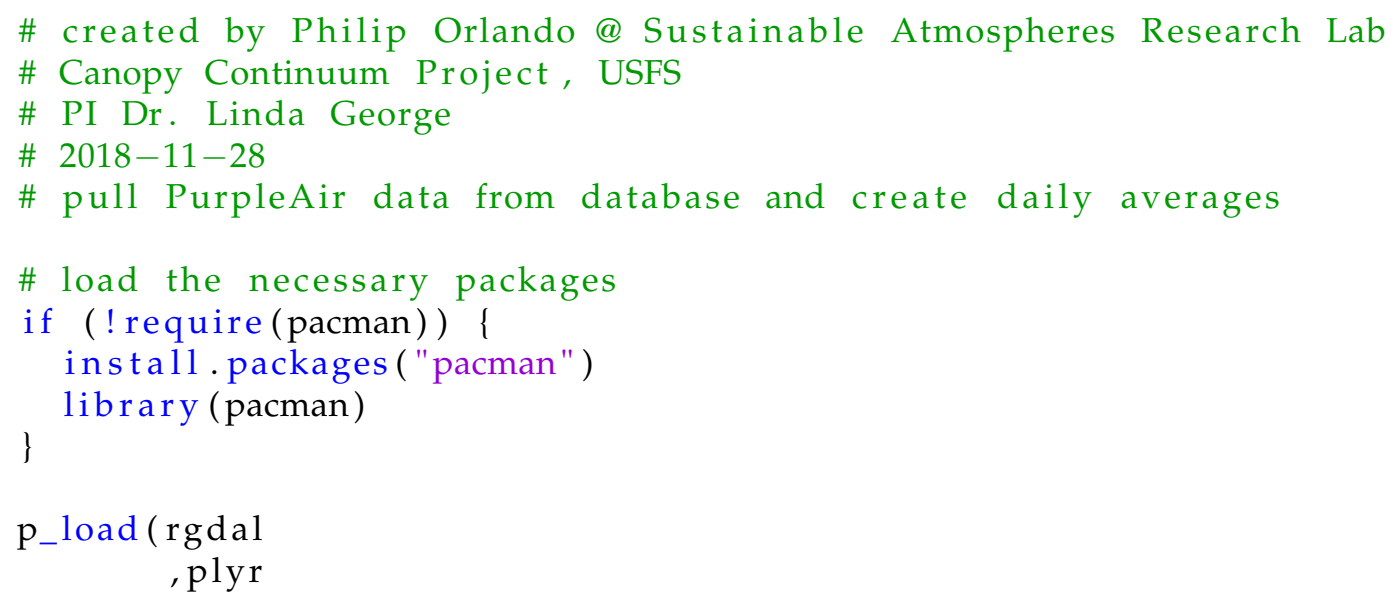




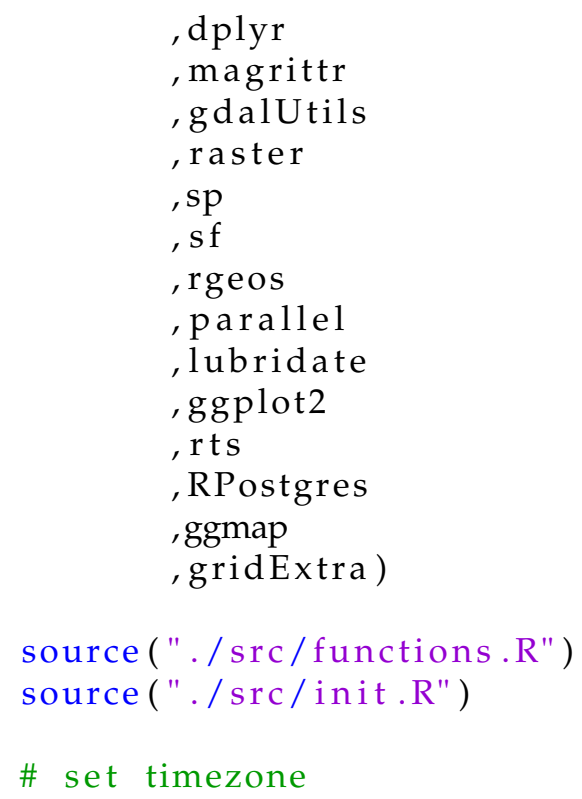


92 


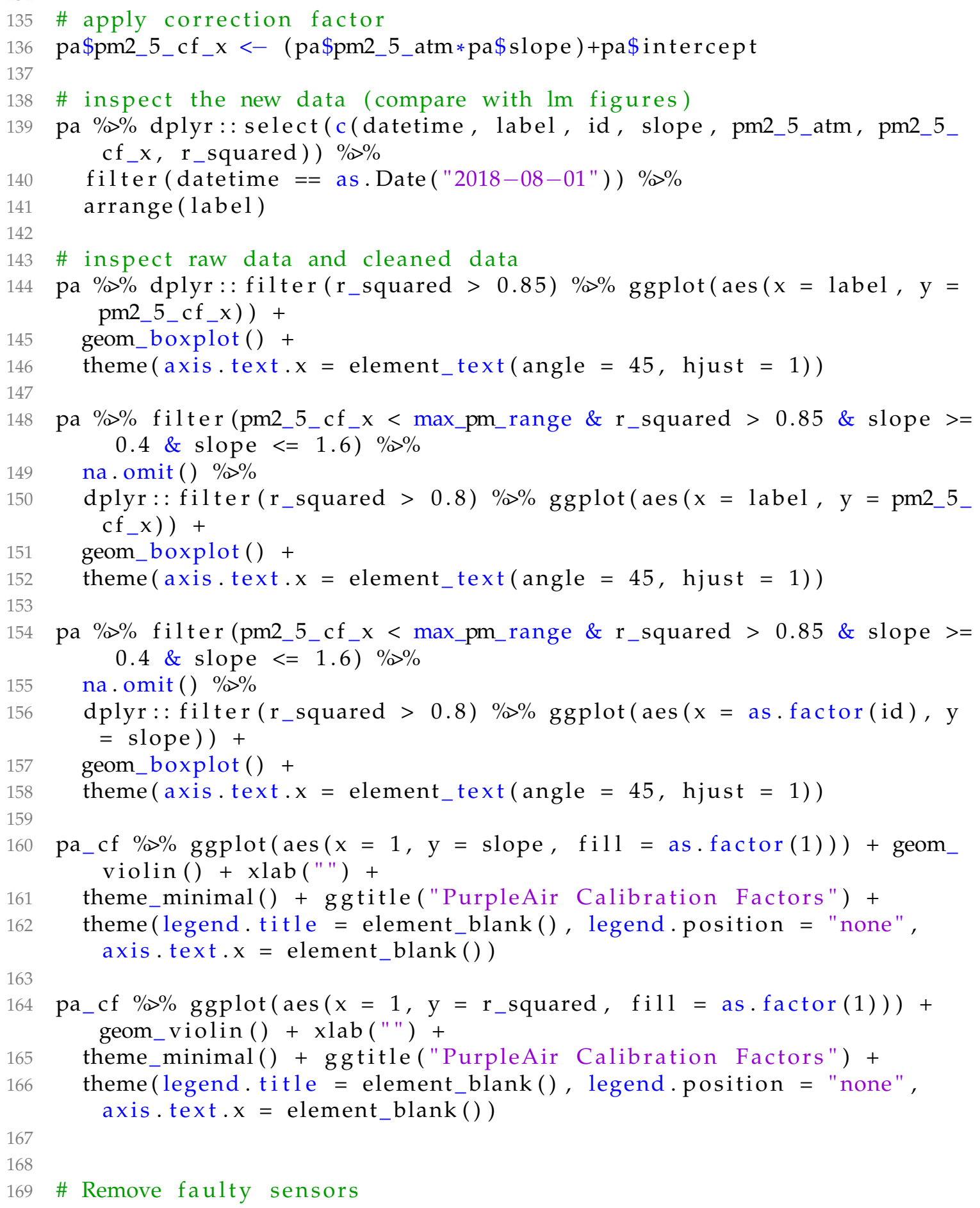




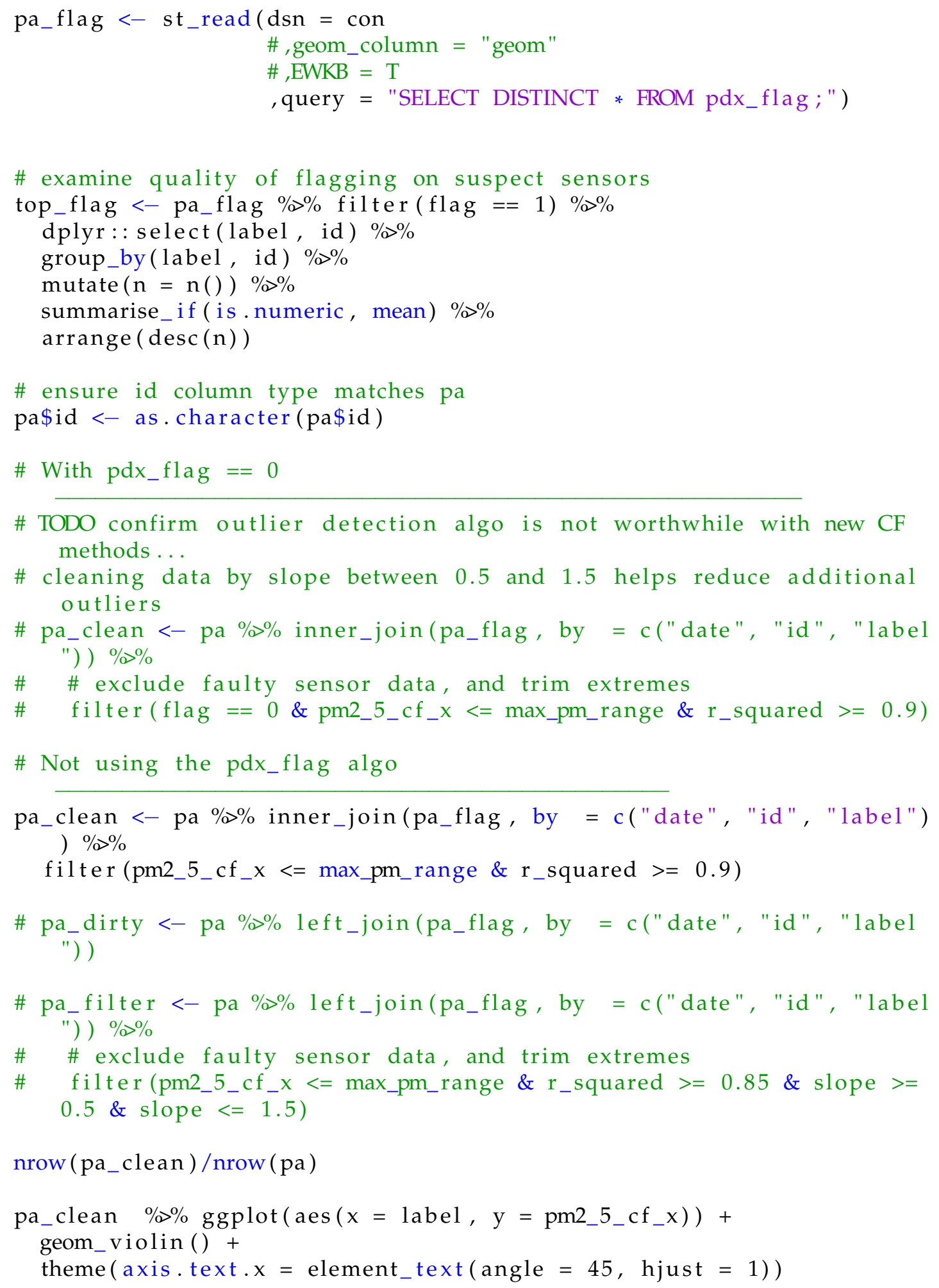




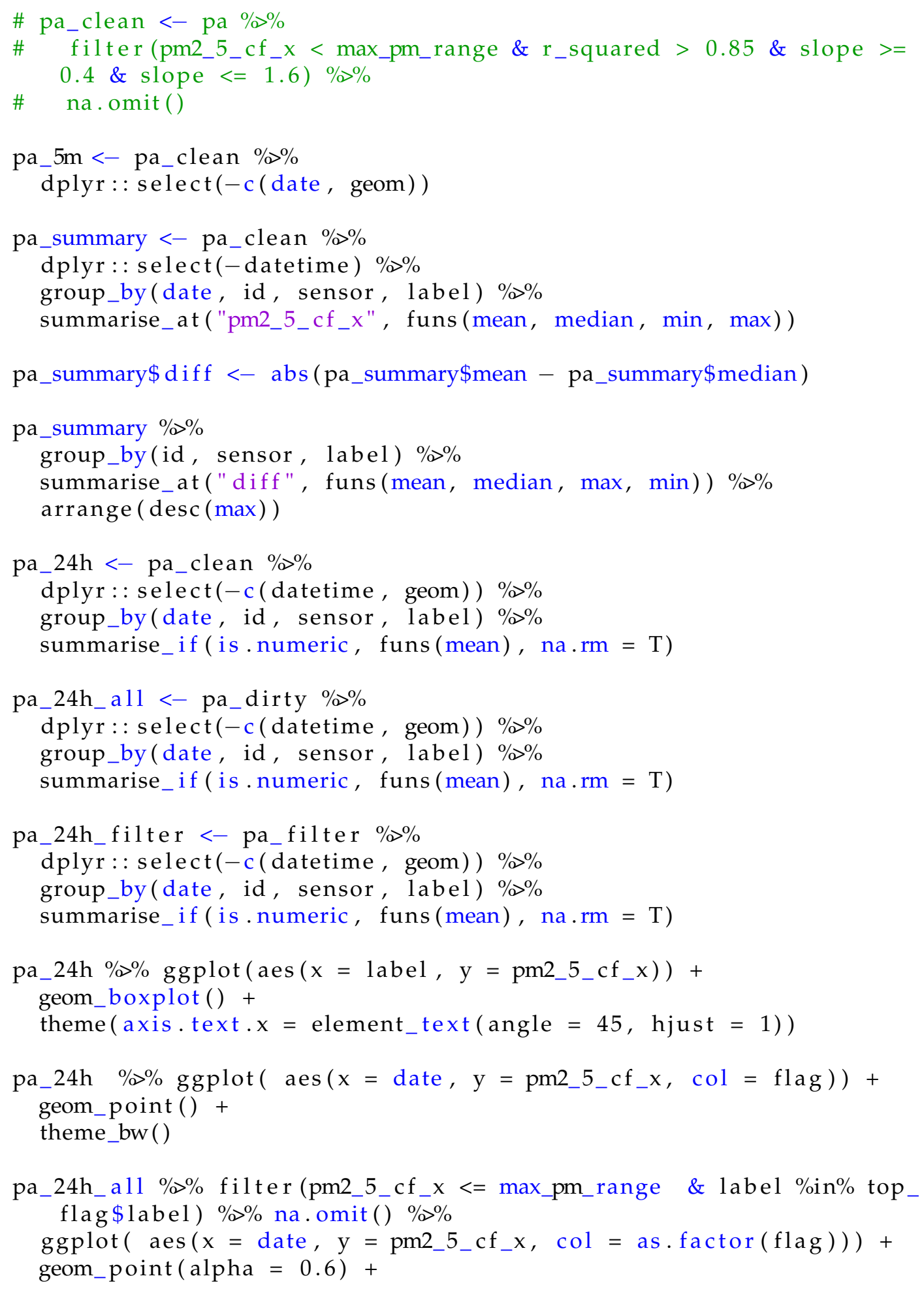


theme_bw () + scale_color_manual $($ values $=c(" \# 000000 ", " \#$ ba2525") $)+$ labs $(y=$ "PM2.5 (ug/m3)", $x=" "$, color $=$ "Outlier Flag", title = " Modified Z-Score Outlier Detection") + theme (legend . position="bottom")

pa_24h_al1 \%\% filter (date $>=$ "2018-01-01" \& date $<=" 2018-03-01 ")$ $\% \%$

filter (pm2_5_cf_x < = max_pm_range \& label \%in\% top_flag $\$$ label $) \% \%$ na.omit () $\% \%$

$\operatorname{ggplot}(\operatorname{aes}(\mathrm{x}=$ date, $y=$ pm2_5_cf_x, col $=$ as.factor $($ flag $)))+$ geom_point $($ alpha $=0.6)+$

theme_bw() + scale_color_manual $($ values $=c(" \# 000000 ", " \#$ ba2525") $)+$ labs $(y=$ PM2.5 (ug/m3)", $x=" ", \operatorname{color}=$ "Outlier Flag", title = " Modified Z-Score Outlier Detection") + theme (legend . position="bottom")

ggsave( last_plot() , filename = paste0 $(" . /$ figures/outlier_detection $/ "$, print_clean ) _time (), "-Jan-Mar-z-score-outlier-ts .png")

pa_24h_all \%\% filter (date $>=$ "2018-07-01" \& date $<=" 2018-09-01 ")$ $\% \%$

filter (pm2_5_cf_x <= max_pm_range \& label \%in\% top_flag $\$$ label $) \% \%$ na.omit () $\% \%$

$\operatorname{ggplot}(\operatorname{aes}(\mathrm{x}=$ date, $\mathrm{y}=$ pm2_5_cf_x, col = as.factor $($ flag $)))+$ geom_point $($ alpha $=0.6)+$

theme_bw ()$+$ scale_color_manual(values $=c(" \# 000000 ", " \#$ ba2525") $)+$

labs $(y=$ "PM2.5 (ug/m3)", $x=" ", \operatorname{color}=$ "Outlier Flag", title = " Modified Z-Score Outlier Detection") +

theme (legend . position="bottom" )

ggsave (last_plot () ,filename = paste0 $(" . /$ figures/outlier_detection $/ "$, print_clean _time (), "-Jul-Sep-z-score-outlier-ts .png")

)

pa_24h_filter $\% \%$ na.omit ()$\% \% \operatorname{ggplot}(\operatorname{aes}(\mathrm{x}=$ date, $y=$ pm2_5_cf_x, $\mathrm{col}=$ as.factor $(\mathrm{flag})))+$

geom_point $($ alpha $=0.25)+$

theme_bw () + scale_color_manual(values = c("\#000000","\#ba2525")) + labs $(y=$ "PM2.5 (ug/m3)", $\mathrm{x}="$ ", color = "Outlier Flag", title = " Modified Z-Score Outlier Detection") + 


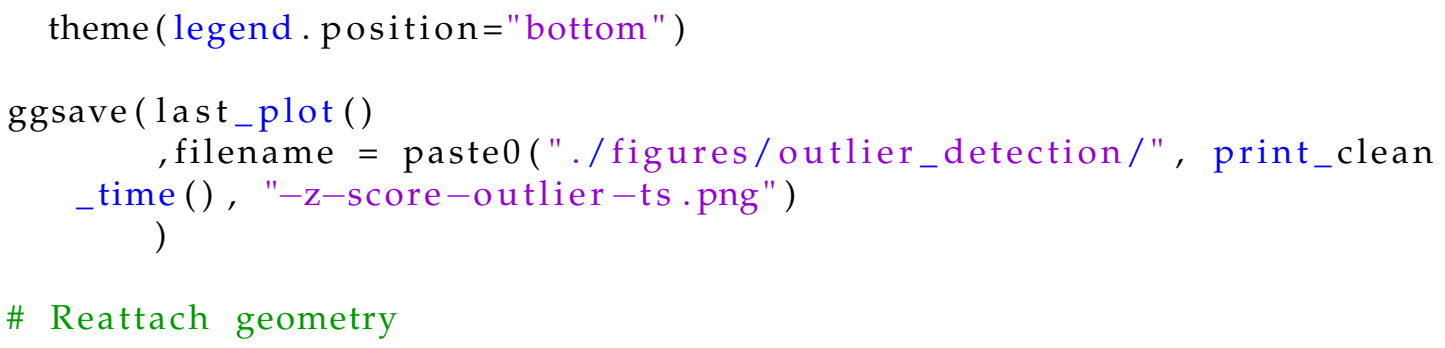




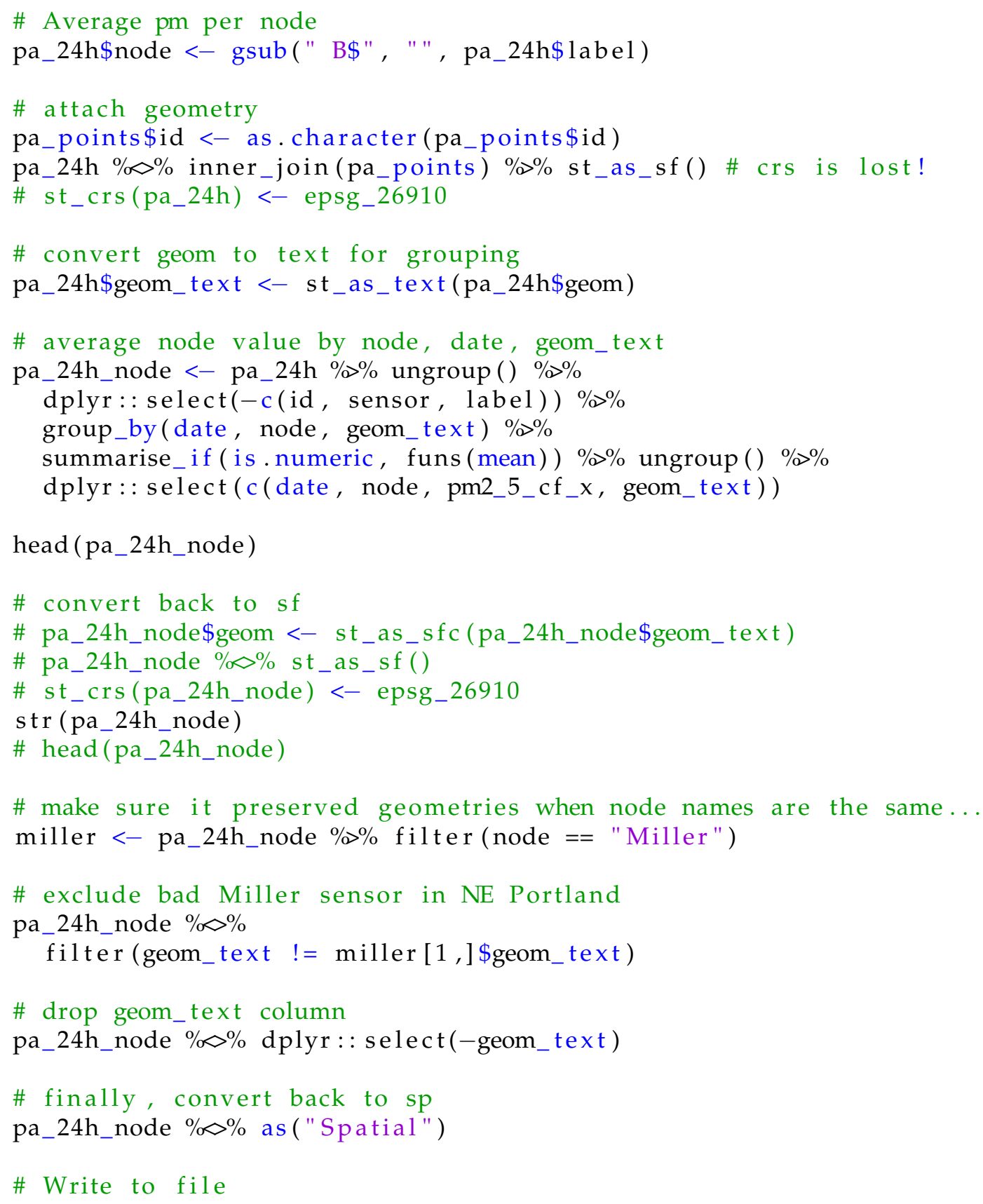


\# writeOGR(pa_24h, "./data/purpleair/", layer = "pm_df_24h", driver = "ESRI Shapefile", overwrite = T)

\section{Source/buffer.R}

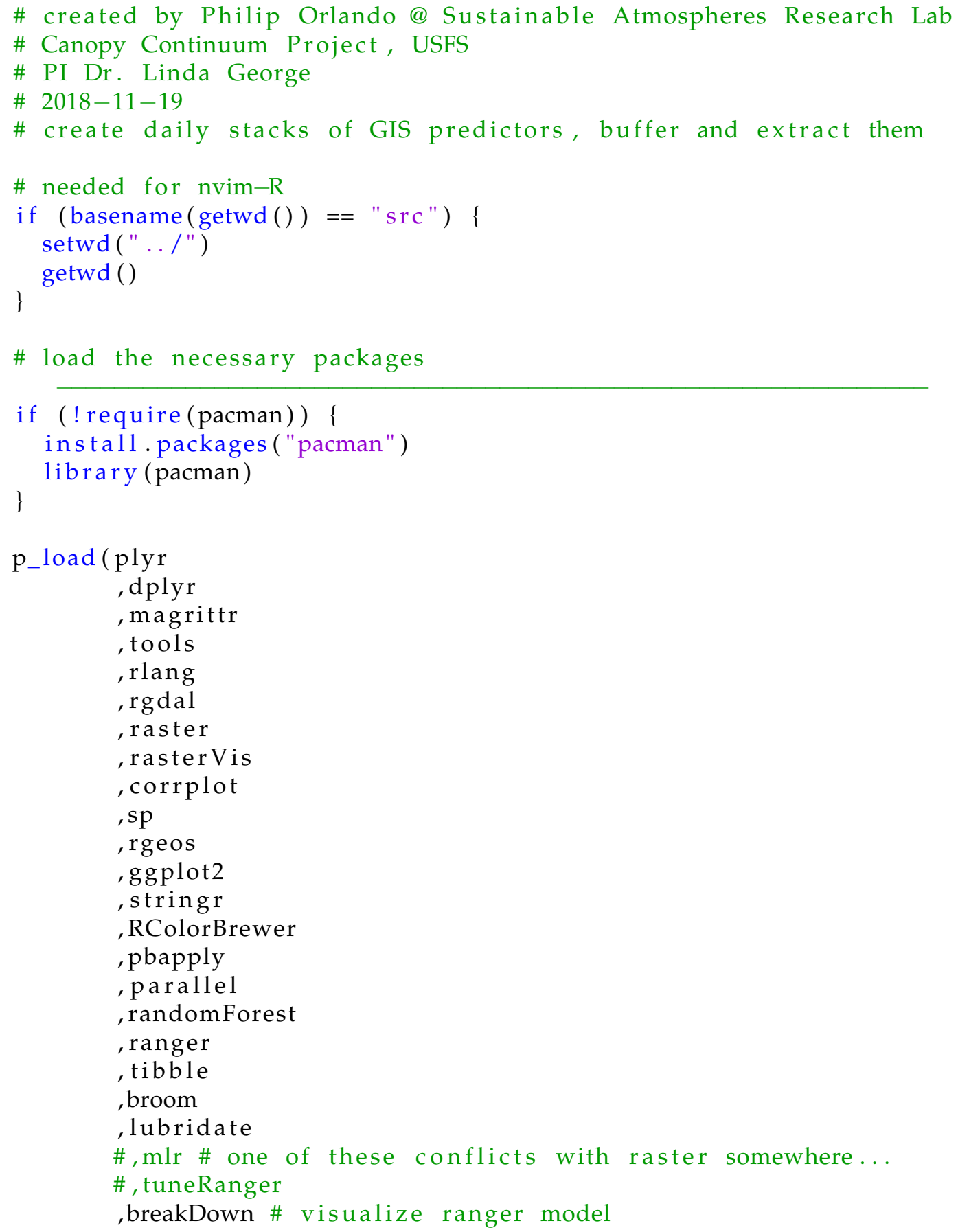




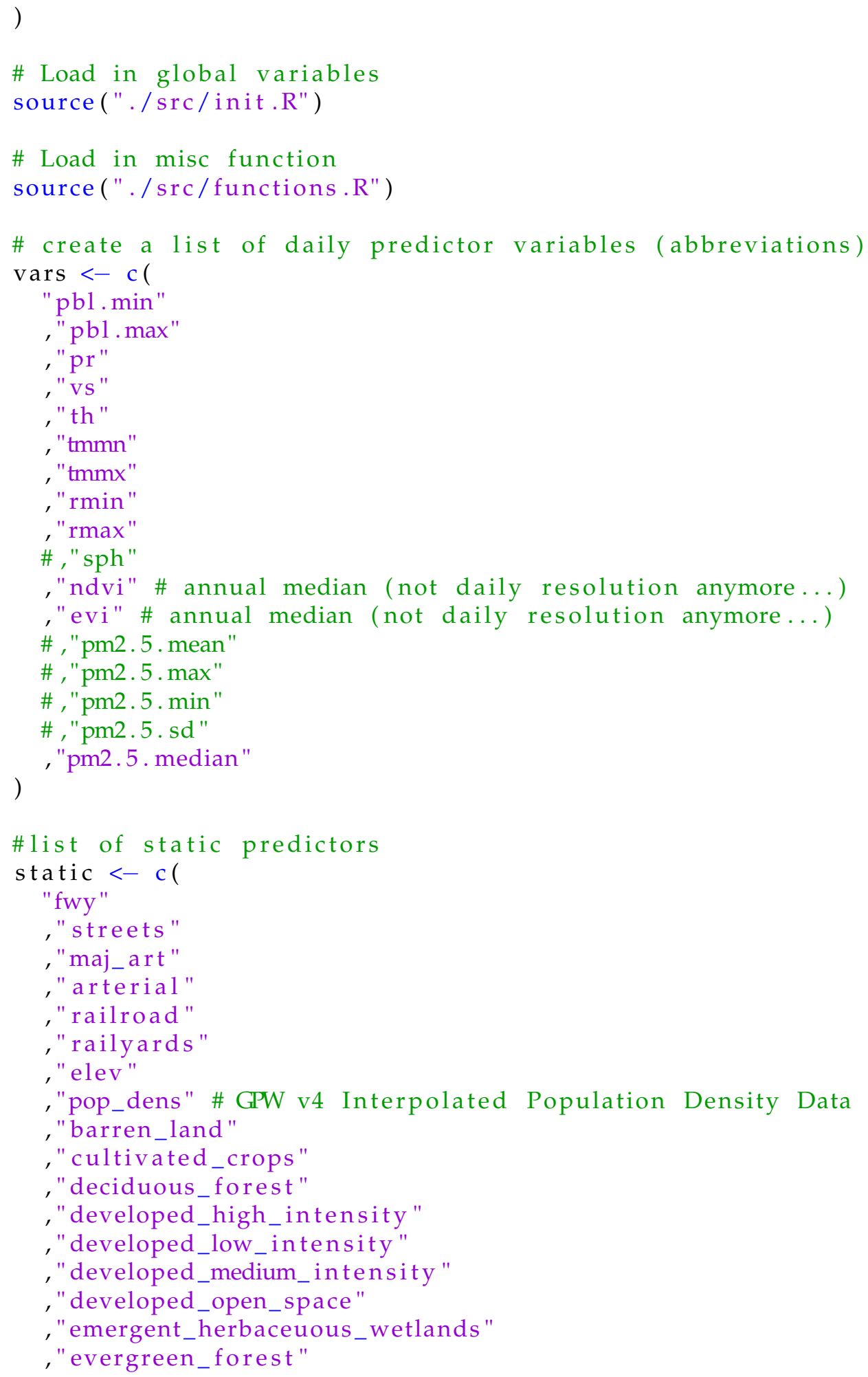




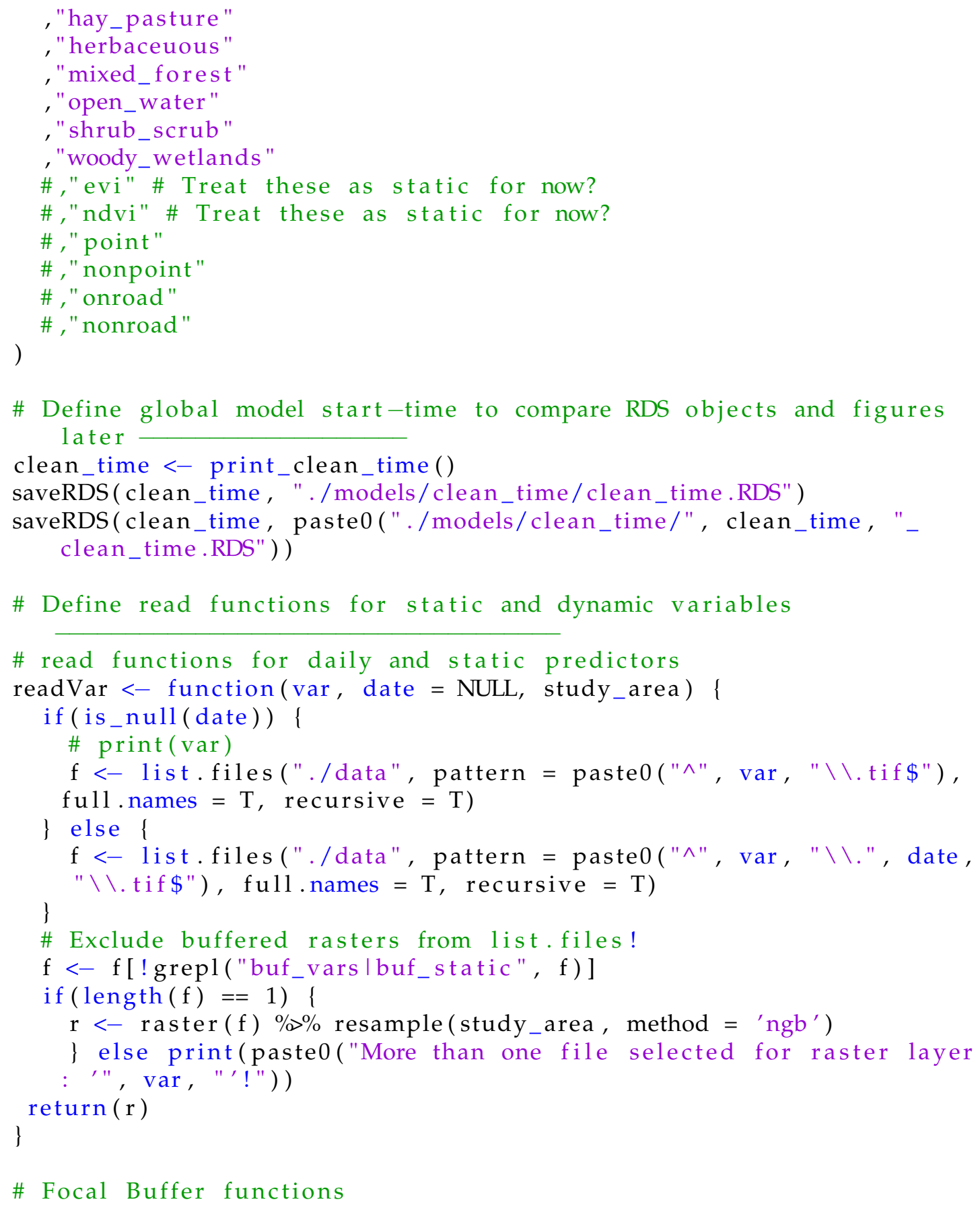




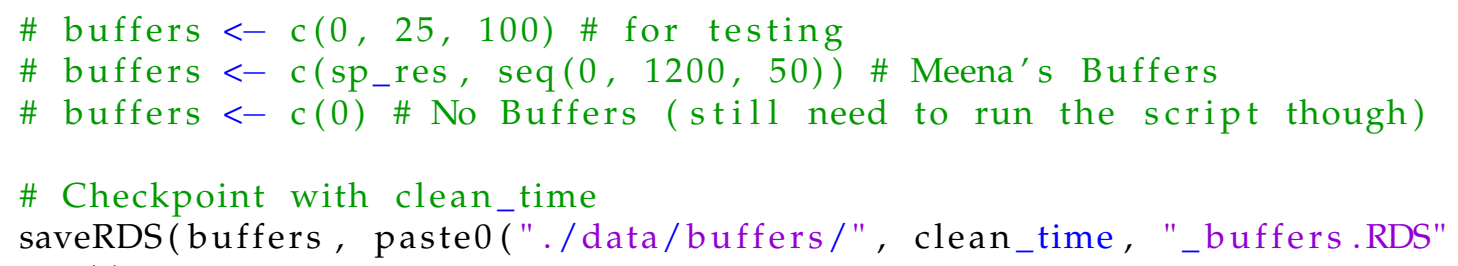




$$
\text { return (buf) }
$$




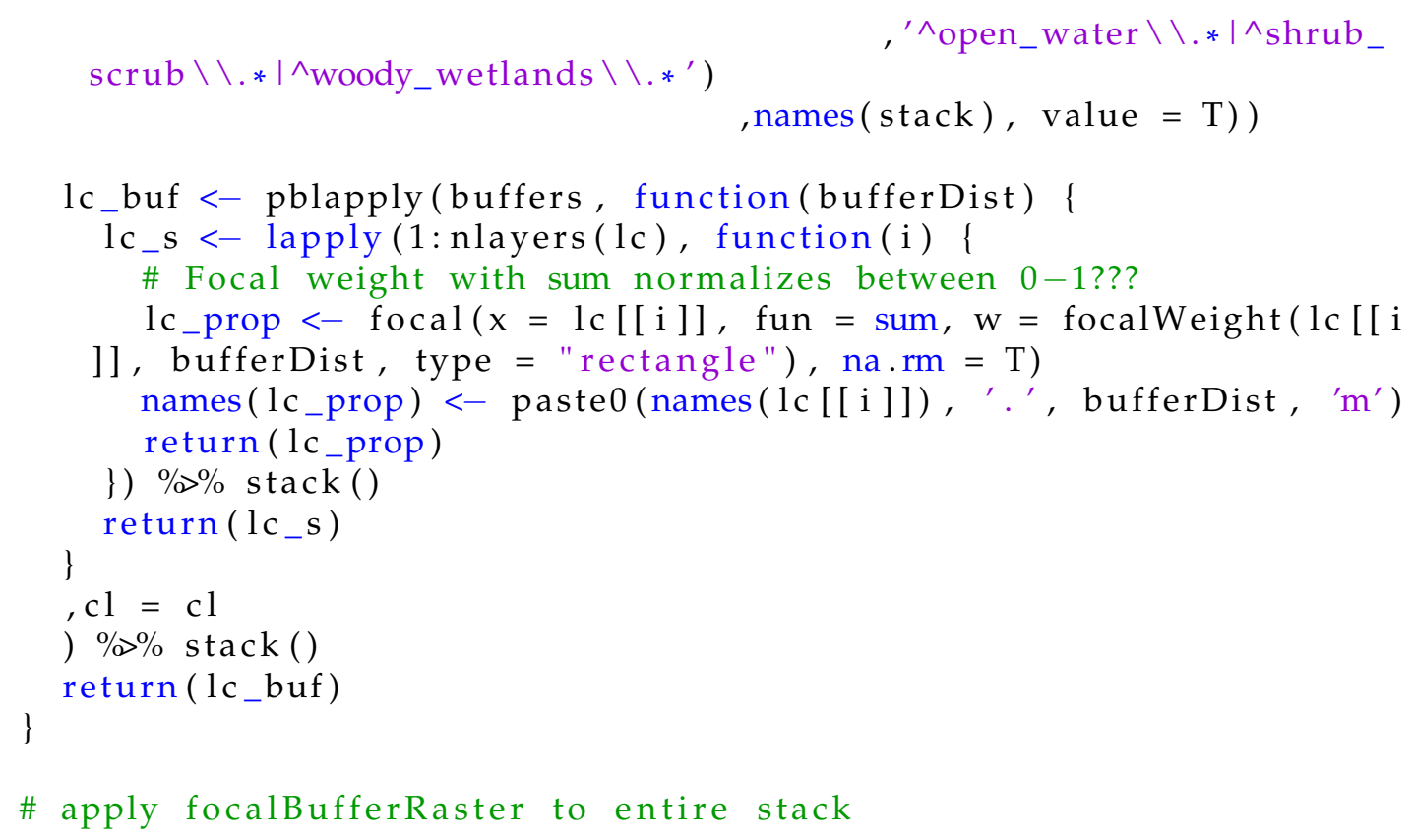


s_list <- pblapply(buffers, function(x) focalBufferStack (stack, bufferDist $=\mathrm{x}), \mathrm{cl}=\mathrm{cl}$ )

\} else \{

s_list <- lapply(buffers, function(x) focalBufferStack (stack, bufferDist $=x)$ )

\}

$\mathrm{s}_{<-} \mathrm{s}_{-}$list [!vapply(s_list, is.null, logical(1))] \% $\$$ stack()

return (s)

\}

\# function resamples and combines all GIS predictors into single raster stack

stackPredictors $<-$ function (date=NULL, crs, study_area, vars) \{ if (rlang:: is_null(date) $)\{$ $\mathrm{s}<-$ lapply (vars, function $(\mathrm{x})$ readVar $(\operatorname{var}=\mathrm{x}$, study_area $=$ study -area ) $\% \%$ stack ()

\} else \{

$\mathrm{s}<-$ lapply(vars, function $(\mathrm{x})$ readVar $(\operatorname{var}=\mathrm{x}$, date $=$ date, study _area $=$ study_area $)$ ) $\% \%$ stack () 
\# Raster processing functions

\# Combine all predictors for a given day

bufferPredictors <- function(date, crs, study_area, bb, vars) \{

\# Study Area Extent Raster used for resampling...

a $<-$ readRDS(study_area) $\% \%$ spTransform (CRSobj $=$ crs) $\% \%$ raster ( res $=$ sp_res, vals $=0$ )

\# Final bounding box

$\mathrm{b}<-\operatorname{readRDS}(\mathrm{bb}) \% \%$ spTransform $($ CRSobj $=\mathrm{crs}) \% \%$ raster $\left(\right.$ res $=\mathrm{sp}_{-}$ res, vals $=0$ )

\# Create Raster Stack

$\mathrm{s}<-$ stackPredictors $($ date $=$ date

, crs $=$ crs

, study_area $=a$

, vars = vars )

\# Buffering

s_buf $<-$ createBuffers $(s$, buffers = buffers $)$

\# Add PBL and Neph data (not buffered)

pbl $<-$ raster : : subset $\left(s, \operatorname{grep}\left({ }^{\prime \wedge} \operatorname{pb} 1 \backslash \backslash . *^{\prime}\right.\right.$, names $(\mathrm{s})$, value $\left.\left.=\mathrm{T}\right)\right)$

\# neph <- raster : : subset $\left(\mathrm{s}\right.$, grep $\left({ }^{\prime} \wedge \mathrm{pm} 2 \backslash \backslash .5 \backslash \backslash . * '\right.$, names $(\mathrm{s})$, value $=$ T) )

neph $<-$ raster:: subset $\left(\mathrm{s}, \operatorname{grep}\left({ }^{\wedge} \mathrm{pm} 2 \backslash \backslash .5 \backslash \backslash\right.\right.$.median*', names $(\mathrm{s})$, value $=\mathrm{T})$ )

s_buf $\% \diamond \%$ raster : : stack (pbl, neph)

\# strip date from daily predictor layer names

names $($ s_buf $)<-$ gsub $(x=$ names $($ s_buf $)$, pattern $=$ paste $0(" \backslash \backslash . "$, date ), replacement $=" "$ )

\# Rename Om buffer to match non-buffered layers

names $\left(s_{-}\right.$buf $)<-$gsub $\left(x=\right.$ names $\left(s_{-}\right.$buf $)$, pattern $=$paste0 $(" \backslash \backslash .0 \mathrm{~m} \$ ")$, replacement $=" "$ )

\# Crop extent to original bounding box (10 times smaller than buffer boundary)

318 s_buf $\% \diamond \%$ raster $:$ : $\operatorname{crop}\left(b\right.$, snap $={ }^{\prime}$ near' $) \% \%$ resample $(b$, method $=$ 'ngb')

319 s_buf $\% \diamond \%$ raster : : mask(b) 
\# Write s_buf to file

\# return a cropped raster (using the largest buffer distance to cut from )

writeRaster(s_buf

$$
\text { (s_buf), ".tif") }
$$




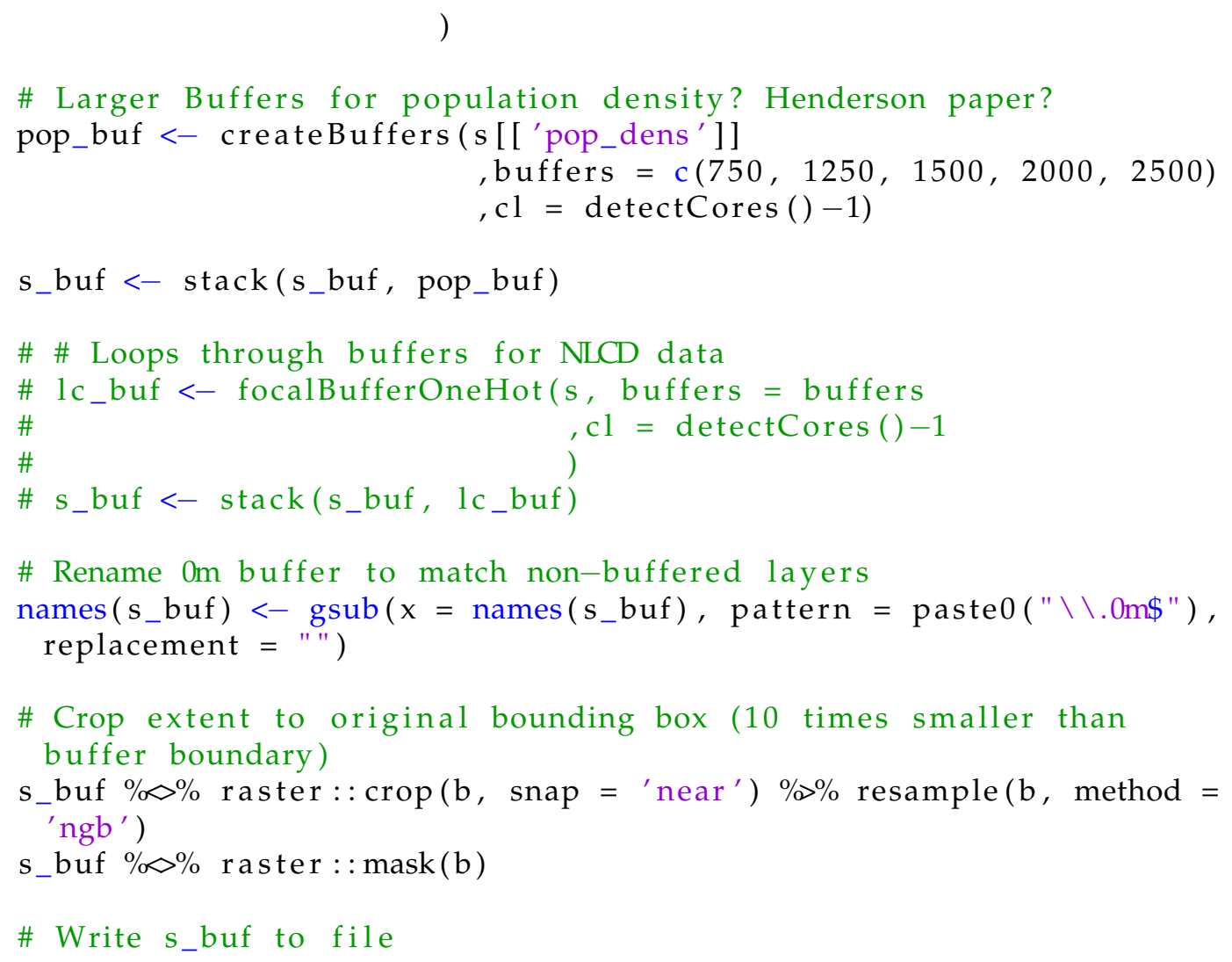




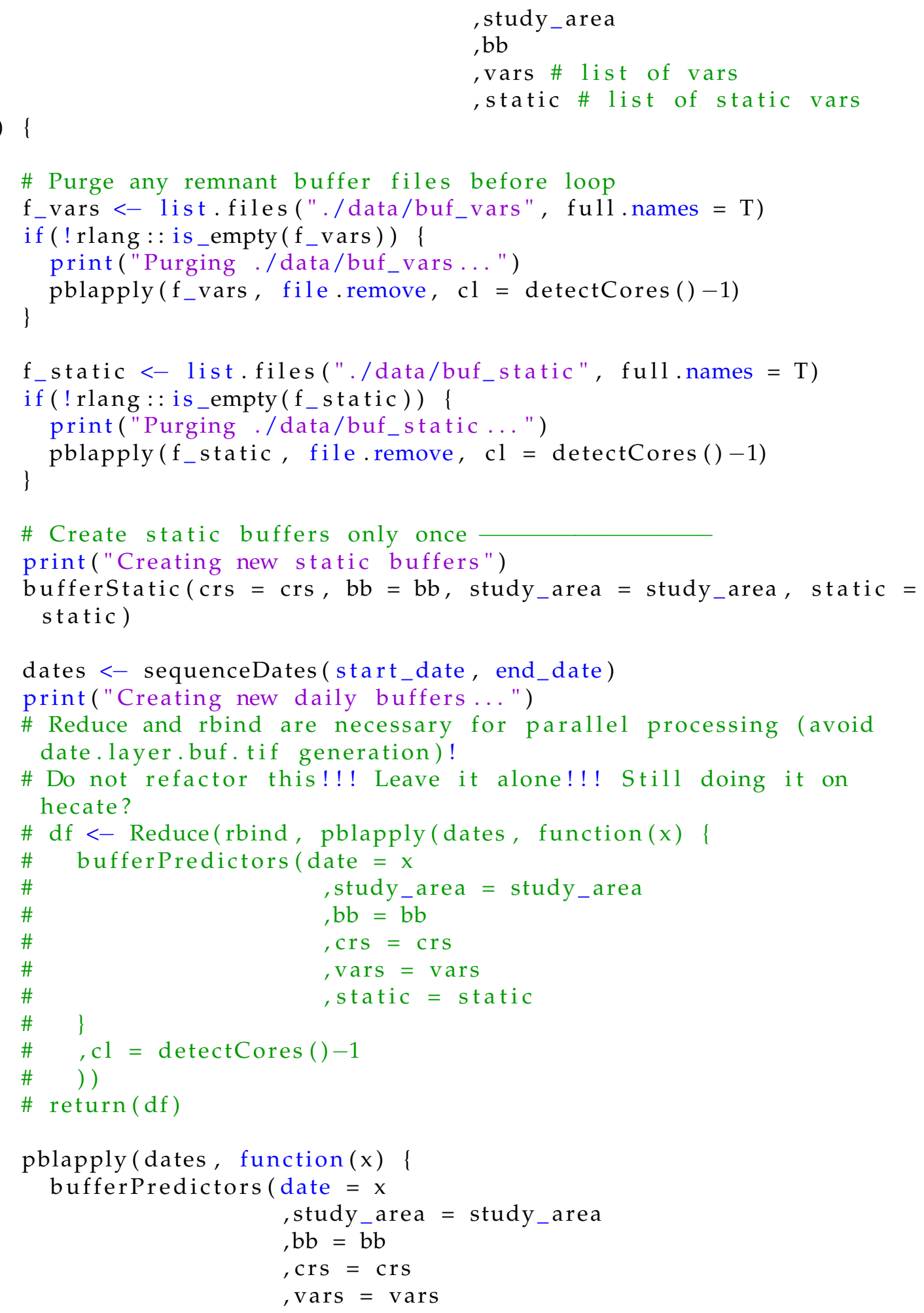




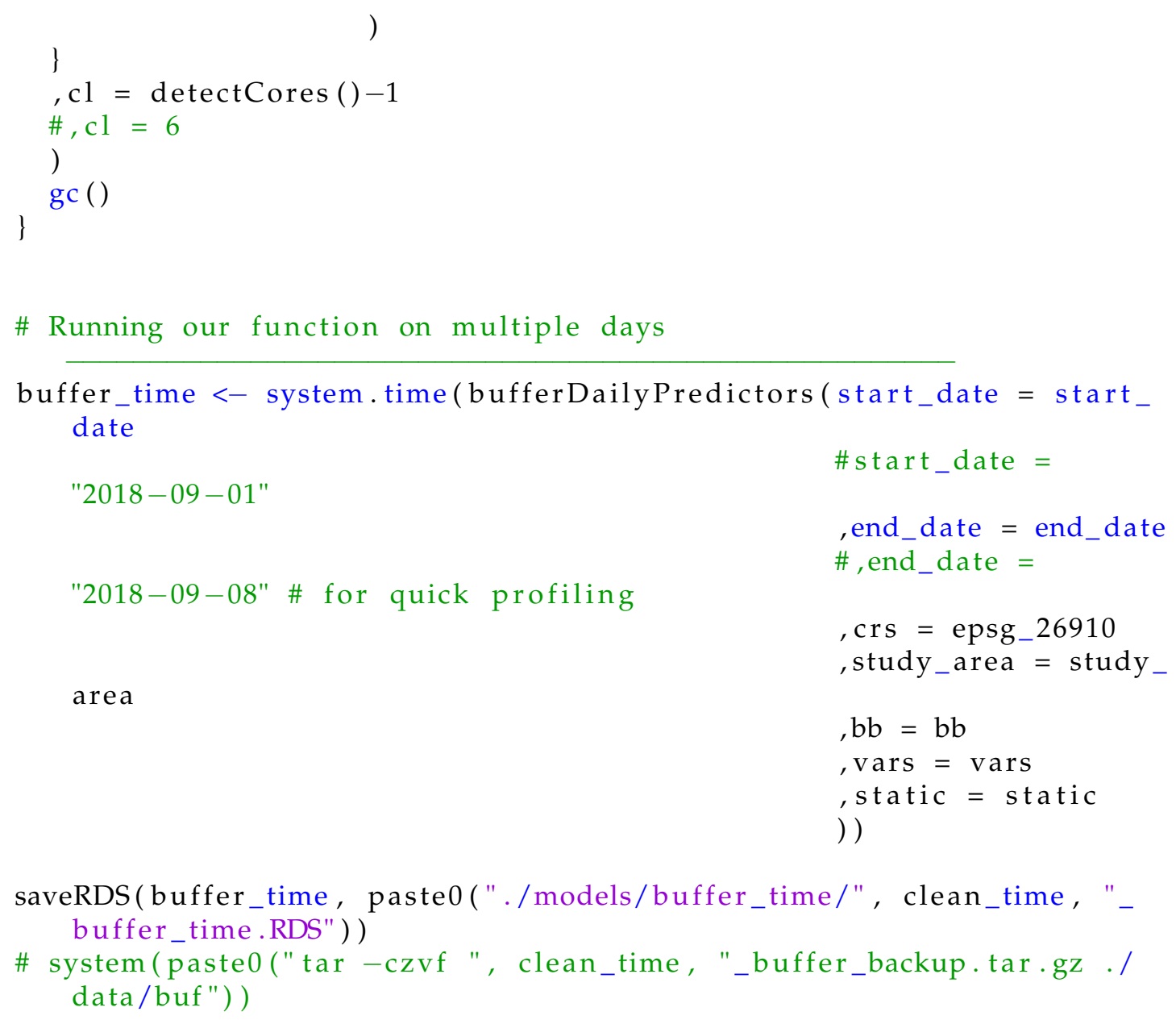

\section{Source/extract.R}

\# created by Philip Orlando@ Sustainable Atmospheres Research Lab

\# Canopy Continuum Project, USFS

\# PI Dr. Linda George

\# 2018-11-19

\# create daily stacks of GIS predictors, buffer and extract them

\# needed for nvim-R

if $($ basename $(\operatorname{getwd}())=="$ src" $)$ \{

setwd ("../")

getwd ()

\}

\# load the necessary packages

14 if (!require (pacman)) \{ 


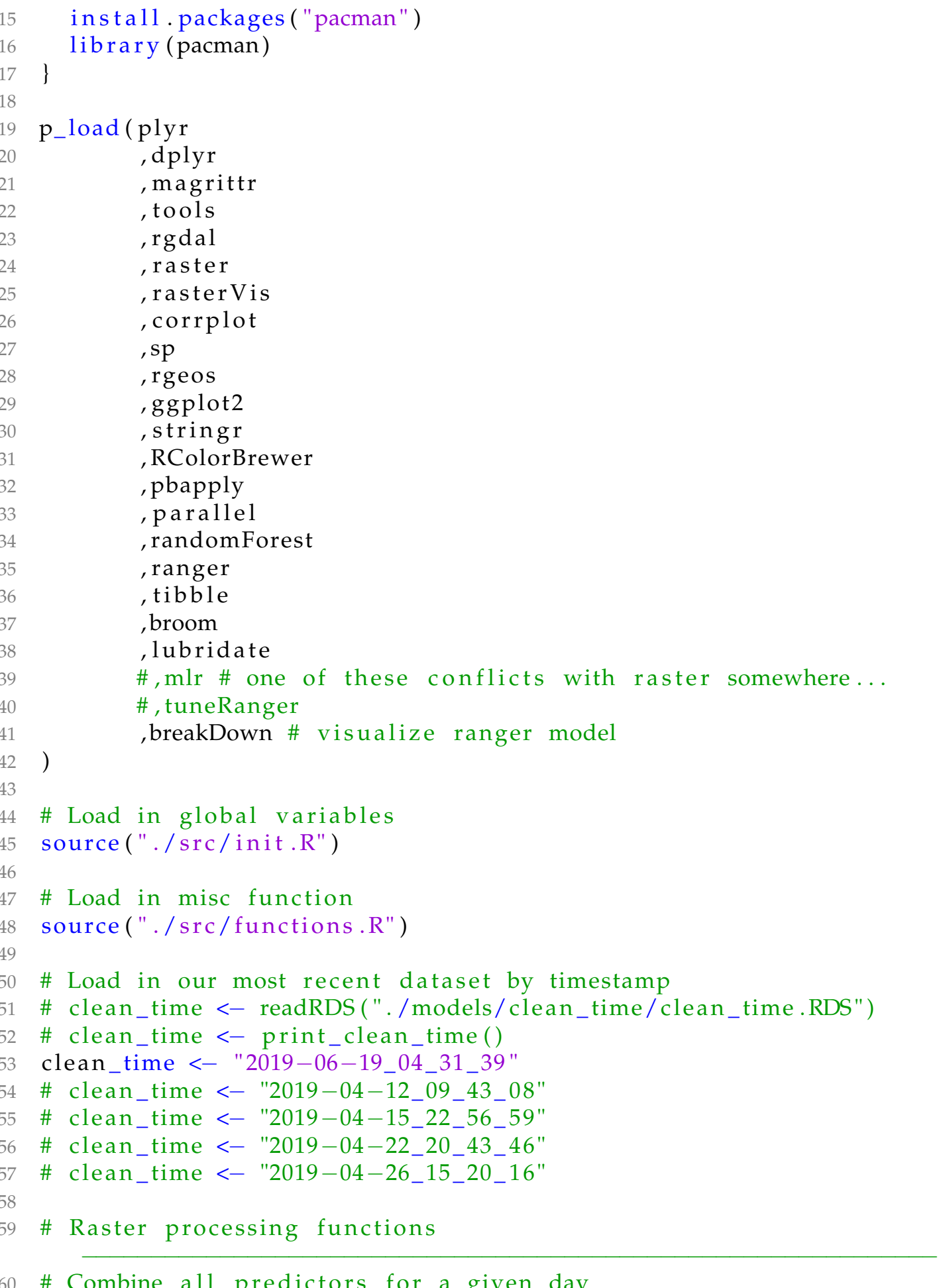


extractPredictors <- function(date, crs, study_area, bb, pm_df, static, scale_neph = scale_neph) \{

\# Study Area Extent Raster used for resampling...

a $<-$ readRDS(study_area) $\% \%$ spTransform $($ CRSobj $=$ crs $) \% \%$ raster ( res $=$ sp_res, vals $=0$ )

\# read PurpleAir data

pa $<-$ readRDS $($ pm_df $) \% \%$ spTransform $($ CRSobj $=$ crs $)$

\# Final bounding box

$\mathrm{b}<-\operatorname{readRDS}(\mathrm{bb}) \% \%$ spTransform $($ CRSobj $=\mathrm{crs}) \% \%$ raster $\left(\right.$ res $=\mathrm{sp}_{-}$ res, vals $=0$ )

\# Read in our variable buffers

s_buf_vars <- raster::stack(list.files ("./data/buf_vars", pattern = paste0 $($ date, $" * \backslash \backslash$.tif $\$ ")$, full.names $=\mathrm{T}))$

\# strip date from daily predictor layer names

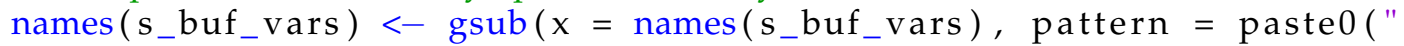
$\wedge X^{\prime}$, date, $\left." \backslash \backslash . "\right)$, replacement $\left.=" "\right)$

\# \# Read in our static buffers

\# s_buf_static <- raster::stack(list.files("./data/buf_static", pattern $=" . * \backslash \backslash$.tif $\$ "$, full. names $=\mathrm{T})$ )

\# Combine static and variable buffer stack

s_buf $<-$ stack $($ s_buf_vars, static)

\# \# Reclassify NA that buffer script missed

\# s_buf [is.na(s_buf) $]<-0$

\# \# Add $\mathrm{x}$ and $\mathrm{y}$ values as predictors to raster layer

\# lat $<-$ lon $<-$ subset (s_buf, grep ("pm2.5. median", names(s_buf), value $=\mathrm{T})$ )

\# $x y<-$ coordinates (s_buf)

\# lon [] $<-x y[, 1]$

\# lat []$<-x y[, 2]$

\# names(lon) $<-$ "x"

\# names $(1$ at $)<-$ "y"

\# plot (stack(lon, lat))

\# s_buf $\% \diamond \%$ stack (lon, lat)

\# \# Compare differences between s_buf layers and df attributes

\# colnames (df $[$, which (colnames (df) \%! in\% names(s_buf)) ]) 
pa $<-$ pa [which $($ pa\$date $==\operatorname{gsub}(" \backslash \backslash . ", " \backslash \backslash-"$, date $))$,

\# Simple extract grabs only the intersecting cell value

\# s_extract_simple <- raster : : extract (s_buf, pa, method = 'simple' , $\mathrm{sp}=\mathrm{T}, \mathrm{na} \cdot \mathrm{rm}=\mathrm{T}$ )

s_extract $<$ - raster: : extract $\left(s_{-}\right.$buf, pa, method = 'bilinear', sp = , $\mathrm{na} \cdot \mathrm{rm}=\mathrm{T}$ )

\# Convert to data.frame

df $<-$ raster::as.data.frame (s_extract) $\% \%$ na.omit ()

\# Rename lat and lon to re-convert to raster later... names $(\mathrm{df})[\operatorname{names}(\mathrm{df})==$ "coords. $\mathrm{x} 1 "]<-$ "lon"

names $(\mathrm{df})[$ names $(\mathrm{df})==$ "coords.x2"] $<-$ "lat"

\# Attach temporal variables

\# These are categorical variables that get converted into integers during modeling

\# Add DayOfWeek variable

df\$weekday $<-$ lubridate $:$ wday $($ df $\$$ date $) \% \%$ factor $($ levels $=c(1: 7))$

\# Add Season variable

df\$season $<-$ getSeason $($ df $\$$ date $) \% \%$ factor $($ levels $=c(1: 4))$

\# \# Add Month Variable? Captures phenological differences in PM from vegetation?

df $\$$ month $<-$ lubridate $:$ month $($ df $\$$ date $) \% \%$ factor $($ levels $=\mathrm{c}(1: 12))$

\# Weekend/Weekday Captures differences in on-road mobile emissions?

df $\$$ weekend <- ifelse (lubridate : :wday (df\$date) \%in\% c $(6,7), 1,0)$ $\% \%$ factor $(1$ evels $=\mathrm{c}(0: 1))$

\# strip date from daily predictor layer names

\# names $(\mathrm{df})<-$ gsub $(x=\operatorname{names}(\mathrm{df})$, pattern $=$ paste $0(" \backslash \backslash . "$, date $)$, replacement $=" "$ )

\# Convert back to sp (used later)

\# $x y<-d f[, c(" x ", " y ")]$

\# $\mathrm{sp}<-$ SpatialPointsDataFrame $(\operatorname{coords}=\mathrm{xy}, \mathrm{data}=\mathrm{df})$

\# proj4string $(\mathrm{sp})<-$ proj4string (s_extract) 
\# Convert Absolute PM to Neph-scaled PM

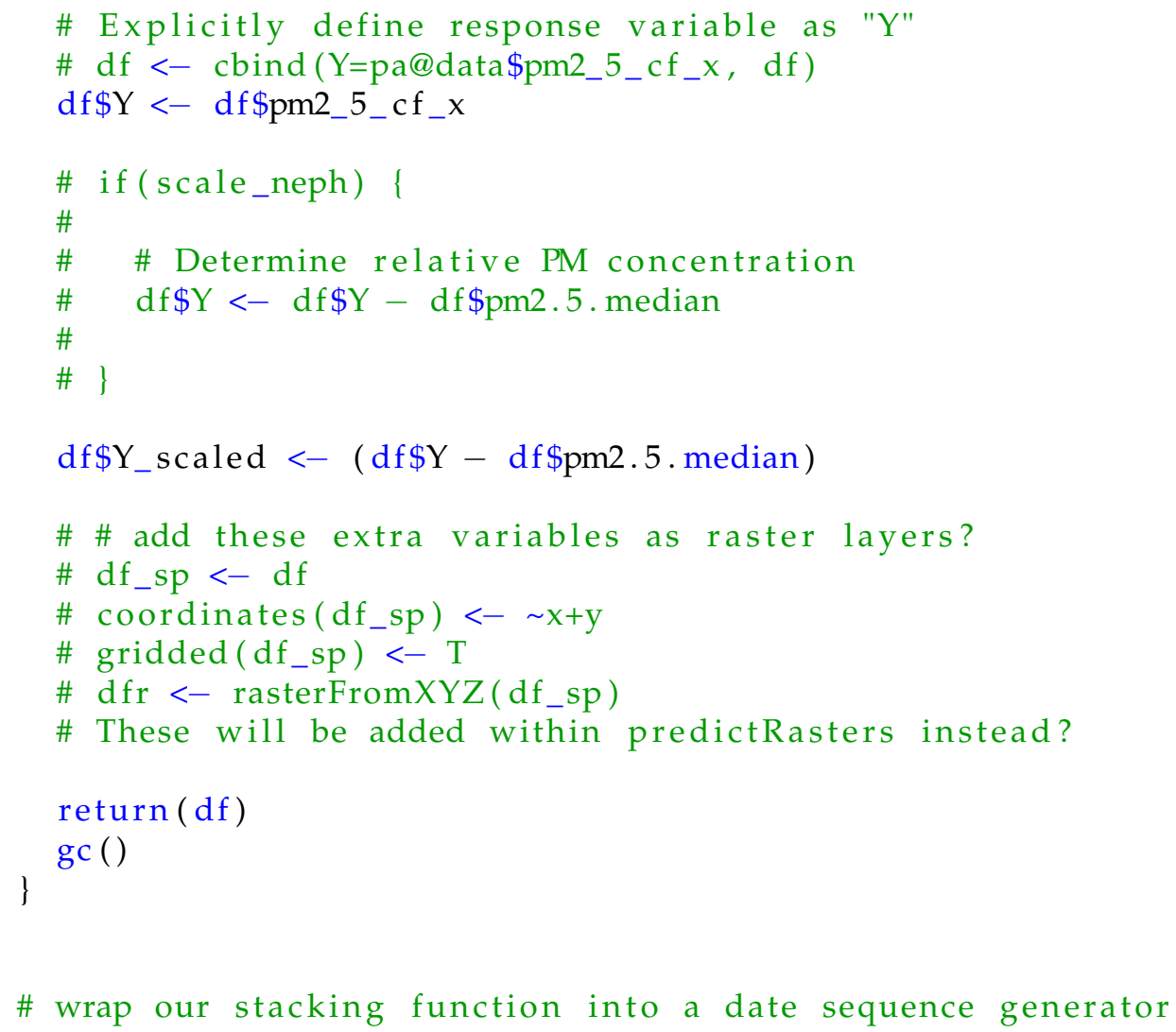




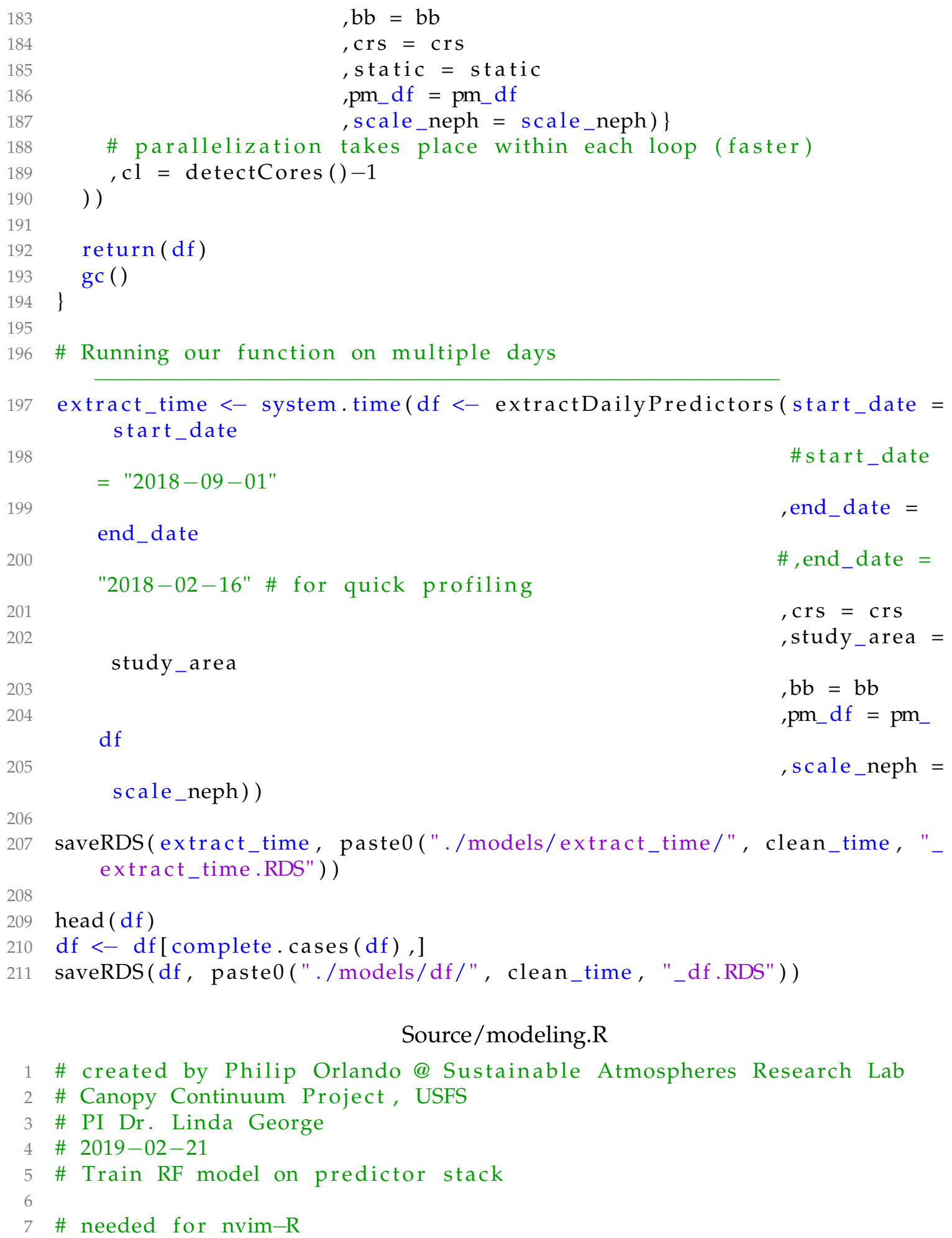




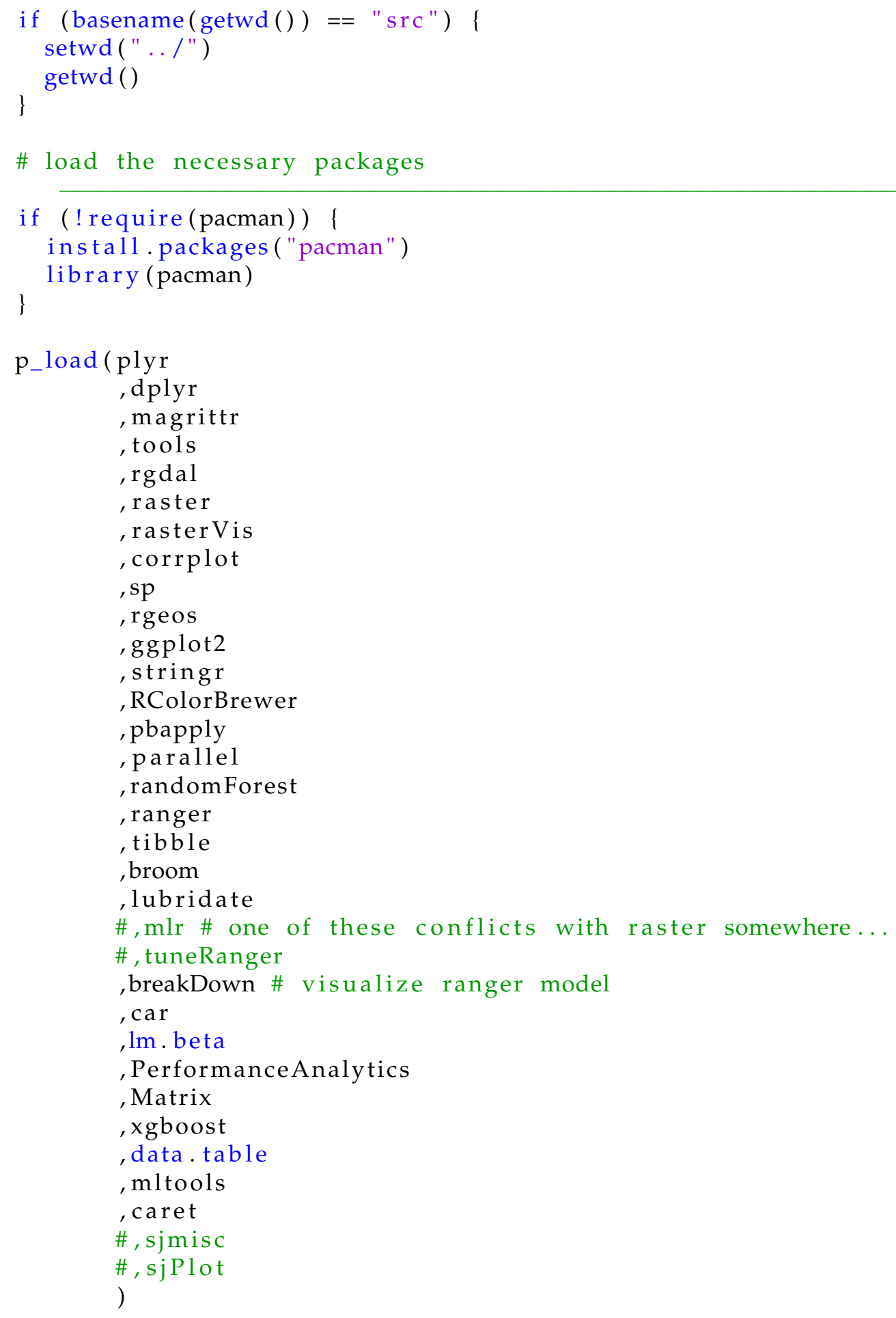




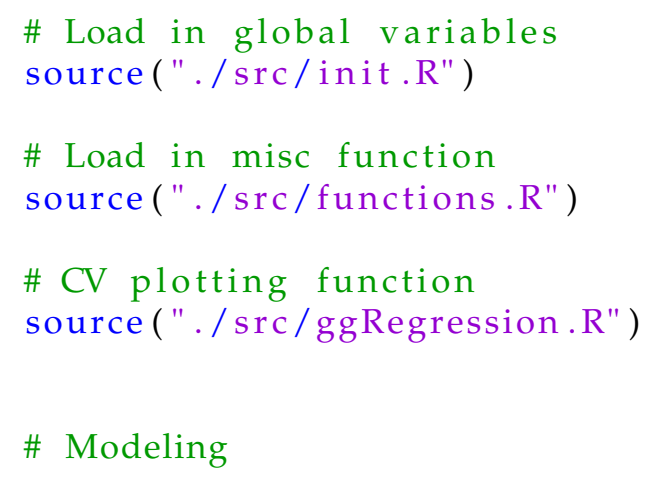




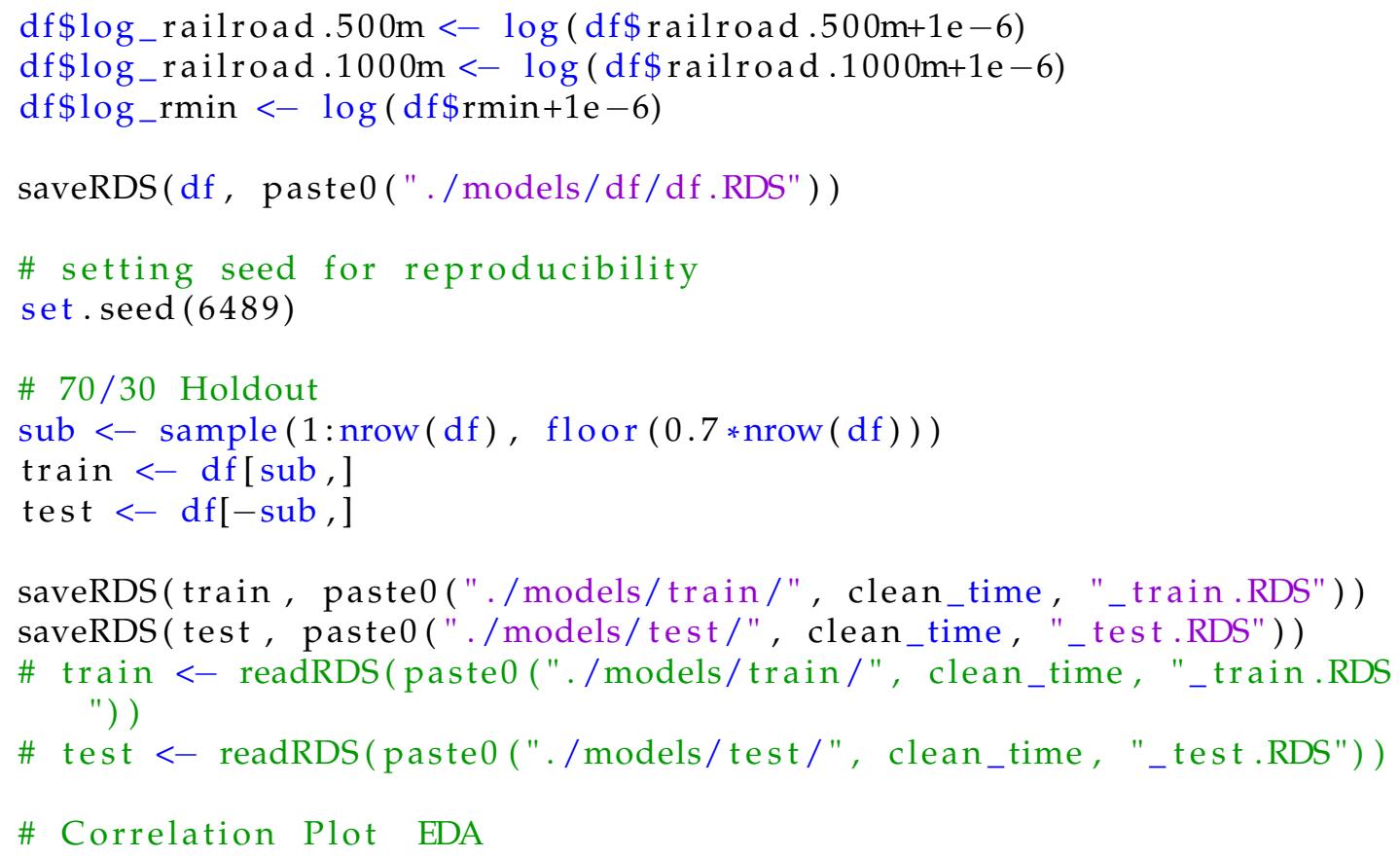

\# Loop through each base var and develop invidual corrplot

vars $<-$ df $\% \%$ select_if( is .numeric) $\% \%$ colnames () $\% \%$

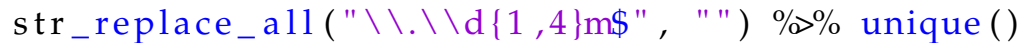

lapply(vars, function(i) \{

$\operatorname{try}(\{$ cmatrix <- data.frame $(\mathrm{df}[$, grepl $(\operatorname{paste} 0(" \wedge \mathrm{Y} \$ \mid \wedge ", \mathrm{i})$, names $(\mathrm{df}))])$ names (cmatrix) [names $($ cmatrix $)==$ 'pm2.5. median'] $<-$ 'neph' \# Exclude columns that all contain zero (produces $\mathrm{NaN}$ error later ) cmatrix $<-$ cmatrix $[$, colSums (cmatrix $!=0)>0]$ col <- colorRampPalette $($ c("\#BB4444", "\#EE9988", "\#FFFFFF", "\#77 AADD", "\#4477AA") ) res1 $<-$ cor.mtest $($ cmatrix, conf.level $=0.95$, na.rm=T) p. mat $<-$ cor. mtest (cmatrix) \$p corrplot $(\operatorname{cor}(\operatorname{cmatrix}), \operatorname{col}=\operatorname{rev}(\operatorname{col}(200))$, order $=$ "hclust", number. cex $=0.7$

$$
\begin{aligned}
& \text {, addCoef.col = "black" } \\
& \text {, } \mathrm{tl} \cdot \mathrm{col}=\text { "black", } \mathrm{tl} \cdot \mathrm{srt}=90 \\
& \text {,p. mat }=\text { p. mat } \\
& \text {, sig.level = 0.05, insig = "blank", diag }=\mathrm{F} \text { ) } \\
& \text { png }\left(\text { filename } = \text { paste } 0 \left({ }^{\prime} . / \text { figures } / \operatorname{corrplot} /{ }^{\prime},\right.\right. \text { clean_time, '_', i, }
\end{aligned}
$$
'_corrplot.png') 


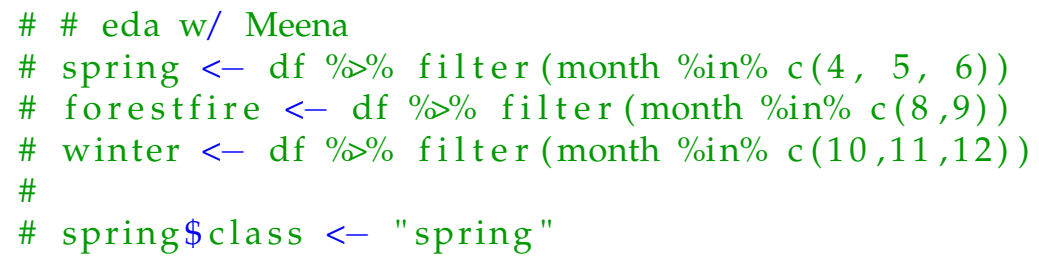




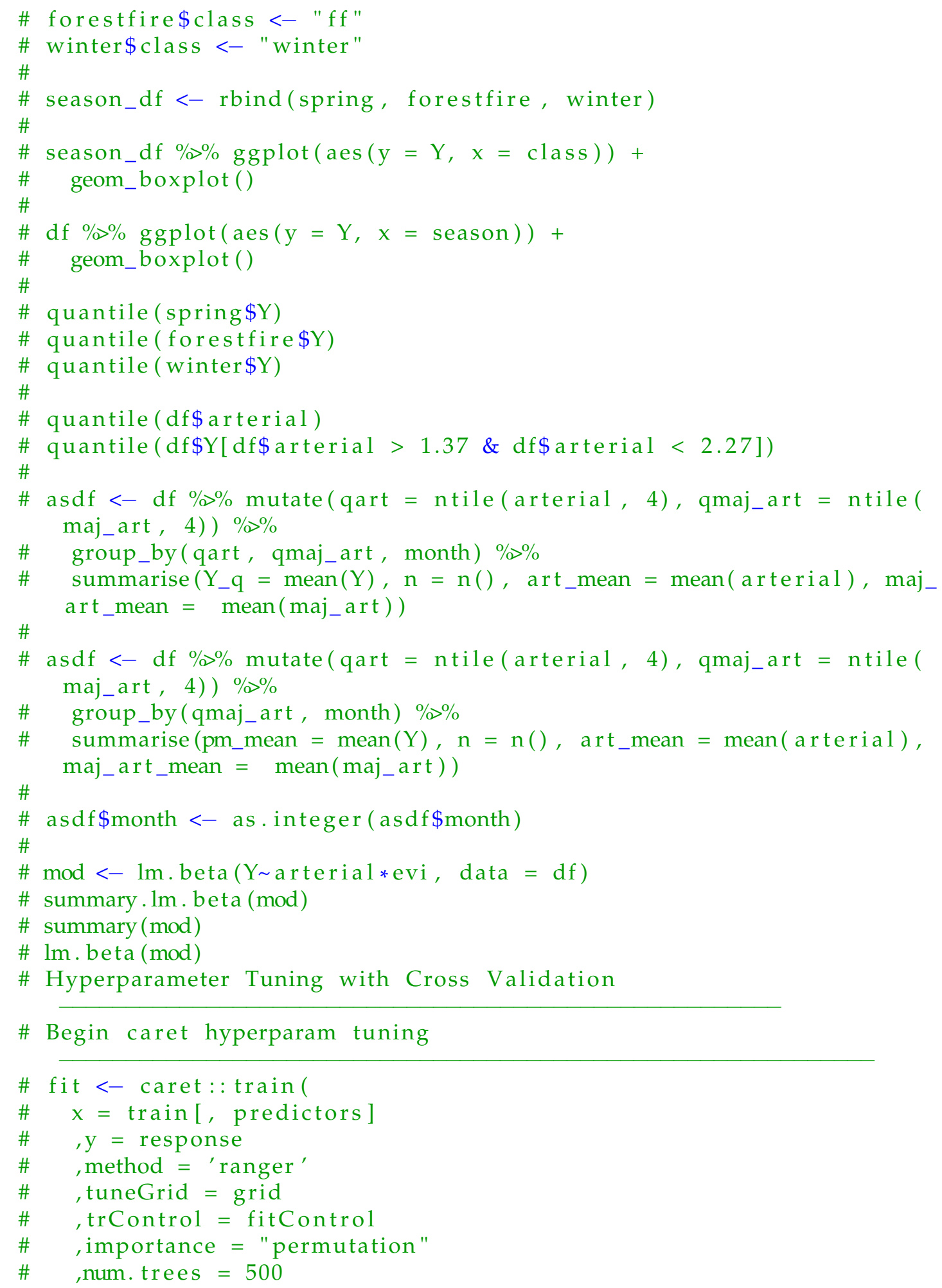




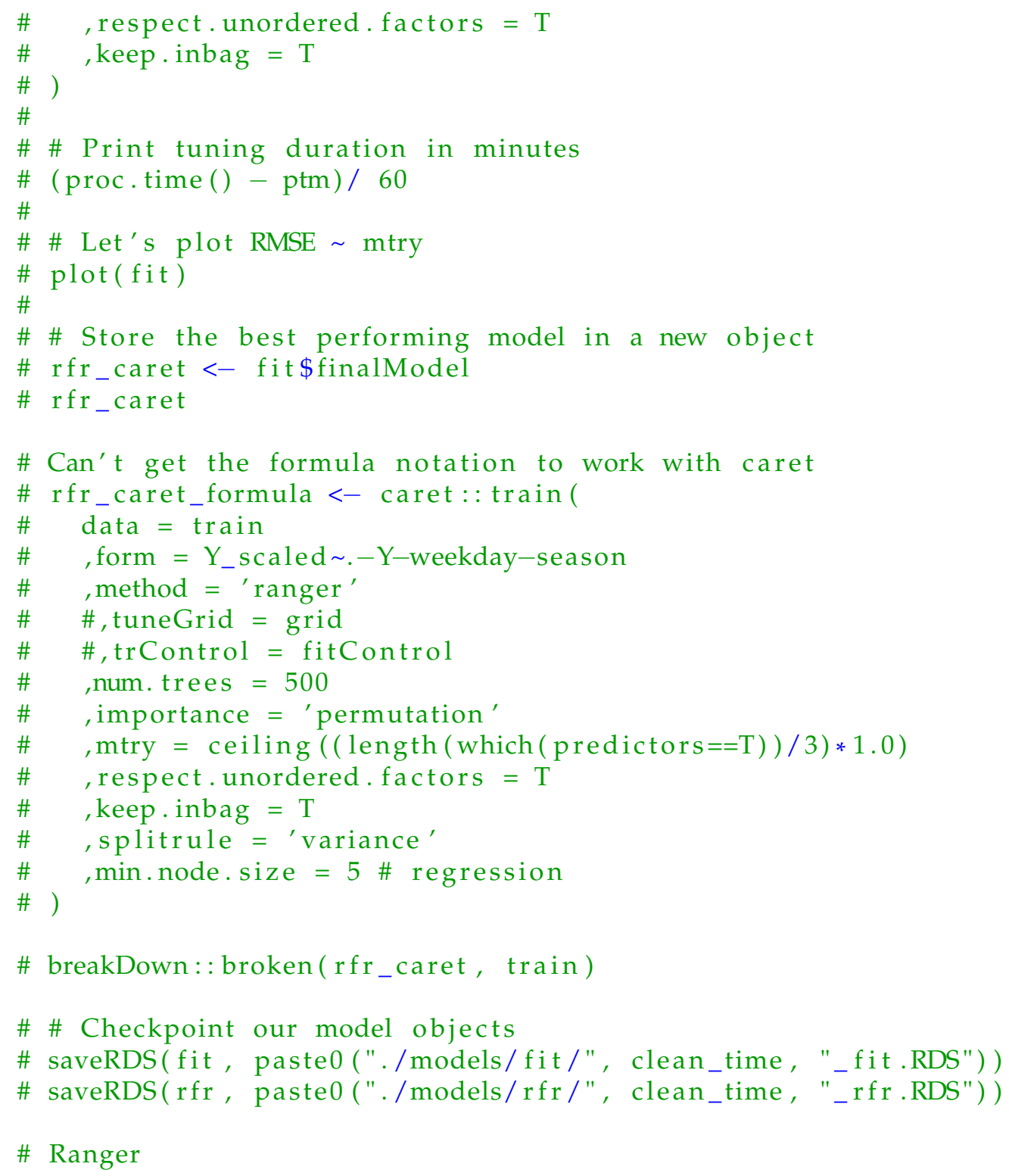

\# Following the DataCamp course principles for repeatedcv

fitControl <- caret : trainControl (method = "repeatedcv"

, number $=5 \# \mathrm{~K}-$ folds

, repeats $=3$

\# store start time

ptm $<-$ proc.time () 
\# Train model differently depending on scale_neph

\# Manually select predictors to train model on (scale_neph handles pm2.5. median)

\# Select single buffer for each predictor based on the strongest correlation and instictive sign

initial_predictor_names <- c("fwy.100m" \# largest buffer to smooth data

,"elev.300m" \# maybe smooth this out to larger buffer prevent overfitting?

,"streets.100m" \# include or not?

\#," arterial" \# negative relationship all buffers

,"pr" \# pr is one of the better correlated

predictors, but may be introducing noise...

\#," th" \# ignore wd because of resolution!

relationship for buffers \#," maj_art" \# negative relationship/non-

noise to the model

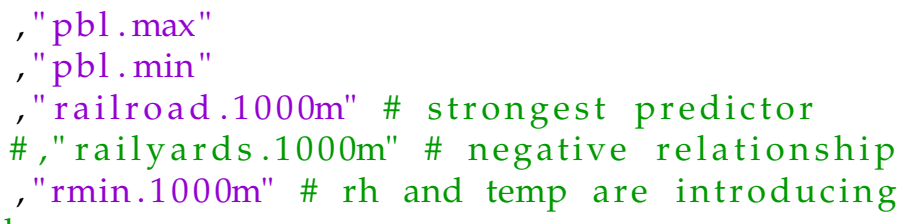

with PM

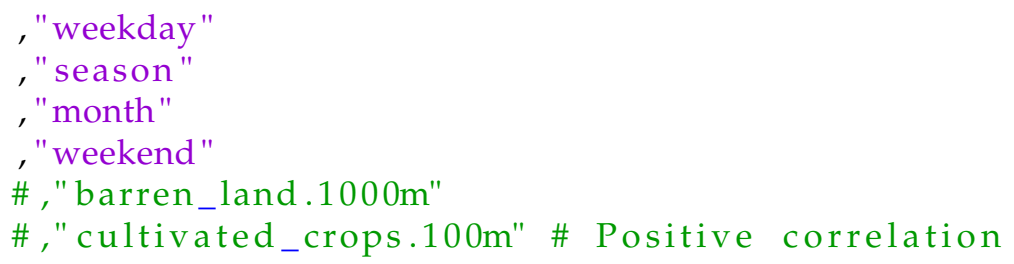




\section{4}

$$
\text { correlation }
$$

exclude? ,"ndvi.100m" \# largest buffer with negative , "evi.100m" \# largest buffer with negative

,"deciduous_forest.1000m"

," evergreen_forest. $1000 \mathrm{~m} "$

, "mixed_forest.1000m"

\#,"shrub_scrub.100m" \# Exclude?

\#,"hay_pasture.1000m" \# positive correlation? positive at $1000 \mathrm{~m}$

$$
\text { \#,"herbaceuous.500m" \# negative at 500m, }
$$

\#,"emergent_herbaceuous_wetlands.1000m"

\#,"open_water.100m" \# LUR overfits this! ( correlated with railroads...) \#,"woody_wetlands" \# positive correlation for all buffers

$$
\begin{aligned}
& , \text { "1on " } \\
& , \text { "1at " }
\end{aligned}
$$

\# Use hclust to determine which predictor to select for each collinear group

\# cmatrix <- data.frame (df $[$, grepl(paste $0(" \wedge Y \$ \mid \wedge "$, paste 0 (initial_ predictor_names, collapse $\left.\left.={ }^{\prime} \mid{ }^{\prime}\right), " \$ "\right)$, names $\left.\left.(\mathrm{df})\right)\right]$ ) cmatrix <- df $[$, names $(\mathrm{df}) \%$ in\% c("Y", "pm2.5.median", initial_ predictor_names)] names (cmatrix) [names $($ cmatrix $)==$ 'pm2.5. median'] $<-$ 'neph' \# Exclude columns that all contain zero (produces $\mathrm{NaN}$ error later) cmatrix <- cmatrix [, colSums (cmatrix $!=0)>0]$ cmatrix <- dplyr:: select_if (cmatrix, is .numeric) col <- colorRampPalette (c("\#BB4444", "\#EE9988", "\#FFFFFF", "\#77AADD", "\#4477AA" ) ) res $1<-$ cor.mtest (cmatrix, conf.level $=0.95$, na.rm=T) sp.mat $<-$ cor.mtest (cmatrix) $\$ \mathrm{p}$ saveRDS(cor (cmatrix), paste0 ("./models/cmatrix/", clean_time, "_ cmatrix.RDS"))

\# Checkpoint our correlation matrix cdf $<-$ as.data.frame (as.table (cor (cmatrix))) cdf $\% \diamond \%$ dplyr:: filter (Var1 != Var2 \& Var1 == "Y") \% $\%$ arrange(desc ( Freq))

\# Figure out something better later ggplot(cdf, aes (reorder(Var2, Freq, sum), Freq)) + 


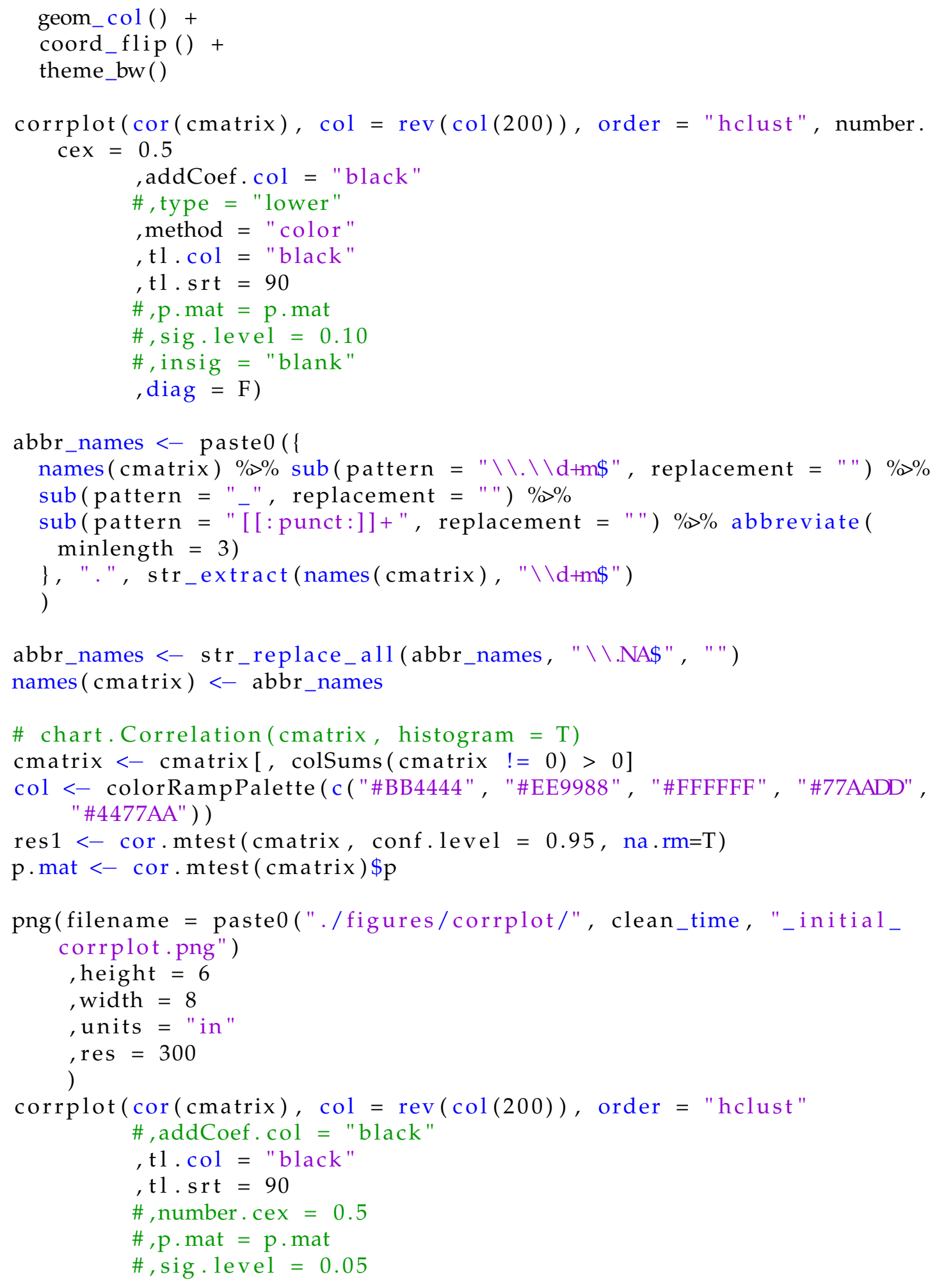




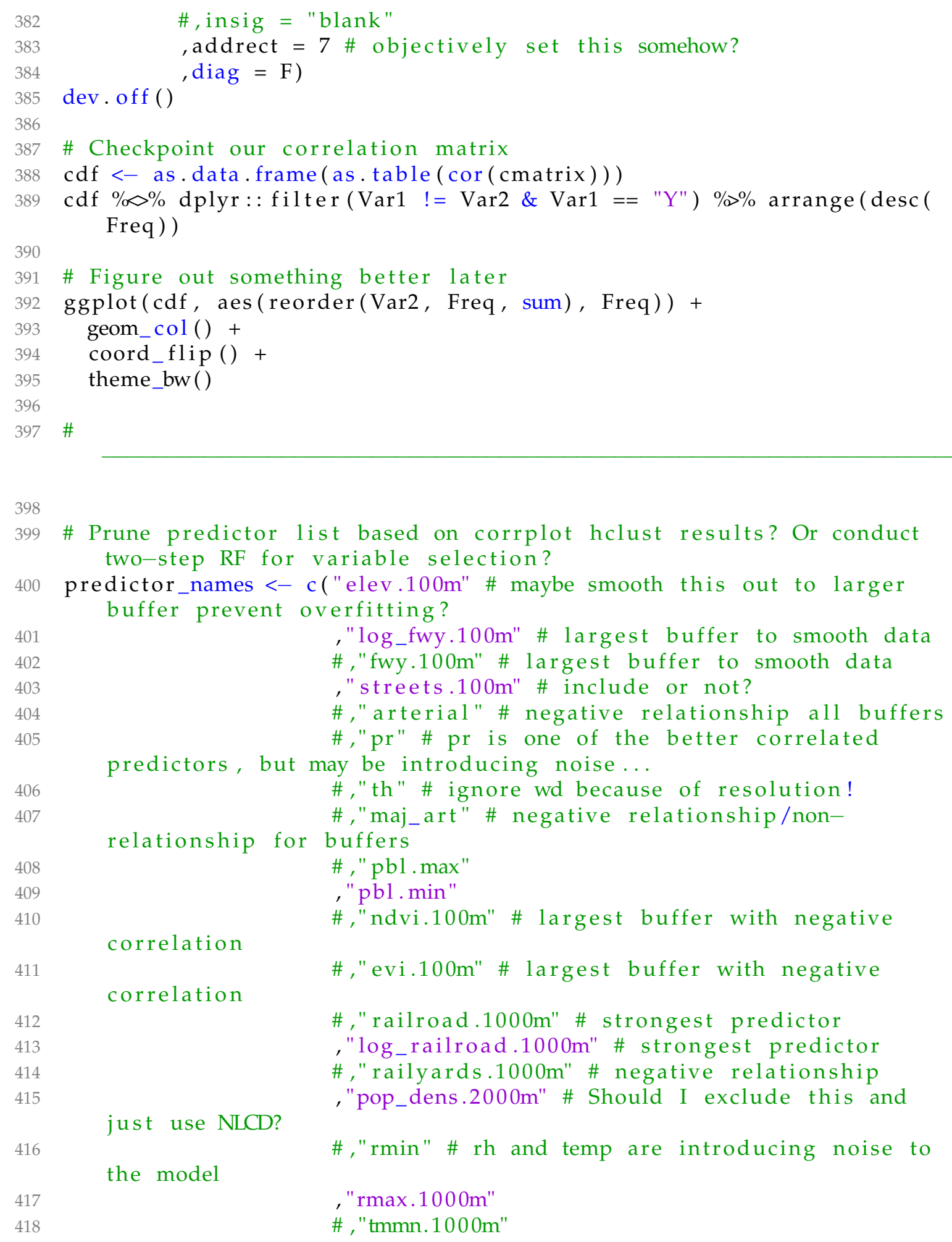


resolution?

\author{
, "tmmx.1000m" \\ \# , "vs.1000m" \\ \#,"th.1000m" \# omit entirely due to poor
}

\#,"weekday"

, "season"

\# , "month"

,"weekend"

\# ," barren_land.1000m"

\#,"cultivated_crops.100m" \# Positive correlation

with PM

correlation with PM

$$
\begin{aligned}
& \text { \#,"deciduous_forest.1000m" } \\
& \text { \#,"developed_high_intensity.500m" \# no }
\end{aligned}
$$

\#,"developed_low_intensity.100m" \# positive correlation at $500 \mathrm{~m}$, negative at $1000 \mathrm{~m}$

\#,"developed_medium_intensity.300m" \# positive correlation for all buffers

\#,"developed_open_space.1000m" \# take out later?

\#, " evergreen_forest.1000m"

\#,"hay_pasture.1000m" \# positive correlation?

exclude?

\#,"herbaceuous.500m" \# negative at 500m, positive at $1000 \mathrm{~m}$

\#,"emergent_herbaceuous_wetlands.1000m"

\#," mixed_forest.1000m"

\#," open_water.100m" \# overfits?

\#," shrub_scrub.100m"

\#,"woody_wetlands" \# positive correlation for

all buffers

$$
\begin{aligned}
& \text {,"lon " } \\
& \text {,"lat " }
\end{aligned}
$$

if (scale_neph) \{

predictors <- names(train) \%in\% predictor_names

form <- as.formula (paste0 ('Y_scaled ' , paste (predictor_names, collapse $\left.\left.={ }^{\prime}+{ }^{\prime}\right)\right)$ )

\} else \{

predictors <- names(train) \%in\% c("pm2.5.median", predictor_names) form $<-$ as.formula (paste0 ('Y pm2.5.median+', paste (predictor_names, \} collapse $\left.\left.={ }^{\prime}+{ }^{\prime}\right)\right)$ )

\# Temporary kitchen-sink formula!

\# form <- as.formula ("Y .-1at-1on-Y_scaled-pm2_5_cf_x-date-node") 


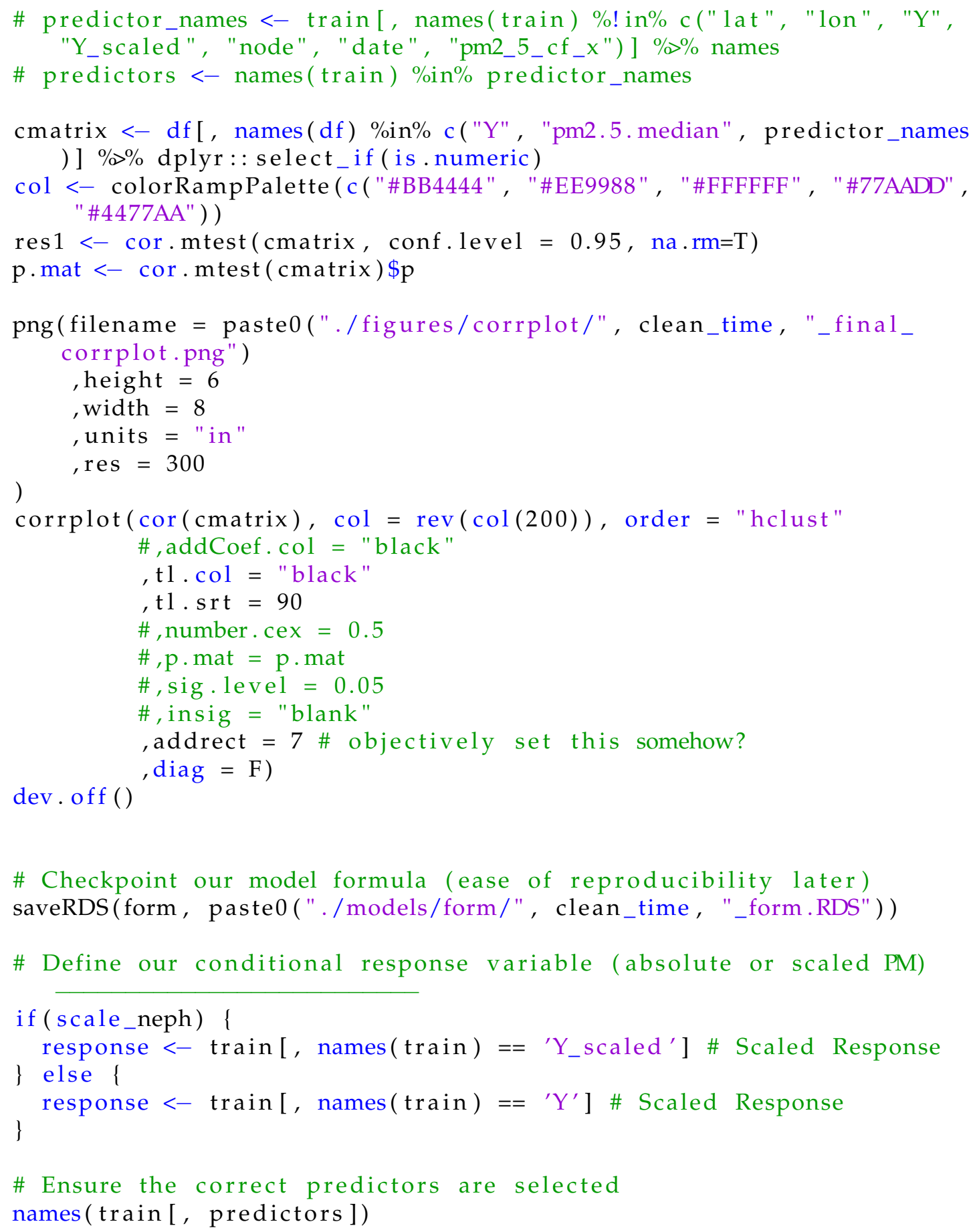


\# LUR

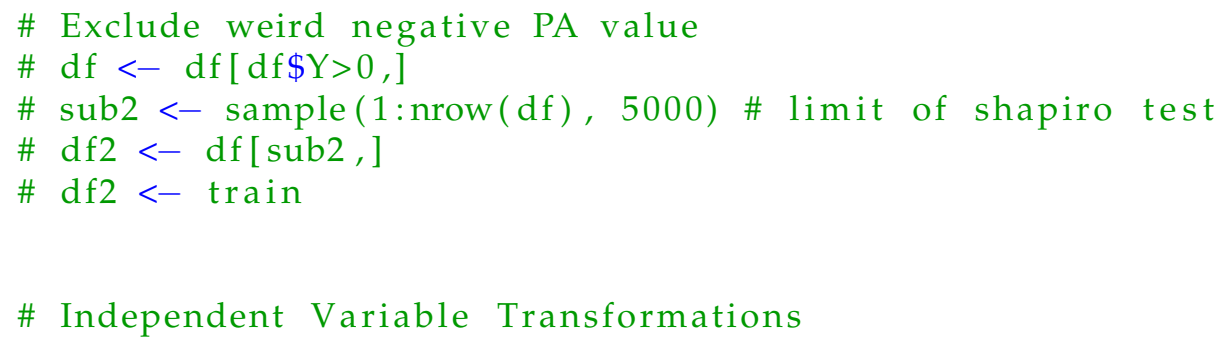


\# Response variable's residuals normally distributed? qqnorm ( train $\$ Y$ )

qqline (train $\$ Y$, col $=$ "red", lwd $=2$ )

\# TODO make sure this still makes sense...

\# Formula for indpendent variables

\# iv <- "log_pm2.5.median+log_fwy.100m+log_elev.100m+log_pbl.min+ndvi $.100 \mathrm{~m}+\log _{-}$railroad.100m+pop_dens.100m+log_rmin+month"

iv <- "pm2.5. median+log_fwy.100m+elev.100m+pbl.min+ndvi.100m+log_ railroad.100m+pop_dens.100m+rmin+season"

\# Create a landuse regression model too?

\# form_raw $<-$ paste0 ("Y ", iv)

\# $\operatorname{lm}<-\operatorname{lm}($ form_raw, data = train)

$\operatorname{lm}<-\operatorname{lm}($ form, data $=$ train $)$

summary $(1 \mathrm{~m})$

$\operatorname{par}(\mathrm{mfrow}=\mathrm{c}(2,2))$

plot $(\operatorname{lm})$

$\operatorname{par}(\mathrm{mfrow}=\mathrm{c}(1,1))$

\# Box-Cox Transformation

bc <- MASS: : boxcox $($ object $=1 \mathrm{~m}$, data $=$ train $)$

best_lambda $<-$ bc $\$ x[$ which $(b c \$ y==\max (b c \$ y))]$

train $\$ Y \_b c<-$ train $\$ Y^{\wedge}$ best_lambda

qqnorm (train $\$ Y \_b c$ )

qqline (train\$Y_bc, $\operatorname{col}=$ "red", lwd $=2$ )

\# form_bc <- as.formula (paste0 ('Y_bc pm2.5. median ${ }^{\prime}$ ', paste (predictor names, collapse $\left.\left.={ }^{\prime}+{ }^{\prime}\right)\right)$ )

\# form_bc <- "Y_bc log_pm2.5. median+log_fwy.100m+log_elev.100m+log_ pbl.min+ndvi.100m+log_railroad.100m+pop_dens.100m+log_rmin+month"

form_bc <- paste0 ("Y_bc " , iv)

lm_bc $<-\operatorname{lm}($ form_bc, data = train $)$

summary $\left(\operatorname{lm}_{-} \mathrm{bc}\right)$

$\operatorname{par}(\operatorname{mfrow}=\mathrm{c}(2,2))$

plot $\left(\operatorname{lm}_{-} b c\right)$

$\operatorname{par}(\mathrm{mfrow}=\mathrm{c}(1,1))$

\# Log Transformation

train $\$ Y_{-} \log <-\log ($ train $\$ Y+1 e-6)$

\# form_log <- "Y_log log_pm2.5.median+log_fwy.100m+log_elev.100m+log_ pbl.min+ndvi.100m+log_railroad.100m+pop_dens.100m+log_rmin+season+ month"

\# form_log <- as.formula (paste0 ('Y_log pm2.5.median+', paste ( predictor_names, collapse $\left.\left.={ }^{\prime}+{ }^{\prime}\right)\right)$ )

form_log <- paste0 ("Y_log ", iv) qqnorm ( train $\left.\$ Y \_l o g\right)$ qqline (train $\$ Y_{-} \log , \quad \mathrm{col}=$ "red", lwd = 2) 


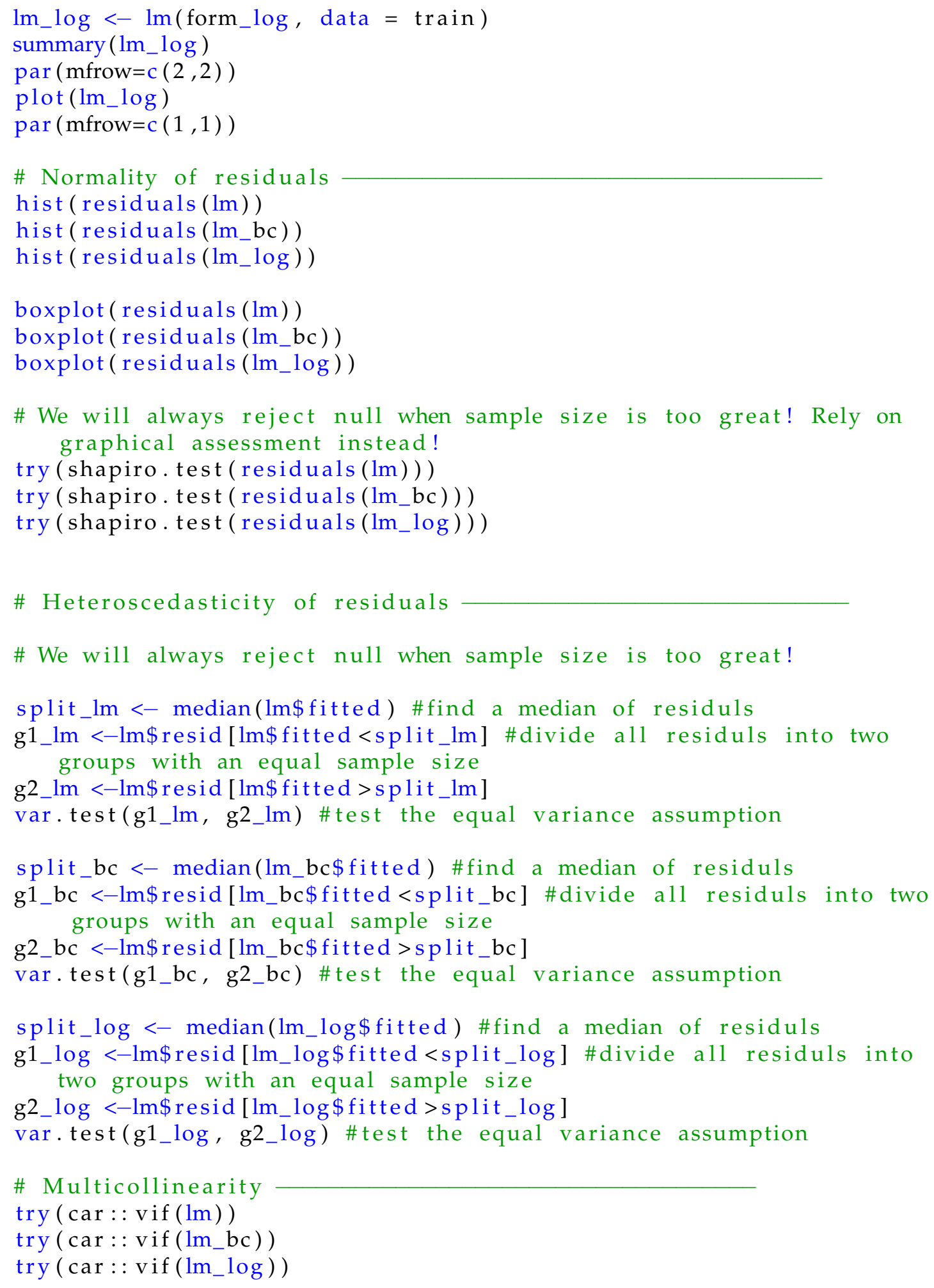




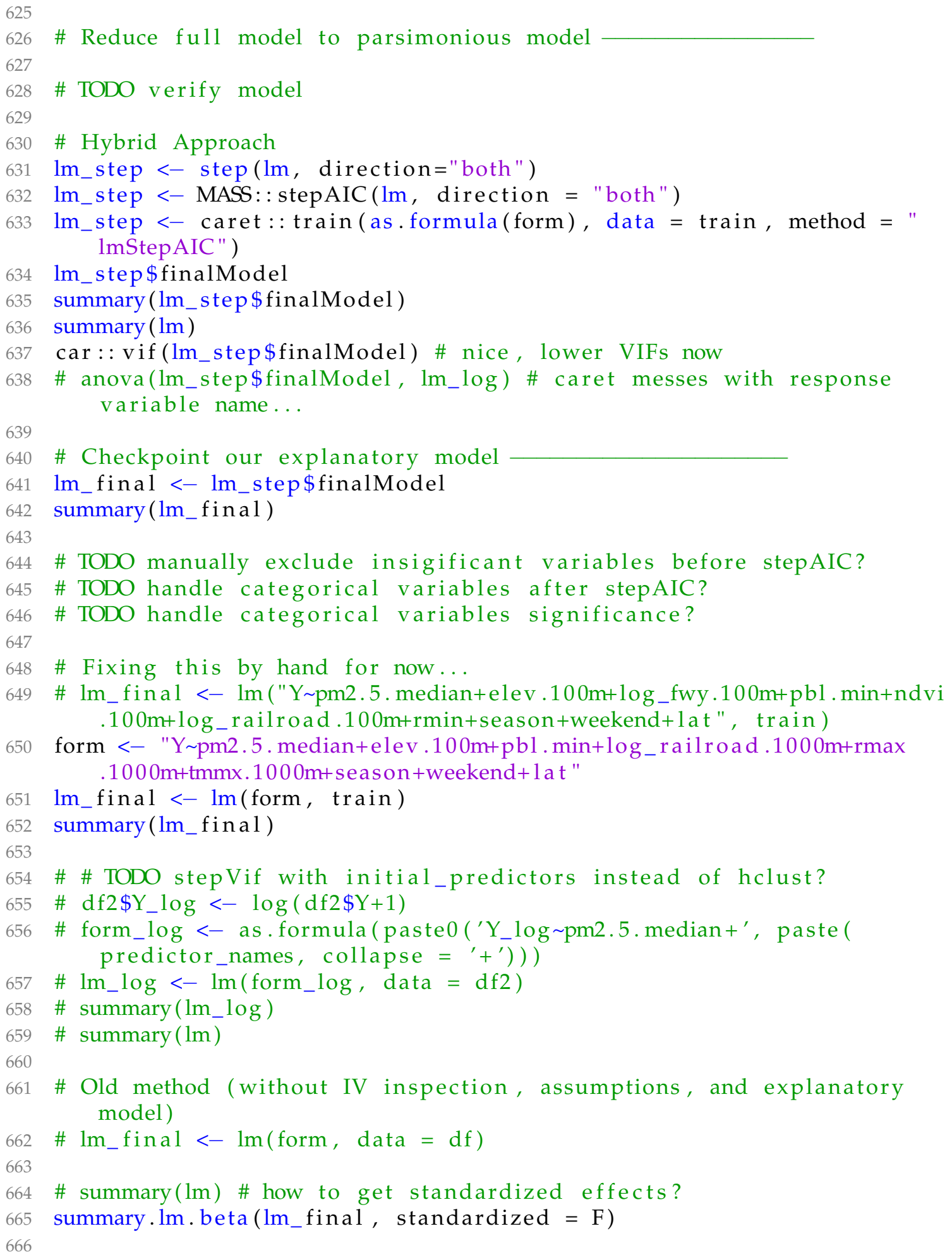


\# Checkpoint our LUR

saveRDS $\left(1 m \_f i n a l, p a s t e 0(" . /\right.$ models $/ 1 m / "$, clean_time, "_lm.RDS") )

\# Build grid of possible hyperparam combos

grid $<-$ expand grid $($ mtry $=c($ ceiling $(($ length $($ which $($ predictor $s==T)) / 3)$ $* 1.0)$

$3) * 1.5)$

$3) * 2.0)$

$3) * 2.5)$

$3) * 2.7)$ ) 


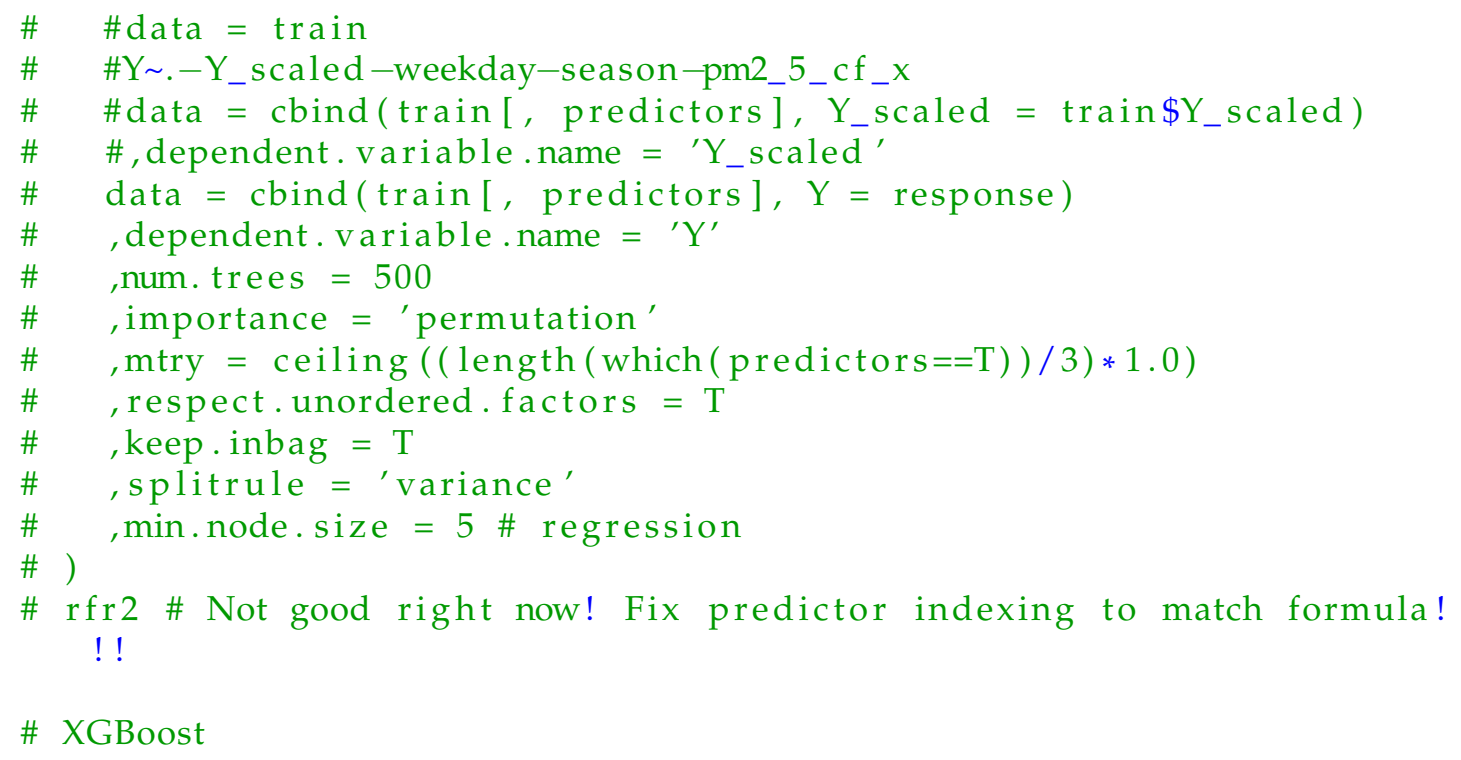

$x_{-}$train $<-$train [, predictors] $\% \%$ as data.table ()$\% \%$ mltools : : one hot (dropUnusedLevels $=\mathrm{F}$ )

$y_{-}$train $<-$train $\$ Y$

$x_{-}$test $<-$test [, predictors] $\% \%$ as.data.table () $\% \%$ mltools: : one_hot ( dropUnusedLevels $=\mathrm{F}$ )

$y_{-}$test $<-$test $\$ Y$

\# Test that factor encoding is preserved with month subset

\# df_dt_test $<-\mathrm{df}_{-} \mathrm{dt} \% \%$ filter (month $==" 1 "$ ) $\% \%$ as.data.table ()

\# df_oh_test $<-$ mltools:: one_hot $\left(\mathrm{df}_{-}\right.$dt_test, dropUnusedLevels $\left.=\mathrm{F}\right)$

$x g b_{-}$trcontrol = trainControl (

method $=" \mathrm{cv} "$,

number $=5$, allow Parallel = TRUE, verboseIter = FALSE, returnData $=$ FALSE

)

xgbGrid <- expand.grid (nrounds $=c(100,200), \quad \#$ this is n_estimators in the python code above

$5)$

$$
\text { sklearn-api . }
$$

max_depth $=c(10,15,20,25)$, colsample_bytree $=\operatorname{seq}(0.5,0.9$, length.out $=$ \#\# The values below are default values in the eta $=0.1$, gamma $=0$, 


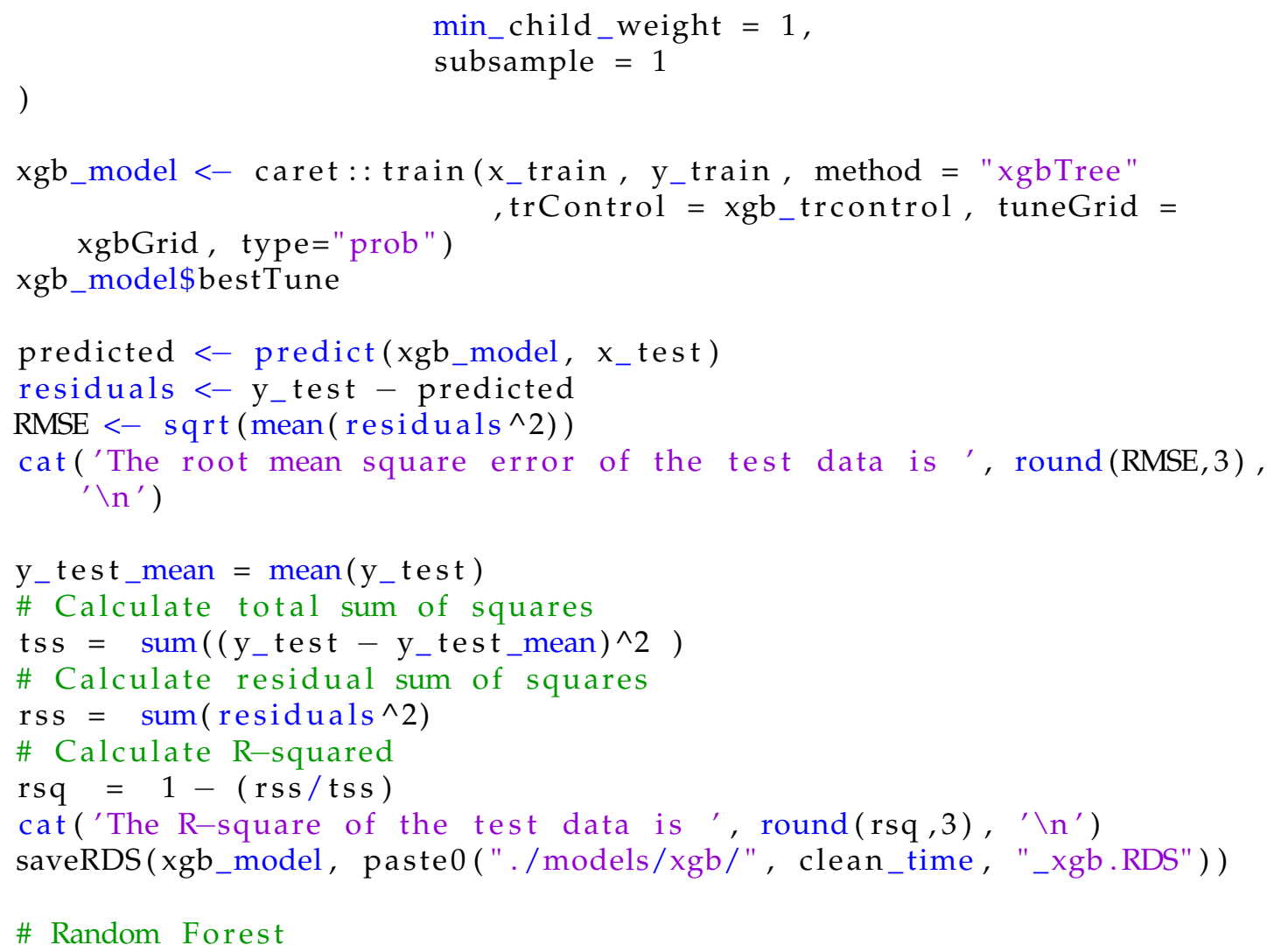




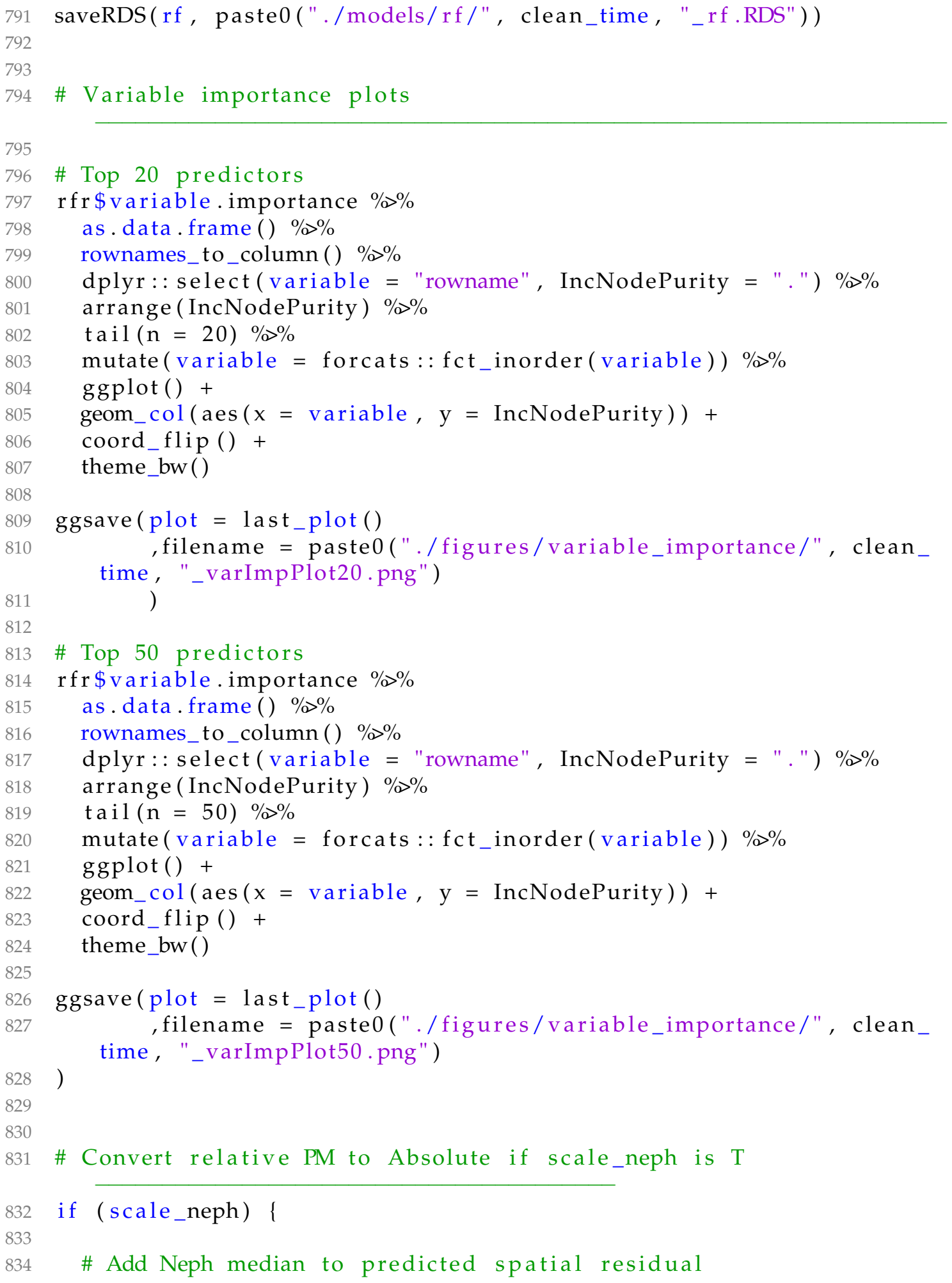


predictions <- predict $(\mathrm{rfr}$, test [, predictors $]) \$$ predictions+test $\$ \mathrm{pm} 2$ .5. median predict_full <- predict $(\mathrm{rfr}, \mathrm{df}[$, predictors $]) \$$ predictions + df $\$$ pm2. 5 . median

\# Add Neph median to observed spatial residual

test $\$ X<-$ test $\$ Y_{-}$scaled + test $\$ p m 2.5$. median

df $\$ X<-$ df $\$ Y \_s c a l e d+$ df $\$ p m 2.5$. median

\# Build test regression dataframe

cvr $<-$ data.frame $(x=$ test $\$ X, y=$ predictions $)$

cvr_full<-data.frame $(x=d f \$ X, y=$ predict_full $)$

\# Create test regression model

$\mathrm{rfr}_{-} \mathrm{cv}<-\operatorname{lm}(\mathrm{y} \sim \mathrm{x}$, data $=\mathrm{cvr})$

$\mathrm{rfr}_{-} \mathrm{Cv}_{-} \mathrm{fu}$ fll $<-\operatorname{lm}\left(\mathrm{y} \sim \mathrm{x}\right.$, data $\left.=\mathrm{cvr}_{-} \mathrm{full}\right)$

\} else \{

\# Build test regression dataframe cvr $<-$ data.frame $(x=$ test $\$ Y, y=\operatorname{predict}(r f r$, test [, predictors $]) \$$ predictions )

cvr_full <- data.frame $(x=d f \$ Y, y=\operatorname{predict}(\operatorname{rfr}, \operatorname{df}[$, predictors $]) \$$ predictions )

\# Create regression validation model

$\mathrm{rfr}_{-} \mathrm{Cv}<-\operatorname{lm}(\mathrm{y} \sim \mathrm{x}$, data $=\mathrm{cvr})$

rfr_clf $r_{-}$ull $<-\operatorname{lm}\left(y \sim x\right.$, data $\left.=\mathrm{cvr}_{-} f u l l\right)$

\# Build another test regression with $1 \mathrm{~m}$ instead of $\mathrm{rfr}$ $\mathrm{Cv}_{-} \mathrm{lm}<-$ data.frame $\left(\mathrm{x}=\right.$ test $\$ \mathrm{Y}, \mathrm{y}=$ predict $\left(\mathrm{lm}_{-}\right.$final, test $[$, predictors ]) )

$c v_{-} x g b<-$ data.frame $\left(x=y_{-}\right.$test,$y=$ predicted $)$

\# rank-deficit warning ignored for now...

\}

\# Store summary of model as new object

modr $<-$ summary $\left(\mathrm{rfr}_{-} \mathrm{cv}\right)$

modr

\# modr_full $<-\operatorname{summary}\left(\mathrm{rfr}_{-} \mathrm{CV}_{-}\right.$full $)$

\# modr_full

\# Checkpoint our regression validation model object summary saveRDS(modr, paste0 ("./models/mod_summary/", clean_time, "_mod_ summary.RDS") ) 
\# modr $<-$ readRDS(paste0 ("./models/mod_summary/", clean_time, "_mod_ summary.RDS") )

\# TODO verify p-value thing ... $\operatorname{lmp}()$ should be suffice

\# Compute RMSE on test data versus predictions

rmse $<-\operatorname{sqrt}\left(\right.$ mean $\left.\left((\operatorname{cvr} \$ y-\operatorname{cvr} \$ x)^{\wedge} 2\right)\right)$

rmse

\# Plot Validation Regression

testRegression $(\mathrm{df}=\mathrm{cvr}, \mathrm{x}=$ "x", $\mathrm{y}=$ "y", outdir $="$./figures/lm_obs _pred/", clean_time $=$ paste0 $($ clean_time, $"$ _rf"), reduced $=F$ )

testRegression $\left(\mathrm{df}=\mathrm{cv} \_\mathrm{xgb}, \mathrm{x}=\right.$ "x", $\mathrm{y}=$ "y", outdir $=$ "./figures/lm_ obs_pred/", clean_time = paste0 (clean_time, "_xgb"), reduced $=F$ )

testRegression $\left(\mathrm{df}=\mathrm{cv} \_\mathrm{lm}, \mathrm{x}=" \mathrm{x} ", \mathrm{y}=\right.$ "y", outdir $="$./figures $/ \mathrm{lm}_{-}$ obs_pred/", clean_time $=$ paste0 $($ clean_time, $"$ _lm" $)$, reduced $=F)$

\# testRegression $(\mathrm{df}=\mathrm{cvr}, \mathrm{x}=$ "x", $\mathrm{y}=$ "y", outdir $="$./figures/misc $/ "$, clean_time $=$ clean_time, reduced $=\mathrm{F}$ )

\# testRegression $\left(\mathrm{df}=\mathrm{cvr}_{-}\right.$full, $\mathrm{x}=$ "x", $\mathrm{y}=$ "y", outdir $=$ "./figures /lm_obs_pred/full_", clean_time $=$ clean_time, reduced $=\mathrm{F}$ )

\# Plot regression with train and test data

\# TODO Store model summary stats in table for safe keeping

\# LOOCV

\# Determine viable nodes

n_obs_node $<-$ train $\% \%$ group_by (node) $\% \%$ summarise $(\mathrm{n}=\mathrm{n}())$

n_threshold $<-100$

$\mathrm{p}<-\operatorname{ggplot}\left(\mathrm{n} \_\right.$obs_node, aes $(\mathrm{x}=\operatorname{reorder}($ node,$\left.-n), \mathrm{y}=\mathrm{n})\right)+$ geom_col ()$+$ geom_hline (yintercept $=n_{-}$threshold, $\operatorname{col}=$ "firebrick", linetype $=$ "dashed") + theme_minimal ()$+$ theme $($ axis.text. $x=$ element_text $($ angle $=90$, vjust $=0.5)$ , axis.title.x = element_blank ())

$\mathrm{p}$

png ("./figures/loocv/n_threshold/n_threshold.png"

, width $=8$

, height $=6$

, units = "in" 


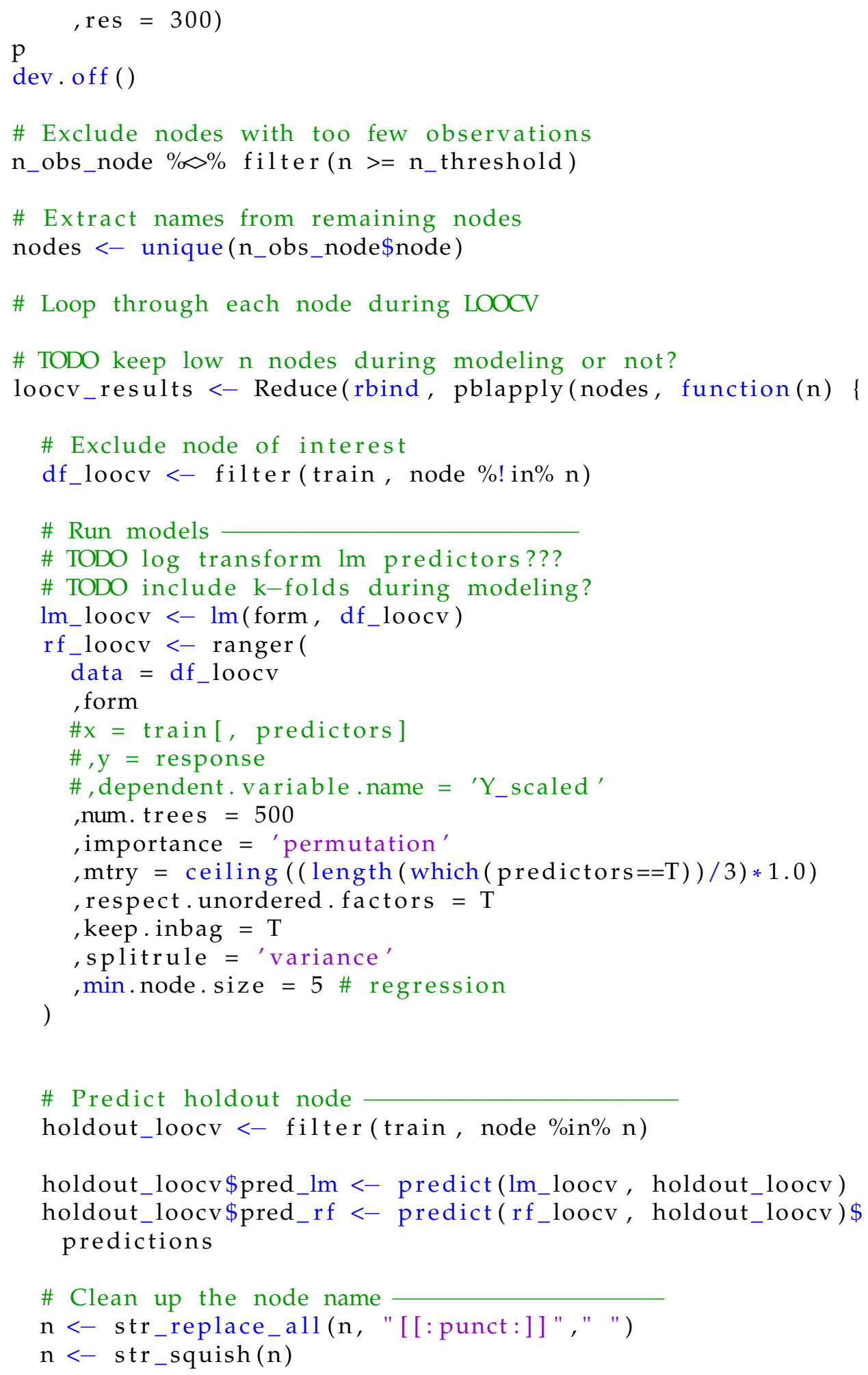




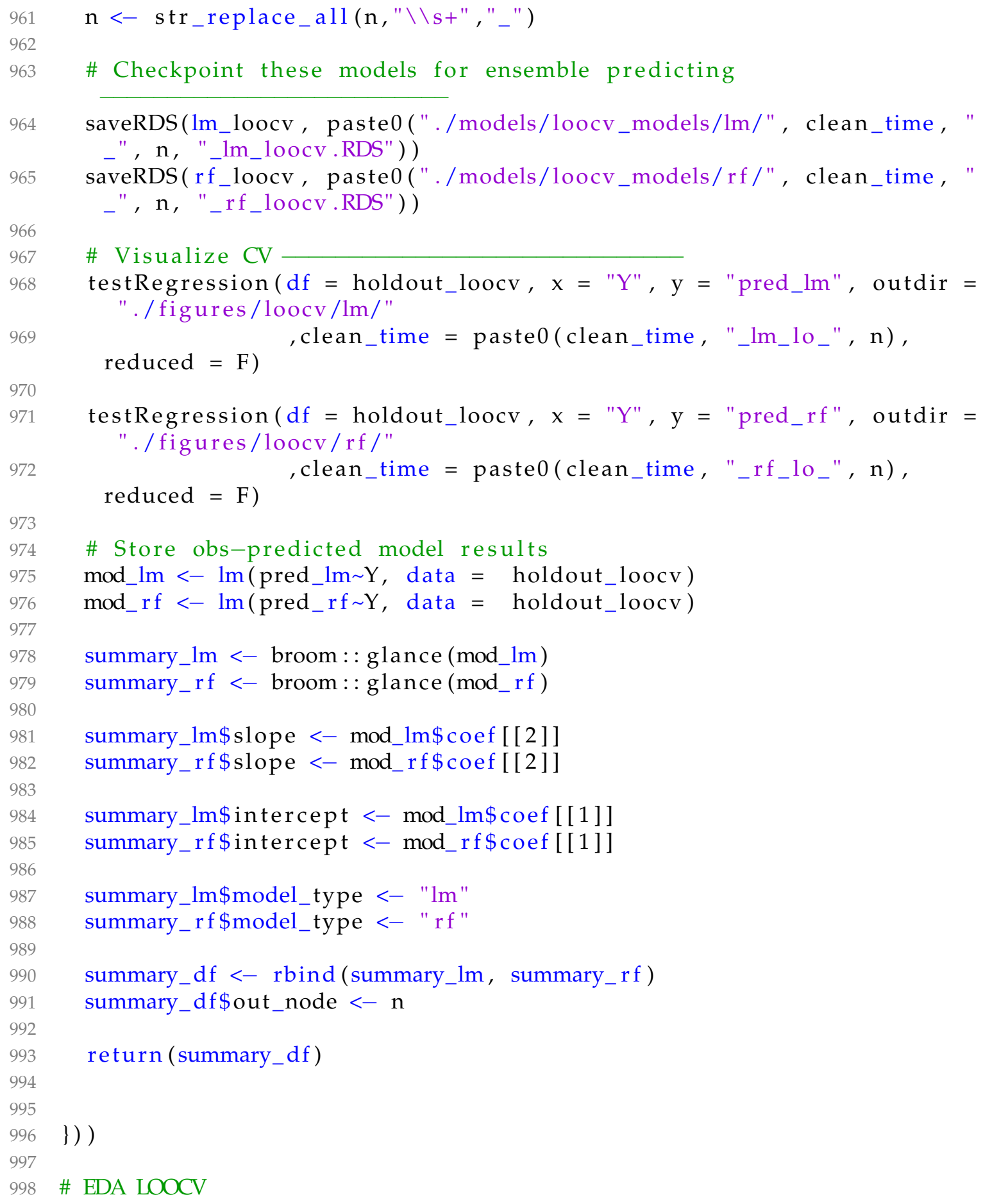




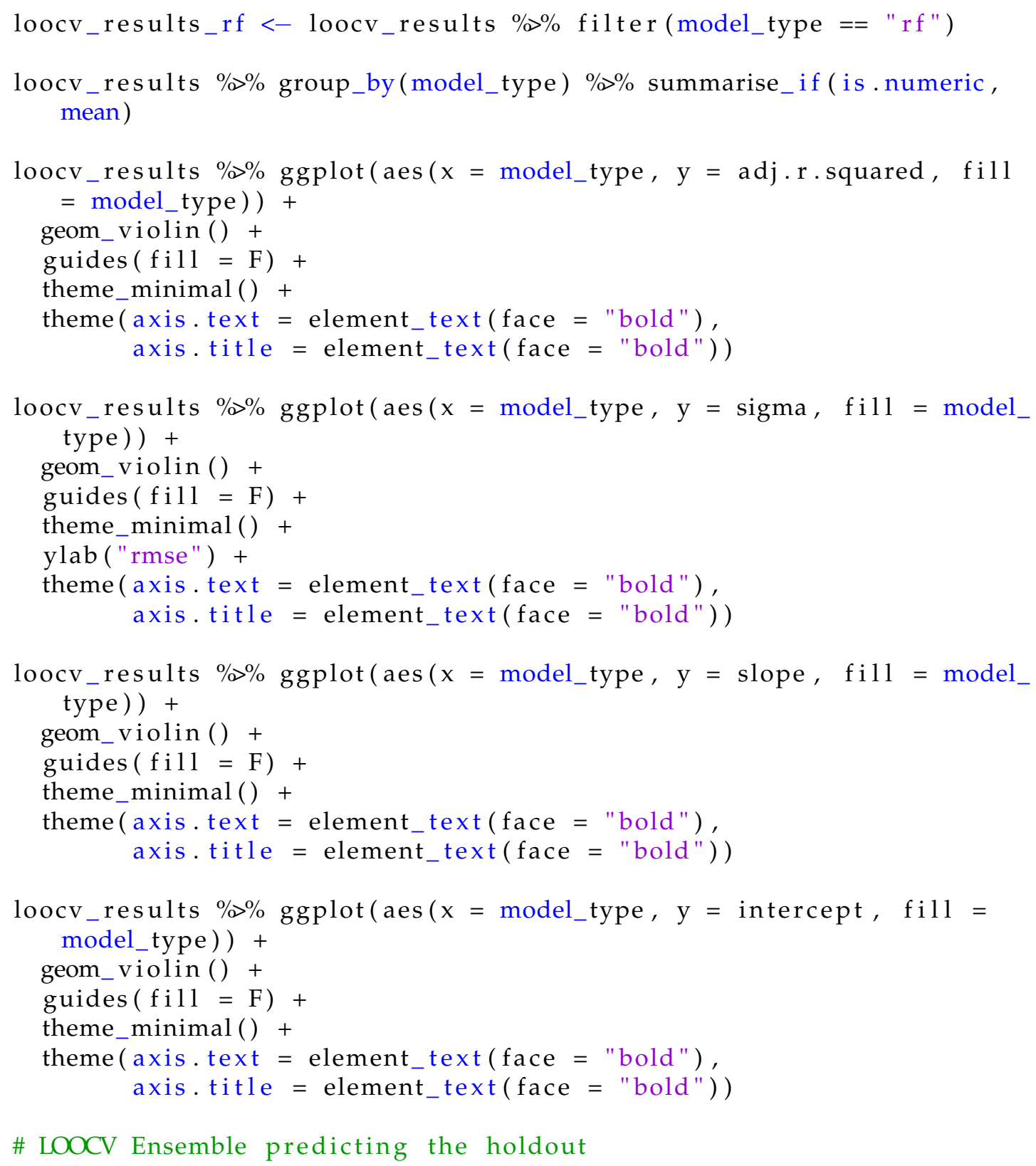




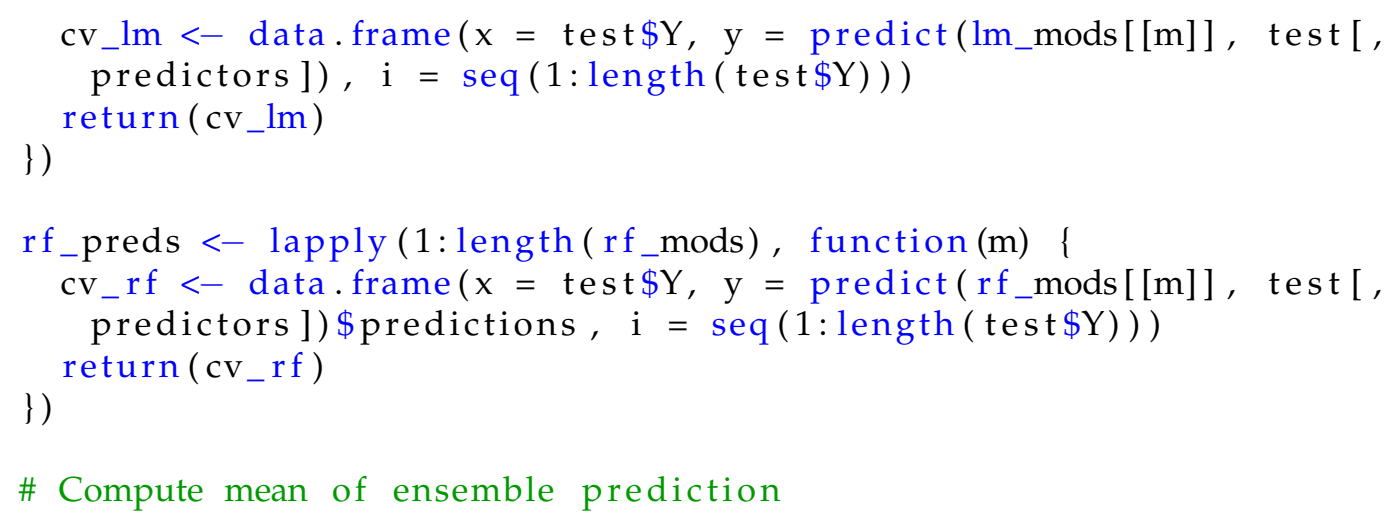

ens_lm_cv $<-$ data.table : : rbindlist (lm_preds) [, lapply (.SD, mean), list (i) ]

ens_rf_cv $<-$ data.table:: rbindlist (rf_preds) [, lapply (.SD, mean), list (i ) ]

testRegression $(\mathrm{df}=$ ens_lm_cv, $\mathrm{x}=$ "x", $\mathrm{y}=$ "y", outdir $=$ "./figures/ lm_obs_pred/", clean_time $=$ paste0 (clean_time, "_lm_ensemble"), reduced $=\mathrm{F}$ )

testRegression $(\mathrm{df}=$ ens_rf_cv, $\mathrm{x}=$ "x", $\mathrm{y}=$ "y", outdir $=$ "./figures/ lm_obs_pred/", clean_time $=\operatorname{paste} 0$ (clean_time, "_rf_ensemble"), reduced $=\mathrm{F}$ )

\# testRegression $(\mathrm{df}=$ ens_xgb_cv, $x=" x ", y=" y "$, outdir $=" . /$ figures/lm_obs_pred/", clean_time = paste0(clean_time, "_xgb_ ensemble"), reduced $=F$ )

\section{Source/predict.R}

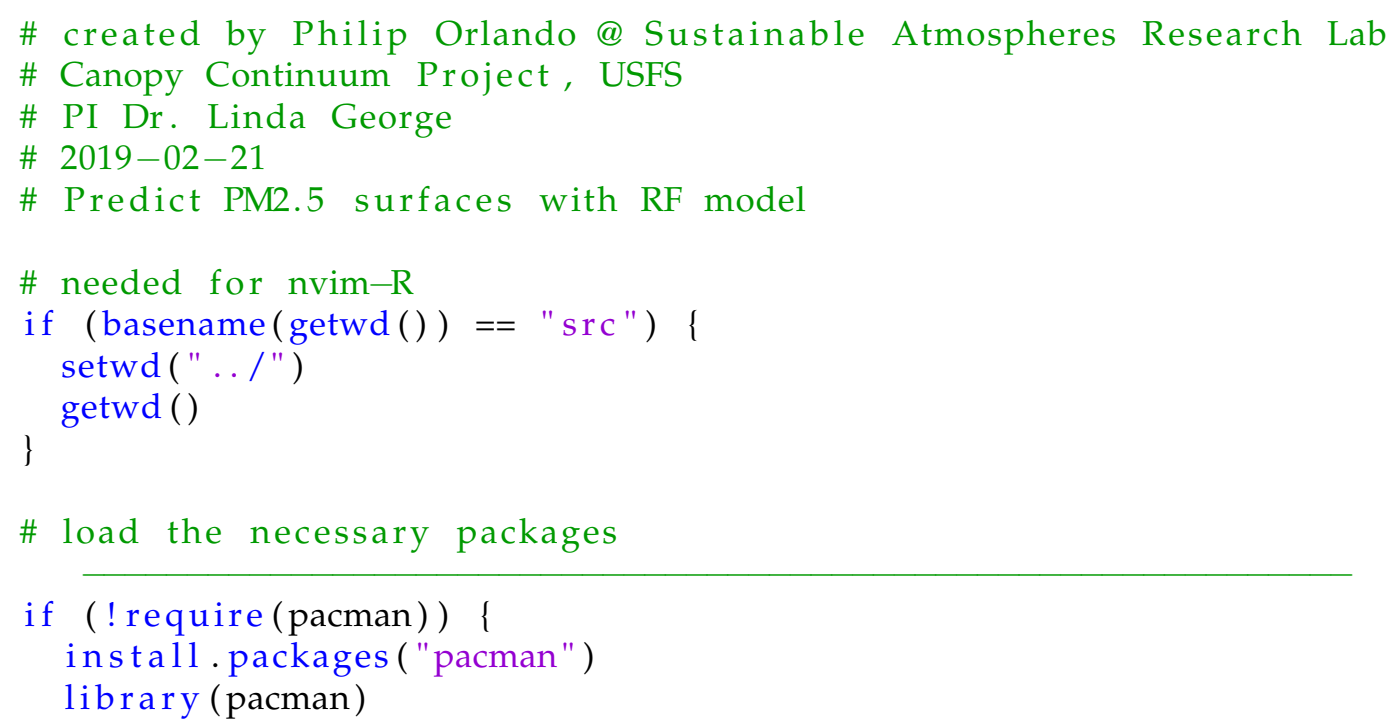




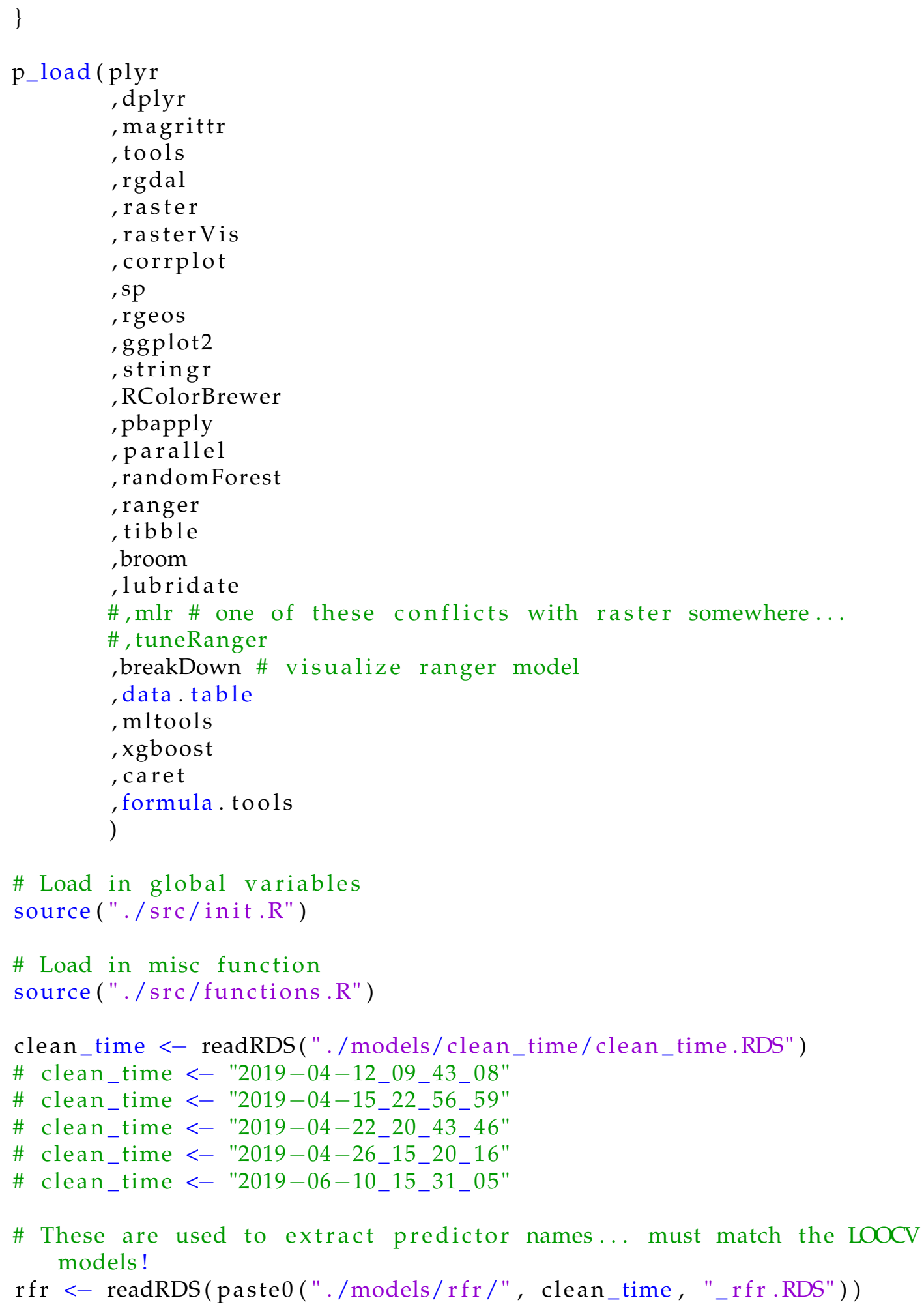




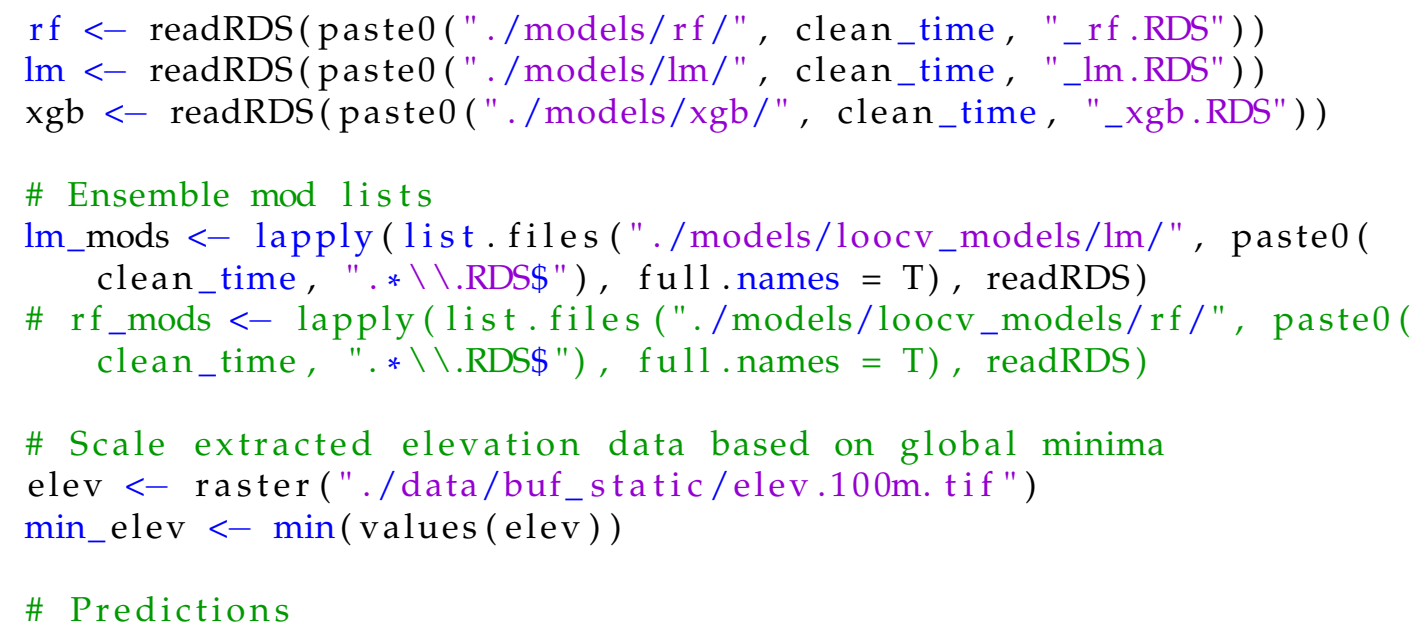




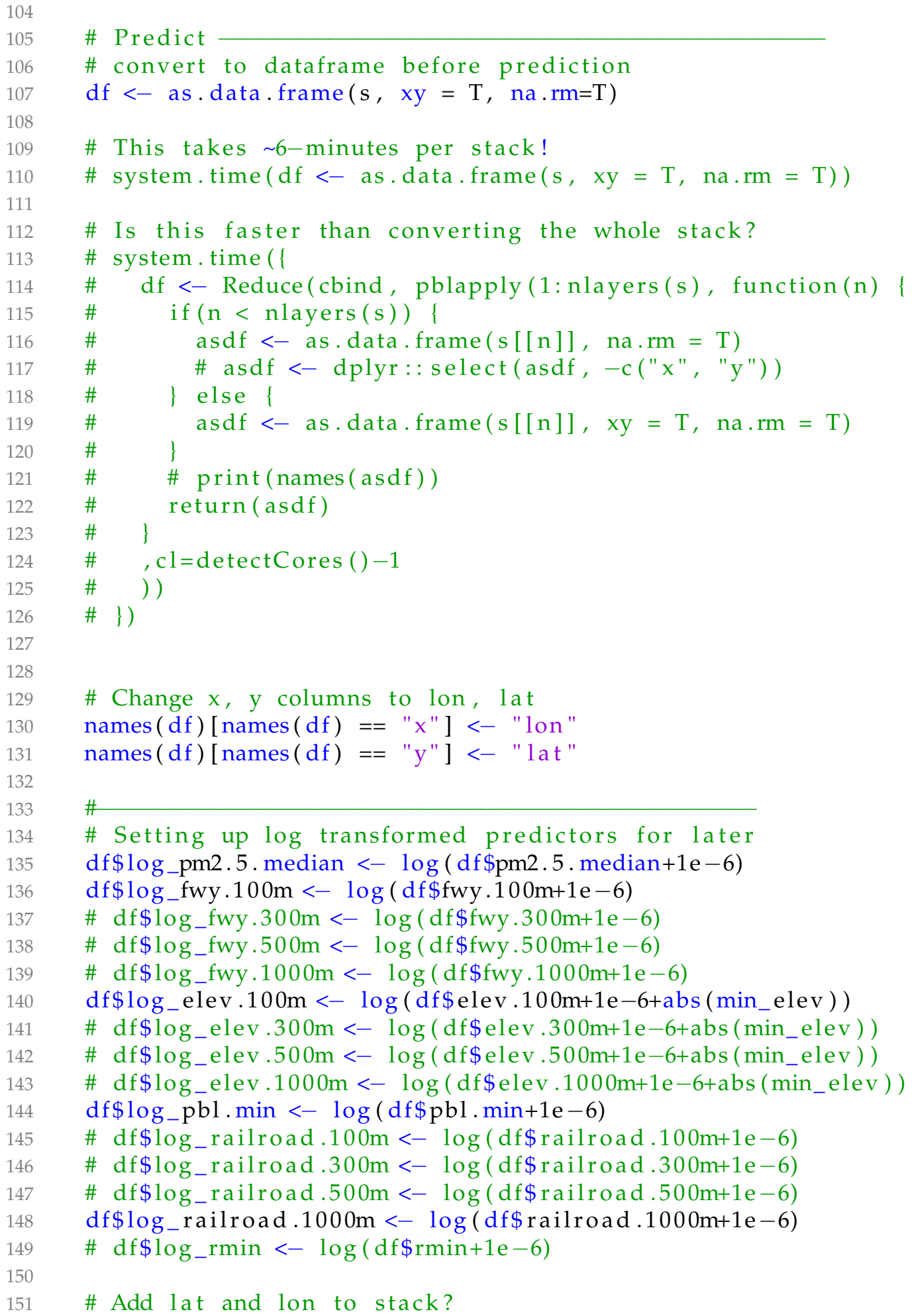




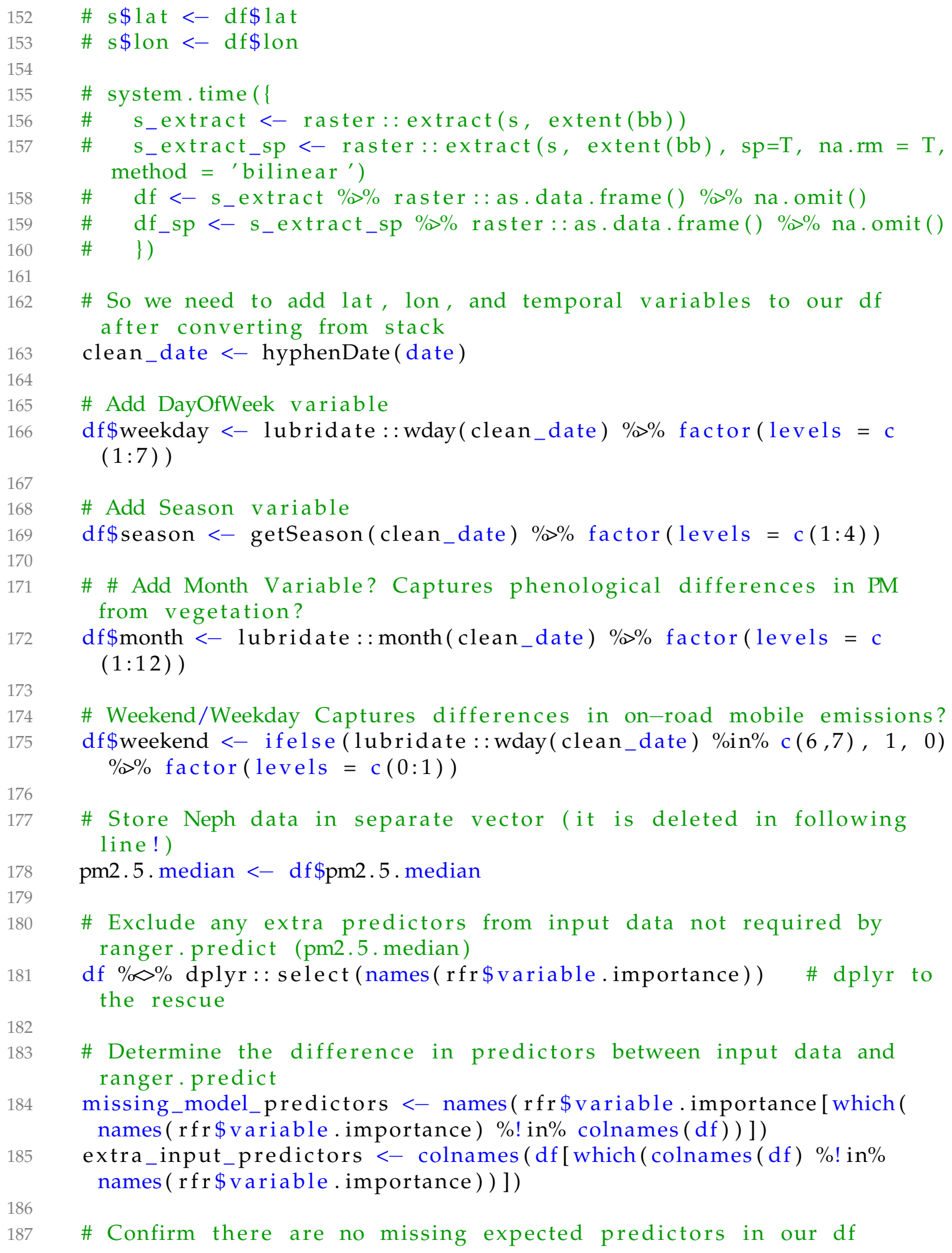




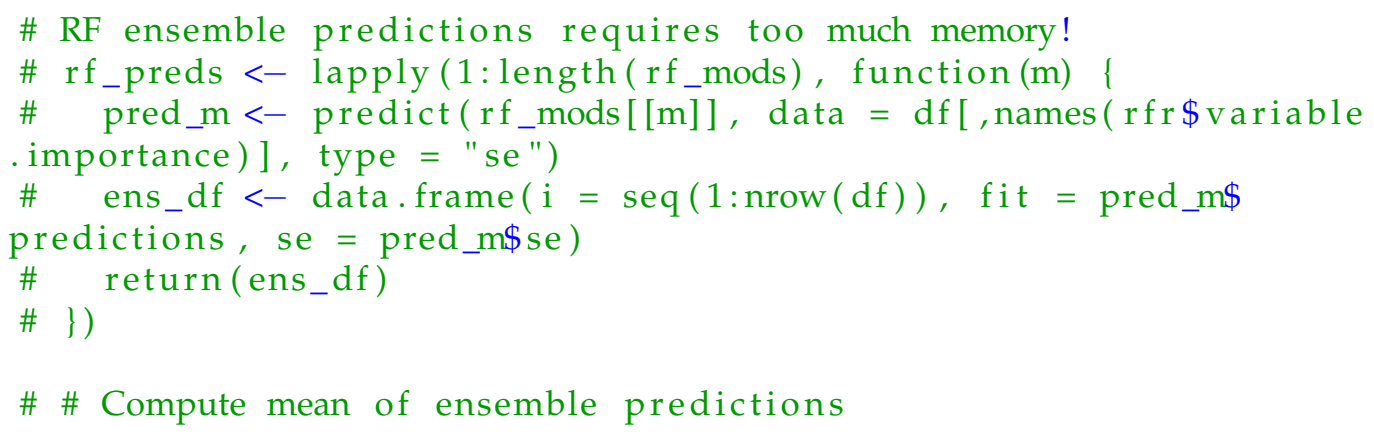




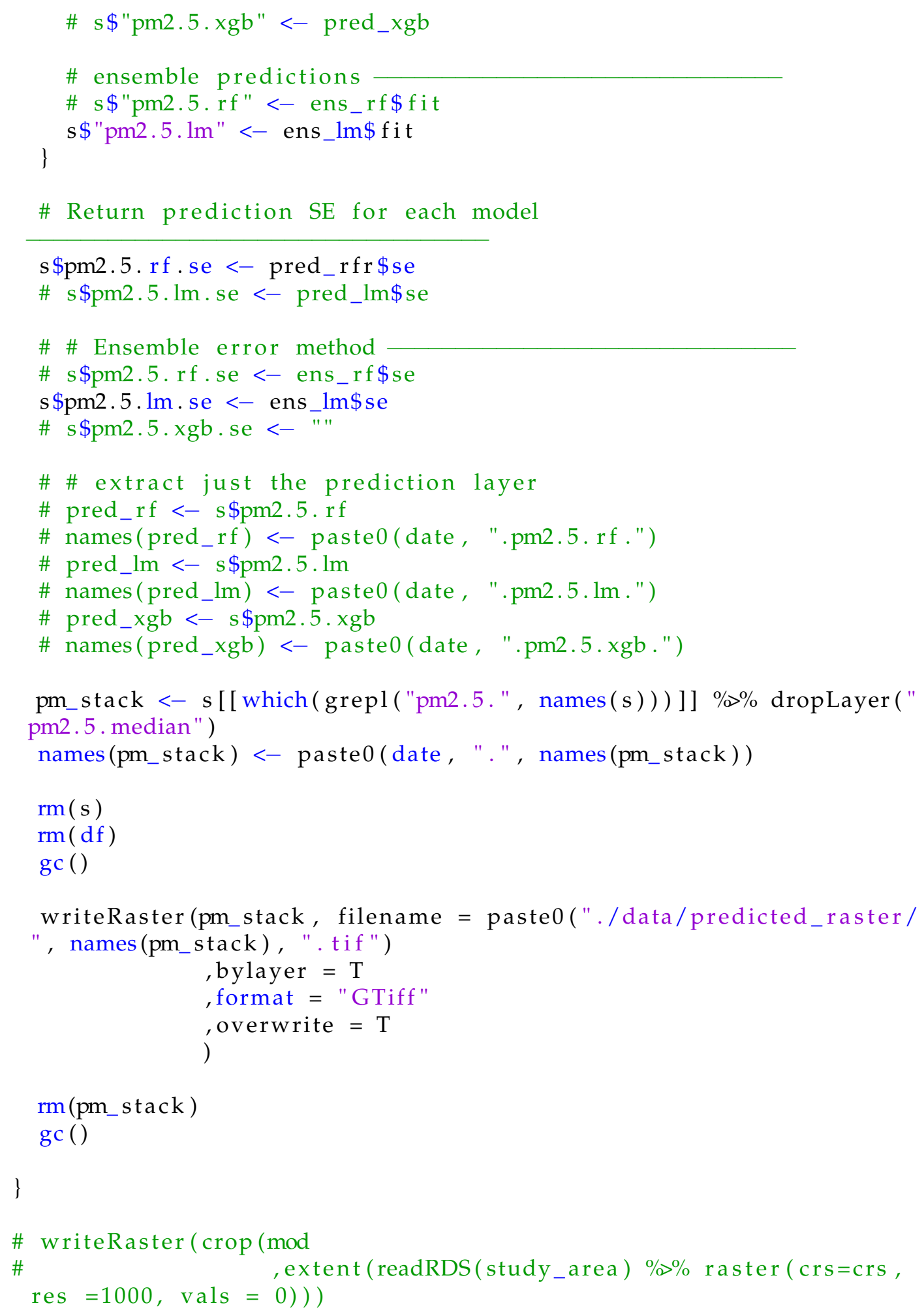




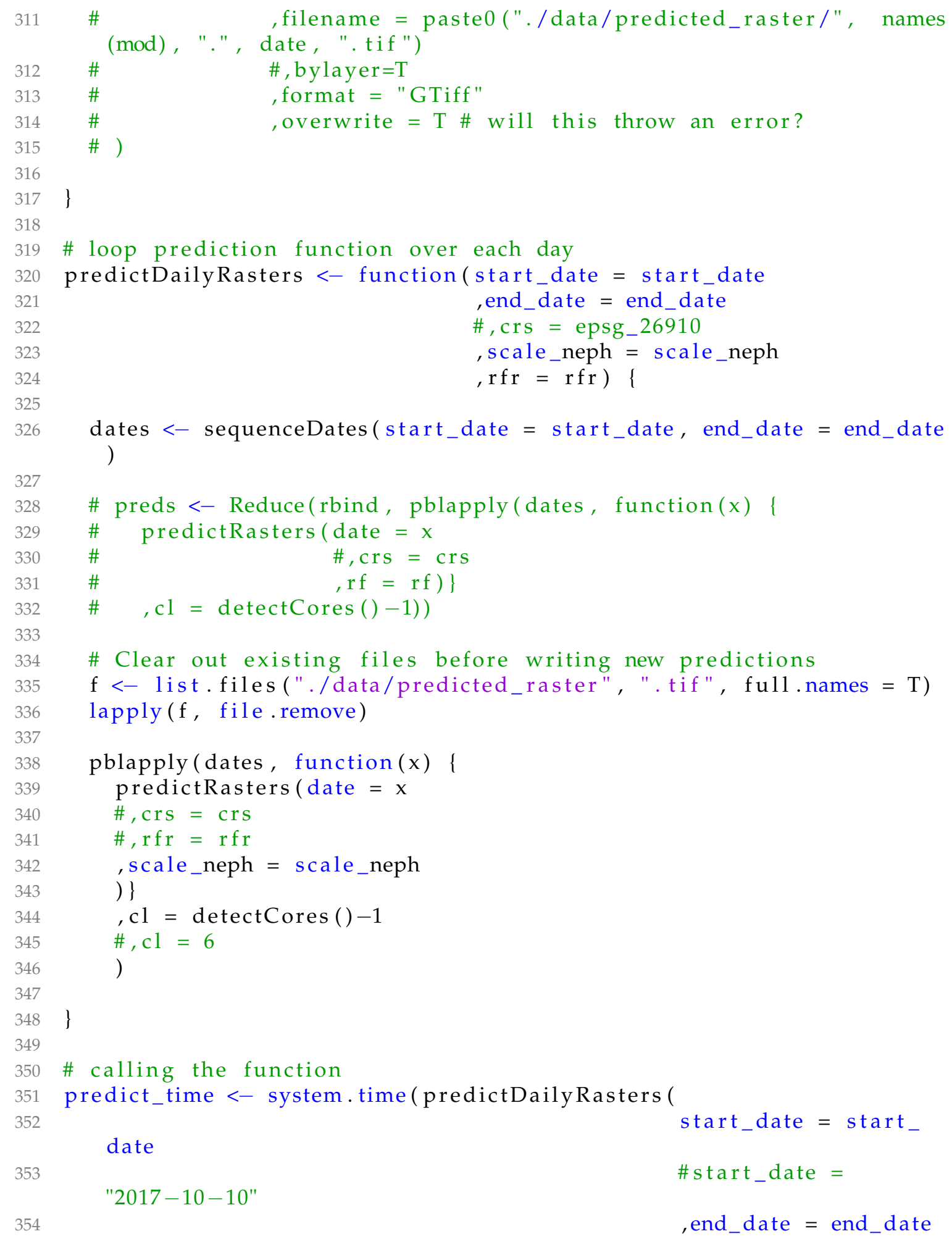




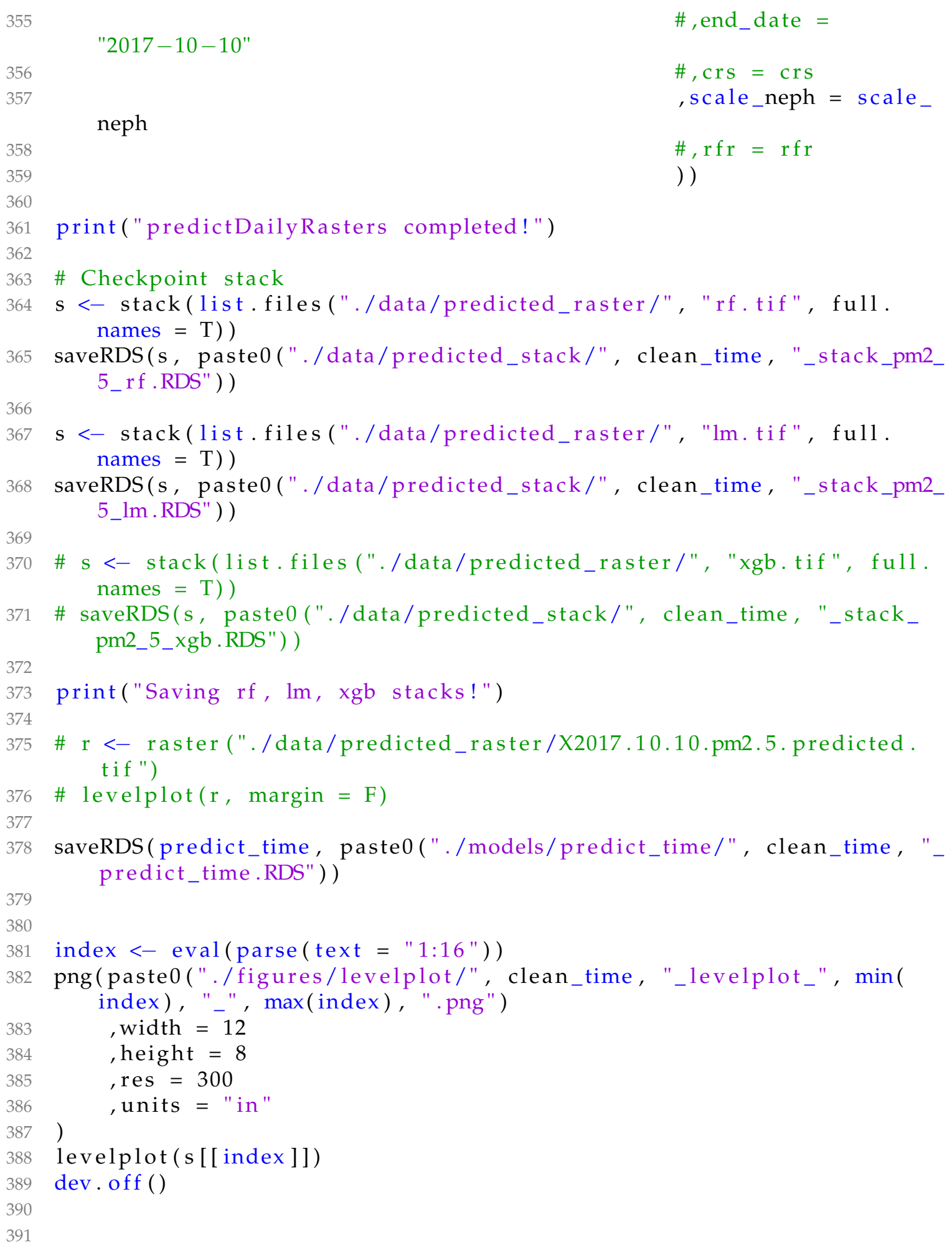

$$
\text { "2017-10-10" }
$$

neph 


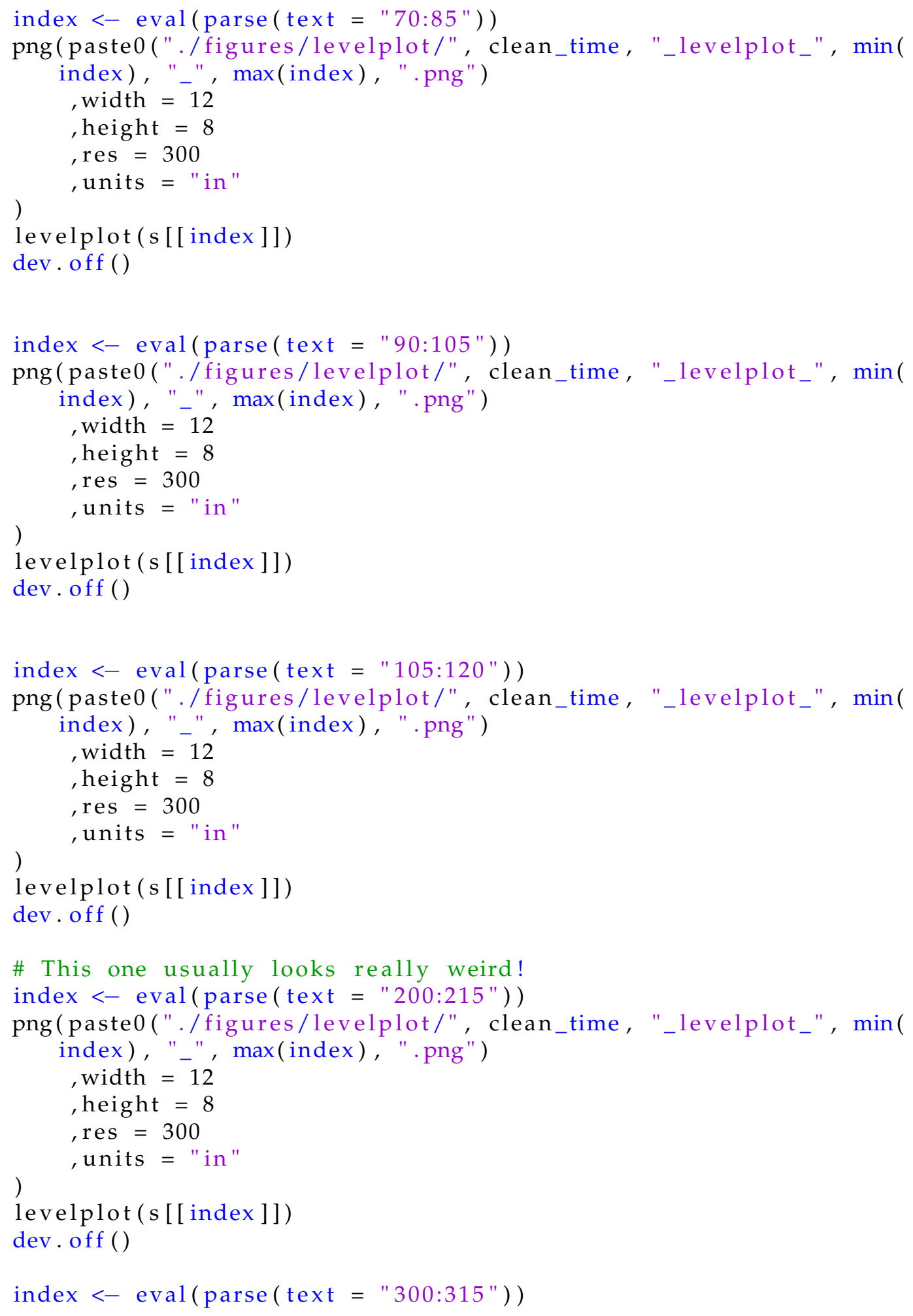




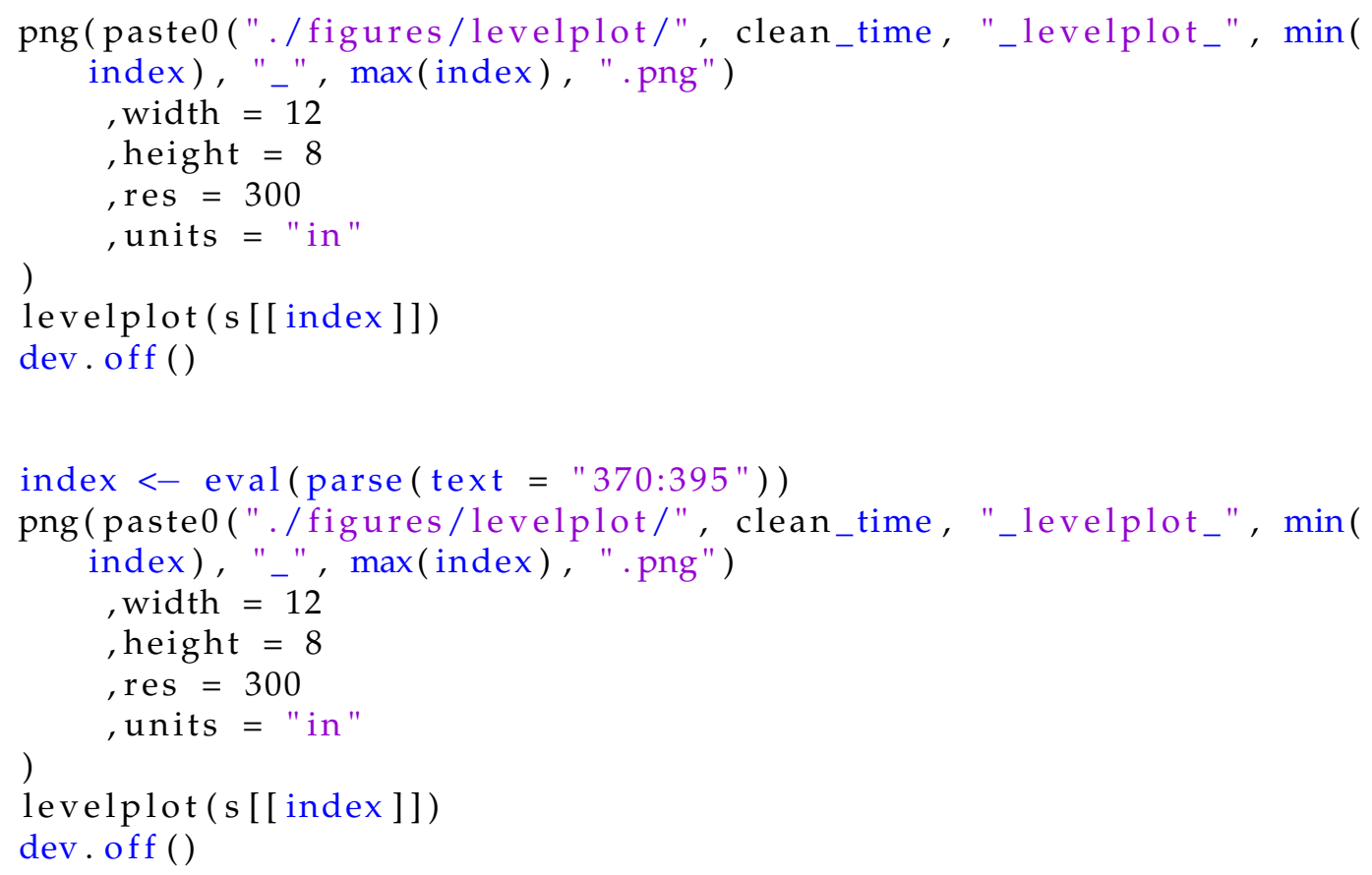

\section{Source/vectorToRaster.R}

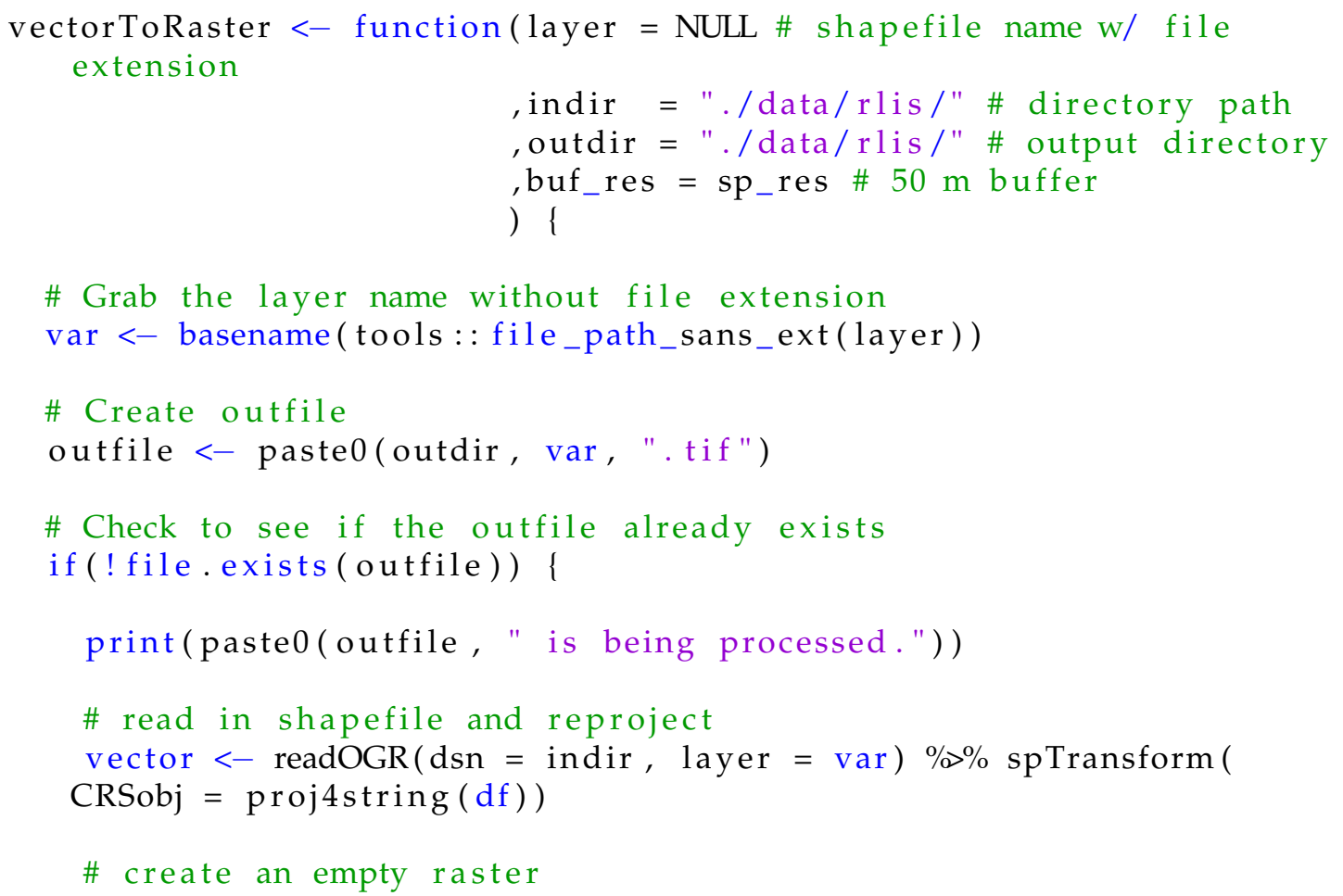




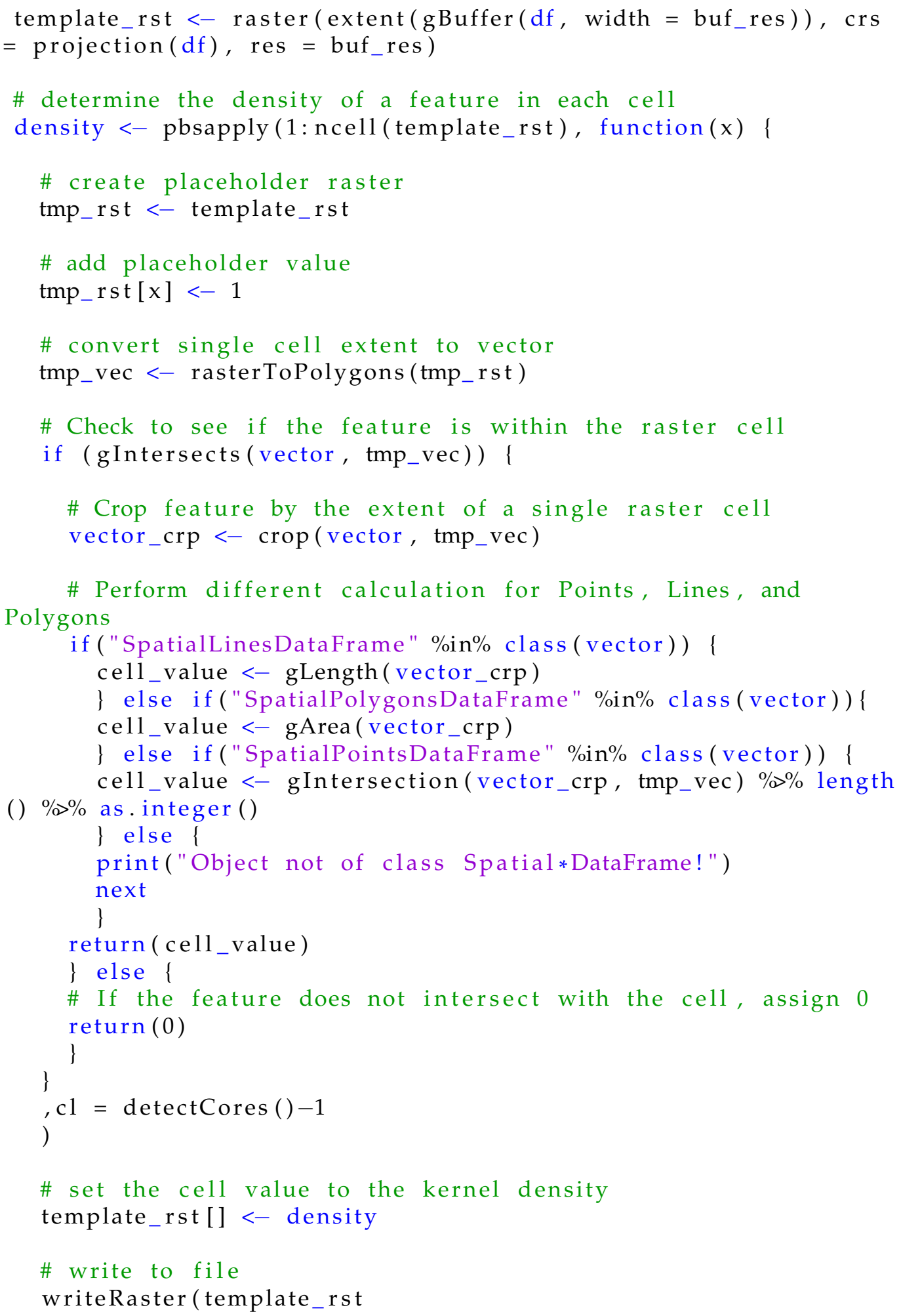




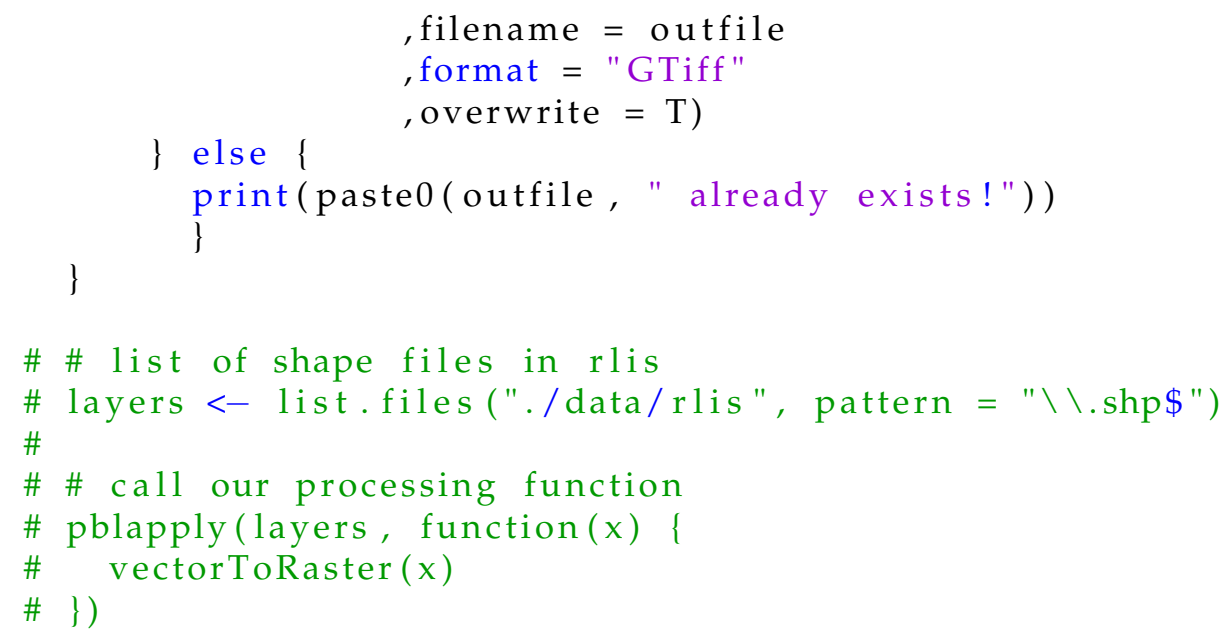

Source/purpleair_id_key.py

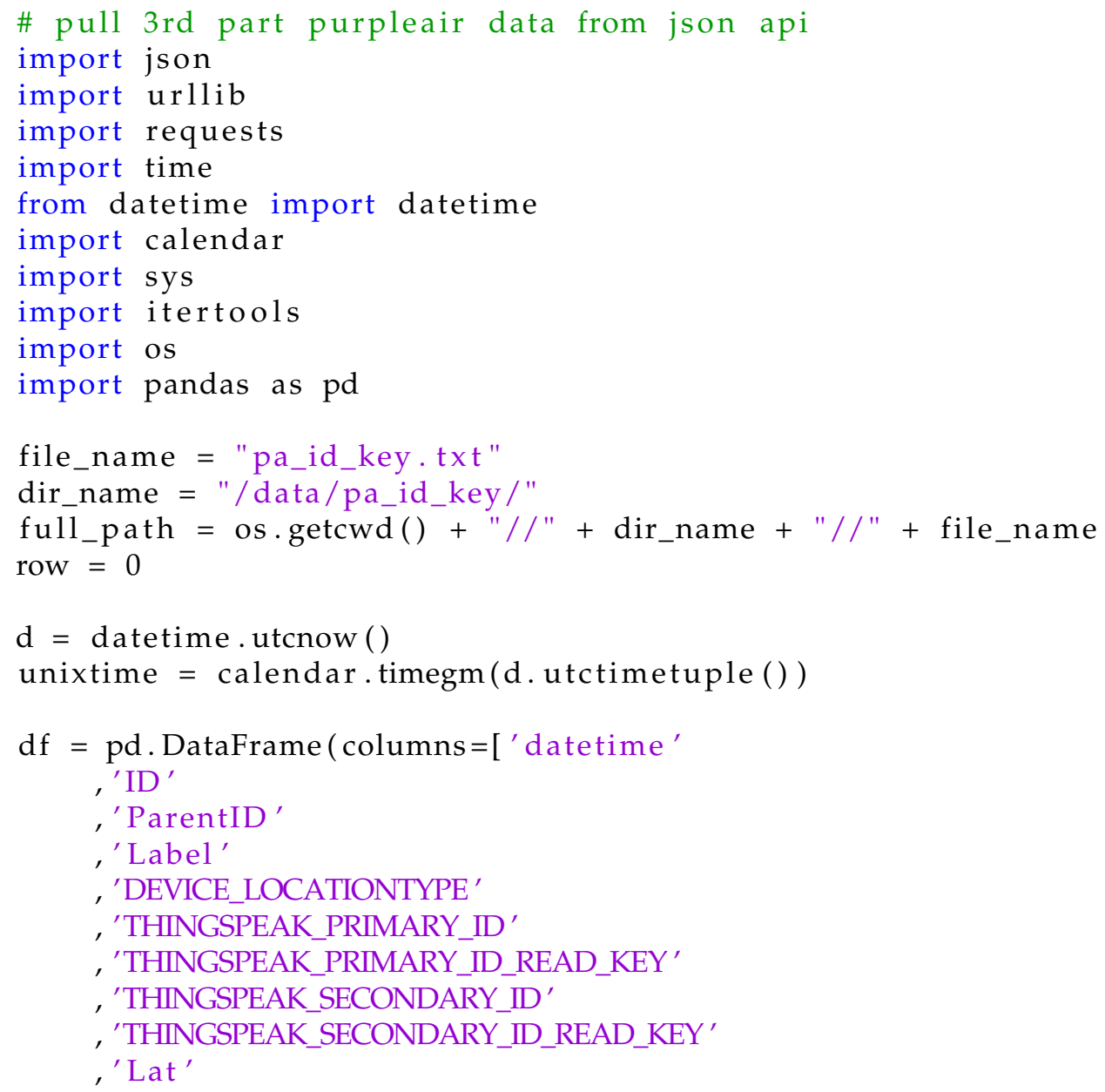




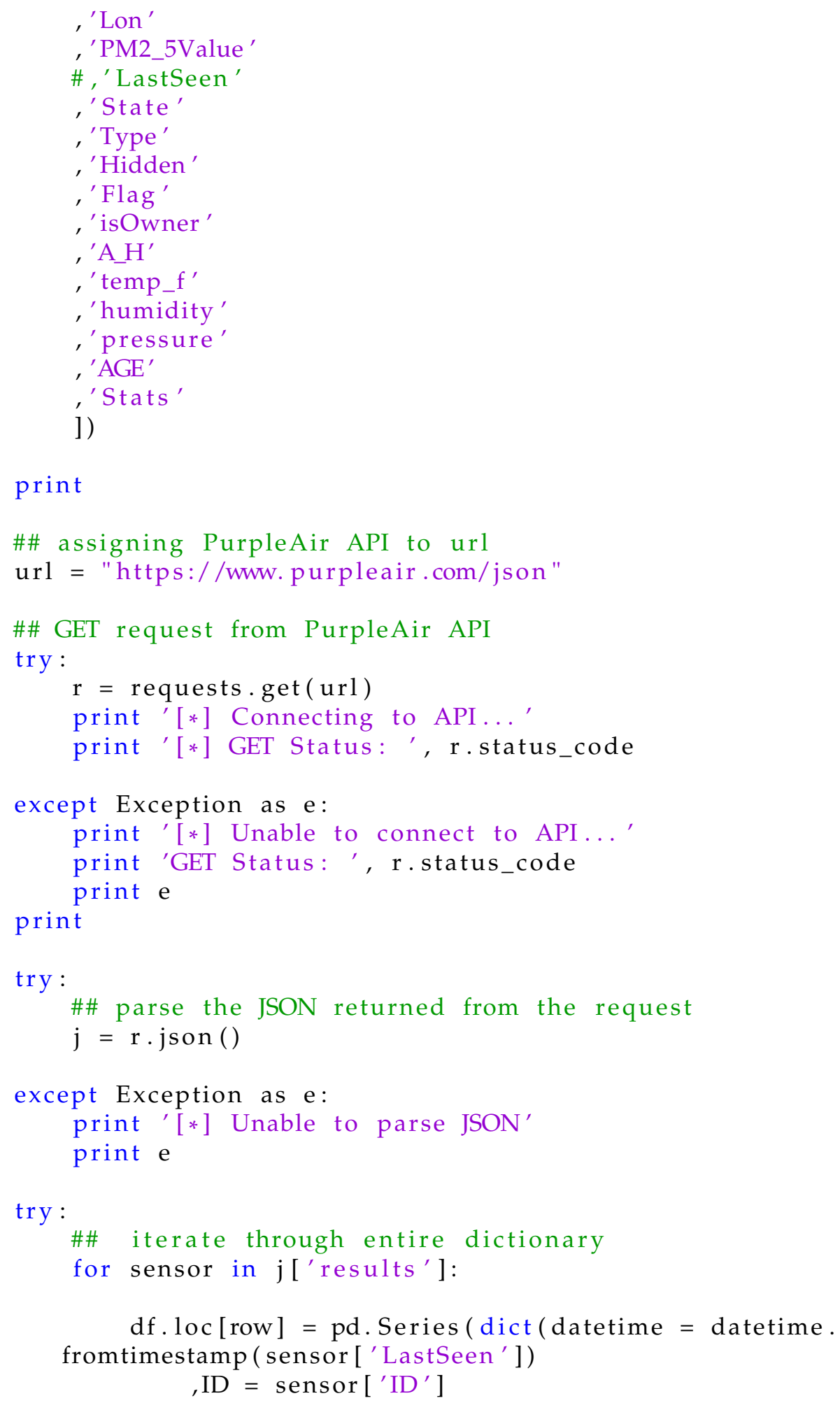




$$
\begin{aligned}
& 3 \\
& 4 \\
& 5 \\
& 6 \\
& 7 \\
& 8 \\
& 9
\end{aligned}
$$

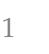

\section{Source/create_observation.sql}

create table observation (

created_at varchar (20)

,id varchar(10)

, sensor char(1)

, label varchar(100)

, temp_f numeric , humidity numeric ,pm1_0_atm numeric ,pm2_5_atm numeric ,pm10_0_atm numeric 
,pm1_0_cf_1 numeric ,pm2_5_cf_1 numeric ,pm10_0_cf_1 numeric ,p_0_3_um numeric ,p_0_5_um numeric ,p_1_0_um numeric ,p_2_5_um numeric ,p_5_0_um numeric ,p_10_0_um numeric ,geom geometry );

Source/create_pdx_cf.sql

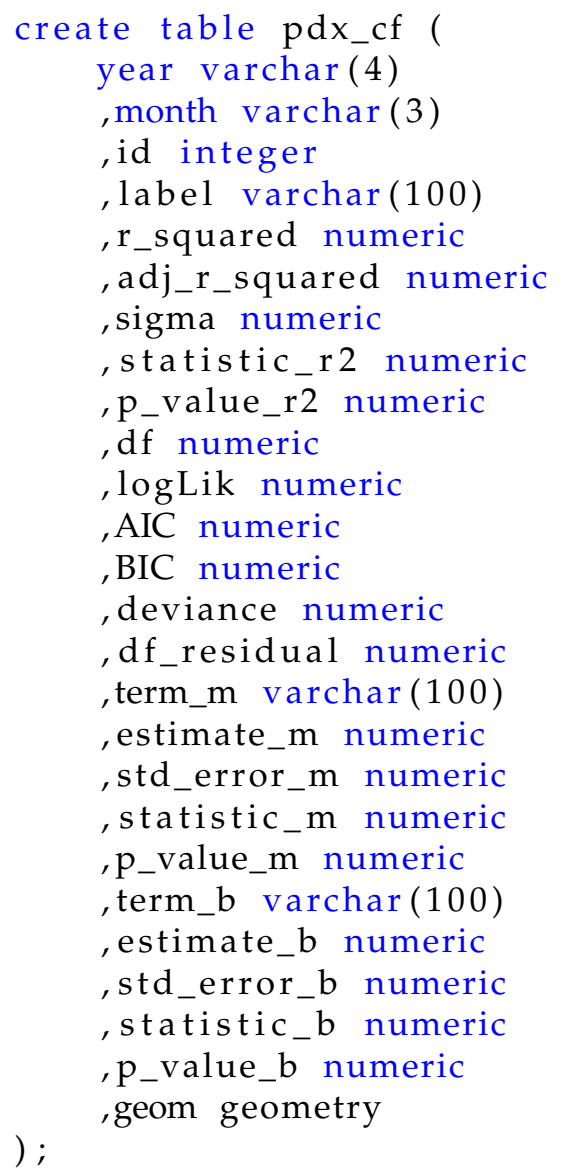

Source/thingspeakCollect.R

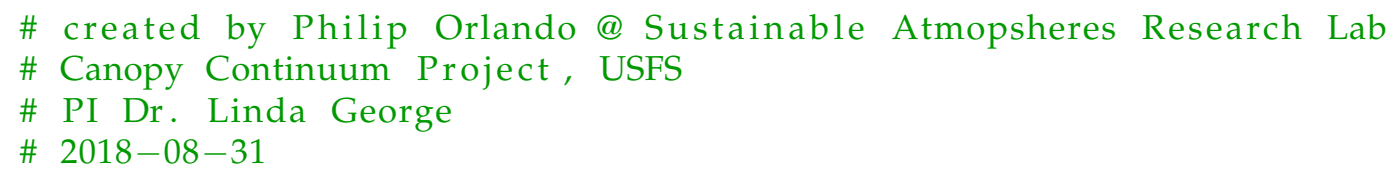




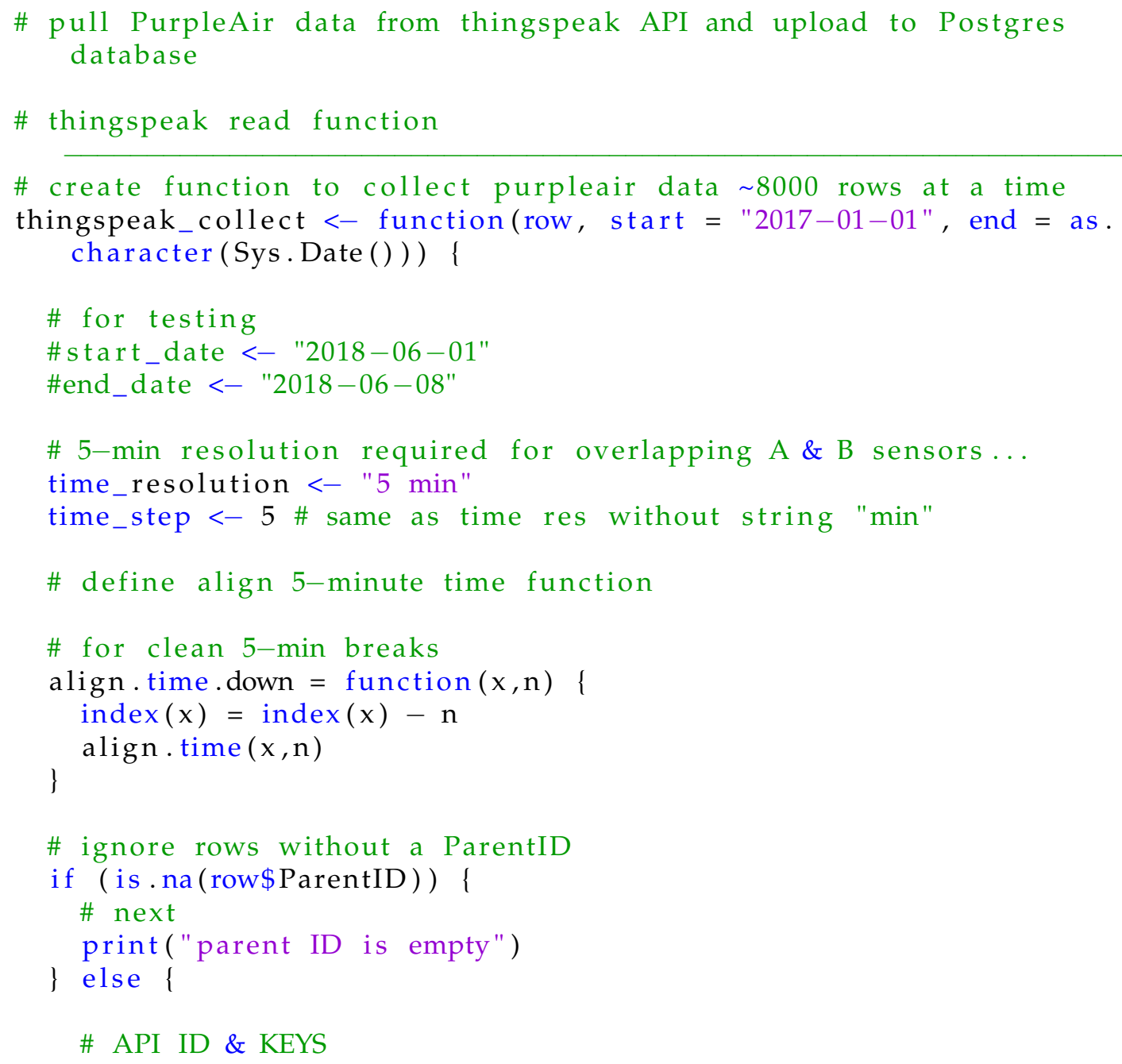




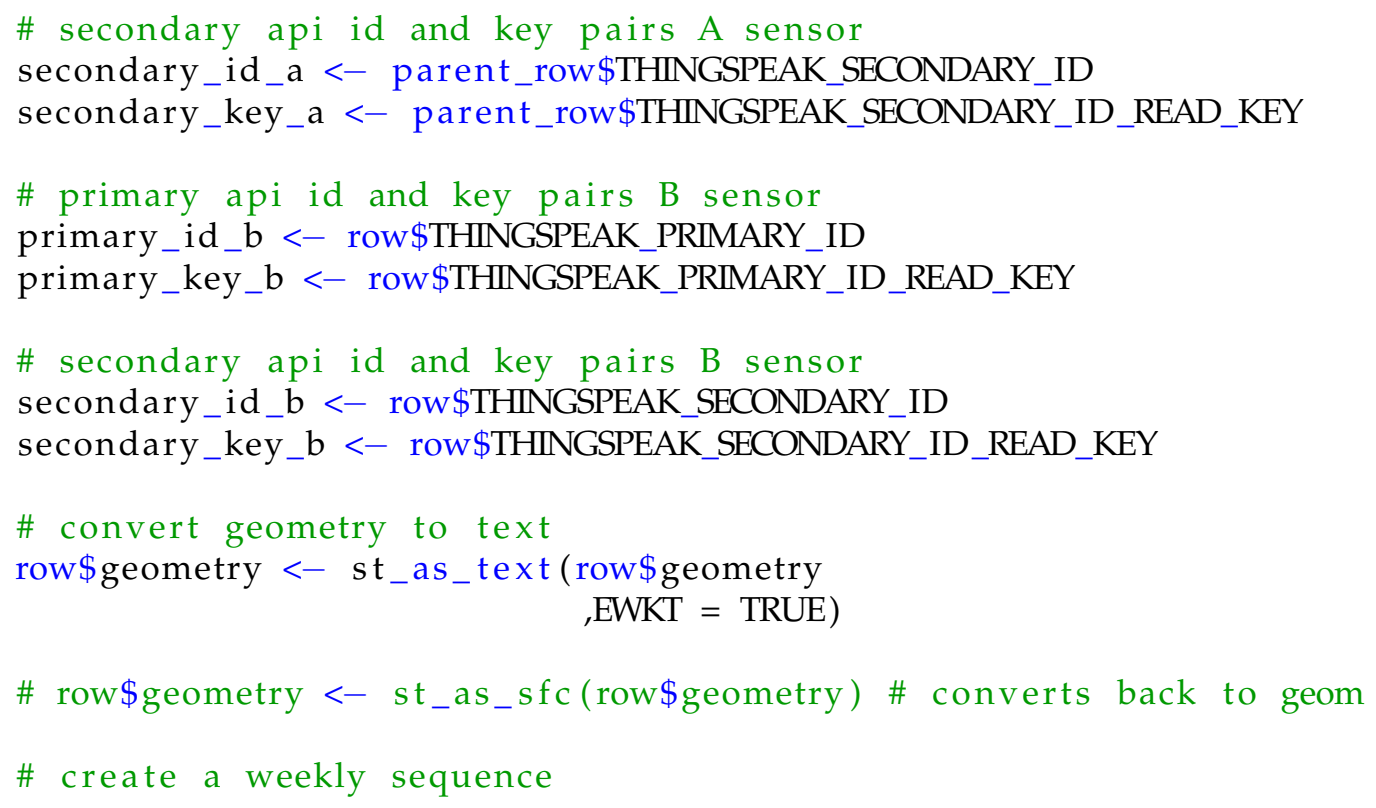




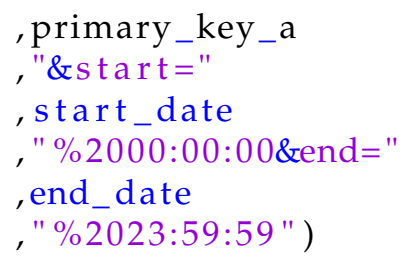




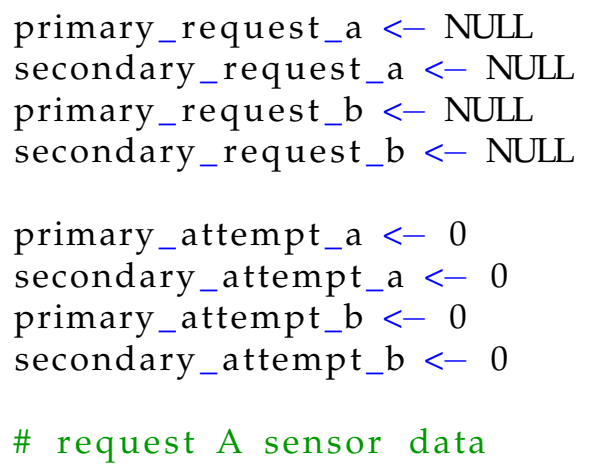


\{

while (is.null(secondary_request_b) \&\& secondary_attempt_b $<=3$ )

)) try (secondary_request_b $<-$ jsonlite : fromJSON (secondary_url_b print (paste0 ("Secondary Attempt B: ", secondary_attempt_b)) secondary_attempt_b $<-$ secondary_attempt_b +1

if (is_empty(primary_request_a\$feeds) I is_empty(secondary_ request_a $\$$ feeds) | is_empty(primary_request_b $\$$ feeds) I is_empty( secondary_request_b\$feeds) ) \{

print (paste0 (start_date, "-", end_date, " ", parent_row $\$$ Label , " is empty..."))

next

\} else \{

print (paste0 (start_date, "-", end_date, " ", parent_row $\$$ Label

, " A \& B are being processed..."))

\# channel A field names

secondary_fields_a $<-c\left(" c r e a t e d_{-} a t "\right.$

, "entry_id"

, "p_0_3_um"

,"p_0_5_um"

, "p_1_0_um"

, "p_2_5_um"

,"p_5_0_um" 


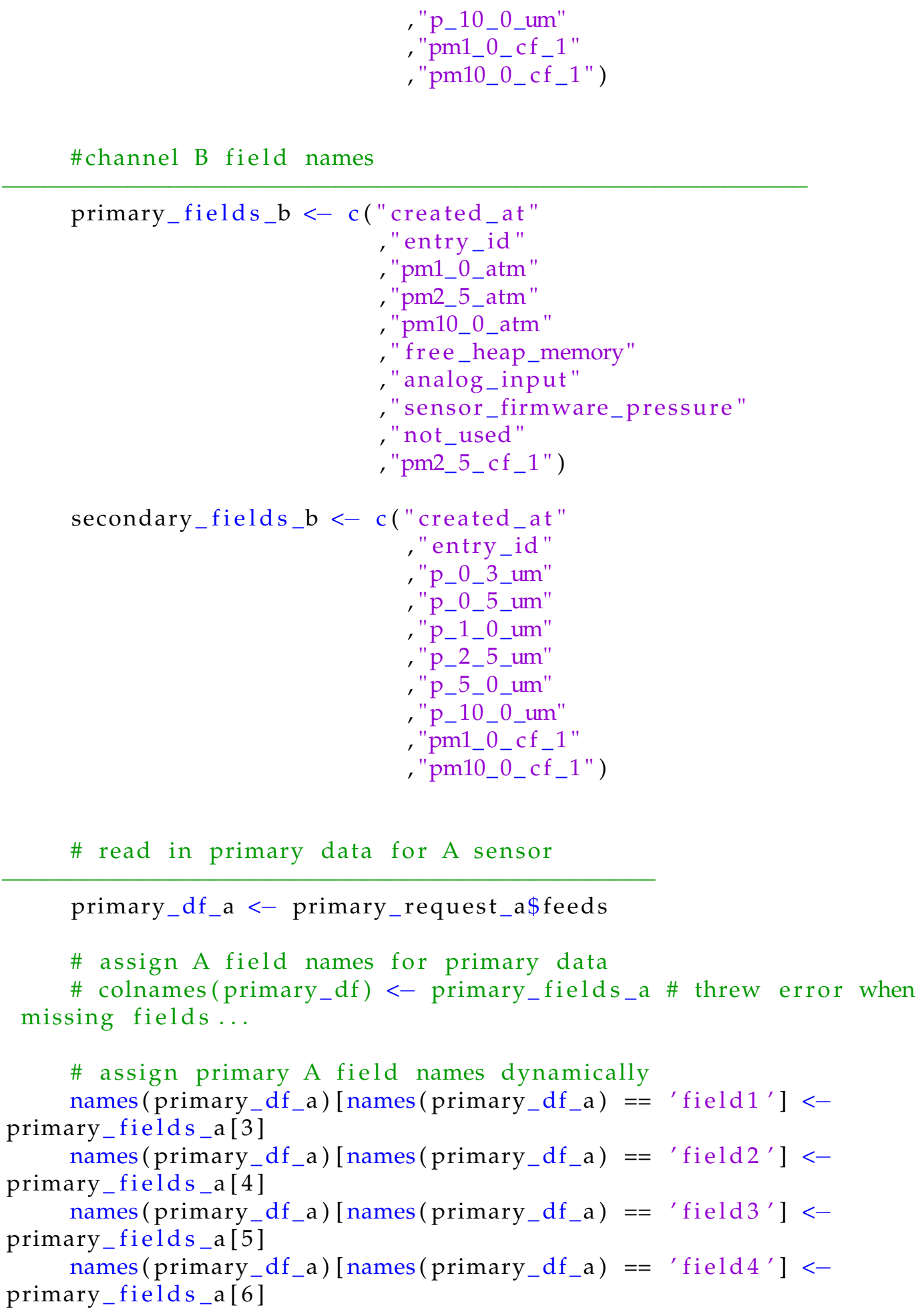


names $($ primary_df_a $)\left[\right.$ names $($ primary_df_a $)==$ 'field5 $\left.^{\prime}\right]<-$ primary_fields_a[7] names $($ primary_df_a $)\left[\right.$ names $($ primary_df_a $)=={ }^{\prime}$ field $\left.6^{\prime}\right]<-$ primary_fields_a[8] names (primary_df_a) [names $($ primary_df_a $)=={ }^{\prime}$ field7'] $<-$ primary_fields_a[9] names (primary_df_a) [names $($ primary_df_a $)=={ }^{\prime}$ field $\left.8^{\prime}\right]<-$ primary_fields_a[10]

\# remove non-numeric columns before grouping by date primary_df_a $<-$ primary_df_a $\% \%$ dplyr : : select(-c(entry_id, uptime_min, rssi_wifi_strength))

\# cast from character to numeric class $\operatorname{try}(\{$

) primary_df_a\$pm1_0_atm $<-$ as .numeric (primary_df_a\$pm1_0_atm primary_df_a\$pm2_5_atm $<-$ as . numeric (primary_df_a\$pm2_5_atm ) atm ) primary_df_a\$pm10_0_atm $<-$ as.numeric $($ primary_df_a\$pm10_0_ primary_df_a $\$$ temp_f $<-$ as.numeric (primary_df_a\$temp_f) primary_df_a\$humidity $<-$ as.numeric (primary_df_a\$humidity) - 1) primary_df_a\$pm2_5_cf_1 $<-$ as.numeric (primary_df_a\$pm2_5_cf

\} , silent $=$ TRUE)

\# aggregate by clean 5 -min breaks primary_xts_a $<-x t s($ primary_df_a $[,-1]$ , as.POSIXct (primary_df_a\$created_at SZ" , format $=$ "\%Y-\%m-\%dT\%H:\%M:\%

)) , $\mathrm{tz}=$ "GMT"

primary_means_a $<-$ period.apply (primary_xts_a, endpoints ( primary_xts_a, "mins", $\mathrm{k}=$ time_step), mean) primary_rounded_a $<-$ align.time.down(primary_means_a, time_ step $* 60$ ) primary_df_a $<-$ fortify (primary_rounded_a) names $($ primary_df_a $)[$ names $($ primary_df_a $)==$ "Index"] $<-"$ created_at" at )

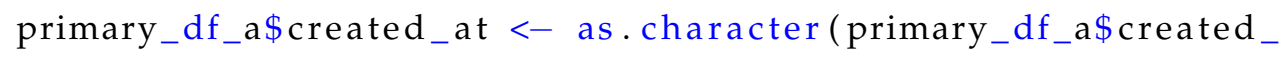


\# read in secondary data for A sensor

secondary_df_a $<-$ secondary_request_a $\$$ feeds

\# assign A field names for secondary data

\# colnames(secondary_df) $<-$ secondary_fields_a \# breaks when fields are missing

\# assign secondary A field names dynamically

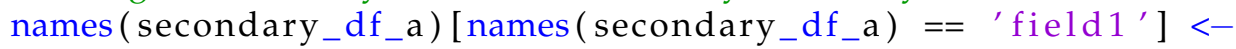
secondary_fields_a[3] names ( secondary_df_a) [names (secondary_df_a) == 'field2'] $<-$ secondary_fields_a[4] names ( secondary_df_a) [names (secondary_df_a) $==$ 'field3 $\left.^{\prime}\right]<-$ secondary_fields_a[5]

names (secondary_df_a) [names (secondary_df_a) == 'field4'] $<-$ secondary_fields_a[6] names (secondary_df_a) [names (secondary_df_a) $=={ }^{\prime}$ field5'] $<-$ secondary_fields_a[7] names ( secondary_df_a) [names (secondary_df_a) $=={ }^{\prime}$ field6 $\left.6^{\prime}\right]<-$ secondary_fields_a[8] names ( secondary_df_a) [names (secondary_df_a) $=={ }^{\prime}$ field7'] $<-$ secondary_fields_a[9] names ( secondary_df_a) [names (secondary_df_a) $=={ }^{\prime}$ field $\left.8^{\prime}\right]<-$ secondary_fields_a[10]

\# remove non-numeric columns before grouping id ) ) secondary_df_a $<-$ secondary_df_a $\% \%$ dplyr:: select $(-c($ entry

\# cast from character to numeric class

$\operatorname{try}(\{$

um) secondary_df_a $\$ p \_0 \_3 \_u m<-$ as. numeric $\left(\operatorname{secondary} \_d f \_a \$ p \_0 \_3\right.$ um) secondary_df_a\$p_0_5_um $<-$ as.numeric ( secondary_df_a\$p_0_5_ secondary_df_a\$p_1_0_um $<-$ as.numeric $\left(\operatorname{secondary} \_d f \_a \$ p \_1 \_0 \_\right.$ um) um) secondary_df_a\$p_2_5_um $<-$ as.numeric $\left(\operatorname{secondary} \_d f \_a \$ p \_2 \_5 \_\right.$ um) secondary_df_a\$p_5_0_um $<-$ as.numeric $\left(\operatorname{secondary} \_\mathrm{df} \_\mathrm{a} \$ \mathrm{p} \_0 \_5 \_\right.$ secondary_df_a $\$ p_{-} \_10_{-} 0 \_u m<-$ as. numeric $\left(\right.$ secondary_df_a $\$ p_{-} 10_{-}$ 0_um) $0 \_$cf_1) secondary_df_a\$pm1_0_cf_1 $<-$ as.numeric (secondary_df_a\$pm1_ 


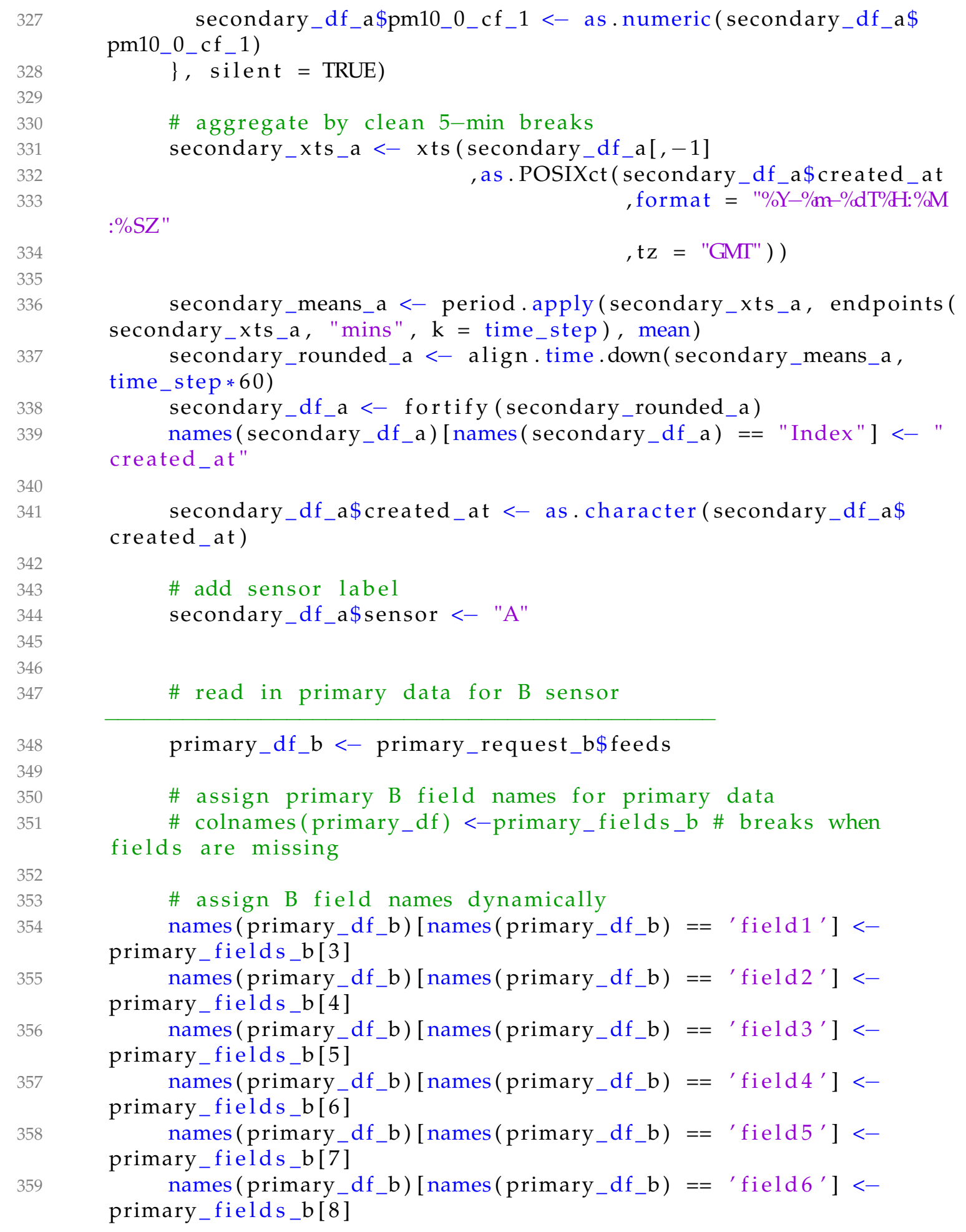




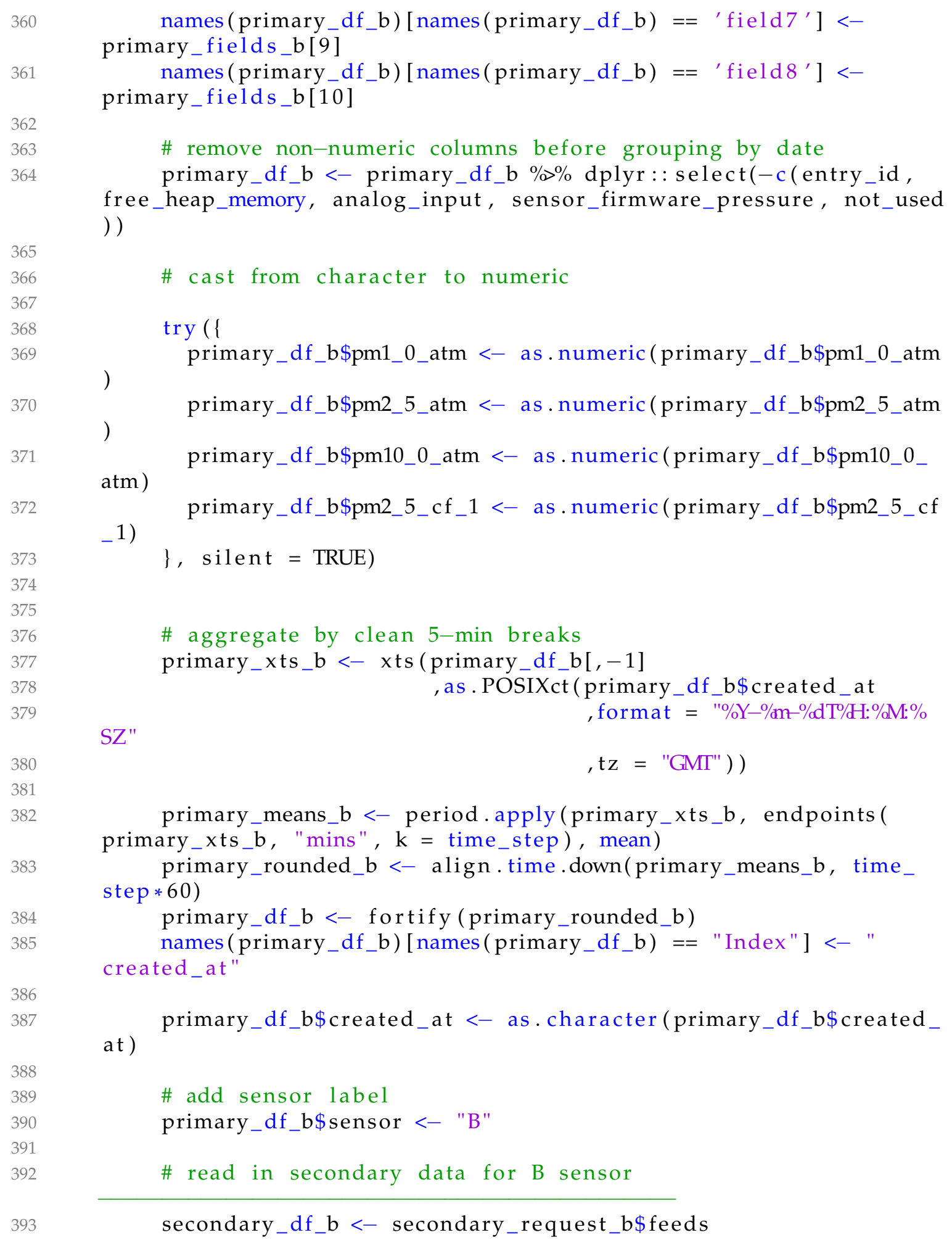

names $\left(\right.$ primary_df_b) [names $($ primary_df_b $)==$ 'field7 $\left.^{\prime}\right]<-$ primary_fields_b[9]

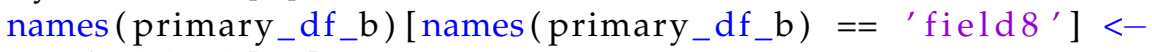
primary_fields_b[10]

\# remove non-numeric columns before grouping by date

primary_df_b $<-$ primary_df_b \% $\%$ dplyr : : select(-c(entry_id,

free_heap_memory, analog_input, sensor_firmware_pressure, not_used ))

\# cast from character to numeric

$\operatorname{try}(\{$ primary_df_b\$pm1_0_atm $<-$ as.numeric $($ primary_df_b\$pm1_0_atm

) primary_df_b\$pm2_5_atm $<-$ as . numeric $($ primary_df_b\$pm2_5_atm

) atm) primary_df_b\$pm10_0_atm $<-$ as . numeric $($ primary_df_b\$pm10_0_ -1) primary_df_b\$pm2_5_cf_1 $<-$ as. numeric $\left(\right.$ primary_df_b $\$ p m 2 \_5 \_c f$ \}, silent $=$ TRUE)

\# aggregate by clean 5 -min breaks primary_xts_b $<-x t s($ primary_df_b $[,-1]$ , as.POSIXct (primary_df_b $\$$ created_at SZ" , format $=" \%$ Y $-\% m-\% d T / \%: \% M: \%$

$$
, \mathrm{tz}=\text { "GMT") ) }
$$

primary_means_b $<-$ period.apply (primary_xts_b, endpoints ( primary_xts_b, "mins", $k=$ time_step), mean) primary_rounded_b $<-$ align.time.down(primary_means_b, time_ step $* 60)$ primary_df_b $<-$ fortify (primary_rounded_b) names $($ primary_df_b $)[$ names $($ primary_df_b $)==$ "Index"] $<-"$ created_at" at ) primary_df_b $\$ c r e a t e d_{-} a t<-$ as. character $\left(\right.$ primary_df_b $\$ c r e a t e d_{-}$

\# add sensor label

primary_df_b\$sensor $<-$ "B"

\# read in secondary data for B sensor

secondary_df_b $<-$ secondary_request_b $\$$ feeds 
\# assign B field names for secondary sensor

\# colnames (secondary_df) $<-$ secondary_fields_b

\# assign B field names dynamically names (secondary_df_b) [names(secondary_df_b) == 'field1'] $<-$ secondary_fields_b[3] names (secondary_df_b) [names(secondary_df_b) $=={ }^{\prime}$ field $\left.2^{\prime}\right]<-$ secondary_fields_b[4] names ( secondary_df_b) [names (secondary_df_b) $==$ 'field3 '] $<-$ secondary_fields_b[5] names ( secondary_df_b) [names (secondary_df_b) == 'field4'] $<-$ secondary_fields_b[6] names (secondary_df_b) [names (secondary_df_b) $=={ }^{\prime}$ field5'] $<-$ secondary_fields_b[7]

names (secondary_df_b) [names (secondary_df_b) $=={ }^{\prime}$ field $\left.6^{\prime}\right]<-$ secondary_fields_b[8]

names (secondary_df_b) [names(secondary_df_b) $=={ }^{\prime}$ field7'] $<-$ secondary_fields_b[9] names (secondary_df_b) [names (secondary_df_b) $=={ }^{\prime}$ field $\left.8{ }^{\prime}\right]<-$ secondary_fields_b[10]

\# remove non-numeric columns before grouping id ) )

secondary_df_b $<-$ secondary_df_b \% $\%$ dplyr: : select $(-c($ entry

\# cast character to numeric class

$\operatorname{try}(\{$

um)

secondary_df_b\$p_0_3_um $<-$ as.numeric $($ secondary_df_b\$p_0_3_

um) secondary_df_b\$p_0_5_um $<-$ as.numeric ( secondary_df_b\$p_0_5_

um) secondary_df_b\$p_1_0_um $<-$ as.numeric $\left(\operatorname{secondary} \_d f \_b \$ p \_1 \_0 \_\right.$ um) secondary_df_b\$p_2_5_um $<-$ as.numeric $\left(\operatorname{secondary} \_d f \_b \$ p \_2 \_5 \_\right.$ um) secondary_df_b\$p_5_0_um $<-$ as . numeric ( secondary_df_b\$p_5_0_ secondary_df_b $\$ p \_10 \_0 \_u m<-$ as . numeric $\left(\operatorname{secondary} \_d f \_b \$ p \_10 \_\right.$ 0_um) $0 \_$cf_1) secondary_df_b\$pm1_0_cf_1 $<-$ as.numeric(secondary_df_b\$pm1_ secondary_df_b\$pm10_0_cf_1 $<-$ as.numeric (secondary_df_b\$ pm10_0_cf_1) \} , silent $=$ TRUE) 


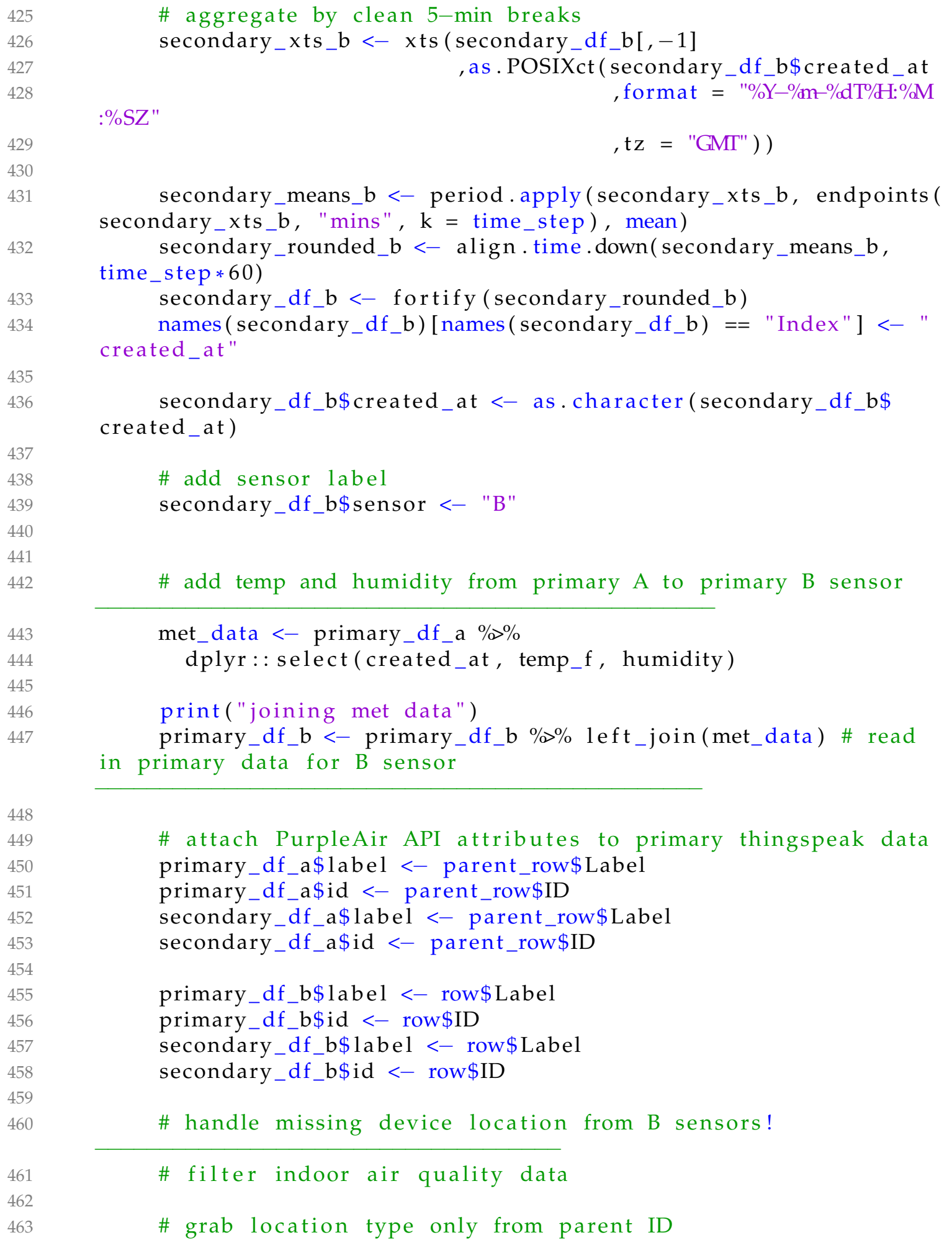




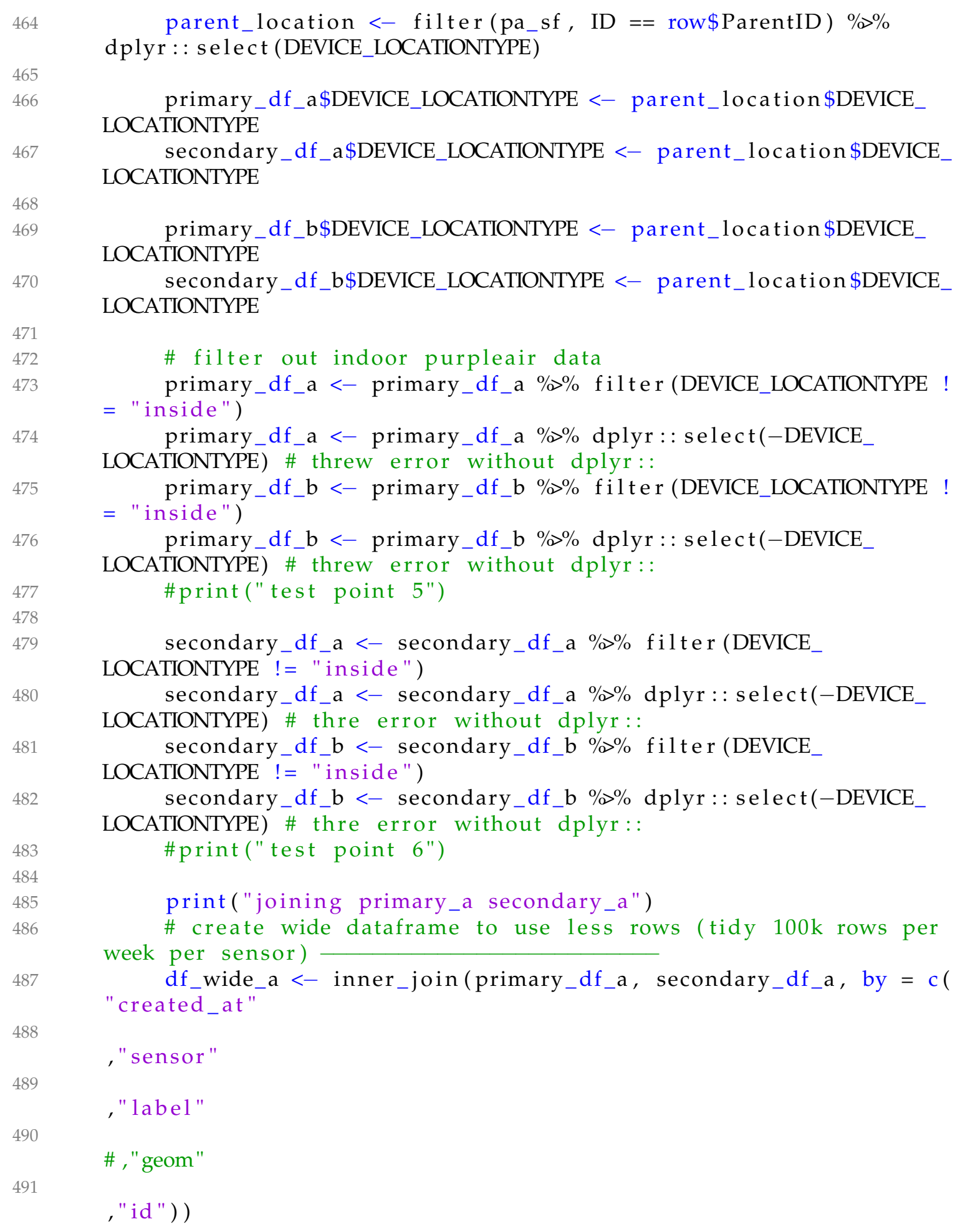

parent_location $<-$ filter $\left(\mathrm{pa} \_\right.$sf , ID $==$row $\$$ ParentID) $\% \%$ dplyr : : select (DEVICE_LOCATIONTYPE)

\section{primary_df_a\$DEVICE_LOCATIONTYPE <- parent_location \$DEVICE_} LOCATIONTYPE

LOCATIONTYPE 


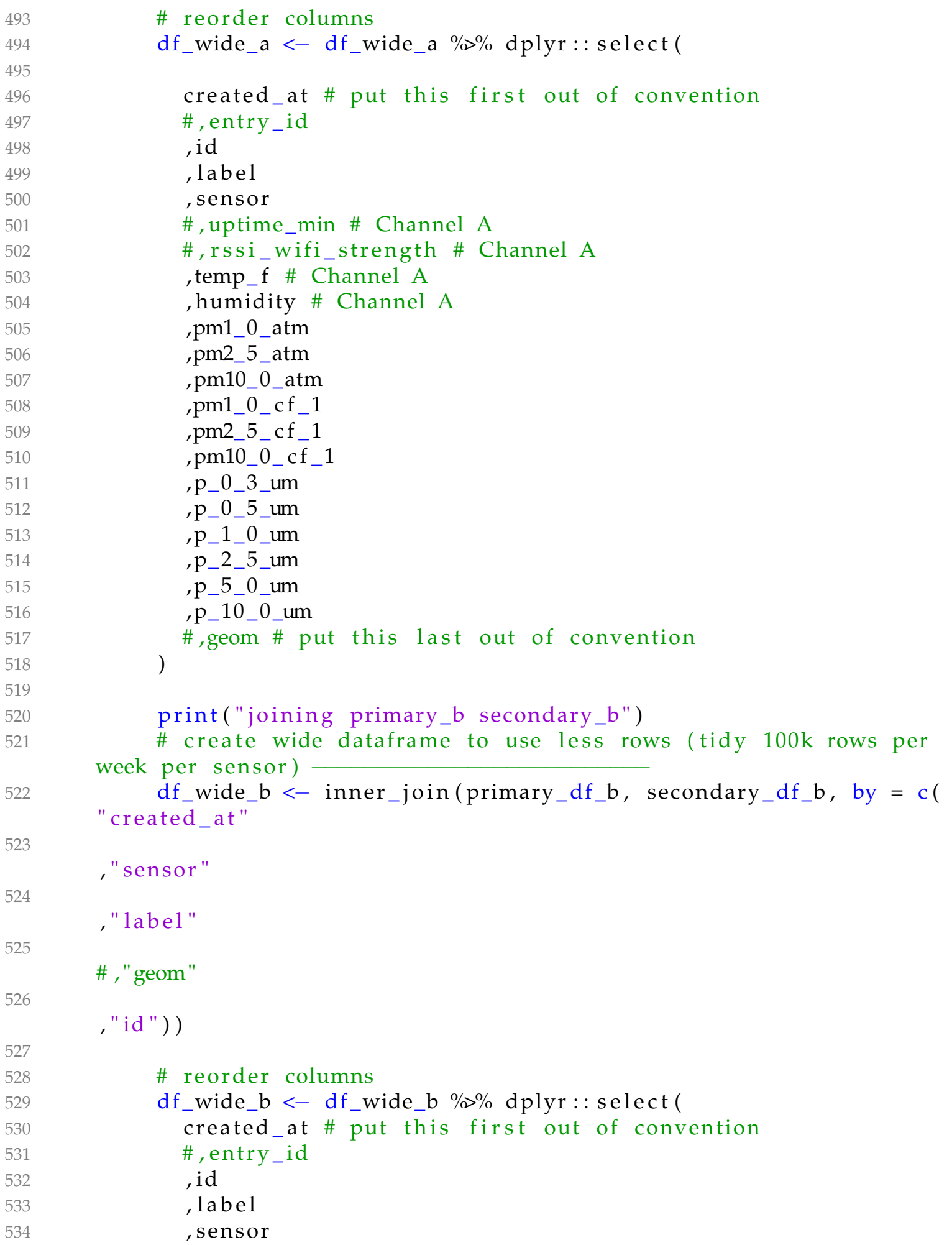




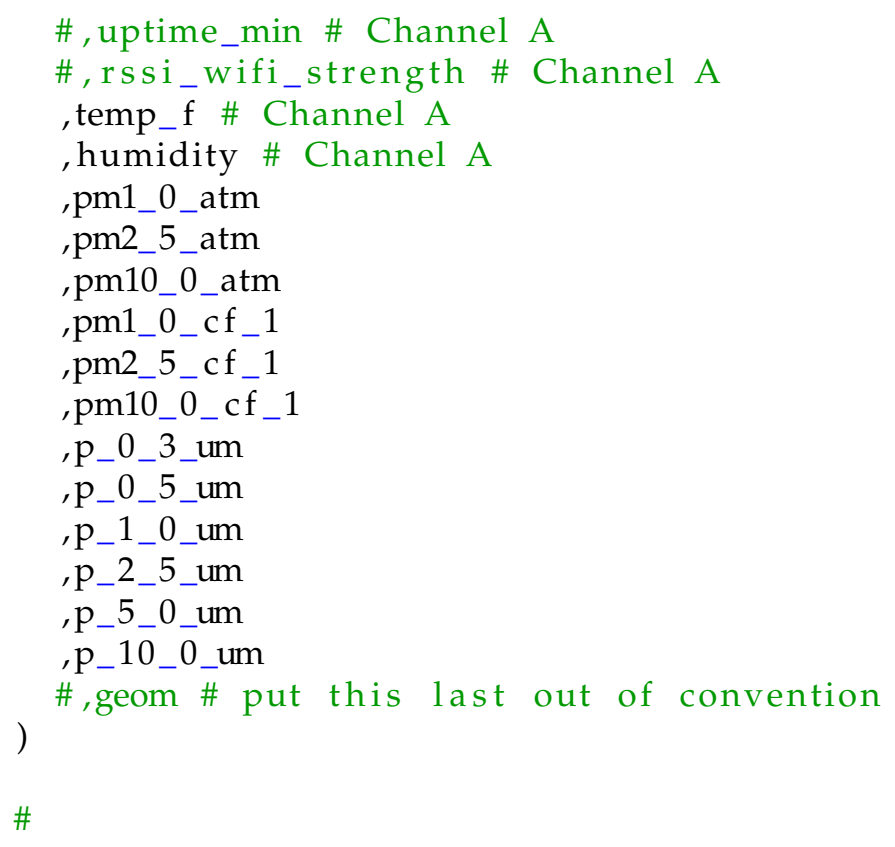

\# Join Primary and Secondary data and omit NAs df_wide $<-$ bind_rows ( df_wide_a, df_wide_b) 


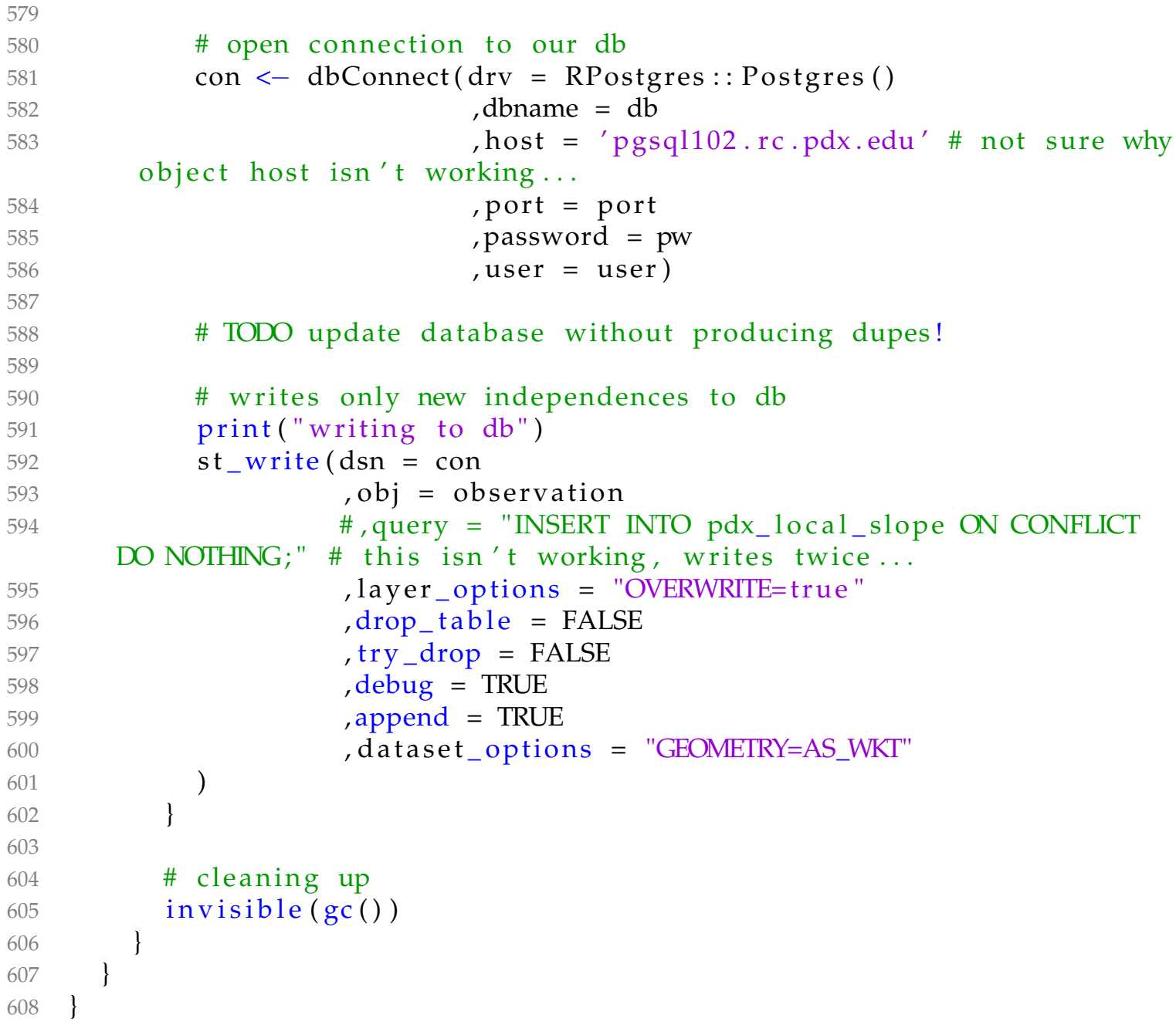

\section{Source/uploadDailyThingspeak.R}

\# created by Philip Orlando @ Sustainable Atmopsheres Research Lab

\# Canopy Continuum Project, USFS

\# PI Dr. Linda George

\# 2018-07-16

\# pull PurpleAir data from thingspeak API and upload to Postgres database

\# set up environment

\# load the necessary packages

if (! require (pacman)) \{

install.packages ("pacman") 


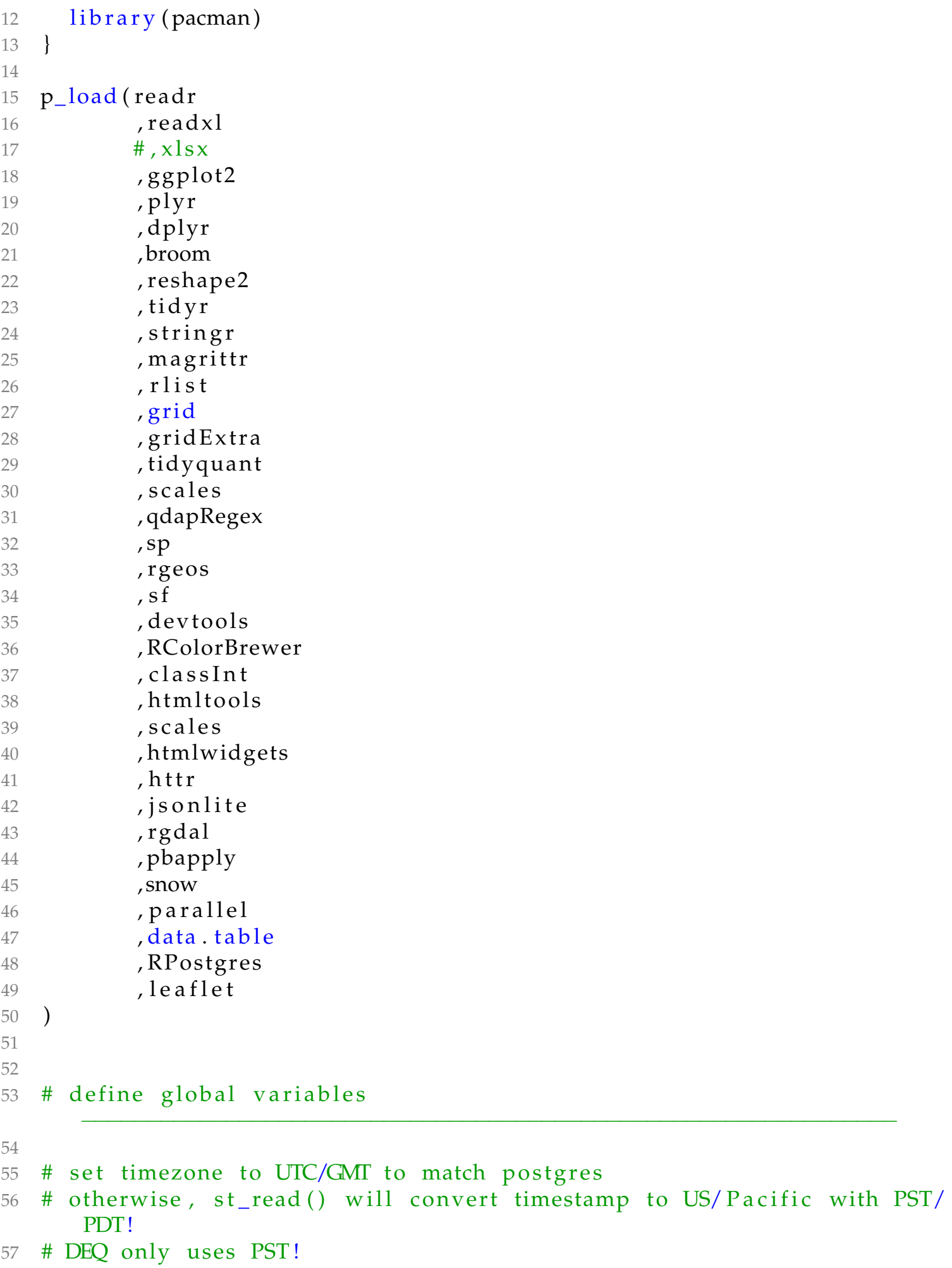




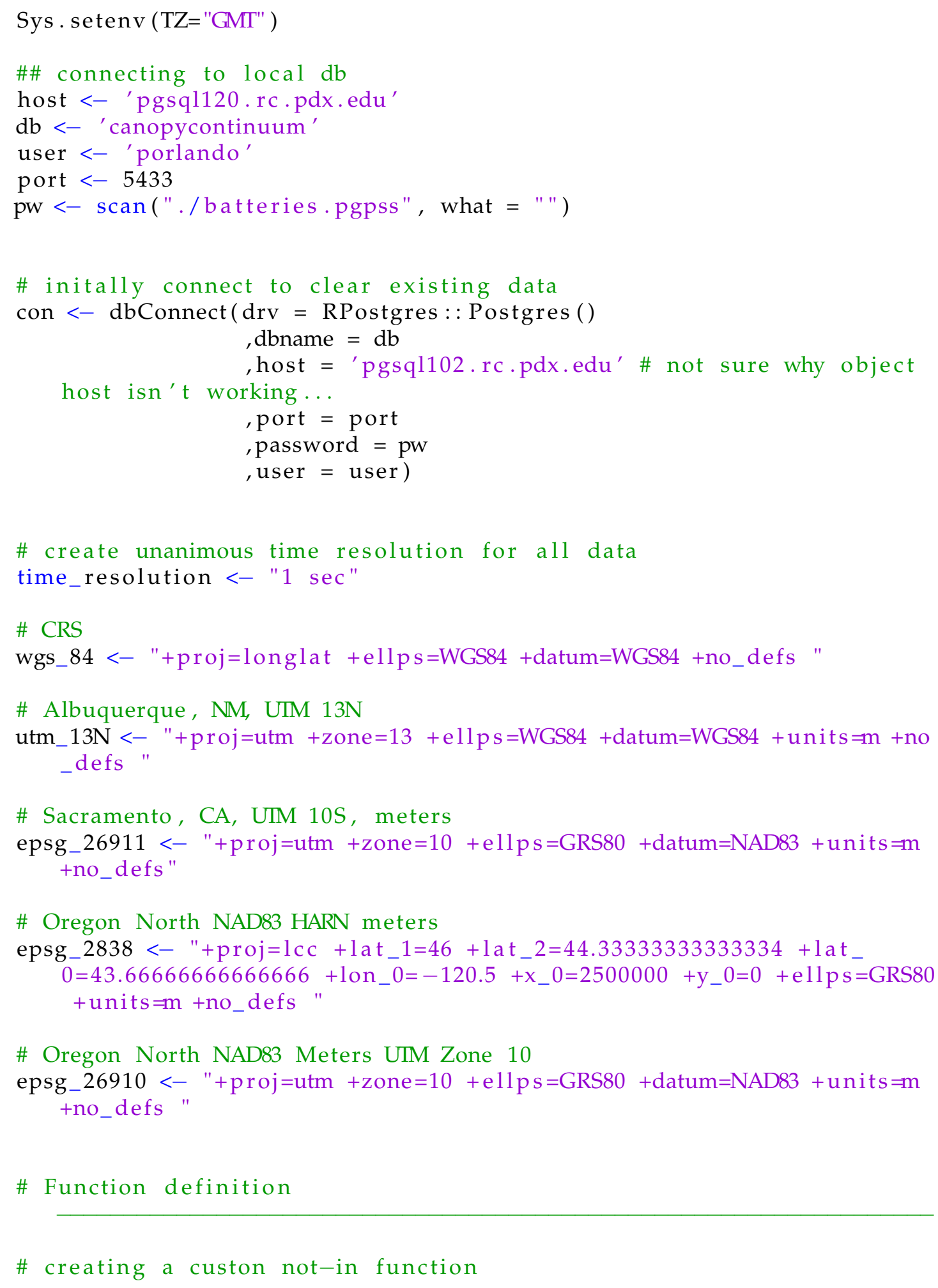




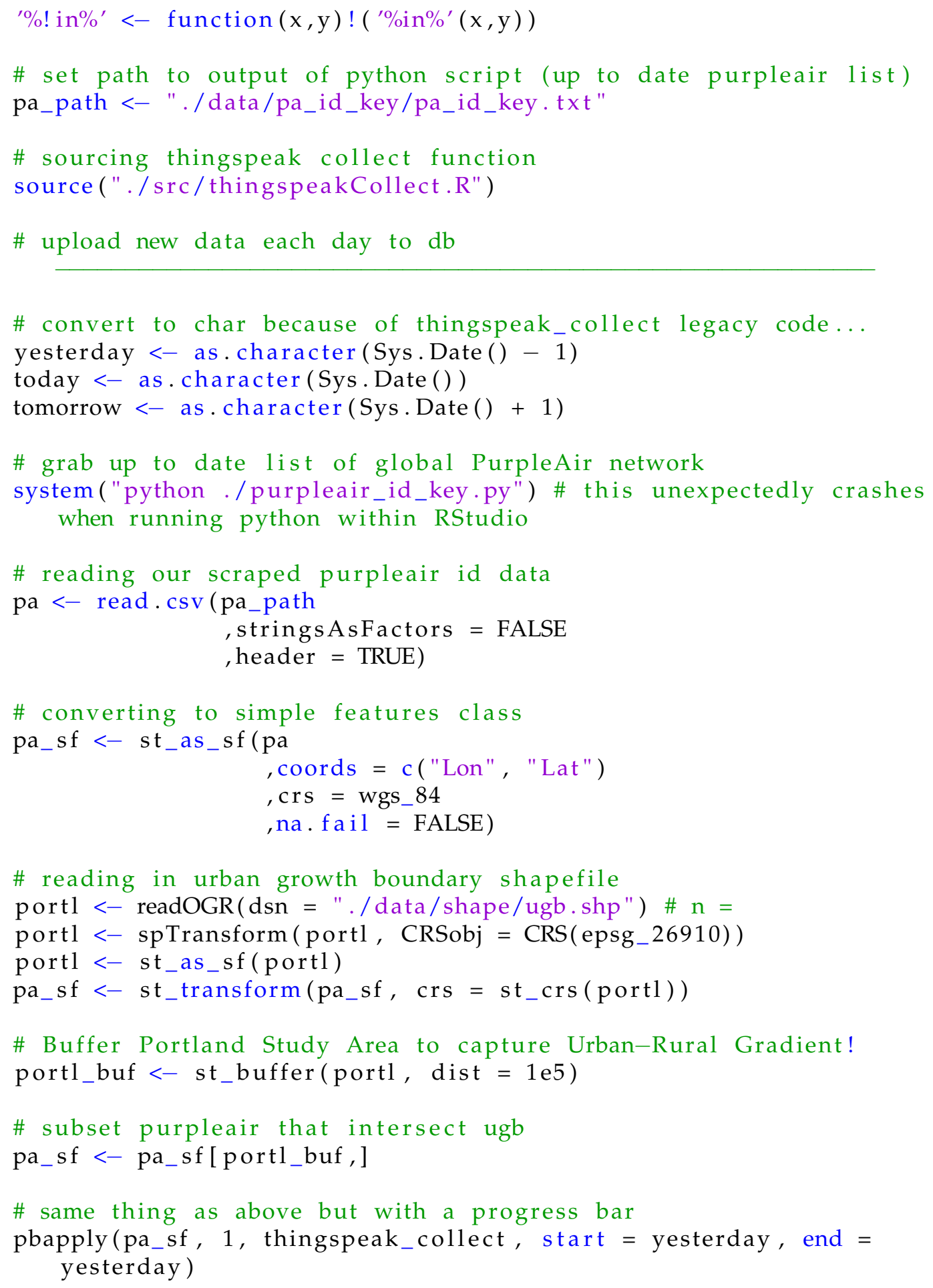

Source/uploadHistoricalThingspeak.R 


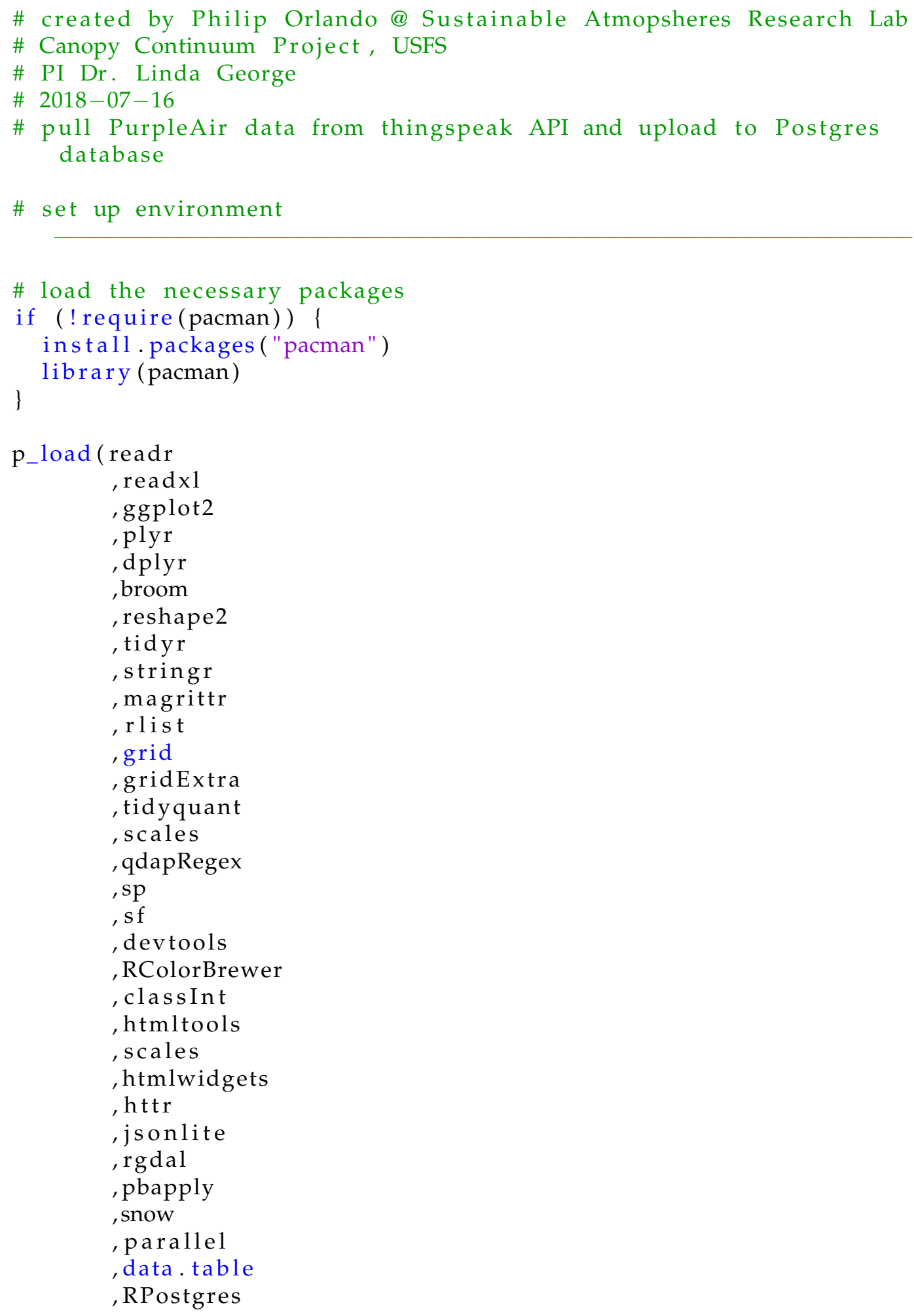




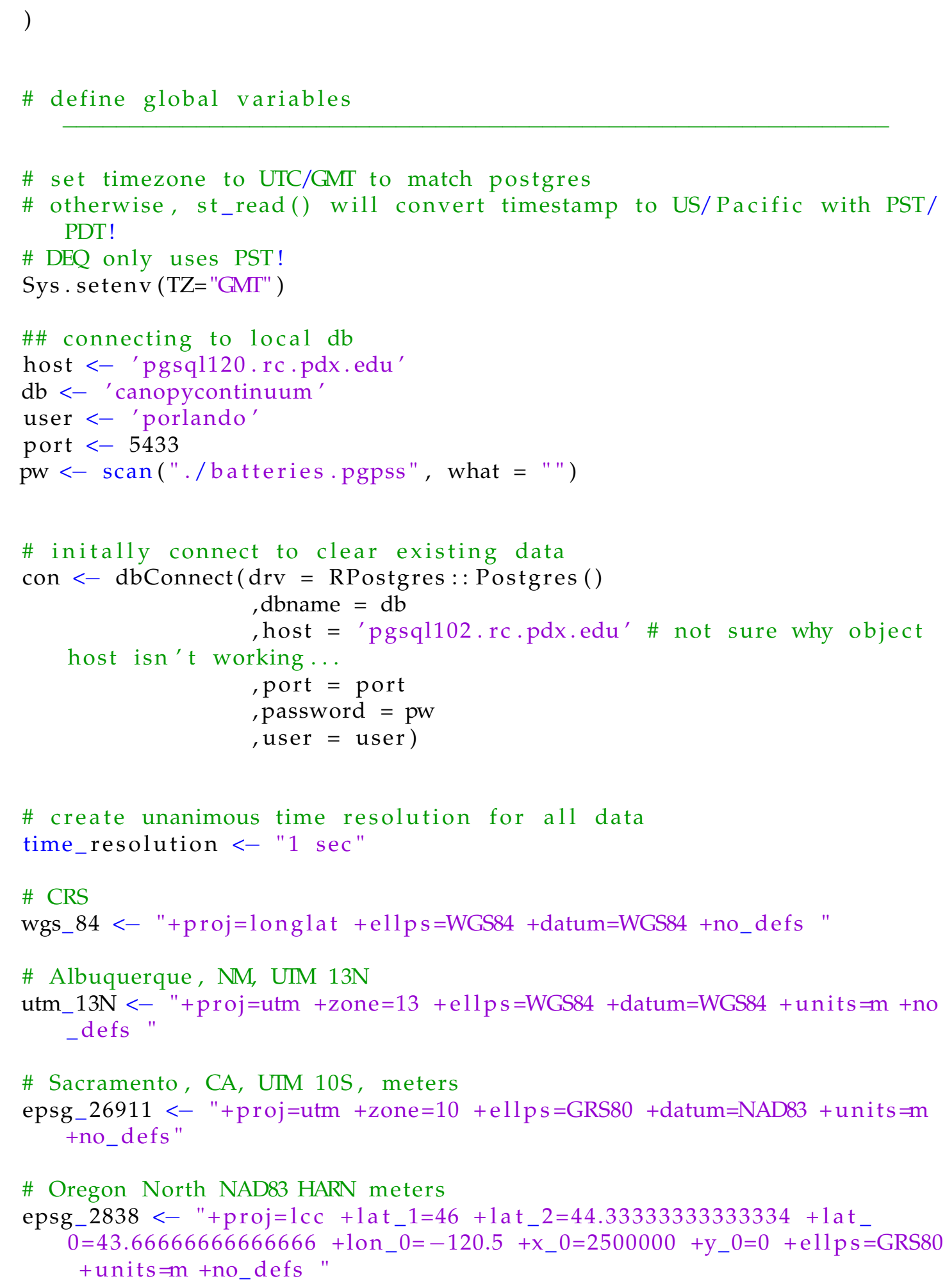


\# Oregon North NAD83 Meters UTM Zone 10

epsg_26910<- "+proj=utm +zone=10 +ellps=GRS80 +datum=NAD83 +units=m +no_defs "

\# Function definition

\# creating a custon not-in function

'\%! in\%' <- function $(x, y) !\left({ }^{\prime} \%\right.$ in $\%$ ' $\left.(x, y)\right)$

\# set path to output of python script (up to date purpleair list)

pa_path $<-"$./data/pa_id_key/pa_id_key.txt"

\# sourcing thingspeak collect function

source ("./src/thingspeakCollect.R")

\# loop begin

\# upload new data each day to db

\# grab up to date list of global PurpleAir network

system("python ./purpleair_id_key.py") \# this unexpectedly crashes when running python within RStudio

\# reading our scraped purpleair id data

pa $<-$ read.csv (pa_path , stringsAsFactors = FALSE , header = TRUE)

\# converting to simple features class

pa_sf $<-$ st_as_sf $(p a$

, coords $=c($ "Lon", "Lat")

, $\mathrm{crs}=$ wgs_84

, na.fail = FALSE)

\# reading in urban growth boundary shapefile

portl $<-$ readOGR $($ dsn $="$./data/shape/ugb.shp" $) \# n=$

portl <- spTransform $\left(\right.$ portl, CRSobj = CRS $\left.(\text { epsg_26910 })^{2}\right)$

portl $<-$ st_as_sf $($ portl $)$

pa_sf $<-$ st_transform (pa_sf, crs $=s_{-} t_{-}$crs $($portl $\left.)\right)$

\# Buffer Portland Study Area to capture Urban-Rural Gradient!

portl_buf $<-$ st_buffer $($ portl, dist $=1$ e 5$)$

\# subset purpleair that intersect ugb

pa_sf $<-$ pa_sf [portl_buf, ] 


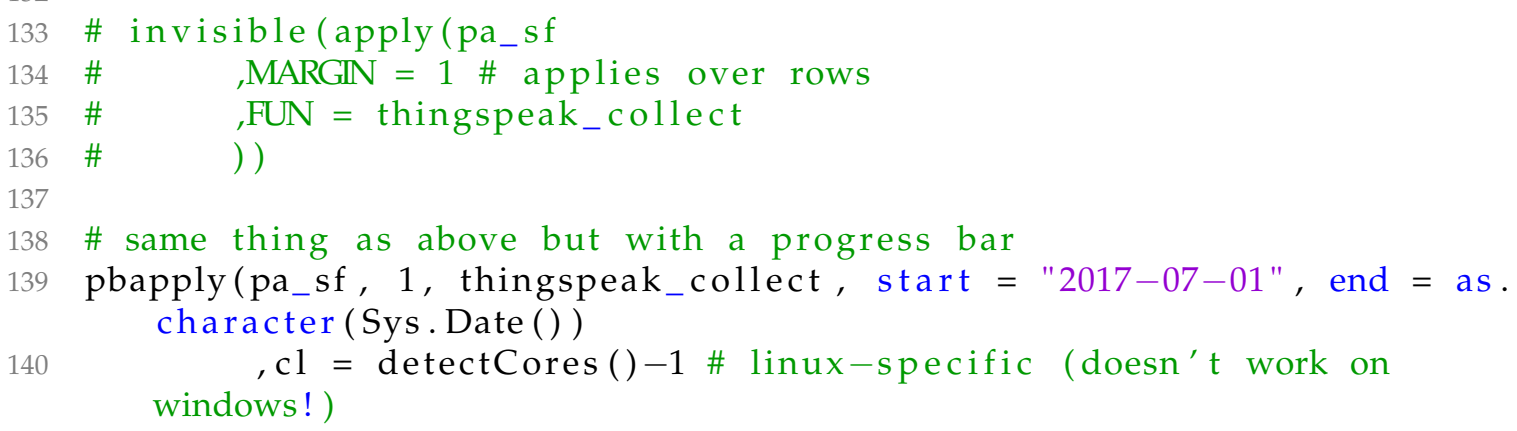

Source/purpleair_watchdog.py 


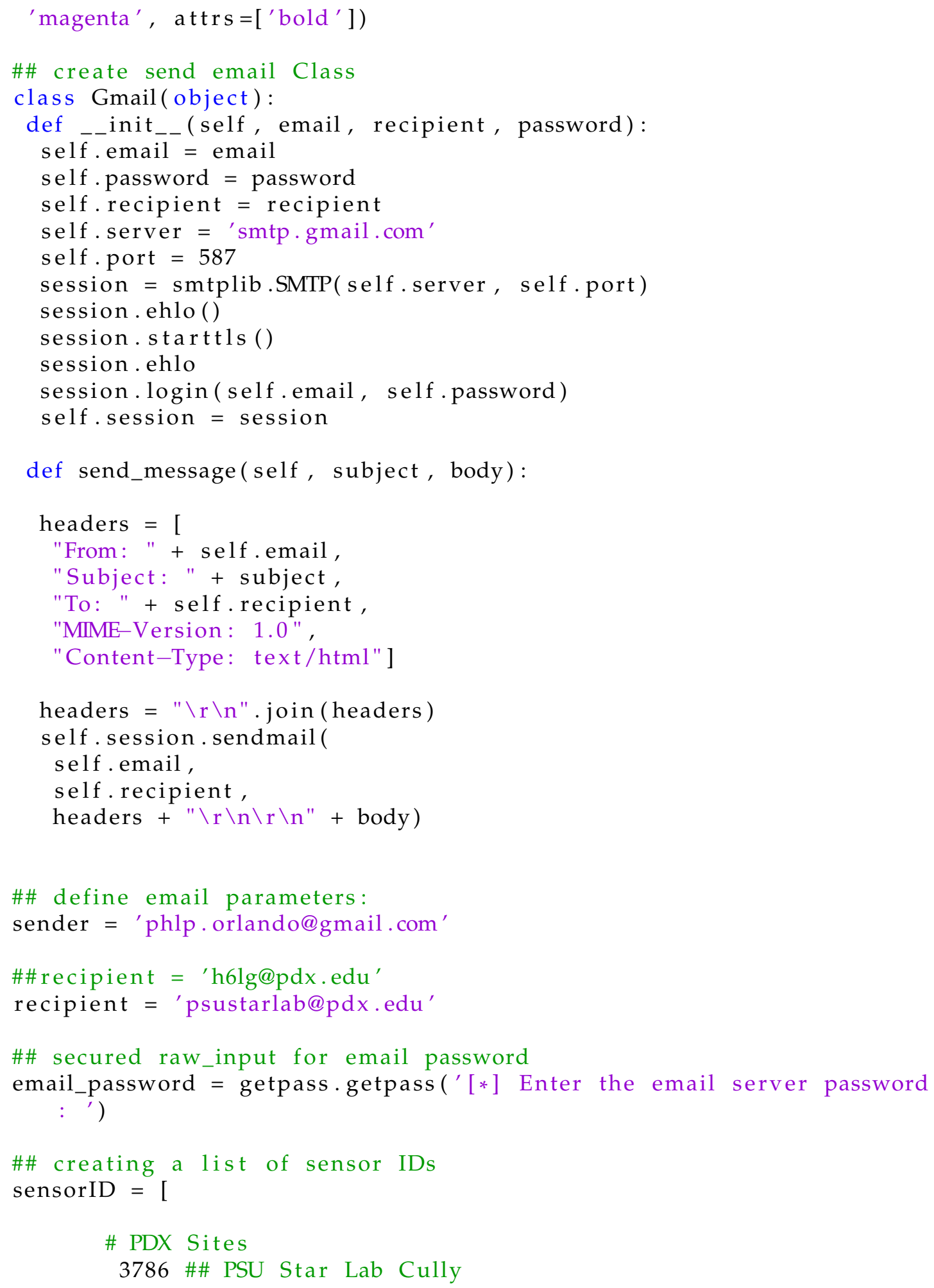




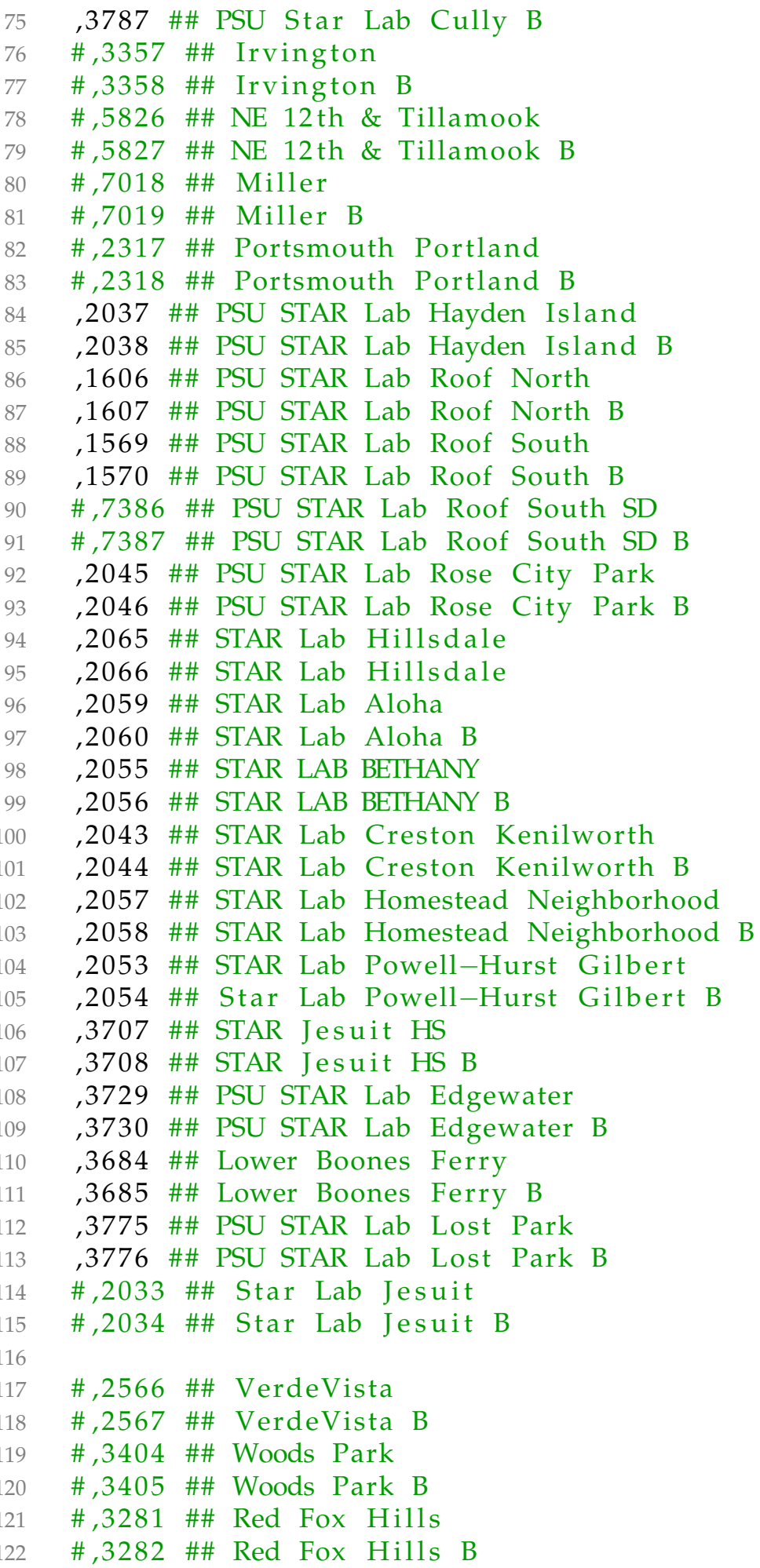




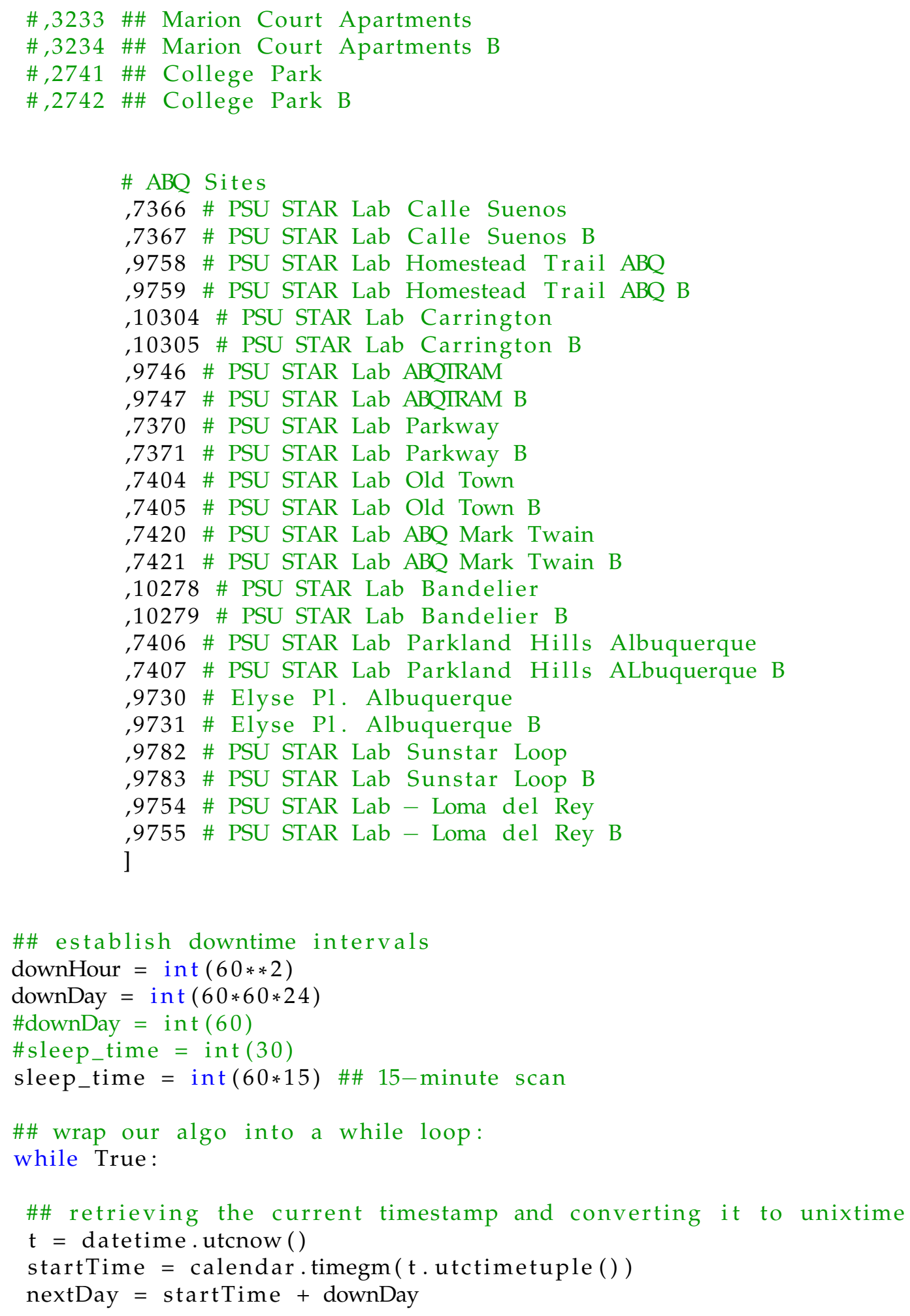




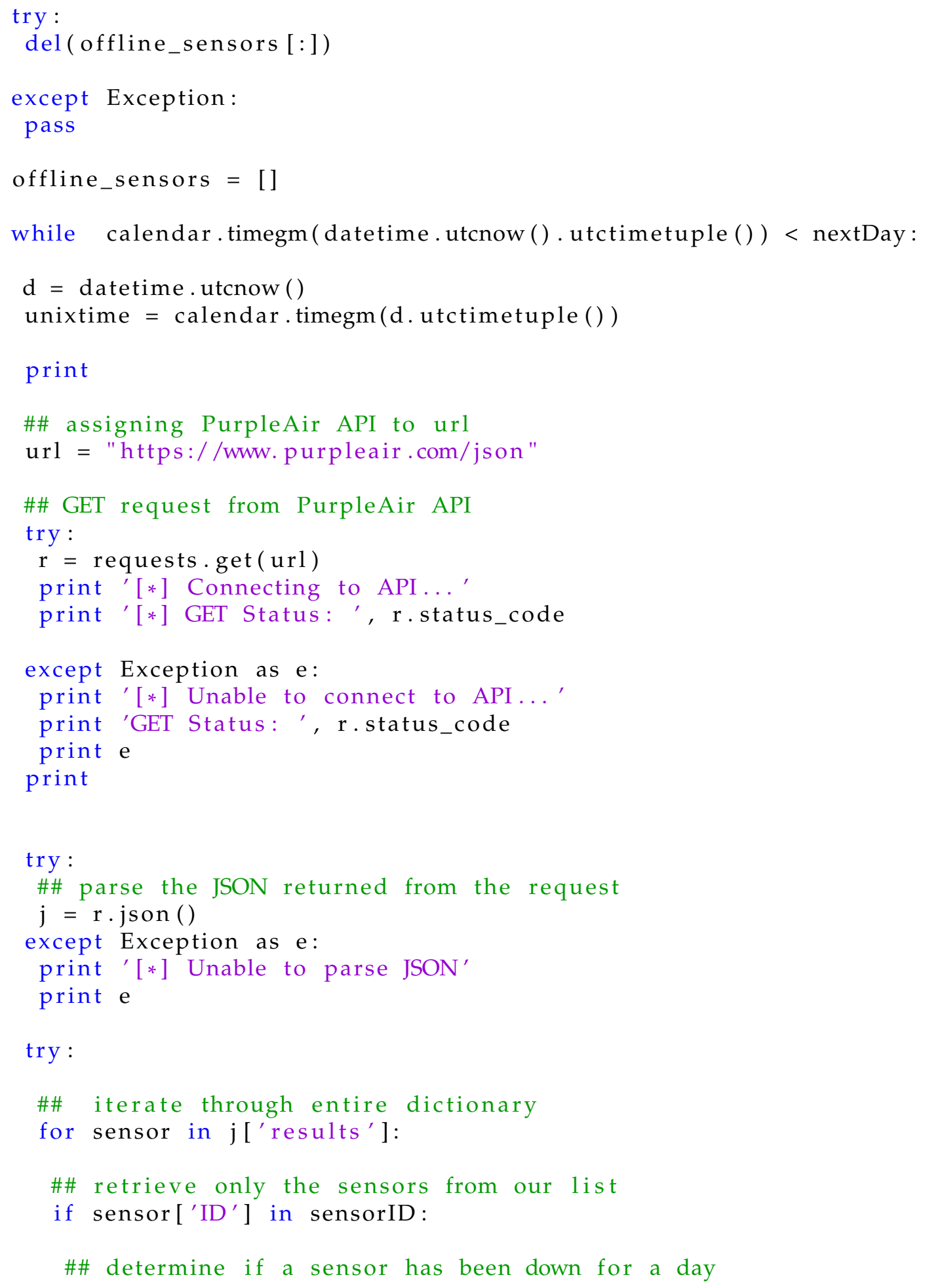


if (unixtime - int (sensor['LastSeen'])) > downHour and sensor ['

ID'] not in [int(y) for $x$ in offline_sensors for $y$ in $x$.split()]: print '[*] Sensor', sensor['Label'], 'went offline at',

datetime . fromtimestamp ( sensor [ 'LastSeen']) down_sensor $=\operatorname{str}($ sensor ['Label'])

down_ID = str $($ sensor [ 'ID'])

offline_sensors.append(down_ID) \#\# this could be better handled with files, avoiding dupe emails when restarting the program more than once per day (if needed due to an exception)

\#\# send email

msg $=$ 'has been down since' + str (datetime. fromtimestamp (

sensor [ 'LastSeen']))

gm = Gmail(sender, recipient, email_password)

gm.send_message(down_sensor, msg)

print '[*] Notification sent.'

elif (unixtime - int (sensor['LastSeen'])) > downHour and sensor [

'ID'] in [int(y) for $x$ in offline_sensors for $y$ in $x$.split()]: print '[*] Sensor', sensor['Label'], 'has been down since', datetime . fromtimestamp (sensor ['LastSeen']) print ' $[*]$ Notification has already been sent to', recipient

else :

print '[*]', $\operatorname{str}($ sensor ['Label']) + ':', sensor ['ID'], lastSeen', datetime.fromtimestamp(sensor['LastSeen'])

try:

offline_sensors.remove(sensor['ID']) \# clear a sensor once it goes back online!

except:

pass

print

print '[*] nextScan', datetime.fromtimestamp (unixtime + sleep_time )

print '[*] startTime', datetime.fromtimestamp (startTime)

print '[*] unixtime', datetime fromtimestamp (unixtime)

print '[*] nextDay', datetime.fromtimestamp (nextDay)

time.sleep (sleep_time)

except Exception as e:

print '[*] Delivery to ', recipient, 'failed!'

print e

print '[*] Will try again in one hour....'

time.sleep (sleep_time)

\# empty our list of offline sensors each day

\# offline_sensors [:] = [] 


\section{Appendix B}

\section{ThingSpeak PurpleAir Field Descriptions}

Channel A and B, primary and secondary ThingSpeak channels together provide 32 fields for each sensor. There are six $\mu \mathrm{gm}^{-3}$ values and six particle counts for each channel (laser) as well as temperature, humidity, WiFi signal (RSSI), sensor uptime, free memory and analog input.

\section{B.1 Channel A}

\section{B.1.1 PrimaryData}

field1: PM1.0 (CF=ATM) ug $/ \mathrm{m} 3$

field2: PM2.5 (CF=ATM) ug $/ \mathrm{m} 3$

field3: PM10.0 (CF=ATM) ug/m3

field4: Uptime (Minutes)

field5: RSSI (WiFi Signal Strength)

field6: Temperature $(\mathrm{F})$

field7: Humidity (\%)

field8: PM2.5 $(\mathrm{CF}=1) \mathrm{ug} / \mathrm{m} 3$ This is the field to use for PM2.5 


\section{B.1.2 SecondaryData}

field1: 0.3um particles/deciliter

field2: 0.5um particles/deciliter

field3: 1.0um particles/deciliter

field4: 2.5um particles/deciliter

field5: 5.0um particles/deciliter

field6: 10.0um particles/deciliter

field7: PM1.0 ( $\mathrm{CF}=1) \mathrm{ug} / \mathrm{m} 3$ This is the field to use for PM1.0

field8: PM10 $(\mathrm{CF}=1) \mathrm{ug} / \mathrm{m} 3$ This is the field to use for PM10

\section{B.2 Channel B}

\section{B.2.1 PrimaryData}

field1: PM1.0 (CF=ATM) ug/m3

field2: PM2.5 (CF=ATM) ug $/ \mathrm{m} 3$

field3: PM10.0 (CF=ATM) ug/m3

field4: Free HEAP memory

field5: ADC0 (analog input) voltage

field6: SENSOR FIRMWARE 2.5 and up: Atmospheric Pressure

field7: (NOT USED)

field8: PM2.5 ( $\mathrm{CF}=1) \mathrm{ug} / \mathrm{m} 3$ This is the field to use for PM2.5 


\section{B.2.2 SecondaryData}

field1: 0.3um particles/deciliter

field2: 0.5um particles/deciliter

field3: 1.0um particles/deciliter

field4: 2.5um particles/deciliter

field5: 5.0um particles/deciliter

field6: 10.0um particles/deciliter

field7: PM1.0 ( $\mathrm{CF}=1) \mathrm{ug} / \mathrm{m} 3$ This is the field to use for PM1.0

field8: PM10 $(\mathrm{CF}=1) \mathrm{ug} / \mathrm{m} 3$ This is the field to use for PM10 